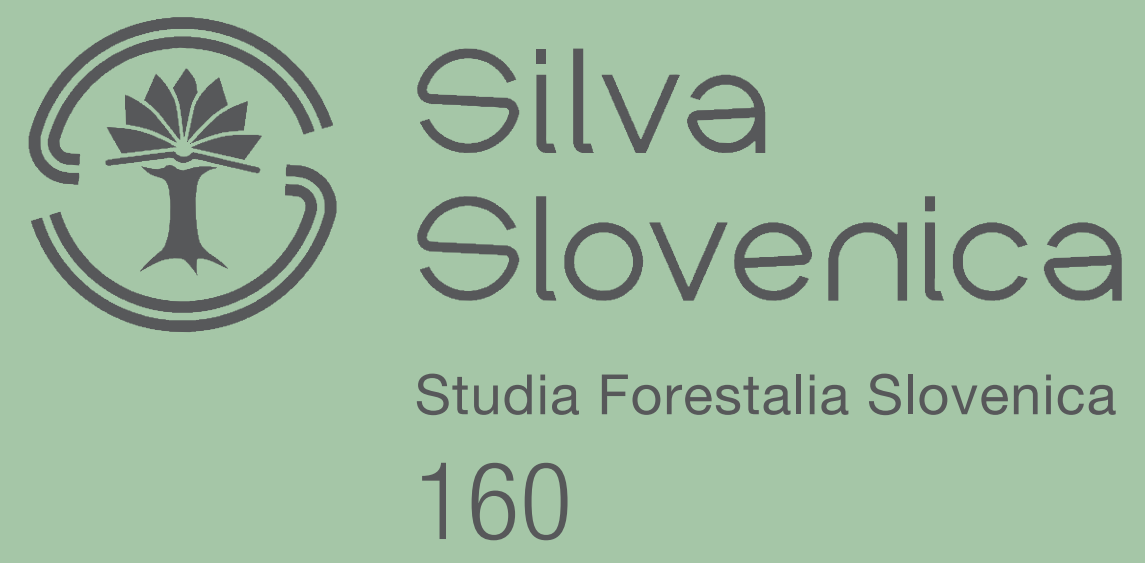

\title{
TECHNICAL GUIDELINES FOR MOLECULAR GENETIC ANALYSIS IN NON-NATIVE FOREST TREE SPECIES OF EUROPE
}

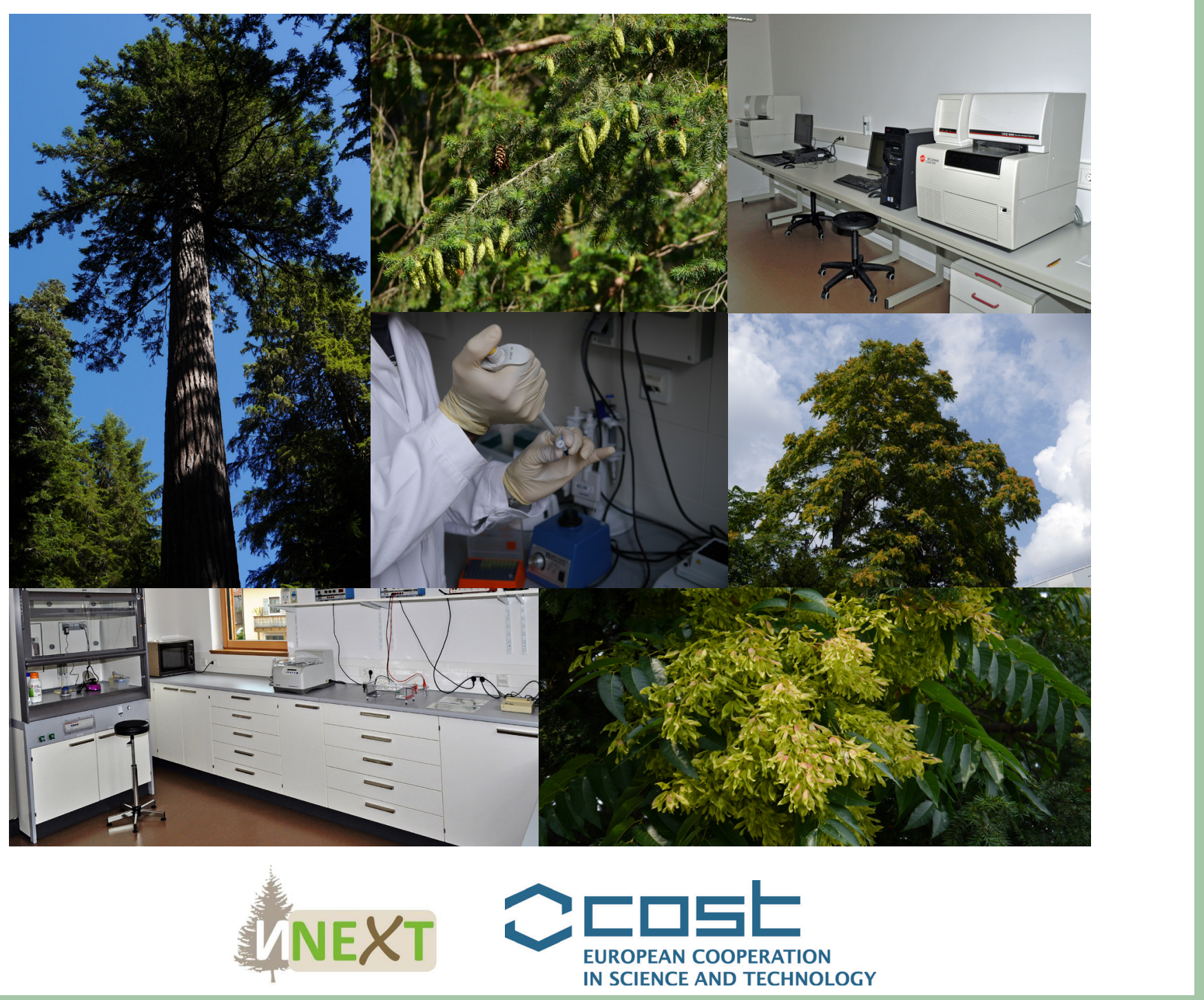


CIP - Kataložni zapis o publikaciji

Narodna in univerzitetna knjižnica, Ljubljana

$577.21: 582.4$

TECHNICAL guidelines for molecular genetic analysis in non-native forest tree species of Europe / [chapters edited by Charalambos Neophytou and Monika Konnert]. - 1st ed. - Ljubljana : Slovenian Forestry Institute, Silva Slovenica Publishing Centre, 2018. - (Studia Forestalia Slovenica, ISSN 0353-6025; 160)

ISBN 978-961-6993-43-2

1. Neophytou, Charalambos 296199168 


\section{Silva}

\section{Slovenica}

Studia Forestalia Slovenica, 160

ISSN 0353-6025

ISBN 978-961-6993-43-2

Publisher: Slovenian Forestry Institute,

Silva Slovenica publishing centre, Ljubljana 2018

Title: Technical Guidelines for Molecular Genetic Analysis in Non-Native Forest

Tree Species of Europe

Chapters edited by: Dr. Charalambos

Neophytou and Dr. Monika Konnert

Technical editor: Dr. Peter Železnik

Front page photos: Dr. Charalambos Neophytou, Dr. Monika Konnert, Dr.

Mathias Neumann

Edition: $1^{\text {st }}$ edition

Circulation: 200 copies

Price: free

Electronic download:

DOI 10.20315/SFS. 160

Acknowledgement:

The work has been carried out within the COST Action FP1403 Nonnative tree species for European forests experiences, risks and opportunities (NNEXT), Working group "Pathways".

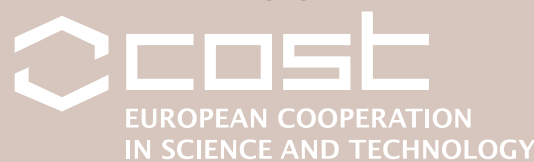

Disclaimer:

The short reviews were not subjected to external peer review. The responsibility lies solely with the authors.
2 Editorial

3 Molecular markers used for genetic studies 3 in Grand fir (Abies grandis (Douglas ex D.Don) Lindl.)

Eva Cremer, Monika Konnert

9 Molecular markers used for genetic studies in Cedrus spp.

Monika Konnert, Muhidin Šeho

19 Molecular markers used for genetic studies in Japanese Larch (Larix kaempferi (Lamb.) Carr.)

Jean-Charles Bastien, Vanina Guerin, Anna-Maria Szasz-Len, Monika Konnert

38 Molecular markers used for genetic studies in Lodgepole pine (Pinus contorta (Dougl. ex. Loud.))

Marjana Westergren

48 Molecular markers used for genetic studies in Eastern white pine (Pinus strobus L.) Paraskevi Alizoti

69) Molecular markers used for genetic studies in Douglas-fir (Pseudotsuga menziesii (Mirb.) Franco)

Monika Konnert, Anna-Maria Szasz-Len, Marcela van Loo

95 Molecular markers used for genetic studies in Sitka spruce (Picea sitchensis (Bong.) Carr.)

Branislav Cvjetković, Jason Holliday, Monika Konnert, Anna-Maria Szasz-Len

114 Molecular markers used for genetic studies in tree of heaven (Ailanthus altissima (Mill.) Swingle)

Marcela van Loo, Vlatko Andonovski

119 Molecular markers used for genetic studies in Northern red oak (Quercus rubra L.)

Charalambos Neophytou, Branislav Cvjetković

137 Molecular markers used for genetic studies in black locust (Robinia psendoacacia L.) Charalambos Neophytou, Marcela van Loo Marcin Klisz 


\section{Editorial}

The last two centuries were marked by an increased use of non-native tree species in European forestry. For much of this time, the question of seed origin and genetic variability had received little attention. In many, if not in most cases, it is unknown where forest stands of introduced species come from. Practical experience and research in the field of forest genetics have shown that both provenance (the geographic origin) and genetic diversity are of utmost importance for the survival and growth performance of forest trees. Knowledge about these factors is even more crucial for introduced species, given the lack of autochthonous, locally adapted provenances. This is why the NNEXT action puts a special focus on origin identification and genetic variability of non-native forest tree species.

The development and application of molecular markers has opened avenues for studying population genetic variation in forest trees. Advances in this field have been rapid and the availability of tools for answering various questions has been increasing steadily. Nowadays, markers from the nuclear and organelle genomes of forest trees enable us, for instance, to assess their genetic diversity and to trace back the origin of forest stands and forest reproductive material. Especially the latter is of particular importance for non-native species.

With the "Technical Guidelines for Molecular Genetic Analysis in Non-Native Forest Tree Species of Europe", we aim to provide a comprehensive collection of available molecular markers for Abies grandis, Cedrus spp., Larix spp., Picea sitchensis, Pinus strobus, Pinus contorta, Pseudotsuga menziesii, Ailanthus altissima, Quercus rubra and Robinia pseudoacacia, as well as technical details for their use in a molecular genetics laboratory. Extensive literature about all analysis steps and the most important results of case studies from across the world, in summary, complete the picture. While we do not claim completeness, we intend to supply forest geneticists with a basic manual for their work with non-native tree species and to facilitate their search of suitable molecular markers for this purpose.

The „Technical Guidelines“ were compiled by members of the Working Group 2-'Pathways' of the Cost Action FP 1403 NNEXT (Non-Native Tree Species for European Forests: EXperiences, Risks and OpporTunities).

May 30th, 2018

Charalambos Neophytou, Monika Konnert 


\title{
Molecular markers used for genetic studies in Grand fir (Abies grandis (Douglas ex D.Don) Lindl.)
}

\author{
Eva Cremer and Monika Konnert
}

Bavarian Office for Forest Seeding and Planting, Forstamtsplatz 1, 83317 Teisendorf, Germany

\section{General remarks}

Among the true firs of the western United States, Grand fir is the species that is able to grow under the most diverse site conditions. Grand fir has a split distribution along the Pacific Coast from Southern British Columbia (Canada) to Northern California. In the continental interior it occurs from the Okanagan and Kootenay lakes region of British Columbia in the north to eastern Oregon, central Idaho, and western Montana in the south. Grand fir can hybridize with white fir (Abies concolor). A broad zone of hybridogenous grand $x$ white fir populations occurs from northeastern Washington and Oregon southwards to northern California and eastwards to west-central Idaho (Steinhoff 1978). However, there seems to be a reproductive isolation between Abies grandis and the Mediterranean Abies species, minimizing the danger of hybridization among Abies grandis and the local Abies species when introduced to Europe (Kormutak 2004). There are no recognized varieties of grand fir, although a green coastal form and gray interior form are often assumed (Figure 1).

Grand fir grows frequently in mixed forests of coniferous and hardwood species, but also occurs in pure stands. Depending on the region, other fir species as Abies lasciocarpa, Abies amabilis, Abies magnifica and Abies procera grow together with Abies grandis in mixed stands (Howard and Aleksoff 2000, Marvin et al. 2003).

While other fir species, as for example Abies alba and Abies procera, have been extensively studied with various genetic markers, there are very few genetic studies for Abies grandis. It is, however, assumed that genetic markers, which have been successfully used in other fir species, can also be used for Abies grandis. For example, Postolache et al. (2014) have tested the transferability of Abies alba developed transcriptome-derived expressed sequence tags (EST)SSR-markers to 17 congeneric taxa including one sample of Abies grandis. Thirteen of these markers worked also in grand fir (see also chap. 4.a). Moreover, seven nSSRmarkers developed for Abies fraseri could successfully be transferred to Abies grandis, but they have not yet been used for population genetic studies (Josserand et al. 2006). However, continuing test runs and case-by-case modifications of the methodology are necessary to obtain an optimized DNA-marker-set for the grand fir.

Since there are hardly any publications on genetic investigations for Abies grandis, selected references on genetic studies carried out in other fir species are cited in the current technical guidelines. The citations mentioned above provide information on the analytical methodology used.

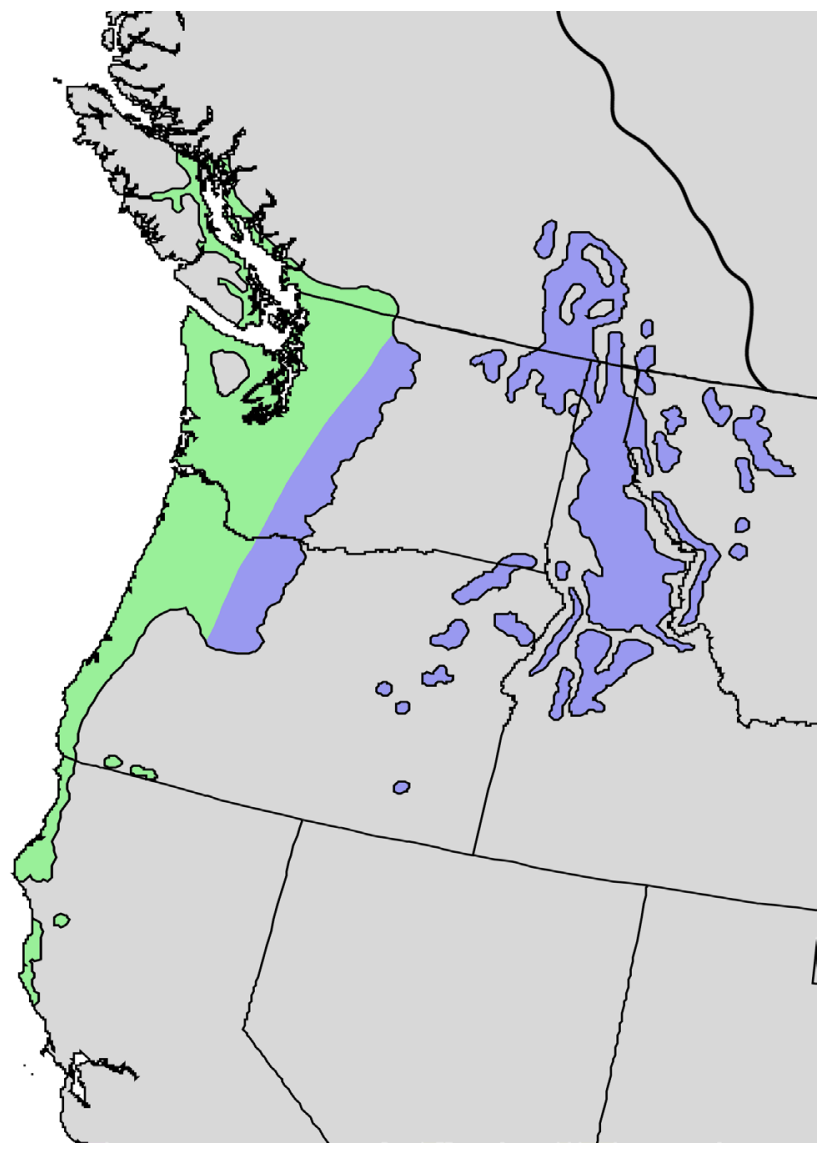

Figure 1. Native distribution range of Grand fir. The coastal form denoted with green and the interior form denoted with blue (source: USGS, USA). 


\section{Isozymes}

Only one publication (Konnert and Ruetz 1997) dealing with isozyme analysis for Abies grandis is to our knowledge up to now. Konnert and Ruetz (1997) used isozyme analysis to investigate the genetic structure of eleven Abies grandis provenances tested in a provenance trial in Southern Germany.

The list of enzymes, number of loci and number of obtained alleles are presented in Table 1.

Table 1: List of enzymes, scored loci and number of alleles reported by Konnert and Ruetz (1997)

\begin{tabular}{|l|c|c|c|}
\hline \multicolumn{1}{|c|}{ Enzyme system } & $\begin{array}{c}\text { E.C. } \\
\text { Number }\end{array}$ & Scored loci & $\begin{array}{c}\text { No. of } \\
\text { alleles }\end{array}$ \\
\hline $\begin{array}{l}\text { Isocitrate } \\
\text { dehydrogenase }\end{array}$ & 1.1 .1 .42 & IDH-A,-B & 1,4 \\
\hline $\begin{array}{l}\text { Glutamate oxaloacetate } \\
\text { transaminase }\end{array}$ & 2.6 .1 .1 & GOT-A,-B,-C & $3,1,3$ \\
\hline $\begin{array}{l}\text { Leucine } \\
\text { aminopeptidase }\end{array}$ & 3.4 .11 .1 & LAP-A,-B & 3,3 \\
\hline $\begin{array}{l}\text { Phosphoglucose } \\
\text { isomerase }\end{array}$ & 5.3 .1 .9 & PGI-A,-B & 1,2 \\
\hline Phosphoglucomutase & 2.7 .5 .1 & PGM-A & 3 \\
\hline Diaphorase & 1.6 .4 .3 & DIA-A & 2 \\
\hline
\end{tabular}

\section{Material for protein extraction}

Proteins were extracted from dormant buds or seed endosperm (Konnert and Ruetz 1997).

\section{Protein extraction and separation protocols}

The extraction procedure and separation protocols are described in Konnert and Ruetz (1997).

\section{Important results}

- Clear differences in the genetic structure between provenances were found at the polymorphic gene loci Idh-B and Pgm-A.

- Considering allgene loci, a clear regional differentiation between the provenances from Washington/British Columbia and western Oregon was observed.

- The northern provenances had higher genetic diversity and heterozygosity.

- The provenance Post $(1450 \mathrm{~m})$ from central Oregon (Interior) had by far the highest genetic variability and a very specific genetic structure, and could not be assigned to any of the two regional groups.

\section{Organelle markers (chloroplast (cp)DNA, mitochondrial (mt)DNA)}

Different studies have used so far organelle DNA-

Table 2: PCR-RFLP- markers (cpDNA) for Grand fir

\begin{tabular}{|c|c|c|c|}
\hline Locus & $\begin{array}{c}\text { Primer sequence } \\
(\mathrm{F}=\text { forward, } \mathrm{R}=\text { reverse })\end{array}$ & $\begin{array}{c}\text { Restriction } \\
\text { Enzymes }\end{array}$ & References \\
\hline rbcL1-rbcL2 & $\begin{array}{l}\text { F: TGTCACCAAAAACAGAGACT } \\
\text { R: TTCCATACTTCACAAGCAGC }\end{array}$ & $\begin{array}{c}\text { Hind III, } \\
\text { VSP I, } \\
\text { Dra I }\end{array}$ & 1,2 \\
\hline $\operatorname{trnS}$-psbC & $\begin{array}{l}\text { F: GTTTGGAATCCCTCTCTCTC } \\
\text { R: GGTCGTGACCAAGAAACCAC }\end{array}$ & $\begin{array}{c}\text { Hae III, } \\
\text { Taq I, } \\
\text { Tru 1I } \\
\end{array}$ & 2,3 \\
\hline $\operatorname{trn} L-\operatorname{trn} V$ & $\begin{array}{l}\text { F: CTGCTTCCTAAGAGCAGCGT } \\
\text { R: TTGACATGGTGGAAGTCATCA }\end{array}$ & Msp I, & 2,4 \\
\hline $\operatorname{trnK}$ & $\begin{array}{l}\text { F: AACCCGGAACTAGTCGGATG } \\
\text { R: TCAATGGTAGAGTACTCGGC }\end{array}$ & $\begin{array}{l}\text { Hpa II, } \\
\text { Hinf I, }\end{array}$ & 2,5 \\
\hline rpoC1 & $\begin{array}{l}\text { F: TCGATTGAAACGAGTACGACC } \\
\text { R: CACTGGAGGGCCAATACCTA }\end{array}$ & $\begin{array}{l}\text { Msp I, } \\
\text { Taq I, }\end{array}$ & 2,6 \\
\hline $\mathrm{rpl} 2$ & $\begin{array}{l}\text { F: AAAGGTCGTAATGCCAGAGGAAT } \\
\text { R: TTCCAAGYGCAGGATAACCCCA }\end{array}$ & Msp I, & 2,7 \\
\hline psbB & $\begin{array}{l}\text { F: ATGGTTTTGCCTTGGTATCGTGTTCATAC } \\
\text { R: CCAAAAGTRAACCAACCCCTTGGAC }\end{array}$ & Msp I & 2,4 \\
\hline psbD & $\begin{array}{l}\text { F: ATGACTGGTTACGRAGGGACCA } \\
\text { R: CATAACCRAAGAAKGGAAAAGAATC }\end{array}$ & Cfr 131 & 2,4 \\
\hline
\end{tabular}


markers in Abies grandis, aiming primarily at species differentiation/identification and clarification of the taxonomy of the different Abies species, e.g. as attendance of interspecific crossing experiments (e.g. Kormutak et al. 2004).

\section{Loci and primers used}

Analysis of Kormutak et al. 2004 (see Table 2)

- Restriction fragment length polymorphism (RFLP)chloroplast marker: rbcL1-rbcL2, trnS-psbC, trnL$\operatorname{trnV}, \operatorname{trnK}, \operatorname{rpoC1}, \operatorname{rpl} 2, \mathrm{psbB}, \mathrm{psbD}$ using the restriction enzymes Msp I, Cfr131, Hinf I, Tag I, Hpa III, Trn II, Hind III, VSP I, Dra I,

Analysis at ASP, Teisendorf (unpublished, see Table 3)

- mitochondrial marker Nad5.4 (Liepelt et al. 2002, Jaramillo-Correa et al. 2013);

- chloroplast simple sequence repeats (SSR)-marker Pt71936, Pt30141, Pt30249 (Vendramin et al. 1996).

\section{Material for DNA-extraction}

In the mentioned studies (Kormutak et al. 2004, ASP unpublished) DNA was extracted from fresh or silicagel dried needles.

\section{DNA-extraction protocols}

For DNA isolation at the ASP Teisendorf the CTABmethod of Dumolin et al. (1995) was used. Kormutak et al. (2014) used a slightly modified protocol of Murray and Thompson (1980) in which only $0.5 \mathrm{~g}$ of needles per individual were used instead of prescribed $10 \mathrm{~g}$ of needle material. This also reduced the amount of extracting buffer to $7 \mathrm{ml}$ instead of $200 \mathrm{ml}$.

\section{Important results}

- It is possible to distinguish different groups in the genus Abies using the above mentioned organelle DNA markers, especially by using the mitochondrial marker NAD5.4 to identify even closely related Abies species (Jaramillo-Correa et al. 2013, Ziegenhagen et al. 2005).

- Phylogenetic relationships among Abies species could also be constructed by using chloroplast-markers (e.g. Kormutak at al. 2004).

\section{Nuclear DNA markers (nSSRs, EST-SSRs, SNPs)}

\section{a) nSSRs (putatively neutral microsatellites) and EST- SSRs (expressed sequence tag derived microsatellites)}

Ten nuclear microsatellite markers were recently used for Abies grandis in an internal analysis at ASP Teisendorf for seed source identification purposes and species differentiation (between Abies alba and Abies grandis). The analyses were carried out within the scope of controlling the rules of the law for forest reproductive material in Germany (Cremer 2016, internal report ASP, unpublished).

\section{Loci and primers used}

The following nuclear microsatellite markers developed for Abies nordmanniana and Abies alba were successfully transferred to Abies grandis (see Table 4):

- NFF3, NFF7, NFH3 from A. nordmaniana (Hansen et al. 2005). These markers are simple sequence repeats (SSRs);

- Aat01, Aat04, Aat05, Aat06, Aat10, Aat11, Aat15 from Abies alba (Postolache et al. 2014). These markers are expressed-sequence-tag (EST-SSRs).

Table 3: Primer information of the mtDNA markers and cpSSRs used for the genetic analysis of Abies grandis $(\mathrm{Ta}=$ annealing temperatures, $\mathrm{Na}=$ number of alleles scored $)$

\begin{tabular}{|c|c|c|c|c|c|c|c|}
\hline Locus & Motif & Type of marker & $\begin{array}{c}\text { Primer sequence } \\
(\mathrm{F}=\text { forward, } \mathrm{R}=\text { reverse })\end{array}$ & $\begin{array}{c}\mathrm{Ta} \\
\left({ }^{\circ} \mathrm{C}\right)\end{array}$ & $\mathbf{N}_{\mathrm{a}}$ & $\begin{array}{l}\text { Size } \\
\text { (bp) }\end{array}$ & References \\
\hline NAD5.4 & $\begin{array}{l}2-4 \text { imper- } \\
\text { fect STRs, } \\
\text { repeats } 0-8\end{array}$ & $\begin{array}{c}\text { mt-intron-DNA } \\
\text { marker } \\
\text { (maternally inherited) }\end{array}$ & $\begin{array}{l}\text { F: GGACAATGACGATCCGAGATA } \\
\text { R: CATCCCTCCCATTGCATTAT }\end{array}$ & 52 & 2 & & 1,2 \\
\hline Pt71936 & (T) 22 & \multirow{3}{*}{$\begin{array}{c}\text { cp-SSR-marker } \\
\text { (paternally inherited) }\end{array}$} & $\begin{array}{l}\text { F: TTCATTGGAAATACACTAGCCC } \\
\text { R: AAAACCGTACATGAGATTCCC }\end{array}$ & 55 & & $124-148$ & 3 \\
\hline Pt30141 & & & $\begin{array}{l}\text { F: CCGAAGACAACGACTGTATTTT } \\
\text { R: CATTAAACTAGAGATACAAGGG }\end{array}$ & 55 & & & 3 \\
\hline Pt30249 & & & $\begin{array}{l}\text { F: CCCTTGTATCTCTAGTTTAATG } \\
\text { R: CTAGTTAGGCTTGGTCAACTAA }\end{array}$ & 55 & & & 3 \\
\hline
\end{tabular}

1-Liepelt et al. 2002, 2-Jaramillo-Correa et al. 2013, 3-Vendramin et al. 1996 


\section{Material used for extraction}

DNA was extracted from silicagel dried needles (ASP Teisendorf, unpublished)

\section{DNA-extraction protocols}

DNA extraction was performed using the method of Dumolin et al. (1995).

\section{Important results}

- The ten nSSRs that could be transferred from other Abies species and were only used in a case study of A. grandis (ASP, Teisendorf 2016) are a promising tool for continuing analyses of further populations of grand fir.

- Differentiation between $A$. alba and A. grandis is clearly possible with the nSSRs used; species-specificalleles/variants exist.

- The diversity (genetic diversity and heterozygosity) of a grand fir plant- and seed lot show slightly lower values when compared to those found in $A$. alba populations by using the same nSSR-markers.

\section{b) SNPs (single-nucleotide polymorphisms)}

There is no publication on SNP-analysis for Abies grandis.

\section{References}

Demesure, B., Sodzi, N., Petit, R.J. (1995): A set of universal primers for amplification of polymorphic noncoding regions of mitochondrial and chloroplast DNA in plants. Molecular Ecology, 4: 129-131.

Dumolin, S., Demesure, B., Petit, R.J. (1995): Inheritance of chloroplast and mitochondrial genomes in pedunculated oak investigated with an efficient PCR method. Theoretical and Applied Genetics, 91: 12531256.

Graham, S.W., Olmstead, R.G. (2000): Utility of 17 chloroplast genes for inferring the phylogeny of the basal angiosperms. American Journal of Botany, 87: 17121730 .

Table 4: Primer sequences, annealing temperatures ( $\mathrm{Ta}$ ), allele length in base pairs (bp), number of alleles scored $(\mathrm{Na})$ and references for nSSR markers available for genetic analyses in Abies grandis. $\left({ }^{*}=\mathrm{nSSR} ;{ }^{* *}=\mathrm{EST}-\mathrm{SSR}\right)$

\begin{tabular}{|c|c|c|c|c|c|c|}
\hline Locus & Motif & $\begin{array}{c}\text { Primer sequence } \\
(\mathrm{F}=\text { forward, } \mathrm{R}=\text { reverse })\end{array}$ & $\begin{array}{c}\mathrm{Ta} \\
\left({ }^{\circ} \mathrm{C}\right) \\
\end{array}$ & $\begin{array}{l}\text { Size } \\
(\mathbf{b p})\end{array}$ & $\mathbf{N}_{\mathbf{a}}$ & References \\
\hline NFF3* & - & $\begin{array}{l}\text { F: CCAATGGGTTGTCAGAGTGTT } \\
\text { R: GGCATTCGAGATTGCTTGAT }\end{array}$ & 58 & $109-123$ & 8 & 1 \\
\hline NFF7* & - & $\begin{array}{l}\text { F: CCCAAACTGGAAGATTGGAC } \\
\text { R: ATCGCCATCCATCATCAGA }\end{array}$ & 57 & $126-156$ & 15 & 1 \\
\hline NFH3* & - & $\begin{array}{l}\text { F: TTGCCATCAAATTAAAAATGCTT } \\
\text { R: CATCATTCTCTCTATCCCCATCA }\end{array}$ & 58 & $95-139$ & 22 & 1 \\
\hline Aat $01^{* *}$ & $(\mathrm{GCG}) 10$ & $\begin{array}{l}\text { F: CCATGTCTCCGATTTCCAGT } \\
\text { R: GGCCTAACGAAAGCAGAATC }\end{array}$ & 57 & $96-11$ & 4 & 2 \\
\hline Aat $04^{* *}$ & $(\mathrm{CAG}) 11$ & $\begin{array}{l}\text { F: CCATGTATGGTGCTCCTCCT } \\
\text { R: CCTTCATTGCAGAAAAGCAA }\end{array}$ & 57 & $147-159$ & 2 & 2 \\
\hline Aat $05^{* *}$ & (GCA)7 & $\begin{array}{l}\text { F: AGCATCCACATTCCGTAACC } \\
\text { R: AGTTGACCGTTGGAGAGCAG }\end{array}$ & 57 & $192-201$ & 3 & 2 \\
\hline Aat $06^{* *}$ & $(\mathrm{GCA}) 8$ & $\begin{array}{l}\text { F: TTATGCGGAGCAGTTCTGTG } \\
\text { R:TGTTGCTGGCGTACTGGTAG }\end{array}$ & 57 & $198-207$ & 4 & 2 \\
\hline Aat $10^{* *}$ & (AT) 12 & $\begin{array}{l}\text { F: GAGCACGATGAAGAGGAAGC } \\
\text { R: AAAACCCCCACGCGGTAT }\end{array}$ & 57 & $219-235$ & 8 & 2 \\
\hline Aat $11^{* *}$ & $(\mathrm{AAC}) 9$ & $\begin{array}{l}\text { F: AGCGTTGATTGGAAGCAGTC } \\
\text { R: GAAGCATGGTGTCGTTGTTG }\end{array}$ & 57 & $266-269$ & 2 & 2 \\
\hline Aat15** & $(A G A) 8$ & $\begin{array}{l}\text { F: AGGAGGAGGTTCAGCATGTC } \\
\text { R: CTTGCTCTCTGACCCAGTTG }\end{array}$ & 57 & 358 & 1 & 2 \\
\hline
\end{tabular}


Hansen, O.K., Vendramin, G.G., Sebastiani, F., Edwards, K.J. (2005): Development of microsatellite markers in Abies nordmanniana (Stev.) Spach and cross-species amplification in the Abies genus. Molecular Ecology Notes, 5: 784-787.

Hipkins, V.D., Tsai, C.H., Strauss, S.H. (1990): Sequence of the gene for large subunit of ribulose 1, 5-biphosphate carboxylase from a gymnosperm, Douglas fir. Plant Molecular Biology, 15: 505-507.

Hiratsuka, J., Shimada, H., Whittier, R., Ishibashi, T., Sakamoto, M., Mori, M., Kondo, C., Honji, Y., Sun, C.R., Meng, B.Y., Li, L.Q., Kanno, A., Nishizawa, Y., Hirai, A., Shinozaki, K., Sugiura, M. (1989): The complete sequence of the rice (Oryza sativa) chloroplast genome: intermolecular recombination between distinct tRNA genes accounts for a major plastid DNA inversion during the evolution of cereals. Molecular Genes and Genetics, 217: 185-194.

Howard, J.L., Aleksoff, K.C. (2000): Abies grandis. In: Fire Effects Information System, [Online]. U.S. Department of Agriculture, Forest Service, Rocky Mountain Research Station, Fire Sciences Laboratory (Producer). http://www. fs.fed.us/ database/feis/plants/tree/abigra/all.html [2017, January 30].

Jaramillo-Correa, J.P., Aguirre-Planter, E., Eguiarte, L.E., Khasa, D.P., Bousquet, J. (2013): Evolution of an ancient microsatellite hotspot in the conifer mitochondrial genome and comparison with other plants. Journal of Molecular Evolution, 76(3): 146-157.

Josserand, S.A., Potter, K.M., Johnson, G., Bowen, J.A., Frampton, J., Nelson, C.D. (2006): Isolation and characterization of microsatellite markers in Fraser fir (Abies fraseri). Molecular Ecology Notes, 6: 65-68.

Konnert, M., Ruetz, W.R. (1997): Genetic variation among provenances of Abies grandis from the Pacific Northwest. Forest Genetics, 4(2): 77-84.

Kormutak, A. (2004): Crossability relationships between some representatives of the Mediterranean, North American and Asian firs (Abies sp.). Veda, Bratislava

Kormutak, A., Vookova, B., Ziegenhagen, B., Kwon, H.Y., Hong, Y.P. (2004): Chloroplast DNA Variation in some Representatives of the Asian, North American and Mediterranean Firs (Abies spp.), Silvae Genetica, 53(3): 99-104.

Liepelt, S., Bialozyt, R., Ziegenhagen, B. (2002): Winddispersed pollen mediates post-glacial gene flow among refugia. Proc Natl Acad Sci USA, 99: 14590-14594.

Marvin, W.F., Russel T.G., David F.O.Jr. (2003): Grand Fir Abies grandis (Dougl. ex D. Don) Lindl. https://www. na.ff.fed.us/spfo/pubs/silvics_manual/Volume_1/ abies/ grandis.htm
Murray, M. G., Thompson, W. F. (1980): Rapid isolation of high molecular weight DNA. Nucleic Acid Research, 8: 4231-4235.

Parducci, L., Szmidt, A.E. (1999): PCR-RFLP analysis of cpDNA in the genus Abies. Theoretical and Applied Genetics, 98: 802-808.

Postolache, D., Leonarduzzi, C., Piotti, A., Spanu, I., Roig, A., Fady, B., Roschanski, A., Liepelt, S., Vendramin, G.G. (2014): Transcriptome versus Genomic Microsatellite Markers: Highly Informative Multiplexes for Genotyping Abies alba Mill. and Congeneric Species. Plant Molecular Biology Reporter, 32: 750-760.

Steinhoff, R.J. (1978): Distribution, ecology, sivicultural characteristics and genetics of the Abies grandis-Abies concolor complex. In: Proceedings of the IUFRO joint meeting of working parties VIII, Vancouver, B.C. Canada, B.C. Ministry of Forests, 123-132.

Vendramin, G.G., Lelli, L., Rossi, P., Morgante, M. (1996): A set of primers for the amplification of 20 chloroplast microsatellites in Pinaceae. Molecular Ecology, 5: 595-598.

Wang, X.R., Szmidt, A.E., Nguyng, H.N. (2000): The phylogenetic position of the endemic flat-needle pine Pinus krempfii (Pinaceae) from Vietnam, based on PCRRFLP analysis of chloroplast DNA. Plant Systematics and Evolution, 220: 21-36.

Wu, J., Krutovskii, K., Strauss, S.H. (1998): Abundant mitochondrialgenome diversity, population differentiation and convergent evolution in pines. Genetics, 150: 16051614.

Ziegenhagen, B., Fady, B., Kuhlenkamp, V., Liepelt, S. (2005): Differentiating groups of Abies species with a simple molecular marker. Silvae Genetica, 54: 123-126.

\section{Selected references referring to genetic investigations on related Abies species}

The cited publications contain information on markers and methodological aspects for genetic investigations in different Abies species.

\section{Isozymes}

Abies alba

Breitenbach-Dorfer, M., Pinsker, W., Hacker, R., Müller, F. (1992): Clone identification and clinal allozyme variation in populations of Abies alba from the Eastern Alps (Austria). Plant Systematics and Evolution, 181: 109-120.

Cremer, E., Liepelt, S., Ziegenhagen, B., Hussendörfer, E. (2003): Microsatellite and isozyme markers for seed source identification in silver fir. Forest Genetics, 10(3): 165-171. 
Hussendörfer, E., Konnert, M., Bergmann, F. (1995): Inheritance and linkage of isozyme variants of silver fir (Abies alba Mill.). Forest Genetics (Arbora Publishers), 2(1): 29-40.

Konnert, M., Bergmann, F. (1995): The geographical distribution of genetic variation of silver fir (Abies alba, Pinaceae) in relation to its migration history. Plant Systematics and Evolution, 181: 109-120.

Konnert, M., Maurer, W. (1995): Isozymic Investigations on Norway Spruce (Picea abies (L.) Karst.) and European Silver Fir (Abies alba Mill.): A Practical Guide to Separation Methods and Zymogram Evaluation. From the German Federal-State Working Group "Conservation of Forest Gene Resources”, ISBN 3-00-000042-9.

Schroeder, S. (1989): Isozyme Polymorphisms in Silver Fir (Abies alba Mill.). Silvae Genetica, 38(3-4): 130-133.

\section{Abies amabilis}

Davidson, R.H. (1990): Patterns of variation of Pacific silver fir (Abies amabilis (Dougl.) Forbes) on Vancouver Island. Ph.D.Thesis, University of British Columbia, pp.206.

Davidson, R.H., El-Kassaby, Y.A. (1997): Genetic diversity and gene conservation of Pacific silver fir (Abies amabilis) on Vancouver Island, British Columbia. Forest Genetics, 4(2): 85-98.

\section{Abies balsamea}

Neale, D.B., Adams, W.T. (1980): Inheritance of isozyme variants in seed tissue of balsam fir (Abies balsamea). Canadian Journal of Botany, 59: 1285-1291.

Neale, D.B., Adams, W.T. (1986): Allozyme and mating system variation in balsam fir (Abies balsamea) across a continuous elevational gradient. Canadian Journal of Botany, 63: 2448-2453.

\section{Abies concolor}

Westfall, R.D., Conkle, M.T. (1992): Allozyme markers in breeding zone designation. New Forests, 6: 279-309.

\section{Abies fraseri}

Diebel, K.E., Feret, P.P. (1991): Isozyme variation within the Fraser fir (Abies fraseri (Pursh) Poir.) population on Mount Rogers, Virginia: Lack of microgeographic differentiation. Silvae Genetica, 40(2): 79-85.

\section{Abies lasiocarpa}

Ettle, G.J., Peterson, D.L. (2001): Genetic variation of Subalpine fir (Abies lasiocarpa (HOOK.) NUTT.) in the Olympic Mountains, WA, USA. Silvae Genetica, 50(3-4): 145-153.

Shea, K.L. (1988): Segregation of allozyme loci in megagametophytes of Engelmann spruce and subalpine fir. Genome 30: 103-107.

\section{Abies procera}

Siegismund, H.R., Kjaer, E.D. (1997): Outcrossing rates in two Stands of Noble fir (Abies procera REHD.) in
Denmark. Silvae Genetica, 46(2-3): 144-146.

\section{Molecular markers (cpDNA, mtDNA)}

Abies alba

Cremer, E., Liepelt, S., Ziegenhagen, B., Hussendörfer, E. (2003): Microsatellite and isozyme markers for seed source identification in silver fir. Forest Genetics, 10(3): 165-171.

Fady, B., Forest, I., Hochu, I., Ribiollet, A., de Beaulieu, J.L., Pastuzska, P. (1999): Genetic differentiation in Abies alba populations from south-eastern France. Forest Genetics, 6:129-138.

Gömöry, D., Paule, L., Krajmerová, D., Romšáková, I., Longauer, R. (2012): Admixture of genetic lineages of different glacial origin: a case study of Abies alba Mill. in the Carpathians. Plant Systematics and Evolution, 298: 703-712.

\section{Molecular markers (nSSR, SNP)}

\section{Abies alba}

Cremer, E., Liepelt, S., Sebastiani, F., Buonamici, A., Michalczyk, I.M., Ziegenhagen, B., Vendramin, G.G. (2006): Identification and characterization of nuclear microsatellite loci in Abies alba Mill. Molecular Ecology Notes, 6: 374-376.

Cremer, E., Ziegenhagen, B., Schulerowitz, K., Mengel, C., Donges, K., Bialozyt, R., Hussendörfer, E., Liepelt, S. (2012): Local seed dispersal in European silver fir (Abies alba Mill.): lessons learned from a seed trap experiment. Trees, 26: 987-996.

Gömöry, D., Paule, L., Krajmerová, D., Romšáková, I., Longauer, R. (2012): Admixture of genetic lineages of different glacial origin: a case study of Abies alba Mill. in the Carpathians. Plant Syst Evol, 298: 703-712.

Liepelt, S., Cheddadi, R., de Beaulieu, J.L., Fady, B., Gömöry, D., Hussendörfer, E., Konnert, M., Litt, T., Longauer, R., Terhürne-Berson, R., Ziegenhagen, B. (2009): Postglacial range expansion and its genetic imprints in Abies alba (Mill.) - A synthesis from palaeobotanic and genetic data. Review of Paleobotany and Palynology, 153: 139-149.

Roschanski, A., Ery, K., Liepelt, S., Odou-Muratorio, S., Ziegenhagen, B., Huard, F., Ullrich, K.K., Postolache, D., Vendramin, G.G., Fady, B. (2016): Evidence of divergent selection for drought and cold tolerance at landscape and local scales in Abies alba Mill. in the French Mediterranean Alps. Molecular Ecology, 25: 776-794.

\section{Abies fraseri}

Josserand, S.A., Potter, K.M., Johnson, G., Bowen, J.A., Frampton, J., Nelson, C.D. (2006): Isolation and characterization of microsatellite markers in Fraser fir (Abies fraseri). Molecular Ecology Notes, 6: 65-68. 


\title{
Molecular markers used for genetic studies in Cedrus spp.
}

\author{
Monika Konnert and Muhidin Šeho
}

\begin{abstract}
Bavarian Office for Forest Seeding and Planting, Forstamtsplatz 1, 83317 Teisendorf, Germany
\end{abstract}

\section{General remarks}

The Genus Cedrus includes three species native to Mediterranean mountains and one restricted to the Himalayas.

\section{Cedrus atlantica}

Atlas ceder (Cedrus atlantica) is highly fragmented and native to the northern African regions of Morocco and Algeria. In Morocco regions with Cedrus atlantica are Rif, Middle-Atlas and High-Atlas. The species optimum is the Middle-Atlas region in altitudes between 1600 and $2200 \mathrm{~m}$. In Algeria the main regions are the TellAtlas and the Sahara-Atlas. The whole distribution range covers an area of 145.000 ha.

\section{Cedrus brevifolia}

Cedrus brevifolia is a narrow endemic island tree species of Cyprus. It is divided into five neighboring sites (geographic regimes) on the mountains of the Paphos forest. C. brevifolia ranges from $900-1400 \mathrm{~m}$ above sea level. On all five sites are growing approximately 15.800 trees with a diameter at breast height over $12 \mathrm{~cm}$.

\section{Cedrus deodara}

The natural distribution range of Cedrus deodara is restricted from Afghanistan to the southern slopes of the western Himalayas. The optimum of Cedrus deodora is the high range of Kashmir from 2000-2500 m. This tree species is reaching heights from $40-50 \mathrm{~m}$ and diameter up to $3 \mathrm{~m}$.

\section{Cedrus libani}

Cedrus libani can be divided into two main distribution areas: Turkey, with South- and Southwest-Anatolia and the geographical distinct occurrence in Lebanon and Syria. In Turkey this includes large parts of the westand middle Taurus and the Amanos-mountain area, with around 400.000 ha. Due to heavy depletion, the occurrence of Cedrus libani is scattered in many small populations. Larger populations can be found mainly in the western Taurus. In Lebanon the natural distribution range has declined from 500.000 ha, to 1.700 ha for the same reasons as in Turkey.

The native distribution of the Cedrus ssp. is depicted in Figure 1.

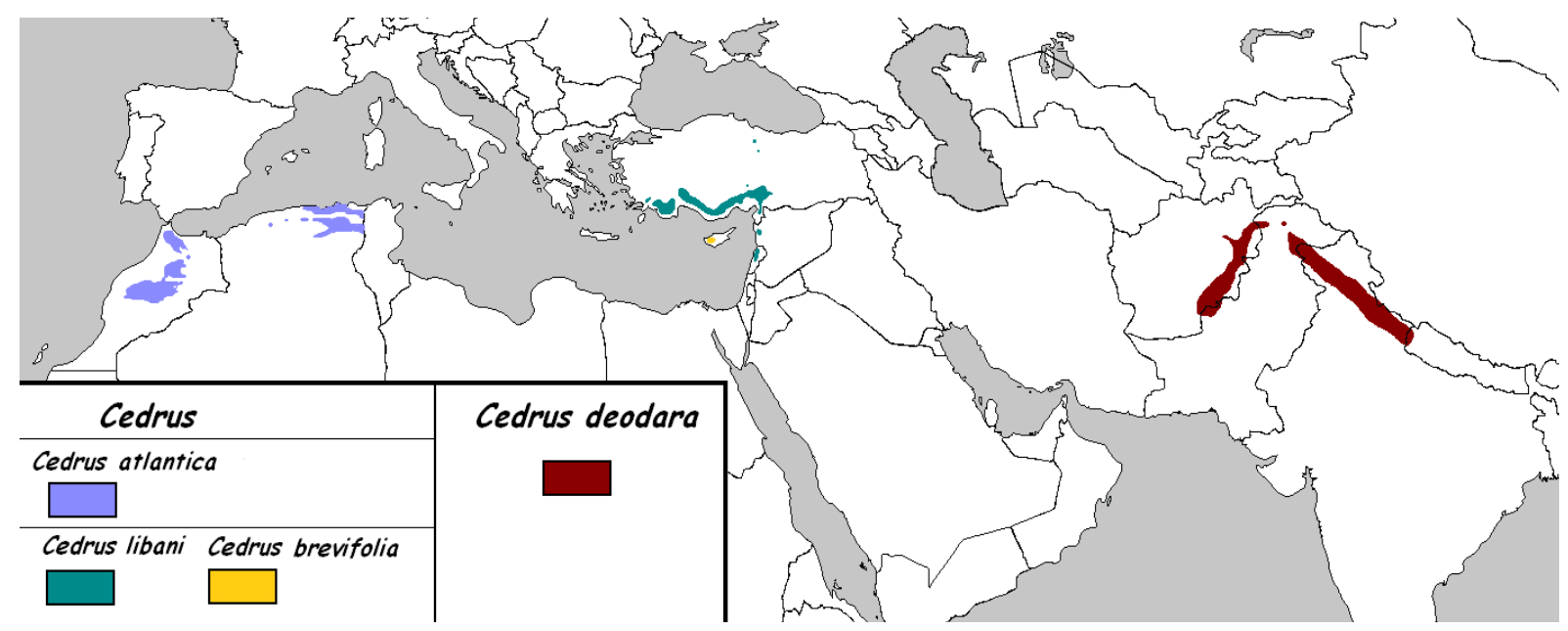

Figure 1. Native distribution range of Cedrus ssp. (modified after: https://commons. wikimedia.org/wiki/ File:Cedrus_map.png) 


\section{Isozymes}

First publication on isozymes for Cedrus species appeared in 1992 (Panetsos et al. 1992). Further on isozymes were used to determine the allozyme differentiation and phylogeny of cedar species (Bariteau et al. 1999, Scaltsoyiannes 1999, Fady et al. 2000) or to see the genetic variation in natural populations of the same species in a restricted part of the natural distribution range (Yahyaoglu et al. 1997, Gülbaba and Özkurt 2002, Kurt et al. 2008).

The list of enzymes, number of loci and references are presented in table 1. In some studies (e.g. Kurt et al. 2008) loci are indicated by numbers (e.g. IDH-1), in other studies by letters (e.g. IDH-A) (e.g. Scaltsoyiannes 1999). Given this situation it has to be considered that IDH-A in table 1 is identical with IDH-1.

\section{Material for protein extraction}

Proteins were extracted from different tissues as for example seed (megagametophytes) (e.g. Kurt et al. 2007, Panetsos et al. 1992, Fady et al. 2008), radicle from germinated seed (Scaltsoyiannes 1999) and dormant buds (Panetsos et al. 1992, Scaltsoyiannes 1999).

\section{Protein extraction and separation protocols}

Details on extraction procedures and starch gel electrophoretic analysis are given in Panetsos et al. (1992), Scaltsoyiannes (1999) and Kurt et al. (2008). In general the separation buffers and staining recipes from Fady and Conkle (1992), Conkle et al. (1982) and Cheliak and Pitel (1984) were applied.

\section{Important results}

- Cedrus libani in Turkey exhibits a high intrapopulation variation but a low genetic variation

Table 1: List of enzymes, scored loci and number of alleles for Cedrus species

\begin{tabular}{|c|c|c|c|c|}
\hline Enzyme system & E.C. Number & Scored loci & $\begin{array}{l}\text { No. of } \\
\text { alleles }\end{array}$ & References \\
\hline Acid phosphatase & 3.1.3.2 & ACP-A,-B & 2,2 & $1,2,6$ \\
\hline Alcohol dehydrogenase & 1.1 .1 .1 & ADH-A & 1 & 1 \\
\hline Aspartate aminotransferase & 2.6.1.1 & $\begin{array}{c}\text { AAT-A,-C resp. } \\
\text { GOT-A,-C }\end{array}$ & 4,3 & $1,2,3,7$ \\
\hline Diaphorase & 1.6.4.3 & Dia-A* & 4 & $1,4,5$ \\
\hline Glutamate dehydrogenase & 1.4.1.2 & GDH-A & 2 & 1,2 \\
\hline Isocitrate dehydrogenase & 1.1 .1 .42 & IDH-A & 3,5 & $1,2,5,6,8$ \\
\hline Leucine aminopeptidase & 3.4 .11 .1 & LAP-A,-B & 3,4 & $1,4,5,8$ \\
\hline Malate dehydrogenase & 1.1 .1 .37 & MDH-A,-B & 2,2 & 1,4 \\
\hline Menadione reductase & 1.6 .99 .2 & MNR-A,-B,-C & $2,4,3$ & 1 \\
\hline 6-Phosphogluconate dehydrogenase & 1.1 .1 .44 & 6PGDH-A,-B & 3,6 & $1,2,4,5,8$ \\
\hline Phosphoglucose isomerase & 5.3.1.9 & PGI-A,-B & 1,6 & $1,4,5,8$ \\
\hline Phosphoglucomutase & 2.7.5.1 & $\mathrm{PGM}^{* *}$ & & 9 \\
\hline Superoxide dismutase & 1.15 .1 .1 & SOD-A & 1 & 1 \\
\hline Shikimate dehydrogenase & 1.1 .1 .25 & $\mathrm{SKDH}^{* *}$ & & 9 \\
\hline
\end{tabular}

* corresponds to the MNR-A locus (Scaltsoyiannes 1999); ** no indication on loci resp. allele numbers

1-Kurt et al. 2008, 2-Gülbaba and Özkurt 2002, 3-Panetsos et al. 1994, 4-Panetsos et al. 1992, 5-Scaltsoyiannes 1999, 6-Fallour et al. 2001, 7-Yahyaoglu et al. 1997,

8-Bariteau et al. 1999, 9-Fady et al. 2008 
among populations (Kurt et al. 2008). In contrary, in Lebanon high differentiation and genetic drift effects were found for the same species with a combination of molecular markers and microsatellites (see also chapter 3). Populations from Turkey and Lebanon constitute two genetically isolated groups (Fady et al. 2008). For planting outside the natural range seed from Turkish populations have to be clearly preferred.

- Based on isozyme markers distinction between different Cedrus species is possible in great part (Panetsos et al. 1992, Scaltsoyiannes 1999). Differences are in allele frequencies but also in species specific alleles (e.g. Scaltsoyiannes 1999, Bariteau et al. 1999, Fady et al. 2000). Genetic distances and cluster analysis illustrate the clear genetic differences between the Cedrus species (Scaltsoyiannes 1999, Fady et al. 2008).

- For Cedrus libani alleles with a particular geographic pattern (e.g. 6-PGDH-B4) were found (Scaltsoyiannes 1999).

- Great variation was observed in heterozygosity levels among species with low values in Cedrus deodara and high values in Cedrus brevifolia (Scaltsoyiannes 1999).

\section{Organelle DNA markers (chloroplast (cp) DNA, mitochondrial (mt)DNA)}

\section{Loci and primers used}

All chloroplast microsatellites applied in Cedrus genetic studies were originally designed for the Pinus thunbergii chloroplast genome (Wakasugi et al. 1994). Nine primers out of twenty (Vendramin et al. 1996) could be amplified in different Cedrus taxa (Table 2). Studies with these primers refer to gene flow among different taxonomic units (Fady et al. 2003), conservation studies (Fady et al. 2008), identification of glacial refugia (Cheddadi et al. 2009) and genetic characterization of natural populations (Terrab et al. 2006, Eliades et al. 2011).

\section{Material for DNA-extraction}

In all studies DNA was extracted from fresh or frozen needles (Fady et al. 2003, 2008, Terrab et al. 2007, Cheddadi et al. 2009, Eliades et al. 2011).

Table 2: Primer sequences, annealing temperatures (Ta), allele length in base pairs (bp) and references for cpSSRs available for genetic analyses in Cedrus species

\begin{tabular}{|c|c|c|c|c|c|}
\hline Locus & $\begin{array}{l}\text { Primer sequence }\left[5^{\prime}-3^{\prime}\right] \\
F=\text { Forward, } R=\text { Reverse }\end{array}$ & $\begin{array}{c}\mathrm{Ta} \\
\left({ }^{\circ} \mathrm{C}\right)\end{array}$ & $\begin{array}{l}\text { Size } \\
(\mathbf{b} p)^{*}\end{array}$ & Variability & References \\
\hline Pt15169 & $\begin{array}{l}\text { F:CTTGGATGGAATAGCAGCC } \\
\text { R:GGAAGGGCATTAAGGTCATTA }\end{array}$ & 55 & 118 & $\begin{array}{l}\text { Polymorphic among } \\
\text { and within species }\end{array}$ & $1,2,3,4,5$ \\
\hline Pt63718 & $\begin{array}{l}\text { F:CACAAAAGGATTTTTTTTCAGTG } \\
\text { R:CGACGTGAGTAAGAATGGTTG }\end{array}$ & 55 & 93 & $\begin{array}{l}\text { Polymorphic among } \\
\text { and within species }\end{array}$ & $1,2,3,4,5$ \\
\hline Pt71936 & $\begin{array}{l}\text { F:TTCATTGGAAATACACTAGCCC } \\
\text { R:AAAACCGTACATGAGATTCCC }\end{array}$ & 55 & 148 & $\begin{array}{l}\text { Polymorphic among } \\
\text { and within species }\end{array}$ & $1,2,3,4,5$ \\
\hline Pt87268 & $\begin{array}{l}\text { F:GCCAGGGAAAATCGTAGG } \\
\text { R:AGACGATTAGACATCCAACCC }\end{array}$ & 55 & 165 & $\begin{array}{l}\text { Polymorphic among } \\
\text { species }\end{array}$ & $1,2,4$ \\
\hline Pt26081 & $\begin{array}{l}\text { F:CCCGTATCCAGATATACTTCCA } \\
\text { R:TGGTTTGATTCATTCGTTCA }\end{array}$ & 55 & 112 & monomorphic & $1,2,4$ \\
\hline Pt36480 & $\begin{array}{l}\text { F:TTTTGGCTTACAAAATAAAAGAGG } \\
\text { R:AAATTCCTAAAGAAGGAAGAGCA }\end{array}$ & 55 & 147 & monomorphic & $1,2,4$ \\
\hline Pt110048 & $\begin{array}{l}\text { F:TAAGGGGACTAGAGCAGGCTA } \\
\text { R:TTCGATATTGAACCTTGGACA }\end{array}$ & 55 & 88 & monomorphic & $1,2,3$ \\
\hline Pt30204 & $\begin{array}{l}\text { TCATAGCGGAAGATCCTCTTT } \\
\text { CGGATTGATCCTAACCATACC }\end{array}$ & 55 & 145 & - & 1,3 \\
\hline Pt109567 & $\begin{array}{l}\text { F:TATTATCGAACAACGAGAATAATCC } \\
\text { R:TCACTGTCACTCTACAAAACCG }\end{array}$ & 55 & 115 & - & 1,3 \\
\hline
\end{tabular}

* values for Pinus thunbergii

1-Vendramin et al. 1996, 2-Fady et al. 2003, 3-Terrab et al. 2006, 4-Fady et al. 2008, 5-Eliades et al. 2011 


\section{DNA-extraction and amplification protocols}

Total DNA was extracted from ground needle tissue using:

- the DNeasy 96 Plant Kit from QIAGEN (Eliades et al. 2011, Fady et al. 2008)

- the Doyle and Doyle (1990) protocol (Fady et al. 2003)

- the CTAB miniprep protocol of Cullings (1992) (Terrab et al. 2006)

For amplification the following PCR-protocols were used:

- $95^{\circ} \mathrm{C}$ for 3 min followed by 25 cycles of $94^{\circ} \mathrm{C}$ for $1 \mathrm{~min}, 55^{\circ} \mathrm{C}$ for $1 \mathrm{~min}, 72^{\circ} \mathrm{C}$ for $1 \mathrm{~min}, 1$ cycle at $72^{\circ} \mathrm{C}$ (Terrab et al. 2006)

- $\quad 95^{\circ} \mathrm{C}$ for 5 min followed by 25 cycles of $94^{\circ} \mathrm{C}$ for 1 $\min , 55^{\circ} \mathrm{C}$ for $1 \mathrm{~min}, 72^{\circ} \mathrm{C}$ for $1 \mathrm{~min}$ with a final extension step of $72^{\circ} \mathrm{C}$ for 8 min (Fady et al. 2003, 2008)

\section{Important results}

- Chloroplast DNA was found to be paternally inherited in Cedrus. Therefore chloroplast markers are useful to monitor male gene flow in forests through the genotyping of single trees and their open-pollinated progeny (Fady et al. 2003). It seems that no strong reproductive barrier exists between the Mediterranean Cedrus species (atlantica, brevifolia and libani) (Fady et al. 2003).

- Cedrus libani populations from Turkey and Lebanon constitute two genetically isolated groups (Fady et al. 2008), probably because they arose from two different glacial refugia. Turkish populations have high genetic variation inside populations and low differentiation between them. In Lebanon, Cedrus populations are highly differentiated and show sever cases of genetic drift due to strong human influence and reduction of population size. For planting outside the natural range seed from Turkish populations have to be clearly preferred. Similar results were found in the same study with isozymes (see chapter 2).

- Cedrus atlantica in Morocco appears to maintain a high level of haplotypic diversity, which is mostly within populations. Differences between populations are low. No clear center of genetic diversity in Cedrus altlantica was identified (Terrab et al. 2006). High genetic diversity is found in the High Atlas Mountains. To preserve the species under climate change it is suggested to transfer genetic material from this region to western High Atlas (Cheddadi et al. 2009).

- Cedrus brevifolia, a narrow endemic island tree species from Cyprus, has high genetic diversity both at the nuclear and plastid level (see also chapter 4), suggesting no drift effects or genetic bottleneck (Eliades et al. 2011).

\section{Randomly amplified polymorphic DNA markers (RAPD)}

\section{Loci and primers used are listed in Table 3}

\section{Material for DNA-extraction}

For RAPD-analysis DNA was extracted from needles collected from seedlings (Fady et al. 2003) or adult trees (Renau-Morata et al. 2005, Semaan and Dodd 2008) and from seed (megagametophytes) (Kayihan et al. 2006).

\section{DNA-extraction and amplification protocols}

Total DNA was extracted from needle tissue using

- the Doyle and Doyle (1990) protocol (Semaan and Dodd 2008)

- the DNeasy 96 Plant Kit from QIAGEN (RenauMorata et al. 2005).

For the extraction of DNA from megagametophytes Kayihan et al. (2006) used a combination of DNA extraction procedure from Dellaporta et al. (1983) and Kreike (1990).

For amplification the following PCR-protocols were used:

- 45 cycles of $94^{\circ} \mathrm{C}$ for $20,40{ }^{\circ} \mathrm{C}$ for $20 \mathrm{sec}$, and $72{ }^{\circ} \mathrm{C}$ for $90 \mathrm{sec}$ with a final extension step at $72^{\circ} \mathrm{C}$ for 10 min (Semaan and Dodd 2008).

- Renau-Morata et al. (2005) performed the DNAamplification as described in Neubauer et al. (2000): 1 min denaturation at $94^{\circ} \mathrm{C}$, followed by 45 cycles of $30 \mathrm{sec}$ at $94^{\circ} \mathrm{C}, 30 \mathrm{sec}$ annealing at $39^{\circ} \mathrm{C}$ and a 90 -sec extension at $72^{\circ} \mathrm{C}$, with a last step of an additional $7 \mathrm{~min}$ extension at $72^{\circ} \mathrm{C}$.

- Fady et al. (2003) used PCR, separation and staining protocols form Lefebvre et al. (1995).

\section{Important results}

- RAPD markers in combinations with cpSSR are a good tool to study hybridization between species. 
Table 3: Primers employed and RAPD markers obtained from DNA amplification in Cedrus species $(\mathrm{Ta}=$ annealing temperature $)$

\begin{tabular}{|c|c|c|c|c|c|c|}
\hline \multirow{2}{*}{ Locus } & \multirow{2}{*}{ Sequence $5^{\prime}-3^{\prime}$} & \multirow{2}{*}{$\begin{array}{c}\mathbf{T a} \\
\left({ }^{\circ} \mathbf{C}\right)\end{array}$} & \multirow{2}{*}{ Size (bp) } & \multicolumn{2}{|c|}{ Number of bands } & \multirow{2}{*}{ References } \\
\hline & & & & polymorphic & monomorphic & \\
\hline OPA18* & AGGTGACCGT & 39 & $550-2100$ & 22 & 2 & 1,2 \\
\hline OPA20* & GTTGCGATCC & 39 & $630-2000$ & 18 & 0 & 1,2 \\
\hline OPB7* & GGTGACGCAG & 39 & $480-1600$ & 18 & 0 & 1,2 \\
\hline OPB8* & GTCCACACGG & 39 & $750-2250$ & 18 & 0 & 1,2 \\
\hline OPB12* & CCTTGACGCA & 39 & $500-2200$ & 23 & 1 & 1,2 \\
\hline $\mathrm{OPC}^{*}$ & GTGAGGCGTC & 39 & $400-1700$ & 38 & 0 & 1,2 \\
\hline OPA7** & GAAACGGGTG & 37.4 & $400-2500$ & 22 & 0 & 3 \\
\hline OPA $10^{* *}$ & GTGATCGCAG & 37.4 & $400-2500$ & 22 & 0 & 3 \\
\hline OP $17^{* *}$ & GACCGCTTGT & 37.4 & $400-2500$ & 15 & 1 & 3 \\
\hline $\mathrm{A} 15^{* * *}$ & TTCCGAACCC & & & & & 4 \\
\hline $\mathrm{A} 20^{* * *}$ & GTTGCGATCC & & & & & 4 \\
\hline $\mathrm{B} 12^{* * *}$ & CCTTGACGCA & & & & & 4 \\
\hline $\mathrm{C} 16^{* * *}$ & САСАСТССАG & & & & & 4 \\
\hline $\mathrm{E} 9^{* * *}$ & CCTCACCCGA & & & & & 4 \\
\hline $\mathrm{P} 14^{* * *}$ & CCAGCCGAAC & & & & & 4 \\
\hline Q5*** & CCGCGTCTTG & & & & & 4 \\
\hline
\end{tabular}

- Intensive gene flow between Cedrus atlantica and Cedrus libani was observed in open pollinated seedlings in two plantation forests (see also AFLP) (Fady et al. 2003).

- For Cedrus libani from Turkey no clear geographic differentiation pattern was observed. For one geographically isolated stand based on RAPD the putative origin was traced back (Kayihan et al. 2006).

- For Cedrus libani from Libanon RAPDs show considerable variation within populations and low population differentiation. Inbreeding effects or genetic drift was not observed. Correlation between genetic diversity and climatic conditions (temperature, humidity) was observed (Semaan and Dodd 2008).

- Genetic diversity of Cedrus atlantica in Morocco analyzed using RAPD markers was high and comparable to that revealed by isozymes. No differences in genetic diversity of natural and managed populations were observed (Renau-Morata et al. 2005). 


\section{Nuclear DNA markers (AFLPs, nSSRs, SNPs)}

a) AFLPs (Amplified Fragment Length Polymorphisms)

\section{Loci and primers used}

In both studies AFLP primers contained one selective nucleotide for pre-amplification and three selective nucleotides for amplification. Dagher-Kharrat et al. (2006) generated a total of 107 polymorphic amplification products with eleven selective AFLP primer pairs. In total 388 bands appear. Among them 107 AFLP showed clear polymorphic bands. Information on used primer combinations is given in Table 4 .

\section{Material for DNA-extraction}

For AFLP-analysis DNA was extracted from frozen needles collected from seedlings (Fady et al. 2003) and megagametophytes (haploid) (Dagher-Kharrat et al. 2006).

\section{DNA-extraction and amplification protocols}

Total DNA was extracted following the Doyle and Doyle (1990) protocol (Fady et al. 2003). For megagametophytes Dagher-Kharrat et al. (2006) modified this protocol slightly by the addition of $2 \% \mathrm{PVP} \mathrm{mW} 40.000$ to the extraction buffer.
AFLP analyses were carried out using the "AFLP Analysis System I" kit from "Life Technologies". For details on the analysis procedures see Fady et al. (2003) and DagherKharrat et al. (2006).

\section{Important results}

- There was no indication of strong reproductive isolating barriers between the Mediterranean Cedrus species (atlantica, brevifolia and libani). An intensive gene flow between Cedrus atlantica and Cedrus libani was observed in open pollinated seedlings in two plantation forests (Fady et al. 2003).

- Himalayan cedar (Cedrus deodara) is genetically clearly different from the Mediterranean cedars. 13 out of 25 species specific bands distinguished Cedrus dodara from the other Cedrus species (see also Table 5). Within the Mediterranean cedars Cedrus atlantica is different from Cedrus libani and brevifolia. The latter two species are genetically similar (DagherKharrat et al. 2006).

- Cedrus brevifolia, a narrow endemic island tree species from Cyprus, has high genetic diversity (same results as for chloroplast markers) (DagherKharrat et al. 2006).

Table 4: Primer combinations used to produce AFLP fingerprints (Dagher-Kharrat et al. 2006)

\begin{tabular}{|c|c|c|c|c|c|c|c|c|c|c|c|c|c|}
\hline & \multirow{2}{*}{ Sequence } & \multicolumn{12}{|c|}{$\mathrm{NNN}^{\prime} \mathbf{3}^{\prime}$} \\
\hline & & I & II & III & IV & $\mathrm{V}$ & VI & VII & VIII & IX & $\mathbf{X}$ & $\mathrm{XI}$ & Total \\
\hline$E c o \mathrm{RI}+\mathrm{NNN}-3^{\prime}$ & 5'-GACTGCGTACCAATTC & ACC & ACA & ACT & AAC & ACT & ACT & AAC & ACA & ACG & ACG & ACG & \\
\hline MseI + NNN-3' & 5'-GATGAGTCCTGAGTAA & СTT & CAA & CTA & CTT & CAC & CTT & CAT & CAG & СТС & CTA & CAG & \\
\hline $\begin{array}{l}\text { No of variable } \\
\text { bands }\end{array}$ & & 1 & 2 & 2 & 2 & 4 & 3 & 5 & 23 & 52 & 63 & 66 & 223 \\
\hline No of fixed bands & & 16 & 24 & 13 & 11 & 17 & 11 & 9 & 28 & 18 & 9 & 9 & 165 \\
\hline Total band number & & 17 & 26 & 15 & 13 & 21 & 14 & 14 & 51 & 70 & 72 & 75 & 388 \\
\hline $\begin{array}{c}\text { Level of } \\
\text { polymorphism in \% }\end{array}$ & & 5.9 & 7.7 & 13.2 & 15.4 & 19 & 21.3 & 35.6 & 45.1 & 74.3 & 87.5 & 88 & 57.4 \\
\hline
\end{tabular}




\section{b) nSSRs (putatively neutral microsatellites)}

\section{Loci and primers used}

Chaib et al. (2006) developed primers for the amplification of six polymorphic nuclear microsatellites in Mediterranean Cedrus taxa. Microsatellites originated from two Cedrus atlantica genomic libraries enriched for TC (four markers) and TG (two markers) motifs. Eliades et al. (2011) tested the transferability and polymorphism of these primers in Cedrus brevifolia. Four out of the six nSSR developed for Cedrus atlantica showed amplification also in Cedrus brevifolia, namely CatITgD4, CatXITcE11, CatXITcD12, CatXIITcC6 (see Table 6). CatXIIITcA3, CatITgD4, CatXITcD12 also amplified in the Himalayan cedar (Cedrus deodara).

\section{Material for DNA-extraction}

In both studies DNA was extracted from needle tissues (Chaib et al. 2006, Eliades et al. 2011).

\section{DNA-extraction and amplification protocols}

Total DNA was extracted from ground needle tissue using:

- the Doyle and Doyle (1990) protocol (Chaib et al. 2006)

- the DNeasy 96 Plant Kit from QIAGEN (Eliades et al. 2011)

For amplification the following PCR-protocols were used:

Cedrus atlantica (Chaib et al. 2006)

5 min at $95^{\circ} \mathrm{C}$ followed by 35 cycles of 1 min at $94^{\circ} \mathrm{C}$, $1 \mathrm{~min}$ at the annealing temperature (Table 6), $1 \mathrm{~min}$ at $72^{\circ} \mathrm{C}$ and a final extension of $5 \mathrm{~min}$ at $72^{\circ} \mathrm{C}$.

Cedrus brevifolia (Eliades et al. 2011)

- primers CatITgD4 and CatXITcD12: $95^{\circ} \mathrm{C}$ for $15 \mathrm{~min}$, followed by 1 cycle at $80^{\circ} \mathrm{C}$ for $4 \mathrm{~min}, 30$ cycles at $94^{\circ} \mathrm{C}$ for $1 \mathrm{~min}, \mathrm{Ta}$ (annealing temperature) $\left(53^{\circ} \mathrm{C}\right.$ and $\left.55,5^{\circ} \mathrm{C}\right)$ for $1 \mathrm{~min}, 72^{\circ} \mathrm{C}$ for $1 \mathrm{~min}$, extension $72^{\circ} \mathrm{C}$ for 30 $\min$

Table 5: Primer combinations giving species- or populations-specific markers (Dagher-Kharrat et al. 2006)

\begin{tabular}{|c|c|c|c|c|c|c|}
\hline \multirow{2}{*}{$\begin{array}{c}\text { Primer } \\
\text { combination }\end{array}$} & \multicolumn{6}{|c|}{ Number of markers for the identification of } \\
\hline & C. atlantica & C. libani & C. brevifolia & C. deodara & $\begin{array}{l}\text { C. libani and } \\
\text { C. brevifolia } \\
\end{array}$ & $\begin{array}{c}\text { Mediterranean } \\
\text { cedars }\end{array}$ \\
\hline E-ACT/M-CTT & 1 & \begin{tabular}{|c|}
1 (Turkish \\
populations) \\
\end{tabular} & & 1 & & 1 \\
\hline E-ACC/M-CTT & & & & & & 1 \\
\hline E-AAC/M-CTT & & & & 2 & & \\
\hline E-ACC/M-CAT & 1 & $\begin{array}{c}\text { (Lebanese } \\
\text { populations) }\end{array}$ & 1 & & 1 & \\
\hline E-ACT/M-CAC & $\begin{array}{c}1 \text { (Algerian and } \\
\text { French populations) }\end{array}$ & $\begin{array}{c}1 \text { (Turkish } \\
\text { populations) }\end{array}$ & 1 & & & \\
\hline E-ACT/M-CTA & 1 & & & 1 & 1 & \\
\hline E-ACA/M-CAA & & & & & & 1 \\
\hline E-ACA/M-CAG & & & & 1 & & 1 \\
\hline E-ACG/M-CAG & & & & & 1 & \\
\hline E-ACG/M-CTC & & & & 3 & & 1 \\
\hline
\end{tabular}




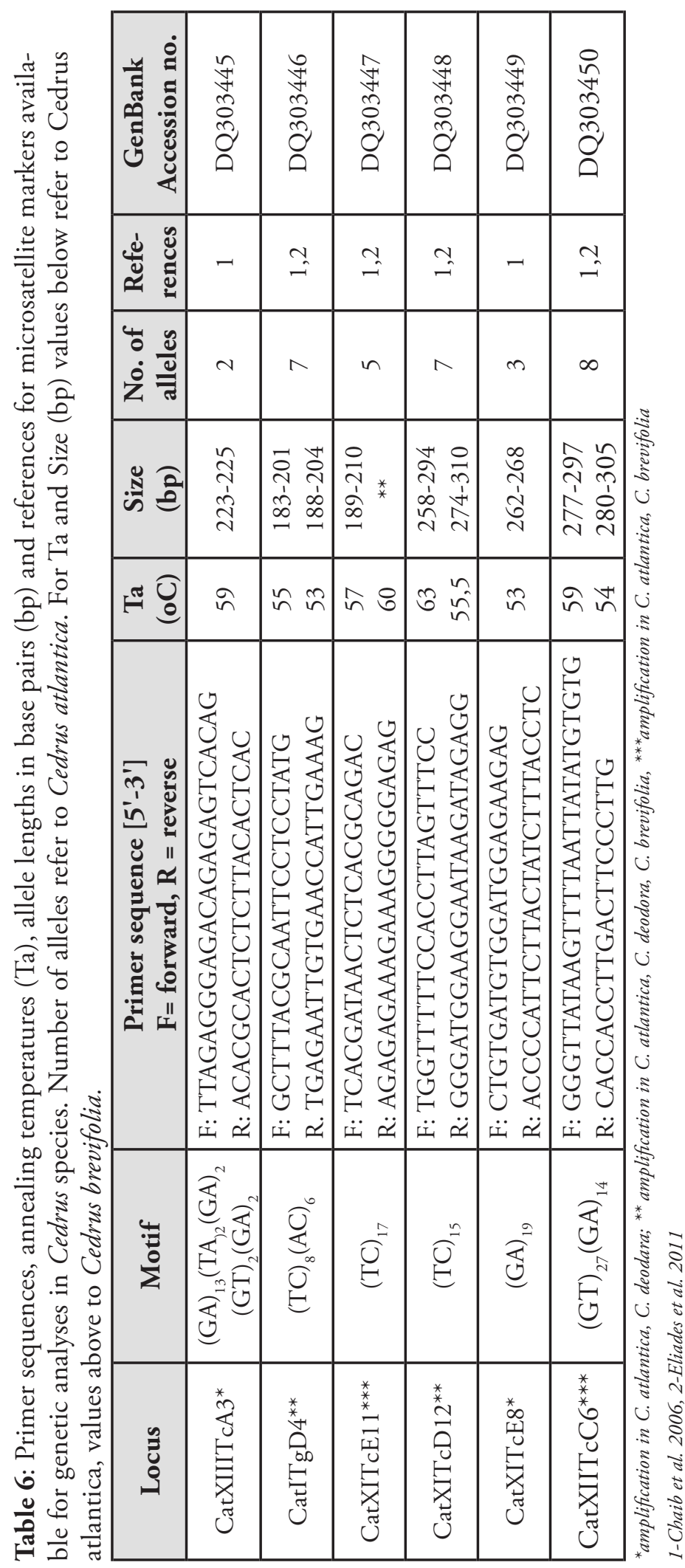


- primer CatXIITcC6:

$95^{\circ} \mathrm{C}$ for $5 \mathrm{~min}$, followed by 1 cycle at $80^{\circ} \mathrm{C}$ for 4 min, 30 cycles at $94^{\circ} \mathrm{C}$ for $1 \mathrm{~min}, 54^{\circ} \mathrm{C}$ for 1 min, $72^{\circ} \mathrm{C}$ for $1 \mathrm{~min}$, extension $72^{\circ} \mathrm{C}$ for $30 \mathrm{~min}$ - primer CatXITcE11:

Touch down PCR: $95^{\circ} \mathrm{C}$ for $15 \mathrm{~min}$, followed by 6 cycles at $94^{\circ} \mathrm{C}$ for $1 \mathrm{~min}, 60^{\circ} \mathrm{C}$ for $1 \mathrm{~min}$ (about $1^{\circ} \mathrm{C}$ per cycle) and $72^{\circ} \mathrm{C}$ for $1 \mathrm{~min}$, followed by 35 cycles at $94^{\circ} \mathrm{C}$ for $1 \mathrm{~min}, 54^{\circ} \mathrm{C}$ for $1 \mathrm{~min}$ and $72^{\circ} \mathrm{C}$ for $1 \mathrm{~min}$, extension $72^{\circ} \mathrm{C}$ for $30 \mathrm{~min}$

\section{Important results}

- The biparentally inherited codominant genetic nSSR markers should be helpful for species identification, diversity studies, parentage analysis and genome mapping (Chaib et al. 2006).

- Cedrus brevifolia, a narrow endemic island tree species from Cyprus, has high genetic diversity both at the nuclear and plastid level (see also chapter 2), suggesting no drift effects or genetic bottleneck (Eliades et al. 2011).

\section{c) SNPs (single-nucleotide polymorphisms)}

\section{Loci and primers used}

Restriction site associated DNA sequencing (RADsequencing) and mRNA sequencing were used in a recent genome mapping study of Cedrus atlantica conducted by Karam et al. (2015) in a panel of one single individual and three pools of three individuals each.

17,348 single nucleotide polymorphisms (SNPs) were identified in the RADseq data set and 5,714 simple sequence repeats (SSRs) in the transcriptome. Various stringent filters were applied resulting in 400 highquality SNPs along with 192 SSRs. A subset of 282 SNPs was validated using the Fluidigm genotyping technology. The 192 in silicodetected high-quality SSRs still need to be tested for validation by sequencing. The authors consider that the high-quality molecular markers they developed constitute a valuable resource for future population genetic studies.

Detailed information on the four hundred SNPs selected within the RADseq dataset, functional annotation of the contigs where they are located and results of the Fluidigm genotyping is provided as supplementary material (Appendix S4) by Karam et al. (2015). Appendix S5 from the same publication contains information for SSRs as the pure SSR and clean flanking regions.

\section{Important results}

The high-quality molecular markers developed constitute a valuable resource for future population genetic studies.

\section{References}

Bariteau, M., Panetsos, K.P., M'hint, O., Scaltsoyianos, A. (1999): Genetic variation of Atlas Cedar compared to that of other Mediterranean cedars. Forets Mediterranneennes, 4: 175-190.

Chaib, J., Danan, S., Jouaud, B., Hagen, L.S., Lefèvre, F., Fady, B. (2006): Identification and characterization of nuclear microsatellites in Mediterranean cedars (Cedrus sp.). Molecular Ecology Resourses, 6(3): 840-842.

Cheddadi, R., Fady, B., Francois, L., Hajar, L., J.P. Suc, Huang, K., Demarteau, M., Vendramin, G.G., Orti, E. (2009): Putative glacial refugia of Cedrus atlantica deduced from Quaternary pollen records and modern genetic diversity. Journal of Biogeography, 36: 13611371.

Cheliak, W.M., Pitel, J.A. (1984): Techniques for starch gel electrophoresis of enzymes from forest tree species. Information Report PI-X-42. Petawawa National Forestry Institute, Chalk River, Ontario, p. 49.

Conkle, M.T., Hodgskiss, P.O., Nunnally, L.B., Hunter, S.C. (1982): Starch gel electrophoresis of conifer seeds: A laboratory manual. U.S.D.A. Gen. Techn. Rept. PSW64, p. 18.

Cullings, K.W. (1992): Design and testing of a plantspecific primer for ecological and evolutionary studies. Molecular Ecology, 12: 2087-2097.

Dagher-Kharrat, M.B., Mariette, S., Lefèvre, F., Fady, B., Grenier-de March, G., Plomion, Ch., Savouré, A. (2006): Geographical diversity and genetic relationships among Cedrus species estimated by AFLP. Tree Genetics \& Genomes, 3(3): 275-285.

Dellaporta, S.L., Wood, J., Hicks, J.B. (1983): A plant DNA minipreparation: version II. Plant Molecular Biology Reporter, 1(4): 19-21.

Doyle, J.J., Doyle, J.L. (1990): Isolation of plant DNA from fresh tissue. Focus, 12: 13-15.

Eliades, N.G.H., Gailing, O., Leinemann, L., Fady, B., Finkeldey, R. (2011): High genetic diversity and significant population structure in Cedrus brevifolia Henry, a narrow endemic Mediterranean tree from Cyprus. Plant Systematics and Evolution, 294(3): 185198. 
Fady, B., Bariteau, M., Fallour, D., Giroud, E., Lefèvre, F. (2000): Isozyme gene markers and taxonomy of Mediterranean Cedrus species. - In: Panetsos, K. (ed.): Adaptation and selection of Mediterranean Pinus and Cedrus for sustainable afforestation of marginal lands. Giahoudi-Giapouli, Thessaloniki, Greece, p. 21-26.

Fady, B., Conkle, M.T. (1992): Segregation and linkage in allozymes in seed tissues of the hybrid Greek fir Abies borisii regis Mattfeld. Silvae Genetica, 41: 273-278.

Fady, B., Lefèvre, F., Reynaud, M., Vendramin, G.G., Dagher-Kharrat, M.B., Anzidei, M., Pastorelli, R., Savouré, A., Bariteau, M. (2003): Gene flow among different taxonomic units: evidence from nuclear and cytoplasmic markers in Cedrus plantation forests. Theoretical and Applied Genetics, 107: 1132-1138. DOI $10.1007 / s 00122-003-1323-z$

Fady, B., Lefèvre, F., Vendramin, G.G., Ambert, A., Régnier, C., Bariteau, M. (2008): Genetic consequences of past climate and human impact on eastern Mediterranean Cedrus libani forests. Implications for their conservation. Conservation Genetics, 9(1): 85-95.

Fallour, D., Fady, B., Lefèvre, F. (2001): Evidence of variation in segregation patterns within a Cedrus population. J Heredity, 92: 260-266.

Gülbaba, A.G., Özkurt, N. (2002): Isozyme diversity in Cedar (Cedrus libani A. Rich) populations sampled from Bolkar mountains. Ministry of Forestry. Publication No. 191 Eastern Mediteranean Forestry Research Institute. Publ. No. 23, Tech. Bull. 14, p. 30 (in Turkish, English summary).

Karam, M.J., Lefevrè, F., Dagher-Kharrat, M.B., Pinosio, S., Vendramin, G.G. (2015): Genomic exploration and molecular marker development in a large and complex conifer genome using RADseq and mRNAseq. Molecular Ecology Resources, 15: 601-612.

Kayihan, G.C., Kaya, Z., Kandemir, G., Önde, S. (2006): The genetic structure of Cedrus libani A. Rich seed stands determined by random amplified polymorphic DNA markers. Forest Genetics, 12(3): 181-190.

Kreike, J. (1990): Genetic Analysis of Forest Tree Populations: Isolation of DNA from spruce and fir apices. Plant Molecular Biology, 14(5): 877-879.

Kurt, Y., Kaya, N., Isik, K. (2008): Isozyme variation in four natural populations of Cedrus libani A. Rich. in Turkey. Tur J Agric For, 32: 137-145.

Lefebvre, V., Palloix, A., Caranta, C., Pochard, E. (1995): Construction of an intraspecific integrated linkage map of pepper using molecular markers and doubled haploid progenies. Genome, 38(1): 112-121.
Neubauer, S.G., Del Castillio-Agudo, L., Segura, J. (2000): An assesment of genetic relationships within the genus Digitalis based on PCR-generated RAPD markers. Theoretical and Applied Genetics, 100: 1209-1216.

Panetsos, K.P., Christou, A., Scaltsoyiannes, A. (1992): First Analysis on Allozyme Variation in Cedar Species (Cedrus sp.). Silvae Genetica, 41(6): 339-342.

Panetsos, K.P., Scaltsoyiannes, A., Tsaktsira, M. (1994): Genetic variation in allozymes of Cedrus libani A. Rich and Cedrus atlantica Manetti. Annales de la Recherche Forestiere au Maroc, 27: 420-434.

Renau-Morata, B., Nebauer, S.G., Sales, R., Allainguillaume, J., Caligari, P., Segura, J. (2005): Genetic diversity and structure of natural and managed populations of Cedrus atlantica (Pinaceae) assessed using random amplified polymorphic DNA. American Journal of Botany, 92(5): 875-884.

Scaltsoyiannes, A. (1999): Allozyme differentiation and phylogeny of cedar species. Silvae Genetica, 48: 61-68.

Semaan, M.T., Dodd, R.S. (2008): Genetic variability and structure of the remnant natural populations of Cedrus libani (Pinaceae) of Lebanon. Tree Genetics \& Genomes, 4(4): 757-766.

Terrab, A., Paun, O., Talavera, S., Tremetsberger, K., Arista, M., Stuessy, T.F. (2006): Genetic diversity and population structure in natural populations of Moroccan Atlas cedar (Cedrus atlantica; Pinaceae) determined with cpSSR markers. American Journal of Botany, 93(9): 1274-1280.

Vendramin, G.G., Lelli, L., Rossi, P., Morgante, M. (1996): A set of primers for the amplification of 20 chloroplast microsatellites in Pinaceae. Molecular Ecology, 5(4): 595-598.

Wakasugi, T., Tsudzuki, S.I., Shibata, M., Sugiura, M. (1994): A physical map and clone bank of the black pine (Pinus thunbergii) chloroplast genome. Plant Molecular Biology Reporter, 12: 395-420.

Yahyaoglu, Z., Turna, I., Cakmak, F. (1997): Genetic analysis of isozymes variation in Lebanon Cedar (Cedrus libani A. Rich). Abstracts Proc. XI World Forestry Congress, 13-22 Oct. Antalya, pp. 230. 


\title{
Molecular markers used for genetic studies in Japanese Larch (Larix kaempferi (Lamb.) Carr.)
}

\author{
Jean-Charles Bastien ${ }^{1}$, Vanina Guerin ${ }^{1}$, Anna-Maria Szasz-Len², Monika Konnert ${ }^{2}$ \\ ${ }^{1}$ INRA Centre Val de Loire - Integrated biology for the valorisation of tree and forest diversity, 2163 Avenue de la Pomme de Pin, CS 40001 \\ ARDON-45075 Orleans Cedex 2, France \\ ${ }^{2}$ Bavarian Office for Forest Seeding and Planting, Forstamtsplatz 1, 83317 Teisendorf, Germany
}

\section{General remarks}

Larch is one of the most abundant conifers in the northern hemisphere where it grows at both high latitudes and high elevations. It comprises around ten species distributed over North America, Europe and Asia.

Japanese larch is native on Honshu Island, where it grows at 1300 to $2900 \mathrm{~m}$ elevation. Faster growing than European larch at early stage, it has usually been preferred in Western Europe's oceanic areas. Presently, hybrid larch (Larix x eurolepis, hybrid between European and Japanese larches), which is more resistant to cold and drought, tends to replace Japanese larch in the reforestation of northern Europe.

Since 2009, infection of Japanese larch by the Phytophtora ramorum has led to a severe decline of the species in the British Isles. For this reason, the species is no more planted there.

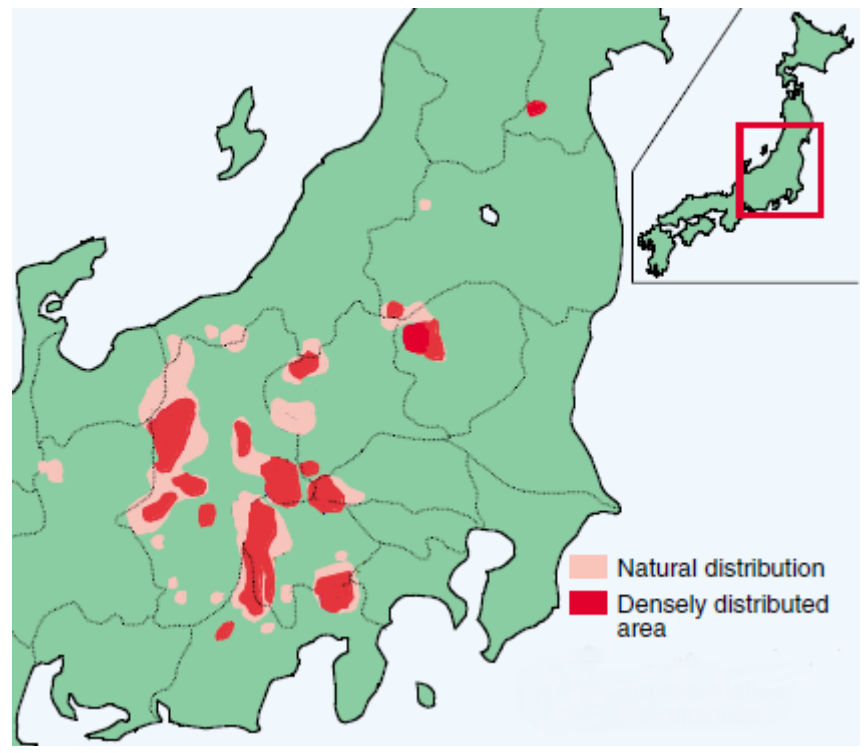

Figure 1. Natural distribution of Japanese Larch in Japan (in Hoshi 2004)
Due to literature scarcity on the use of molecular markers to implement genetic studies on Larix kaempferi, references in the present document are often given for related species (Larix sibirica, Larix gmelinii, Larix decidua, Larix eurolepis) when available.

\section{Isozymes}

Genetic studies on Larix kaempferi based on isozyme markers have been generally used to identify interspecific hybrid seeds (embryos) between Larix kaempferi and Larix decidua (e.g. Bergmann and Ruetz 1987, Häcker and Bergmann 1991, Tröber and Hasemann 2000). Isozyme patterns are very similar for the two species in the number of scored loci, but for some loci the relative position of bands differs. For both species the same analysis method can be applied. As detailed studies on genetic variation for Larix kaempferi are missing, references in Table 1 refer not only to Larix kaempferi, but also to Larix decidua.

\section{Material for protein extraction (only Larix kaempferi)}

Proteins were extracted from dormant buds and seeds (both endosperm and embryos) (Bergmann and Ruetz 1987, Häcker and Bergmann 1991, Tröber and Hasemann 2000).

\section{Protein extraction and separation protocols}

Isozyme extraction, separation by starch gel electrophoresis and staining of gels were carried out based on standard procedures decribed by Siciliano and Shaw (1976), Cheliak and Pitel (1985), Häcker and Bergmann (1991), Müller-Starck and Starke (1993) and Konnert and Maurer (1995).

\section{Important results (only Larix kaempferi and hybrids)}

Estimation of the proportion of hybrid seed from seed orchards consisting of Larix kaempferi and Larix decidua 
clones was possible (Bergmann and Ruetz 1987, Häcker and Bergmann 1991, Tröber and Hasemann 2000) based on the loci SKDH-A and NADH-A. At these two loci the two larch species could be unambiguously distinguished by the position of bands in the zymogram and, therefore, the proportion of hybrids and selfings (individual and clonal) could be exactly determined.

Table 1: List of enzymes, scored loci and number of alleles for Larix decidua and Larix kaempferi

\begin{tabular}{|c|c|c|c|c|}
\hline Enzyme system & E.C. Number & Scored loci & $\begin{array}{l}\text { No. of } \\
\text { alleles* }\end{array}$ & References \\
\hline Aspartate aminotransferase & 2.6.1.1 & AAT-A,-B -C & $3,4,3$ & $1,5,9$ \\
\hline Diaphorase & 1.8.1.4 & Dia & & 9 \\
\hline Esterase & 3.1 .1 .2 & EST-A,-C & 2,4 & $1,3,4,9$ \\
\hline Glutamate dehydrogenase & 1.4.1.2 & GDH-A & 3 & $2,3,4,5,9$ \\
\hline Glucose-6-phospate dehydrogenase & 1.1.1.49 & G6PDH-A & 3 & $2,3,4,9$ \\
\hline Glyceraldehyde-3-phosphate-dehydrogenase & 1.2.1.9 & G3PDH-A,-B, & 4,2 & 1 \\
\hline Isocitrate dehydrogenase & 1.1.1.42 & IDH-A,-B & 1,3 & $1,2,3,4,5,9$ \\
\hline Leucine aminopeptidase & 3.4.11.1 & LAP-A,-B & 4,4 & $3,4,5$ \\
\hline Malate dehydrogenase & 1.1.1.37 & MDH-A,-B,-C,-D & $3,3,4,3$ & $1,2,3,4$ \\
\hline Menadione reductase & 1.6.99.2 & MNR-B,-C,-D & $1,4,2$ & $1,3,4$ \\
\hline Phosphoglucose isomerase & 5.3.1.9 & PGI-A,-B & 2,3 & $1,5,6$ \\
\hline Phosphoglucomutase & 2.7.5.1 & PGM-A & 6 & $1,5,9$ \\
\hline 6-Phosphogluconate dehydrogenase & 1.1.1.44 & 6PGDH-A,-B & 3,3 & $1,2,5,9$ \\
\hline Triose-phosphate isomerase & 5.3.1.1 & TPI-A,-B & 1,2 & 1 \\
\hline Shikimate dehydrogenase & 1.1.1.25 & SKDH-A & 6 & $\begin{array}{c}1,2,3,4,5 \\
6,7,8\end{array}$ \\
\hline Superoxide dismutase & 1.15.1.1. & SOD-A,-B & 1,3 & $3,4,9$ \\
\hline Sorbitol dehydrogenase & 1.1.1.14 & SrDH-A & 2 & 3,4 \\
\hline NADH dehydrogenase & 1.6.99.3 & NDH-A & 2 & 6 \\
\hline
\end{tabular}

*-values for Larix decidua except SKDH-A and NADH-A which refer also to Larix kaempferi

1-Beletti et al. 1996, 2-Maier 1992, 3-Lewandowski and Mejnartowicz 1988, 4- Lewandowski and Mejnartowicz 1992, 5-Müller-Starck and Felber 2010, 6-Häcker and Bergmann 1991, 7-Bergmann and Ruetz 1987, 8-Tröber and Haasemann 2000, 9-Semerikov and Lascoux 1999 


\section{Organelle DNA markers (chloroplast (cp) DNA, mitochondrial (mt)DNA)}

Detailed information on markers used for DNA analyses from organelle and nuclear DNA from Larix species is given in Heinze et al. (2012). In the present guidelines, the focus is on Larix kaempferi. Thus, only primers working also for this species are included.

\section{Loci and primers used}

According to Heinze et al. (2012), chloroplast DNA (cpDNA) variation has been studied based on PCRRFLPs (Semerikov and Lascoux 2003, Acheré et al. 2004), sequence variation (Wei and Wang 2003, Gros-Louis et al. 2005) and microsatellites (Semerikov and Lascoux 2003). Mitochondrial DNA (mtDNA) variation has been studied using direct PCR, PCR-RFLPs (Semerikov and Lascoux 2003, Acheré et al. 2004, Semerikov and Polezhaeva 2007) and sequencing (Gros-Louis et al. 2005).

Acheré et al. (2004) applied PCR-RFLP markers on cpDNA (paternally inherited) and mtDNA (maternally inherited) to identify European $\mathrm{x}$ Japanese larch hybrids. They used universal primers (Taberlet et al. 1991, Demesure et al. 1995, Dumolin-Lapégue et al. 1997, Petit et al. 1998). For cpDNA, ten out of 22 tested primer pairs gave clear amplification products in Larix kaempferi and Larix decidua. Only these primers are introduced in Table 2. Amplification products were digested with five restriction enzymes - TaqI, HapII, HhaI, HaeIII and $B c$ II. For mtDNA eight of the eleven tested primer pairs amplify (see also table 2).

For PCR-RFLPs, Semerikov et al. (2003, 2006) and Semerikov and Lascoux (2003) used also published universal primers (Taberlet et al. 1991, Demesure et al. 1995, Dumolin-Lapégue et al. 1997, Parducci and Szmidt 1999) to amplify cpDNA and mtDNA fragments. cpDNA amplified fragments were cut with AluI, HaeIII, Hinfl, HpaII, MboI, RsaI, Sfl3.

\section{Material for DNA-extraction}

DNA was extracted from buds, needles or germinated seed (Semerikov and Lascoux 2003, Semerikov et al. 2003, Acheré et al. 2004, Gros-Louis et al. 2005, Wei and Wang 2003, Polezhaeva et al. 2010, San Jose-Maldia et al. 2009).

\section{DNA-extraction and amplification protocols}

Total DNA was extracted from the mentioned tissue using:

- the CTAB protocol of Devey et al. (1996) cited in Ostrowska et al. (1998) (Semerikov and Lascoux 2003)

- the CTAB protocol of Rogers and Bendich (1988) (Wei and Wang 2003)

- the QIAGEN DNeasy Kit (Acheré et al. 2004, GrosLouis et al. 2005, Pluess 2011)

- NucleoSpin Plant II (Macherey Nagel, used in INRA lab, unpublished)

Examples for amplification protocols (PCR-RFLP)

- $\quad 94^{\circ} \mathrm{C}$ for $6 \mathrm{~min}$ followed by 35 cycles of $94^{\circ} \mathrm{C}$ for 45 $\mathrm{s}, 55^{\circ} \mathrm{C}$ for $45 \mathrm{~s}, 70^{\circ} \mathrm{C}$ for $3 \mathrm{~min}, 30 \mathrm{~s}$ (Acheré et al. 2004).

- $\quad 94^{\circ} \mathrm{C}$ for 5 min followed by 35 cycles of $94^{\circ} \mathrm{C}$ for $30 \mathrm{~s}, 65^{\circ} \mathrm{C}$ for $45 \mathrm{~s}$ (UBC460), $50^{\circ} \mathrm{C}$ for nad $4-3 / 4$, $55^{\circ} \mathrm{C}$ for nad5-1/2, atpA1-R, elongation 3 min for UBC 460 and 2 min for the rest of primers at $72^{\circ} \mathrm{C}$, final elongation at $72^{\circ} \mathrm{C}$ for $10 \mathrm{~min}$ (Polezhaeva et al. 2010).

Examples for amplification protocols (cpSSR):

- $\quad 94^{\circ} \mathrm{C}$ for $5 \mathrm{~min}$ followed by 35 cycles of $94^{\circ} \mathrm{C}$ for 30 s, $55^{\circ} \mathrm{C}$ for $45 \mathrm{~s}, 72^{\circ} \mathrm{C}$ for 30 s; final elongation at $72^{\circ}$ $\mathrm{C}$ for $10 \mathrm{~min}$ (Polezhaeva et al. 2010).

- $\quad 94^{\circ} \mathrm{C}$ for $5 \mathrm{~min}$ followed by 35 cycles of $94^{\circ} \mathrm{C}$ for 30 $\mathrm{s}, 55^{\circ} \mathrm{C}$ for $30 \mathrm{~s}, 72^{\circ} \mathrm{C}$ for $30 \mathrm{~s}$; final elongation at $72^{\circ}$ C for 6 min (Semerikov and Lascoux 2003).

Example for amplification protocols (cpDNA sequencing)

- $\quad 94^{\circ} \mathrm{C}$ for $3 \mathrm{~min}$ followed by 35 cycles of $94^{\circ} \mathrm{C}$ for 1 $\min , 56^{\circ} \mathrm{C}$ for $1 \mathrm{~min}, 72^{\circ} \mathrm{C}$ for $1 \mathrm{~min}$ and $20 \mathrm{~s}$; final elongation at $72^{\circ} \mathrm{C}$ for $10 \mathrm{~min}$ (Gros-Louis et al. 2005).

Example for amplification protocols ( $m$ tDNA sequencing)

- $\quad 94^{\circ} \mathrm{C}$ for $2 \mathrm{~min}$ followed by 35 cycles of $94^{\circ} \mathrm{C}$ for 30 $\mathrm{s}, 60^{\circ} \mathrm{C}$ for $30 \mathrm{~s}, 72^{\circ} \mathrm{C}$ for $1 \mathrm{~min}$; final elongation at $72^{\circ} \mathrm{C}$ for $10 \mathrm{~min}$ (Gros-Louis et al. 2005).

\section{Important results}

- Four cpDNA (matK, trnL-intron, trnT-trnL trnLtrnF) and five mtDNA markers (cox1-1, matR1, nad1-b/c, nad3-1 and nad5-1) were developed to distinguish unambiguously four larch species (Larix laricina, Larix decidua, Larix kaempferi, and Larix sibirica) used in intensive forestry in Western Europe or eastern North America and trace forest reproductive material (Acheré et al. 2004, GrosLouis 2005). 
- By combining the mitochondrial PCR-RFLP marker f13 and the chloroplast PCR-RFLP marker rbcLTaqI, Larix decidua and Larix kaempferi could be discriminated (Acheré et al. 2004). The two markers are sufficient to identify first-generation hybrid individuals.

- Japanese larch is found to be closely related to populations of Larix kamtschatica inhabiting the Kuril Islands and South Sakhalin (Polezhaeva et al. 2010).

- Despite the restricted natural distribution of Japanese larch, the mtDNA showed geographic structure (San Jose-Maldia et al. 2009).

Table 2: PCR-RFLP markers (cpDNA, mtDNA) used for Larix kaempferi and other larix species

\begin{tabular}{|c|c|c|c|c|}
\hline Type & $\begin{array}{c}\text { Amplified } \\
\text { region }\end{array}$ & $\begin{array}{c}\text { Primer sequence } \\
5^{c}-3^{c} \\
\end{array}$ & References & Source of primer pairs \\
\hline \multirow{13}{*}{ cpDNA } & $\operatorname{trn} T-\operatorname{trnF}$ & $\begin{array}{l}\text { CATTACAAATGCGATGCTCT } \\
\text { ATTTGAACTGGTGACACGAG }\end{array}$ & 1,3 & Taberlet et al. (1991) \\
\hline & rpl20-trnW & $\begin{array}{l}\text { T3 + TTTTCGAACTGCTAACCAACG } \\
\text { (T3 = AATTAACCCTCACATAAGGG) } \\
\text { T7 + ACCTACGGCATCAGGTTTTG } \\
\text { (T7=GTAATACGACTCACTATAGGGC) }\end{array}$ & 1,2 & $\begin{array}{l}\text { Parducci and Szmidt } \\
\text { (1999), modified by } \\
\text { Semerikov et al. (2006) }\end{array}$ \\
\hline & $\operatorname{trnL}-\operatorname{trn} V$ & $\begin{array}{l}\text { CTGCTTCCTAAGAGCAGCGT } \\
\text { TTGACATGGTGGAAGTCATCA }\end{array}$ & 1 & $\begin{array}{l}\text { Parducci and Szmidt } \\
\text { (1999) }\end{array}$ \\
\hline & psbC-trnS & $\begin{array}{l}\text { GGTCGTGACCAAGAAACCAC } \\
\text { GGTTCGAATCCСTCTCTCTC }\end{array}$ & 1,3 & $\begin{array}{l}\text { Parducci and Szmidt } \\
\text { (1999) }\end{array}$ \\
\hline & psbD-16S & $\begin{array}{l}\text { CCACAAAAACGAAACGGTCT } \\
\text { ACTAACTAATCAGACGCGAGCC }\end{array}$ & 1 & $\begin{array}{l}\text { Parducci and Szmidt } \\
\text { (1999) }\end{array}$ \\
\hline & $\mathrm{rbcL}$ & $\begin{array}{l}\text { ATGTCACCACAAACAGAAACTAAAGCAAGTA } \\
\text { CTTCACAAGCAGGAGCTAGTTCAGGACTCC }\end{array}$ & 3 & Petit et al. (1998) \\
\hline & $\operatorname{trnK}$ & $\begin{array}{l}\text { GGGTTGCCCGGGACTCGAAC } \\
\text { CAACGGTAGAGTACTCGGCTTTTA }\end{array}$ & 3 & Demesure et al. (1995) \\
\hline & $\operatorname{trnK}-\operatorname{trn} Q$ & $\begin{array}{l}\text { TAAAAGCCGAGTACTCTACCGTTG } \\
\text { CTATTCGGAGGTTCGAATCCTTCC }\end{array}$ & 3 & $\begin{array}{l}\text { Dumolin-Lapégue et al. } \\
\text { (1997) }\end{array}$ \\
\hline & $\operatorname{trn} Q-\operatorname{trn} R$ & $\begin{array}{l}\text { GGGACGGAAGGATTCGAACC } \\
\text { ATTGCGTCCAATAGGATTTGAA }\end{array}$ & 3 & $\begin{array}{l}\text { Dumolin-Lapégue et al. } \\
\text { (1997) }\end{array}$ \\
\hline & $\operatorname{trnS}-\operatorname{trnfM}$ & $\begin{array}{l}\text { GAGAGAGAGGGATTCGAACC } \\
\text { CATAACCTTGAGGTCACGGG }\end{array}$ & 3 & Demesure et al. (1995) \\
\hline & $\operatorname{trnS}-\operatorname{trn} \mathrm{T}$ & $\begin{array}{l}\text { CGAGGGTTCGAATCCCTCTC } \\
\text { AGAGCATCGCATTTGTAATG }\end{array}$ & 3 & Demesure et al. (1995) \\
\hline & atpF-rps2 & Primer sequence not published & 3 & Acheré et al. (2004) \\
\hline & trnR-atpF & Primer sequence not published & 3 & Acheré et al. (2004) \\
\hline
\end{tabular}




\begin{tabular}{|c|c|c|c|c|}
\hline Type & $\begin{array}{l}\text { Amplified } \\
\text { region }\end{array}$ & $\begin{array}{c}\text { Primer sequence } \\
5^{c}-3^{c} \\
\end{array}$ & References & Source of primer pairs \\
\hline \multirow{21}{*}{ mtDNA } & nad5-1/2 & $\begin{array}{l}\text { TTTTTTCGGACGTTTTCTAG } \\
\text { TTTGGCCAAGTATCCTACAA }\end{array}$ & 1 & Wu et al. (1998) \\
\hline & nad4-3c/4r & $\begin{array}{l}\text { GGAGCTTTCCAAAGAAATAG } \\
\text { GCCATGTTGCACTAAGTTAAC }\end{array}$ & 1 & $\begin{array}{l}\text { Dumolin-Lapégue et al. } \\
\text { (1997) }\end{array}$ \\
\hline & F13 & $\begin{array}{l}\text { CTGTTGGTAACTTGGGG } \\
\text { GCGCCTCTTTCGGAATAG }\end{array}$ & 3 & Acheré et al. (2004) \\
\hline & UBC460 & $\begin{array}{l}\text { AACCTAGAGCCAACAGCAGCACCT } \\
\text { CCCAACTTCCTCGAAAGCAGATG }\end{array}$ & 4,5 & Semerikov et al. (2006) \\
\hline & C8 & $\begin{array}{l}\text { GGATCGTAGCGTGGGAACTA } \\
\text { AGGGAACTTGTGAACGTTGG }\end{array}$ & 4 & Semerikov et al. (2006) \\
\hline & B11 & $\begin{array}{l}\text { TACCCGCCTTAACCGTAAGA } \\
\text { GACCCGTAGTTTGGCTGAGA }\end{array}$ & 4 & Semerikov et al. (2006) \\
\hline & R11 & $\begin{array}{l}\text { CATCCCGTCGCTTGTTTAAT } \\
\text { CCGGTTGGCACCTTAAATAGA }\end{array}$ & & Semerikov et al. (2006) \\
\hline & $\operatorname{Cox} 2$ & $\begin{array}{l}\text { TTTTCTTCСТСАТТСТКАТTT } \\
\text { ССАСТСТАТТGTССАСТТСТА }\end{array}$ & 3 & $\begin{array}{l}\text { Dumolin-Lapégue et al. } \\
\text { (1997) }\end{array}$ \\
\hline & nad $1-2 / 3$ & $\begin{array}{l}\text { GCATTACGATCTGCAGCTCA } \\
\text { GGAGCTCGATTAGTTTCTGC }\end{array}$ & 3,4 & Demesure et al. (1995) \\
\hline & Nad3-rps 12 & $\begin{array}{l}\text { AATTGTCGGCCTACGAATGTG } \\
\text { GCTCG }(\mathrm{A}=\mathrm{I}) \text { GTACGGTC(C=I)GTGCG }\end{array}$ & 3 & Wu et al. (1998) \\
\hline & Nad4-1/2 & $\begin{array}{l}\text { CAGTGGGTTGGTCTGGTATG } \\
\text { TCATATGGGCTACTGAGGAG }\end{array}$ & 3,4 & Demesure et al. (1995) \\
\hline & Nad4-2/3 & $\begin{array}{l}\text { CTCCTCAGTAGCCCATATGA } \\
\text { AACCAGTCCATGACTTAACA }\end{array}$ & 3 & $\begin{array}{l}\text { Dumolin-Lapégue et al. } \\
\text { (1997) }\end{array}$ \\
\hline & Nad4-3/4 & $\begin{array}{l}\text { GGAGCTTTCCAAAGAAATAG } \\
\text { GCCATGTTGCACTAAGTTAC }\end{array}$ & 5 & $\begin{array}{l}\text { Dumolin-Lapégue et al. } \\
\text { (1997) }\end{array}$ \\
\hline & Nad4-2/4 & $\begin{array}{l}\text { TGTTTCCCGAAGCGACACTT } \\
\text { GGAACACTTTGGGGTGAACA }\end{array}$ & 4 & Demesure et al. (1995) \\
\hline & Nad5-1/2 & $\begin{array}{l}\text { GAAATGTTTGATGCTTCTTGGG } \\
\text { ACCAACATTGGCATAAAAAAAGT }\end{array}$ & $3,4,5$ & Wu et al. (1998) \\
\hline & Rps14-cob & $\begin{array}{l}\text { CACGGGTCGCCCTCGTTCCG } \\
\text { GTGTGGAGGATATAGGTTGT }\end{array}$ & 3 & Demesure et al. (1995) \\
\hline & Mh02 & $\begin{array}{l}\text { TTTTAGGGCCATTTGCCTGC } \\
\text { TCTATGGACAAGAGCCCGACCT }\end{array}$ & 4 & Jeandroz et al. (2002) \\
\hline & Mh09‘ & $\begin{array}{l}\text { CCATCCAGCCATGTCTCATC } \\
\text { AGGGCTTCACATAGAGCATC }\end{array}$ & 4 & Jeandroz et al. (2002) \\
\hline & Mh27 & $\begin{array}{l}\text { TGCTTTCCAATTTACCACGAG } \\
\text { GATACGCTTTCCTGGCATAC }\end{array}$ & 4 & Jeandroz et al. (2002) \\
\hline & Mh50 & $\begin{array}{l}\text { AGAATGGCAGCAACTAATAAGC } \\
\text { ACTATGCACTTCCСТCССTCA }\end{array}$ & 4 & Jeandroz et al. (2002) \\
\hline & Atp1-R & $\begin{array}{l}\text { GCTGGCAAATTCAACCATTT } \\
\text { GCAATTAGGCTGGCTTTCC }\end{array}$ & 5 & Polezhaeva et al. (2010) \\
\hline
\end{tabular}


Table 3: Primer information for amplification of chloroplast microsatellites (cpSSRs) and variable fragments for sequencing (cpDNA and mtDNA) in genetic analysis of Larix species (including Larix kaempferi) $(\mathrm{Ta}=$ annealing temperature)

\begin{tabular}{|c|c|c|c|c|c|c|}
\hline Locus & Type & $\begin{array}{l}\text { Primer sequence }\left[5^{\prime}-3^{\prime}\right] \\
F=\text { forward, } R=\text { reverse }\end{array}$ & $\begin{array}{cc}\mathbf{T} & \mathbf{a} \\
\left({ }^{\circ} \mathrm{C}\right)\end{array}$ & $\begin{array}{l}\text { Size } \\
(\mathbf{b p})\end{array}$ & Ref. & $\begin{array}{c}\text { Source of } \\
\text { primer pa- } \\
\text { irs }\end{array}$ \\
\hline Pt9383 & \multirow{7}{*}{ cpSSR } & $\begin{array}{l}\text { F: AGAATAAACTGACGTAGATGCCA } \\
\text { R: AATTTTCAATTCCTTTCTTTCTCC }\end{array}$ & 48 & 118 & 1 & $\begin{array}{l}\text { Vendramin et } \\
\text { al. (1996) }\end{array}$ \\
\hline $\mathrm{Pt} 9393$ & & \begin{tabular}{|l} 
F:GACGTAGATGCTATGGGTACG \\
R:GAGAGCGGTATGAGGGAAGA \\
\end{tabular} & 55 & 135 & 2 & $\begin{array}{l}\text { Polezhaeva et } \\
\text { al. (2010) }\end{array}$ \\
\hline Pt9833 & & $\begin{array}{l}\text { F:GACGATGGACGCTCTTTCTC } \\
\text { R:GATCGGGCGGGATAATGTA } \\
\end{array}$ & 55 & 84 & 2 & $\begin{array}{l}\text { Polezhaeva et } \\
\text { al. (2010) }\end{array}$ \\
\hline $\mathrm{Pt} 30$ & & $\begin{array}{l}\text { F:TCAATCCTAACCATATCAGGTG } \\
\text { R:TCATAGCGGAAGATCCTCTTT }\end{array}$ & 55 & 139 & 2 & $\begin{array}{l}\text { Polezhaeva et } \\
\text { al. (2010) }\end{array}$ \\
\hline Pt26081 & & \begin{tabular}{|l} 
F:CCCGTATCCAGATATACTTCCA \\
R:TGGTTTGATTCATTCGTTCAT
\end{tabular} & 55 & 112 & 1 & $\begin{array}{l}\text { Vendramin et } \\
\text { al. (1996) }\end{array}$ \\
\hline TrnLV & & $\begin{array}{l}\text { F:AAATACCACGGGCCTCCTA } \\
\text { R:TTGACATGGTGGAAGTCATCAT }\end{array}$ & 55 & 86 & 2 & $\begin{array}{l}\text { Polezhaeva } \\
\text { et al. (2010) }\end{array}$ \\
\hline Pt30204 & & \begin{tabular}{|l} 
TCATAGCGGAAGATCCTCTTT \\
CGGATTGATCCTAACCATACC
\end{tabular} & 55 & 145 & 1 & $\begin{array}{l}\text { Vendramin } \\
\text { et al. (1996) }\end{array}$ \\
\hline matK & \multirow{5}{*}{$\begin{array}{c}\text { cpDNA } \\
\text { amplification } \\
\text { and } \\
\text { sequencing }\end{array}$} & $\begin{array}{l}\text { F:GAACTCGTCGGATGGAGTG } \\
\text { R:GAGAAATCTTTTTCATTACTACAGTG }\end{array}$ & 56 & & 3 & $\begin{array}{l}\text { Wang et al. } \\
(1999)\end{array}$ \\
\hline trnL Intron & & $\begin{array}{l}\text { F:CGAAATCGGTAGACGCTACG } \\
\text { R:GGGGATAGAGGGACTTGAAC } \\
\end{array}$ & 56 & & 3 & $\begin{array}{l}\text { Taberlet et } \\
\text { al. (1991) }\end{array}$ \\
\hline $\operatorname{trn} T-\operatorname{trnL}$ & & $\begin{array}{l}\text { F:CATTACAAATGCGATGCTCT } \\
\text { R:CGAAATCGGTAGACGCTACG }\end{array}$ & 56 & & 3 & $\begin{array}{l}\text { Taberlet et } \\
\text { al. (1991) }\end{array}$ \\
\hline $\operatorname{trnL}-\operatorname{trnF}$ & & $\begin{array}{l}\text { F:CGAAATCGGTAGACGCTACG } \\
\text { R:ATTTGAACTGGTGACACGAG }\end{array}$ & 56 & & 3 & $\begin{array}{l}\text { Taberlet et } \\
\text { al. (1991) }\end{array}$ \\
\hline $\operatorname{trn} T-\operatorname{trn} F$ & & $\begin{array}{l}\text { CATTACAAATGCGATGCTCT } \\
\text { ATTTGAACTGGTGACACGAG }\end{array}$ & 48 & & 4 & $\begin{array}{l}\text { Taberlet et } \\
\text { al. (1991) }\end{array}$ \\
\hline Cox1-1 & \multirow{5}{*}{$\begin{array}{c}\text { mtDNA } \\
\text { amplification } \\
\text { and } \\
\text { sequencing }\end{array}$} & \begin{tabular}{|l} 
F:TTATTATCACTTCCGGTACT \\
R:AGCATCTGGATAATCTGG \\
\end{tabular} & 60 & & 3 & $\begin{array}{l}\text { Lu et al. } \\
(1998)\end{array}$ \\
\hline matR-1 & & $\begin{array}{l}\text { F:CGACAGAAGCACGAAATTCC } \\
\text { R:ACCCGACGATAACTAGCTTC }\end{array}$ & 60 & & 3 & $\begin{array}{l}\text { Qiu et al. } \\
(1999)\end{array}$ \\
\hline nad1-b/c & & $\begin{array}{l}\text { F:GCATTACGATCTGCAGCTCA } \\
\text { R:GGAGCTCGATTAGTTTCTGC } \\
\end{array}$ & 60 & & 3 & $\begin{array}{l}\text { Demesure et } \\
\text { al. }(1995)\end{array}$ \\
\hline nad3-1 & & \begin{tabular}{|l} 
F:CAGAAGTCGTTTCGATATACG \\
R:ATTGATTCGATGTAGGCATCG \\
\end{tabular} & 60 & & 3 & $\begin{array}{l}\text { Soranzo et } \\
\text { al. (1999) }\end{array}$ \\
\hline nad5-1 & & $\begin{array}{l}\text { F:AGTCCAATAGGGACAGCACAC } \\
\text { R:GCTTTGATAGCTGCTTTATCTGC }\end{array}$ & 60 & & 3 & $\begin{array}{c}\text { Jaramillo- } \\
\text { Correa et al. } \\
(2003)\end{array}$ \\
\hline
\end{tabular}

1-Semerikov and Lascoux 2003, 2- Polezhaeva et al. (2010), 3- Gros-Louis et al. 2005, 4-Wei and Wang 2003 


\section{Randomly amplified polymorphic DNA (RAPD) markers}

\section{Primer used and important results}

Scheepers et al. (2000) analyzed the following 11 markers that differentiated Larix decidua and Larix kaempferi. Two of these markers were mitochondrial (maternally inherited) (DeVerno et al. 1993).

- $\mathrm{OPH}-11-2,2 \mathrm{~kb}-100 \%$ presence in Larix decidua

- OPD-15 - 1,4 kb - $100 \%$ presence in Larix decidua (mtDNA)

- OPE-17 - $0.8 \mathrm{~kb}-100 \%$ presence in Larix decidua

- OPF-05 - $2.25 \mathrm{~kb}-100 \%$ presence in Larix decidua

- OPG-12 - $1.3 \mathrm{~kb}-100 \%$ presence in Larix kaempferi

- OPH-14 - $1.45 \mathrm{~kb}-100 \%$ presence in Larix kaempferi

- OPC-16 - $1.38 \mathrm{~kb}-100 \%$ presence in Larix kaempferi

- OPC-06 - $0.93 \mathrm{~kb}-100 \%$ presence in Larix kaempferi

- OPR-08 - $1.2 \mathrm{~kb}-100 \%$ presence in Larix kaempferi (mtDNA)

- OPD-10 - $1.2 \mathrm{~kb}-100 \%$ presence in Larix kaempferi

- OPF-13-1.0 kb-100\% presence in Larix kaempferi

The following four markers were sufficient to estimate the F1 hybrid (Larix X eurolepis) fraction in a seed lot: OPH-14, OPC-06, OPH-11, OPF-05.

For DNA-amplification the following PCR-protocol was used:

- 1 cycle of $3 \mathrm{~min}$ at $93^{\circ} \mathrm{C}, 1 \mathrm{~min}$ at $37^{\circ} \mathrm{C}$ and $2 \mathrm{~min}$ at $72^{\circ} \mathrm{C}, 35$ cycles of $1 \mathrm{~min}$ at $93^{\circ} \mathrm{C}, 1 \mathrm{~min}$ at $37^{\circ} \mathrm{C}$ and 2 min at $72^{\circ} \mathrm{C}$, followed by a final cycle of 10 $\min$ at $72^{\circ} \mathrm{C}$.

Semerikov et al. (2003) used 4 RAPD primers to develop PCR-based mitochondrial DNA markers useful for phylogenetic studies in larch species. The following four RAPD primers produced fragments considered for further analysis:

UBC460 - 5'-ACTGACCGGC-3"

OPB11 - 5'-GTAGACCCGT-3،

OPC8 - 5'-TGGACCGGTG-3

OPR11 - 5'-GTAGCCGTCT-3'

The RAPD fragments were cut out of a $1 \%$ agarose gel, purified using a gel extraction kit (Qiagen), cloned into pGEM-T easy plasmid (Promega) and sequenced.
Gros-Louis et al. (2005) tested 130 RAPD-primers using the following kits from Operon Biotechnologies, Alameda, CA:

- OPL-OPQ,

- OPC-6,

- OPD-10, OPD-15,

- OPE-17,

- OPF05, OPF-13,

- OPG-12,

- OPH-11, OPH-14,

- OPR-18.

For DNA-amplification the following PCR-protocol was used:

- 1 cycle of $1 \mathrm{~min}$ at $94^{\circ} \mathrm{C}, 20$ cycles of $15 \mathrm{~s}$ at $94^{\circ} \mathrm{C}$, $15 \mathrm{~s}$ at $35^{\circ} \mathrm{C}$, and $1 \mathrm{~min} 30 \mathrm{~s}$ at $72^{\circ} \mathrm{C}$ followed by 25 cycles of $15 \mathrm{~s}$ at $94^{\circ} \mathrm{C}, 15 \mathrm{~s}$ at $35^{\circ} \mathrm{C}$, and $1 \mathrm{~min} 30 \mathrm{~s}$ at $72^{\circ} \mathrm{C}$, with a ramp at this extension step of $5 \mathrm{~s}$ per cycle, final extension of $10 \mathrm{~min}$ at $72^{\circ} \mathrm{C}$.

Amplification products $(6 \mu \mathrm{l})$ were separated into a $0.5 \%$ Synergel (Gordon Technologies, Mississauga, Ontario) plus $1.0 \%$ agarose gels using $0.75 \times$ Tris-phosphateEDTA (TPE) running buffer. Amplification products were stained with ethidium bromide and visualized under UV light.

\section{Nuclear DNA markers (AFLPs, nSSRs, EST- SSRs, SNPs)}

a) AFLPs (Amplified Fragment Length Polymorphism)

Semerikov and Lascoux (2003) and Semerikov et al. (2003) used besides other markers the AFLP technique (Vos et al. 1995) for analyzing larch species differentiation at the nuclear level.

DNA was digested with EcoRI and $M s e I$. Three selective nucleotides were used in the case of the EcoRI primer and four for the $M s e I$ primer. The EcoRI primer was labeled by g ${ }^{33}$ P-ATP.

The following primer combinations were used:

- Eco1+ ACG x Mse1+CCCA, Mse1+CCAC, Mse 1+CCAG (Semerikov and Lascoux 2003)

- Eco1+ ACG x Mse1+CCTC, Mse1+CCCA, Mse $1+\mathrm{CCAC}, \quad M s e 1+\mathrm{CCAG}, \quad M s e 1+\mathrm{CCTG}$, Mse 1+CCAG (Semerikov et al. 2003)

Arcade et al. (2000) analysed 114 AFLPs resulting from 5 AFLP primer combinations and constructed a singletree genetic linkage map of European and Japanese larch. 
b) nSSRs (putatively neutral microsatellites) and ESTSSRs (expressed sequence tag derived microsatellites)

\section{Loci and primers used}

More then 200 nuclear microsatellites were developed for Larix kaempferi: 19 polymorphic simple sequence repeats (SSR) markers in Isoda and Watanabe (2006), 165 SSR marker in Chen et al. (2015, Supplementary Material), six expressed sequence tags (EST-SSRs) in Yang et al. (2011).

28 microsatellite markers were amplified in cross-species transferability tests for Larix kaempferi (6 SSR marker in Khasa et al. 2000, 13 SSR marker in Wagner et al. 2012 and 9 SSR marker in Zhang et al. 2015) (Table 4). Wagner et al. (2012) designed multiplexes for Larch SSRs. Gros-Louis et al. (2005) tested the transferability of EST-SSRs developed by Perry and Bosquet (1998) to Larix species, among them also Larix kaempferi.

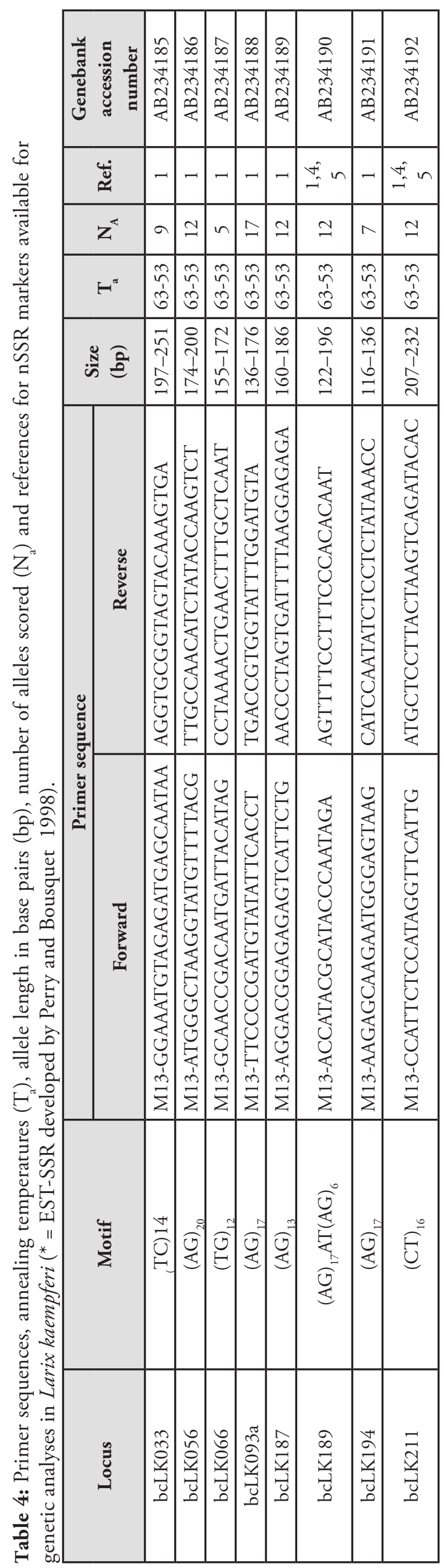




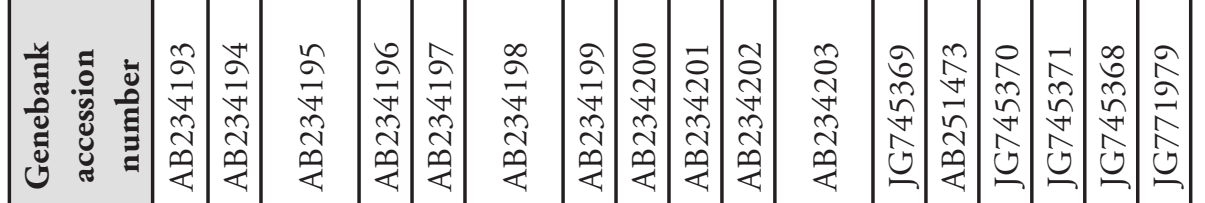

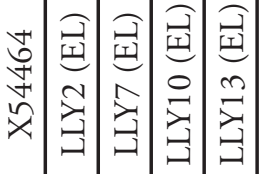

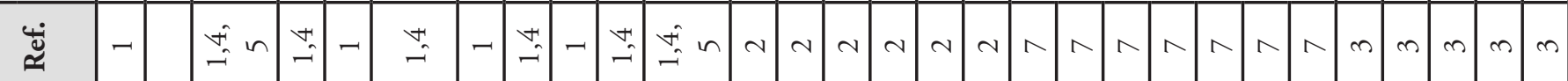

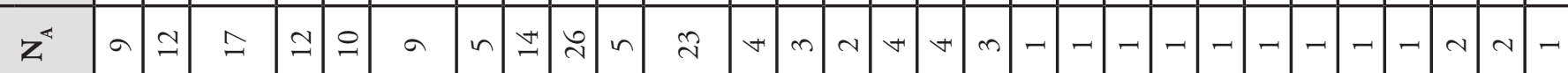

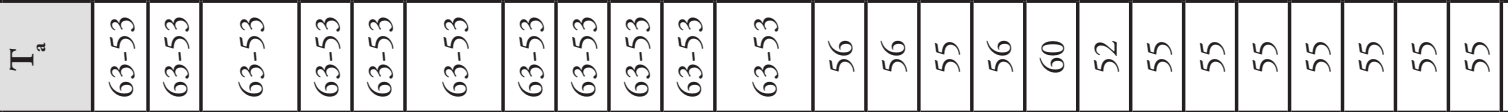

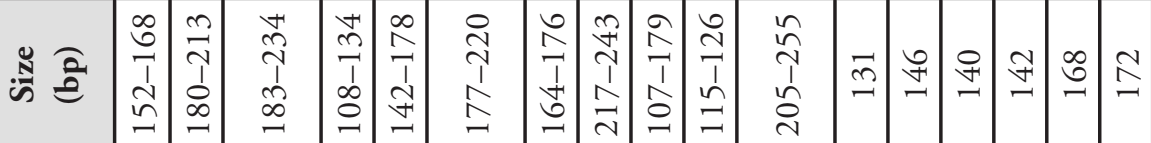

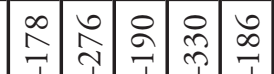

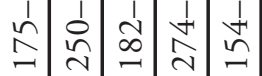

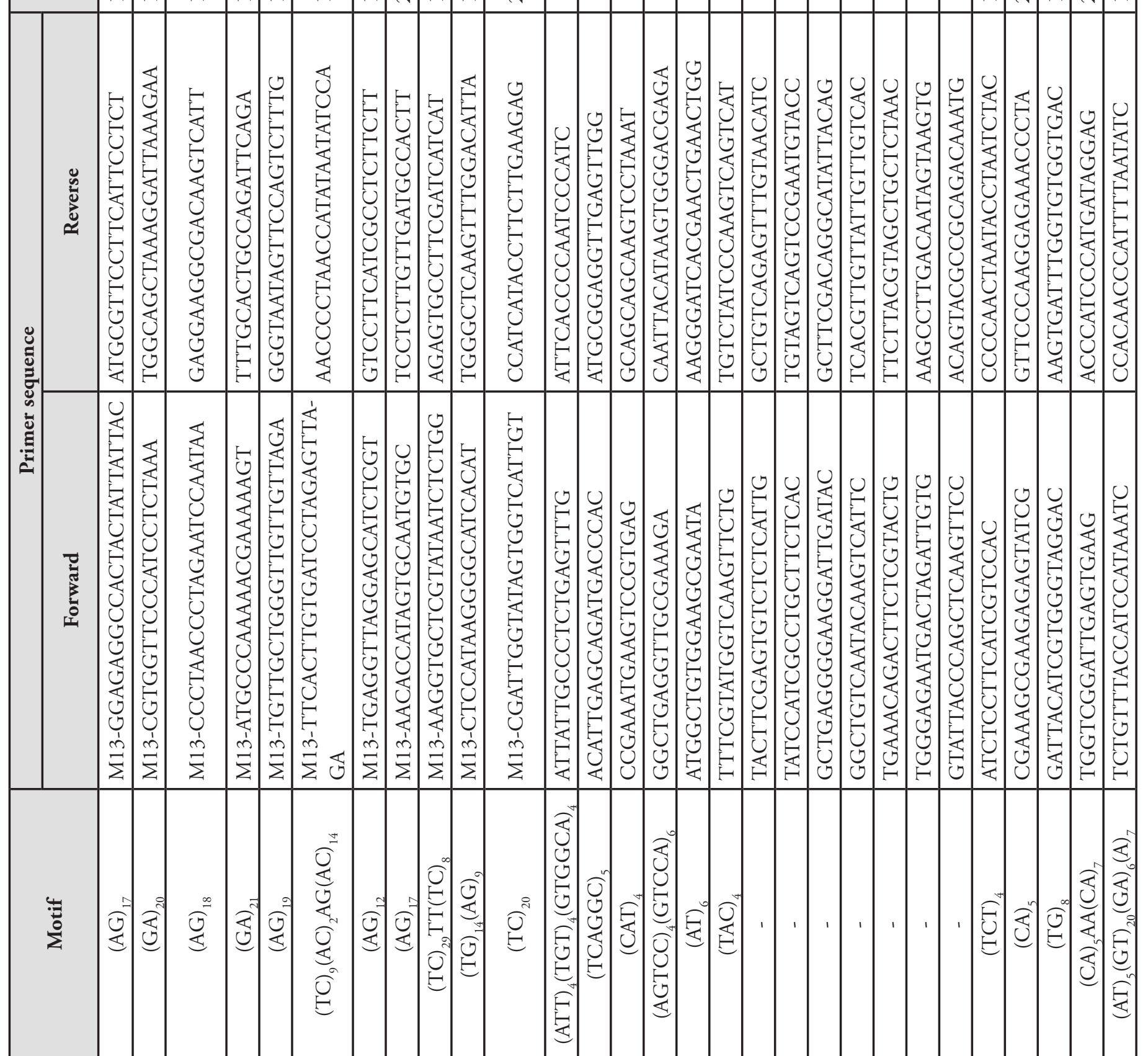

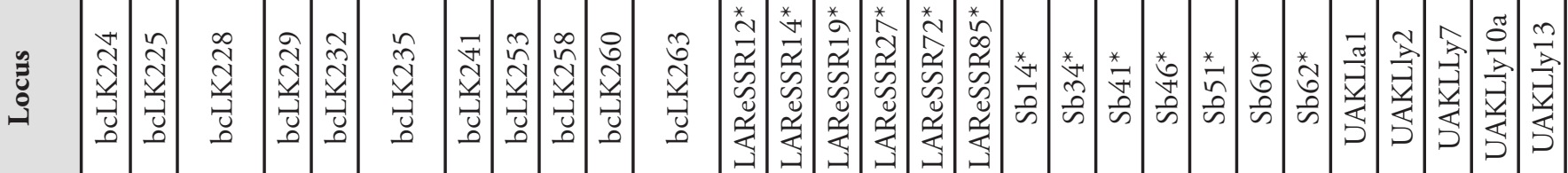




\begin{tabular}{|c|c|c|c|c|c|c|c|c|c|c|c|c|c|c|c|c|c|c|c|c|c|c|c|c|c|}
\hline 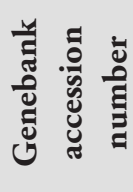 & 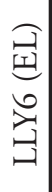 & ' & 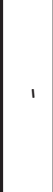 & ' & & I & 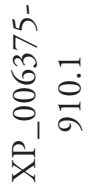 & 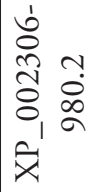 & ' & 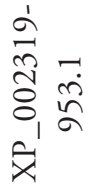 & $\begin{array}{ll}1 & 1 \\
0 & \\
0 & -1 \\
\infty & - \\
0 & 0 \\
0 & 0 \\
i & 0 \\
\dot{x} & \end{array}$ & 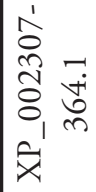 & & 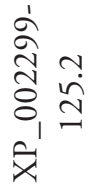 & 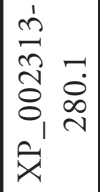 & 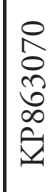 & 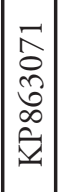 & 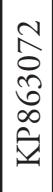 & 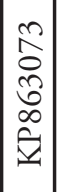 & 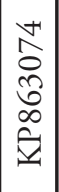 & \begin{tabular}{l|}
$n$ \\
$\hat{0}$ \\
$\tilde{\sigma}$ \\
$\infty$ \\
$\infty$ \\
$\tilde{v}$
\end{tabular} & 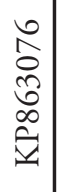 & 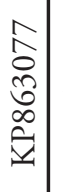 & 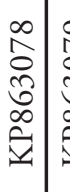 & \\
\hline$\ddot{\mathscr{\Xi}}$ & $\begin{array}{l}y_{1} \\
n_{0}\end{array}$ & $n$ & in & in & $i$ & $n$ & ○ & ○ & ○ & ○ & ○ & ○ & ○ & ○ & ○ & $\infty$ & $\infty$ & $\infty$ & $\infty$ & $\infty$ & $\infty$ & $\infty$ & $\infty$ & $\infty$ & \\
\hline$z$ & - & $\infty$ & $\simeq$ & 6 & $\lambda$ & $a$ & $m$ & $N$ & $N$ & - & $\sim$ & - & - & $=$ & $m$ & $\wedge$ & $n$ & $\neg$ & -1 & $n$ & $\sim$ & - & $m$ & $n$ & \\
\hline$H^{\prime \prime}$ & & & & & & & $\stackrel{m}{n}$ & $\stackrel{\infty}{i}$ & & $\stackrel{\vec{\forall}}{\circ}$ & $\stackrel{\infty}{\stackrel{n}{n}}$ & $\frac{n}{\not}$ & & 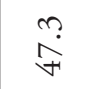 & $\stackrel{\leftrightarrow}{\stackrel{\leftrightarrow}{\sigma}}$ & $\stackrel{0}{n}$ & 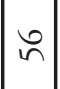 & 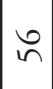 & 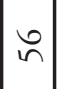 & 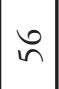 & 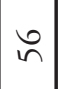 & 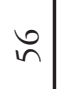 & 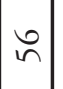 & $\stackrel{゚}{\curvearrowleft}$ & \\
\hline is & 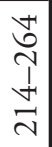 & 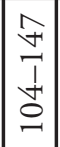 & 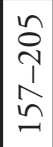 & $\begin{array}{l}\vec{a} \\
1 \\
\hat{\sigma} \\
-1\end{array}$ & 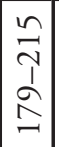 & 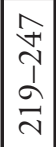 & \begin{tabular}{l}
$\stackrel{8}{0}$ \\
$\stackrel{1}{7}$ \\
\multirow{J}{7}{}
\end{tabular} & 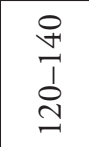 & $\stackrel{8}{n}$ & $\begin{array}{l}\stackrel{0}{1} \\
\stackrel{1}{1} \\
\text { t+ }\end{array}$ & 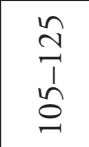 & 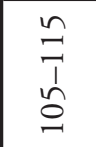 & 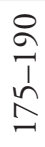 & 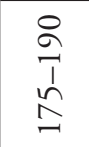 & $\begin{array}{l}8 \\
\infty \\
1 \\
\stackrel{1}{+1}\end{array}$ & $\begin{array}{l}\stackrel{0}{n} \\
1 \\
\stackrel{1}{=}\end{array}$ & 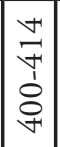 & $\stackrel{\infty}{\varrho}$ & $\begin{array}{r}\tilde{\imath} \\
\mathbf{2}\end{array}$ & $\begin{array}{l}\exists \\
\bar{\sigma} \\
\tilde{\sigma}\end{array}$ & 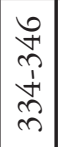 & $\stackrel{\mathbb{N}}{\widehat{N}}$ & 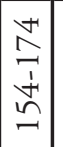 & $\begin{array}{l}\text { के } \\
\text { के } \\
\text { ले } \\
\text { ले }\end{array}$ & \\
\hline
\end{tabular}

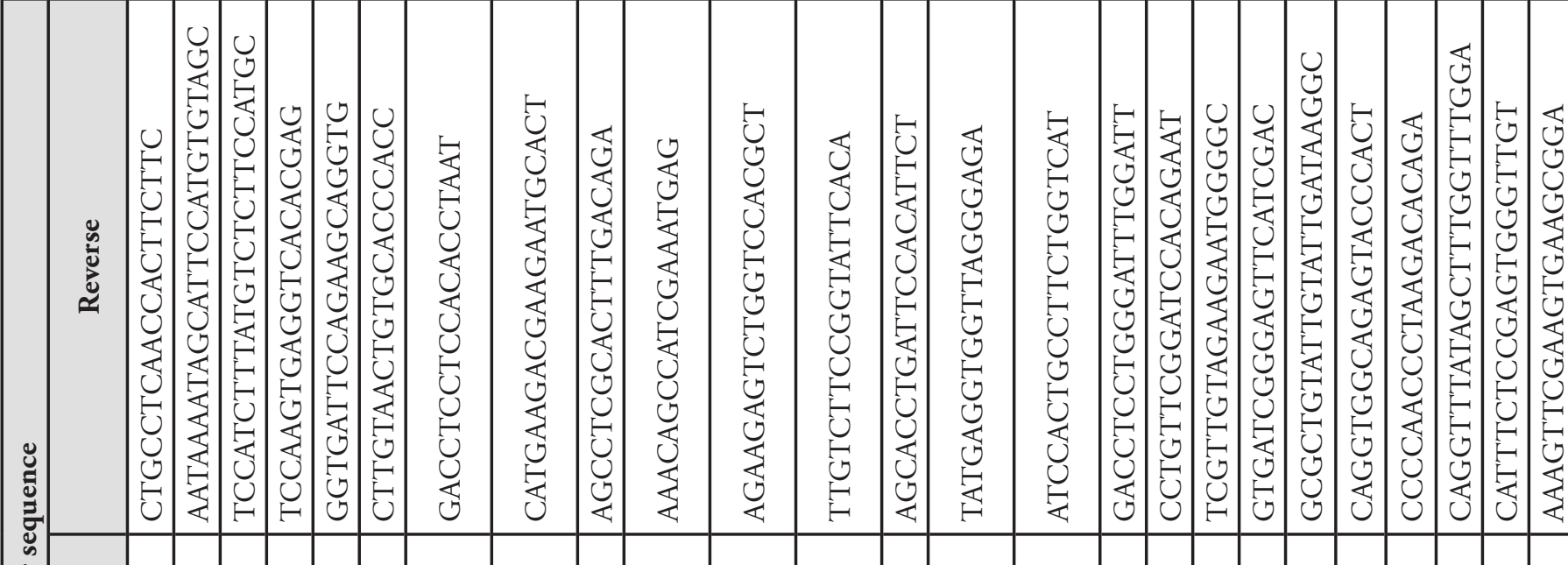

苞

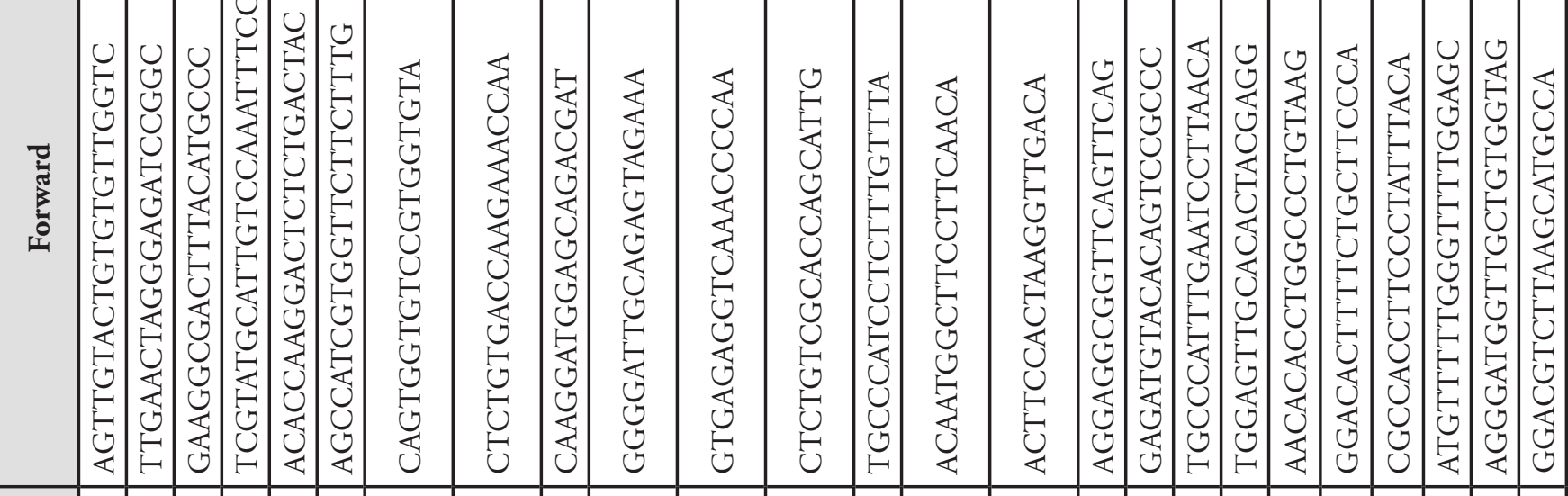

蓉

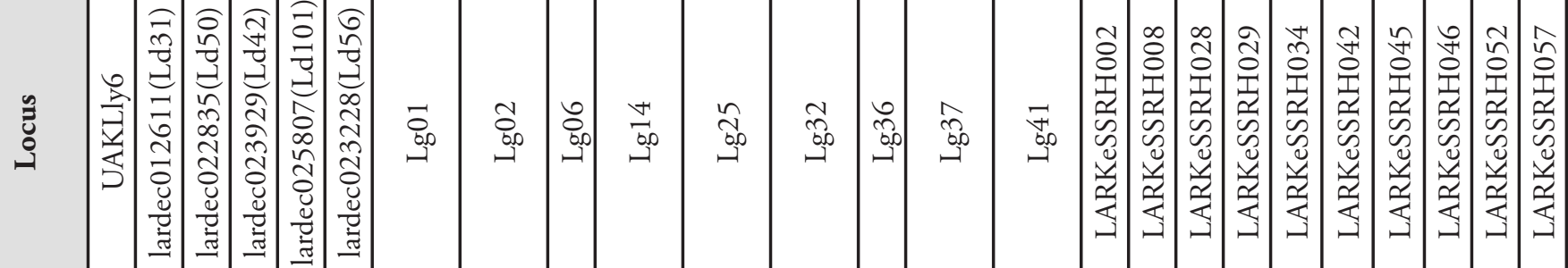




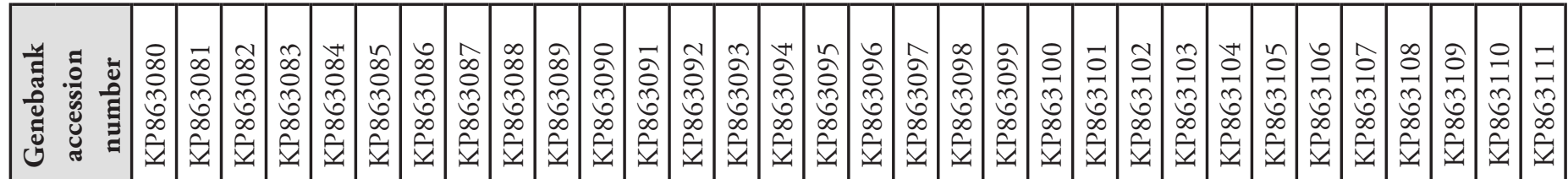

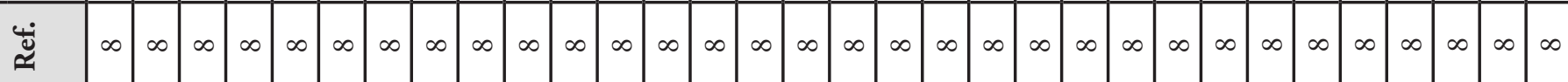

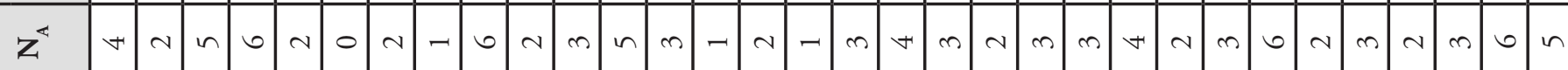

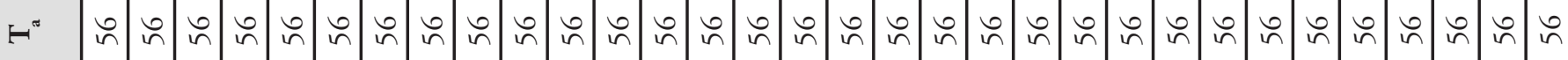

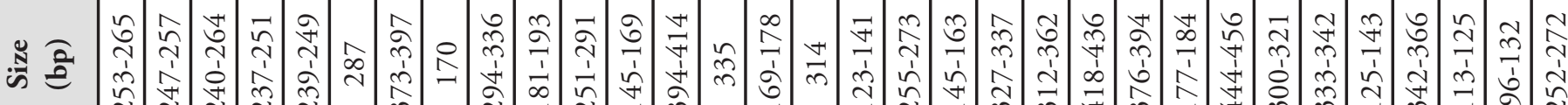

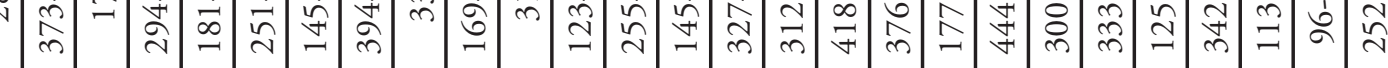

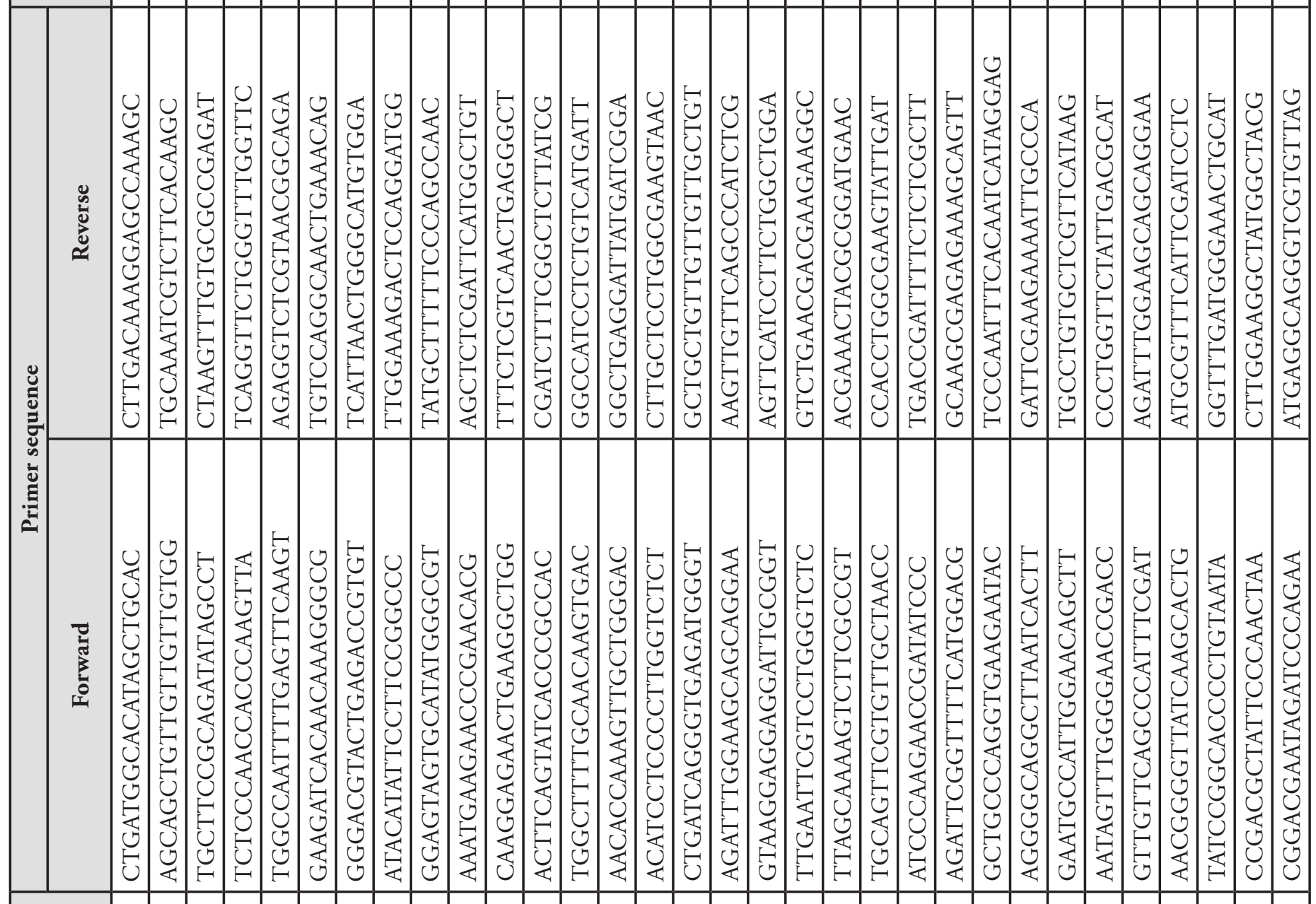

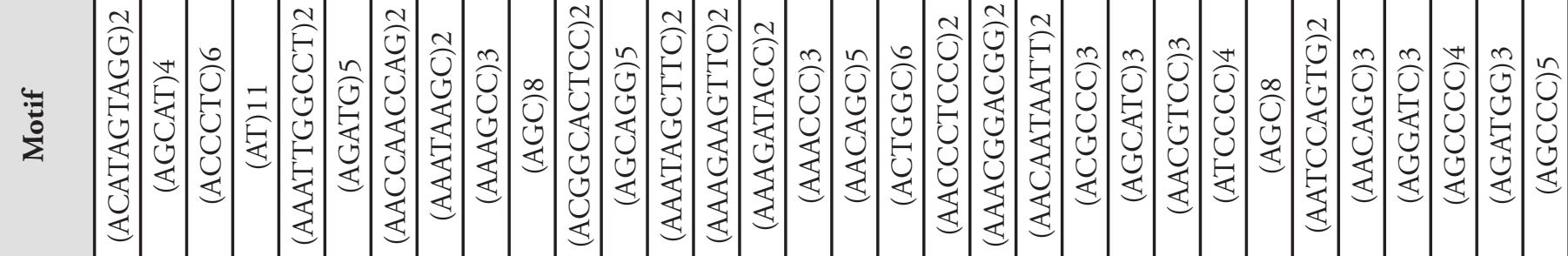

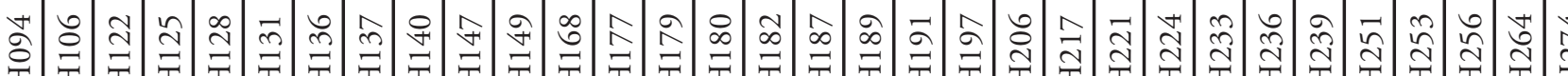

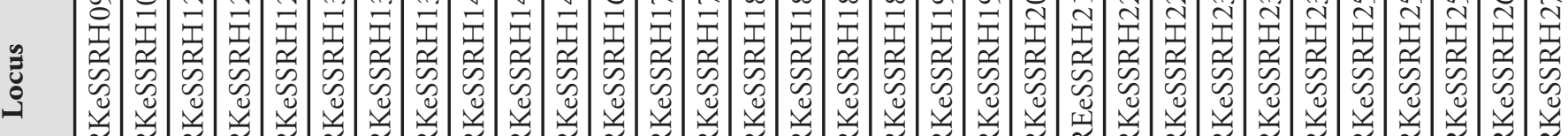

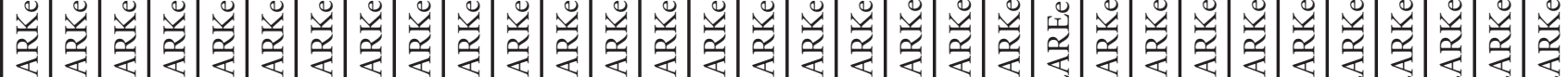




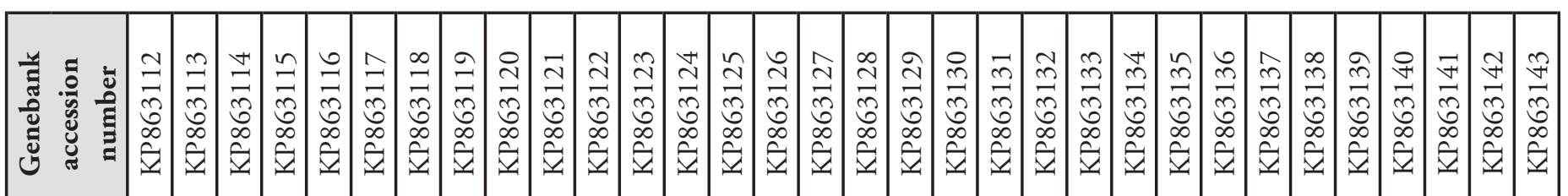

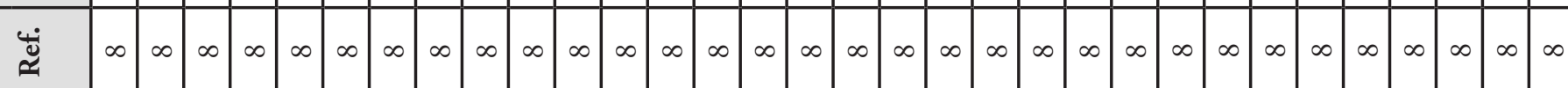

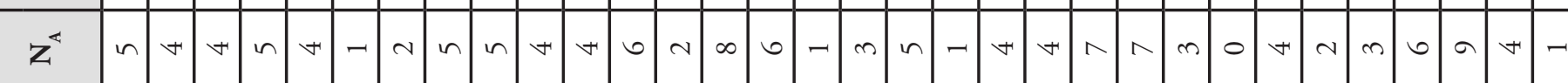

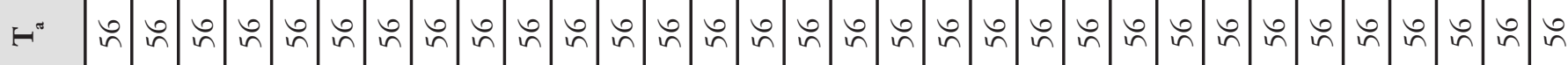

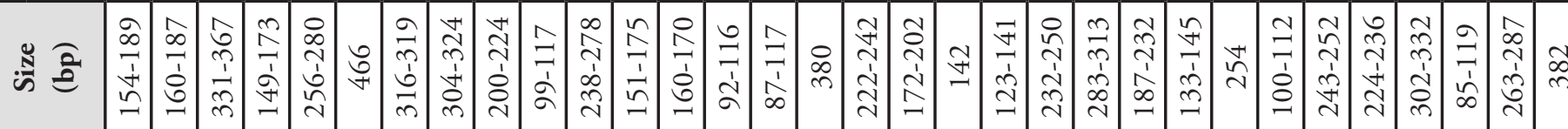

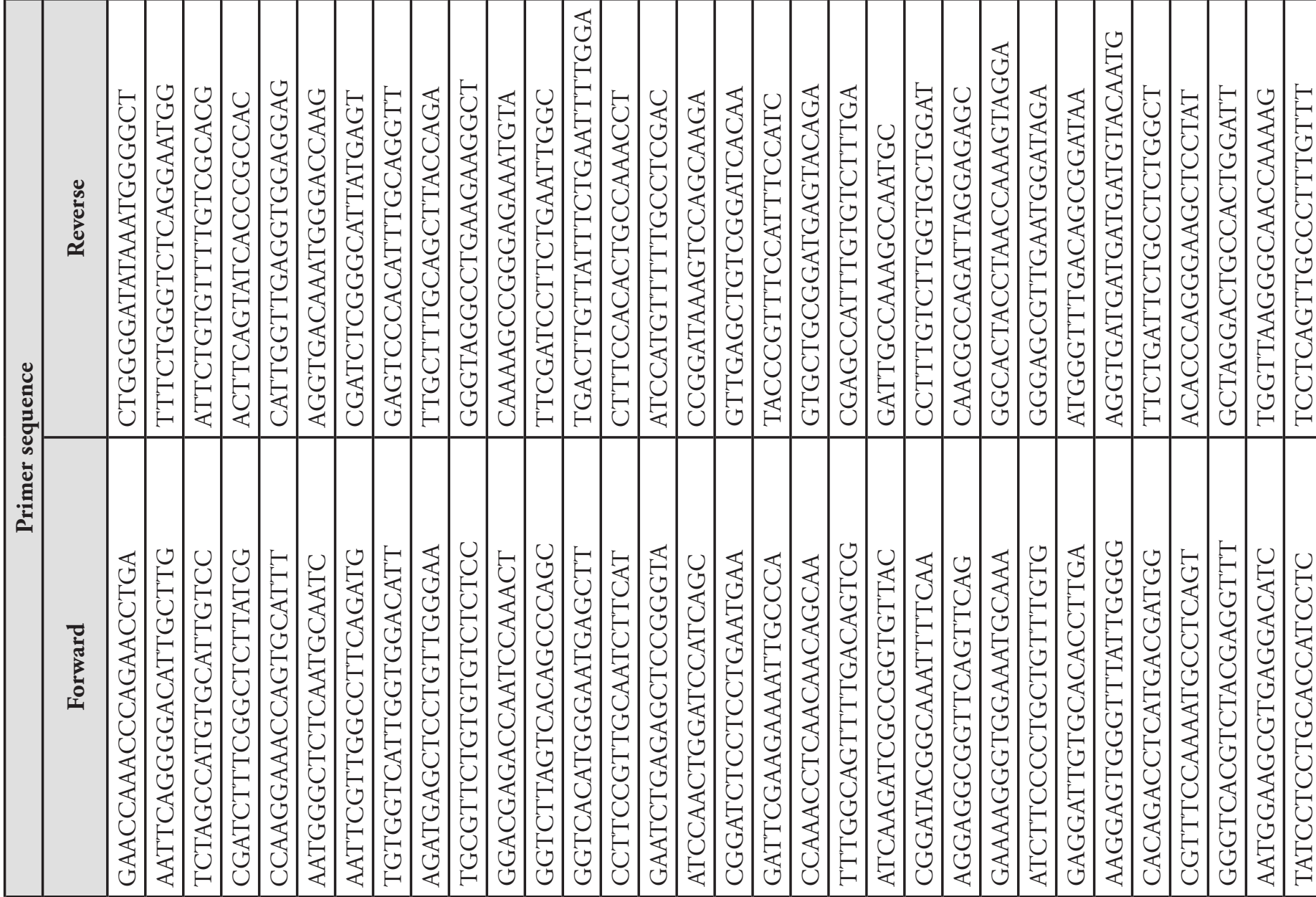

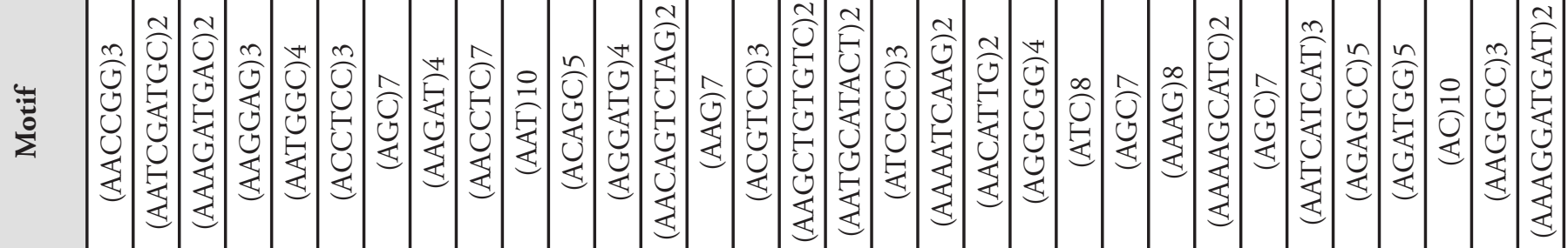
શ)

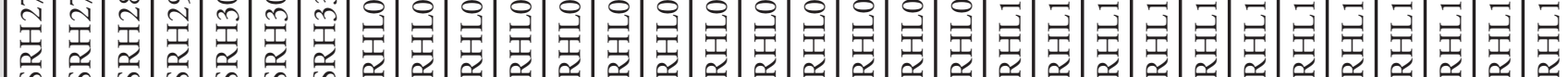

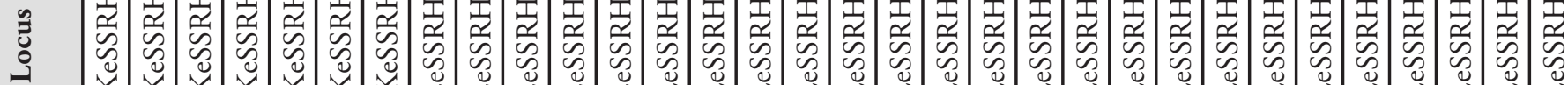

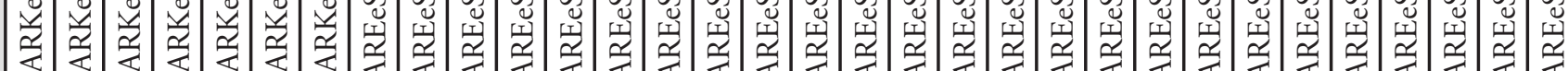

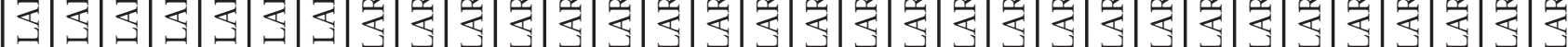




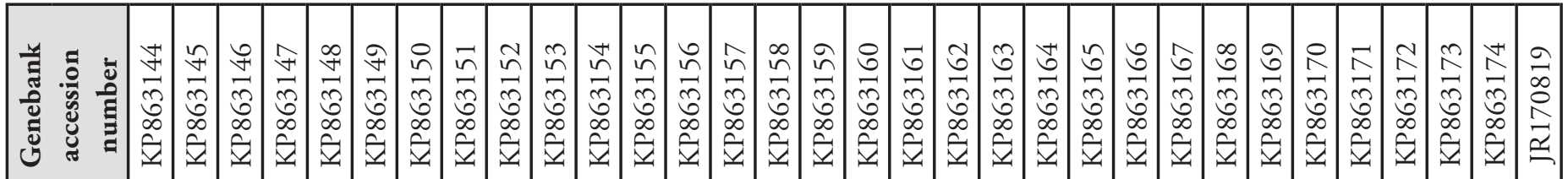

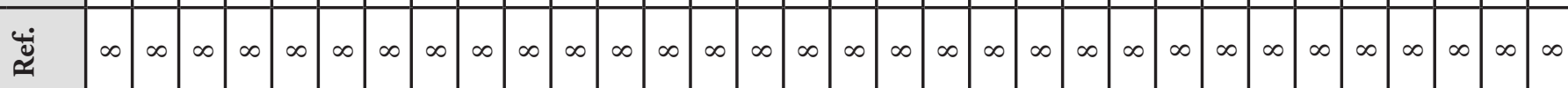

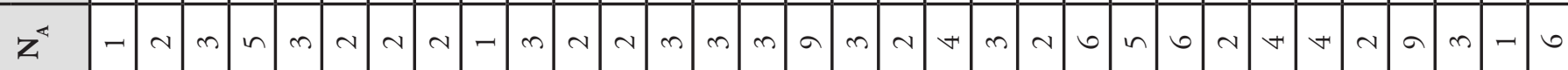

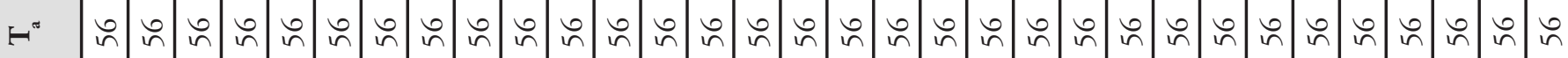

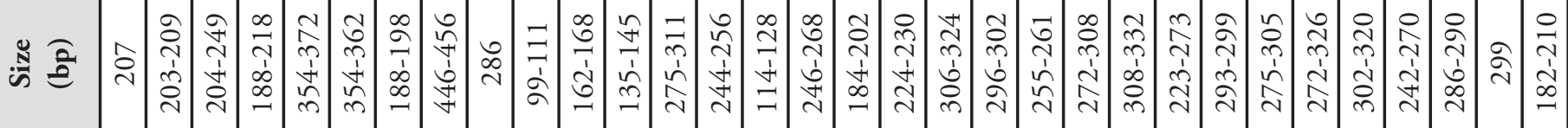

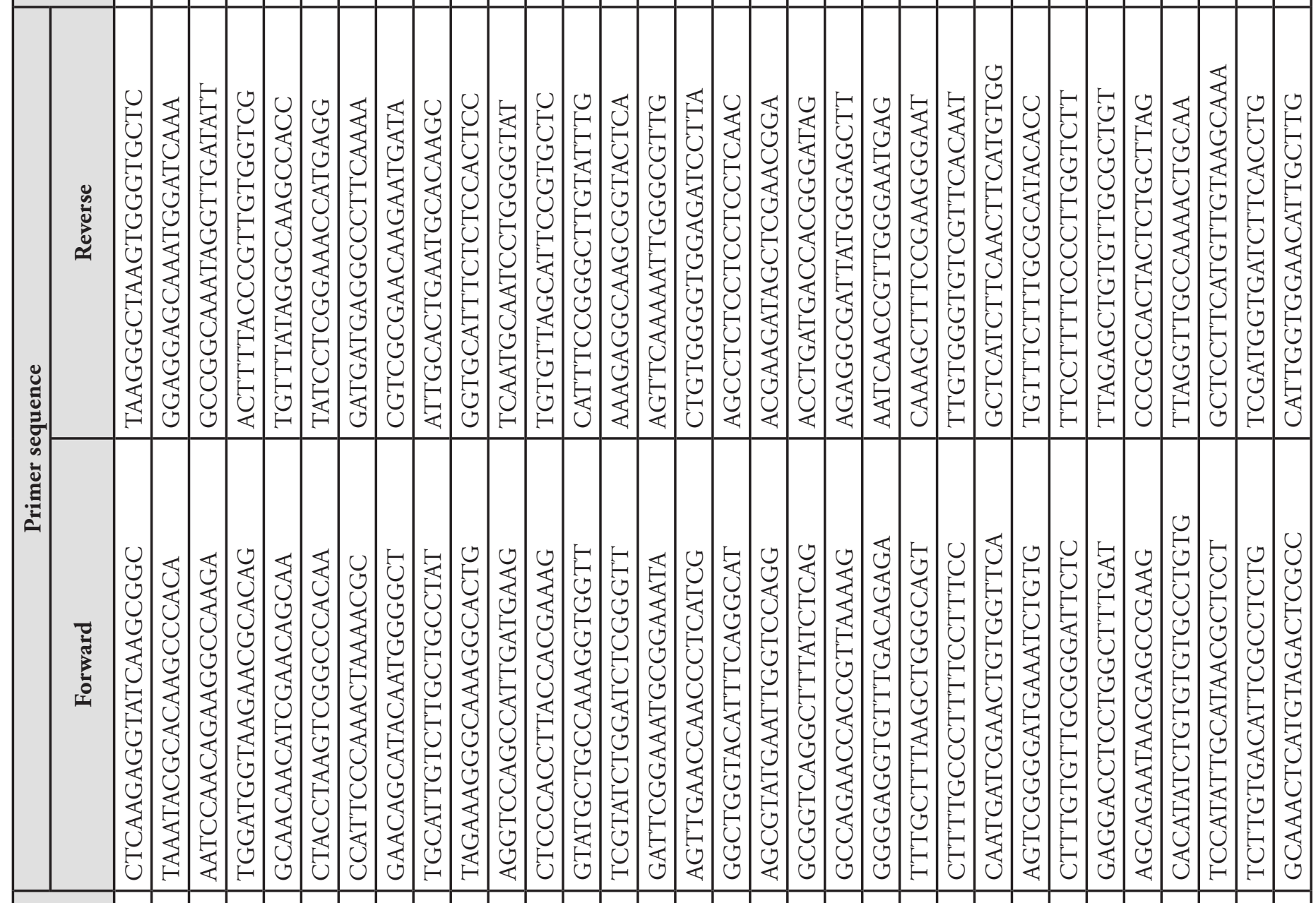

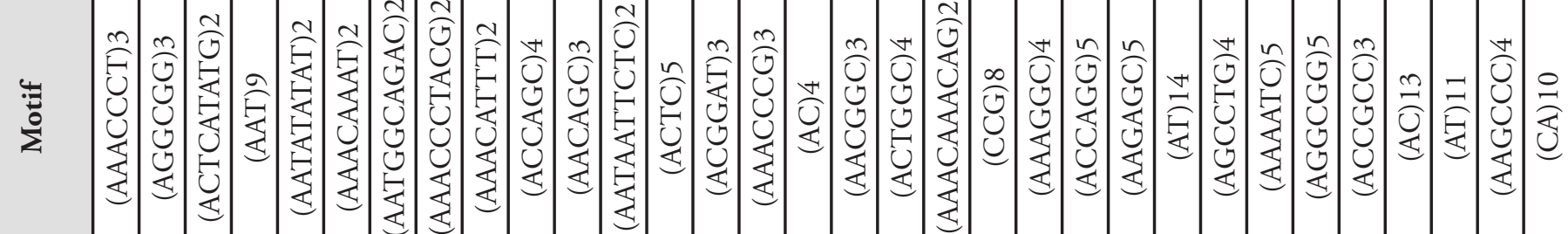

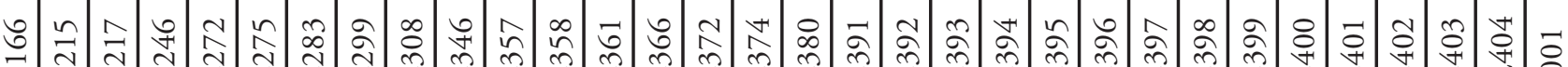

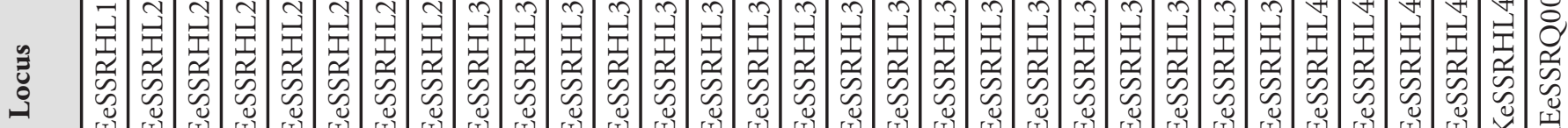

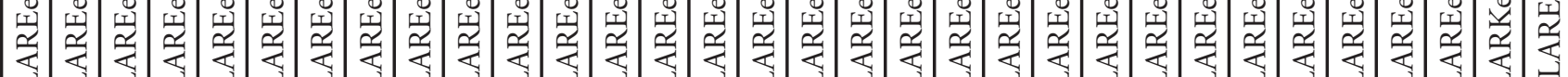




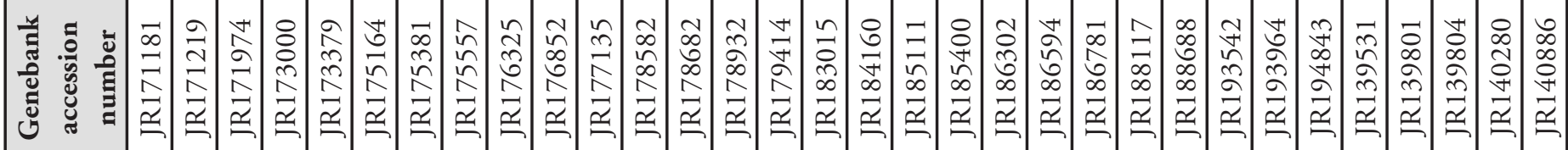

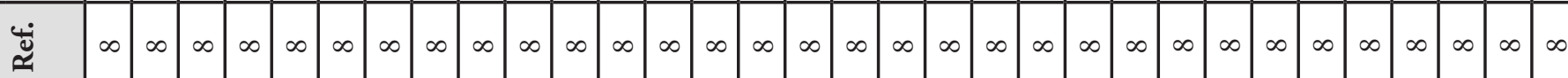

$z$

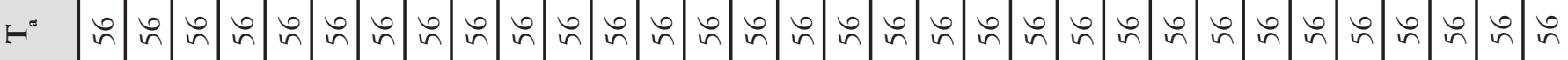

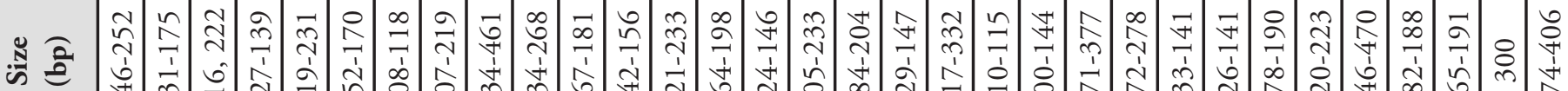

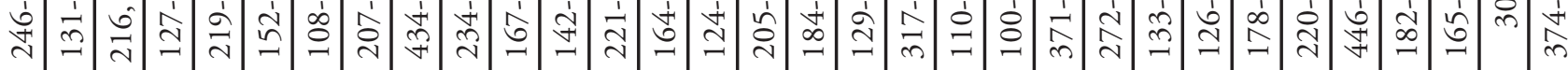

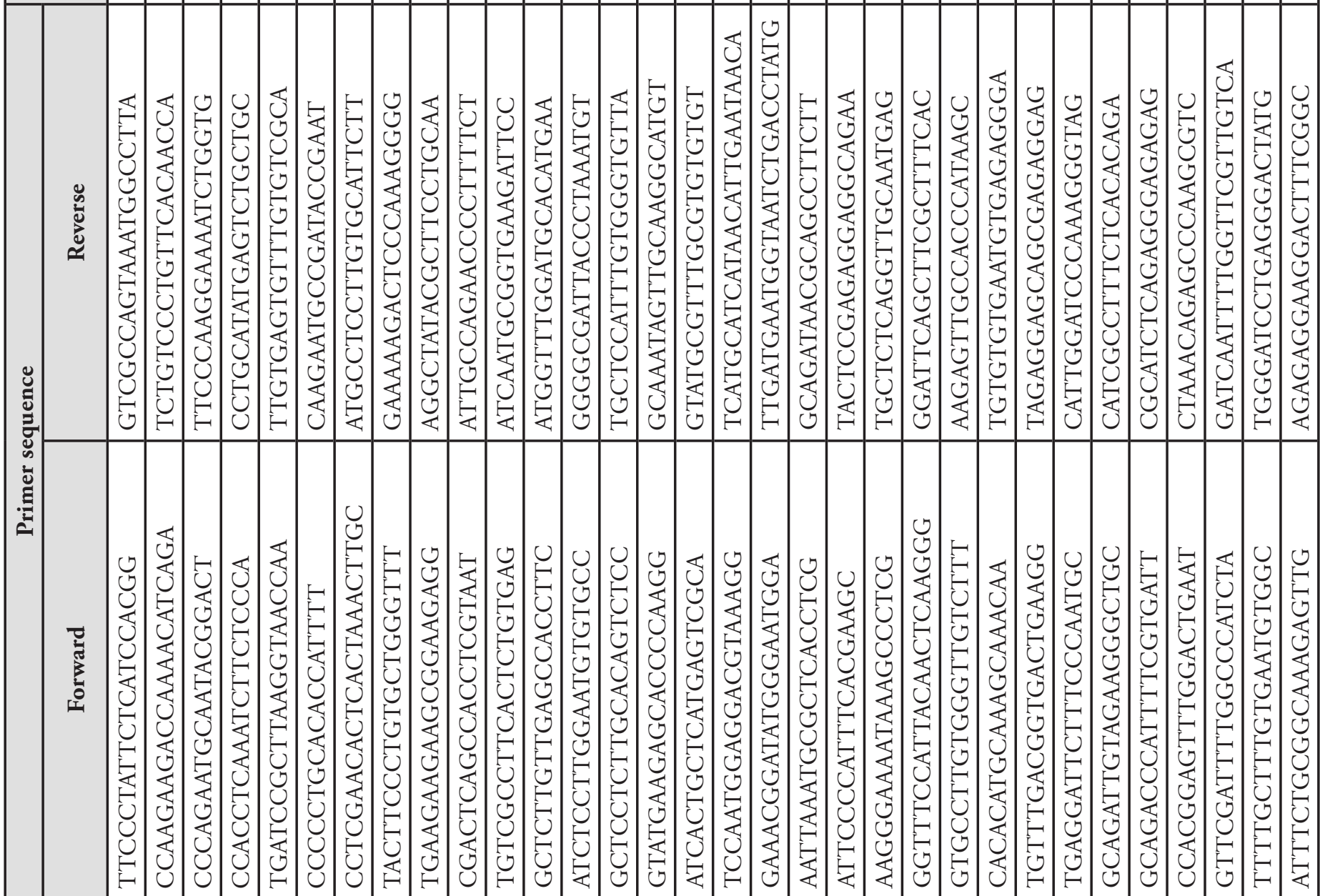

：

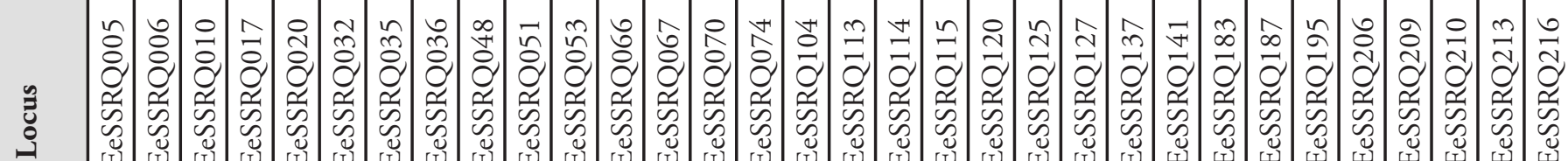
产 


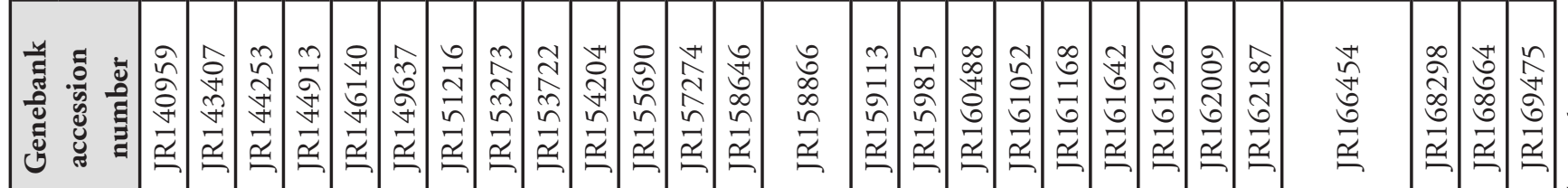

\begin{tabular}{|c|c|c|c|c|c|c|c|c|c|c|c|c|c|c|c|c|c|c|c|c|c|c|c|}
\hline & & & & & & & & & & & & & & & & & & & & & & & \\
\hline$\ddot{\tilde{\Xi}}$ & $\infty$ & $\infty$ & $\infty$ & $\infty$ & $\infty$\begin{tabular}{c|c}
$\infty$ \\
$\infty$
\end{tabular} & \begin{tabular}{l|l}
$\infty$ & $\infty$
\end{tabular} & $\infty$ & $\infty$ & $\infty$ & 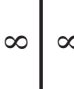 & $\infty$ & $\infty$ & $\infty$ & $\infty$ & $\infty \mid \infty$ & $\infty$ & $\infty$ & $\infty \quad \infty$ & $\infty \quad \infty$ & $\infty$ & $\infty$ & $\infty$ & \\
\hline 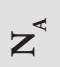 & 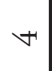 & $n$ & $\stackrel{m}{=}$ & $\infty$ & \begin{tabular}{r|r}
$*$ & $r$
\end{tabular} & $\sim$ & $\sim$ & $m$ & $n$ & $n=$ & $m$ & 0 & - & $a$ & -0 & $\sim$ & $\sigma$ & $+v$ & \begin{tabular}{l|l}
0 & $\infty$
\end{tabular} & $a$ & -1 & $\sim$ & in \\
\hline & 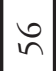 & $\stackrel{\bullet}{\curvearrowleft}$ & $\stackrel{\imath}{\curvearrowleft}$ & 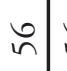 & 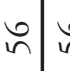 & 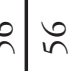 & 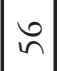 & $\stackrel{\diamond}{\curvearrowleft}$ & & $\stackrel{\sim}{\bullet}$ & $\stackrel{\bullet}{\curvearrowleft}$ & 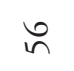 & 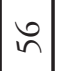 & & 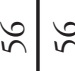 & 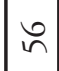 & $\stackrel{\bullet}{\circ}$ & 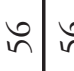 & ^) & $\stackrel{i}{n}$ & $\bullet$ & 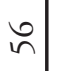 & 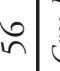 \\
\hline
\end{tabular}

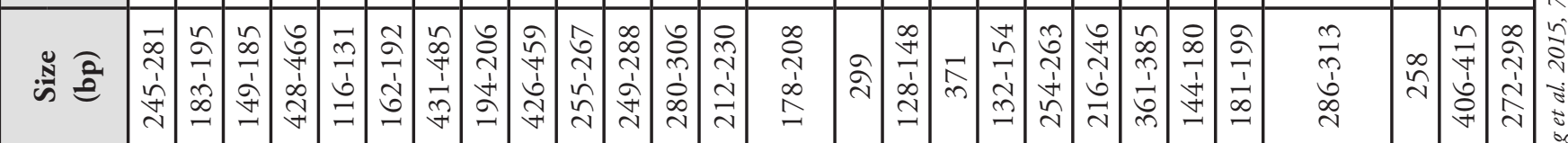

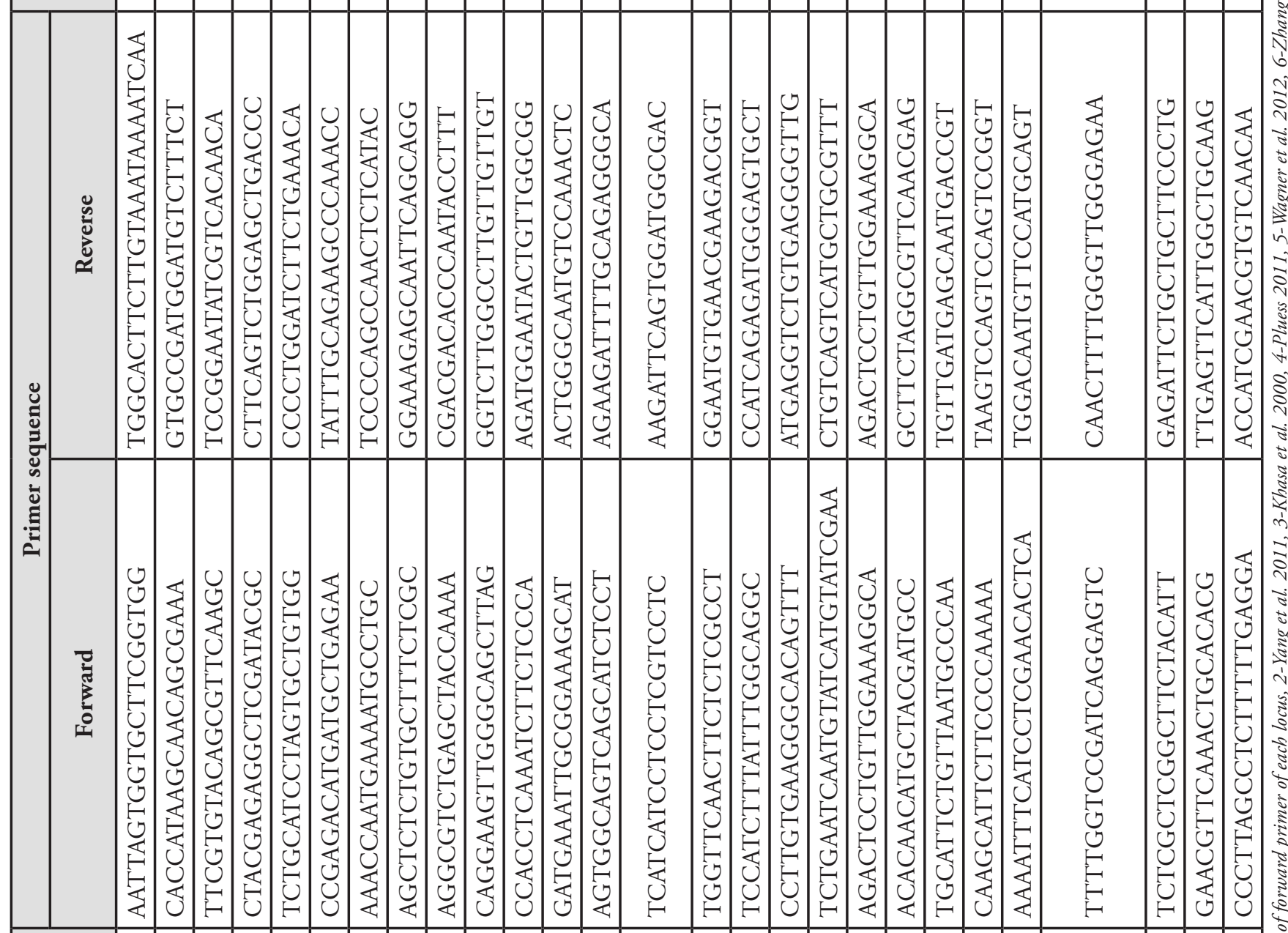

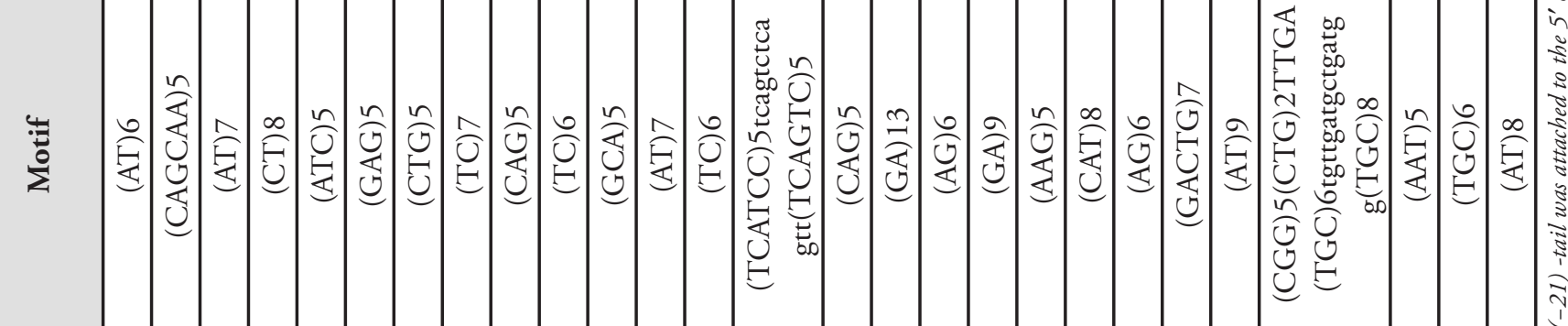

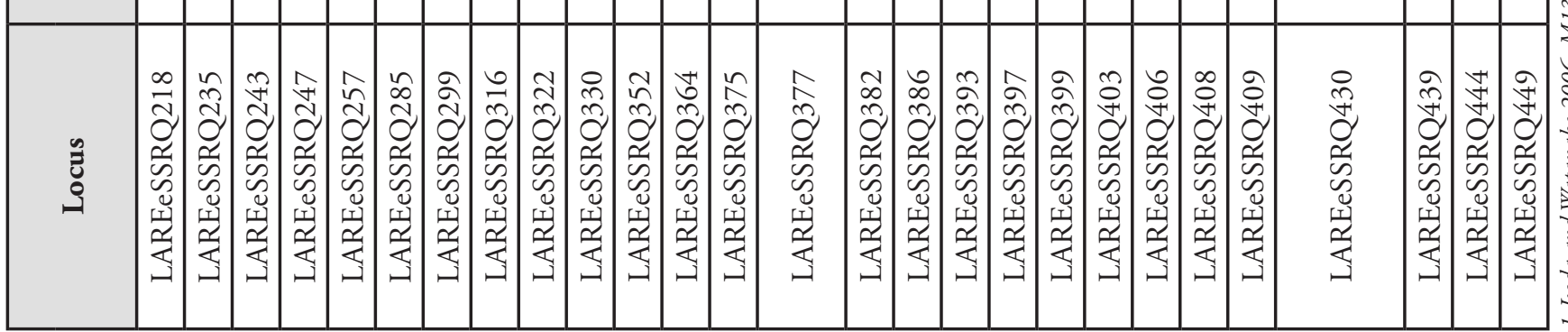




\section{Material for extraction}

Plant tissue used for extraction included seed, buds, leaves (in general frozen needles), e.g. Isoda and Watanabe (2006), Wagner et al. (2012), Nishimura and Setoguchi (2011), Gros-Louis et al. (2005), Chen et al. (2015), phloem (Wagner et al. (2012) and cambium (Khasa et al. 2000).

\section{DNA-extraction and amplification protocols}

Total DNA was extracted from the mentioned tissue using:

- NucleoSpin Plant II (Macherey Nagel, used in INRA lab)

- the QIAGEN DNeasy Plant Mini Kit (Gros-Louis et al. 2005, Pluess 2011, Zhang et al. 2015)

- a CTAB protocol after Shiraishi and Watanabe (1995) (Isoda and Watanabe (2006) and after Doyle and Doyle (1990) (Yang et al. 2011, Chen at al. 2015)

\section{Examples for amplification protocols (nSSR)}

- $\quad 94^{\circ} \mathrm{C}$ for $1 \mathrm{~min}$ followed by 10 cycles of $94^{\circ} \mathrm{C}$ for 30 $\mathrm{s}, 63-53^{\circ} \mathrm{C}\left(-1^{\circ} \mathrm{C}\right.$ at each cycle) for $45 \mathrm{~s}$, followed by 25 cycles of $94^{\circ} \mathrm{C}$ for $30 \mathrm{~s}, 53^{\circ} \mathrm{C}$ for $30 \mathrm{~s}, 72^{\circ} \mathrm{C}$ for 1 min; final elongation at $72^{\circ} \mathrm{C}$ for $10 \mathrm{~min}$ (Isoda and Watanabe 2006).

- $\quad 94^{\circ} \mathrm{C}$ for 4 min followed by 30 cycles of $94^{\circ} \mathrm{C}$ for 45 $\mathrm{s}, 56^{\circ} \mathrm{C}$ for $45 \mathrm{~s}, 72^{\circ} \mathrm{C}$ for $45 \mathrm{~s}$; final elongation at $72^{\circ} \mathrm{C}$ for $7 \mathrm{~min}$ (Chen et al. 2015).

Examples for amplification protocols (EST-SSR):

- $94^{\circ} \mathrm{C}$ for $3 \mathrm{~min}$ followed by 40 cycles of $94^{\circ} \mathrm{C}$ for $30 \mathrm{~s}$, Ta (Table 4) for $45, \mathrm{~s} 72^{\circ} \mathrm{C}$ for $1 \mathrm{~min}$; final elongation at $72^{\circ} \mathrm{C}$ for min (Yang et al. 2011).

- $\quad 95^{\circ} \mathrm{C}$ for 5 min followed by 25 cycles of $95^{\circ} \mathrm{C}$ for 30 s, Ta (Table 4) for $45 \mathrm{~s}, 72^{\circ} \mathrm{C}$ for 60 s; final elongation at $72^{\circ} \mathrm{C}$ for $20 \mathrm{~min}$ (Zhang et al. 2015).

- $\quad 94^{\circ} \mathrm{C}$ for 4 min followed by 40 cycles of $95^{\circ} \mathrm{C}$ for 30 $\mathrm{s}, 55^{\circ} \mathrm{C}$ for $30 \mathrm{~s}, 72^{\circ} \mathrm{C}$ for $1 \mathrm{~min}$; final elongation at $72^{\circ} \mathrm{C}$ for $10 \mathrm{~min}$ (Gros-Louis et al. 2005).

SSR - Multiplexing (Wagner et al. 2012)

- Multiplex 1 - Ld31, bcLK211, Ld30, bcLK228, Ld50, bcLK189, bcLK253

- Multiplex 2 - Ld58, Ld45, Ld42, bcLK263, Ld101, Ld56

- Amplification protocol for multiplexes 1 and 2:

- $95^{\circ} \mathrm{C}$ for $15 \mathrm{~min}$ followed by 35 (multiplex 1)/30 (multiplex 2) cycles of $94^{\circ} \mathrm{C}$ for $30 \mathrm{~s}, \mathrm{~T}_{\mathrm{a}}$ (Table 4) $56^{\circ} \mathrm{C}$ for $1 \mathrm{~min}, 72^{\circ} \mathrm{C}$ for $1 \mathrm{~min}$; final elongation at $60^{\circ} \mathrm{C}$ for $30 \mathrm{~min}$

\section{Important results}

- New EST-SSR markers were developed for Larix kaempferi (Gros-Louis et al. 2005, Yang et al. 2015, Zang et al. 2015). The markers are transferable also to other Larix species.

- New SSR markers were developed and identified as highly polymorphic in Larix kaempferi. Most of them could be amplified in related Larix species (Larix olgensis, Larix gmelinii, Larix principi-rupprechtii) (165 nSSR between them 145 polymorphic developed by Chen et al. 2015, 20 primer pairs between them 19 polymorphic developed by Isoda and Watanabe 2006).

- In a Larix kaempferi Danish seed orchards, SSR markers were used to evaluate the selfing rate, the paternal contribution to the progenies and the pollution rate from external larch sources (Hansen 2008).

- A $34 \%$ introgression rate by spontaneous hybridization between L. kaempferi and L. laricina was observed in Québec (Canada), suggesting to take into consideration the proximity of this exotic species in the management of natural genetic resources (Meirmans et al. 2014).

\section{c) SNPs (single-nucleotide polymorphisms)}

- Gros-Louis et al. (2005) used SNP in a study aiming at distinguishing larch species (Larix decidua, Larix sibirica, Larix kaempferi and Larix laricina). The results were the identification of three gene loci (Sb14, Sb48, Sb51) with fixed interspecific polymorphisms implicating 17 SNPs and 2 indels.

- Li et al. (2014) identified many single-nucleotide polymorphisms (SNPs) in a genome-wide marker development for Larix kaempferi. Among these SNPs, 364227 (78.6\%) were determined from transcripts with annotation information, and they were distributed in 32453 known genes.

\section{References}

Acheré, V., Rampant, P.F., Paques, L.E., Prat, D. (2004): Chloroplast and mitochondrial molecular tests identify European x Japanese larch hybrids. Theoretical and Applied Genetics, 108(8): 1643-1649.

Arcade, A., Faivre-Rampant, P., Le Guerroue, B., Paques, L.E., Prat, D. (1996): Heterozygosity and hybrid performance in larch. Theoretical and Applied Genetics, 8: 1274-1281. 
Beletti, P., Lanteri, S., Leonardi, S. (1996): Genetic Variability among European larch (Larix decidua Mill.) populations in Piedmont, North-West Italy. Forest Genetics, 4(3): 113-121.

Bergmann, F., Ruetz, W. (1987): Identifizierung von Hybridlärchensaatgut aus Samenplantagen mit Hilfe eines Isoenzym-Markers. Silvae Genetica, 36(2): 102105.

Cheliak, W.M., Pitel, J.A. (1985): Inheritance and linkage of allozymes in Larix laricina. Silvae Genetica, 34: 142-148.

Chen, X.B., Xie, Y.H., Sun, X.M. (2015): Development and Characterization of polymorphic Genic-SSR markers in Larix kaempferi. Molecules, 20(4): 6060-6067.

Demesure, B., Sodzi, N.,. Petit, R.J. (1995): A set of universal primers for amplification of polymorphic noncoding regions of mitochondrial and chloroplast DNA in plants. Molecular Ecology, 4: 129-131.

DeVerno, L.L., Charest, P.J., Bonen, L. (1993): Inheritance of mitochondrial DNA in the conifer Larix. Theoretical and Applied Genetics, 86(2-3), 383-388.

Devey, M.E., Bell, J.C., Smith, D.N., Neale, D.B., Moran, G.F. (1996): A genetic linkage map for Pinus radiata based on RFLP, RAPD, and microsatellite markers. Theoretical and Applied Genetics, 92: 673-679.

Doyle, J.J, Doyle, J.L. (1990): Isolation of plant DNA from fresh tissue. Focus, 12: 13-15.

Dumolin-Lapégue, S., Pemonge, M.H., Petit R.J. (1997): An enlarged set of consensus primers for the study of organelle DNA in plants. Molecular Ecology, 6: 393-397.

Gros-Louis, M.C., Bousquet, J., Paques, L.E., Isabel, N. (2005): Species-diagnostic markers in Larix spp. based on RAM and nuclear, cpDNA, and mtDNA gene sequences, and their phylogenetic implications. Tree Genetics \& Genomes, 1(2): 50-63.

Häcker, M., Bergmann, F. (1991): The proportion of hybrids in seed from a seed orchard composed of two larch species (L. europaed and L. leptolepis). Annales des Sciences Forestières, 48(6): 631-640.

Hansen, O.K. (2008): Mating patterns, genetic composition and diversity levels in two seed orchards with few clones - Impact on planting crop. Forest Ecology and Management, 256(5): 1167-1177.

Heinze, B. et 11 contributors: Fussi, B., Belle, C., Konnert, M., Blanc-Jolivet, C., Liesebach, M., Buiteveld, J., Piotti, A., Vendramin, G.G., Wagner, S., Petit, R.J., Jahn, D., Heinze, B. (2012): Report on review of available and tested methods for identification and on new marker development. Designing Trees for the Future project $\mathrm{N}^{\circ}$ 284181. Deliverable D71. p. 160.

Hoshi, H. (2004): Forest Tree Genetic Resources Conservation Stands of Japanese Larch (Larix kaempferi (Lamb.) Carr.). For. Tree Gen. Res. Inf., Special Issue No.1

Isoda, K., Watanabe, A. (2006): Isolation and characterization of microsatellite loci from Larix kaempferi. Molecular Ecology Resources, 6(3): 664-666.

Jaramillo-Correa, J.P., Bousquet, J., Beaulieu, J., Isabel, N., Perron, M., Bouillé, M. (2003): Cross-species amplification of mitochondrial DNA sequence-taggedsite markers in conifers: the nature of polymorphism and variation within and among species in Picea. Theor Appl Genet, 106: 1353-1367.

Jeandroz, S., Bastien D., Chandelier A., Du J., Favre J.M. (2002): A set of primers for amplification of mitochondrial DNA in Picea abies and other conifer species. Molecular Ecology Notes, 2: 389-392.

Khasa, D.P., Newton, C.H., Rahman, M.H., Jaquish, B., Dancik, B.P. (2000): Isolation, characterization, and inheritance of microsatellite loci in alpine larch and western larch. Genome, 43: 439-448.

Konnert, M., Maurer, W. (1995): Isozymic Investigations on Norway Spruce (Picea abies (L.) Karst.) and European Silver Fir (Abies alba Mill.): A Practical Guide to Separation Methods and Zymogram Evaluation. From the German Federal-State Working Group "Conservation of Forest Gene Resources”, ISBN 3-00-000042-9

Lewandowski, L., Mejnartowicz, L. (1988): Inheritance of allozymes in Larix decidua Mill, Silvae Genetica, 39: 184-188.

Lewandowski, L., Mejnartowicz, L. (1992): Lavels and patterns of allozyme variation in some European larch (Larix decidua) populations. Hereditas, 115: 107-109.

Li, W.F., Han, S.Y., Qi, L.W., Zhang, S.G. (2014): Transcriptome resources and genome-wide marker development for Japanese Larch (L. kaempferi). Frontiers of Agricultural Science and Engineering, 1(1): 77-84.

Lu, M.-Z., Szmidt, A.E., Wang, X.-R. (1998): RNA editing in gymnosperms and its impact on the evolution of the mitochondrial cox 1 gene. Plant Molecular Biology, 37: 225-234.

Maier, J. (1992): Genetic variation in European larch (Larix decidua). Annales des Sciences Forestières, 49: 3947.

Meirmans, P.G., Gros-Louis, M.C., Lamothe, M., Perron, M., Bousquet, J., Isabel, N. (2014): Rates of spontaneous hybridization and hybrid recruitment in 
co-existing exotic and native mature larch populations. Tree Genetics \& Genomes, 10(4): 965-975.

Müller-Starck, G., Felber, F. (2010): Genetische Variation in Altbeständen der Lärche und ihrer natürlichen Verjüngung im Alpenraum (Genetic variation in adult stands of European larch and its natural regeneration in the alpine habitat). Schweizerische Zeitschrift für Forstwesen, 161(6): 223-230.

Müller-Starck, G., Starke, R. (1993): Inheritance of isoenzymes in European beech (Fagus sylvatica L.). Journal of Heredity, 84: 291-296.

Nishimura, M., Setoguchi, H. (2011): Homogeneous genetic structure and variation in tree architecture of Larix kaempferi along altitudinal gradients on Mt. Fuji. Journal of Plant Research, 124(2), 253-263.

Ostrowska, E., Muralitharan, M., Chandler, S., Volker, P., Hetherington, S., Unshea, F.D. (1998): Optimizing conditions for DNA isolation from Pinus radiata. In Vitro Cellular and Developmental Biology-Plant, 34: 108-111.

Parducci, L., Szmidt A.E. (1999): PCR-RFLP analysis of cpDNA in the genus Abies. Theoretical and Applied Genetics, 98: 802-808.

Perry, D.J., Bousquet, J. (1998): Sequence-tagged-site (STS) markers of arbitrary genes: the utility of black spruce-derived STS primers in other conifers. Theoretical and Applied Genetics, 97:735-743.

Petit, R.J., Demesure, B., Dumolin, S. (1998): cpDNA and mtDNA primers in plants. In: Karp, A., Isaac, P.G., Ingram, D. (eds): Molecular tools for screening biodiversity: plants and animals. Chapman and Hall, London, pp. 256-261.

Pluess, A.R. (2011): Pursuing glacier retreat: genetic structure of a rapidly expanding Larix decidua population. Molecular Ecology, 20: 473-485.

Polezhaeva, M.A., Lascoux, M., Semerikov, V.L. (2010): Cytoplasmic DNA variation and biogeography of Larix Mill. in Northeast Asia. Molecular Ecology, 19(6): 12391252.

Qiu, Y.L., Lee, J., Bernasconi-Quadroni, F., Soltis, D.E., Soltis, P.S., Zanis, M., Zimmer, E.A., Chen, Z., Savolainen, V., Chase, M.W. (1999): The earliest angiosperms: evidence from mitochondrial, plastid and nuclear genomes. Nature, 402: 404-407.

Rogers, S.O., Bendich, A.J. (1988): Extraction of DNA from plant tissues. Plant Molecular Biology Manual A6: 1-10.

San Jose-Maldia, L., Uchida, K., Tomaru, N. (2009):
Mitochondrial DNA variation in natural populations of Japanese larch (Larix kaempferi). Silvae Genetica, 58(16): 234-241.

Scheepers, D., Eloy, M.C., Briquet, M. (2000): Identification of larch species (Larix decidua, Larix kaempferi and Larix $\mathrm{x}$ eurolepis) and estimation of hybrid fraction in seed lots by RAPD fingerprints. Theoretical and Applied Genetics, 100(1): 71-74.

Semerikov, V.L., Lascoux, M. (1999): Genetic relationship among Eurasian and American Larix species based on allozymes. Heredity, 83: 62-70.

Semerikov, V.L., Lascoux, M. (2003): Nuclear and cytoplasmatic variation within and between Eurasian Larix (Pinaceae) species. American Journal of Botany, 90: 1113-1123.

Semerikov, V.L., Polezhaeva, M.A. (2007): Mitochondrial DNA variation pattern in larches of eastern Siberia and the far east. Russian Journal of Genetics, 43: 646-652.

Semerikov, V.L., Vendramin, G.G., Sebastiani, F., Lascoux, M. (2006): RAPD-derived, PCR-based mitochondrial markers for Larix species and their usefulness in phylogeny. Conservation Genetics, 7(4): 621-625.

Semerikov, V.L., Zhang, H., Sun, M., Lascoux, M. (2003): Conflicting phylogenies of Larix (Pinaceae) based on cytoplasmic and nuclear DNA. Molecular Phylogenetics and Evolution, 27: 173-184.

Shiraishi, S., Watanabe, A. (1995): Identification of chloroplast genome between Pinus densiflora Sieb. et Zucc. and $P$. thunbergii Parl. based on the polymorphism in rbcL gene. Journal of Japanese Forestry Society, 77: 429-436.

Siciliano, M.J., Shaw, C.R. (1976): Separation and vizualization of enzymes on gels. In: J.Smith (ed.) Chromatographic and Electrophoretic Techniques. W. Heinemann Med.Books Ltd. London, pp. 185- 209.

Soranzo, N., Provan, J., Powell, W. (1999): An example of microsatellite length variation in the mitochondrial genome of conifers. Genome, 42: 158-161.

Taberlet, P., Geilly, L., Pautou, G., Bouvet, J. (1991): Universal primers for amplification of 3 noncoding regions of chloroplast DNA. Plant Molecular Biology, 17: 1105-1109.

Tröber, U., Haasemann, W. (2000): Pollination Effects in a Larch Hybrid Seed Orchard. Forest Genetics, 7: 7782.

Vos, P., Hogers, R., Bleeker, M., Reijans, M., Van der Lee, T., Hornes, M., Frijters, A., Pot, J., Peleman, J., Kuiper, M., Zabeau, M. (1995): AFLP: a new technique 
for DNA fingerprinting. Nucleic Acids Research, 23: 4407-4414.

Wagner, S., Gerber, S., Petit, R.J. (2012): Two highly informative dinucleotide SSR multiplexes for the conifer Larix decidua (European larch). Molecular Ecology Resources, 12(4): 717-725.

Wang, X.-R., Tsumura, Y., Yoshimaru, H., Nagasaka, K., Szmidt, A.E. (1999): Phylogenetic relationships of Eurasian pines (Pinus, Pinaceae) based on chloroplast rbcL, matK, rpl20-rps18 spacer, and trnV intron sequences. American Journal of Botany, 86: 1742-1753.

Wei, X.X., Wang, X.Q. (2003): Phylogenetic split of Larix: evidence from paternally inherited cpDNA trnTtrnF region. Plant Systematics and Evolution, 239(1-2): 67-77.

Wu, J., Krutovskii, K.V., Strauss, S.H. (1998): Abundant mitochondrial genome diversity population differentiation and convergent evolution in pines. Genetics, 150: 1605-1614.

Yang, X., Sun, X., Zhang, S. (2011): Short note: Development of six EST-SSR markers in Larch. Silvae Genetica, 60(1-6): 161-163.

Zhang, G., Sun, Z., Zhou, D., Xiong, M., Wang, X., Yang, J., Wei, Z. (2015): Development and Characterization of Novel EST-SSRs from Larix gmelinii and Their CrossSpecies Transferability. Molecules, 20: 12469-12480.

\section{Selected references referring to genetic investigations on related Larix species}

\section{Isozymes}

\section{Larix decidua}

Beletti, P., Lanteri, S., Leonardi, S. (1996): Genetic Variability among European larch (Larix decidua Mill.) populations in Piedmont, North-West Italy. Forest Genetics, 4(3): 113-121.

Konnert, M., Behm, A. (2006): Proof of identity of forest reproductive material based on reference samples. Mitteilungen der Bundesforschungsanstalt für Forstund Holzwirtschaft. Hamburg, pp. 61-71.

Lewandowski, L., Burzyk, J., Mejnartowicz, L. (1990): Genetic structure and the Mating System in an Old Stand of Polish Larch. Silvae Genetica, 40(2): 75-79.

Lewandowski, L., Mejnartowicz, L. (1988): Inheritance of allozymes in Larix decidua Mill, Silvae Genetica, 39: 184-188.

Lewandowski, L., Mejnartowicz, L. (1992): Levels and patterns of allozyme variation in some European larch (Larix decidua) populations. Hereditas, 115: 107-109.

Maier, J. (1992): Genetic variation in European larch (Larix decidua). Annales des Sciences Forestières, 49: 3947.

Mejnartowicz, L., Bergmann, F. (1975): Genetic studies on European larch (Larix decidua Mill.) employing isozyme polymorphisms. Genetica Polonica, 16: 29-35.

Müller-Starck, G., Felber, F. (2010): Genetische Variation in Altbeständen der Lärche und ihrer natürlichen Verjüngung im Alpenraum (Genetic variation in adult stands of European larch and its natural regeneration in the alpine habitat). Schweizerische Zeitschrift für Forstwesen, 161(6): 223-230.

\section{Larix gmelinii}

Larionova, A.Y., Yakhneva, N.V., Abaimov, A.P. (2004): Genetic diversity and differentiation of Gmelin larch Larix gmelinii populations from Evenkia (Central Siberia). Genetika, 40(10): 1127-1133.

\section{Larix laricina:}

Cheliak, W.M., Pitel, J.A. (1985): Inheritance and Linkage of Allozymes in Larix laricina Silvae Genetica, 34(4-5): 142-148.

Cheliak, W.M., Wang, J., Pitel, J.A. (1988): Population structure and genic diversity in tamarack, Larix laricina (DuRoi) K. Koch. Can. J. For. Res., 18(10): 1318-1324.

Ying, L., Morgenstern, E.K. (1991): The population structure of Larix laricina in New Brunswick, Canada Silvae Genetica, 40(5/6): 180-184.

\section{DNA-markers}

With few exceptions references cited for Larix kaempferi refer also to many other Larix species, since generally species-diagnostic markers were developed and the crossspecies transferability tested.

\section{Larix decidua}

Wagner, S., Gerber, S., Petit, R.J. (2012): Two highly informative dinucleotide SSR multiplexes for the conifer Larix decidua (European larch). Molecular Ecology Resources, 12(4): 717-725.

\section{Larix gmelinii}

Zhang, G., Sun, Z., Zhou, D., Xiong, M., Wang, X., Yang, J., Wei, Z. (2015): Development and Characterization of Novel EST-SSRs from Larix gmelinii and Their CrossSpecies Transferability. Molecules, 20: 12469-12480.

\section{Larix decidua and Larix occidentalis}

Khasa, P.D., Newton, C.H., Rahman, M.H., Jaquish, B., Dancik, B.P. (2000): Isolation, characterization and inheritance of microsatellite loci in alpine larch and western larch. Genome, 43: 439-448. 


\title{
Molecular markers used for genetic studies in Lodgepole pine (Pinus contorta (Dougl. ex. Loud.))
}

\author{
Marjana Westergren
}

Slovenian Forestry Institute, Večna pot 2, 1000 Ljubljana, Slovenia

\section{General remarks}

Lodgepole pine is widely distributed in Western North America, from Mexico to Alaska and from the Pacific eastwards to South Dakota. It is ecologically and economically important, occurring as a dominant tree species from costal to subalpine forests.

It is divided into four morphologically distinct subspecies (ssp. latifolia, contorta, murrayana and bolanderi) (Critchfield 1957). Each subspecies grows in a separate part of the species' range and is hypothesized to be adapted to local climate and environmental conditions (Ying and Liang 1994, Rehfeldt et al. 1999, 2001). The geographic distributions of the subspecies do not overlap except for the subspecies contorta and bolanderi in California.

High gene flow among subspecies and various divisions of population structure has been deduced from population genetic analysis (Wheeler and Guries 1982a, Fazekas and Yeh 2006, Bisbing 2013). In general, no correlation between genetic structure and differences among subspecies were observed while there was discontinuity between coastal and montane stands and a north-south differentiation along the coast and the Rockies revealed by isozymes, RAPDs, mtDNA and cpDNA SSRs (reviewed in Jaramillo-Correa et al. 2009 but see Bisbing 2013 for an example where such correlation was observed).

\section{Isozymes}

Isozymes were used in single-locus (Wheeler and Guries 1982a) and multi-locus (Yang and Yeh 1993) genetic diversity studies as well to compare six quantitative traits and isozyme variation in five populations (Yang et al. 1996). Yang and Yeh (1995) conducted a range-wide isozyme variation study with 21 loci (14 enzyme systems) in 66 populations from the three subspecies to study the gene flow patterns and the genetic structure among the three subspecies contorta, latifolia and murrayana.

\section{Material for protein extraction}

Proteins were extracted from seed (megagametophytes) (Wheeler and Guries 1982 a,b).

\section{Protein extraction and separation protocols}

Sample preparation, extraction and separation protocols are described in Guries and Ledig (1978) and O'Malley et al. $(1979,1980)$. Staining and gel-buffer recipes were described by Shaw and Prasad (1970), Harris and Hopkinson (1976) and Siciliano and Shaw (1976).

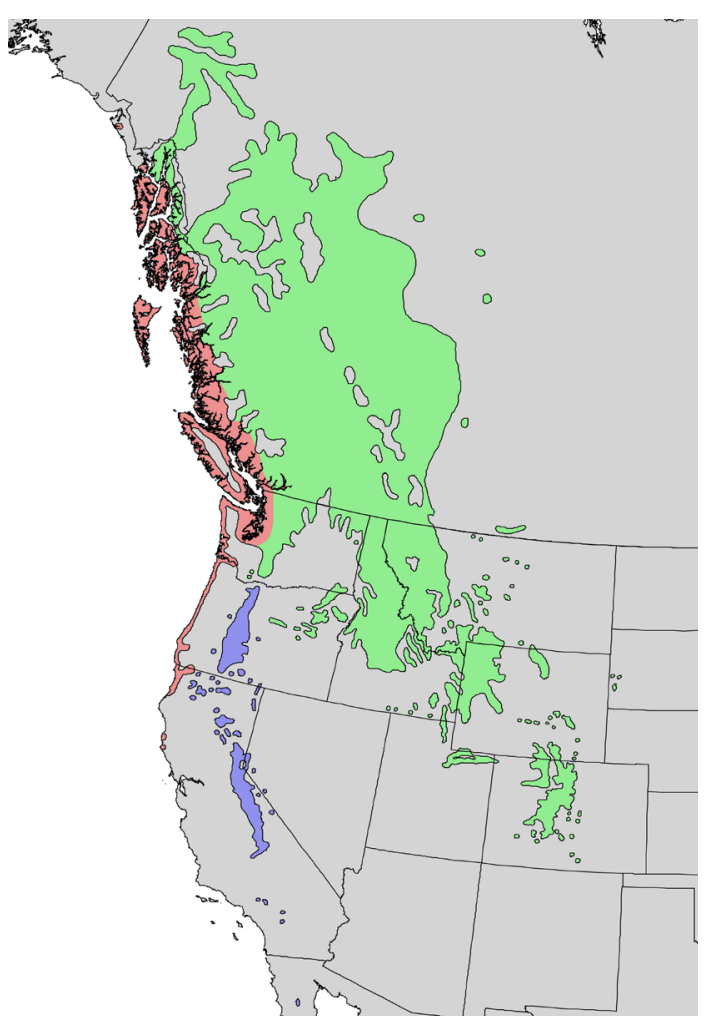

Figure 1. Native distribution range of Lodgepole pine. Subspecies latifolia denoted with green, subspecies contorta denoted with red and subspecies murrayana marked with blue (Source: USGS, USA) 
Table 1: List of enzymes, scored loci and number of alleles for Lodgepole pine

\begin{tabular}{|l|l|l|l|}
\hline \multicolumn{1}{|c|}{ Enzyme system } & \multicolumn{1}{|c|}{ E.C. Number } & \multicolumn{1}{c|}{ Scored loci } & \multicolumn{1}{c|}{ References } \\
\hline Aspartate aminotransferase & 2.6 .1 .1 & AAT-1,-2 & $1,2,3$ \\
\hline Acid phosphatase & 3.1 .3 .2 & ACP-1 & 1 \\
\hline Aconitase & 4.2 .1 .3 & ACO-1 & $1,2,3$ \\
\hline Alcohol dehydrogenase & 1.1 .1 .1 & ADH-1,-2,-3 & $1,2,3$ \\
\hline Alcaline phosphatases & 3.1 .3 .1 & APH & 2,3 \\
\hline Adenylate kinase & 2.7 .4 .3 & AK-1 & 1 \\
\hline Aldolase & 4.1 .2 .13 & ALD-1,-2 & 1 \\
\hline alpha-Galactosidase & 3.2 .1 .22 & alph-GAL-1,-2 & 1 \\
\hline Diaphorase & 1.6 .2 .2 & DIA-1,-2,-3,-4 & $1,2,3$ \\
\hline Hexosdiphosphatase & 3.1 .3 .11 & HDP-1 & 1 \\
\hline Fumarase & 4.2 .1 .1 & FUM-1 & 1 \\
\hline 4-Methylumbelliferyl acetate & 3.1 .1 .56 & 4-MUA-1 & 1 \\
\hline 4-Methylumbelliferyl butyrate & 3.1 .1 .56 & 4-MUB-1 & 1 \\
\hline Glycerate dehydrogenase & 1.1 .1 .29 & GLD-1 & 1 \\
\hline Glutamate dehydrogenase & 1.4 .1 .2 & GDH-1 & $1,2,3$ \\
\hline Alanine aminotransferase & 2.6 .1 .2 & ALAT-1,-2 & 1 \\
\hline Glucose-6-phosphate dehydrogenase & 1.1 .1 .49 & G6PD-1 & $1,2,3$ \\
\hline 6-Phosphogluconate dehydrogenase & 1.1 .1 .44 & 6PGD-1,-2 & $1,2,3$ \\
\hline Isocitrate dehydrogenase & 1.1 .1 .42 & IDH-1 & $1,2,3$ \\
\hline Malate dehydrogenase & 1.1 .1 .37 & MDH-1,-2,-4,-5 & $1,2,3$ \\
\hline Malic enzyme & 1.1 .1 .40 & ME-1 & $1,2,3$ \\
\hline Mannosephosphate isomerase & 5.3 .1 .8 & MPI-1 & 1 \\
\hline Phosphoglucose isomerase & 5.3 .1 .9 & PGI-1,-2 & $1,2,3$ \\
\hline Phosphoglucomutase & 2.7 .5 .1 & PGM-1 & $1,2,3$ \\
\hline Leucyl-tyrosine peptidase & 3.4 .13 .11 & PEPLT-1,-2,-3 & 1 \\
\hline Valyl-leucyl peptidase & 3.4 .13 .11 & PEPVL-4 & 1 \\
\hline Sorbitol dehydrogenase & 1.1 .1 .14 & SDH-1 & 1 \\
\hline 1-Wheeler and Guries 1982a, 2-Yang and Yeh $1993,3-Y a n g$ and Yeh 1995 & \\
\hline & & & \\
\hline
\end{tabular}

\section{Important results}

- Yang and Yeh $(1993,1995)$ observed that loci Pep1 and Pep-2 were monomorphic in all populations, while the remaining 19 loci were polymorphic.

- The mean number of polymorphic loci and the mean heterozygosity over 19 polymorphic loci were, respectively, 13 and 0.194 in ssp. latifolia, 12 and 0.196 in ssp. murrayana, and 12 and 0.180 in ssp. contorta (Yang and Yeh 1993).

- Yang and Yeh (1995) observed the lack of isolation by distance in spp. latifolia but a slightly signifcant pattern of isolation by distance in spp. contorta. They observed an extensive gene flow among populations in each subspecies.

- Low differentiation between populations $(6 \%)$ as well as between subspecies (3\%) was detected.

\section{Organelle DNA markers (chloroplast (cp) DNA, mitochondrial (mt)DNA)}

\section{Loci and primers used are listed in Table 1}

\section{Material for DNA extraction}

DNA was extracted from buds (Marshall et al. 2002) and needles (Godbout et al. 2005, 2008).

\section{DNA-extraction and amplification protocols}

Total DNA was extracted from $40 \mathrm{mg}$ of ground needle tissue using the DNeasy Plant Mini Kit (QIAGEN) (Godbout et al. 2005, 2008).

Total genomic DNA extraction from buds followed the standard CTAB (cetyl trimethylammonium bromide) procedures (Doyle and Doyle 1987) in Marshall et al. (2002). 
For amplification the following PCR-protocols were used:

- $\quad$ cpSSR (Stoehr and Newton 2002)

$95^{\circ} \mathrm{C}$ for $3 \mathrm{~min}$ followed by 25 cycles of $94^{\circ} \mathrm{C}$

for $30 \mathrm{~s}, 55^{\circ} \mathrm{C}$ for $30 \mathrm{~s}, 72^{\circ} \mathrm{C}$ for $1 \mathrm{~min}$.

- $\quad$ mtDNA-marker (Godbout et al. 2008)

$94^{\circ} \mathrm{C}$ for 2 min followed by 35 cycles of $94^{\circ} \mathrm{C}$ for $30 \mathrm{~s}, 69^{\circ} \mathrm{C}$ for $15 \mathrm{~s}$, and $72^{\circ} \mathrm{C}$ for $1 \mathrm{~min}$, followed by a final extension step at $72^{\circ} \mathrm{C}$ for 5 $\min$

\section{Important results}

- High levels of cpDNA variation were obtained; in 69 orchard parents genotypes, 46 multilocus haplotypes were detected. The number of alleles per locus ranged from two to seven with gene diversity levels of 0.44-0.72. cpSSR loci 8/87, 10FR, 96FR, L2T1 in I1A2 evolve primarily by a stepwise mutation model (Stoehr and Newton 2002). Coalescent simulations point to a mutation rate of about $10^{-3}$ for chloroplast microsatellites underlying the stepwise mutation model (Marshall et al. 2002).

- mtDNA analysis revealed no correlation between genetic structure and differences among subspecies. However, three major genetic clusters were identified (Godbout et al. 2008).

Table 2: Primer information of the organelle DNA-markers used for DNA sequencing in the genetic analysis of Lodgepole pine

\begin{tabular}{|c|c|c|c|c|c|}
\hline \multirow{2}{*}{ Locus } & \multicolumn{2}{|c|}{ Primer sequence } & \multirow{2}{*}{$\mathbf{T}_{\mathrm{a}}$} & \multirow{2}{*}{ Type } & \multirow{2}{*}{ Ref. } \\
\hline & Forward & Reverse & & & \\
\hline G2R1 & AGATCGGGACAATGTATGCC & TGTCCTATCCATTAGACGAT & 55 & \multirow{6}{*}{$\operatorname{cpSSR}$} & \multirow{6}{*}{1,2} \\
\hline $8 / 87$ & ACTGCAAGGAACAGTAGAAC & CGGAACGTTTTCTGATGCAC & 55 & & \\
\hline 10FR & CAGAAGCCCAAGCTTATGGC & CGGATTGATCCTAACCATAC & 55 & & \\
\hline $96 \mathrm{FR}$ & TTTCGGGCTCCACTGTTATC & CGTACTCAATTTGTTACTAC & 55 & & \\
\hline $\mathrm{L} 2 \mathrm{~T} 1$ & ACCAATTCCGCCATATCCCC & CTAGGGGAGGATAATAACATTGC & 55 & & \\
\hline $\mathrm{I} 1 \mathrm{~A} 2$ & TTCAAGTCCAGGATAGCCCA & CTACCAACTGAGCTATATCC & 55 & & \\
\hline $\begin{array}{c}\text { nad7 } \\
\text { intron } 1\end{array}$ & GAGGGACAACCCTGGAATACT & AAGGCCTCTCCATTTCCAAT & 69 & mtDNA & 3,4 \\
\hline
\end{tabular}

1-Stoehr and Newton 2002, 2-Marshall et al. 2002, 3-Godbout et al. 2005, 4-Godbout et al. 2008, $T_{a}=$ annealing temperature

\section{Nuclear DNA markers (nSSRs, EST-SSRs, SNPs)}

a) nSSRs (putatively neutral microsatellites), ESTSSRs (expressed sequence tag derived microsatellites

\section{Loci and primers used}

In the following table Pico, LOP and PtTX2164 markers are EST-SSRs, the others are nSSRs. 


\begin{tabular}{|c|c|c|c|c|c|c|c|c|c|c|c|c|c|c|c|c|c|}
\hline$\ddot{\widetilde{\Xi}}$ & - & $a_{7}$ & & & & & 1 & & & & & & & & & & \\
\hline$F^{*}$ & & $i n$ & & & & & & & & & & $i n$ & 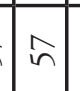 & & & & $\pi i n$ \\
\hline
\end{tabular}

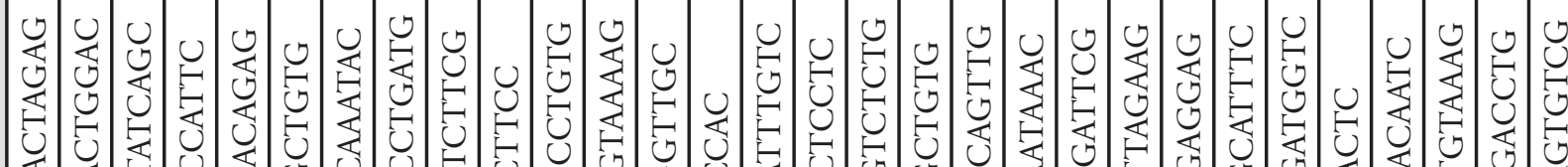

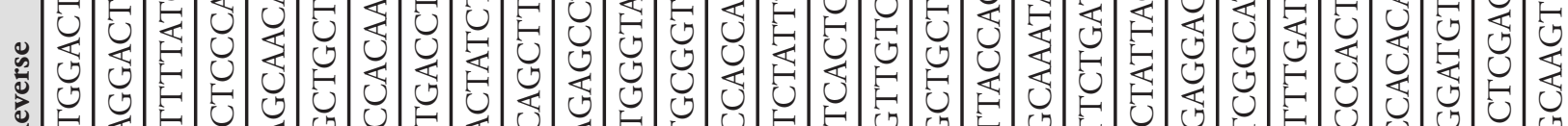
$\approx$ 至

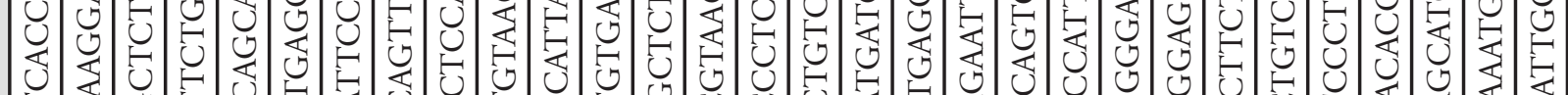

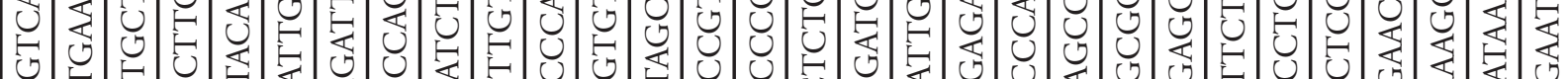
ะ

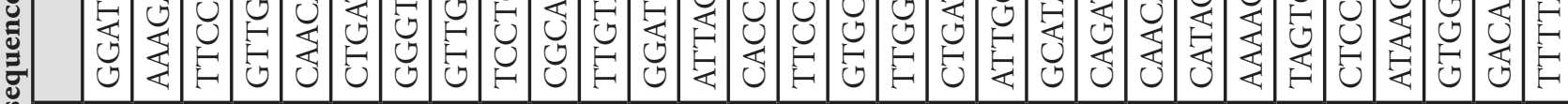

正

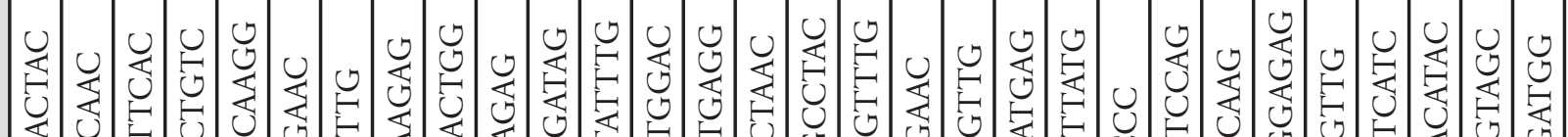
च

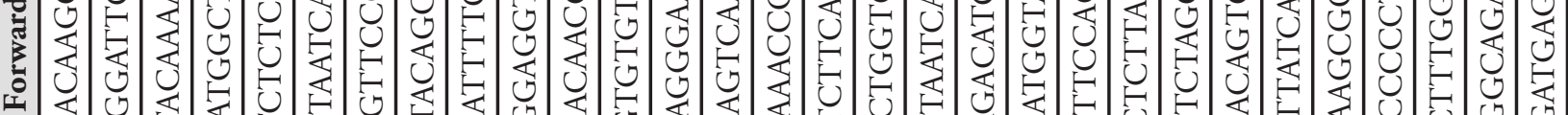

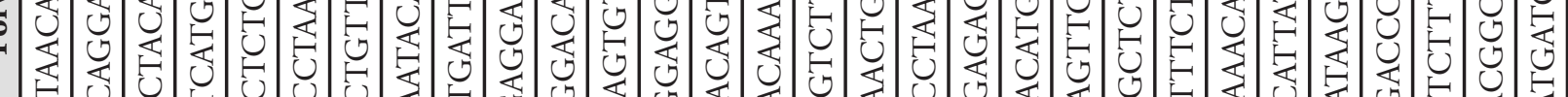
(1)

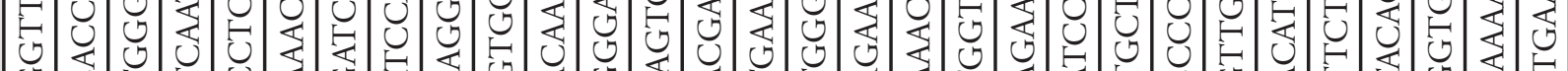

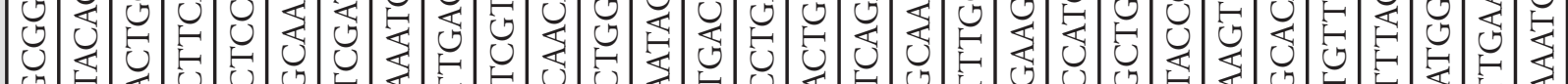

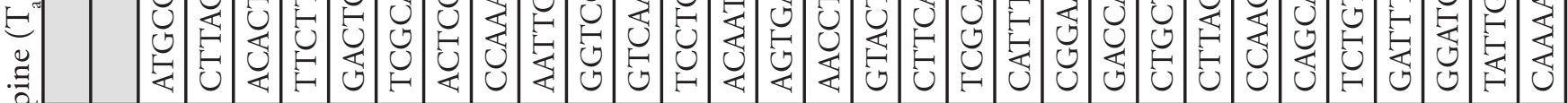

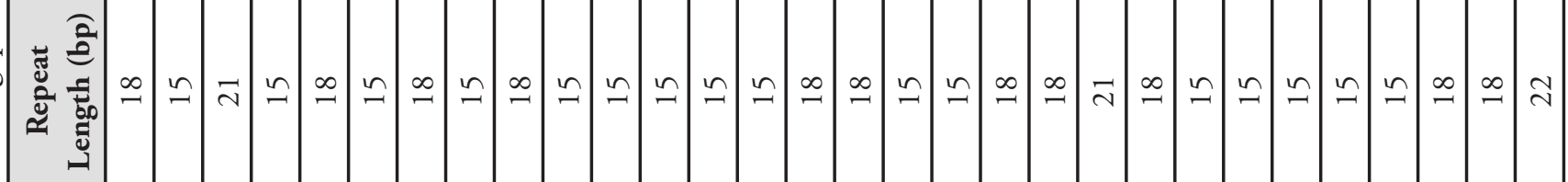

)

:

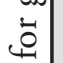

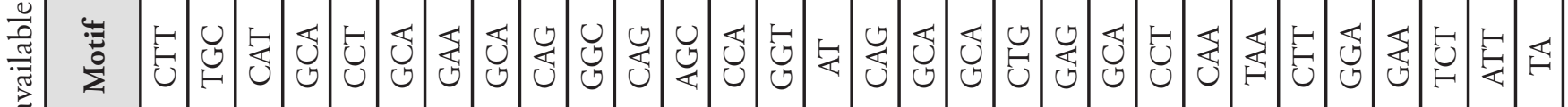

产

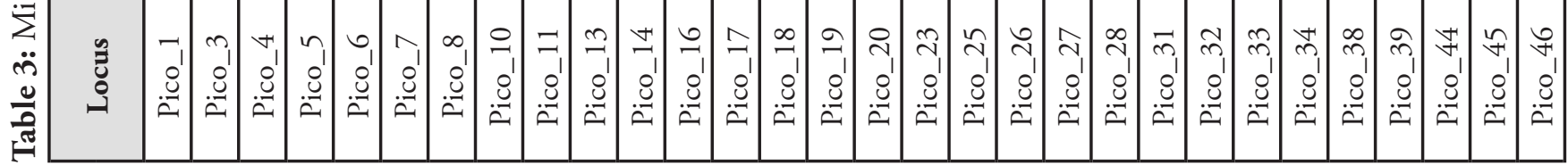




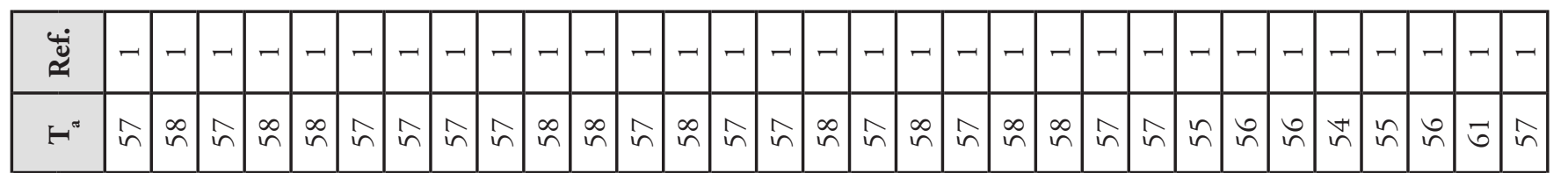

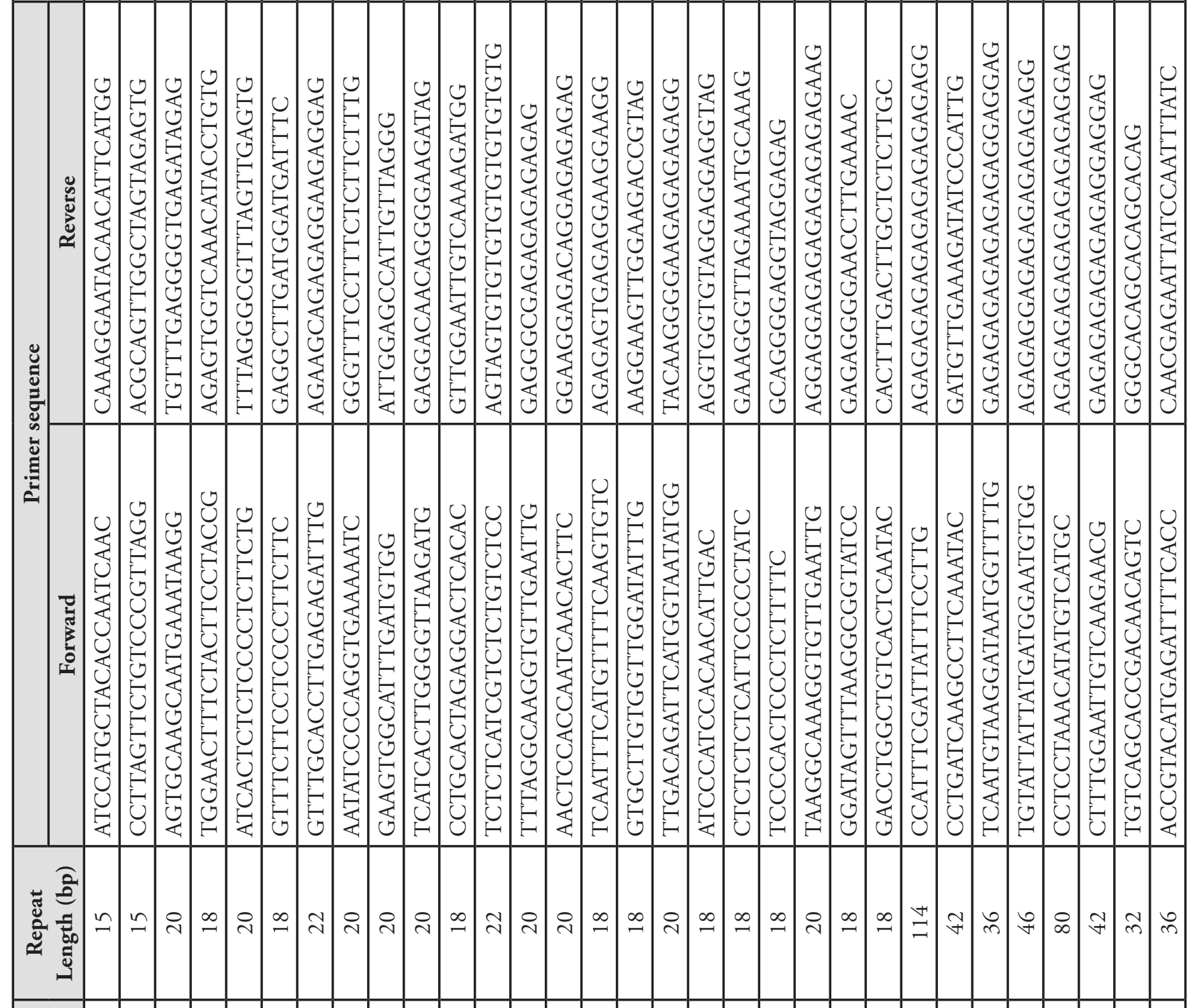

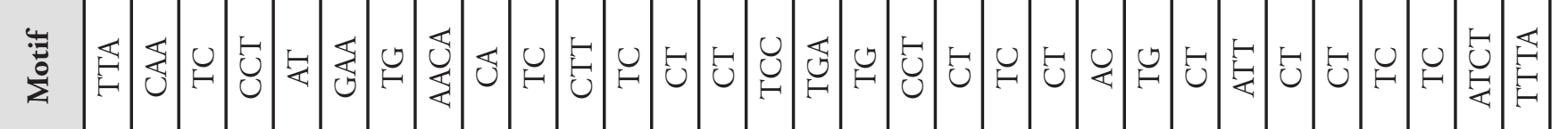

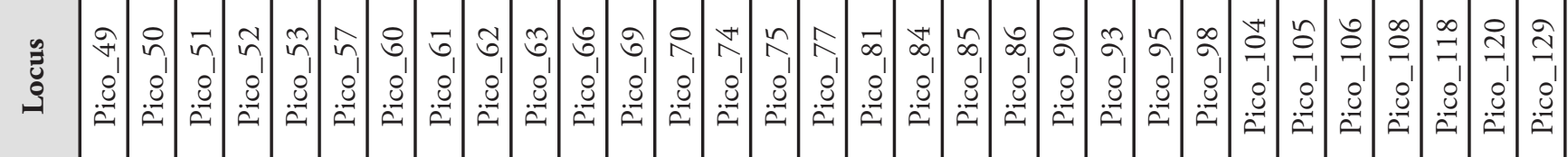




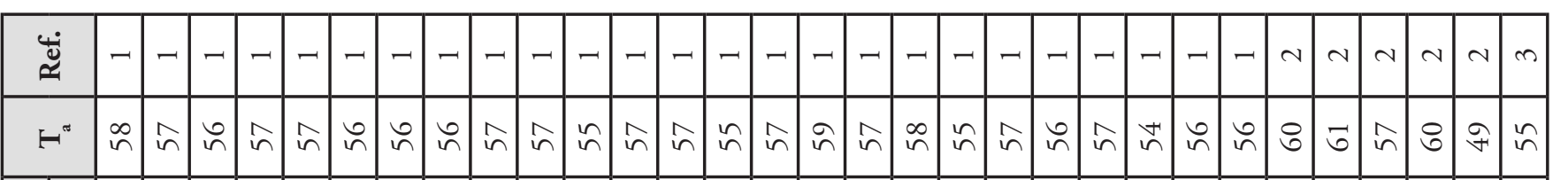

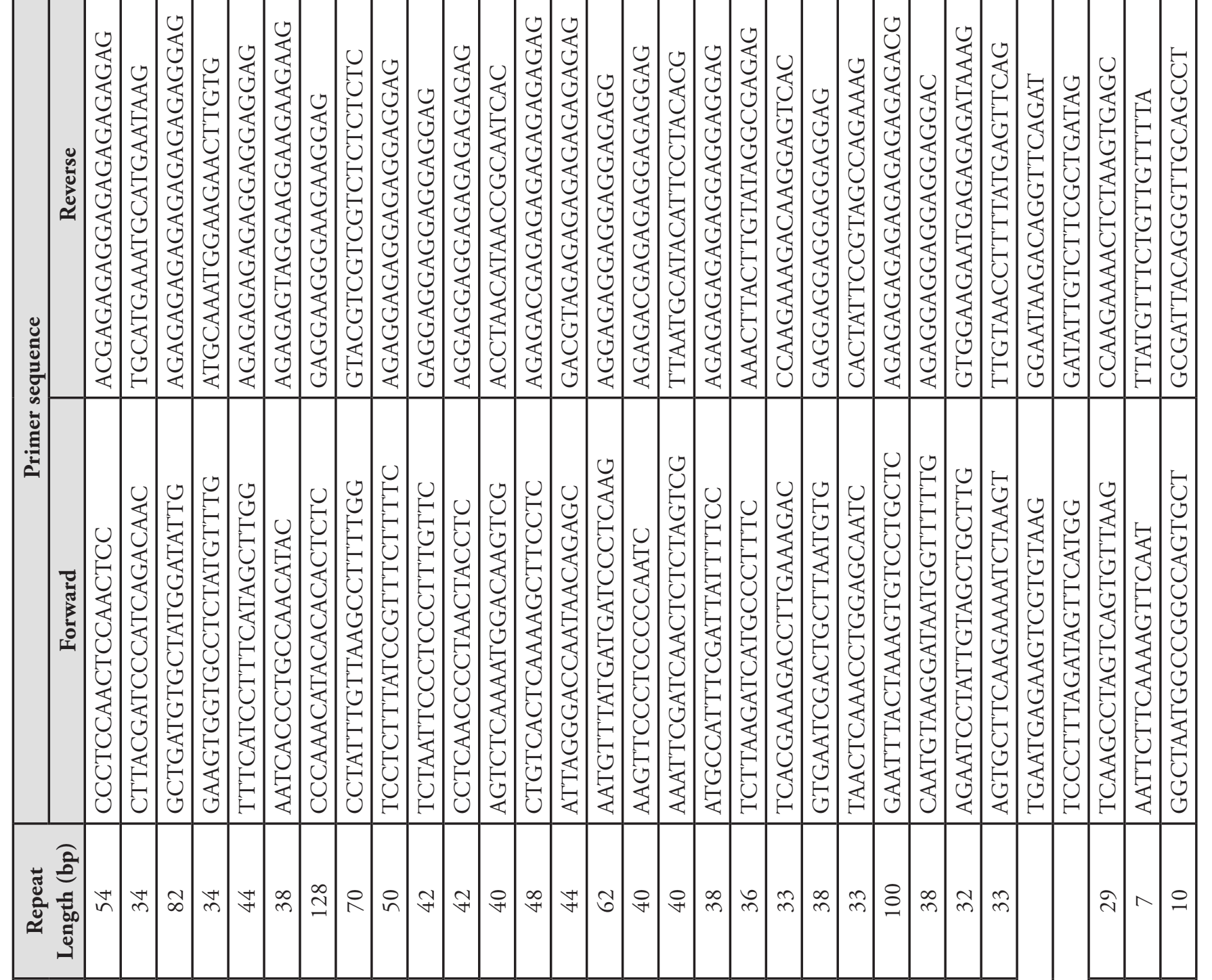

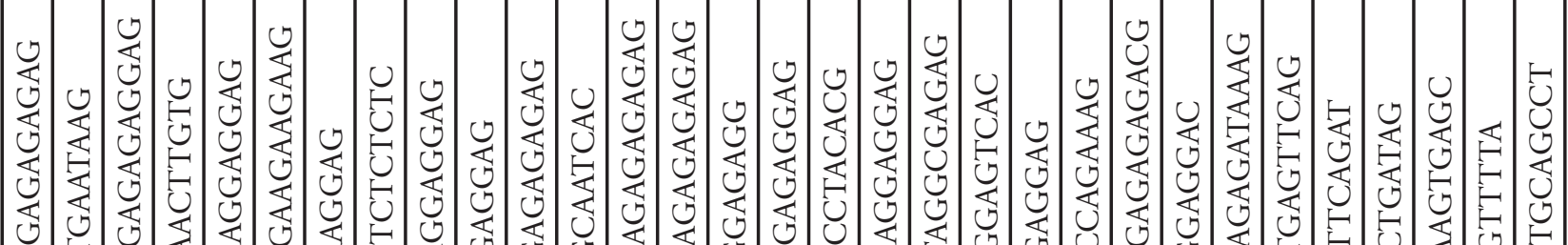

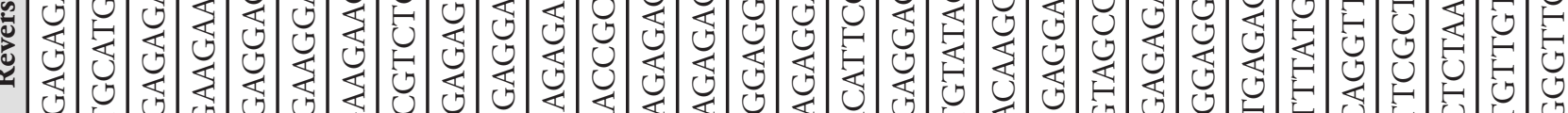

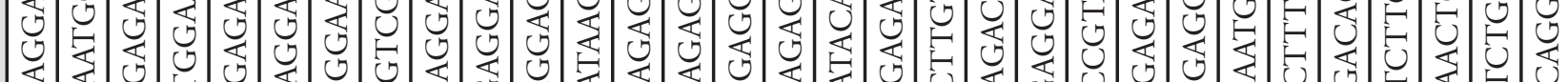

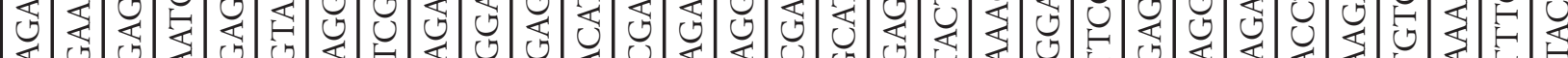

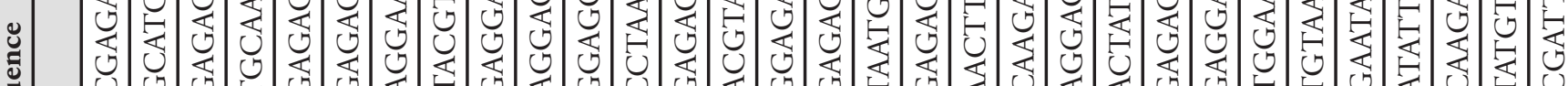

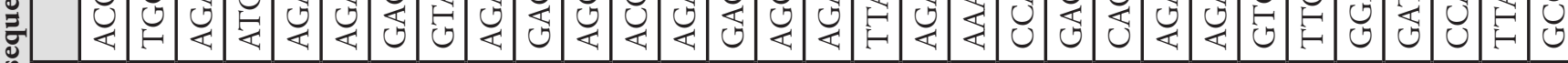

1.

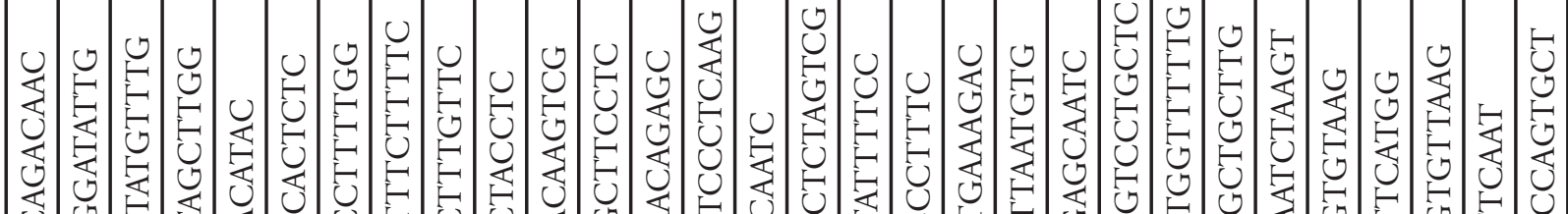

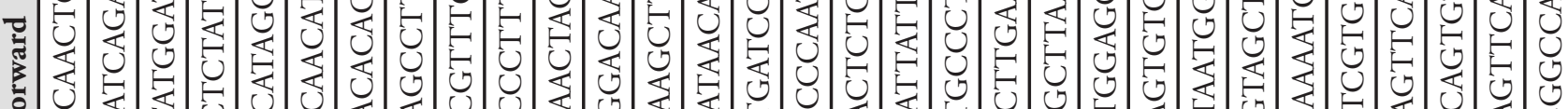

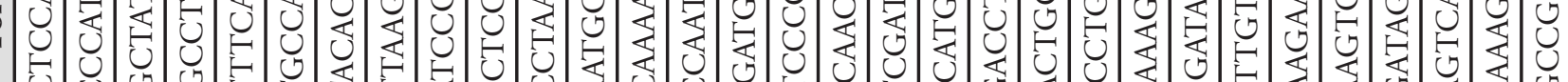

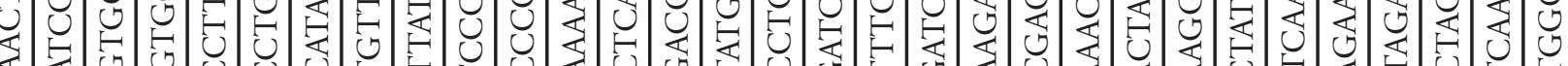
作

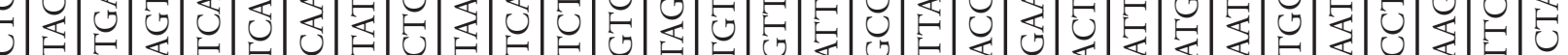

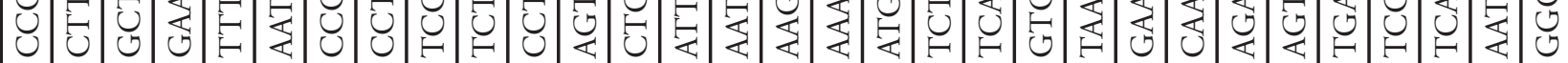

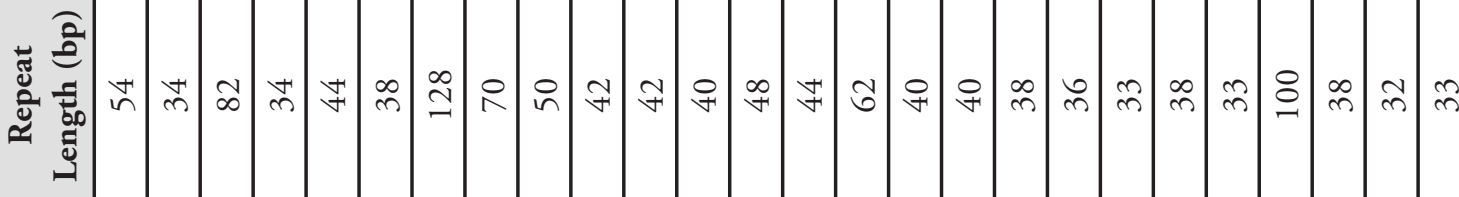

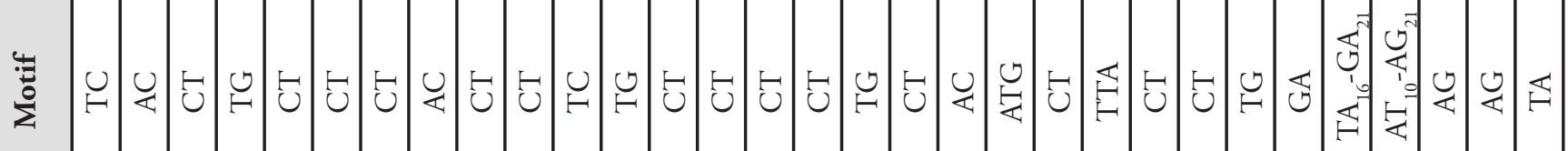

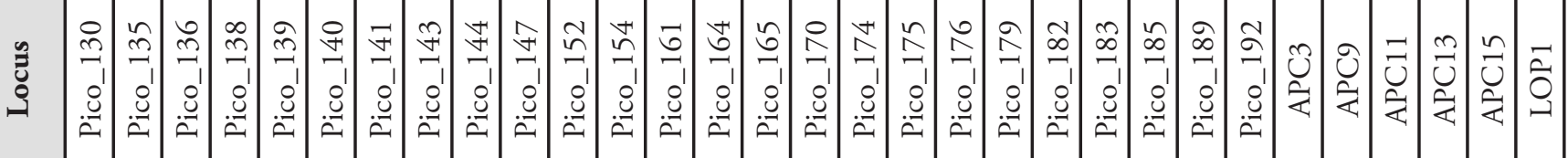




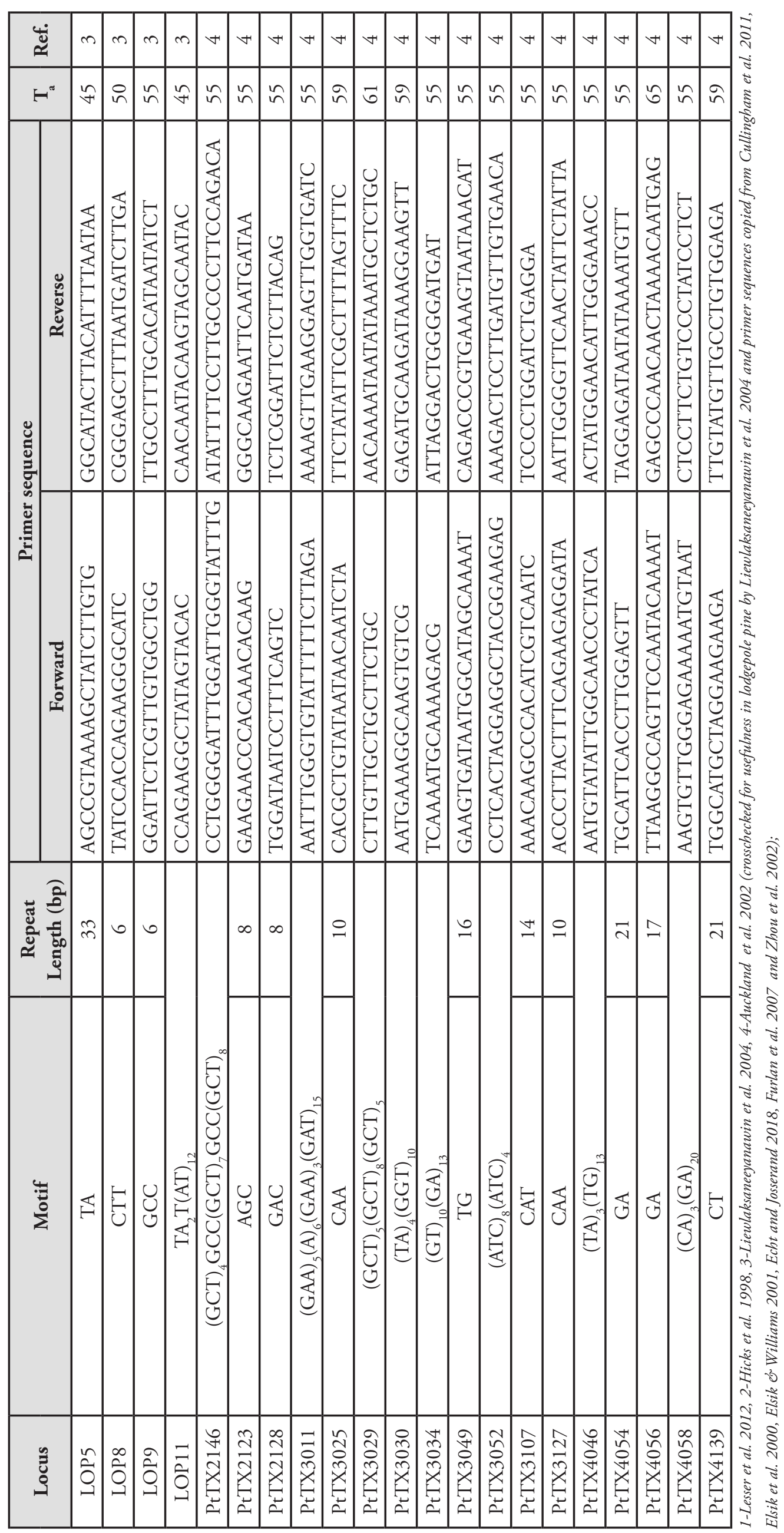




\section{Material for DNA-extraction}

DNA was extracted from needles (Hicks et al. 1998, Lesser et al. 2012).

\section{DNA-extraction and amplification protocols}

Total genomic DNA extraction followed the standard CTAB (cetyl trimethylammonium bromide) procedures (Doyle and Doyle 1987) in Lesser et al. (2012).

For amplification the following PCR-protocols were used (Lesser et al. 2012):

- $\quad 94^{\circ} \mathrm{C}$ for $5 \mathrm{~min}$, followed by 32 cycles of $94^{\circ} \mathrm{C}$ for 1 min, $45^{\circ} \mathrm{C}$ for $1 \mathrm{~min}$ and $72^{\circ} \mathrm{C}$ for $1 \mathrm{~min}$, followed by a final extension step of $72^{\circ} \mathrm{C}$ for $3 \mathrm{~min}$.

\section{Important results}

- All listed markers are polymorphic, exhibit good amplification and clean patterns (no multiple bands). Listed LOP and PtTX markers did not exhibit null alleles in the original publications.

- In her thesis, Bisbing (2013) identified three genetic clusters that coincided with three of the four subspecies (contorta, latifolia, and murrayana) using markers 69FR, PICO1, PICO4, PICO7, PICO77, PICO104, PICO109, PICO138, PICO154 and PICO179. This supports treatment of these three subspecies as separate taxonomic units. Subspecies bolanderi populations were assigned

\section{b) SNPs (single nucleotide polymorphisms)}

Loci and primers used are listed in Table 4.

Table 4: Primers for amplification of SNP loci used in Cullingham et al. (2013)

\begin{tabular}{|c|c|c|c|c|c|}
\hline Locus & $\begin{array}{l}\text { SNP poly- } \\
\text { morphism }\end{array}$ & Null & $\begin{array}{c}\text { Primer sequence } \\
F=\text { Forward, } R=\text { Reverse }\end{array}$ & $\mathbf{T}_{\mathbf{a}}$ & Annotation \\
\hline C26372-P562 & $\mathrm{G} / \mathrm{C}$ & 8 & $\begin{array}{l}\text { F:GAGCAGCCTCTGCTAGTGAA } \\
\text { R:ACAAAGAACTAGCTCACTTGTAC }\end{array}$ & 60 & $\begin{array}{l}\text { Calcium-dependent lipid } \\
\text { binding family protein }\end{array}$ \\
\hline C35213-P325 & $\mathrm{C} / \mathrm{T}$ & 1 & $\begin{array}{l}\text { F:GCCAAGGGACCACACGCTCT } \\
\text { R:CCTTGACTTGCTAATTGTGATGGCA }\end{array}$ & 65 & $\begin{array}{c}\text { Eukaryotic aspartyl protease } \\
\text { family protein }\end{array}$ \\
\hline C39371-P429 & $\mathrm{A} / \mathrm{G}$ & 41 & $\begin{array}{l}\text { F:CACTTGCTGTTGGGTGGCTGT } \\
\text { R:GCCCAGCAGGATTAATGAACTCA }\end{array}$ & 65 & $\begin{array}{l}\text { Protein of unknown } \\
\text { function (DUF3353) }\end{array}$ \\
\hline C54523-P103 & $\mathrm{A} / \mathrm{T}$ & 1 & $\begin{array}{l}\text { F:AGAACTTTTGTACACCTGACAAACT } \\
\text { R:GCGAGGCATCTATCCATAGCTCA }\end{array}$ & 60 & $\begin{array}{l}\text { Translation protein } \mathrm{SH} 3 \text {-like } \\
\text { family }\end{array}$ \\
\hline C55350-P439 & $\mathrm{C} / \mathrm{T}$ & 6 & $\begin{array}{l}\text { F:AGAGCTAAAGGAGTACAATTGTGCA } \\
\text { R:TCAGAGGACTCACTTGGTTCA }\end{array}$ & 60 & $\begin{array}{l}\text { Chaperone protein dnaJ- } \\
\text { related }\end{array}$ \\
\hline C55378-P723 & $\mathrm{T} / \mathrm{G}$ & 2 & $\begin{array}{l}\text { F:GAACGTGGTGGCTGTGGCAA } \\
\text { R:GTGCAGCTGGACAGTACAAGAAA }\end{array}$ & 65 & $\begin{array}{l}\text { Transcription factor jumonji } \\
\text { domain-containing protein }\end{array}$ \\
\hline C55401-P415 & $\mathrm{T} / \mathrm{G}$ & 0 & $\begin{array}{l}\text { F:TGACACTAATATCAGCAATGTGGCA } \\
\text { R:TGGCGCACTTTTCTGACCCA }\end{array}$ & 60 & Transcribed locus \\
\hline C63961-P710 & $\mathrm{C} / \mathrm{T}$ & 1 & $\begin{array}{l}\text { F:CGCTCATCAGTGGCTCTTCTGGT } \\
\text { R:GTGGACGATTCTCCTGGCGCT }\end{array}$ & 65 & \\
\hline C64907-P190 & $\mathrm{A} / \mathrm{C}$ & 0 & $\begin{array}{l}\text { F:AGGTACCGCTCCAATTATTGTGT } \\
\text { R:GTCGGATGATTGCACCTCTA }\end{array}$ & 60 & $\begin{array}{l}\text { Thioredoxin superfamily } \\
\text { protein }\end{array}$ \\
\hline C66807-P512 & $\mathrm{C} / \mathrm{T}$ & 1 & $\begin{array}{l}\text { F:TAAAACTTCTAGTCACGCTG } \\
\text { R:TAGCCATCTCTATCATGACA }\end{array}$ & 60 & $\begin{array}{l}\text { Beta-amylase/glycosyl } \\
\text { hydrolase family } 14\end{array}$ \\
\hline C84852-P331 & $\mathrm{A} / \mathrm{T}$ & 17 & $\begin{array}{l}\text { F:ACCTAATGCAATCCCTTCACCTCC } \\
\text { R:GGACTCTGAACATGACAGGTCCACA }\end{array}$ & 65 & $\begin{array}{c}\text { CRAL/ TRIO } \\
\text { domain/Sep14p-like } \\
\text { phosphatidylinositol transfer } \\
\text { protein }\end{array}$ \\
\hline C85320-P102 & $\mathrm{C} / \mathrm{G}$ & 11 & $\begin{array}{l}\text { F:TGAGCGAACAAACACTTAGGGT } \\
\text { R:CCATTGCCCTGTGACTCCGT }\end{array}$ & 65 & $\begin{array}{c}\text { DEK domain-containing } \\
\text { chromatin associated protein }\end{array}$ \\
\hline C85407-P1002 & $\mathrm{C} / \mathrm{G}$ & 16 & $\begin{array}{l}\text { F:ACGCTTTCTAGATACAGCATG } \\
\text { R:TTTATTTTATATTCACTCACGTCTT }\end{array}$ & 60 & Embryo defective 2737 \\
\hline
\end{tabular}

Null = missing datallocus, $T_{a}=$ annealing temperature used for resequencing 


\section{Material for DNA-extraction}

DNA was extracted from megagametophytes, seedlings, needles (Cullingham et al. 2013).

\section{DNA-extraction and amplification protocol}

SEQUENOM ${ }^{\bullet}$ iPLEX ${ }^{\oplus}$ Gold technology.

Amplification protocol: (Culingham et al. 2013):

- $\quad 94^{\circ} \mathrm{C}$ for $2 \mathrm{~min}$, followed by 35 cycles of $94^{\circ} \mathrm{C}$ for $30 \mathrm{~s}, \mathrm{Ta}\left(50-60^{\circ} \mathrm{C}\right)$ for $45 \mathrm{~s}$, and $72^{\circ} \mathrm{C}$ for $60 \mathrm{~s}$, and a final extension at $72{ }^{\circ} \mathrm{C}$ for $30 \mathrm{~min}$.

\section{Important results}

Using a panel of 7-14 SNPs Cullingham et al. (2013) could differentiate between $P$. contorta, $P$. banksiana and their interspecific hybrids, with minor decreases of assignment accuracy in the second- and third-generation backcrosses.

\section{References}

Auckland, L.D., Bui, T., Zhou, Y., Shepherd, M., Williams, C.G. (2002): Conifer microsatellite handbook. Corporate Press, Raleigh, N.C.

Bisbing, S.M. (2013): From genes to landscapes: the distribution of western conifers. $\mathrm{PhD}$ thesis, Colorado State University, 123 p.

Critchfield, W.B. (1957): Geographic variation in Pinus contorta. Harvard University Cambridge, Massachusetts, USA.

Cullingham, C.I., Cooke, J.E.K., Dang, S., Davis, C.S., Cooke, B.J., Coltman, D.W. (2011): Mountain pine beetle host-range expansion threatens the boreal forest. Molecular Ecology, 20: 2157-2171

Cullingham, C.I., Cooke, J.E.K., Dang, S., Coltman, D.W. (2013): A species-diagnostic SNP panel for discriminating lodgepole pine, jack pine, and their interspecific hybrids. Tree Genetics \& Genomes, 9: 1119-1127.

Doyle, J.J., Doyle, J.L. (1987): A rapid DNA isolation procedure for small quantities of fresh leaf material. Phytochemistry, 19: 11-15.

Echt, C., Josserand, S. (2018): DNA Fingerprinting Sets for Four Southern Pines. E-Research note SRS-24, USDA.

Elsik, C.G., Minihan, V.T., Hall, S.E., Scarpa, A.M.,
Williams, C.G. (2000): Low-copy microsatellite markers for Pinus taeda L. Genome, 43: 550-555.

Elsik, C.G., Williams, C.G. (2001): Low-copy microsatellite recovery from a conifer genome. Theoretical and Applied Genetics, 103:1189-1195.

Fazekas, A.J., Yeh, F.C. (2006): Postglacial colonization and population genetic relationships in the Pinus contorta complex. Canadian Journal of Botany, 84: 223-234.

Furlan, R.A., Seizo MoriI, E., Vagner TambarussiIII, E., Bueno de MoraesII, C., Almeida de Jesus, F., Zimback, L. (2007): Genetic structure in breeding populations of Pinus caribeae var. hondurensis by SSR markers. Bragantia, 66:4

Godbout, J., Fazekas, A., Newton, C.H., Yeh, F.C., Bosquet, J. (2008): Glacial vicariance in the Pacific Northwest: evidence from a lodgepole pine mitochondrial DNA minisatellite for multiple genetically distinct and widely separated refugia. Molecular Ecology, 17: 2463 2475.

Godbout, J., Jaramillo-Correa, J.P., Beaulieu, J., Bosquet, J. (2005): A mitochondrial DNA minisatellite reveals the postglacial history of jack pine (Pinus banksiana), a broad-range North American conifer. Molecular Ecology, 14: 3497-3512.

Guries, R.P., Ledig, F.T. (1978): Inheritance of some polymprphic isoenzymes in pitch pine (Pinus rigida Mill). Heredity, 40:27-32.

Harris, H., Hopkinson, D.A. (1976): Handbook of enzyme electrophoresis in human genetics. NorthHolland Publishing Co., Amsterdam.

Hicks, M., Adams, D., O'Keefe, S., Macdonald, E., Hodgetts, R. (1998): The development of RAPD and microsatellite markers in lodgepole pine (Pinus contorta var. latifolia). Genome, 41: 797-805.

Jaramillo-Correa, J.P., Beaulieu, J., Khasa, D.P., Bosquet, J. (2009): Inferring the past from the present phylogeographic structure of North American forest trees: seeing the forest for the genes. Canadian Journal of Forest Research, 39(2): 286-307.

Lesser, M.R., Parchman, T.L., Buerkle, C.A. (2012): Cross-species transferability of SSR loci developed from transciptome sequencing in lodgepole pine. Molecular ecology resources, 12: 448-455.

Liewlaksaneeyanawin, C., Ritland, C.E., El-Kassaby, Y.A., Ritland, K. (2004): Single-copy, species-transferable microsatellite markers developed from loblolly pine ESTs. Theoretical and Applied Genetics, 109: 361-369.

Marshall, H.D., Newton, C., Ritland, K. (2002): 
Chloroplast phylogeography and evolution of highly polymorphic microsatellites in lodgepole pine (Pinus contorta). Theoretical and Applied Genetics, 104: $367-$ 378.

O'Malley, D.M., Wheeler, N.C., Guries, R.P. (1980): A manual for starch gel electrophoresis. University of Wisconsin Department of Forestry Staff paper No. 11

O’Malley, D.M., Allendorf, F.W., Blake, G.M. (1979): Inheritance of isozyme variation and heterozygosity in Pinus ponderosa. Biochemical Genetics, 17:233-250

Rehfeldt, G., Wykoff, W., Ying, C. (2001): Physiologic plasticity, evolution, and impacts of a changing climate on Pinus contorta. Climatic Change, 50(3): 355-376.

Rehfeldt, G., Ying, C., Spittlehouse, D., Hamilton, D. (1999): Genetic Responses to Climate in Pinus contorta: Niche Breadth, Climate Change, and Reforestation. Ecological Applications, 69: 375-407.

Shaw, C.R., Prasad, R. (1970): Strach gel electrophoresis of enzymes - a compilation of recipes. Biochemical Genetics, 4:297-320.

Siciliano, M.J., Shaw, C.R. (1976): Separation and vizualization of enzymes on gels. In Smith, I. (ed.) Chromatographic and Electrophoretic techniques. Zone elecrtophoresis. Vol. 2. William Heinemann Medical Books Ltd. London.pp_ 185-209.

Stoehr, M.U., Newton, C.H. (2002): Evaluation of mating dynamics in a lodgepole pine seed orchard using chloroplast DNA markers. Canadian Journal of Forest Research, 476: 469-476.

Wheeler, N., Guries, R. (1982a): Population structure, genic diversity, and morphological variation in Pinus contorta Dougl. Canadian Journal of Forest Research, 12: 595-696.

Wheeler, N., Guries, R. (1982b): Biogeography of lodgepole pine. Canadian Journal of Botany, 60: 18051814.

Yang, R.C., Yeh, F.C. (1993): Multilocus structure in Pinus contorta Dougl. Theoretical and applied genetics, 87(5): 568-576.

Yang, R.C., Yeh, F.C. (1995): Patterns of gene flow and geographic structure in Pinus concorta Dougl. International Journal of Forest Genetics, 2(2): 65-75

Yang, R.C., Yeh, F.C., Yanchuk, A.D. (1996): A comparison of isozyme and quantitative genetic variation in Pinus contorta ssp. latifolia by $\mathrm{F}_{\mathrm{ST}}$ Genetics, 142(3), 1045-1052.

Ying, C., Liang, Q. (1994): Geographic pattern of adaptive variation of lodgepole pine (Pinus contorta
Dougl.) within the species' coastal range: field performance at age 20 years. Forest ecology and management, 67: $281-298$.

Zhou, Y., Bui, T., Auckland, L.D., Williams, C.G. (2002): Undermethylated DNA as a source of microsatellites from a conifer genome. Genome, 45: 91-99 


\title{
Molecular markers used for genetic studies in Eastern white pine (Pinus strobus L.)
}

\author{
Paraskevi Alizoti \\ Aristotle University of Thessaloniki, School of Forestry and Natural Environment, 54124 Thessaloniki, Greece
}

\section{General remarks}

Eastern white pine (Pinus strobus L.) is naturally distributed in eastern North America, spanning from Newfoundland to northern Georgia and westward to Manitoba and Minnesota (Figure 1). The species is characterized by a vast phenotypic and genetic variation, resulting from its wide and disjunct distribution in a broad spectrum of environmental niches. Eastern white pine is an ecologically important species and a highvalued one as timber resource. The species is among the most extensively planted American trees, due to its use for the Christmans tree industry, for reforestation and landscaping.

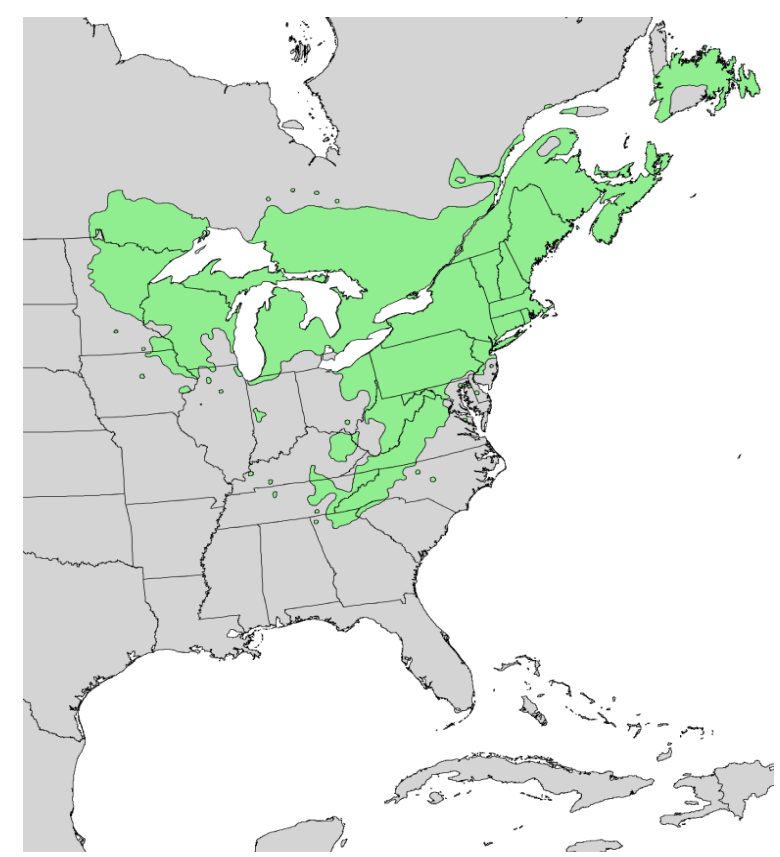

Figure 1. Natural distribution range of Pinus strobus. (Adopted from: http://www. usgs.gov/, Based on: Little 1971).
It grows in cool and humid regions of eastern North America, where the average July temperature ranges from 18 to $23^{\circ} \mathrm{C}$, the annual precipitation from $510 \mathrm{~mm}$ to $2030 \mathrm{~mm}$ and the growing season from 90 to 180 days. The species can withstand a frost depth of above $178 \mathrm{~cm}$, an average annual snowfall from $13 \mathrm{~cm}$ to more than 254 $\mathrm{cm}$ and it can grow on various soils, ranging from sandy ones (where it is a strong competitor) to sandy loams, slit-loams, clay soils and poorly to very poorly drained soils (Wendel 1980, Mader 1985, Wendel and Smith 1990). The species forms pure and mixed forests and can function as a pioneer species, but also as a physiographic climax species on dry and sandy soils, as a long-lived successional species and thus, as a component of the climax forests throughout its range.

Eastern white pine may hybridize with other native and exotic to North America pines such as: Pinus monticola, Pinus peuce, Pinus griffithii, Pinus parviflora, Pinus flexilis and Pinus ayacahuite.

\section{Isozymes}

Isozyme studies focused mainly on the study of the genetic variation present among and within populations of the species. Populations could be under different management practices (Epperson and Chung 2001), or natural populations declining for over a century (Rajora et al. 1997) or under harvesting effect (Buchert et al. 1997). For enzymes encoded by multiple loci, the loci were numbered from anodal to cathodal direction, as described in Buchert et al. 1997 (Epperson and Chung 2001).

\section{Material for isozyme extraction}

- Haploid megagametophytes (Rajora et al. 1997, Chagala 1996, Buchert et al. 1997)

- Needles (Epperson and Chung 2001, Chagala 1996, Buchert et al. 1997, Myers et al. 2007) 
Table 1: List of enzymes, E.C. number, scored loci and relative references for eastern white pine.

\begin{tabular}{|c|c|c|c|c|}
\hline Enzyme system & E.C. Number & Scored loci & $\begin{array}{c}\text { Number of alleles per } \\
\text { locus }\end{array}$ & References \\
\hline Acid phosphatase & 3.1 .3 .2 & APH-1,-2,-3,-4 & $5,2,1,2$ & $1,3,4$ \\
\hline Adenylate kinase & 2.7 .4 .3 & $\mathrm{AK}-1,-2$ & 3,2 & $3,4,6$ \\
\hline Alcohol dehydrogenase & 1.1.1.1 & $\mathrm{ADH}-1,-2,-3$ & $4,4,2$ & 4,5 \\
\hline Aldolase & 4.1 .2 .13 & ALD-1,-2-,3,-4 & $2,2,3,2$ & 3,4 \\
\hline Aconitase & 4.2 .1 .3 & ACO- $1,-2$ & $*, 3$ & 3,6 \\
\hline $\begin{array}{l}\text { Aspartate aminotransferase or } \\
\text { Glutamate oxaloacetate transaminase }\end{array}$ & 2.6.1.1 & AAT(GOT)-1,-2,-3 & $2,3,3$ & $1,2,3,4,5,6$ \\
\hline Diaphorase & 1.6 .4 .3 & DIA-1,-2,-3,-4, 5 & $2,2,1,2,2$ & $1,3,4,5$ \\
\hline Fumarase & 4.2 .1 .2 & FUM-1 & 2 & 1,6 \\
\hline Formate dehydrogenase & 1.2 .1 .2 & $\mathrm{FDH}$ & n.a. & 5 \\
\hline Fluorescent Esterase & 3.1 .1 .1 & $\mathrm{FE}$ & n.a. & 5 \\
\hline Glutamate dehydrogenase & 1.4 .1 .2 & GDH-1,-2,-3.-4.-5 & $3,3,3,2,2$ & 4,5 \\
\hline Glucose-6-phosphate-dehydrogenase & 1.1 .1 .49 & G6PDH-1 & 2 & 6 \\
\hline Hexokinase & 2.7 .1 .1 & Hex-1 & 2 & 6 \\
\hline Isocitrate dehydroenase & 1.1 .1 .42 & IDH-1,-2 & 5,3 & $1,3,4,5$ \\
\hline Leucine aminopeptidase & 3.4 .11 .1 & LAP-1,-2,-3 & $4,4,3$ & $1,3,4,5$ \\
\hline Malate dehydrogenase & 1.1 .1 .37 & MDH-1,-2.-3,-4 & $2,4,4,3$ & $1,2,3,4,5,6$ \\
\hline Malic enzyme & 1.1 .1 .82 & ME-1 & 3 & 1,4 \\
\hline Menadione reductase & 1.6 .99 .2 & MNR-1,-2,-3 & $3,3,3$ & 4 \\
\hline Phosphoglucose isomerase & 5.3 .1 .9 & PGI-1,-2,-3 & $6,5,4$ & $1,2,4,5,6$ \\
\hline Peroxidase & 1.11 .1 & PER-1,-2,-3 & $4,3,3$ & 3 \\
\hline Phosphoglucomutase & 2.7 .5 .1 & PGM-1,-2 & 3,4 & $1,3,4,5,6$ \\
\hline Shikimate dehydrogenase & 1.1 .1 .25 & SDH-1,-2 & 4,4 & $1,3,4$ \\
\hline Triose-phosphate isomerase & 5.3 .1 .1 & TPI-1,-2 & n.a. & 2,5 \\
\hline 6-Phosphogluconate dehydrogenase & 1.1.1.44 & 6-PGDH-1,-2,-3, -4 & $4,3,2,2$ & $1,3,4,5$ \\
\hline
\end{tabular}

*-unclear patterns

1-Rajora et al. 1997, 2-Epperson and Chung 2001, 3-Chagala 1996, 4-Buchert et al. 1997, 5-Myers et al. 2007 6-Beaulieu and Simon 1994

\section{Important results}

Epperson and Chung (2001) reported the structured distribution of genotypes in an old growth population, following an isolation-by-distance pattern, and a random distribution in a logged population, which they attributed to the logging effect. Rajora et al. (1997) reported that the genetic variation among populations from Ontario and Newfoundland was only 6\% and the rest of the variation was harbored within populations, due to the extensive gene flow among the populations. The above authors reported no decline of genetic diversity in the declining in size, for almost a centrury, populations of the species. Buchert et al. (1997) reported on the genetic diversity between pre- and postharvest gene pools of virgin, old growth forest stands. Genetic erosion after harvesting was detected. The authors concluded that gene frequency changes occur in the progeny stands, following harvesting, and they suggest that silvicultural practices need to ensure that the gene pools of remaining old-growth stands have been reconstituted in the regenerated stands. Myers et al. (2007) reported that the allozyme-based spatial genetic structure analysis across the landscape of an isolated island in Lake Michigan revealed the existence of significant spatial genetic structure, which suggests that gene flow via seed dispersal was rather limited across the island.

\section{Organelle DNA markers (chloroplast (cp)DNA)}

\section{Loci and primers used}

Cloutier et al. (2003) used four cpSSRs together with eight putatively neutral nuclear microsatellites (nSSRs) to examine the somatic stability of the microsatellite loci 
within 12 individual genets of Eastern white pine, while Myers et al. (2007) used cpSSRs and allozymes to study the spatial genetic structure across the landscape of an island that is isolated in Lake Michigan, USA. Zinck and Rajora (2016) applied cpSSR markers to investigate postglacial phylogeography and evolution of the species in North America. All primers used in the aforementioned studies had been initially developed for amplification of cpSSR-loci in other Pinus species (Cato and Richardson 1996, Vendramin et al. 1996).

\section{Material for DNA-extraction}

- Plant tissue (Cloutier et al. 2003)

- Needles (Myers et al. 2007, Zinck and Rajora 2016)

\section{DNA-extraction protocols}

- Cato and Richardson (1996), as well as Zinck and Rajora (2016) reported DNA extraction by using a modified CTAB protocol (Doyle and Doyle 1987).

- DNA isolation system "AutoGen 850a" (AutoGen, Inc.) using plant protocol "Plant tissue DNA (system 4 ) is reported by Myers et al. (2007).

\section{Important results}

Cloutier et al. (2003) reported that there was no withinindividual variation among the cpSSR loci they tested in the studied material. Myers et al. (2007) reported that no evidence of spatial genetic structure was found in cpDNA SSR data across an island population isolated in Lake Michigan, USA. It was shown that pollen flow has been sufficient to maintain genetic diversity and prevent differentiation across an isolated island landscape over several thousand years of isolation. Zinck and Rajora (2016) observed a broad consensus between nuclear and chloroplast genetic markers in their phylogeographic study. Results support one single glacial refugium in the mid-Atlantic plain in eastern North America. From there, Pinus strobus spread and colonized its current native range mainly through two major migration routes during the Holocene: one route gave rise to populations at the western margin of the species' range in Minnesota and Ontario; the other one gave rise to central-eastern populations.

Table 2: Chloroplast microsatellite (cpSSR) markers (chloroplast DNA).

\begin{tabular}{|c|c|l|c|c|}
\hline Locus & $\begin{array}{c}\text { Repeat } \\
\text { motif }\end{array}$ & \multicolumn{1}{|c|}{$\begin{array}{c}\text { Primer sequences } \\
\text { F=Forward, R=Reverse }\end{array}$} & Ref. & Source of primer pairs \\
\hline cpSSR 1 & $(\mathrm{~A})_{\mathrm{n}}(\mathrm{G})_{\mathrm{n}}$ & $\begin{array}{l}\text { F:CAACAGAAGCCCAAGCTTATGG } \\
\text { R:TGTATTGTATGCGGATCAACTGG }\end{array}$ & 1 & Cato and Richardson (1996) \\
\hline cpSSR 5 & $(\mathrm{~T})_{\mathrm{n}}$ & $\begin{array}{l}\text { F:TCCAGGATAGCCCAGCTG } \\
\text { R:TATATCCCCCGTACTTGGACC }\end{array}$ & 1 & Cato and Richardson (1996) \\
\hline Pt63718 & $(\mathrm{T})_{\mathrm{n}}$ & $\begin{array}{l}\text { F:CACAAAAGGATTTTTTTTCAGTG } \\
\text { R:CGACGTGAGTAAGAATGGTTG }\end{array}$ & 1 & Vendramin et al. (1996) \\
\hline Pt71936 & $(\mathrm{T})_{\mathrm{n}}$ & $\begin{array}{l}\text { F:TTCATTGGAAATACACTAGCCC } \\
\text { R:AAAACCGTACATGAGATTCCC }\end{array}$ & 1 & Vendramin et al. (1996) \\
\hline Pt9383 & - & $\begin{array}{l}\text { F:AGAATAAACTGACGTAGATGCCA } \\
\text { R:AATTTTCAATTCCTTTCTTTCTCC }\end{array}$ & $2^{*}$ & Vendramin et al. (1996) \\
\hline Pt15169 & - & $\begin{array}{l}\text { F:CTTGGATGGAATAGCAGCC } \\
\text { R:GGAAGGGCATTAAGGTCATTA }\end{array}$ & 2 & Vendramin et al. (1996) \\
\hline Pt26081 & - & $\begin{array}{l}\text { F:CCCGTATCCAGATATACTTCCA } \\
\text { R:TGGTTTGATTCATTCGTTCAT }\end{array}$ & 3 & Vendramin et al. (1996) \\
\hline Pt30204 & - & $\begin{array}{l}\text { F:TCATAGCGGAAGATCCTCTTT } \\
\text { R:CGGATTGATCCTAACCATACC }\end{array}$ & 2 & Vendramin et al. (1996) \\
\hline Pt36480 & - & $\begin{array}{l}\text { F:TTTTGGCTTACAAAATAAAAGAGG } \\
\text { R:AAATTCCTAAAGAAGGAAGAGCA }\end{array}$ & $2^{*}$ & Vendramin et al. (1996) \\
\hline Pt63718 & - & $\begin{array}{l}\text { F:CACAAAAGGATTTTTTTTCAGTG } \\
\text { R:CGACGTGAGTAAGAATGGTTG }\end{array}$ & 2,3 & Vendramin et al. (1996) \\
\hline Pt71936 & - & $\begin{array}{l}\text { F:TTCATTGGAAATACACTAGCCC } \\
\text { R:AAAACCGTACATGAGATTCCC }\end{array}$ & $2^{*}, 3$ & Vendramin et al. (1996) \\
\hline
\end{tabular}

1-Cloutier et al. 2003, 2-Myers et al 2007, 3-Zinck and Rajora 2016, *-no polymorphism found in this study 


\section{Nuclear DNA markers (RAPDs, ISSRs, nSSRs, SNPs)}

a) RAPDs (Randomly amplified polymorphic DNA markers) and ISSRs (Inter-simple sequence repeat polymorphisms)

Molecular markers such as Random Amplification of Polymorphic DNA (RAPD) and Inter-Simple Sequence Repeats (ISSR) were used by Mehes et al. (2007) to study the variation among $P$. strobus populations in Canada, while Nkongolo et al. (2012) used ISSR genetic markers to assess the genetic distance among populations of the species growing in stressed areas of Northern Ontario, Canada (Tables 3, 4).

\section{Material for DNA-extraction}

- Seedling tissue (Mehes et al. 2007)

- Needle tissue (Nkongolo et al. 2012)

\section{DNA-extraction protocols}

- The method reported by Nkongolo (1999) was used for DNA extraction by Mehes et al. (2007).

- Nkonglolo et al. (2012) used also the method of Nkongolo (1999) with some modifications that involved addition of PVP (polyvinylpyrrolidone) and $\beta$-mercaptoethanol to the CTAB extraction buffer.

Table 3: RAPD markers applied in DNA amplification in Pinus strobus

\begin{tabular}{|c|c|c|c|}
\hline $\begin{array}{c}\text { Primer } \\
\text { Identification }\end{array}$ & $\begin{array}{c}\text { Nucleotide } \\
\text { sequence (5'-3') }\end{array}$ & $\begin{array}{l}\text { Fragment size } \\
\text { range (bp) }\end{array}$ & Ref. \\
\hline 9 & ACGACGTAGG & n.a. & 1 \\
\hline 10 & CCGCGGTTC & n.a. & 1 \\
\hline 11 & CCGGCTGGAA & n.a. & 1 \\
\hline 12 & GAGGGCCTGA & n.a. & 1 \\
\hline 13 & GCTCCСССАC & $360-1500$ & 1 \\
\hline 14 & CGATGGCTTT & n.a. & 1 \\
\hline 15 & TAGCCCGCTT & n.a. & 1 \\
\hline 16 & GTAGACGAGC & $480-1500$ & 1 \\
\hline 17 & GTGCGTCCTC & $250-1500$ & 1 \\
\hline 18 & GTTCTCGTGT & n.a. & 1 \\
\hline 19 & AACACACGAG & n.a. & 1 \\
\hline 23 & CCCGCCTTC & $280-3000$ & 1 \\
\hline 63 & TTCCCCGCCC & $450-2200$ & 1 \\
\hline 146 & ATGTGTTGCG & $350-2000$ & 1 \\
\hline 184 & CAAACGGCAC & $220-2400$ & 1 \\
\hline E1 & CCGCCCAAAC & $380-2000$ & 1 \\
\hline E2 & GTGGTCCGCA & $280-1800$ & 1 \\
\hline E3 & GTGGCCGCGC & $400-1500$ & 1 \\
\hline E4 & GAGGCGCTGC & $250-900$ & 1 \\
\hline E5 & CGCCCCCAGT & $400-1800$ & 1 \\
\hline E6 & CGTCGCCCAT & $420-1800$ & 1 \\
\hline E7 & CACGGCGAGT & $260-1800$ & 1 \\
\hline E8 & GGGTAACGCC & $220-2100$ & 1 \\
\hline E9 & GTGATCGCAG & $300-2000$ & 1 \\
\hline E10 & CAGCACCCAC & $350-3000$ & 1 \\
\hline E12 & TTATCGCCCC & $600-900$ & 1 \\
\hline E18 & GGACTGCAGA & $200-1800$ & 1 \\
\hline OPA 1 & CAGGCCCTTC & 500-2000 & 1 \\
\hline OPA 2 & TGCCGAGCTG & $250-2000$ & 1 \\
\hline
\end{tabular}

\begin{tabular}{|c|l|c|c|}
\hline $\begin{array}{c}\text { Primer } \\
\text { Identification }\end{array}$ & $\begin{array}{c}\text { Nucleotide } \\
\text { sequence (5'-3') }\end{array}$ & $\begin{array}{c}\text { Fragment size } \\
\text { range (bp) }\end{array}$ & Ref. \\
\hline OPA 3 & AGTCAGCCAC & $220-2000$ & 1 \\
\hline OPA 4 & AATCGGGCTG & $250-2500$ & 1 \\
\hline OPA 5 & AGGGGTCTTG & $400-2500$ & 1 \\
\hline OPA 6 & GGTCCCTGAC & $300-3000$ & 1 \\
\hline OPA 7 & CAAACGGGTG & $400-2000$ & 1 \\
\hline OPA 8 & GTGACGTAGG & $400-3000$ & 1 \\
\hline OPA 11 & CAATCGCCGT & $200-2000$ & 1 \\
\hline OPA 12 & TCGGCGATAG & n.a & 1 \\
\hline OPA 14 & TCGGCGATAG & $500-1600$ & 1 \\
\hline OPA 15 & TTCCGAACCC & $300-850$ & 1 \\
\hline OPA 16 & AGCCAGCGAA & $350-2000$ & 1 \\
\hline OPA 17 & GACCGCTTGT & $300-1600$ & 1 \\
\hline OPA 18 & AGGTGACCGT & $450-1600$ & 1 \\
\hline OPA 19 & CAAACGTCGG & $400-3000$ & 1 \\
\hline OPA 20 & GTTGCGATCC & $400-2000$ & 1 \\
\hline OPB 1 & GTTTCGCTCC & $500-1600$ & 1 \\
\hline OPB 7 & GGTGACGCAG & $500-900$ & 1 \\
\hline UBC 186 & GTGCGTCGCT & $380-2000$ & 1 \\
\hline UBC 197 & TCCCCGTTCC & $680-1500$ & 1 \\
\hline
\end{tabular}

1-Mehes et al. 2007 
Table 4: ISSR markers applied in DNA amplification in Pinus strobus

\begin{tabular}{|c|c|c|c|}
\hline $\begin{array}{c}\text { Primer } \\
\text { Identification }\end{array}$ & $\begin{array}{c}\text { Nucleotide } \\
\text { sequence }\left(5^{\prime}-3^{\prime}\right)\end{array}$ & $\begin{array}{l}\text { Fragment size } \\
\text { range }(b p)\end{array}$ & References \\
\hline 17898B & CACACACACACAGT & $300-3000$ & 1 \\
\hline 17899A & CACACACACACAAG & $300-1300$ & 1 \\
\hline Echt 1 & AATAATAATCG & n.a. & 1 \\
\hline Echt 1 (2) & GATAGATATG & n.a. & 1 \\
\hline Echt 2 & ATCATCATCCG & $400-3000$ & 1 \\
\hline Echt3 & AACAACAACGC & n.a. & 1 \\
\hline Echt 5 & AGACAGACGC & $350-2000$ & 1,2 \\
\hline Echt 6 & ACTCACTCGC & $500-2000$ & 1 \\
\hline Echt 7 & ACAGACAGCG & $300-2000$ & 1 \\
\hline HB 12 & CACCACCACGC & $320-1300$ & 1 \\
\hline HB 13 & GAGGAGGAGGC & $300-650$ & 1,2 \\
\hline HB15 & GTGGTGGTGGC & $320-1800$ & 1,2 \\
\hline UBC 809 & AGAGAGAGAGAGAGAGG & $300-700$ & 1 \\
\hline UBC 823 & ТСТСТСТСТСТСТСТСС & $500-700$ & 1 \\
\hline UBC 825 & ACACACACACACACACT & $180-580$ & 1,2 \\
\hline UBC 827 & ACACACACACACACACG & $500-1600$ & 1 \\
\hline UBC 829 & TGTGTGTGTGTGTGTGC & $500-650$ & 1 \\
\hline UBC 834 & AGAGAGAGAGAGAGAGYT & $220-1300$ & 1 \\
\hline UBC 841 & GAAGGAGAGAGAGAGAYC & $340-1800$ & 1,2 \\
\hline UBC 849 & GTGTGTGTGTGTGTGTYA & $400-1300$ & 1 \\
\hline ISSR 1 & AGAGAGAGAGAGAGAGRG & n.a. & 2 \\
\hline ISSR 5 & ACGACGACGACGAC & n.a. & 2 \\
\hline ISSR9 & GATCGATCGATCGC & n.a. & 2 \\
\hline 17899A & CACACACACACAAG & n.a. & 2 \\
\hline 17898B & CACACACACACAGT & n.a. & 2 \\
\hline
\end{tabular}

\section{Important results}

Following the Mehes et al. (2007) results, the RAPD primers 23, 63, 184, E6, E12, OPA 2,3,4,11,18, and UBC186 yielded the best amplification results, as well as the ISSR primers 17898B, 17899A, Echt 5, HB 15, UBC 834 and 841 . The study revealed that the level of genetic variation among the $P$. strobus populations was higher than that among the P. monticola ones. The results also indicated that the $P$. strobus populations were not as closely related as the populations of $P$. monticola. Nkongolo et al. (2012) reported a low differentiation among the Pinus strobus populations (planted and natural) they studied. They report that the percentage of polymorphic loci within each $P$. strobus population varied between $22 \%$ and $36 \%$, while the planted populations found to have similar or even significantly higher genetic variation from the natural ones that grew on the same site. b) nSSRs (putatively neutral microsatellites)

\section{Loci and primers used}

Development of SSR markers for Eastern white pine was mainly done by Echt et al. $(1996,1999)$ and Echt and Nelson (1997) (Table 5). 


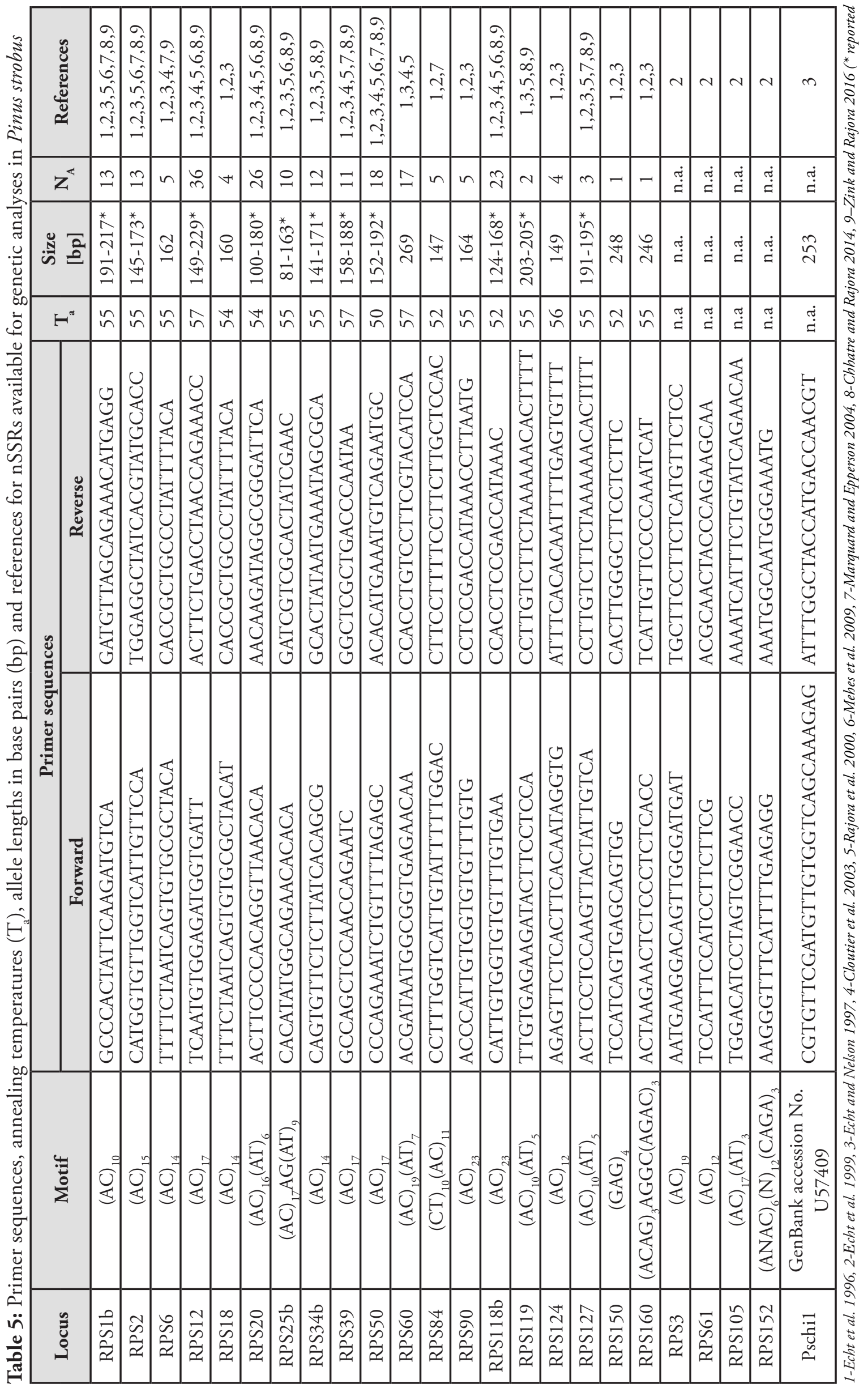




\section{Material for DNA-extraction}

- Haploid megagametophytes (Echt et al. 1996, Echt and Nelson 1997, Cloutier et al. 2003)

- Terminal buds (Echt et al. 1999, Marquardt and Epperson 2004)

- Needles (Rajora et al. 2000, Marquardt and Epperson 2004, Myers et al. 2007, Zinck and Rajora 2016)

- Seedling tissue (Mehes et al. 2009)

\section{DNA-extraction protocols}

- Genomic DNA was extracted by using the DNeasy plant mini-kit (Quiagen, Valencia, CA, USA) (Cloutier et al. 2003, Marquardt and Epperson 2004). Details on the amplification can be found in Marquardt and Epperson (2004) and Cloutier et al. (2003).

- Mehes et al. (2009) used the DNA extraction method described by Nkongolo (1999) with some modifications.

- Echt and Nelson (1997) reported the following protocol for DNA extraction from megagametophytes: "Each megagametophyte was homogenized in $100 \mathrm{mM}$ sodium acetate, $50 \mathrm{mM}$ sodium chloride, $50 \mathrm{mM}$ EDTA, 1.4\% SDS,0.5\% polyvinyl pyrolidone and $60 \mathrm{mM}$ cysteine, $\mathrm{pH}$ 5.3. The homogenate was incubated at $55^{\circ} \mathrm{C}$ for 30 min, followed by the addition of a $0.5 \mathrm{vol}$ of $3.5 \mathrm{M}$ potassium acetate, $\mathrm{pH}$ 5.3. The chilled mixture was cleared by centrifugation, transferred to a fresh tube, and the DNA precipitated in isopropanol. The DNA was re-suspended in $10 \mathrm{mM}$ Tris-Cl, $1 \mathrm{mM}$ EDTA, $\mathrm{pH} 8$, treated with RNase, and extracted twice using Strataclean resin (Stratagene Cloning Systems). For SSR analysis only, $10 \mathrm{ng}$ of each DNA template were transferred into 96-well, V-bottom plates, then dried in a food dehydrator at $50^{\circ} \mathrm{C}$, and stored at $-20^{\circ} \mathrm{C}$ until used for PCR".

- Zinck and Rajora (2016) used a modified CTAB extraction protocol according to Doyle and Doyle (1987).

\section{Important results}

Results reported by Echt et al. (1996) indicate the potential for substantial genetic gains in terms of timber production and wood quality, as well as stresstolerance, following breeding programs that rely on efficient artificial selection. Rajora et al. (2000) studied the impact of harvesting on the genetic diversity of two old-growth stands of the species in Canada and the subsequent loss of genetic variation. They reported reduction in total and mean number of alleles by nearly $26 \%$, after a reduction of tree density of about $75 \%$, while $18-21 \%$ of the low frequency and $76-92 \%$ of the rare alleles were lost from the studied stands. Mehes et al. (2009) studied the potential impact of forest fragmentation on population inbreeding and found that inbreeding levels in the studied populations were low, despite the geographic isolation and the small stand size, concluding that gene flow was high and population differentiation was low for the studied fragmented sites. Marquardt and Epperson (2004) reported results on the genetic diversity of old growth and second growth populations growing in Michigan, USA. They found high genetic diversity within the populations and low inbreeding, and lower spatial structuring in the second growth population due to logging. The presence of higher number of rare alleles in the old growth populations and higher mutation rates were also reported. Chhatre and Rajora (2014) when studying the diversity of marginal versus core populations of the species in Canada found that the central populations had significantly higher allelic and genotypic diversity. The marginal populations were genetically divergent from the central ones, and signatures of natural selection were detected at three loci in the marginal populations; two loci showing divergent selection with directional change in allele frequencies and one balancing selection. Zinck and Rajora (2016) found a high and significant genetic differentiation among 33 populations in the native range $\left(\mathrm{F}_{\mathrm{ST}}=0.104\right)$ and a south to north trend of declining genetic diversity. The latter is consistent with repeated founder effects during post-glacial recolonization. Regarding migration routes, results from nuclear microsatellites are in agreement with findings based on cpSSRs (see above).

\section{c) SNPs (single nucleotide polymorphisms)}

\section{Loci and primers used}

Nadeau et al. (2015) used Single Nucleotide Polymorphisms (SNPs) to characterize the patterns of genetic diversity and population structure across the ranges of two species; namely Pinus strobus (133 populations) and Pinus monticola (61 populations). In the Tables that follow (Table 6a-SNPs for P. strobus and Table $6 \mathrm{~b}-\mathrm{SNPs}$ designed for other species but tested in the above study) SNPs are reported that were used for the analysis of $P$. strobus populations. 


\begin{tabular}{|c|c|c|c|c|c|c|c|c|c|c|c|c|c|c|c|c|c|}
\hline 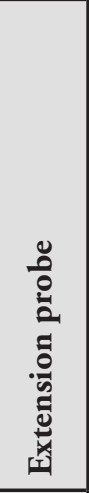 & 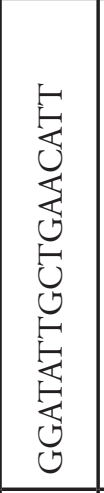 & 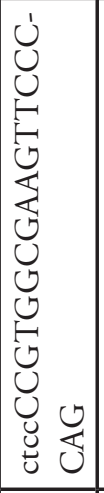 & 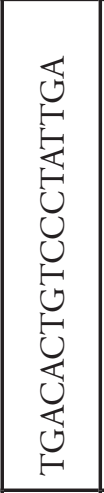 & 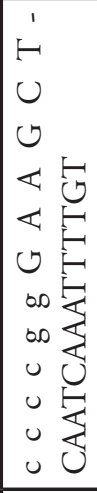 & 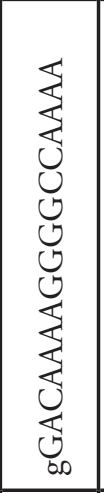 & 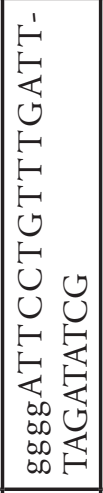 & 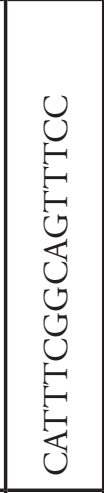 & 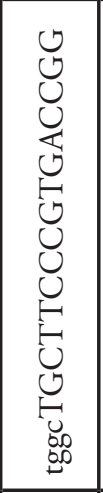 & 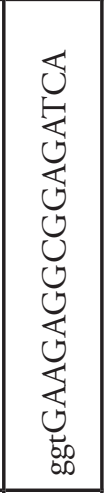 & 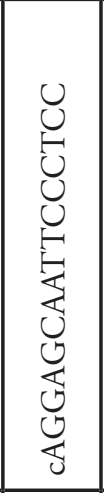 & 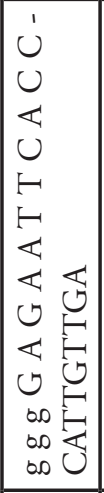 & 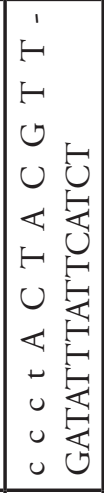 & 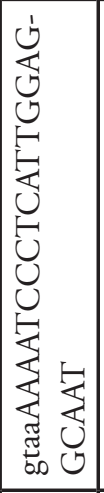 & 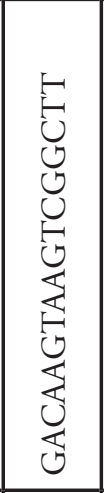 & 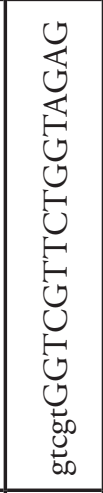 & 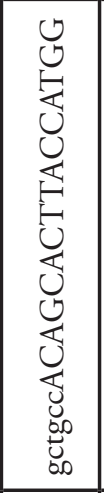 & 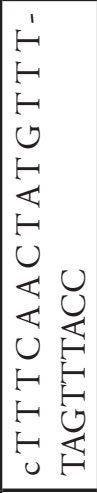 \\
\hline 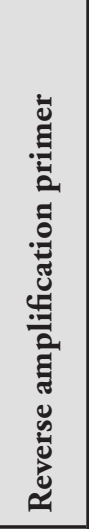 & 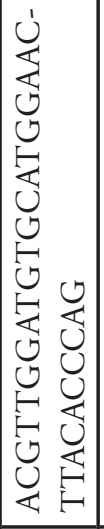 & 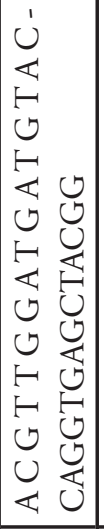 & 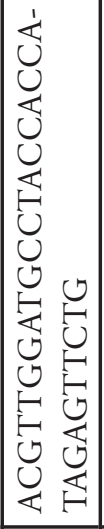 & 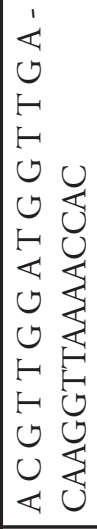 & 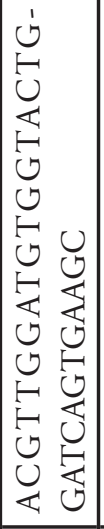 & 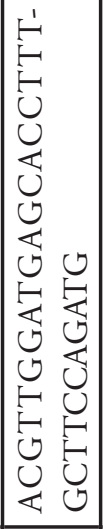 & 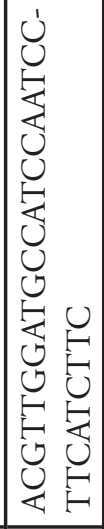 & 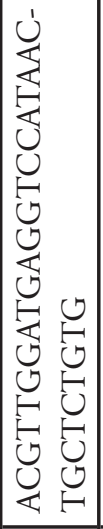 & 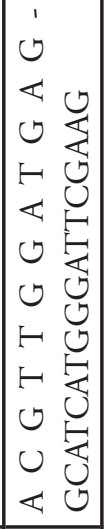 & 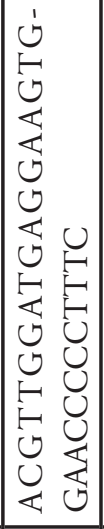 & 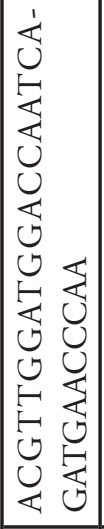 & 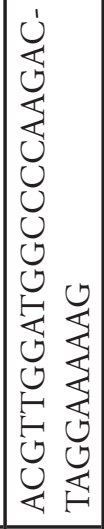 & 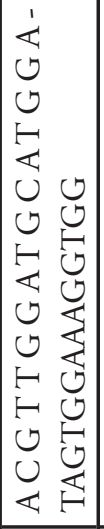 & 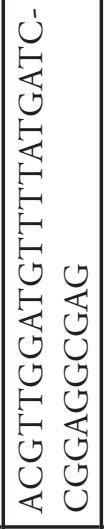 & 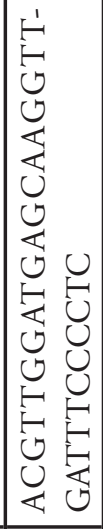 & 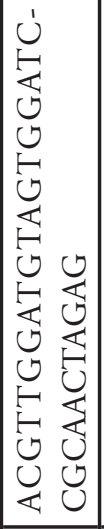 & 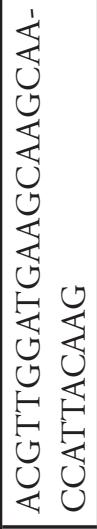 \\
\hline 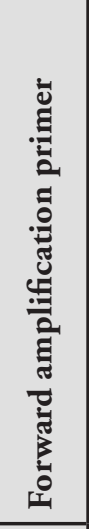 & 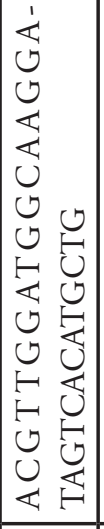 & 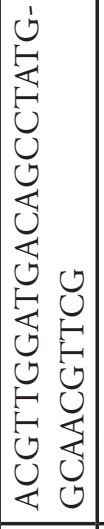 & 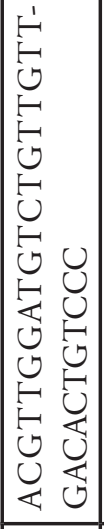 & 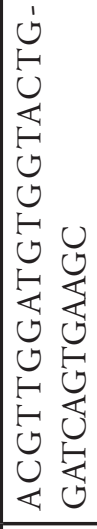 & 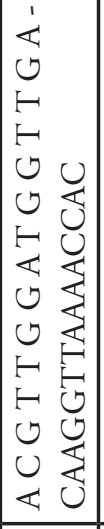 & 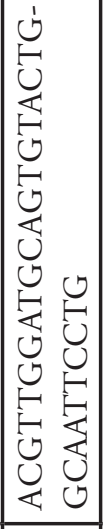 & 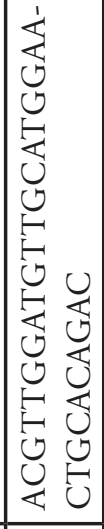 & 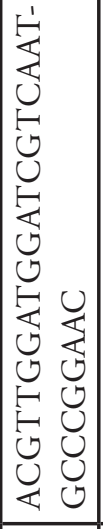 & 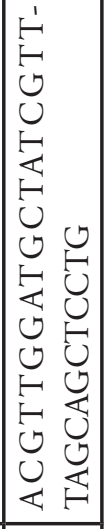 & 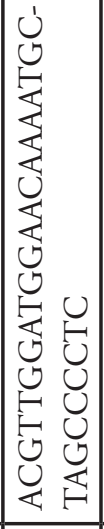 & 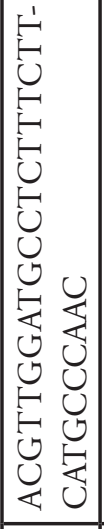 & 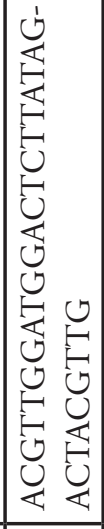 & 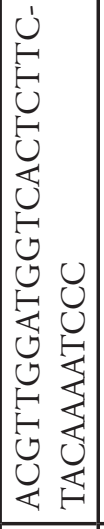 & 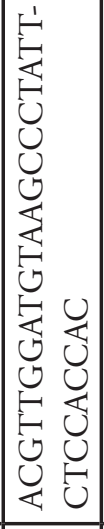 & 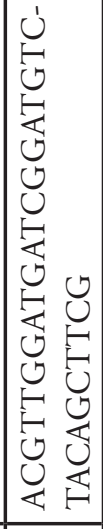 & 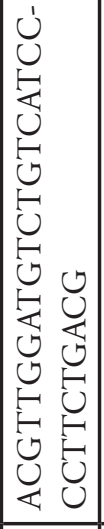 & 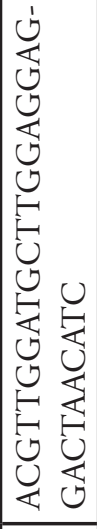 \\
\hline 它 & $\begin{array}{l}\dot{ن} \\
\dot{8} \\
\dot{3}\end{array}$ & 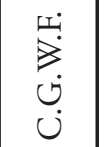 & 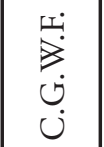 & 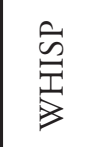 & 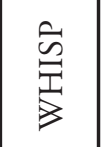 & $\begin{array}{l}\tilde{n} \\
\stackrel{3}{3} \\
\end{array}$ & $\begin{array}{l}\overrightarrow{\tilde{n}} \\
\text { 恶 }\end{array}$ & $\begin{array}{l}\overrightarrow{\tilde{n}} \\
\text { 焉 }\end{array}$ & 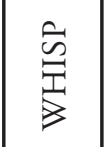 & 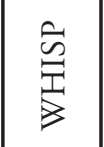 & 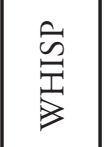 & $\begin{array}{l}\overrightarrow{\tilde{n}} \\
\stackrel{\overrightarrow{1}}{3}\end{array}$ & $\begin{array}{l}\hat{\tilde{\omega}} \\
\stackrel{\overrightarrow{\mid}}{3}\end{array}$ & $\begin{array}{l}\overrightarrow{\tilde{n}} \\
\stackrel{\overrightarrow{1}}{3}\end{array}$ & 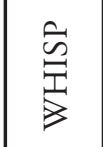 & 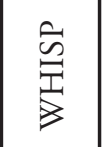 & 急 \\
\hline 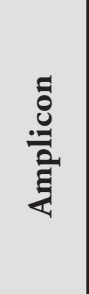 & 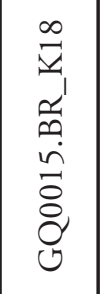 & 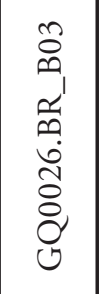 & 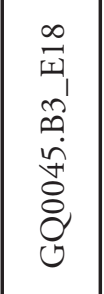 & $\begin{array}{l}\vec{\sigma} \\
\infty \\
\infty \\
\hat{\sigma} \\
\vec{\sigma} \\
\sigma^{\prime}\end{array}$ & $\begin{array}{l}\overrightarrow{\sigma_{1}} \\
\infty \\
\stackrel{0}{\hat{\sigma}} \\
\vec{\sigma} \\
\sigma^{\prime}\end{array}$ & 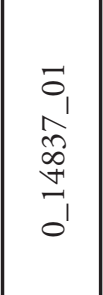 & $\begin{array}{l}\vec{\sigma} \\
\overrightarrow{0} \\
\vec{\omega} \\
\infty \\
\vec{D}^{\prime} \\
\sigma^{\prime}\end{array}$ & 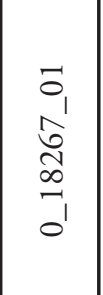 & \begin{tabular}{l}
$0_{1}$ \\
$\hat{\Xi}$ \\
\multirow{\infty}{0}{}
\end{tabular} & $\begin{array}{l}\tilde{o}_{1} \\
\tilde{n}^{\prime} \\
\hat{o} \\
\tilde{n}_{1} \\
0^{\prime}\end{array}$ & $\begin{array}{l}\overrightarrow{\sigma_{1}} \\
\text { n } \\
\hat{\tilde{o}} \\
\tilde{n}_{1} \\
0^{\prime}\end{array}$ & 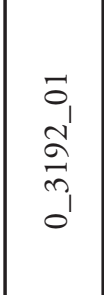 & '? & 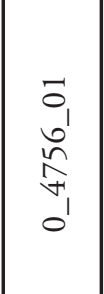 & $\begin{array}{l}\infty \\
\infty \\
\infty \\
0 \\
0\end{array}$ & $\begin{array}{l}\bar{\sigma}_{1} \\
\infty^{\prime} \\
\infty \\
\infty \\
\infty \\
0^{\prime}\end{array}$ & \\
\hline$\stackrel{\text { }}{\circ}$ & ஓ & $\stackrel{\infty}{\sim}$ & $\stackrel{n}{n}$ & & $\stackrel{\infty}{\sim}$ & - & $\widehat{F}$ & $\approx$ & $\bar{m}$ & - & $\underset{\sim}{*}$ & 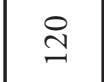 & $n$ & - & $\widetilde{\sim}$ & $\hat{n}$ & $\sim$ \\
\hline 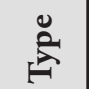 & 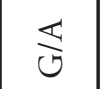 & $\frac{5}{u}$ & $\underset{\circlearrowleft}{\mho}$ & $\stackrel{5}{4}$ & 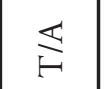 & $\stackrel{\cup}{\ominus}$ & 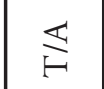 & $\frac{5}{0}$ & $\stackrel{F}{0}$ & $\stackrel{\bigcup}{4}$ & $\frac{5}{\circlearrowleft}$ & $\stackrel{\bigcup}{\ominus}$ & $\frac{\mathbb{U}}{\Delta}$ & $\underset{\circlearrowleft}{\mathbb{U}}$ & $\frac{\mathbb{U}}{U}$ & $\underset{\circlearrowleft}{\circlearrowleft}$ & $\ll$ \\
\hline U & - & - & - & - & - & 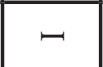 & - & - & - & $\neg$ & $\neg$ & $\neg$ & 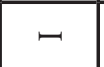 & - & - & - & $\neg$ \\
\hline 离 & 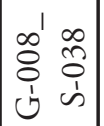 & 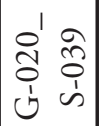 & 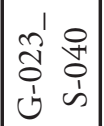 & $\begin{array}{l}\overrightarrow{8} \\
\dot{1}\end{array}$ & $\begin{array}{l}1 \\
\text { oे } \\
\text { i }\end{array}$ & $\begin{array}{l}\text { Oे } \\
\stackrel{1}{1}\end{array}$ & $\begin{array}{l}\stackrel{0}{0} \\
\vdots \\
\dot{1}\end{array}$ & 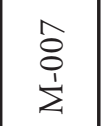 & $\begin{array}{l}\infty \\
8 \\
0 \\
1\end{array}$ & $\sum_{\substack{1 \\
\vdots}}^{1}$ & 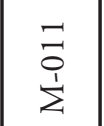 & $\begin{array}{l}\stackrel{1}{7} \\
\stackrel{1}{1} \\
\stackrel{1}{1}\end{array}$ & $\frac{n}{0}$ & 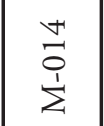 & $\sum_{i}^{0}$ & $\begin{array}{l}0 \\
0 \\
0 \\
\dot{1}\end{array}$ & \\
\hline
\end{tabular}




\begin{tabular}{|c|c|c|c|c|c|c|c|c|c|c|c|c|c|c|c|c|c|}
\hline 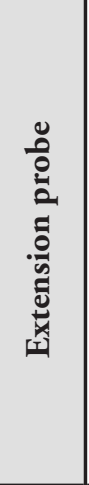 & 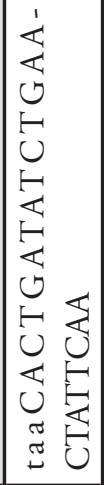 & 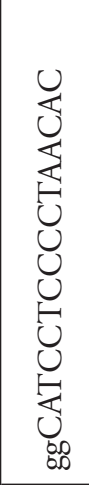 & 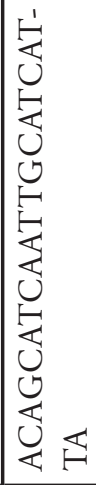 & 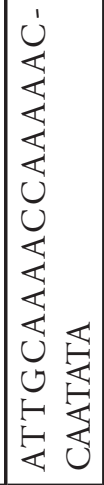 & 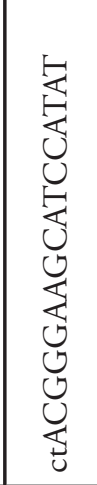 & 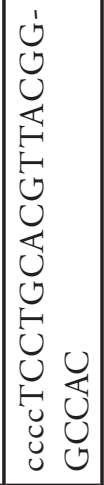 & 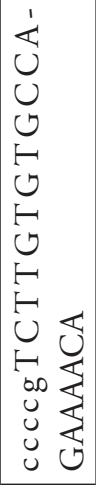 & 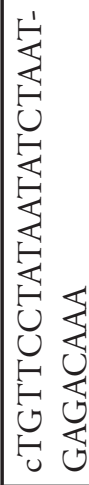 & 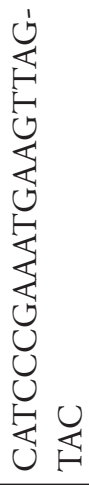 & 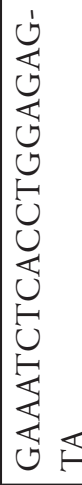 & 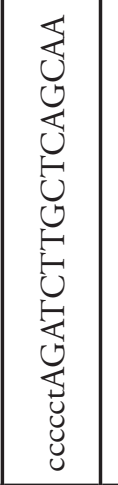 & 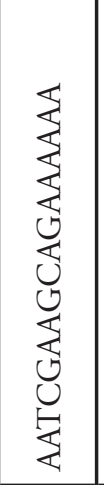 & 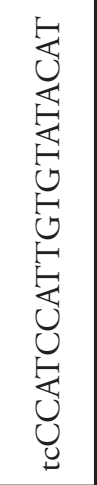 & 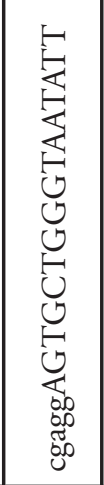 & 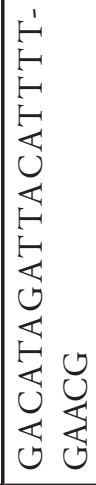 & 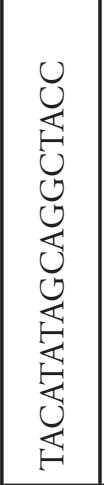 & 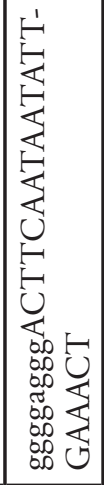 \\
\hline 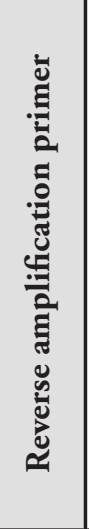 & 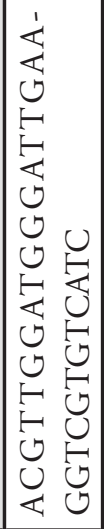 & 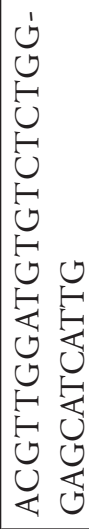 & 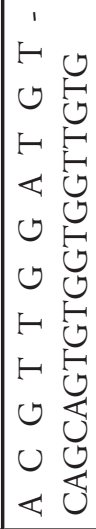 & 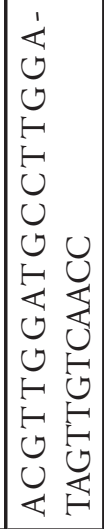 & 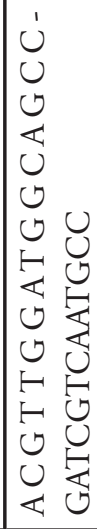 & 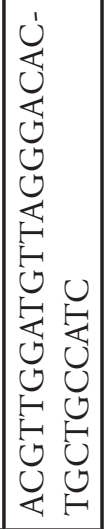 & 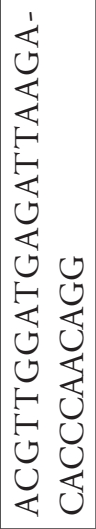 & 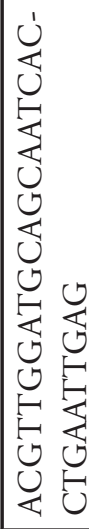 & 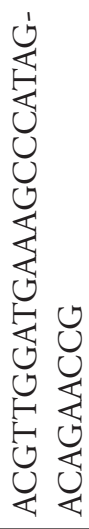 & 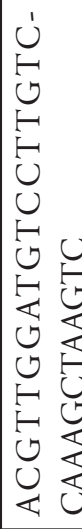 & 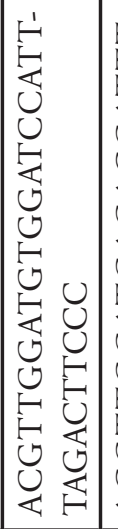 & 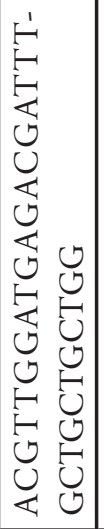 & 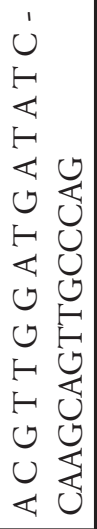 & 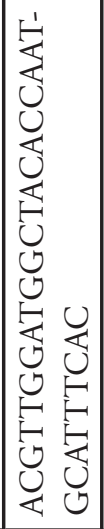 & 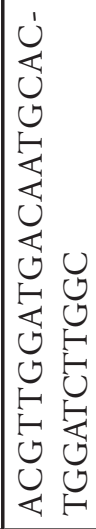 & 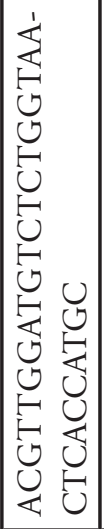 & 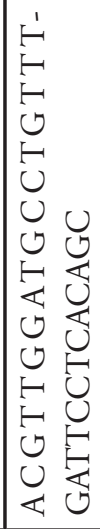 \\
\hline 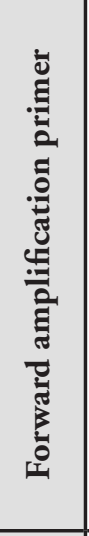 & 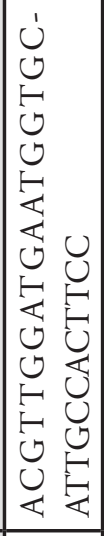 & 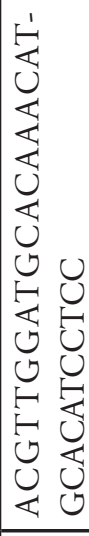 & 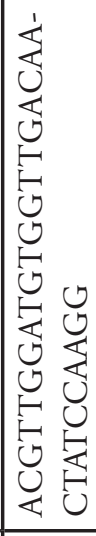 & 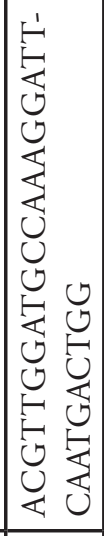 & 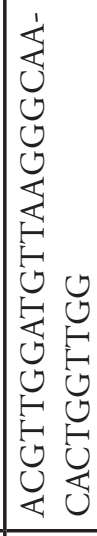 & 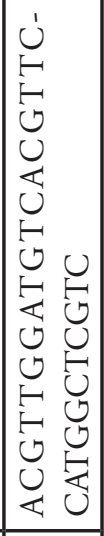 & 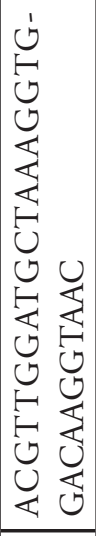 & 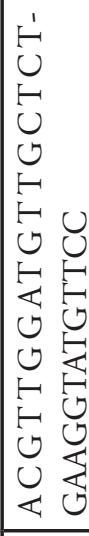 & 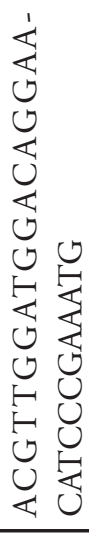 & 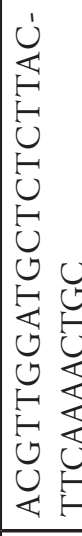 & 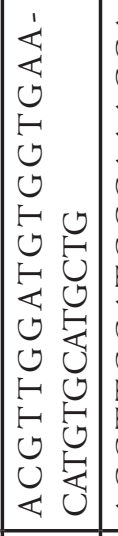 & 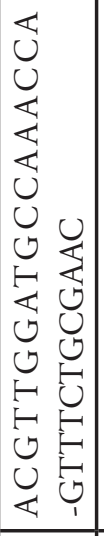 & 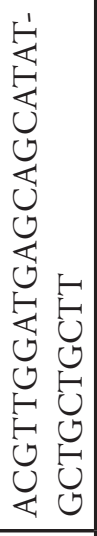 & 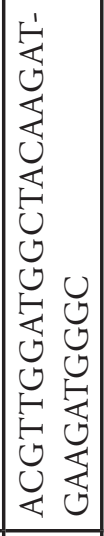 & 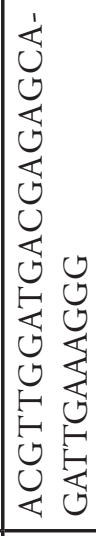 & 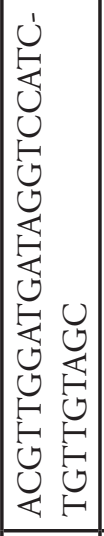 & 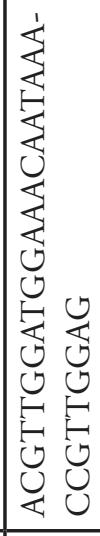 \\
\hline 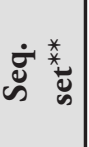 & $\begin{array}{l}\hat{\tilde{\Xi}} \\
\text { 至 }\end{array}$ & 言 & $\begin{array}{l}\hat{\tilde{I}} \\
\vec{\Xi}\end{array}$ & $=$ & $\begin{array}{l}\overline{\hat{m}} \\
\overline{3}\end{array}$ & 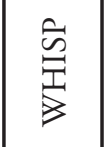 & $\Xi$ & $\hat{\underline{n}}$ & 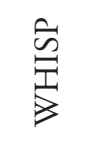 & $\begin{array}{l}\mathrm{E} \\
\mathrm{I}\end{array}$ & f & & نُ ن & نُ ن & & ن ن & \\
\hline 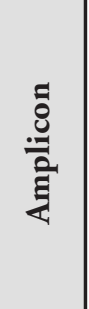 & 0 & 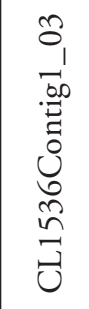 & 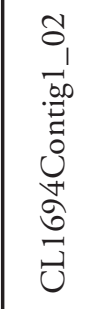 & 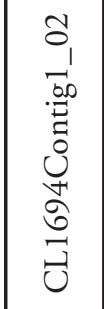 & $\begin{array}{l}\infty \\
0 \\
0\end{array}$ & $\begin{array}{l}0 \\
-1 \\
.00 \\
.00 \\
0 \\
0 \\
0 \\
0 \\
0 \\
0\end{array}$ & 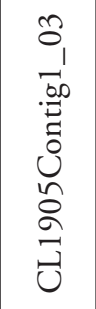 & $\underset{\sim}{ن}$ & 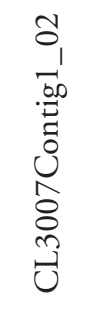 & $\begin{array}{l}\text { 然 } \\
\hat{0} \\
\hat{0} \\
\hat{0}\end{array}$ & $\begin{array}{l}\text { l } \\
v^{\prime} \\
\infty \\
v^{\prime} \\
v^{\prime}\end{array}$ & $\begin{array}{l}\stackrel{1}{N} \\
\text { N } \\
\text { N }\end{array}$ & $\underset{v}{*}$ & 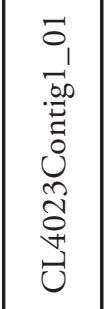 & & 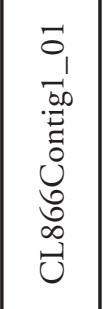 & \\
\hline$\stackrel{\circ}{\circ}$ & aे & $\sigma$ & $\stackrel{\infty}{\infty}$ & & 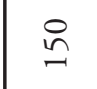 & $\sim$ & i & & $\sim$ & & & $N$ & & & & $\begin{array}{l}\stackrel{\sim}{n} \\
\text { n. }\end{array}$ & \\
\hline 芯 & F & $\stackrel{\cup}{\ominus}$ & $\circlearrowleft$ & & & $\stackrel{\cup}{H}$ & & & & & & & & & & $\frac{\mathbb{U}}{0}$ & \\
\hline U & - & $\neg$ & $\neg$ & 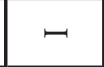 & $\neg$ & 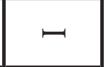 & - & & - & & & - & & & & & \\
\hline $\bar{z}$ & $\bar{i}$ & న్ & 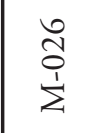 & ชै & $\approx$ & $\begin{array}{l}\text { O } \\
\text { i }\end{array}$ & $\sum_{\substack{1 \\
1}}^{0}$ & $\bar{d}$ & $\begin{array}{l}\tilde{N} \\
\hat{0} \\
i^{2}\end{array}$ & & {$\left[\begin{array}{ll}0 & 0 \\
0 & 0 \\
0 & 0\end{array} \mid\right.$} & 宣 & $H$ & + & & Ô & \\
\hline
\end{tabular}




\begin{tabular}{|c|c|c|c|c|c|c|c|c|c|c|c|c|c|c|c|c|c|}
\hline 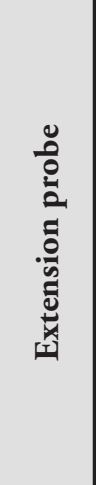 & 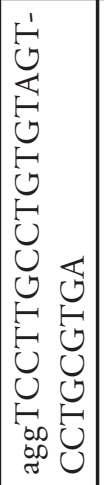 & 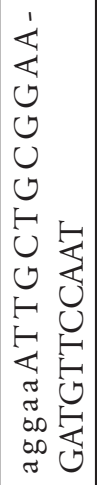 & 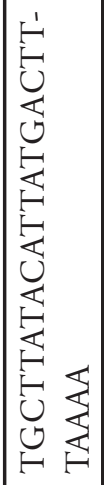 & 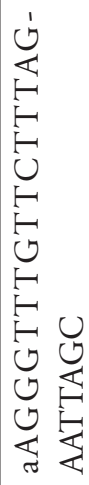 & 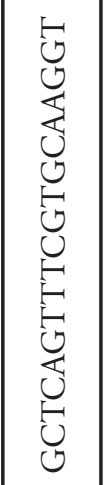 & 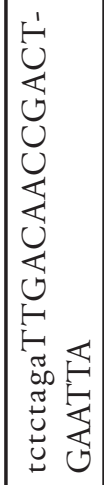 & 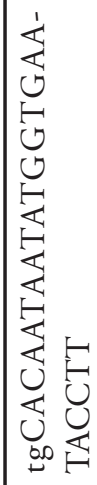 & 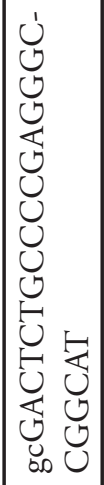 & 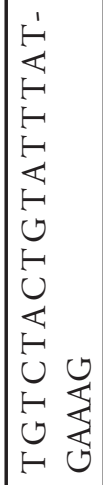 & 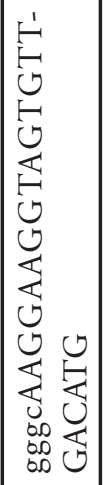 & 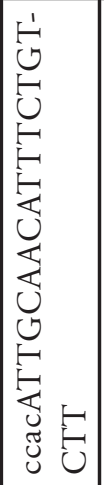 & 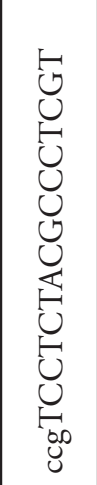 & 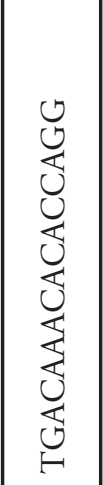 & 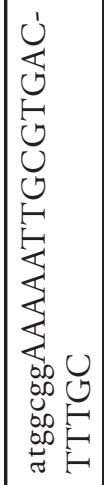 & 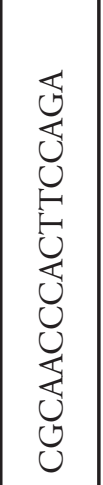 & 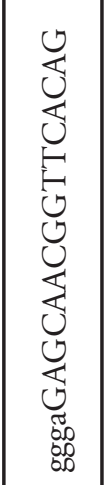 & 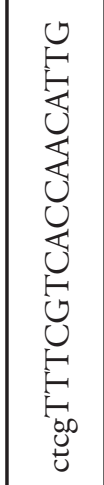 \\
\hline 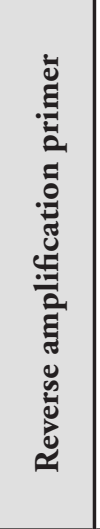 & 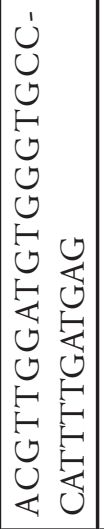 & 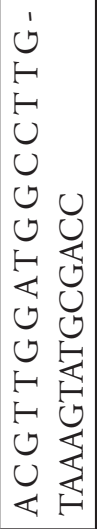 & 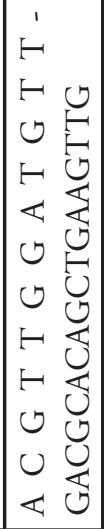 & 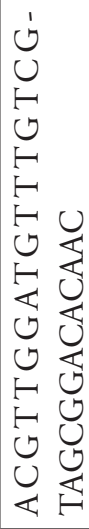 & 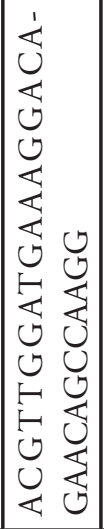 & 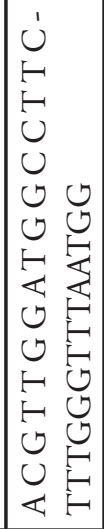 & 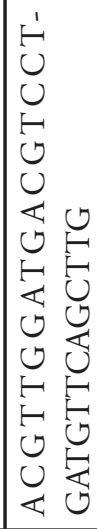 & 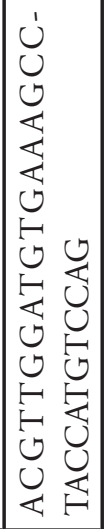 & 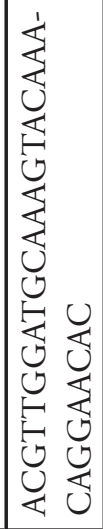 & 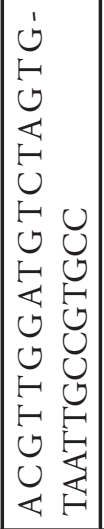 & 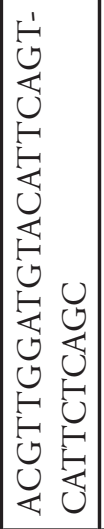 & 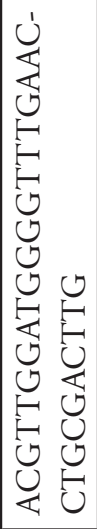 & 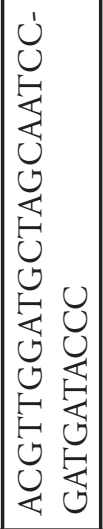 & 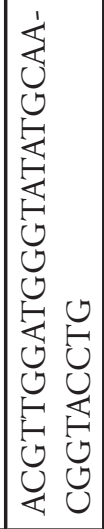 & 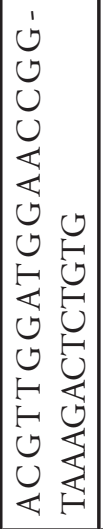 & 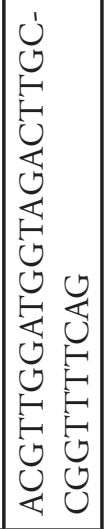 & 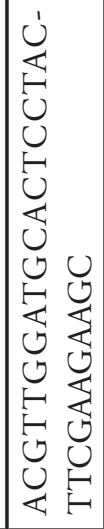 \\
\hline 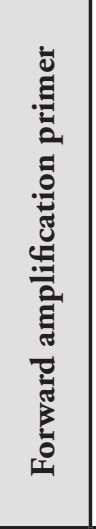 & 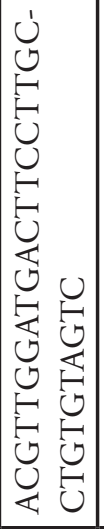 & 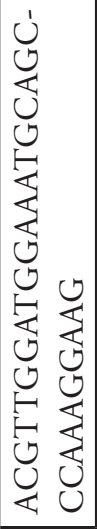 & 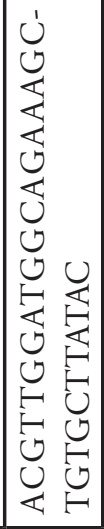 & 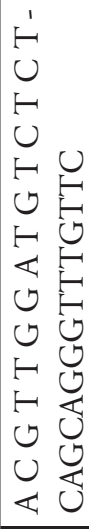 & 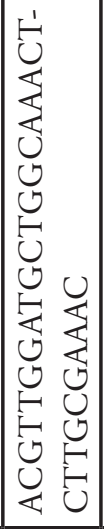 & 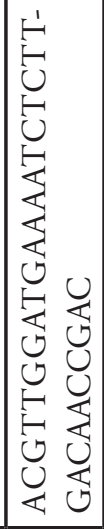 & 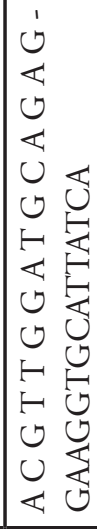 & 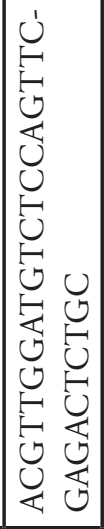 & 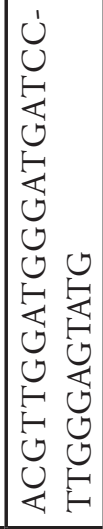 & 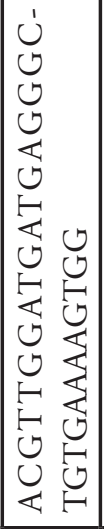 & 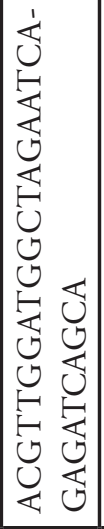 & 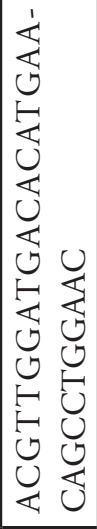 & 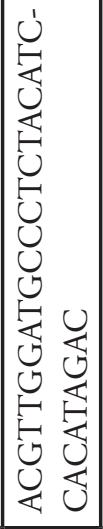 & 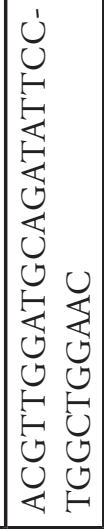 & 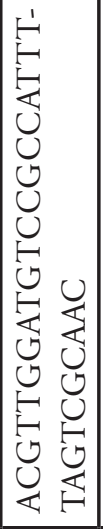 & 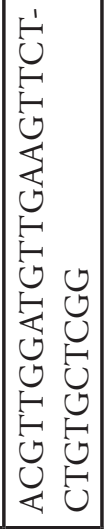 & 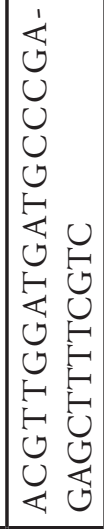 \\
\hline 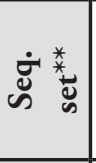 & $\begin{array}{l}8 \\
4\end{array}$ & ن. & $\begin{array}{l}3 \\
0\end{array}$ & 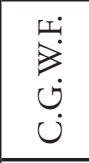 & 4 & . & & 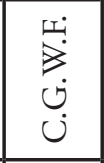 & 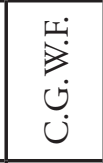 & & & ن & & & $\dot{ن}$ & $\dot{ن}$ & ن \\
\hline 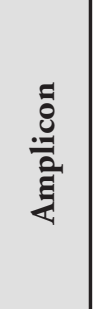 & 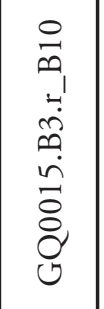 & 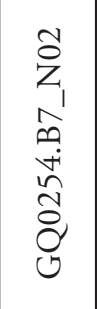 & 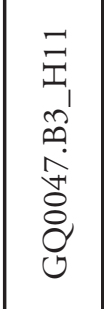 & 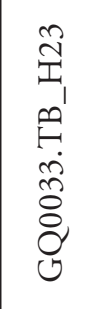 & $\begin{array}{l}\text { ô } \\
0 \\
-1 \\
0 \\
0 \\
\overrightarrow{0} \\
0 \\
0 \\
0\end{array}$ & 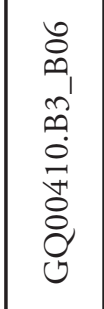 & ڤै. & 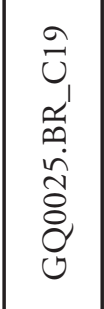 & 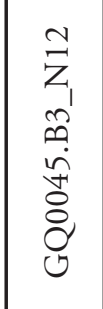 & 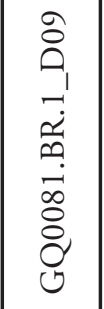 & 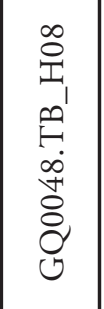 & 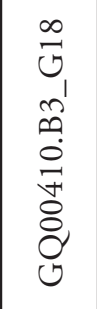 & 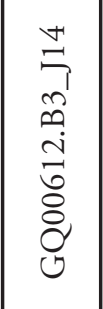 & 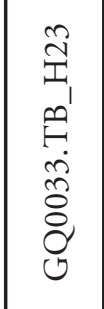 & 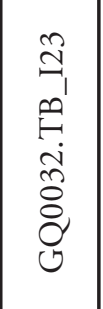 & 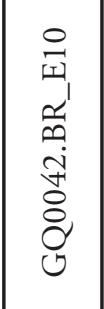 & 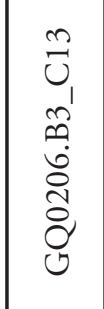 \\
\hline$\stackrel{\circ}{\circ}$ & r & N & & $\stackrel{\longleftarrow}{\sim}$ & in & & & & - & & & $\sim$ & & J & $\stackrel{\infty}{\approx}$ & & \\
\hline 崩 & $\frac{\cup}{U}$ & $\bar{U}$ & $\vec{\omega}$ & & 0 & & & & 0 & & & & & & & & \\
\hline U & $\Xi$ & $\Xi$ & B & $\Xi$ & ヨ & $\Xi$ & $\Xi$ & $=$ & $\exists$ & $\Xi$ & $\Xi$ & 7 & $\Xi$ & & $\Xi$ & $\Xi$ & $\exists$ \\
\hline 芯 & $\begin{array}{l}8 \\
0 \\
1\end{array}$ & $\begin{array}{l}8 \\
0 \\
0\end{array}$ & $\stackrel{8}{1}$ & $\begin{array}{l}8 \\
0 \\
1\end{array}$ & $\begin{array}{l}8 \\
0 \\
1\end{array}$ & 8 & $\begin{array}{l}0 \\
0 \\
ن\end{array}$ & $\mathcal{U}$ & $\begin{array}{l}\overrightarrow{0} \\
\text { d }\end{array}$ & $\begin{array}{l}\mathbb{W} \\
0 \\
\vdots \\
ن\end{array}$ & $\begin{array}{l}\overrightarrow{0} \\
\dot{1}\end{array}$ & $\begin{array}{l}\overrightarrow{0} \\
\dot{u}\end{array}$ & כ & & $\begin{array}{l}\text { Ũ } \\
\vdots \\
\vdots\end{array}$ & 0 & \\
\hline
\end{tabular}




\begin{tabular}{|c|c|c|c|c|c|c|c|c|c|c|c|c|c|c|c|c|c|}
\hline 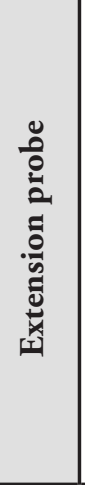 & 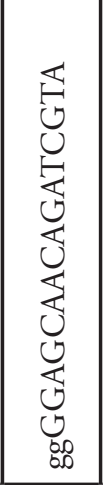 & 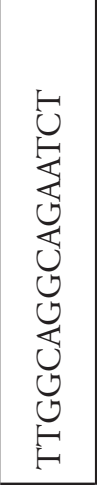 & 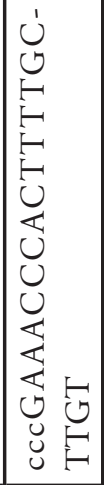 & 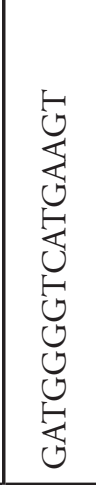 & 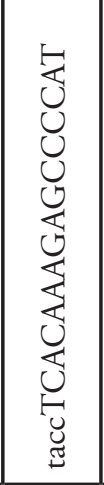 & 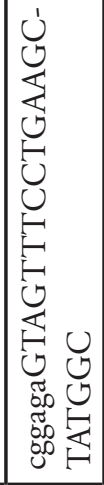 & 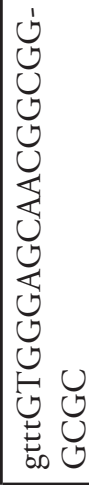 & 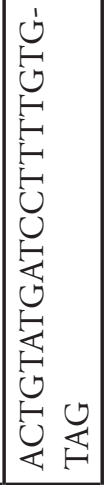 & 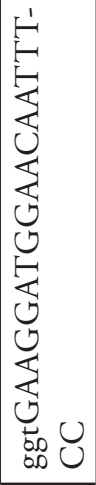 & 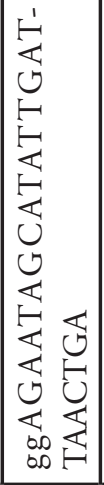 & 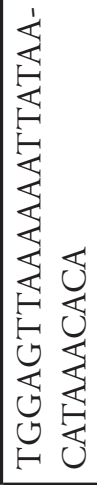 & 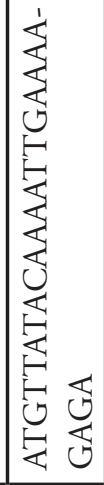 & 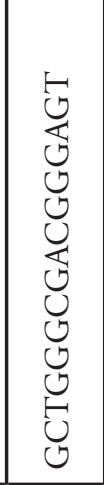 & 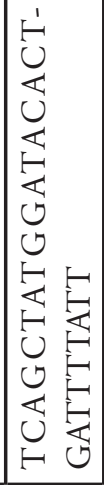 & 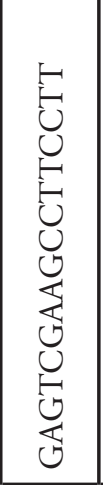 & 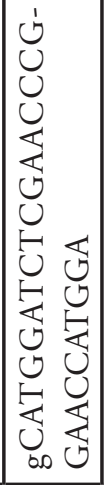 & 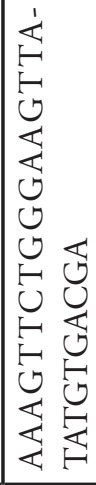 \\
\hline 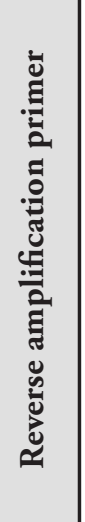 & 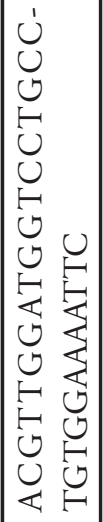 & 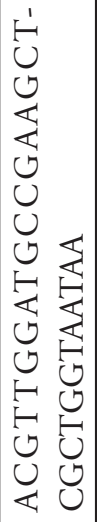 & 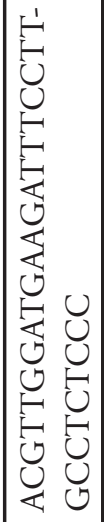 & 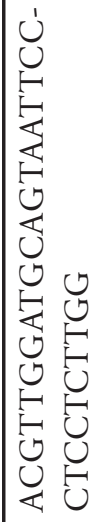 & 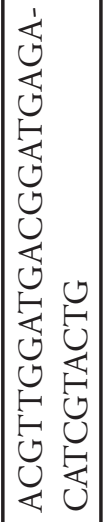 & 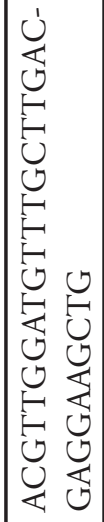 & 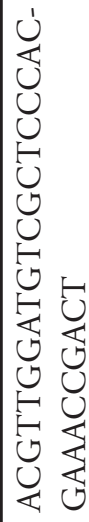 & 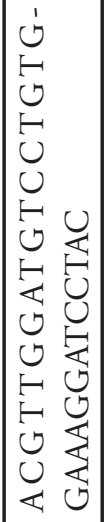 & 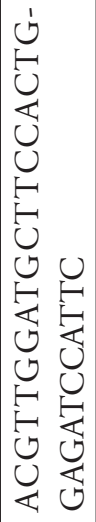 & 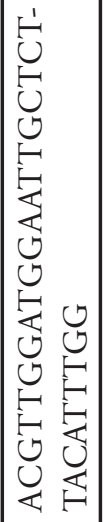 & 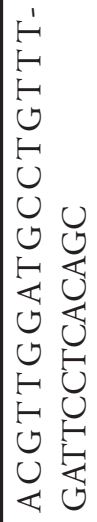 & 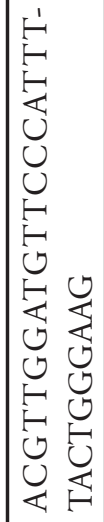 & 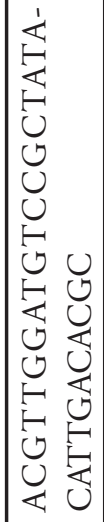 & 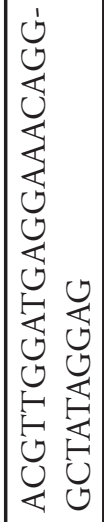 & 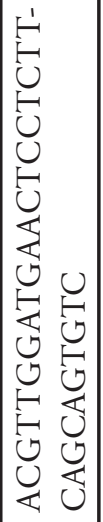 & 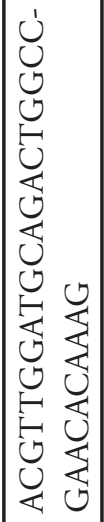 & 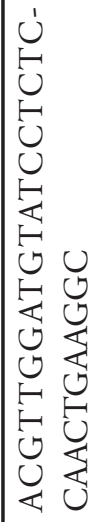 \\
\hline 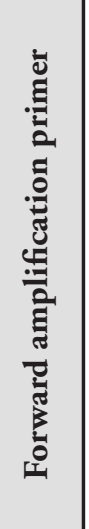 & 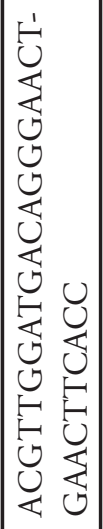 & 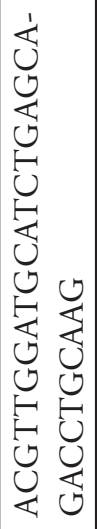 & 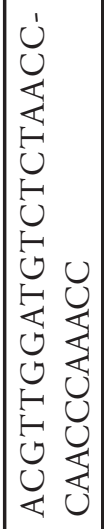 & 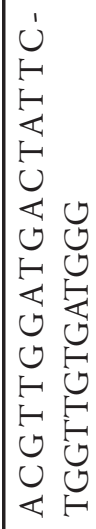 & 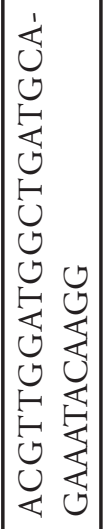 & 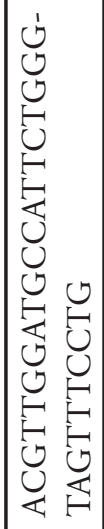 & 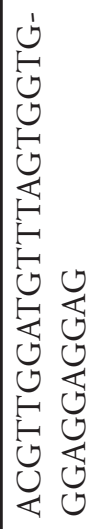 & 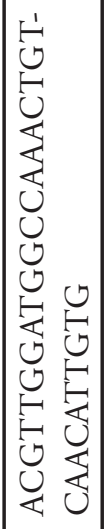 & 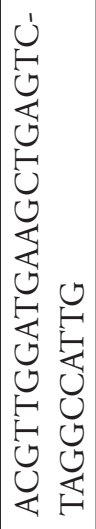 & 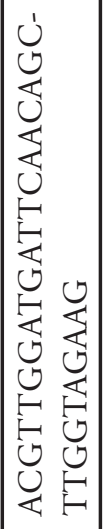 & 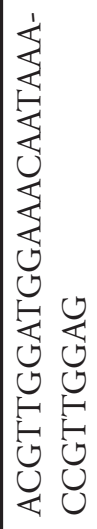 & 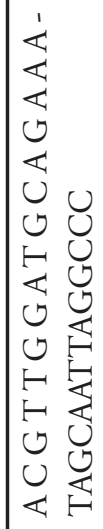 & 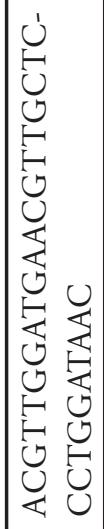 & 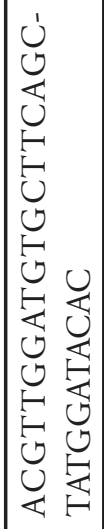 & 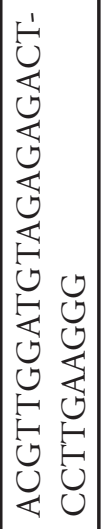 & 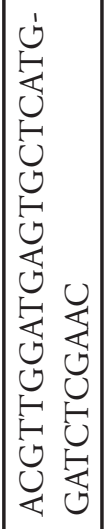 & 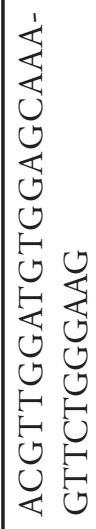 \\
\hline 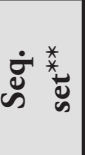 & 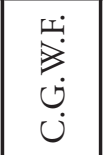 & ن. & $\begin{array}{l}\dot{8} \\
ن\end{array}$ & $\begin{array}{l}3 \\
U\end{array}$ & 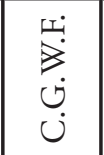 & $c$ & वी & 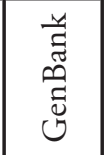 & $\begin{array}{l}\text { ज్ } \\
\text { ज్ } \\
\text { Uू }\end{array}$ & 完 & $\stackrel{3}{3}$ & $\begin{array}{l}\overrightarrow{\tilde{n}} \\
\overrightarrow{\mathbf{F}} \\
\overrightarrow{3}\end{array}$ & $\begin{array}{l}\hat{n} \\
\overrightarrow{3} \\
\overrightarrow{3}\end{array}$ & $=$ & 焉 & 焉 & \\
\hline$\frac{\tilde{0}}{\frac{0}{\pi}}$ & 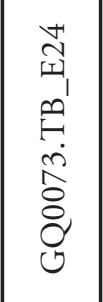 & 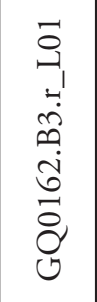 & 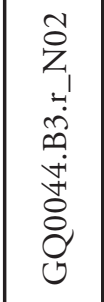 & 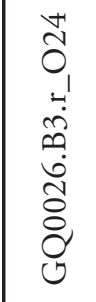 & 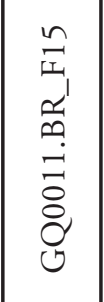 & 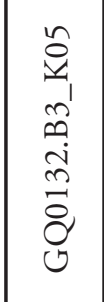 & & $\begin{array}{l}\vec{\Xi} \\
\infty \\
0 \\
\end{array}$ & $\begin{array}{l}\bar{b} \\
\infty \\
0 \\
\underline{I}\end{array}$ & 'ا & $\begin{array}{l}\widetilde{N}^{1} \\
\stackrel{+}{+} \\
0^{1}\end{array}$ & $\approx$ & $\vec{\sim}$ & v & 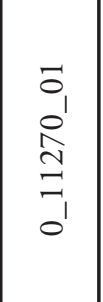 & & \\
\hline$\stackrel{\text { ڤ }}{\circ}$ & तิ & - & $\approx$ & 으 & $\forall$ & $m$ & $\widetilde{\sim}$ & & $m$ & $N$ & $\sim$ & 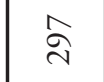 & & v & $\sim$ & & \\
\hline 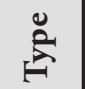 & $\frac{U}{4}$ & ن & $\mathbb{U}$ & & $u$ & & & ن & 0 & & $\cup$ & & & & 0 & & \\
\hline U & $\Xi$ & $\Xi$ & - & $\Xi$ & 水 & - & $\Xi$ & 水 & ヨ & $=$ & $\Xi$ & $\Xi$ & $\Xi$ & $\exists$ & $\exists$ & & \\
\hline 芯 & $\begin{array}{l}0 \\
0 \\
ن\end{array}$ & $\begin{array}{l}\text { d } \\
\text { U. }\end{array}$ & $\begin{array}{l}1 \\
\text { d } \\
\text { bे }\end{array}$ & $\tilde{c}$ & '. & $\begin{array}{l}\tilde{\hat{o}} \\
\hat{b}\end{array}$ & ชู & 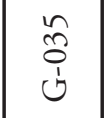 & $\begin{array}{l}\text { ô } \\
\dot{1}\end{array}$ & 8 & ๕ & $\overline{0}$ & a & శె & $\begin{array}{l}8 \\
0 \\
\text { ż }\end{array}$ & $\stackrel{\overbrace{}}{\varrho}$ & \\
\hline
\end{tabular}




\begin{tabular}{|c|c|c|c|c|c|c|c|c|c|c|c|c|c|c|c|c|c|}
\hline 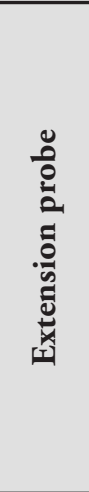 & 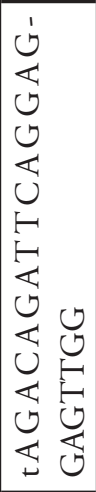 & 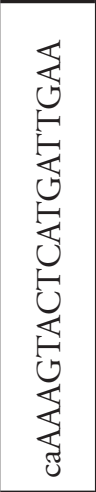 & 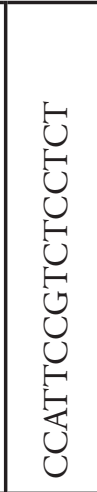 & 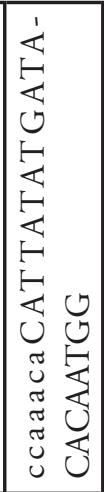 & 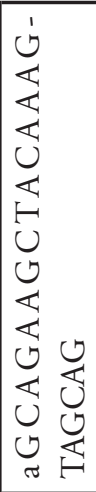 & 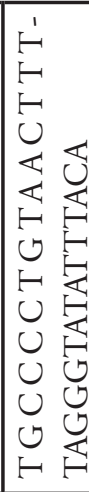 & 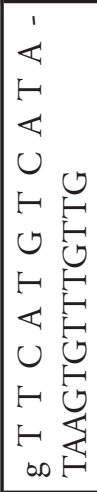 & 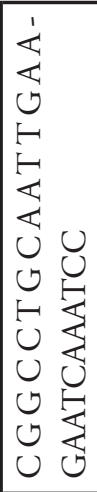 & 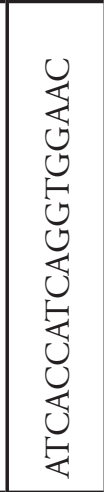 & 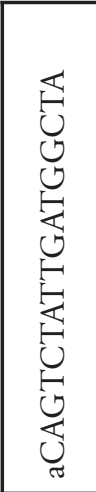 & 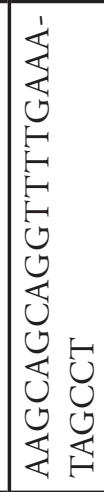 & 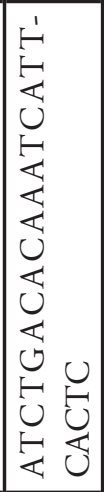 & 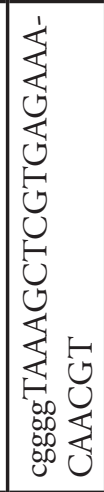 & 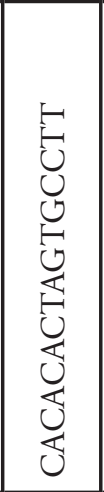 & 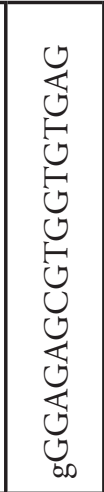 & 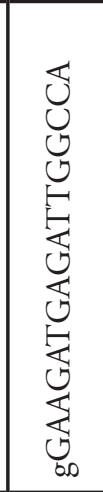 & 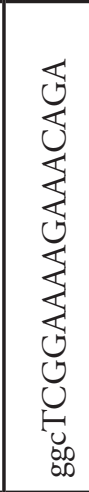 \\
\hline 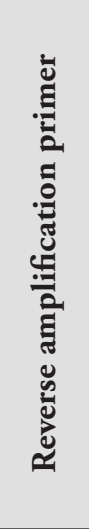 & 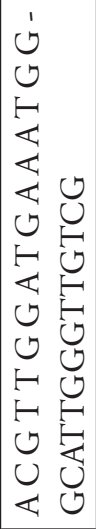 & 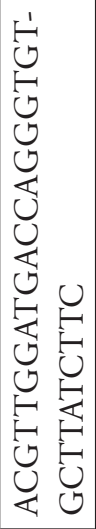 & 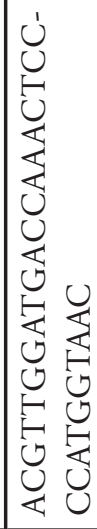 & 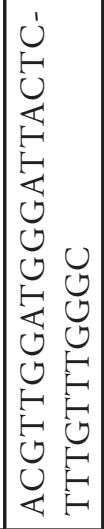 & 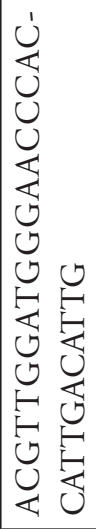 & 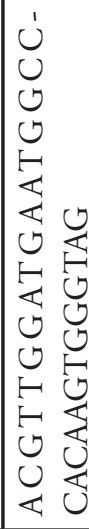 & 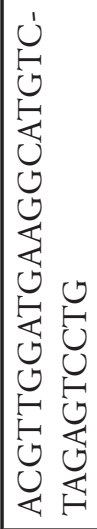 & 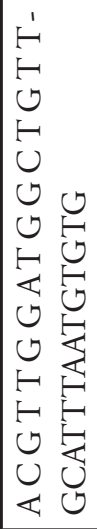 & 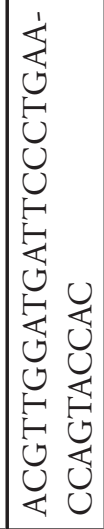 & 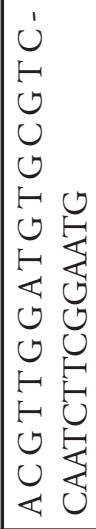 & 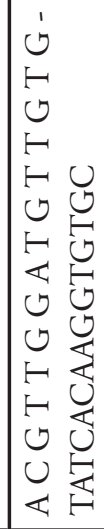 & 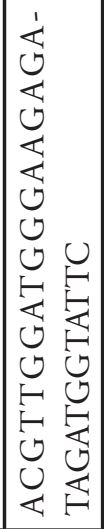 & 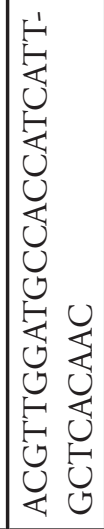 & 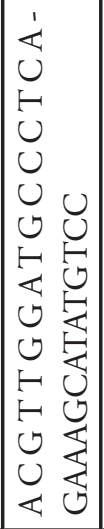 & 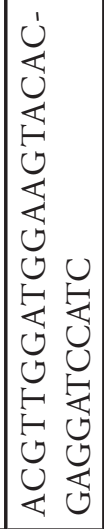 & 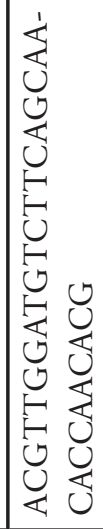 & 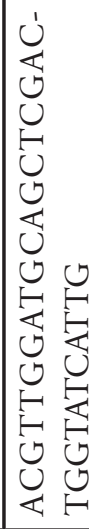 \\
\hline 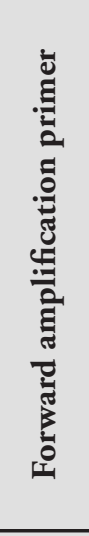 & 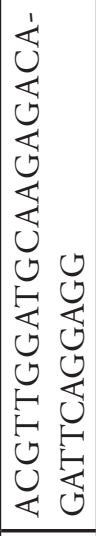 & 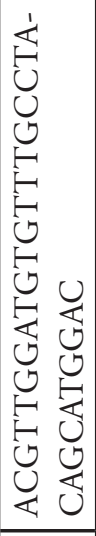 & 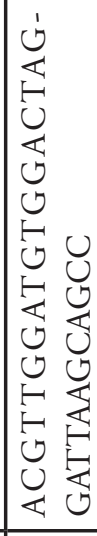 & 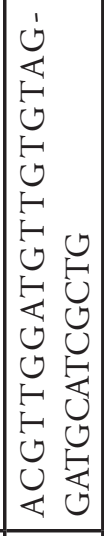 & 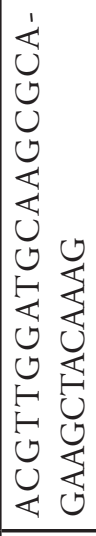 & 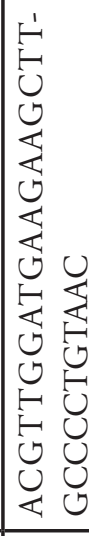 & 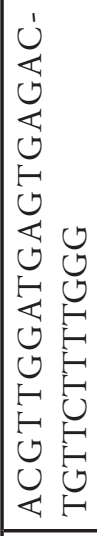 & 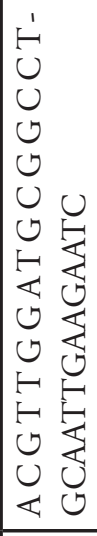 & 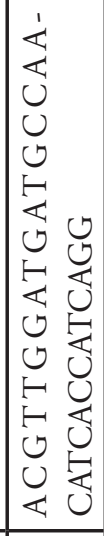 & 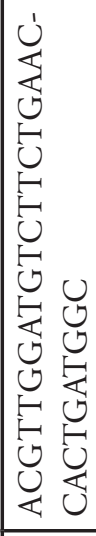 & 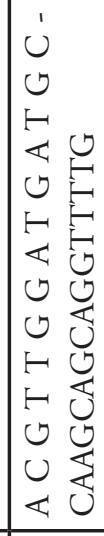 & 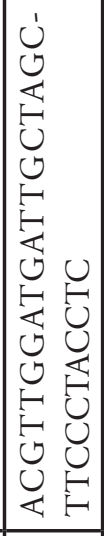 & 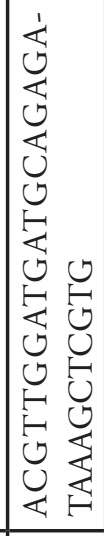 & 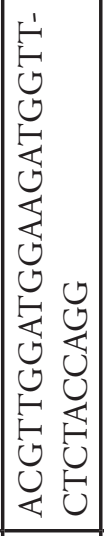 & 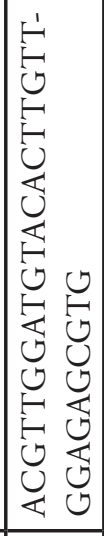 & 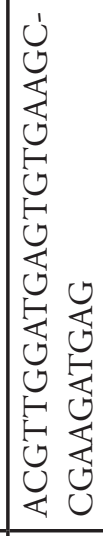 & 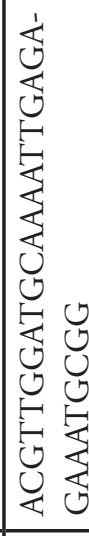 \\
\hline 灾 & $\begin{array}{l}\overrightarrow{\tilde{G}} \\
\text { 焉 }\end{array}$ & $\begin{array}{l}\overrightarrow{\tilde{n}} \\
\text { 焉 }\end{array}$ & 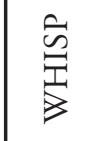 & $\begin{array}{l}\overrightarrow{\tilde{G}} \\
\overrightarrow{3} \\
3\end{array}$ & $\begin{array}{l}\overrightarrow{\tilde{n}} \\
\stackrel{\overrightarrow{1}}{3}\end{array}$ & $\begin{array}{l}\overrightarrow{\tilde{n}} \\
\overrightarrow{3}\end{array}$ & $\begin{array}{l}\overrightarrow{\tilde{n}} \\
\overrightarrow{\mathbf{F}}\end{array}$ & $\begin{array}{l}\overrightarrow{\tilde{n}} \\
\overrightarrow{1} \\
\overrightarrow{3}\end{array}$ & $\begin{array}{l}\overrightarrow{\tilde{n}} \\
\overrightarrow{3} \\
\overrightarrow{3}\end{array}$ & $\begin{array}{l}\overrightarrow{\tilde{n}} \\
\overrightarrow{3}\end{array}$ & 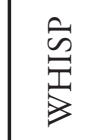 & 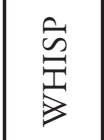 & 王 & 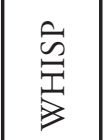 & $\begin{array}{l}\text { 莺 } \\
\text { 焉 }\end{array}$ & 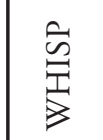 & 焉 \\
\hline 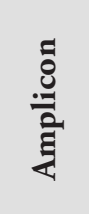 & $\begin{array}{l}\text { ô } \\
\sigma^{\prime} \\
\stackrel{n}{n} \\
\vec{J} \\
-1 \\
0^{\prime}\end{array}$ & 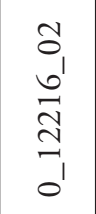 & 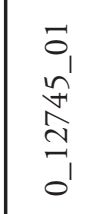 & 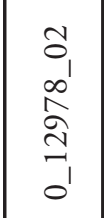 & $\begin{array}{l}\hat{n}_{1} \\
\hat{n} \\
\hat{n} \\
0^{\prime}\end{array}$ & 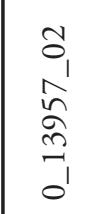 & $\begin{array}{l}\vec{\sigma} \\
\vec{J} \\
\vec{J} \\
\underset{I}{\mid} \\
\overrightarrow{0}\end{array}$ & 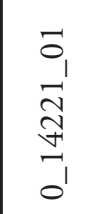 & $\begin{array}{l}\overrightarrow{\sigma_{1}} \\
\hat{\infty}_{\infty}\end{array}$ & $\begin{array}{l}\text { No } \\
\text { No } \\
n \\
0^{\prime}\end{array}$ & $\overrightarrow{o^{\prime}}$ & 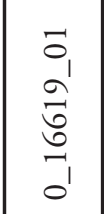 & $0^{\prime}$ & $\begin{array}{l}0 \\
\infty \\
0 \\
0 \\
C^{\prime} \\
0\end{array}$ & $\overrightarrow{0}$ & 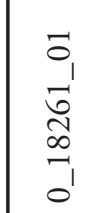 & \\
\hline ڤ̆ & 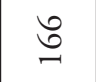 & $\stackrel{\infty}{a}$ & i্ & $\vec{v}$ & 0 & $\stackrel{\infty}{\sim}$ & $\sqrt{6}$ & to & 0 & $\vec{n}$ & $\widehat{\imath}$ & $\stackrel{m}{=}$ & F & $\approx$ & $\widehat{\imath}$ & $\widehat{\imath}$ & $\infty$ \\
\hline 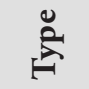 & \& & U & 4 & ৩ & 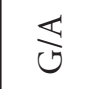 & & - & 0 & J & $\stackrel{5}{v}$ & & 4 & & 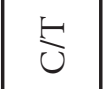 & $c$ & 0 & \\
\hline U & $=$ & $=$ & $=$ & $\exists$ & $\Xi$ & $\exists$ & $=$ & $\Xi$ & $\Xi$ & $\Xi$ & $\exists$ & $\Xi$ & $\Xi$ & $\Xi$ & $\exists$ & 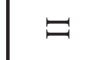 & \\
\hline 玄 & $\begin{array}{l}0 \\
\vdots \\
z\end{array}$ & $\begin{array}{l}\text { Oे } \\
\text { Oे } \\
\text { ż }\end{array}$ & $\begin{array}{l}0 \\
\vdots \\
\text { z }\end{array}$ & $\begin{array}{l}8 \\
\vdots \\
\text { ż }\end{array}$ & $\begin{array}{l}\overrightarrow{0} \\
\dot{i}\end{array}$ & 2 & Z & $\begin{array}{l}\overrightarrow{0} \\
\dot{z}\end{array}$ & $\begin{array}{l}n \\
0 \\
i \\
z\end{array}$ & Z & Z & & & Z & z & $\begin{array}{l}\text { Ô } \\
\dot{1}\end{array}$ & \\
\hline
\end{tabular}




\begin{tabular}{|c|c|c|c|c|c|c|c|c|c|c|c|c|c|c|c|c|c|}
\hline 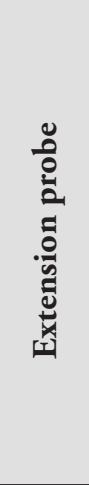 & 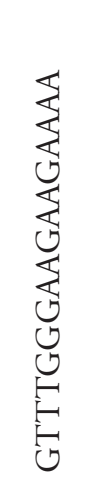 & 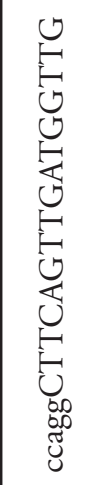 & 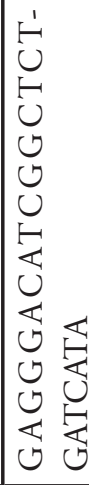 & 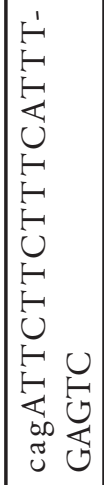 & 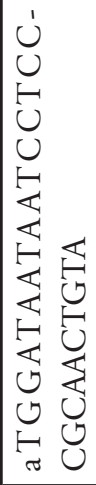 & 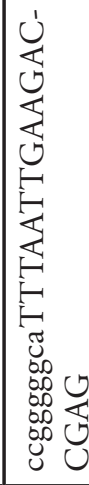 & 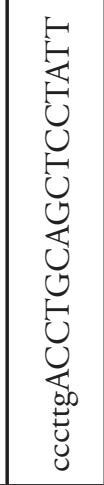 & 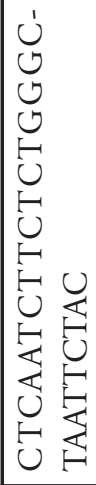 & 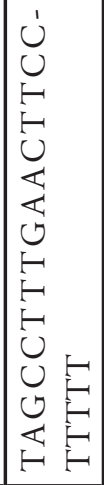 & 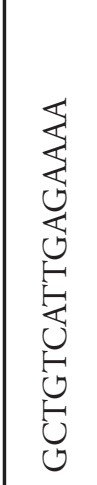 & 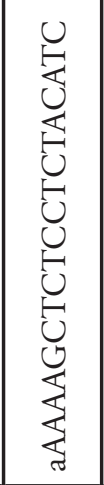 & 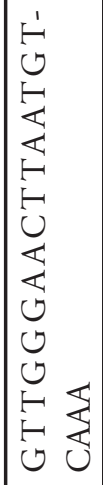 & 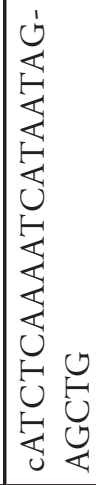 & 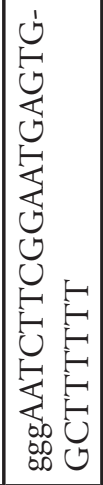 & 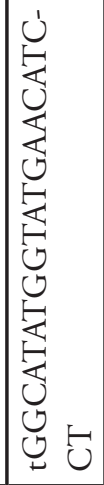 & 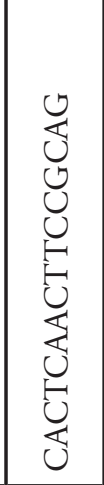 & 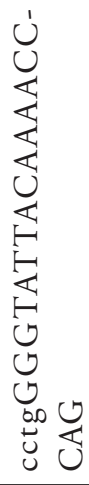 \\
\hline 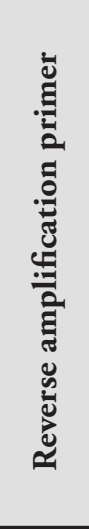 & 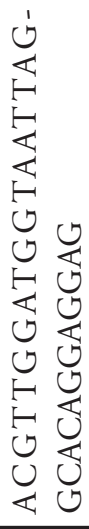 & 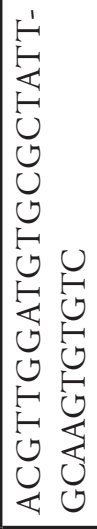 & 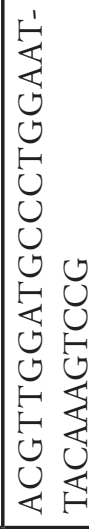 & 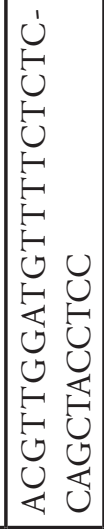 & 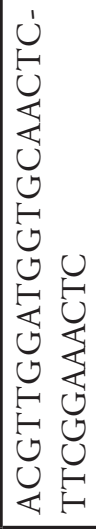 & 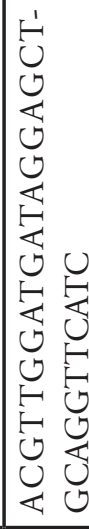 & 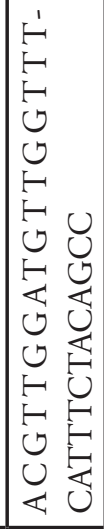 & 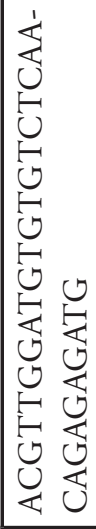 & 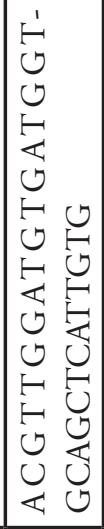 & 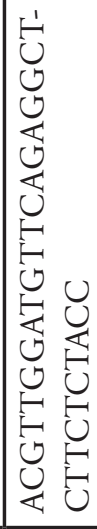 & 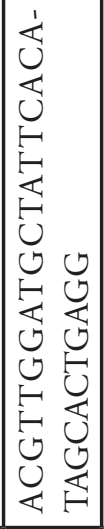 & 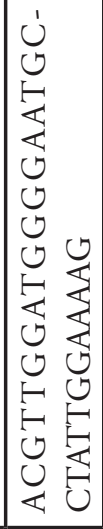 & 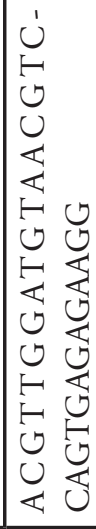 & 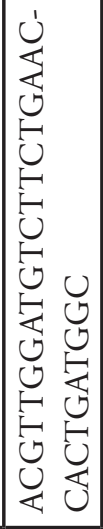 & 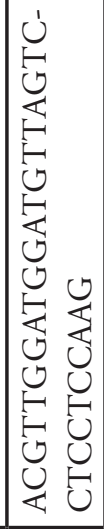 & 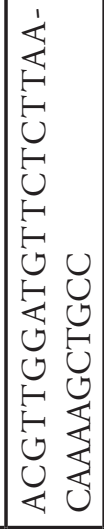 & 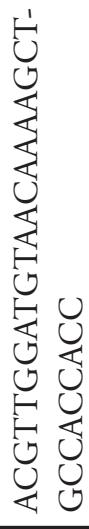 \\
\hline 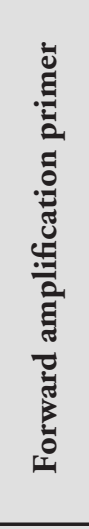 & 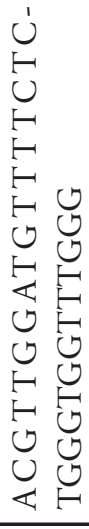 & 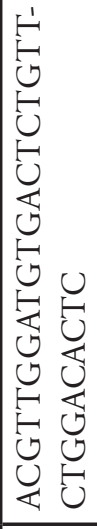 & 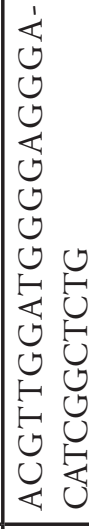 & 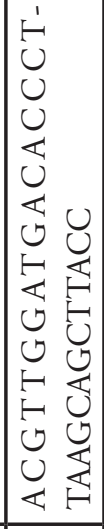 & 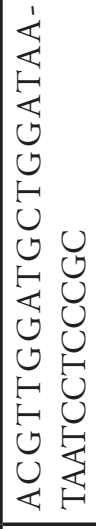 & 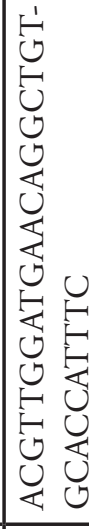 & 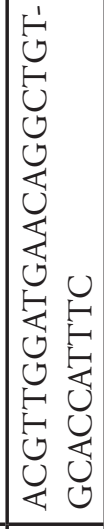 & 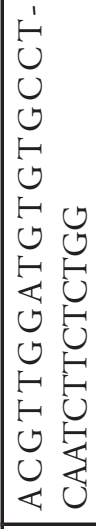 & 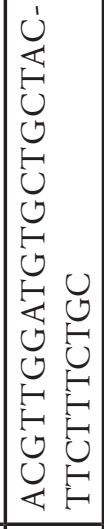 & 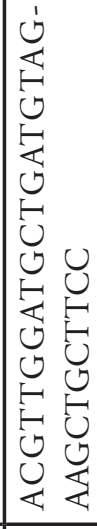 & 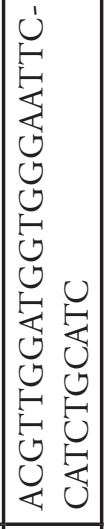 & 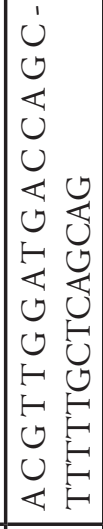 & 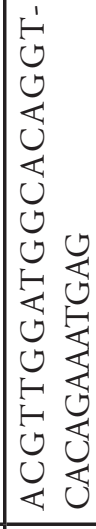 & 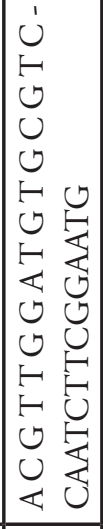 & 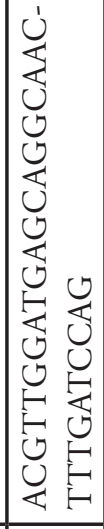 & 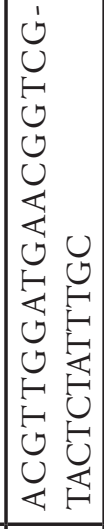 & 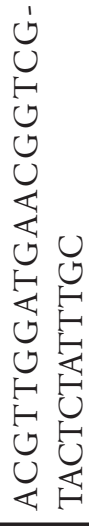 \\
\hline 它 苞 & $\begin{array}{l}\overrightarrow{\tilde{n}} \\
\text { 焉 }\end{array}$ & $\begin{array}{l}\overrightarrow{\tilde{n}} \\
\stackrel{\overrightarrow{1}}{3}\end{array}$ & $\underline{G}$ & $\begin{array}{l}\overrightarrow{\tilde{\Omega}} \\
\stackrel{1}{3}\end{array}$ & 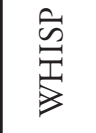 & 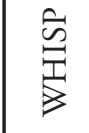 & 3 & 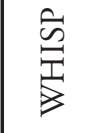 & $\hat{\sigma}$ & 焉 & 焉 & 焉 & $\overrightarrow{\underline{I}}$ & I & $\bar{\nabla}$ & I & \\
\hline $\begin{array}{l}\frac{0}{0.0} \\
\frac{0}{0} \\
\frac{0}{4}\end{array}$ & $\begin{array}{l}\overrightarrow{\sigma_{1}} \\
\tilde{n}^{\prime} \\
\tilde{o}_{1} \\
\tilde{o}^{\prime}\end{array}$ & $\begin{array}{l}\sigma_{1} \\
\text { N } \\
\tilde{o} \\
\stackrel{+}{1} \\
0\end{array}$ & c & 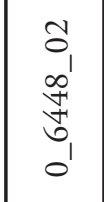 & $\begin{array}{l}0 \\
\infty \\
\infty \\
\infty \\
\infty \\
\sigma_{1} \\
0\end{array}$ & 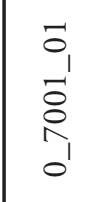 & $\begin{array}{l}\sigma_{1} \\
\vec{o}^{\prime} \\
R_{1} \\
0\end{array}$ & $\hat{0}$ & & $\begin{array}{l}\text { ơ } \\
\text { o } \\
\text { o }\end{array}$ & $\begin{array}{l}\overrightarrow{0} \\
\overbrace{1} \\
\sim_{1}\end{array}$ & $\begin{array}{l}\tilde{D}^{\prime} \\
\underset{N}{1} \\
N^{\prime}\end{array}$ & d & $0^{\prime}$ & & $v^{\prime}$ & \\
\hline$\stackrel{\circ}{\circ}$ & $m$ & $\exists$ & r & $\stackrel{\sim}{\sim}$ & $m$ & $\hat{n}$ & 0 & $\hat{\sigma}$ & 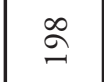 & 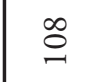 & - & & $\sim$ & & & $\stackrel{\sim}{\sim}$ & \\
\hline 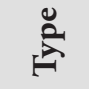 & $\bar{U}$ & ज) & $=$ & $\circlearrowleft$ & $\circlearrowleft$ & & 4 & $\frac{v}{u}$ & $\circlearrowleft$ & & $F$ & $\mathcal{U}$ & 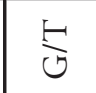 & 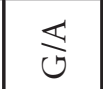 & & जे & \\
\hline U & $\exists$ & $\exists$ & 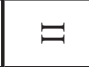 & - & $\exists$ & & 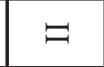 & - & - & & 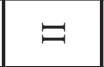 & $\Xi$ & & & & - & \\
\hline$\sum_{n}$ & $\begin{array}{l}\text { o } \\
\text { ż }\end{array}$ & $\begin{array}{l}\text { Oै } \\
\text { Ż }\end{array}$ & 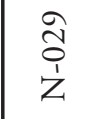 & 0 & $\hat{\jmath}$ & & $\begin{array}{l}\text { ô } \\
\text { ì } \\
\text { z }\end{array}$ & $\begin{array}{l}\text { ô } \\
\dot{z}\end{array}$ & 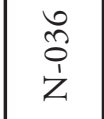 & $\begin{array}{l}\hat{v} \\
\hat{i} \\
z\end{array}$ & 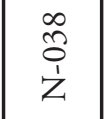 & $\begin{array}{l}\text { ळे } \\
\text { iे }\end{array}$ & & & $c$ & 0 & \\
\hline
\end{tabular}




\begin{tabular}{|c|c|c|c|c|c|c|c|c|c|c|c|c|c|c|c|c|c|}
\hline 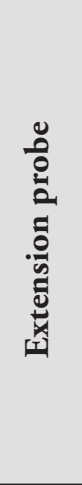 & 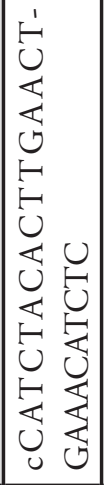 & 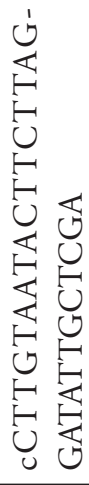 & 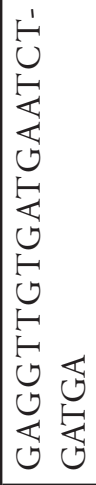 & 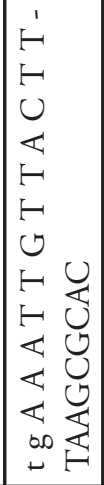 & 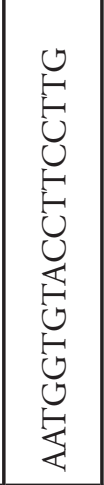 & 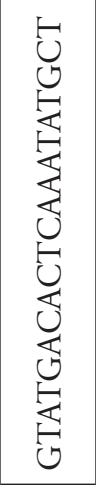 & 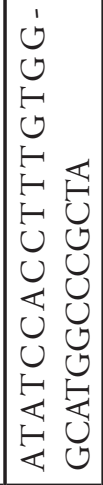 & 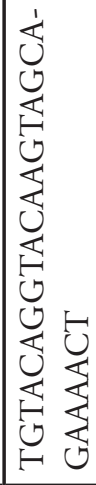 & 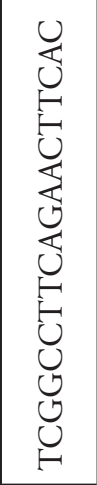 & 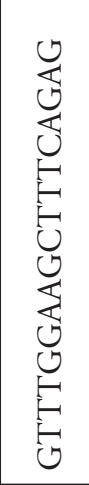 & 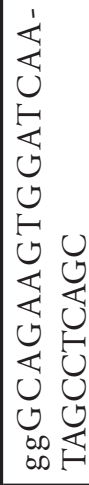 & 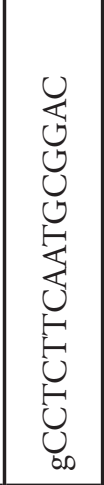 & 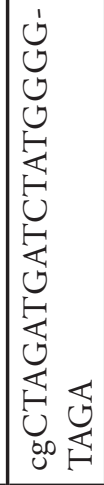 & 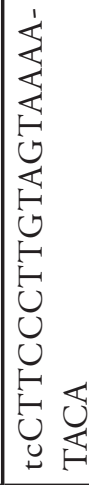 & 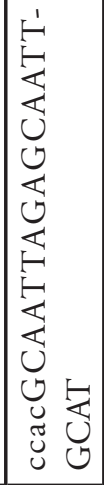 & 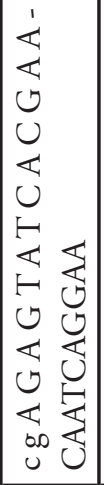 & 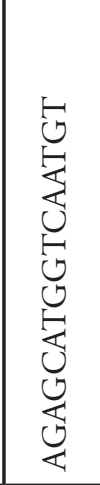 \\
\hline 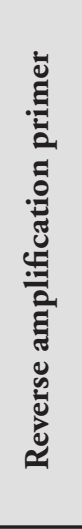 & 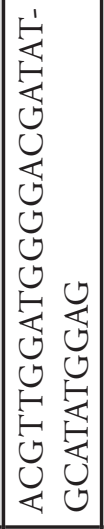 & 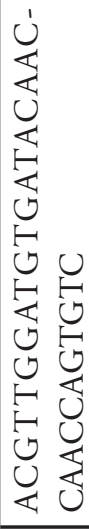 & 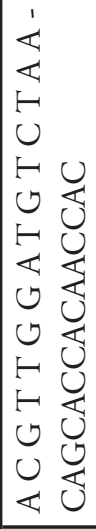 & 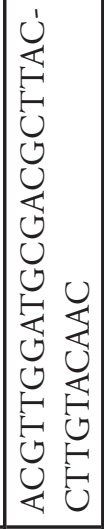 & 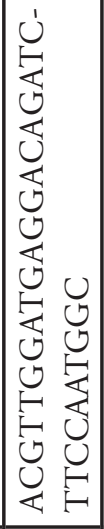 & 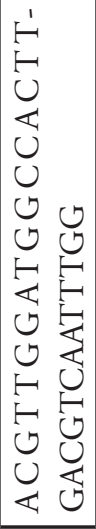 & 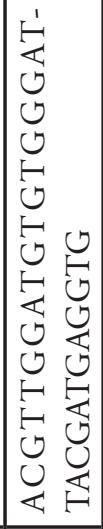 & 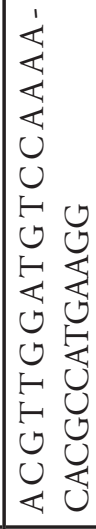 & 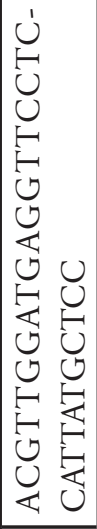 & 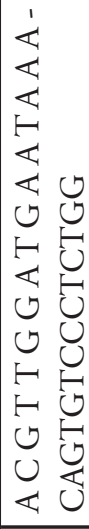 & 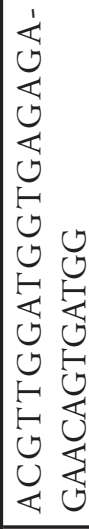 & 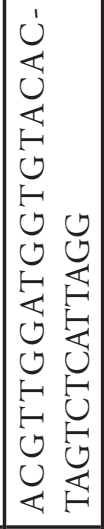 & 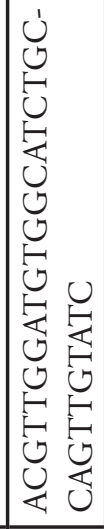 & 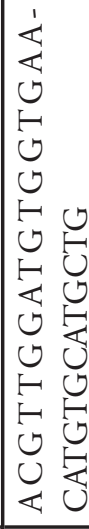 & 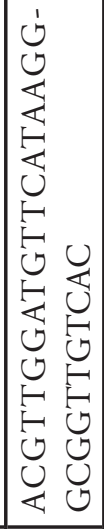 & 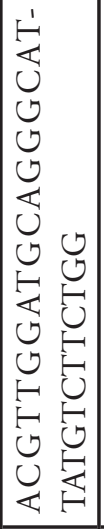 & 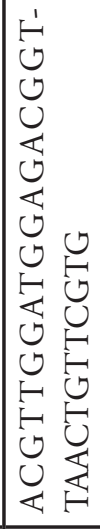 \\
\hline 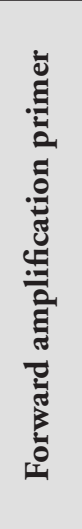 & 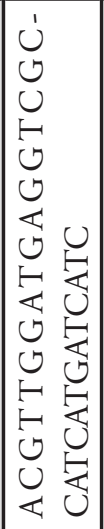 & 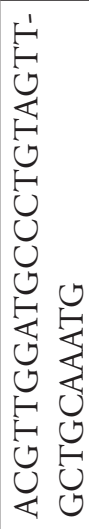 & 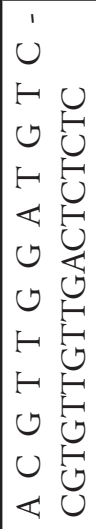 & 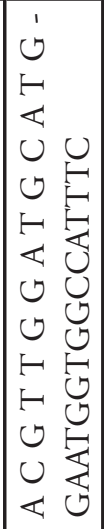 & 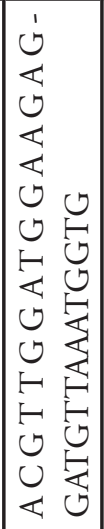 & 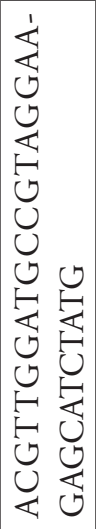 & 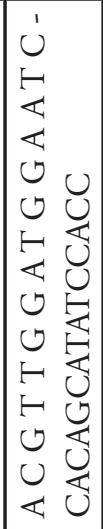 & 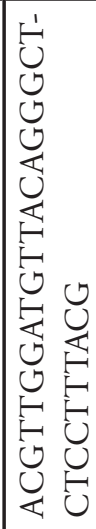 & 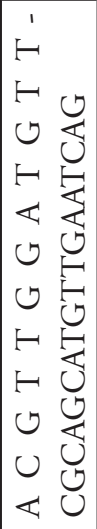 & 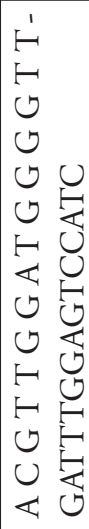 & 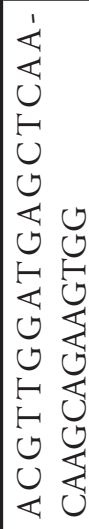 & 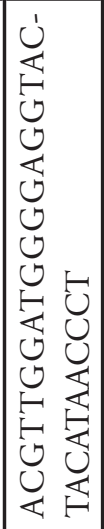 & 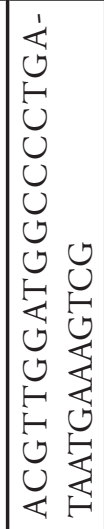 & 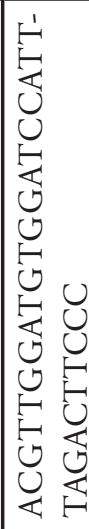 & 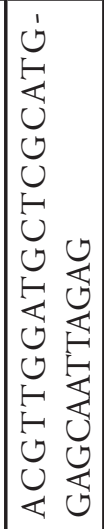 & 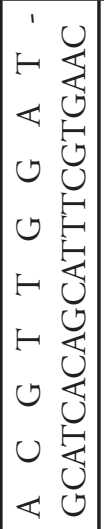 & 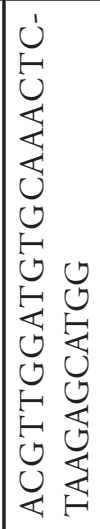 \\
\hline 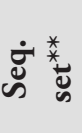 & $\begin{array}{l}\overrightarrow{\tilde{n}} \\
\overrightarrow{1} \\
\overrightarrow{3}\end{array}$ & $\begin{array}{l}\overrightarrow{\tilde{n}} \\
\overrightarrow{1} \\
\overrightarrow{3}\end{array}$ & $\begin{array}{l}\overrightarrow{\tilde{n}} \\
\text { 焉 }\end{array}$ & $\begin{array}{l}\overrightarrow{\tilde{n}} \\
\text { 焉 }\end{array}$ & $\begin{array}{l}\overrightarrow{\tilde{n}} \\
\text { 焉 }\end{array}$ & $\begin{array}{l}\overrightarrow{\tilde{n}} \\
\text { 焉 }\end{array}$ & $\begin{array}{l}\overrightarrow{\tilde{s}} \\
\stackrel{\overrightarrow{\mid}}{3}\end{array}$ & $\hat{n}$ & $\begin{array}{l}\overrightarrow{\tilde{n}} \\
\overrightarrow{1} \\
\overrightarrow{3}\end{array}$ & $\begin{array}{l}\overrightarrow{\tilde{n}} \\
\text { 焉 }\end{array}$ & 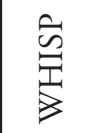 & $\begin{array}{l}\overrightarrow{\tilde{n}} \\
\overrightarrow{3}\end{array}$ & 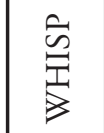 & I & $\begin{array}{l}\mathrm{G} \\
\bar{T}\end{array}$ & 塟 & $\begin{array}{l}\overrightarrow{\tilde{s}} \\
\stackrel{\overrightarrow{\mid}}{3}\end{array}$ \\
\hline 莙 & 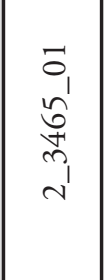 & $\begin{array}{l}\vec{\sigma} \\
v^{\prime} \\
\tilde{\infty} \\
\omega_{1} \\
v\end{array}$ & 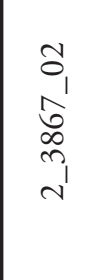 & \begin{tabular}{l} 
ô, \\
o \\
$\infty$ \\
\multirow{2}{*}{}
\end{tabular} & $\begin{array}{l}a \\
\infty \\
0 \\
v \\
v\end{array}$ & 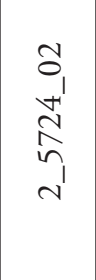 & 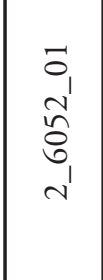 & 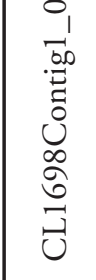 & 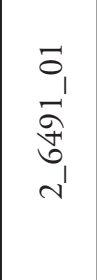 & $\begin{array}{l}\bar{\sigma}_{1} \\
\vec{n} \\
\vec{\sigma}_{1} \\
v^{\prime}\end{array}$ & $\begin{array}{l}\sigma \\
\stackrel{0}{\infty} \\
\infty\end{array}$ & $\begin{array}{l}\sigma_{1} \\
n^{\prime} \\
\stackrel{N}{N} \\
N_{1}\end{array}$ & $y^{\prime}$ & & & 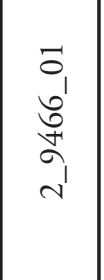 & \\
\hline ڤ̊ & 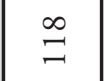 & 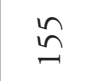 & $\infty$ & & ث્ત & $\stackrel{m}{\sim}$ & 곤 & 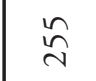 & $\simeq$ & $\begin{array}{l}\infty \\
\stackrel{\sim}{\sim}\end{array}$ & ๙ิ & 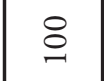 & $\tilde{n}$ & $f$ & & $\stackrel{\text { త్ }}{ }$ & $\exists$ \\
\hline 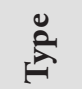 & 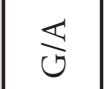 & 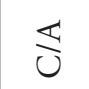 & 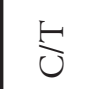 & 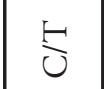 & $\frac{\mathbb{u}}{u}$ & 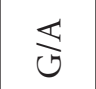 & 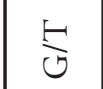 & & 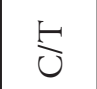 & 2 & & & & & & 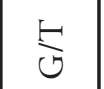 & \\
\hline U & $\Xi$ & 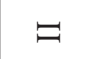 & $=$ & $\Xi$ & ヨ & $\exists$ & - & $=$ & $\Xi$ & $\Xi$ & & $=$ & 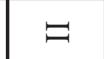 & & & $=$ & \\
\hline 站 & $\begin{array}{l}0 \\
0 \\
0\end{array}$ & $\begin{array}{l}8 \\
0 \\
0\end{array}$ & $\begin{array}{l}8 \\
0 \\
0\end{array}$ & $\tilde{\imath}$ & $\begin{array}{l}\text { o } \\
\text { o }\end{array}$ & i & $\begin{array}{l}m \\
0 \\
0 \\
0\end{array}$ & $\begin{array}{l}\text { Tे } \\
\text { ò }\end{array}$ & $a$ & c & $\begin{array}{l}\hat{a} \\
\dot{1} \\
0\end{array}$ & $\begin{array}{l}\hat{\sigma} \\
\hat{0}\end{array}$ & ல & $\begin{array}{l}\text { ָ̃ } \\
\vdots \\
\vdots\end{array}$ & & $\begin{array}{l}\text { â } \\
\vdots \\
0\end{array}$ & \\
\hline
\end{tabular}




\begin{tabular}{|c|c|c|c|c|c|c|c|c|c|c|c|c|c|c|c|c|c|}
\hline 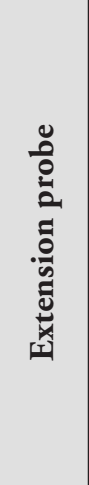 & 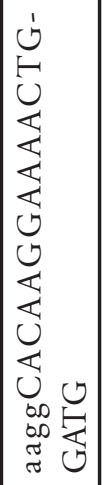 & 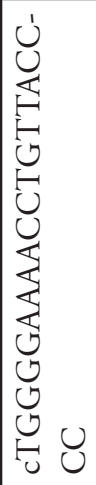 & 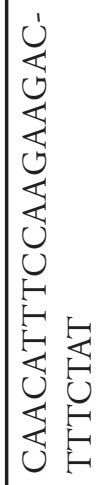 & 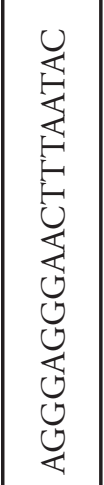 & 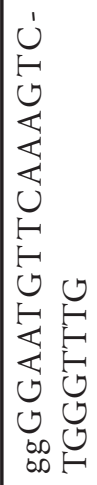 & 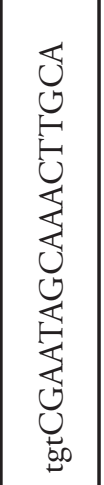 & 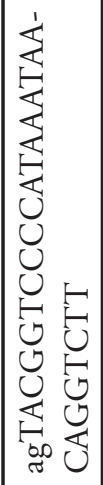 & 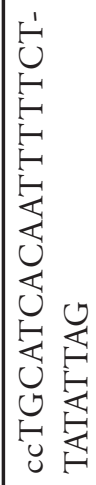 & 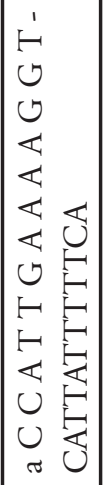 & 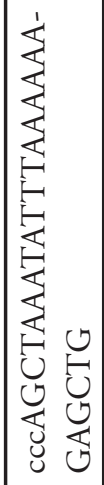 & 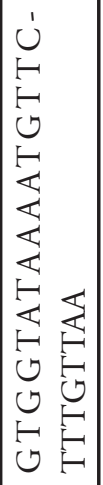 & 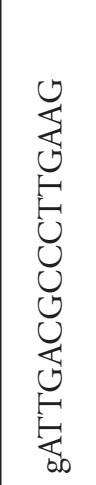 & 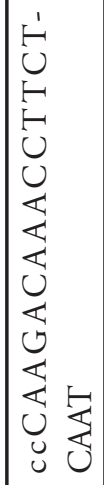 & 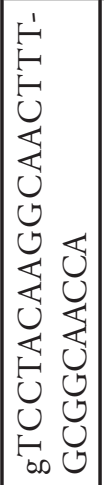 & 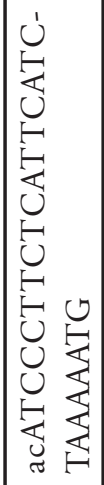 & 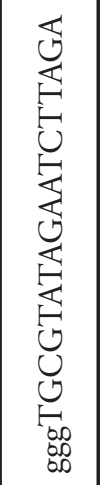 & 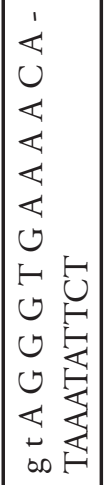 \\
\hline 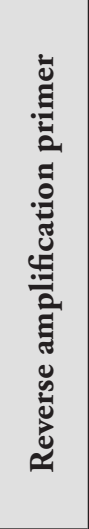 & 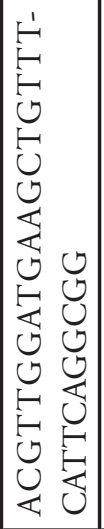 & 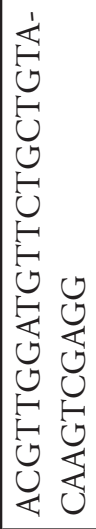 & 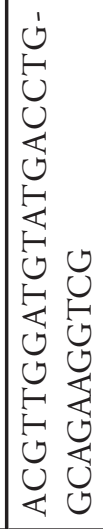 & 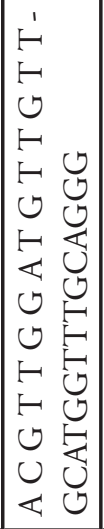 & 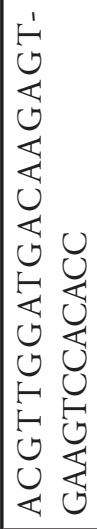 & 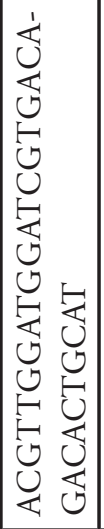 & 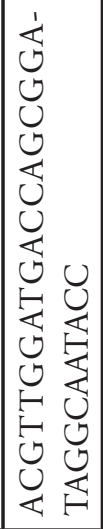 & 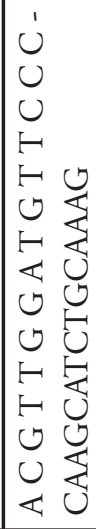 & 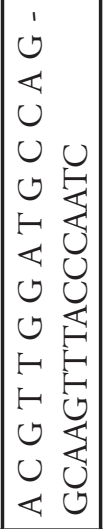 & 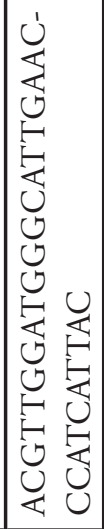 & 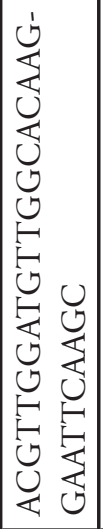 & 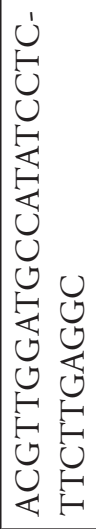 & 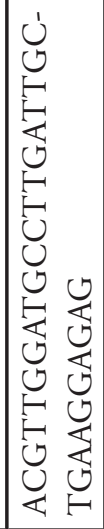 & 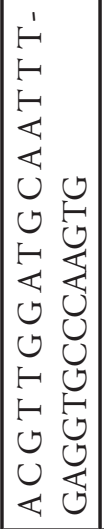 & 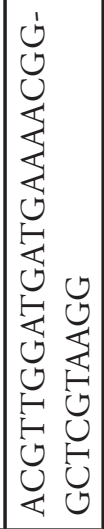 & 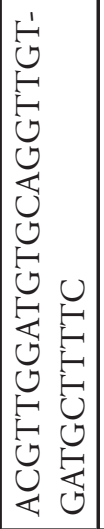 & 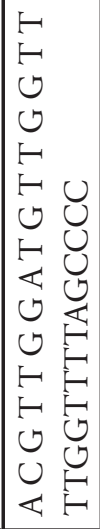 \\
\hline 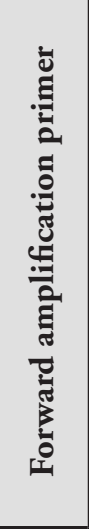 & 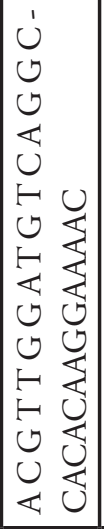 & 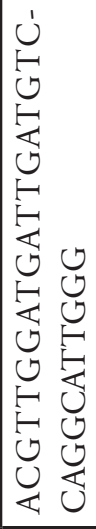 & 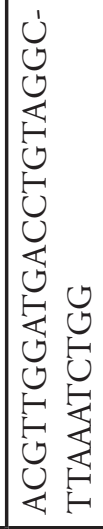 & 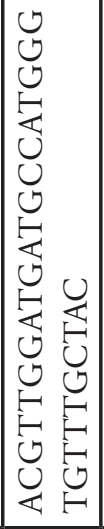 & 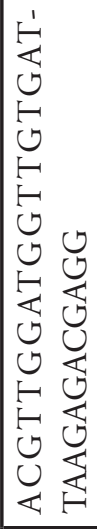 & 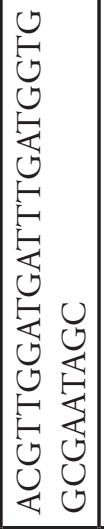 & 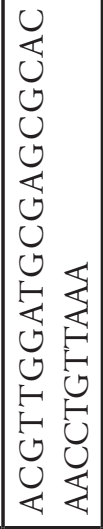 & 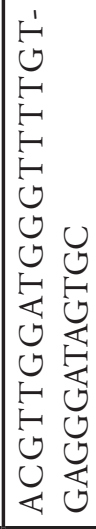 & 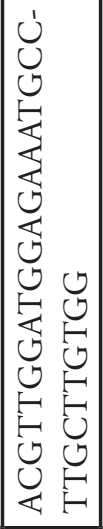 & 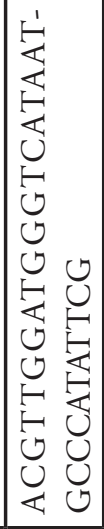 & 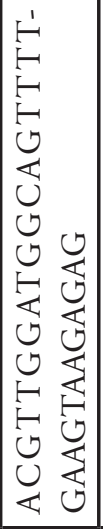 & 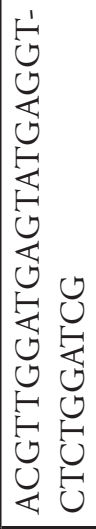 & 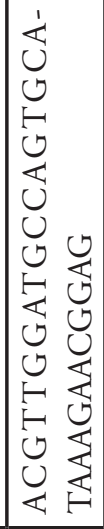 & 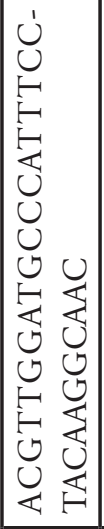 & 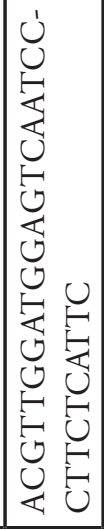 & 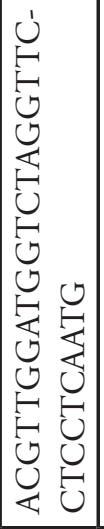 & 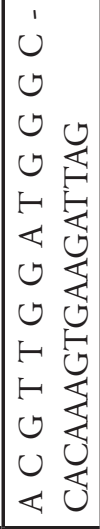 \\
\hline 灾 & $\begin{array}{l}\text { 空 } \\
\text { I }\end{array}$ & $\begin{array}{l}\tilde{\Xi} \\
\vec{\exists}\end{array}$ & $\begin{array}{l}\text { 党 } \\
\text { I }\end{array}$ & $\overrightarrow{\tilde{\Xi}}$ & $\hat{\bar{n}}$ & 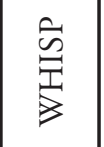 & $\begin{array}{l}\overrightarrow{5} \\
\overrightarrow{3} \\
\overrightarrow{3}\end{array}$ & 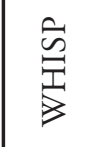 & $\begin{array}{l}\overrightarrow{\widehat{C}} \\
\overrightarrow{3}\end{array}$ & $\bar{I}$ & $=$ & $\begin{array}{l}\hat{\tilde{3}} \\
\overrightarrow{3}\end{array}$ & $z$ & & & نُ ن & \\
\hline$\frac{\tilde{\sigma}}{\frac{\delta}{\bar{z}}}$ & $\begin{array}{l}1 \\
\mathfrak{b}^{\prime} \\
\sigma^{\prime}\end{array}$ & $\begin{array}{l}0 \\
0 \\
\overrightarrow{00} \\
\overrightarrow{0} \\
0 \\
0 \\
0 \\
0 \\
0\end{array}$ & 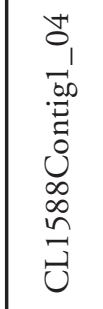 & 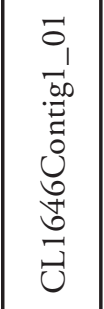 & 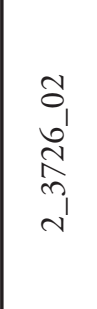 & 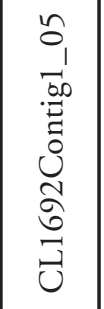 & 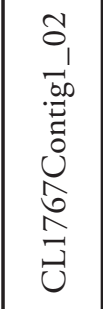 & 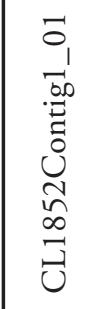 & 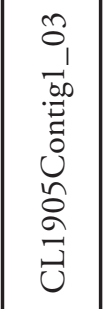 & 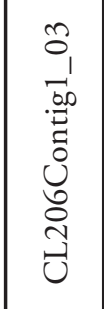 & 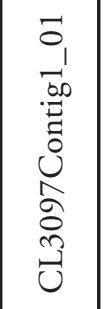 & 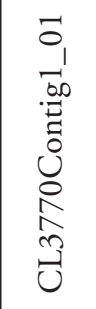 & 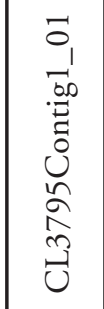 & $\begin{array}{l}i_{1}^{\infty} \\
z^{\prime}\end{array}$ & & $\begin{array}{l}\tilde{o}_{1} \\
\tilde{\vartheta}^{\prime} \\
\delta \\
\delta\end{array}$ & \\
\hline 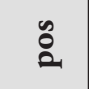 & $\stackrel{n}{n}$ & 1 & & & $\tilde{m}$ & $\forall$ & & & 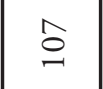 & & & & & & & & \\
\hline 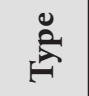 & 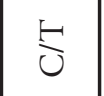 & $\stackrel{\cup}{F}$ & & & & 5 & & & $\mathbb{3}$ & & $\stackrel{F}{U}$ & & & & & & \\
\hline U & $\Xi$ & $\exists$ & $\Xi$ & $\Xi$ & $=$ & - & & & $\exists$ & & & & & & & & \\
\hline 芯 & $\begin{array}{l}\tilde{o} \\
0 \\
0\end{array}$ & రి & $\begin{array}{l}\text { Î } \\
\text { ô }\end{array}$ & के & $\stackrel{n}{0}$ & $\begin{array}{l}\text { ô } \\
\text { ò }\end{array}$ & $\begin{array}{l}\text { Wै } \\
\text { ô } \\
0\end{array}$ & $a$ & ồ & $\begin{array}{l}\hat{\hat{o}} \\
\hat{1}\end{array}$ & 0 & c & 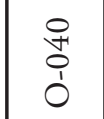 & $\bar{\varepsilon}$ & & & \\
\hline
\end{tabular}




\begin{tabular}{|c|c|c|c|c|c|c|c|c|c|c|c|c|c|c|c|c|c|c|}
\hline 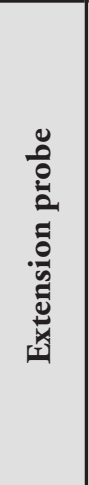 & 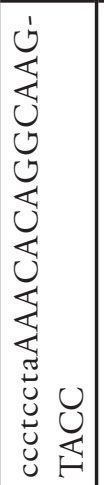 & 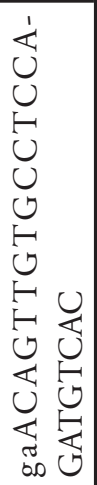 & 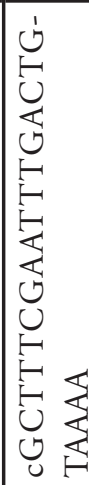 & 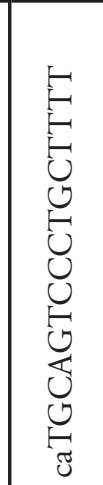 & 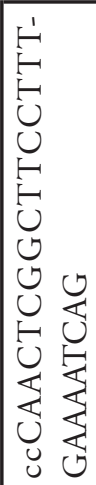 & 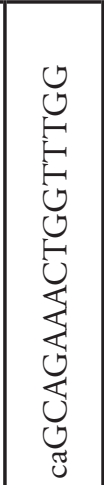 & 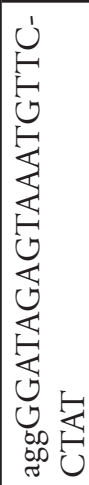 & 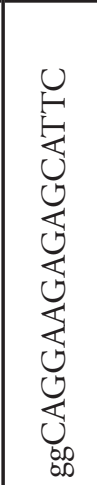 & 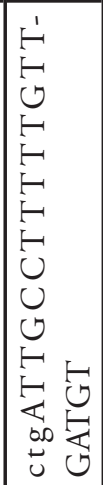 & 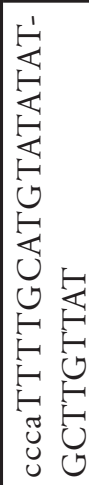 & 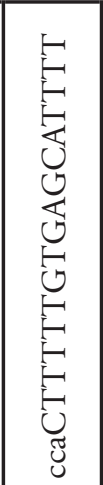 & 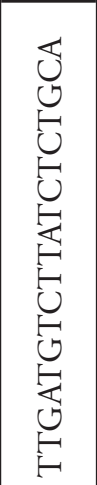 & 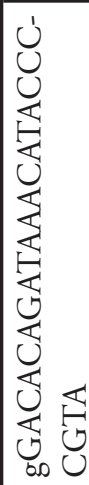 & 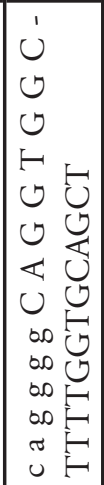 & 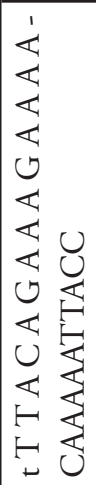 & 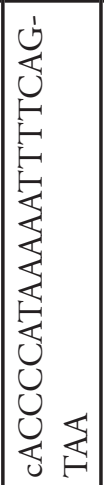 & 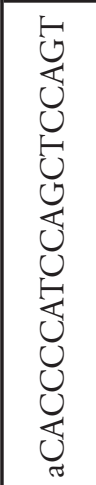 & 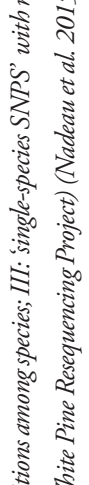 \\
\hline 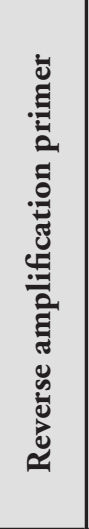 & 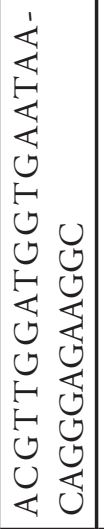 & 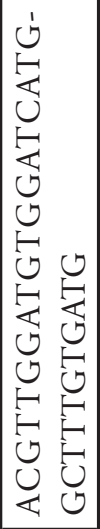 & 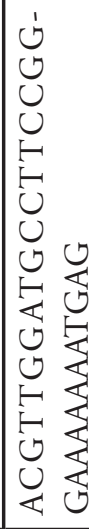 & 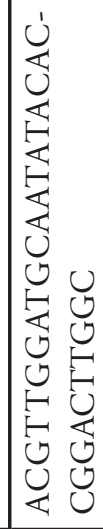 & 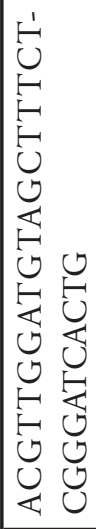 & 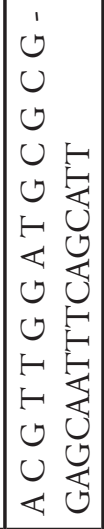 & 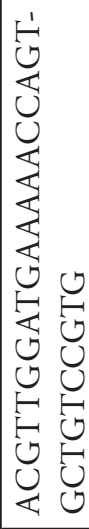 & 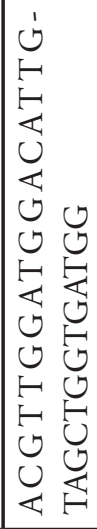 & 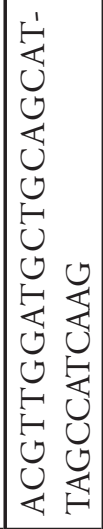 & 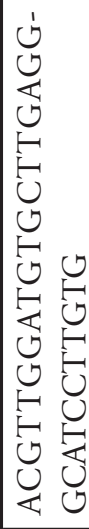 & 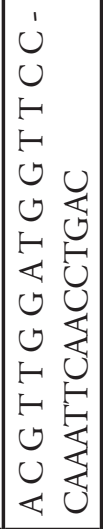 & 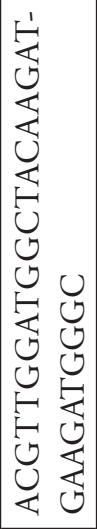 & 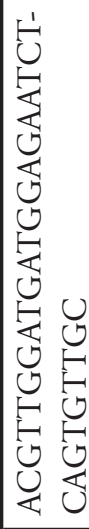 & 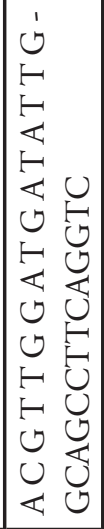 & 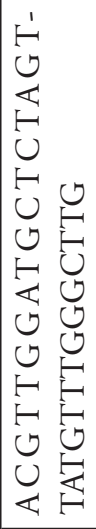 & 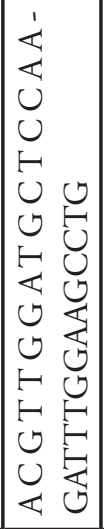 & 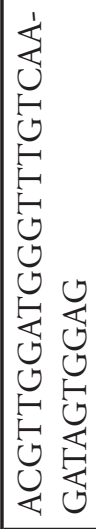 & \\
\hline 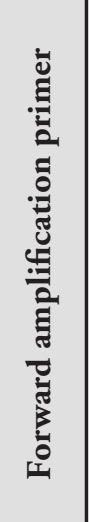 & 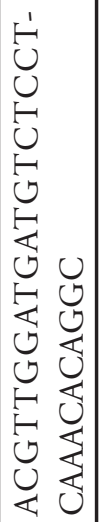 & 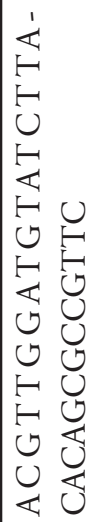 & 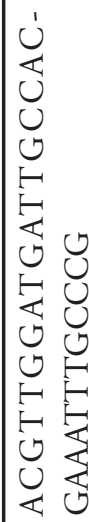 & 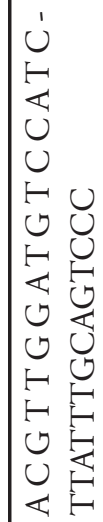 & $\begin{array}{ll}0 \\
0 \\
1 & \\
\varangle & 0 \\
0 & 0 \\
0 & 0 \\
1 & 0 \\
\varangle & 8 \\
0 & 0 \\
0 & 0 \\
0 & 0 \\
1 & 0 \\
1 & 5 \\
0 & 0 \\
0 & 0 \\
0 & 8 \\
4 & 0\end{array}$ & 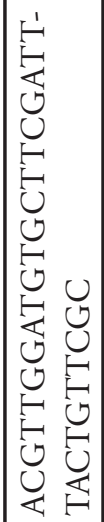 & 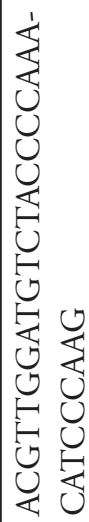 & 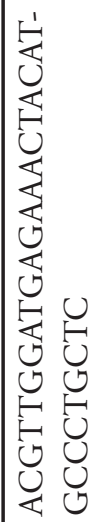 & 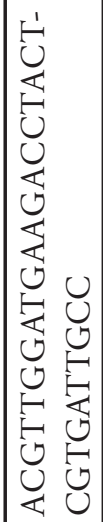 & 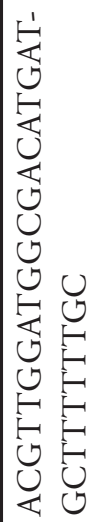 & 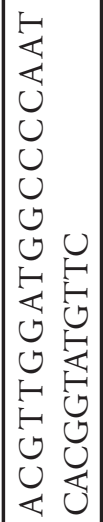 & 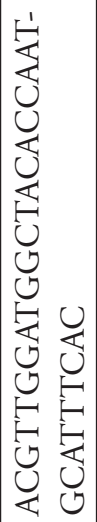 & 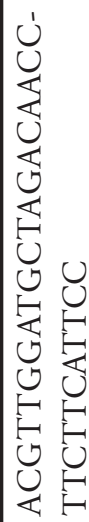 & 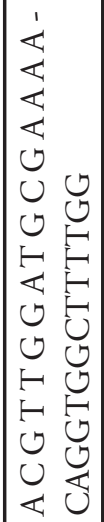 & 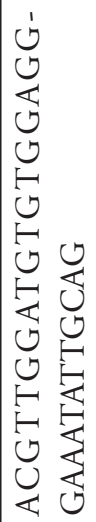 & 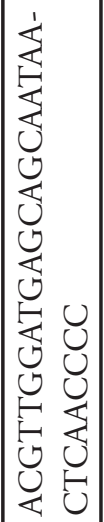 & 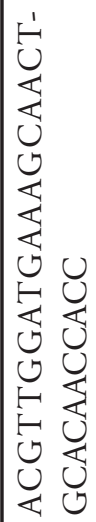 & \\
\hline 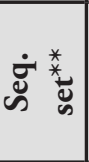 & 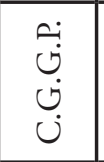 & 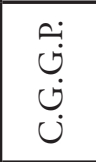 & $\begin{array}{l}\dot{U} \\
\dot{U} \\
\dot{ن}\end{array}$ & 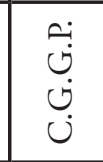 & 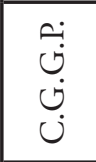 & 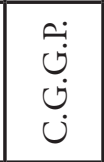 & ن & 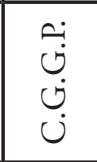 & نُ نُ نُ & 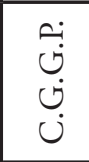 & نُ & نُ نُ & $\bar{I}$ & $\begin{array}{l}\hat{n} \\
\stackrel{3}{3}\end{array}$ & $\begin{array}{l}\overrightarrow{\tilde{s}} \\
\stackrel{\vec{F}}{3}\end{array}$ & 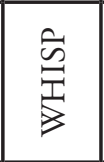 & $\frac{\vec{⿹}}{\overrightarrow{3}}$ & \\
\hline$\frac{\tilde{0}}{\frac{.}{\tilde{Z}}}$ & 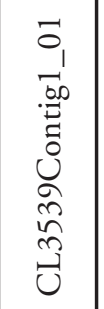 & 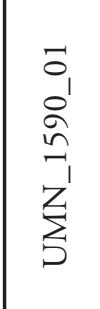 & $\begin{array}{l}\tilde{\sigma}_{1} \\
\vec{\infty}^{\prime} \\
\stackrel{\sim}{*} \\
\sim_{1}\end{array}$ & 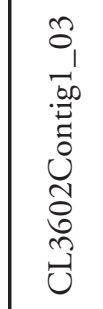 & 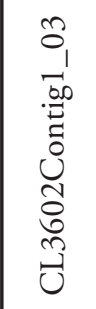 & 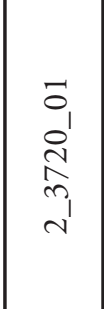 & 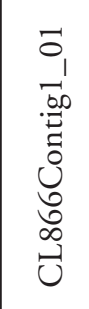 & $\begin{array}{l}\tilde{\sigma}_{1} \\
\hat{\hat{J}} \\
\hat{z}_{1} \\
z_{j}\end{array}$ & 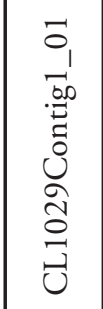 & ठ্் & 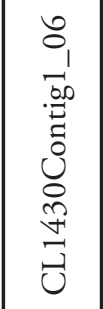 & 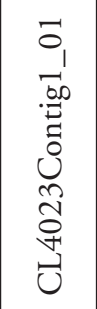 & $\begin{array}{l}0 \\
\infty\end{array}$ & 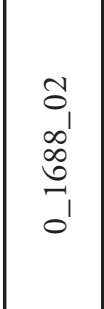 & $\sigma_{1}$ & & $\begin{array}{l}\sigma_{1} \\
N^{\prime} \\
\infty \\
\infty \\
\infty \\
N^{\prime}\end{array}$ & \\
\hline$\ddot{\circ}$ & - & $m$ & $\overrightarrow{\widehat{\lambda}}$ & 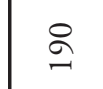 & నె & ते & $\sim$ & 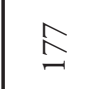 & $\hat{n}$ & & $m$ & $\stackrel{\infty}{\infty}$ & & in & $\widetilde{\sim}$ & & $\hat{n}$ & \\
\hline 兹 & $\frac{5}{\omega}$ & $\stackrel{U}{H}$ & $\underset{U}{\mathbb{U}}$ & $\frac{5}{4}$ & $\frac{5}{U}$ & $\underset{\circlearrowleft}{\mathbb{S}}$ & & $\frac{F}{U}$ & $\frac{\xi}{0}$ & & $\frac{\cup}{U}$ & 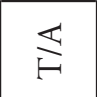 & & 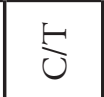 & $\underset{\mho}{\mathbb{S}}$ & & $\stackrel{\bigcup}{\ominus}$ & \\
\hline U & $\exists$ & $\Xi$ & $\Xi$ & $\Xi$ & $\Xi$ & $\Xi$ & $\Xi$ & $\Xi$ & $\Xi$ & $\Xi$ & $=$ & $\exists$ & $\Xi$ & $\exists$ & $\exists$ & $=$ & $\exists$ & \\
\hline 孞 & 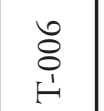 & 足 & $\begin{array}{l}\stackrel{8}{0} \\
1\end{array}$ & $\begin{array}{l}\overrightarrow{0} \\
\stackrel{1}{1}\end{array}$ & $\begin{array}{l}\overrightarrow{0} \\
\stackrel{1}{H}\end{array}$ & 定 & $\begin{array}{l}\overrightarrow{0} \\
1 \\
1\end{array}$ & 涼 & $\begin{array}{l}\text { Oे } \\
\stackrel{1}{H}\end{array}$ & $\stackrel{\sim}{*}$ & 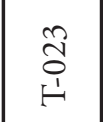 & $\begin{array}{l}\widehat{O} \\
0 \\
H\end{array}$ & 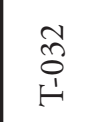 & 勇 & $\begin{array}{l}\hat{0} \\
\stackrel{1}{1}\end{array}$ & $\begin{array}{c}\text { ô } \\
1\end{array}$ & 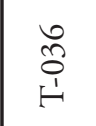 & \\
\hline
\end{tabular}




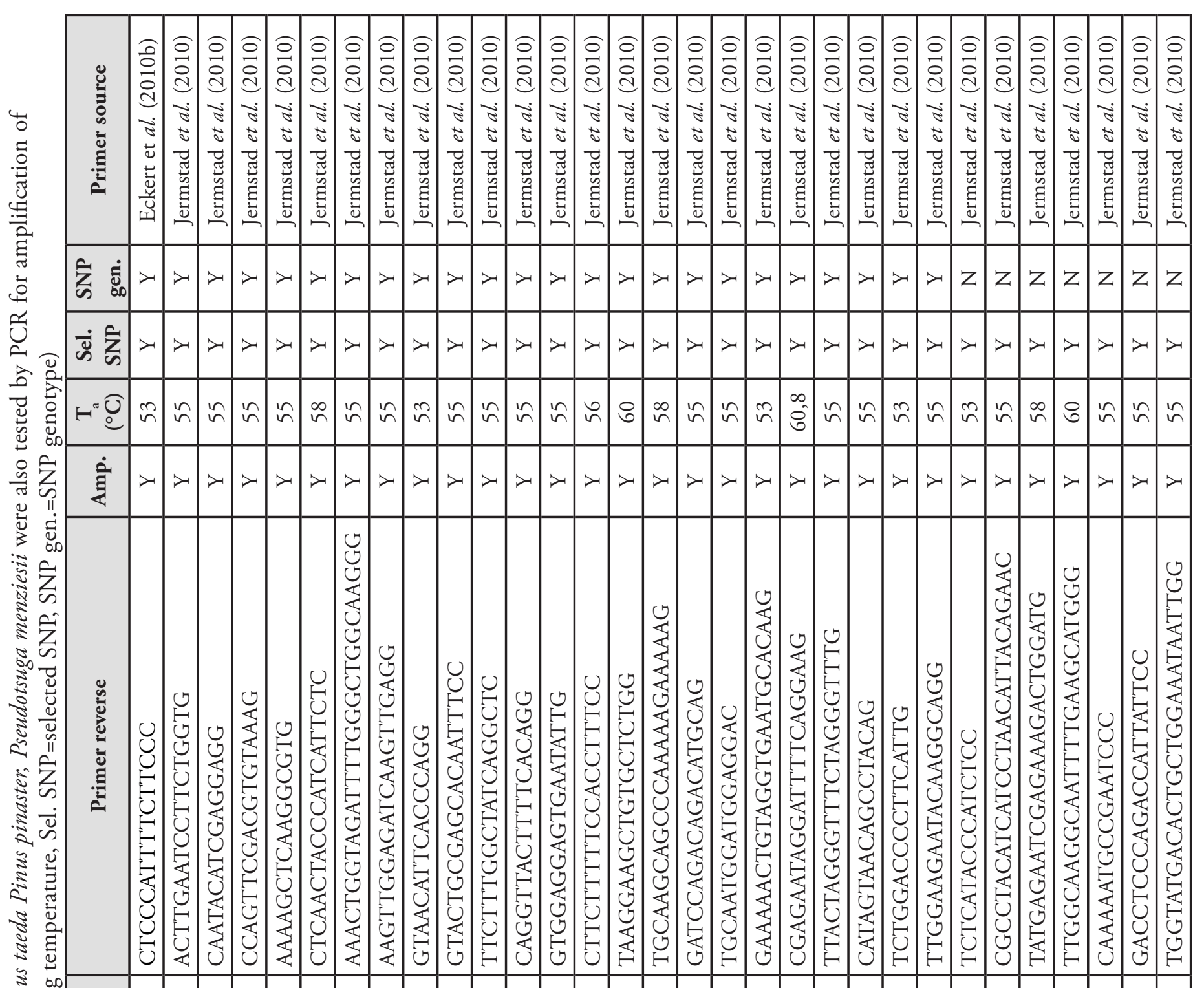

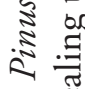

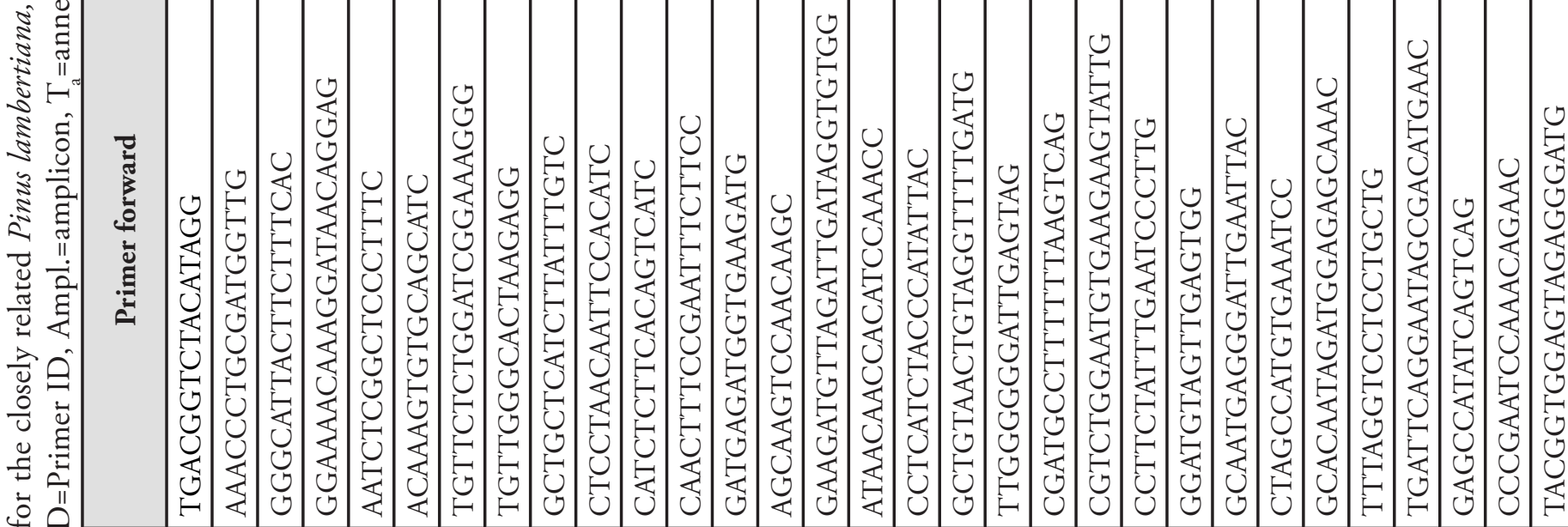

氖

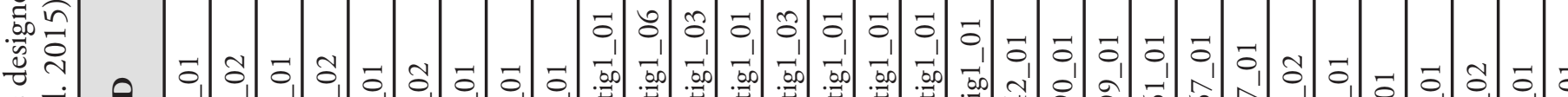

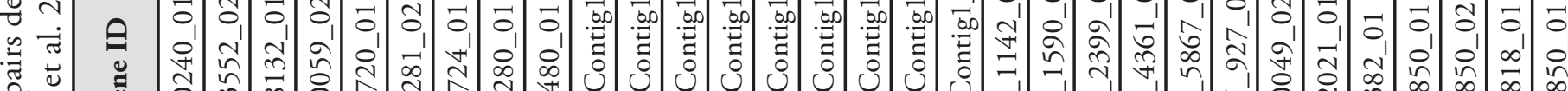

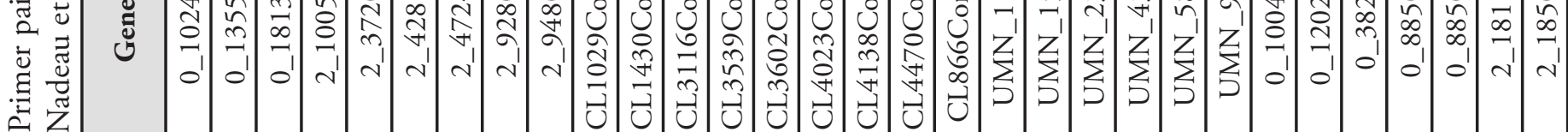

औैं :

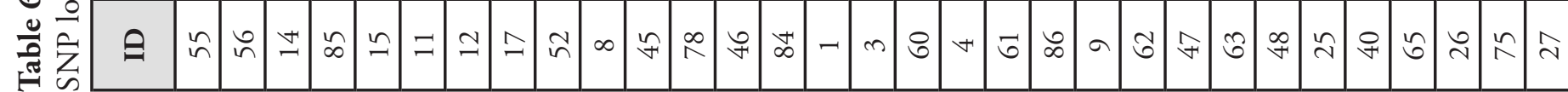




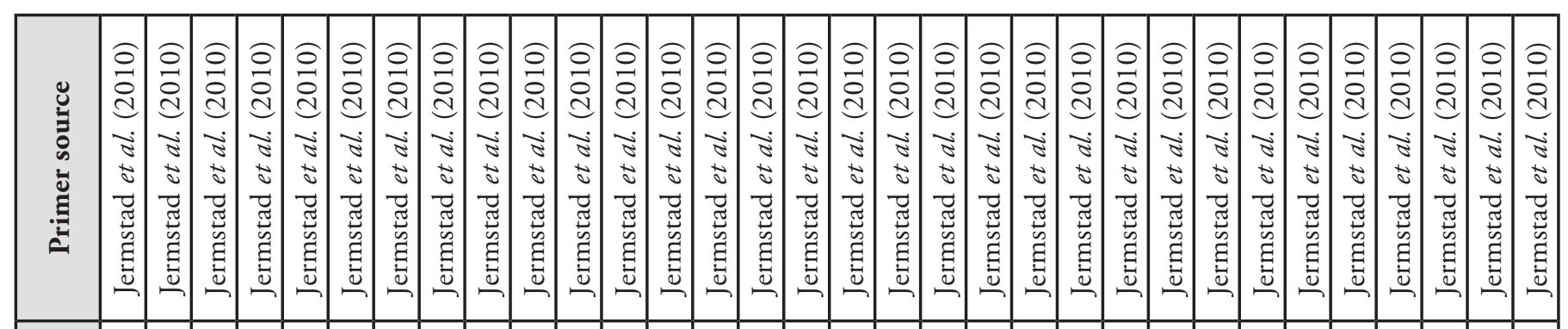

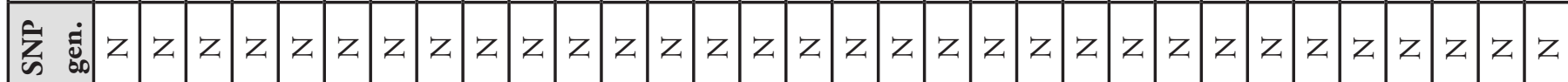

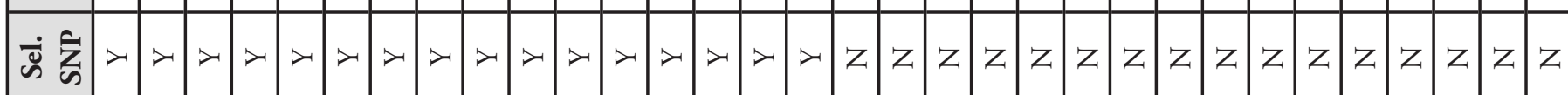
F. O己

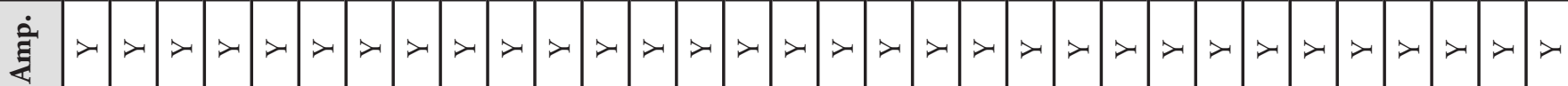
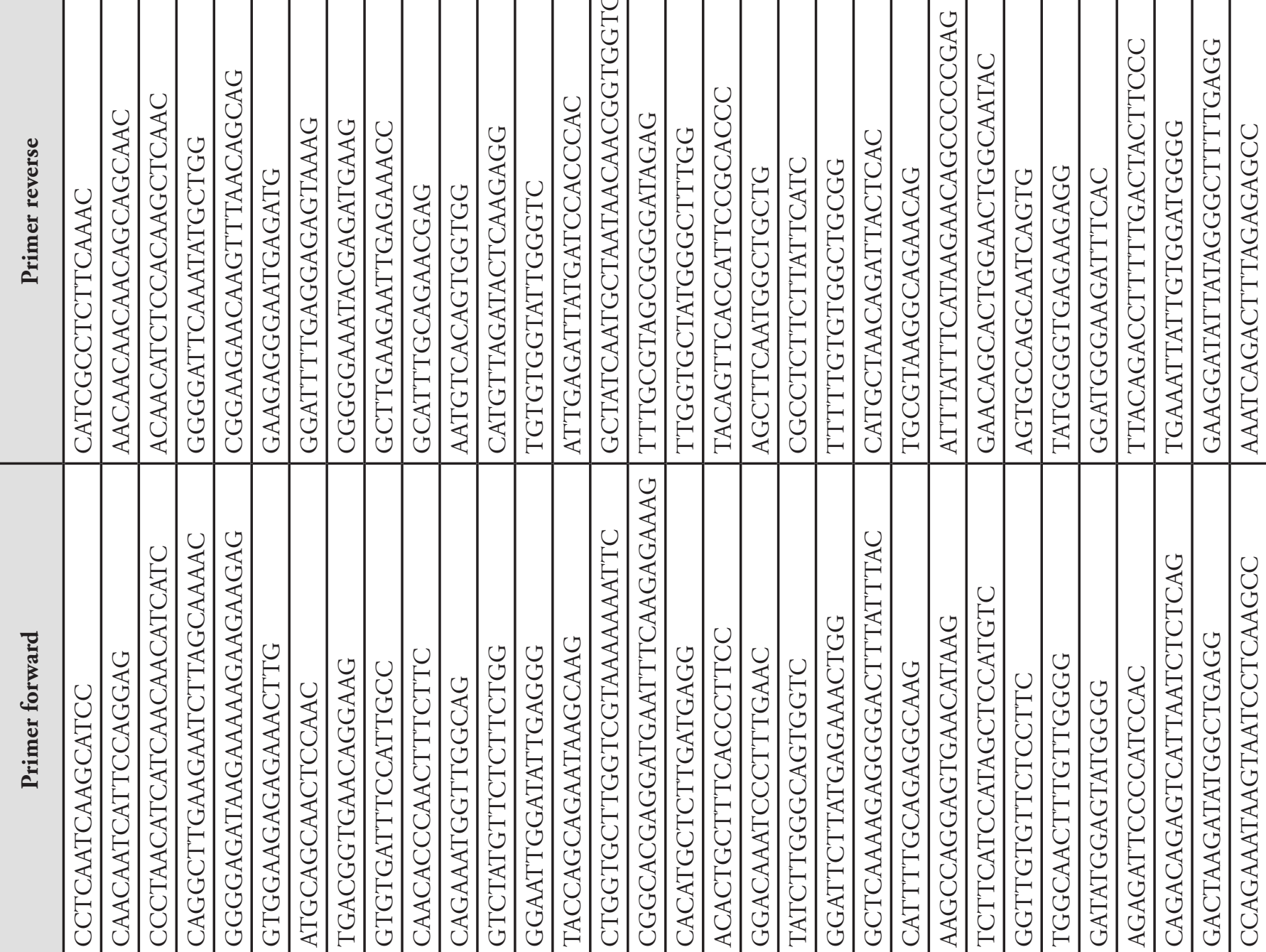

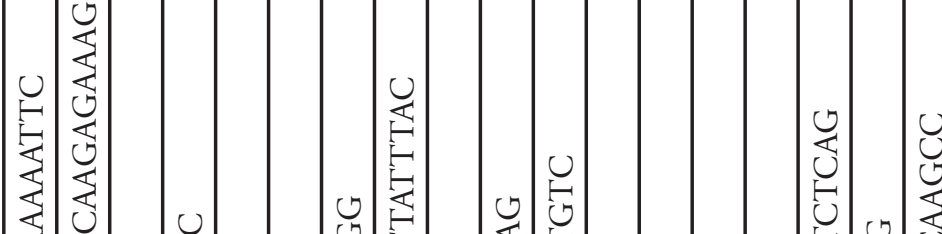
y

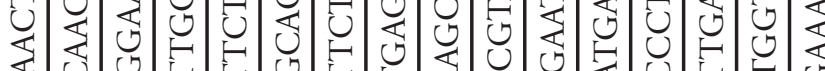

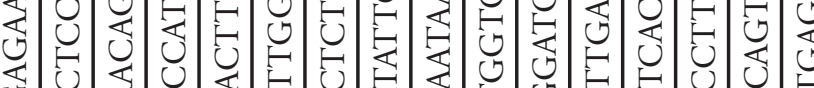
o 可 安

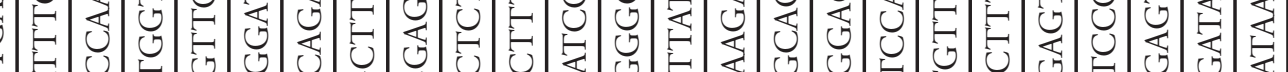

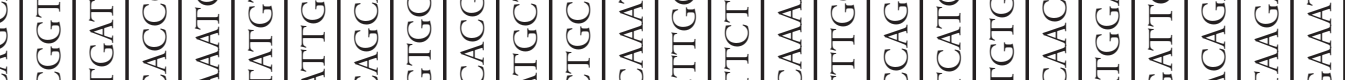
若

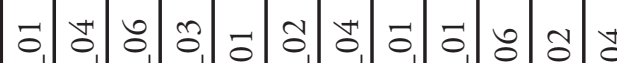

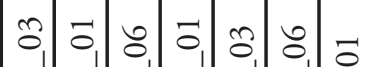

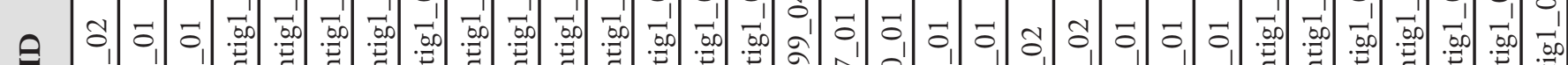

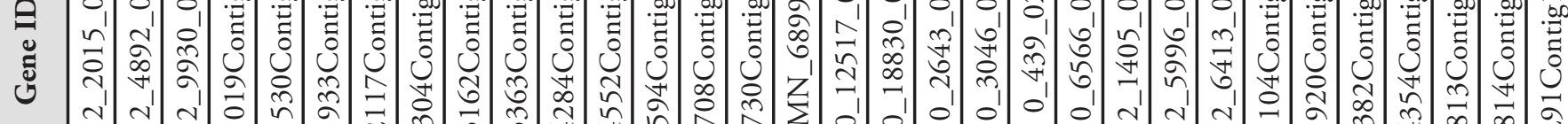

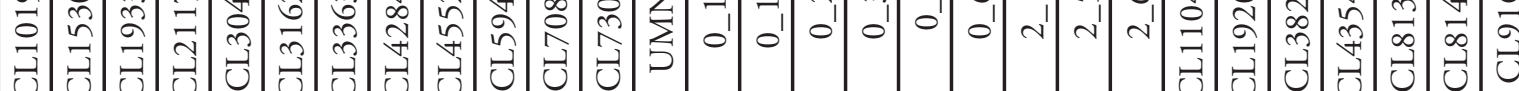

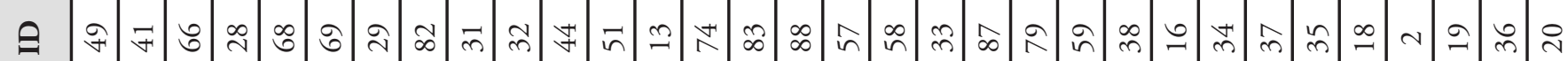




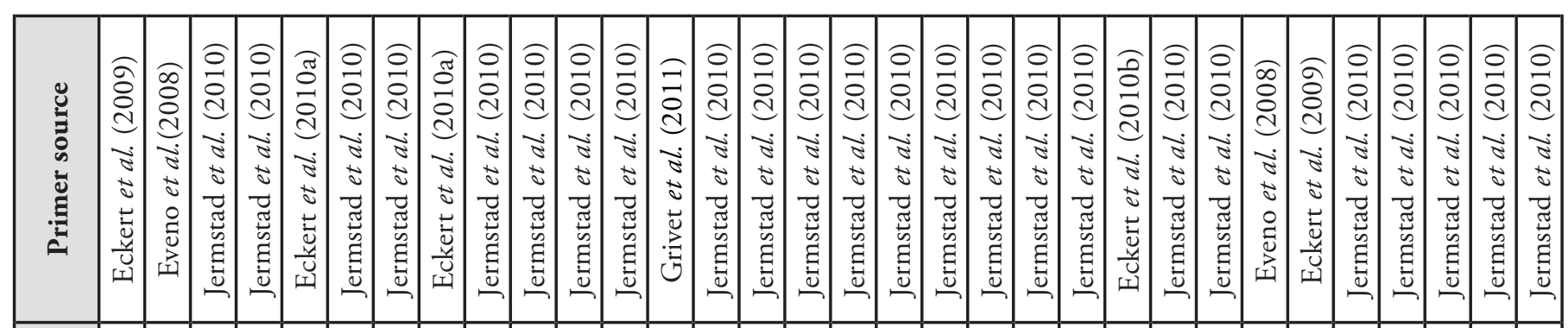
空悹

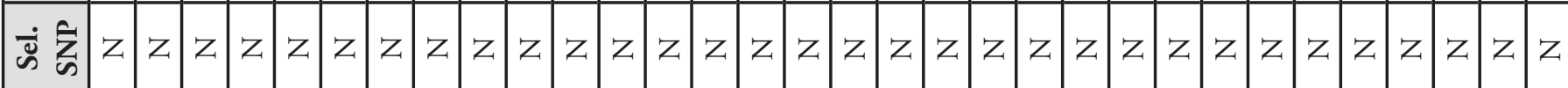
F

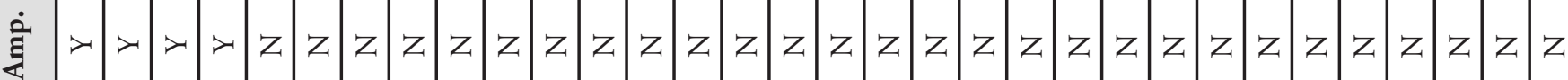

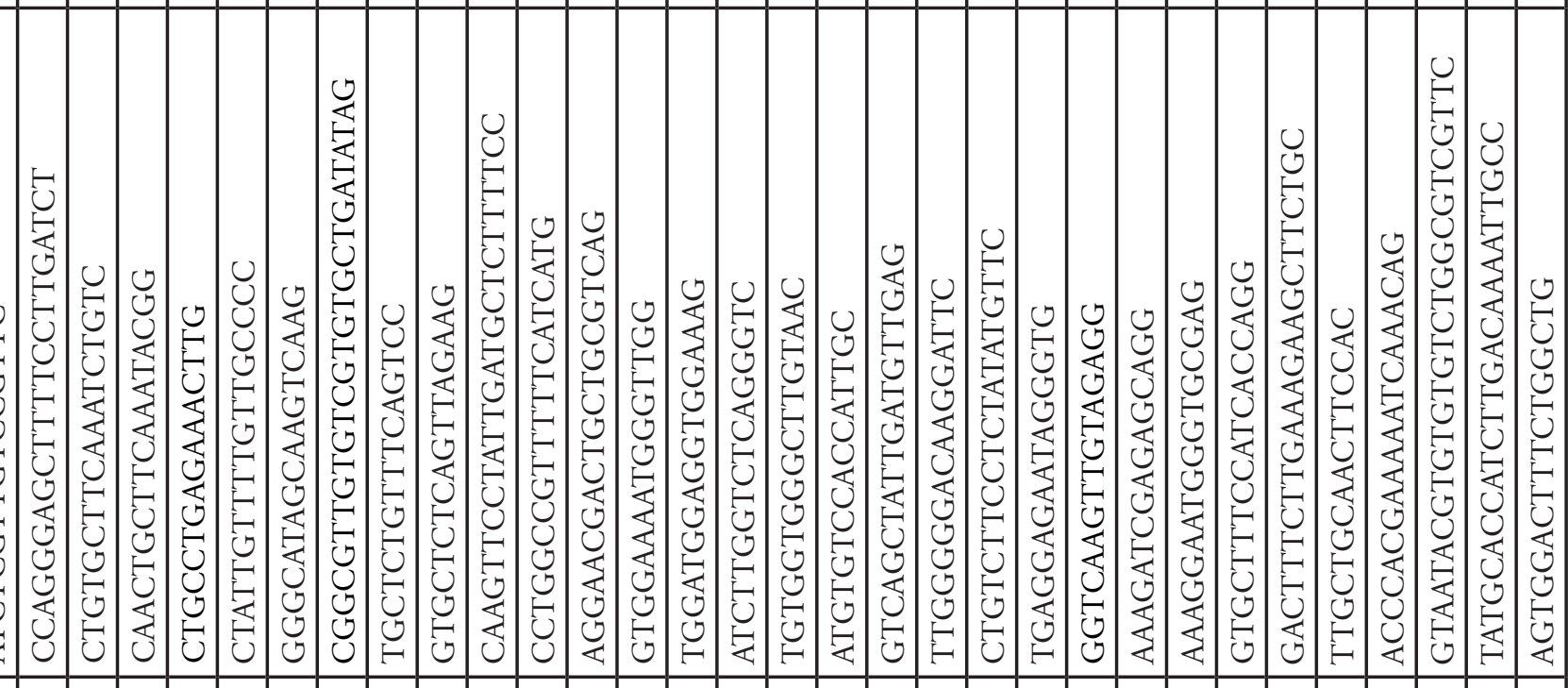

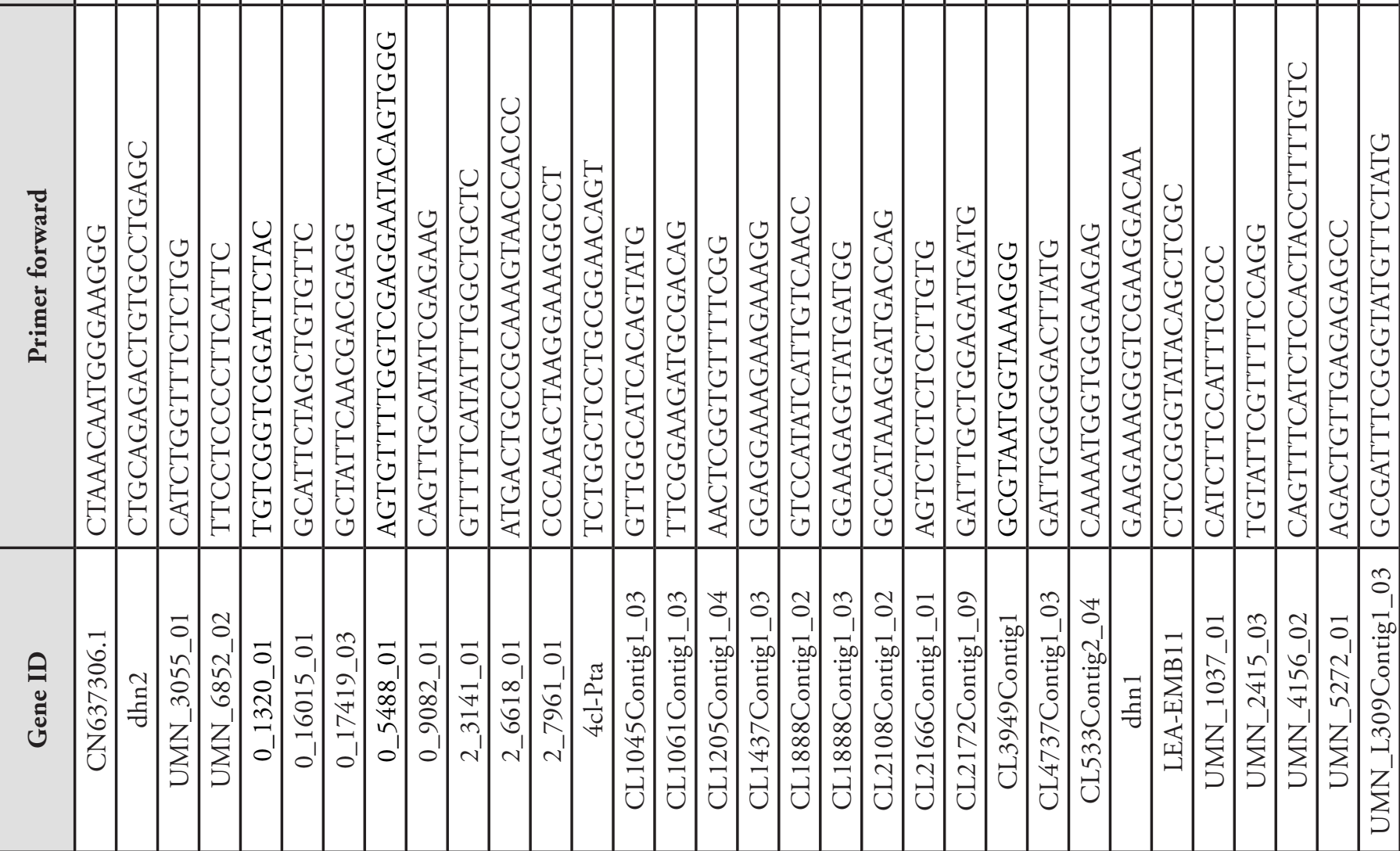

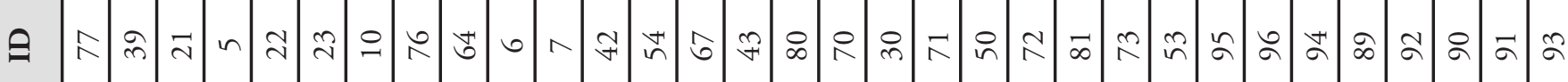




\section{Material for DNA-extraction}

- Haploid megagametophytes (Nadeau et al. 2015)

- Vegetative buds (Nadeau et al. 2015)

- Needles (Nadeau et al. 2015)

\section{DNA-extraction protocols}

Nadeau et al. (2015) reported that 'DNA was extracted from buds or current-year needles using the Nucleospin 96 Plant II kit (Macherey-Nagel, Bethlehem, Pennsylvania, USA) with the following modifications made to the manufacturer's protocol: (1) cell lysis using buffer PL2 at $65^{\circ} \mathrm{C}$ for $2 \mathrm{~h}$ (instead of $30 \mathrm{~min}$ ); and (2) elution with an in-house $0.01 \mathrm{mM}$ Tris- $\mathrm{HCl} \mathrm{pH} 8.0$ buffer'.

\section{Amplification Protocol}

As reported by Nadeau et al. (2015) 'for PCR amplification of the candidate genes, about $15 \mathrm{ng}$ of $P$. strobus megagametophyte DNA was added to a $30 \mu \mathrm{L}$ reaction volume containing $1 \times \mathrm{PCR}$ buffer, $1.66 \mathrm{mM}$ $\mathrm{MgCl} 2,0.133 \mathrm{mM}$ of each dNTP, $0.133 \mu \mathrm{M}$ of each primer, $1 \mathrm{U}$ of Platinum Taq polymerase (Invitrogen, Burlington, Ontario, Canada). DNA regions were amplified using a PTC200 Thermal Cycler (MJ Research, Waltham, Massachusetts, USA) according to the following protocol: an initial 3 min step at $94^{\circ}$ $\mathrm{C} ; 35$ cycles of $1 \mathrm{~min}$ at $94^{\circ} \mathrm{C}, 45 \mathrm{~s}$ at the annealing temperature, $3 \mathrm{~min}$ at $70^{\circ} \mathrm{C}$; and a final $10 \mathrm{~min}$ step at $72^{\circ} \mathrm{C}^{\prime}$.

\section{Important results}

Nadeau et al. (2015) investigated the influence of two physiographic landscapes on the structure of populations and the post glacial colonization of two white pine species growing at contrasting habitats; $P$. monticola, growing in high mountainous regions of western North America, and $P$. strobus occurring in much less mountainous areas of eastern North America. They studied 133 Pinus strobus populations and 61 Pinus monticola ones, by using a big number of Single Nucleotide Polymorphisms (SNPs). They interpreted the steep latitudinal decrease in genetic diversity for Pinus monticola as a possible result of the postglacial colonization that involved long-distance dispersal evens, while the lack of patterns in diversity of $P$. strobus was attributed to recolonization through a gradually advancing front or frequent long-distance dispersal events. For both species, two distinct northern and southern genetic groups were identified that most probably originated from two different glacial lineages. The smaller groups detected by the authors mentioned above were characterized as remnants of cryptic refugia.

\section{Reference list}

Beaulieu J., Simon J.P. (1994): Inheritance and linkage relationships of allozymes in Pinus strobus L. Silvae Genet, 43: 253-261.

Buchert, G.P., Rajora, O.P., Hood, J.V., Dancik B.P. (1997): Effects of harvesting on genetic diversity in old-growth eastern white pine in Ontario, Canada. Conservation Biology, 11(3): 747-755.

Cato, S.A., Richardson T.E. (1996): Inter- and intraspecific polymorphism at chloroplast SSR loci and the inheritance of plastids in Pinus radiata D. Don. Theoretical and Applied Genetics, 93: 587-592.

Chagala, E.M. (1996): Inheritance and linkage of allozymes in Pinus strobus. Silvae Genetica, 45(4):181187.

Chhatre, V.E., Rajora O.P. (2014): Genetic divergence and signatures of natural selection in marginal populations of a keystone, long-lived conifer, eastern white pine (Pinus strobus) from Northern Ontario. PlosONE, 9(5), e97291.

Cloutier, D., Rioux, D., Beaulieu, J., Schoen D.J. (2003): Somatic stability of microsatellite loci in Eastern white pine, Pinus strobus L. Heredity, 90: 247-252.

Doyle, J.J., Doyle J.L. (1987): A rapid DNA isolation procedure for small quantitie of fresh leaf tissue. Phytochemical Bulletin, 19: 11-15.

Echt, C.S., Nelson C.D. (1997): Linkage mapping and genome length in eastern white pine (Pinus strobus L.). Theoretical and Applied Genetics, 94: 1031-1037.

Echt, C.S., May-Marquardt, P., Hseih, M., Zahorchak R. (1996): Characterization of microsatellite markers in eastern white pine. Genome, 39: 1102-1108.

Echt, S.C., Vendramin G.G., Nelson C.D., Marquard P. (1999): Microsatellite DNA as shared genetic markers among conifer species. Canadian Journal of Forest Research, 29: 365-371.

Eckert, A.J., Wegrzyn J.L., Pande, B.,. Jermstad K.D., Lee J.M., Liechty J.D., Tearse B.R., Krutovsky, K.V., Neale, D.B. (2009): Multilocus patterns of nucleotide diversity and divergence reveal positive selection at candidate genes related to cold hardiness in coastal Douglas fir (Pseudotsuga menziesii var. menziesii). Genetics, 183: 289 $-298$.

Eckert, A.J., Bower A.D., González-Martínez S.C., Wegrzyn J.L., Coop G., Neale D.B. (2010a): Back to nature: Ecological genomics of loblolly pine (Pinus taeda, Pinaceae). Molecular Ecology, 19: 3789-3805. 
Eckert, A.J., van Heerwaarden J., Wegrzyn J.L., Nelson C.D., Ross-Ibarra J., González-Martínez S.C., Neale D.B. (2010b): Patterns of population structure and environmental associations to aridity across the range of loblolly pine (Pinus taeda L., Pinaceae). Genetics, 185: 969-982.

Epperson, B.K., Chung, M.G. (2001): Spatial genetic structure of allozyme polymorphisms within populations of Pinus strobus (Pinaceae). American Journal of Botany, 88(6): 1006-1010.

Eveno, E., Collada C., Guevara M.A., Léger V., Soto A., Díaz L., Léger P., González-Martínez, S.C., Teresa Cervera, M., Plomion, C., Garnier-Géré, P.H. (2008): Contrasting patterns of selection at Pinus pinaster Ait. drought stress candidate genes as revealed by genetic differentiation analyses. Molecular Biology and Evolution, 25: $417-437$.

Jermstad, K.D., Eckert, A.J., Wegrzyn, J.L., DelfinoMix, A., Davis, D.A., Burton, D.C., Neale, D.B. (2010): Comparative mapping in Pinus : Sugar pine (Pinus lambertiana Dougl.) and loblolly pine (Pinus taeda L.). Tree Genetics \& Genomes, 7: 457 - 468.

Little, E.L., Jr. (1971): Atlas of United States trees, volume 1, conifers and important hardwoods: U.S. Department of Agriculture Miscellaneous Publication, 1146, 9 p., 200 maps.

Mader, D.L. (1985): Soil-site productivity for natural stands of white pine in the Northeast. In Symposium proceedings-Eastern white pine: Today and tomorrow. pp. 8-31. David T. Funk, comp. U.S.D.A. For. Serv., Gen. Tech. Rep. WO-51. Washington, D.C.

Marquardt, P.E., Epperson B.K. (2004): Spatial and population genetic structure of microsatellites in white pine, Molecular Ecology, 13: 3305-3315.

Mehes, M., Nkongolo, K.K., Michael, P. (2009): Assessing genetic diversity and structure of fragmented populations of eastern white pine (Pinus strobus) and western white pine ( $P$. monticola) for conservation management. Journal of Plant Ecology, 2(3): 143-151.

Mehes, M.S., Nkongolo, K.K., Michael P. (2007): Genetic analysis of Pinus strobus and Pinus monticola populations from Canada using ISSR and RAPD markers: development of genome-specific SCAR markers. Plant Systematics and Evolution, 267: 47-63.

Myers, E.R., Chung, M.Y., Chung M.G. (2007): Genetic diversity and spatial genetic structure of Pinus strobus (Pinaceae) across an island landscape inferred from allozyme and cpDNA markers. Plant Systematics and Evolution, 264: 15-30.
Nadeau, S., Godbout, J., Lamoth, M., Gros-Louis, M.C., Isabel, N., Ritland K. (2015): Contrasting patterns of genetic diversity across the ranges of Pinus monticola and P. strobus: A comparison between eastern and western North American post glacial colonization histories. American Journal of Botany, 102(8): 1342-1355.

Nkongolo, K.K. (1999): RAPD and cytological analyses of Picea spp. from different provenances: Genomic relationships among taxa. Hereditas, 130: 137-144.

Nkongolo, K.K., Narendrula, R., Mehes-Smith, M., Dobrzeniecka, S., Vandeligt, K., Ranger, M., Beckett, P. (2012): Genetic sustainability of fragmented conifer populations from stressed areas in Northern Ontario (Canada): Application of molecular markers. In: Blanco, J.A., Yueh-Hsin, L. (editors) Forest Ecosystems - More than Trees. Publ. IntechOpen. pp. 315-336.

Rajora, O.P., DeVerno L., Moseler A., Innes D.J. (1997): Genetic diversity and population structure of disjunct Newfounland and central Ontario populations of eastern white pine (Pinus strobus). Canadian Journal of Forest Research, 76: 500-508.

Rajora, O.P., Rahman, M.H., Buchert, G.P., Dancik B.P. (2000): Microsatellite DNA analysis of genetic efforts of harvesting in old-growth eastern white pine (Pinus strobus) in Ontario, Canada. Molecular Ecology, 9: 339348.

Vendramin, G.G., Lelli, L., Rossi, P., Morgante M. (1996): A set of primers for the amplification of 20 chloroplast microsattelites in Pinaceae. Molecular Ecology, 5: 595-598.

Wendel, G.W., Smith, H.C. (1990): Pinus strobus L., Eastern White Pine, pp. 476-488. In: Burns R.M., Honkala B.H. (eds.), Silvics of North America, Vol. 1, Conifers, U.S.D.A. For. Serv. Agric. Handbk. 654, Washington, D.C.

Wendel, G.W. (1980): Eastern white pine. In Forest cover types of the United States and Canada. pp. 25-26. F.H. Eyre, ed. Society of American Foresters, Washington, D.C.

Zinck, J.W., Rajora, O.P. (2016): Post-glacial phylogeography and evolution of a wide-ranging highlyexploited keystone forest tree, eastern white pine (Pinus strobus) in North America: single refugium, multiple routes. BMC Evolutionary Biology, 16(1): 56. 


\title{
Molecular markers used for genetic studies in Douglas- fir (Pseudotsuga menziesii (Mirb.) Franco)
}

\author{
Monika Konnert ${ }^{1}$, Anna-Maria Szasz-Len ${ }^{1}$ and Marcela van Loo ${ }^{2}$ \\ ${ }^{1}$ Bavarian Office for Forest Seeding and Planting Teisendorf, Germany \\ ${ }^{2}$ University of Vienna, Department of Botany and Biodiversity Research, Rennweg 14, 1030 Vienna, Austria
}

\section{General remarks}

Douglas-fir (P. menziesii (Mirb.) Franco) has one of the widest natural ranges of any tree species and the largest south-to-north distribution of any commercial conifer in North America, extending from $19^{\circ} \mathrm{N}$ in Mexico to $55^{\circ} \mathrm{N}$ in Western Canada. In Western Oregon and Washington it grows from sea level to $1700 \mathrm{~m}$ (Hermann and Lavender 1990). Within this large geographic area, contrasting climatic conditions are found. Douglas-fir populations seem to have a great adaptive potential and are generally regarded as being adapted to the different environments (St. Clair et al. 2005, St. Clair 2006, Gould et al. 2012). Winter temperatures and frost dates are of great importance for the adaptation of Douglasfir, whereas summer drought is less important (St. Clair et al. 2005). Douglas-fir is a wind-dispersed and windpollinated species with high gene flow and outcrossing rates exceeding 90\% (e.g. El-Kassaby et al. 1981, Shaw and Allard 1982, Neale and Adams 1985, Yeh and Morgan 1987).

Two taxonomic varieties are recognized: the coastal variety (P. menziesii var. menziesii or viridis) found along the North American Pacific Coast and the interior variety (P. menziesii var. glauca) found inland, in the mountains from British Columbia to Central Mexico (Lavender and Hermann 2014). There is no reproductive barrier between them (Gugger et al. 2010, Wei et al. 2011, van Loo et al. 2015). In Europe, a third variety, caesia, is widely recognized as an intermediate type between the coastal (viridis) and the interior (glauca) variety (Schober 1954, Aas 2008).

The varieties differ in a number of important traits: The coastal variety grows faster and gets considerably taller than the interior variety, which tends to be more shade tolerant and more cold-hardy (Lavender and Hermann 2014). Within the coastal variety trees from the coastal areas are less cold hardy than trees from the western slope of the Cascades (e.g. Aitken et al. 1996). Variation in bud-burst, emergence and growth is strongly related to elevation and cool-season temperatures (e.g. St.Clair et al. 2005). Besides the high phenotypic diversity, Douglas-fir shows high genetic variation.

Here, we provide a review of available laboratory techniques that can be used to investigate the genetic diversity and structure in natural and artificial Douglasfir populations and to trace back the origin of European Douglas-fir plantations.

\section{Isozymes}

Since the beginning of 1970s, isozymes have been used for genetic analysis on Douglas-fir. Studies with isozymes focused on:

- investigation of the genetic structure in natural

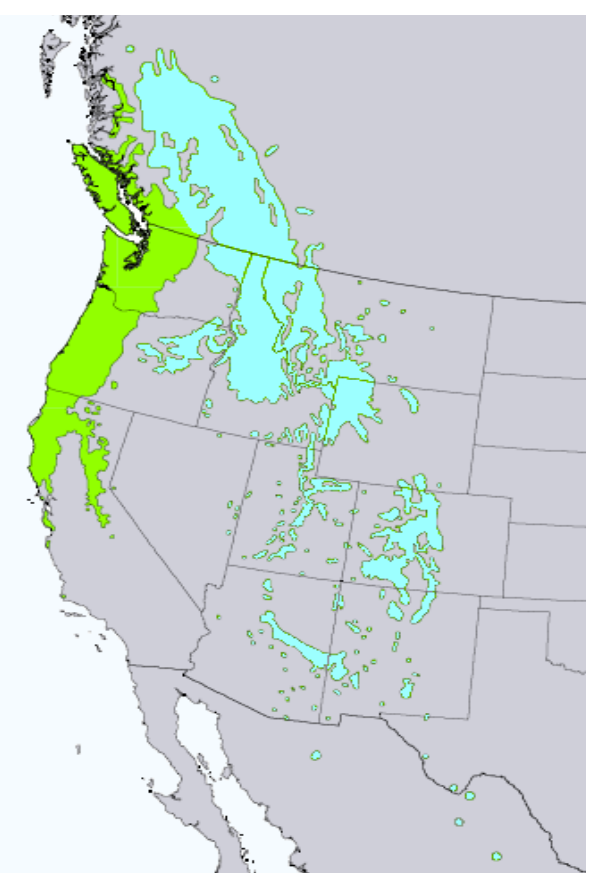

Figure 1. Native distribution range of Douglas-fir (green colour denotes var. menziesii, blue colour denotes var. glauca) (source: USGS, USA) 
populations (e.g. Yang 1974, Neale 1984, El-Kassaby and Ritland 1996a,b, Krutovsky et al. 2009);

- investigation of the genetic structure in planted stands in Europe (Prat and Arnal 1994, Stauffer and Adams 1993, Konnert and Ruetz 2006);

- study of the mating system in Douglas-fir stands (Neale and Adams 1985) and seed orchards (e.g. Shaw and Allard 1982) including determination of effective pollen dispersal distance (e.g. ElKassaby and Davidson 1991, Prat 1994) and pollen contamination rates (Adams et al. 1997);

- delineation of Douglas-fir breeding zones (Westfall and Conkle 1992, Merkle and Adams 1987);

- differentiation of Douglas-fir varieties (Yeh and O’Malley 1980, Klumpp 1999, Leinemann 1996, Leinemann and Maurer 1999, Konnert and Ruetz 2006, Konnert and Fussi 2012, Fussi et al. 2013);

- genetic differences between resistant and susceptible forms of interior Douglas-firs to western spruce budworm (Chen et al. 2001);

- the influence of forest management on the genetic structure of stands (Adams 1998, Neale 1984);

- tracing back the origin of artificial stands in Europe (Klumpp 1999, Konnert and Ruetz 2006, Prat and Burczyk 1998, Fontes et al. 2003).

\section{Material for protein extraction}

Proteins were extracted from the following tissue types:

- seed tissue (megagametophytes, embryo, radicles) (e.g. Adams et al. 1990, El-Kassaby et al. 1981, 1982, El-Kassaby and Davidson 1990, 1991, Fussi et al. 2013, Klumpp 1999, Konnert and Ruetz 2006, Krutovskii et al. 2009, Li and Adams 1989, Merkle and Adams 1987, 1988, Prat and Arnal 1994, Stauffer and Adams 1993, Yeh and O’Malley 1980);

Table 1: List of enzymes, scored loci and number of alleles for genetic studies in Douglas-fir (in the table only references are included where the respective enzymes were described for the first time and/or information on interpretation of zymogrames is given)

\begin{tabular}{|l|c|c|c|c|}
\hline \multicolumn{1}{|c|}{ Enzyme system } & E.C. Number & Scored loci & $\begin{array}{c}\text { No. of } \\
\text { alleles }\end{array}$ & Reference \\
\hline Aconitase & 4.2 .1 .3 & ACO-A,-B & 3,5 & $1,3,4,5,6$ \\
\hline $\begin{array}{l}\text { Aspartate aminotransferase } \\
\text { or Glutamate oxaloacetate transaminase }\end{array}$ & 2.6 .1 .1 & AAT(GOT)-A,-B,-C & $3,6,6$ & $1,2,3,4,5,6$ \\
\hline Acid phosphatase & 3.1 .3 .2$. & APH-B & 4 & 6 \\
\hline Alcohol dehydrogenase & 1.1 .1 .1 & ADH-A & 3 & 4 \\
\hline Aldolase & 4.1 .2 .13 & ALD-A & 3 & 4 \\
\hline Catalase & 1.11 .1 .6 & CAT-A & 6 & 1,5 \\
\hline Diaphorase & 1.6 .2 .2 & DIA-A,-B & 2,5 & $1,3,4,5,6$ \\
\hline Esterase & 3.1 .1 .1 & EST-A & 4 & 4,6 \\
\hline Fluorescent esterase & 3.1 .1 .2 & FEST-A,-B & 4,3 & $1,3,5$ \\
\hline Glucose-6-phosphat dehydrogenase & 1.1 .1 .49 & G6PDH-A & 7 & $1,3,4,5,6$ \\
\hline Glutamate dehydrogenase & 1.4 .1 .2 & GDH-A & 4 & $1,3,4,5$ \\
\hline Glycerate dehydrogenase & 1.1 .1 .29 & GLYD-A & 4 & 1,5 \\
\hline Isocitrate dehydrogenase & 1.1 .1 .42 & IDH-A & 9 & $1,2,3,4,5,6$ \\
\hline Leucine aminopeptidase & 3.4 .11 .1 & LAP-A,-B & 7,6 & $1,2,3,5$ \\
\hline Malate dehydrogenase & 1.1 .1 .37 & MDH-A,-B,-C,-D & $4,4,6,5$. & $1,3,4,5,6$ \\
\hline Malic enzyme & 1.1 .1 .40 & ME-A,-B & 2,3 & 4,6 \\
\hline Peptidase & 3.4 .13 .1 & PEP-B,-C & 3,1 & 1,4 \\
\hline Phosphoglucomutase & 2.7 .5 .1 & PGM-A,-B & 6,7 & $1,3,4,5,6$ \\
\hline Phosphoglucose isomerase & 5.3 .1 .9 & PGI-A,-B & 3,5 & $1,3,4,5,6$ \\
\hline Phosphomanose isomerase & 5.3 .1 .8 & PMI & 3 & 5 \\
\hline Shikimate dehydrogenase & 1.1 .1 .25 & SKDH-A & 5 & $1,2,3$ \\
\hline Superoxide dismutase & 1.15 .1 .1 & SOD-A & 6 & $1,4,5,6$ \\
\hline 6-Phosphogluconate dehydrogenase & 1.1 .1 .44 & 6PGDH-A,-B & $6,4$. & $1,2,3,4,5,6$ \\
\hline
\end{tabular}

1-Adams et al. 1990, 2-Klumpp 1999, 3-Moran and Adams 1989, 4-Yeh and O'Malley 1980, 5-Krutovsky et al. 2009, 6-El-Kassaby et al. 1982 
- buds (e.g. Adams et al. 1990, Fussi et al. 2013, Klumpp 1999, Konnert and Ruetz 2006, Leinemann 1996, Leinemann and Maurer 1999, Moran and Adams 1989);

- needle tissue (Neale et al. 1984);

- bark (inner cambium) (Copes 1978).

\section{Protein extraction and separation protocols}

Details on extraction procedures are given in nearly all references cited below. For electrophoretic procedures and staining recipes, see the following references: Adams and Joly (1980), Adams et al. (1990), Cheliak and Pitel (1984), Conkle et al. (1982), Davis (1981), El-Kassaby et al. (1982), Konnert (2004), Merkle and Adams (1988), Mitton et al. (1979), Neale et al. (1984), Shaw and Prasad (1970), Yeh and O'Malley (1980).

\section{Important results}

- Coastal and interior Douglas-fir can be clearly differentiated based on allozymes. Besides the high genetic distances between populations from the two varieties ( $\mathrm{Li}$ and Adams 1989), the assignment to the coastal or the inland type is possible on the basis of the frequency of specific "marker-alleles", namely 6-PGDH-A3 and A6, PGM-A4 and A6 and LAP-A2 (Yeh and O'Malley 1980, Merkle and Adams 1987, Li and Adams 1989). The allele 6-PGDH-A3 is more common (frequency > $90 \%$ ) in the coastal populations than in the interior ones. On the contrary, the allele 6-PGDH-A6 has larger frequencies $(>60 \%)$ in the interior Douglas-fir populations. The allele PGM-A4 has a frequency of over $80 \%$ in the coastal type. In the interior, type PGM-A4 declines in favour of PGM-A6. These findings can be used to assign artificial European Douglas-fir stands to a specific variety - coastal or interior - by analysing the genetic structure at the loci 6PGDH-A and PGM-A (Stauffer and Adams 1993, Leinemann 1996, Klumpp 1999, Leinemann and Maurer 1999, Konnert and Ruetz 2006, Konnert and Fussi 2012, Fussi et al. 2013, Milenkova et al. 2018), but also to a specific region within a variety (Klumpp 1999, Fussi et al. 2013).

- In its natural range, Douglas-fir has an enormous amount of genetic diversity but more than $95 \%$ of it resides within populations rather than between them (Yeh and O'Malley 1980, Merkle and Adams 1987, $\mathrm{Li}$ and Adams 1989, Moran and Adams 1989, ElKassaby and Ritland 1996a). Artificial populations of Douglas-fir in Europe do not appear strongly different in genetic diversity from the natural ones as long as a sufficient number of trees are involved in the original seed collection (Prat and Arnal 1994, Konnert and Ruetz 2006, Stauffer and Adams 1993 , Prat and Burczyk 1998).

- The two varieties of Douglas-fir, var. glauca and var. menziesii are completely interfertile. The natural regeneration in mixed stands with both Douglas varieties shows a specific genetic composition, which indicates that hybridization between varieties has taken place (Konnert and Fussi 2012).

- As long as the number of parental trees is not too small, forest management has no substantial influence on the genetic structure of the next generation in naturally regenerated stands (Adams et al. 1998, Neale 1985).

- Based on isozyme analysis, the following characteristics of the mating system have been determined: high gene flow (El-Kassaby and Ritland 1986a) and outcrossing rates exceeding 90\% (e.g. El-Kassaby et al. 1981, Shaw and Allard 1982, Neale and Adams 1985, Yeh and Morgan 1987). The effective pollen dispersal distance was estimated to be around $30 \mathrm{~m}$. Within $30 \mathrm{~m}$, mating success is only weakly related to distance (Erickson and Adams 1989). The reproductive phenology affects significantly the mating system and induces a larger part of crossing between individuals of the same phenological (early, intermediate, late flowering) class (El-Kassaby and Ritland 1986b). Crossing between related trees might represent a major part of mating system in natural populations of Douglas-fir. This could also explain the observed fixation index, which is often higher in progenies than in old stands (Prat and Arnal 1994).

- Early selection and breeding of this highly polymorphic species do not reduce genetic variation significantly if the number of clones is not too low (El-Kassaby and Ritland 1996b).

- Allozymes have been succesfully used to estimate pollen contamination in seed orchards (El-Kassaby and Ritland 1986a,b).

- Chen et al. (2001) studied genetic variation of 25 protein loci in interior Douglas-fir trees that were phenotypically resistant or susceptible to defoliation by the western spruce budworm (Choristoneura occidentalis Freeman). They found frequency differences in the most common alleles, as well as differences in the proportion of homozygotes for different allozyme loci (e.g. FEST-1, ACO-1, 6PGDH-1). Chen et al. (2001) suggest that the phenotypic differences in resistance of interior Douglas-fir to Ch. occidentalis defoliation are partly caused by genetic differences among trees. 


\section{Organelle DNA markers (chloroplast (cp)DNA, mitochondrial (mt)DNA)}

\section{Loci and primers used}

CpDNA and mtDNA (RFLP, PCR-RFLP, direct sequencing studies) have been carried out to study phylogeography of Douglas-fir (Gugger et al. 2010, 2011, Wei et al. 2011) to evaluate pollen contamination and natural selfing in a Douglas-fir seed orchard (Stoehr et al. 1998) and to study the gene flow and genetic variability in natural populations of Douglas-fir (Ponoy 1993, Nelson et al. 2003).

Hipkins et al. (1995) analysed a length-mutation hotspot in the chloroplast genome of Douglas-fir, whereas Tsai (1989) used restriction mapping and DNA sequencing to characterize dispersed repetitive DNA in the chloroplast genome of Douglas-fir. Also chloroplast microsatellites (cpSSR), originally designed for Pinaceae by Vendramin et al. (1996) and Provan et al. (1999), have been applied in Douglas-fir genetic studies. Using cpSSRs, Viard et al. (2001) studied the genetic variation in adult natural populations of Douglas-fir from British Columbia in comparison with biparentally inherited markers (isoenzymes, RAPDs), whereas Angelier et al. (2011) investigated the genetic quality of natural regeneration in a mixed stand with Douglas fir, Japanese larch and white fir.

Ponoy et al (1993) digested the cpDNA with the following six restriction enzymes: BamHI, EcoRI, EcoRV, HindIII, SstI, XbaI and used 24 enzyme-probe combinations to evaluate the genetic variation of cpDNA. Primers used for PCR-RFLP and DNA-sequencing are introduced in Table 2 .

\section{Material for DNA-extraction}

DNA was extracted from needle or bud tissue (Tsai 1989, Stoehr et al. 1998, Nelson et al. 2003, Gugger et al. 2010, 2011, Wei et al. 2011), embryos (Stoehr et al. 1998), whole seed (for cpDNA) or megagametophytes (for mtDNA) (Wei et al. 2011, Ponoy 1993).

\section{DNA-extraction and amplification protocols}

Total DNA has been extracted:

- from needles using the NucleoSpin ${ }^{R} 96$ Plant II (Macherey.Nagel) kit (Wei et al. 2011) and the DNeasy Plant Mini Kit (Qiagen) (Guger et al. 2010);

- from seed tissue using DNeasy Plant Mini Kit

Table 2: Primer information of the cpDNA-markers and mtDNA-markers used for PCR- RFLP analysis and DNA sequencing in Douglas-fir

\begin{tabular}{|c|c|c|c|c|c|}
\hline Type & Locus & $\begin{array}{c}\text { Method used/ } \\
\text { restriction enzymes }\end{array}$ & $\begin{array}{c}\text { Primer sequences } \\
5^{c}-3^{c} \\
\end{array}$ & Ref. & $\begin{array}{c}\text { Source of } \\
\text { primer pairs }\end{array}$ \\
\hline \multirow{8}{*}{ cpDNA } & $\begin{array}{l}\operatorname{trnD-GUC} \\
\operatorname{trnS}-\mathrm{GCU}\end{array}$ & PCR_RFLP/EcoRI & $\begin{array}{l}\text { F:TCTAGAAAGGCACTGGCTATCGATC } \\
\text { R:TGATAATTCTAGGCTTTCTAGTTCA }\end{array}$ & 1 & $\begin{array}{l}\text { Hipkins et al. } \\
1995\end{array}$ \\
\hline & $\begin{array}{l}\operatorname{trnD-GUC} \\
\text { trnY-GUA }\end{array}$ & PCR_RFLP/EcoRI & $\begin{array}{l}\text { F:TCTAGAAAGGCACTGGCTATCGATC } \\
\text { R:ATGCCTACGCTGGTTCAAA }\end{array}$ & 1 & $\begin{array}{l}\text { Hipkins et al. } \\
1995\end{array}$ \\
\hline & $\begin{array}{l}\operatorname{trnE-UUC} \\
\operatorname{trnS} \text {-GCU }\end{array}$ & PCR-RFLP/EcoRI & $\begin{array}{l}\text { F:TGCCTCCTTGAAAGAGAGATGTCC } \\
\text { R:TGATAATTCTAGGCTTTCTAGTTCA }\end{array}$ & 1 & $\begin{array}{l}\text { Hipkins et al. } \\
1995\end{array}$ \\
\hline & $\operatorname{trnD}-\operatorname{trn} E$ & PCR-RFLP/TaqI & $\begin{array}{l}\text { F:CTAAATATAAATCTATTGG } \\
\text { R:AAGTATCAATTCATATGG }\end{array}$ & 2 & $\begin{array}{c}\text { Stoehr et al. } \\
1998\end{array}$ \\
\hline & $\begin{array}{l}\text { PM12L- } \\
\text { PM12R }\end{array}$ & $\begin{array}{l}\text { PCR-RFLP/Hind, } \\
\text { BslI, Tsp } 451\end{array}$ & $\begin{array}{l}\text { F:CAGGGCGGTACTCTAACCAA } \\
\text { R:AGATCACGTGCGTGTGAAAA }\end{array}$ & 3 & $\begin{array}{l}\text { Nelson et al. } \\
2003\end{array}$ \\
\hline & rps7-trnL & DNA sequencing & $\begin{array}{l}\text { F:GGTTATTAGGGGCATCTCG } \\
\text { R:CGTGTCTACCATTTCACCATC }\end{array}$ & 4,5 & $\begin{array}{l}\text { Gugger et al. } \\
2010\end{array}$ \\
\hline & rps15-psaC & DNA sequencing & $\begin{array}{l}\text { F:GGTATCCGTGGGCTAAAAAC } \\
\text { R:CAATACATCTGTGGGACAAGC }\end{array}$ & 4,5 & $\begin{array}{l}\text { Gugger et al. } \\
2010\end{array}$ \\
\hline & $\operatorname{trnfM}-\operatorname{trn} S$ & DNA sequencing & $\begin{array}{l}\text { F:CATAACCTTGAGGTCACGGG } \\
\text { R:GAGAGAGAGGGATTCGAACC }\end{array}$ & 6 & $\begin{array}{l}\text { Shaw et al. } \\
2005\end{array}$ \\
\hline \multirow[t]{2}{*}{$\mathrm{mtDNA}$} & $19 \mathrm{~S}$ rDNA V7 & DNA sequencing & $\begin{array}{l}\text { F:GAGCCAAGGAGGCAGATTG } \\
\text { R:ATCCTTGGTCTGATGCTTCG }\end{array}$ & 4,5 & $\begin{array}{l}\text { Gugger et al. } \\
2010 \\
\end{array}$ \\
\hline & nad7-1 & DNA sequencing & $\begin{array}{l}\text { F:ACCTAACAGAACGCACAAGG } \\
\text { R:TTCCAACCAAGAATTGATCC }\end{array}$ & $4,5,6$ & $\begin{array}{l}\text { Gugger et al. } \\
2010\end{array}$ \\
\hline
\end{tabular}

1-Hipkins et al. 1995, 2-Stoehr et al. 1998, 3-Nelson et al. 2003, 4-Gugger et al. 2010, 5-Gugger et al. 2011, 6-Wei et al. 2011 
(Qiagen) (Wei et al. 2011) and the CTAB procedure following Doyle and Doyle (1987) (Stoehr et al. 1998);

- from buds using the protocol of Guillemaut and Maréchal-Drouard (1992), (Nelson et al. 2003), and the CTAB procedure following Doyle and Doyle (1987) (Stoehr et al. 1998).

\section{Examples for amplification protocols (PCR-RFLP)}

- 36 cycles of $20 \mathrm{~s}$ at $94^{\circ} \mathrm{C}, 20 \mathrm{~s}$ at $52^{\circ} \mathrm{C}(\mathrm{cpDNA})$ or $56^{\circ} \mathrm{C}(\mathrm{mtDNA})$ and $1 \mathrm{~min} 30 \mathrm{~s}$ at $72^{\circ} \mathrm{C}$ followed by a final extension step of $6 \mathrm{~min}$ at $72^{\circ} \mathrm{C}$ (Wei et al. 2011).

- $94^{\circ} \mathrm{C}$ for 2 min followed by 35 cycles each at $94^{\circ} \mathrm{C}$ for $1 \mathrm{~min}, 55^{\circ} \mathrm{C}$ for $1 \mathrm{~min}, 70^{\circ} \mathrm{C}$ for $2 \mathrm{~min}$ and a final extension step at $72^{\circ} \mathrm{C}$ for 15 min (Gugger et al. 2011).

- $95^{\circ} \mathrm{C}$ for $2 \mathrm{~min}$, followed by 30 cycles of $94^{\circ} \mathrm{C}$ for $30 \mathrm{~s}, 48^{\circ} \mathrm{C}$ for $30 \mathrm{~s}, 72^{\circ} \mathrm{C}$ for $1 \mathrm{~min}$, and a final extension step at $72^{\circ} \mathrm{C}$ for $5 \mathrm{~min}$ (Stoehr et al. 1998).

\section{Important results}

- By analysing range-wide genetic variation of maternally inherited mtDNA and paternally inherited cpDNA, detailed insights into the evolutionary history of Douglas-fir (ice-age refugia, colonization routes, introgression zones, migration rates) have been obtained (Gugger et al. 2010, 2011, Wei et al. 2011). For example, Gugger et al. $(2010,2011)$ found that the Rocky Mountain (interior variety) Douglas-fir survived in three or even more glacial refugia. In addition, the postglacial colonization of Canada from refugia of both varieties resulted in a wide inter-varietal hybrid zone, formed mainly by pollen exchange and chloroplast DNA introgression.

- Wei et al. (2013) identified 2 mitotypes and 42 chlorotypes. Based on cpDNA, three separated lineages were discovered, which correspond to the Pacific Coast, the Rocky Mountain and Mexican populations. The Mexican lineage is characterized by low genetic diversity and high genetic differentiation. The authors proposed that the Mexican populations originated by southward migration from a refugium of the Rocky Mountain lineage.

- Nelson et al. (2003) suggested that the gene flow from pollen dispersal between coastal and interior Douglas-fir is restricted as no identical cpDNA haplotypes were found in studied trees of both varieties. Out of 31 distinct haplotypes identified in a total of 58 trees, 15 haplotypes were observed in coastal trees while 16 haplotypes were detected in trees from the interior of British Columbia. Gene diversity was in general high, however slightly higher in the coastal sample (0.95) than in the interior one (0.88).

- Similar results were reported by Ponoy (1993) for six British-Columbian Douglas-fir populations. Using six restriction enzymes (BamHI, EcoRI, EcoRV,

Table 3: Primer information of the chloroplast microsatellite markers used in genetic analysis of Douglas-fir $\left(T_{a}=\right.$ annealing temperature)

\begin{tabular}{|c|c|c|c|c|c|c|}
\hline Locus & Motif & $\begin{array}{c}\text { Primer sequences } \\
5^{c}-3^{c}\end{array}$ & Size (bp) & $\begin{array}{c}\mathbf{T}_{\mathbf{a}} \\
\left({ }^{\circ} \mathrm{C}\right)\end{array}$ & Ref. & $\begin{array}{c}\text { Source of primer } \\
\text { pairs }\end{array}$ \\
\hline Pt26081 & $(\mathrm{T})_{14}$ & $\begin{array}{l}\text { F:CCCGTATCCAGATATACTTCCA } \\
\text { R:TGGTTTGATTCATTCGTTCAT }\end{array}$ & $\begin{array}{c}125-126^{1)} \\
99-105^{2)} \\
102-110^{3)} \\
\end{array}$ & 60 & $1,2,3,4$ & $\begin{array}{c}\text { Vendramin et al. } \\
1996\end{array}$ \\
\hline Pt30204 & $(\mathrm{A})_{12}(\mathrm{G})_{10}$ & \begin{tabular}{|l} 
F:TCATAGCGGAAGATCCTCTTT \\
R:CGGATTGATCCTAACCATACC \\
\end{tabular} & $142-145$ & 50 & 3 & $\begin{array}{c}\text { Vendramin et al. } \\
1996\end{array}$ \\
\hline Pt36480 & $(\mathrm{T})_{11}$ & $\begin{array}{l}\text { F:TTTTGGCTTACAAAATAAAAGAGG } \\
\text { R:AAATTCCTAAAGAAGGAAGAGCA }\end{array}$ & $142-147$ & 50 & 3 & $\begin{array}{c}\text { Vendramin et al. } \\
1996\end{array}$ \\
\hline Pt63718 & $(\mathrm{T})_{10}$ & $\begin{array}{l}\text { F:CACAAAAGGATTTTTTTTCAGTG } \\
\text { R:CGACGTGAGTAAGAATGGTTG } \\
\end{array}$ & $\begin{array}{c}110-111^{1)} \\
90-93^{2)} \\
\end{array}$ & 60 & $1,2,4$ & $\begin{array}{c}\text { Vendramin et al. } \\
1996\end{array}$ \\
\hline Pt71936 & $(\mathrm{T})_{16}$ & $\begin{array}{l}\text { F:TTCATTGGAAATACACTAGCCC } \\
\text { R:AAAACCGTACATGAGATTCCC }\end{array}$ & $\begin{array}{l}166-167^{1)} \\
148-151^{2)} \\
143-148^{3)}\end{array}$ & 60 & $1,2,3,4$ & $\begin{array}{c}\text { Vendramin et al. } \\
1996\end{array}$ \\
\hline Pt87314 & $(\mathrm{T})_{16}$ & \begin{tabular}{|l} 
F:TATATCCCCCGTACTTGGACC \\
R:TCCAGGATAGCCCAGCTG \\
\end{tabular} & $98-99$ & 50 & 3 & Provan et al. 1999 \\
\hline Pt110048 & $(\mathrm{T})_{10}$ & $\begin{array}{l}\text { F:TAAGGGGACTAGAGCAGGCTA } \\
\text { R:TTCGATATTGAACCTTGGACA }\end{array}$ & 86 & 60 & 1,4 & $\begin{array}{c}\text { Vendramin et al. } \\
1996\end{array}$ \\
\hline
\end{tabular}

1-Viard et al. 2001, 2-Gugger et al. 2011, 3-Valadon et al. 2011, 4-Angelier et al. 2011 
HindIII, SstI, Xbal) in four cpDNA fragments altogether, 16 haplotypes were detected. 5 haplotypes were found in coastal, 7 in interior Douglas-fir populations. Populations from the transition zone, with 11 haplotypes, show the highest polymorphism rate.

- Angelier et al. (2011) and Viard et al. (2001) found that the universal cpDNA microsatellites Pt26081, Pt36480, Pt71936, Pt63718 show clear patterns, low polymorphism within Douglas-fir populations and no differentiation among populations. Primer Pt36480 was monomorphic for Douglas-fir.

- In a mixed stand with Douglas-fir, Japanese larch and white fir in France, pollen flow from outside the stand was low. The stand was isolated from other Douglas-fir stands. Genetic differentiation of succesfull pollen clouds between Douglas-fir mother trees was significantly lower than for Japanese larch and white fir (Angelier 2011). Genetic differentiation between age classes (adults, natural regeneration) of this stand was low.

- cpDNA markers proved to be very useful in assessing seed orchard mating dynamics and orchard management efficacies for Douglas-fir (Stoehr et al. 1998). In a clonal seed orchard from British Columbia they found the pollen contamination to be of $40 \%$. Natural selfing in six individual clones ranged from 0 to $19 \%$ with an average of $6 \%$. Supplemental mass pollination efficacy was estimated to be $55 \%$, ranging from 39 to $73 \%$, depending on the maternal clone and flowering phenology.

- Hipkins et al. (1995) identified the source of variation in cpDNA within the genus Pseudotsuga as a partially duplicated and an intact trnY-GUA gene. The sequenced Douglas-fir individuals differed from each other only in a single tandem repeat unit, whereas from the Japanese Douglas-fir (Pseudotsuga japonica) they differed in approx. 34 repeat units. RFPLs revealed a $1 \mathrm{~kb}$ variation in length among different Pseudotsuga sp. and $200 \mathrm{bp}$ among Pseudotsuga menziesii individuals from different geographical regions.

\section{Randomly amplified polymorphic DNA markers (RAPD)}

RAPD markers were used to study the inter-varietal and intra-varietal differentiation in natural populations of Douglas-fir (Aagard et al. 1995, 1997, 1998a,b) and for genetic linkage mapping studies (Carlsson et al. 1991, Krutovskii et al. 1998).

\section{Loci and primers used}

Loci and primers used are listed in Table 4. For further markers, see Jermstad et al. (1994), where a list of 80 commercially prepared primers (Operon Technologies) for amplification of RAPD loci are listed. Among them, 29 display Mendelian inheritance. Krutovskii et al. (1998) used 96 10-bp random amplified polymorphic RADP primers. These have been also commercially prepared (Operon Technologies Inc. and the Biotechnology Laboratory of the University of British Columbia).

\section{Material for DNA-extraction}

Total genomic DNA has been extracted from megagametophytes (Krutovskii et al. 1998), embryos (Aagaard et al. 1995, 1997, 1998a,b), needles and buds (Carlson et al. 1991).

\section{DNA-extraction and amplification protocols}

DNA has been extracted with a modified CTAB protocol of Wagner et al. (1987) in which the DNA was further purified by four phenol:chloroform:isoamyl alcohol (25:24:1) extractions and a final ethanol precipitation (Aagard et al. 1995, Carlsson et al. 1991, Tsumura et al. 1996, Krutovskii et al. 1998). A special protocol for extraction of mitochondrial DNA is given in Aagard (1998b). For extraction of DNA from buds, a protocol can be found in Carlsson et al. (1991).

\section{Examples for amplification protocols (RAPD)}

- 7 min denaturation at $94^{\circ} \mathrm{C}$ followed by a total of 45 cycles of 1 min denaturing at $94^{\circ} \mathrm{C}, 1 \mathrm{~min}$ annealing at $35^{\circ} \mathrm{C}$ and 2 min extension at $72^{\circ} \mathrm{C}$, with a final extension step of $72^{\circ} \mathrm{C}$ for $10 \mathrm{~min}$ (Carlson et al. 1991).

- 45 cycles of $93^{\circ} \mathrm{C}$ for $1 \mathrm{~min}, 37^{\circ} \mathrm{C}$ for $1 \mathrm{~min}$, and $72^{\circ} \mathrm{C}$ for 2 min (Aagard et al. 1995, 1997).

\section{Important results}

- From 41 fragments amplified by RAPD markers, 29 showed variability between and within the coastal, north and south interior varieties. 10 bands proved to be variety specific (Aagard et al. 1995).

- RAPD-based linkage maps for Douglas-fir have been constructed by Krutovskii et al. (1998).

- Aagard et al. (1998b) developed a method of screening RAPD markers for the presence of organelle DNA products using enriched organelle DNA. Based on this method, Aagard et al. (1998b) 
Table 4: Primers employed and RAPD markers obtained from DNA amplification in Douglas-fir $\left(\mathrm{T}_{\mathrm{a}}=\right.$ annealing temperature $)$

\begin{tabular}{|c|c|c|c|c|}
\hline $\begin{array}{c}\text { Primer } \\
\text { name }\end{array}$ & $\begin{array}{c}\text { Sequence } \\
5^{\prime}-3^{\prime}\end{array}$ & $\begin{array}{c}\mathbf{T}_{\mathbf{a}} \\
\left({ }^{\circ} \mathbf{C}\right)\end{array}$ & $\begin{array}{l}\text { Total no. of } \\
\text { RAPD bands }\end{array}$ & Ref. \\
\hline UBC 111 & AGTAGACGGG & 37 & 6 & 2 \\
\hline UBC 114 & TGACCGAGAC & 37 & 6 & 2 \\
\hline UBC 197 & TCCCCGTFCC & 37 & 14 & 2 \\
\hline UBC 234 & TCCACGGACG & 37 & 9 & 2,3 \\
\hline UBC 264 & TCCACCGAGC & 37 & 6 & 2 \\
\hline UBC $266^{*}$ & CCACTCACCG & 37 & n.i. & 1 \\
\hline UBC $268^{*}$ & AGGCCGCTTA & 37 & n.i. & 1 \\
\hline UBC $275^{*}$ & CCGGGCAAGC & 37 & n.i. & 1 \\
\hline UBC 285 & GGGCGCCTAG & 37 & 6 & 2 \\
\hline UBC 300 & GGCTAGGGCG & 37 & n.i. & 1 \\
\hline UBC 304 & AGTCCTCGCC & 37 & 9 & 2 \\
\hline UBC 323 & GACATCTCGC & 37 & 13 & 2 \\
\hline UBC 327 & ATACGGCGTC & 37 & 5 & 2 \\
\hline UBC 328 & ATGGCCTTAC & 37 & 5 & 2,3 \\
\hline UBC 330 & GGTGGTTTCC & 37 & 5 & 2,3 \\
\hline UBC 336 & GCCACGGAGA & 37 & 11 & 2,3 \\
\hline UBC 337 & TCCCGAACCG & 37 & 10 & 2 \\
\hline UBC 341 & CTGGGGCCGT & 37 & 15 & 2 \\
\hline UBC 372 & CCCACTGACG & 37 & 13 & 2 \\
\hline UBC 387 & CGCTGTCGCC & 37 & 12 & 2 \\
\hline UBC 409* & TAGGCGGCGG & 37 & n.i. & 1 \\
\hline UBC 411 & GAGGCCCGTT & 37 & 10 & 2,3 \\
\hline UBC 414 & AAGGCACCAG & 37 & n.i. & 1 \\
\hline UBC $419^{*}$ & TACGTGCCCG & 37 & n.i. & 1 \\
\hline UBC $428^{*}$ & GGCTGCGGTA & 37 & 8 & $1,2,3$ \\
\hline UBC $438^{*}$ & AGACGGCCGG & 37 & n.i. & 1 \\
\hline UBC 446 & GCCAGCGTTC & 37 & n.i. & 1 \\
\hline UBC 460 & ACTGACCGGC & 37 & 14 & 2,3 \\
\hline UBC 467 & AGCACGGGCA & 37 & 12 & 2 \\
\hline UBC 497 & GCATAGTGCG & 37 & 10 & 2 \\
\hline UBC 504 & ACCGTGCGTC & 37 & 13 & 2,3 \\
\hline UBC 530 & AATAACCGCC & 37 & 3 & 2 \\
\hline UBC 570 & GGCCGCTAAT & 37 & 9 & 2,3 \\
\hline OP-J1 & CCCGGCATAA & 37 & 13 & 2 \\
\hline FCPI & GCTTACCACC & 35 & n.i. & 4 \\
\hline FCP3 & CCATTCACCG & 35 & n.i. & 4 \\
\hline FCPA3: & TATCGCACGCTA & 35 & n.i. & 4 \\
\hline PRCI: & CGCATCCGA & 35 & n.i. & 4 \\
\hline
\end{tabular}

1-Aagaard et al. 1995, 2-Aagaard 1997, 3-Aagaard et al. 1998a,b, 4-Carlson et al. 1991,source of primers Williams et al. 1990; * - maternal inheritance, mitochondrial origin, n.i. - not indicated 
found that RAPD markers of mitochondrial origin in Douglas-fir show lower genetic diversity than RAPD markers of nuclear origin, but differentiation is much higher for mitochondrial RAPD at both the population and varietal levels.

- A similar level of genetic diversity and differentiation was estimated for Douglas fir populations using RAPD markers of nuclear origin (36 loci) and isozymes (20 loci) by Aagard et al. (1998a).

\section{Nuclear DNA markers (ISSR, nSSR, SNPs)}

\section{a) ISSRs (inter-simple sequence repeat polymorphisms)}

Inter-simple sequence repeat (ISSR) technique allows studying polymorphisms located between microsatellite sequences. The microsatellite sequences serve as primers in a PCR to generate multilocus markers (Zietkiewicz et al. 1994).

\section{Loci and primers used}

Tsumura et al. (1996) examined 96 ISSR primers, from which 22 gave clear banding patterns for Douglas-fir. Out of these, 19 primers showed polymorphism for coastal Douglas-fir and 16 primers for interior Douglasfir (Table 5).

\section{Material for DNA-extraction}

DNA was extracted from megagametophytes (Tsumura et al. 1996).

\section{DNA-extraction and amplification protocol}

DNA was extracted with a modified CTAB protocol of Wagner et al. (1987) in which the DNA was further purified by four phenol:chloroform:isoamyl alcohol (25:24:1) extractions and a final ethanol precipitation (Tsumura et al. 1996).

Table 5: List of 22 ISSR markers and primer sequences ( $\mathrm{T}$-annealing temperature, $\mathrm{N}_{\mathrm{A}}$ number of alleles, R-purine, Y-pyrimidine) after Tsumura et al. (1996).

\begin{tabular}{|c|c|c|c|c|c|}
\hline \multirow{2}{*}{ Primers } & \multirow{2}{*}{$\begin{array}{c}\text { Sequence } \\
\left(5^{\prime}-3^{\prime}\right)\end{array}$} & \multicolumn{2}{|c|}{ Size (bp) } & \multirow{2}{*}{$\begin{array}{c}T_{\mathbf{a}} \\
\left({ }^{\circ} \mathbf{C}\right)\end{array}$} & \multirow{2}{*}{$\begin{array}{l}\text { No of loci } \\
\text { within } \\
\text { varieties* }\end{array}$} \\
\hline & & Coastal variety & Interior variety & & \\
\hline 857 & $(\mathrm{AC})_{8} \mathrm{YG}$ & $440 / 480 / 500 / 680$ & 870 & 52 & \\
\hline 855 & $(\mathrm{AC})_{8} \mathrm{YT}$ & $290 / 650$ & 880 & 52 & \\
\hline 856 & $(\mathrm{AC})_{8} \mathrm{YA}$ & No amplification & $340 / 700$ & 52 & \\
\hline 808 & $(\mathrm{AG})_{8} \mathrm{C}$ & 600 & 1000 & 52 & \multirow{6}{*}{$\begin{array}{l}7 \\
4\end{array}$} \\
\hline 809 & $(\mathrm{AG})_{8} \mathrm{G}$ & $480 / 580$ & No amplification & 52 & \\
\hline 807 & $(\mathrm{AG})_{8} \mathrm{~T}$ & $650 / 1050$ & 460 & 52 & \\
\hline 836 & $(\mathrm{AG})_{8} \mathrm{YA}$ & 400 & No amplification & 52 & \\
\hline 834 & $(\mathrm{AG})_{8} \mathrm{YT}$ & 260 & 560 & 52 & \\
\hline 835 & $(\mathrm{AG})_{8} \mathrm{YC}$ & No amplification & 230 & 52 & \\
\hline 817 & $(\mathrm{CA})_{8} \mathrm{~A}$ & $650 / 850$ & 450 & 52 & \multirow{2}{*}{$\begin{array}{l}3 \\
2\end{array}$} \\
\hline 846 & $(\mathrm{CA})_{8} \mathrm{RT}$ & 600 & 340 & 52 & \\
\hline 844 & $(\mathrm{CT})_{8} \mathrm{RC}$ & $480 / 850$ & $480 / 850$ & 52 & \multirow{2}{*}{$\begin{array}{l}3 \\
2 \\
\end{array}$} \\
\hline 845 & $(\mathrm{CT})_{8} \mathrm{RG}$ & 500 & No amplification & 52 & \\
\hline 812 & $(\mathrm{GA})_{8} \mathrm{~A}$ & 520 & No amplification & 52 & \multirow{6}{*}{$\begin{array}{c}10 \\
4\end{array}$} \\
\hline 811 & $(\mathrm{GA})_{8} \mathrm{C}$ & 280 & 680 & 52 & \\
\hline 810 & $(\mathrm{GA})_{8} \mathrm{~T}$ & $520 / 850$ & No amplification & 52 & \\
\hline 841 & $(\mathrm{GA})_{8} \mathrm{YC}$ & 520 & No amplification & 52 & \\
\hline 842 & $(\mathrm{GA})_{8} \mathrm{YG}$ & $400 / 600 / 700$ & 220 & 52 & \\
\hline 840 & $(\mathrm{GA})_{8} \mathrm{YT}$ & $440 / 650$ & $440 / 800$ & 52 & \\
\hline 850 & $(\mathrm{GT})_{8} \mathrm{YC}$ & $270 / 440 / 520$ & 310 & 52 & \multirow{3}{*}{$\begin{array}{l}6 \\
3\end{array}$} \\
\hline 851 & $(\mathrm{GT})_{8} \mathrm{YG}$ & $130 / 260 / 290$ & 260 & 52 & \\
\hline 849 & $(\mathrm{GT})_{8} \mathrm{YA}$ & No amplification & 800 & 52 & \\
\hline
\end{tabular}


For amplification, the following PCR-protocol was used:

- $\quad 95^{\circ} \mathrm{C}$ for $7 \mathrm{~min}$ followed by 45 cycles of $94^{\circ} \mathrm{C}$ for $30 \mathrm{~s}, 52^{\circ} \mathrm{C}$ for $45 \mathrm{~s}, 72^{\circ} \mathrm{C}$ for $2 \mathrm{~min}$, final $7 \mathrm{~min}$ extension at $72^{\circ} \mathrm{C}$.

PCR products were analyzed on $2 \%$ agarose gels in $1 \mathrm{x}$ TBE buffer, then stained with ethidium bromide, and scored for band presence or absence.

\section{Important results (all results from Tsumura et al. 1996)}

- Coastal variety and interior variety show differences in genetic variability at ISSR loci. The average number of loci per primer was 1.8 for coastal Douglas-fir and 1.12 for interior Douglas-fir, whereas the proportion of polymorphic primers was $92 \%$ to $87 \%$, respectively. The coastal variety shows higher heterozygosity than the interior one, with 35 heterozygote loci from 19 primers when compared to 19 heterozygote loci from 16 primers.

\section{b) nSSRs (putatively neutral microsatellites)}

\section{Loci and primers used}

Amarasinghe and Carlson (2002) developed 50 nuclear microsatellite (nSSR) markers for Douglas-fir, among which 48 loci showed polymorphism with a mean of 7.5 alleles per locus. Mendelian inheritance and genetic variability was confirmed by analyzing 24 unrelated Douglas-fir trees. In addition, 22 nSSR markers were developed by Slavov et al. (2004). From this set, 15 proved to be a valuable tool in genetic identification and parentage analysis (see Table 6). These markers were used in different studies on genetic variability of Douglas-fir populations (e.g. Krutovsky et al. 2009, Fussi et al. 2013), to trace back the origin of European Douglas-fir stands (Fussi et al. 2013, Eckhart et al. 2017, Hintsteiner et al. 2018) and to analyse the mating system in Douglas-fir seed orchards (Slavov et al. 2005, Sk Lai et al. 2010, Kess and El-Kassaby 2015, Korecký and El-Kassaby 2016).

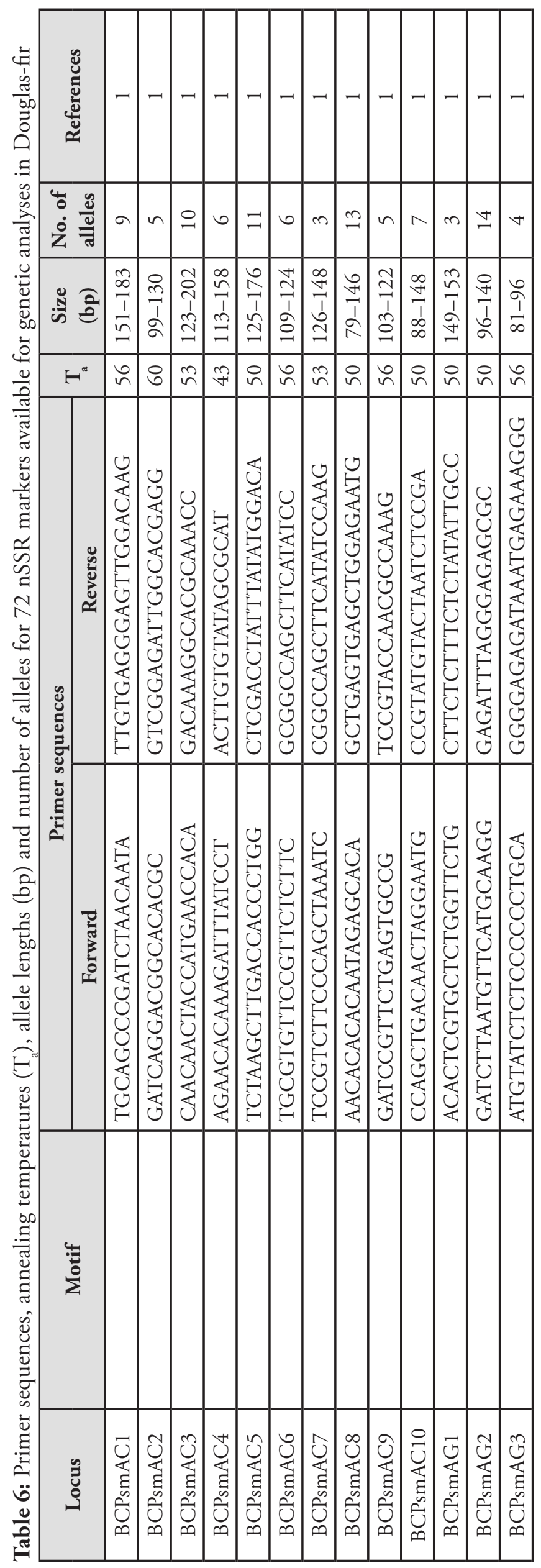




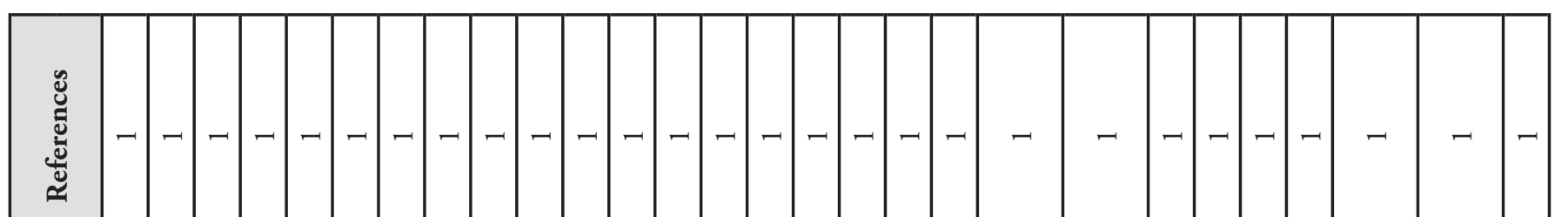

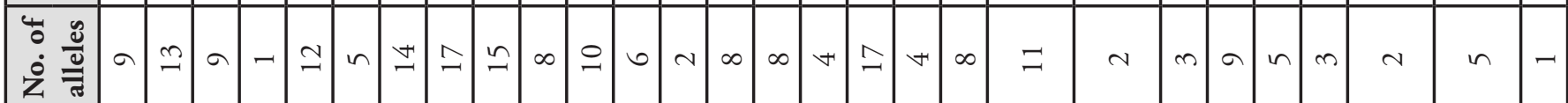

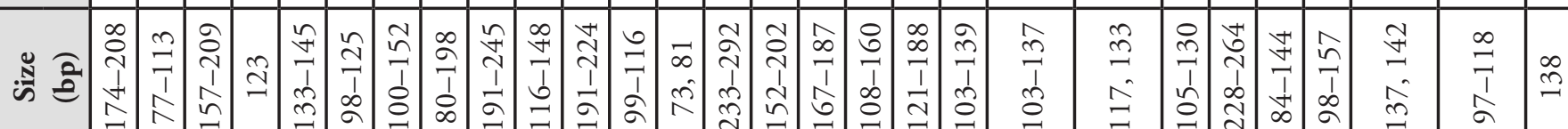
$\because$ *

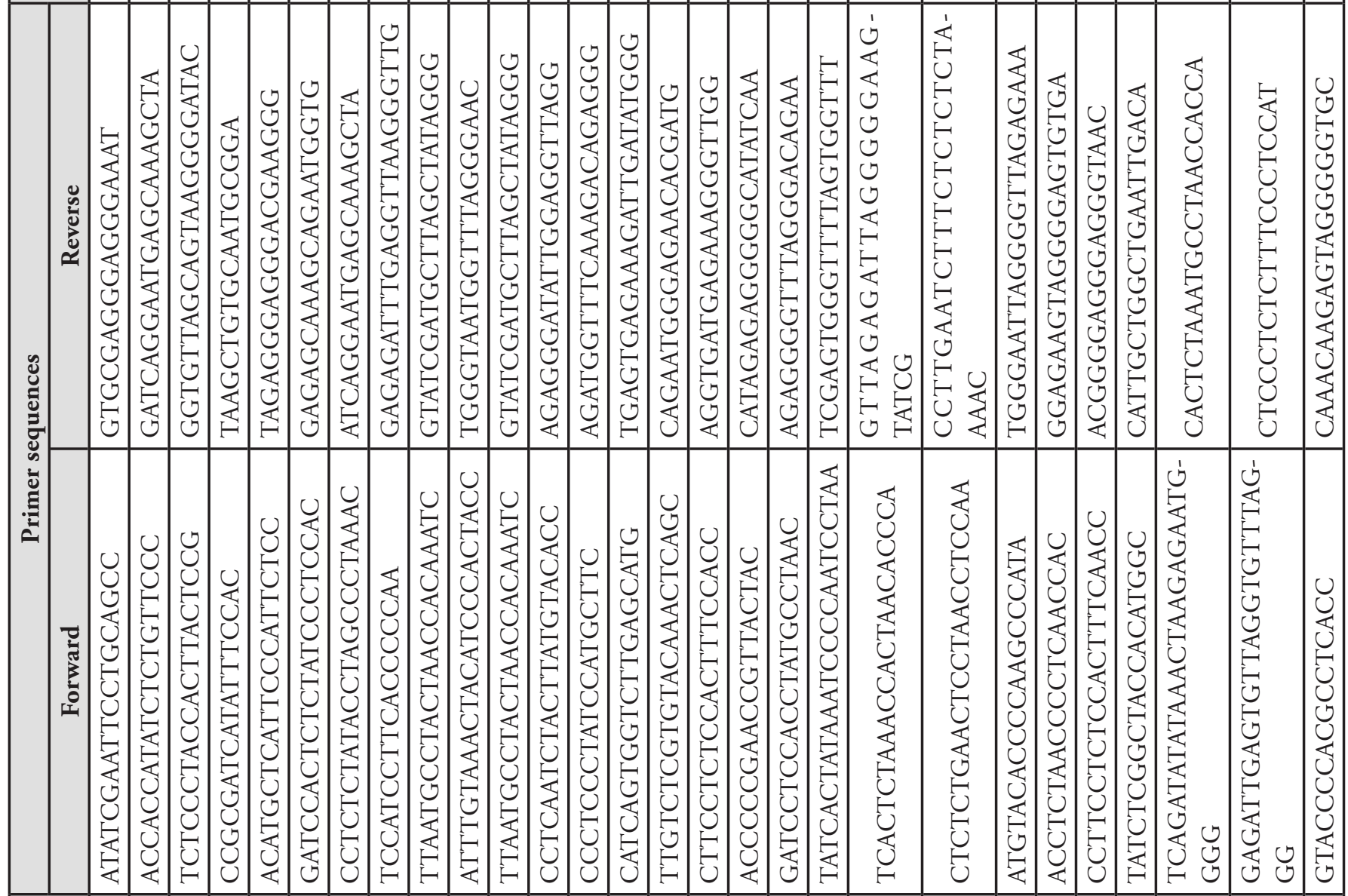

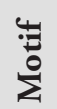

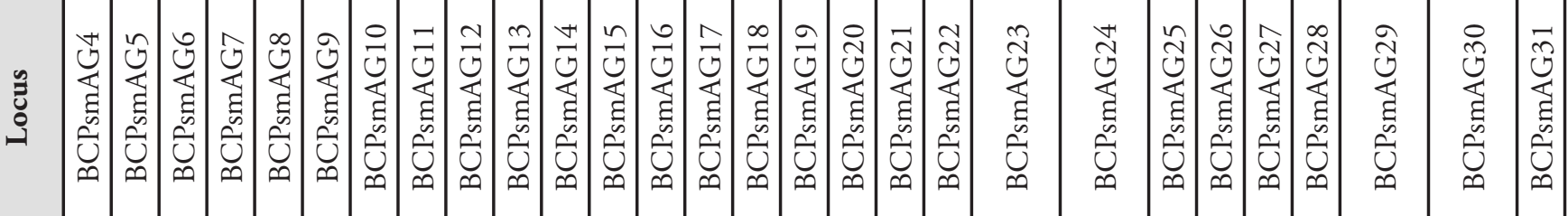




\begin{tabular}{|c|c|c|c|c|c|c|c|c|c|c|c|c|c|c|c|c|c|c|c|c|c|c|c|c|}
\hline 芯 & - & -1 & -1 & - & -1 & -1 & - & -1 & $\neg$ & 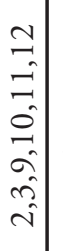 & 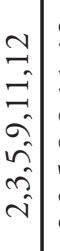 & 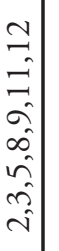 & $\stackrel{m}{i}$ & 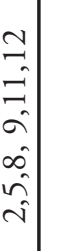 & 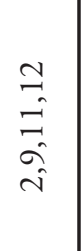 & 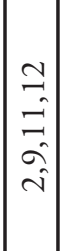 & 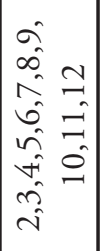 & 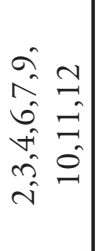 & 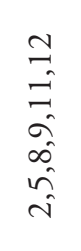 & $\left|\begin{array}{l}\simeq \\
= \\
= \\
0 \\
0 \\
0 \\
0 \\
0\end{array}\right|$ & 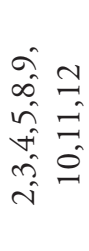 & $\begin{array}{l}0 \\
0 \\
0 \\
\hat{0} \\
0 \\
\hat{n} \\
\hat{n} \\
\hat{n} \\
\hat{v}\end{array}$ & 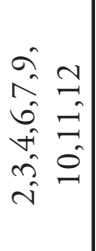 & $\begin{array}{l}\infty \\
2 \\
2 \\
c\end{array}$ \\
\hline $\begin{array}{l}\dot{\overrightarrow{0}} \\
\dot{0} \\
\dot{z}\end{array}$ & $\forall$ & 6 & $\sim$ & $\sim$ & $n$ & $\approx 1$ & $\exists$ & ते & $\infty$ & $\stackrel{\infty}{\sim}$ & $\stackrel{m}{m}$ & $\simeq$ & $\approx$ & m) & లి & $\infty$ & $\stackrel{\bullet}{\simeq}$ & $\widehat{\lambda}$ & $\approx$ & $\beth$ & নి & $\pi$ & m & 0 \\
\hline के है & $\begin{array}{l}0 \\
\cdots \\
1 \\
\infty \\
\infty\end{array}$ & 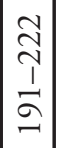 & 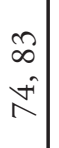 & $\begin{array}{l}\infty \\
\hat{n} \\
\hat{n}\end{array}$ & 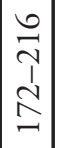 & $\begin{array}{l}\wedge \\
\sim \\
\nu \\
\sim \\
\infty \\
-1\end{array}$ & $\begin{array}{l}\tilde{D} \\
1 \\
\vdots \\
0\end{array}$ & 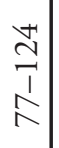 & 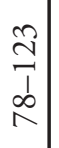 & 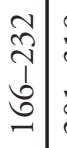 & $\begin{array}{l}\stackrel{a}{\vec{\tau}} \\
\vec{\sim}\end{array}$ & 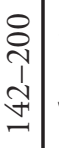 & 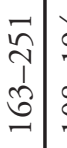 & 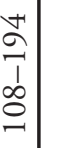 & 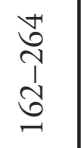 & 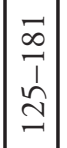 & 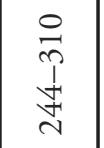 & $\begin{array}{l}\stackrel{0}{1} \\
1 \\
\infty \\
\infty\end{array}$ & $\begin{array}{l}\stackrel{\sim}{\mathbb{N}} \\
\stackrel{1}{1} \\
\stackrel{\Xi}{=}\end{array}$ & 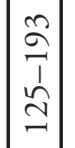 & $\begin{array}{l}\stackrel{0}{+} \\
\stackrel{+}{t} \\
\stackrel{+}{t}\end{array}$ & 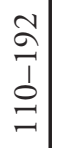 & $\begin{array}{l}\stackrel{+}{+} \\
\stackrel{1}{1} \\
\stackrel{2}{2}\end{array}$ & \begin{tabular}{|l|}
$\infty$ \\
0 \\
1 \\
$\infty$ \\
0 \\
$n$
\end{tabular} \\
\hline$F^{\infty}$ & 이 & 이 & $\stackrel{\circ}{n}$ & in & 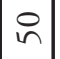 & ㄴ & $\stackrel{\circ}{n}$ & $\stackrel{\circ}{n}$ & $\stackrel{n}{n}$ & in: & $n$ & ৪) & ins & in & $\stackrel{\Downarrow}{n}$ & $\hat{n}$ & $\vec{n}$ & જे & Fे & in & in & $\vec{n}$ & $\stackrel{\infty}{+}$ & $\stackrel{\infty}{\stackrel{\infty}{+}}$ \\
\hline $\begin{array}{l}\bar{y} \\
\bar{z} \\
\bar{\Xi} \\
\bar{z}\end{array}$ & 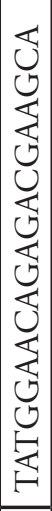 & \begin{tabular}{|l|}
$\mid$ \\
0 \\
0 \\
0 \\
0 \\
0 \\
0 \\
0 \\
0 \\
0 \\
0 \\
0 \\
0 \\
0 \\
0 \\
0 \\
0 \\
\end{tabular} & 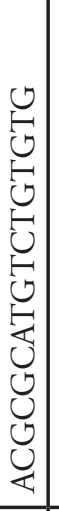 & 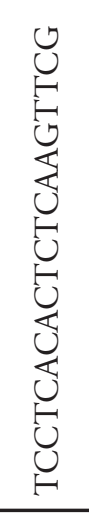 & 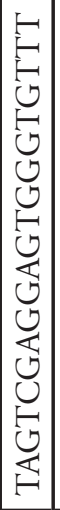 & 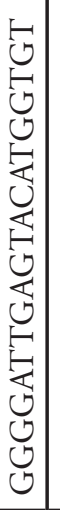 & 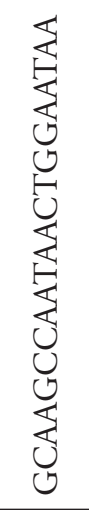 & 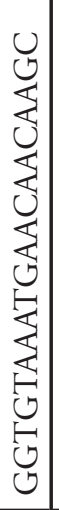 & 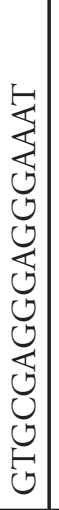 & 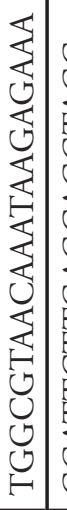 & 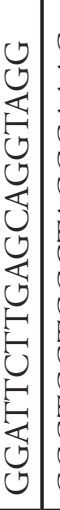 & 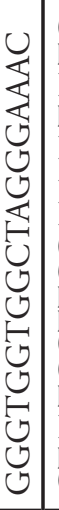 & 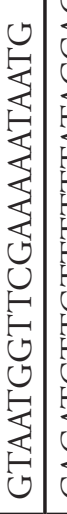 & 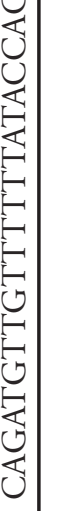 & 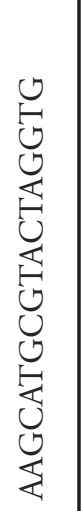 & 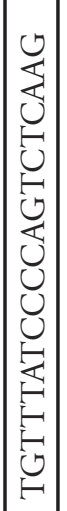 & 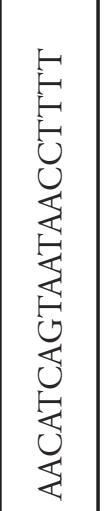 & 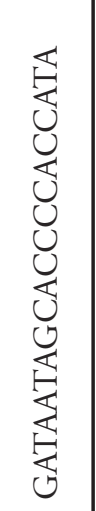 & 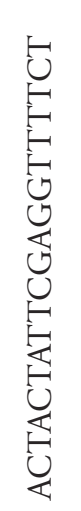 & 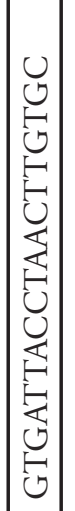 & 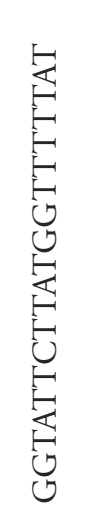 & 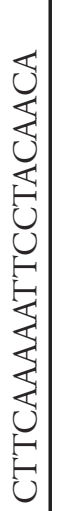 & 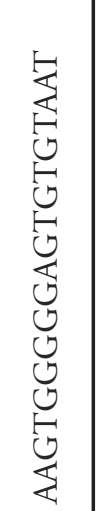 & 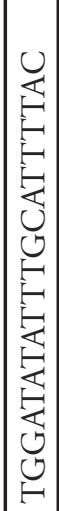 \\
\hline 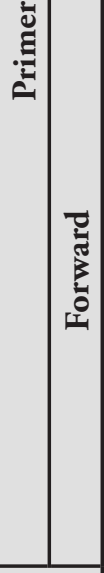 & 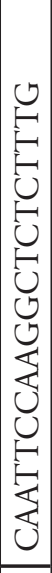 & 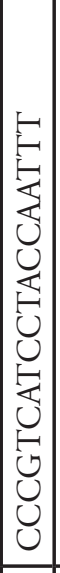 & 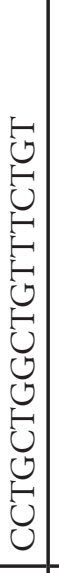 & 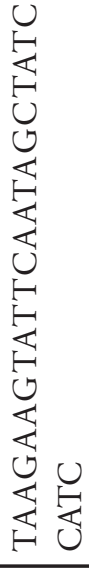 & 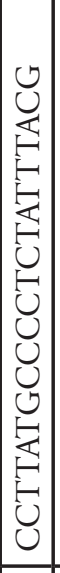 & 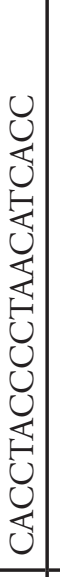 & 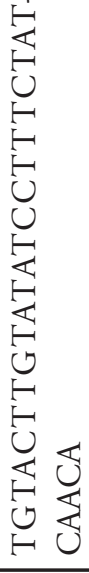 & 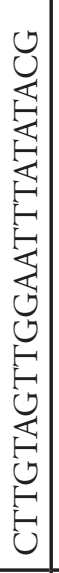 & 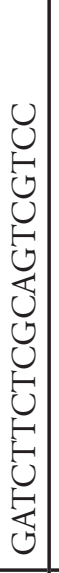 & 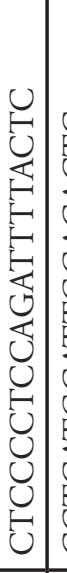 & 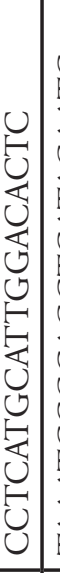 & 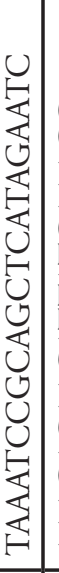 & 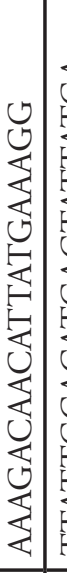 & 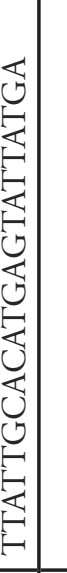 & 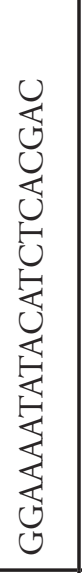 & 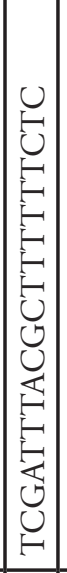 & 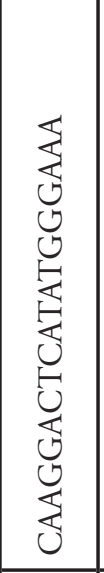 & 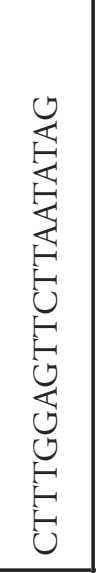 & 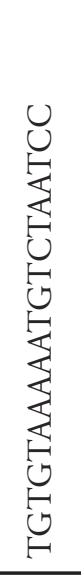 & 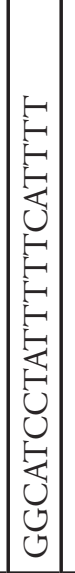 & 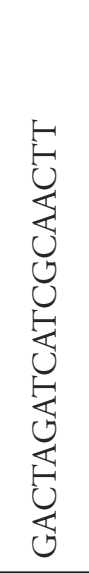 & 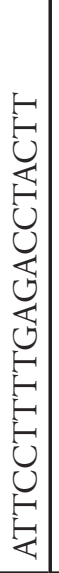 & 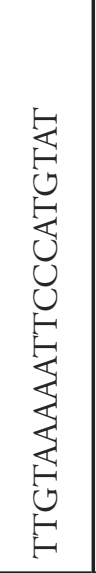 & 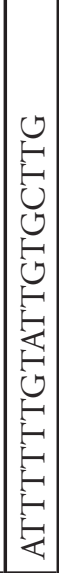 \\
\hline 菅 & & & & & & & & & & (্) & 3 & 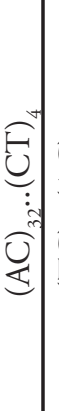 & 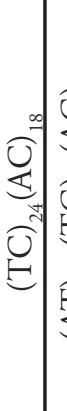 & 苞 & 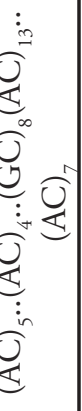 & 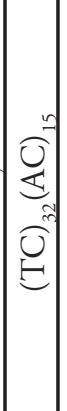 & 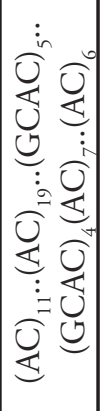 & 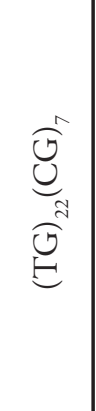 & 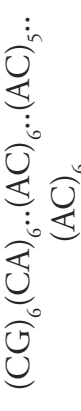 & 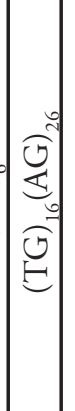 & 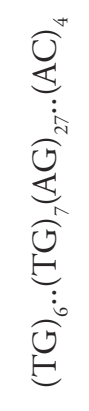 & 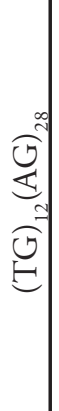 & 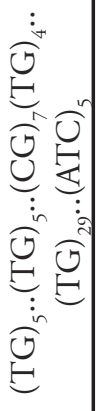 & 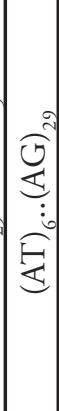 \\
\hline 号 & ปै & 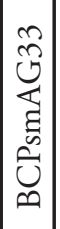 & 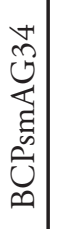 & 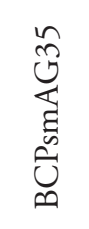 & 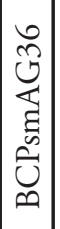 & 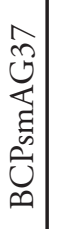 & 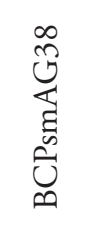 & 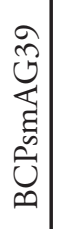 & 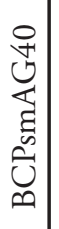 & $\begin{array}{l}3 \\
-1 \\
\vdots \\
0 \\
0 \\
\vdots \\
\vdots \\
\vdots\end{array}$ & 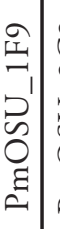 & 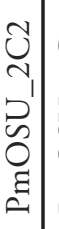 & 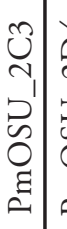 & 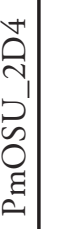 & $\begin{array}{l}\overbrace{1}^{0} \\
D_{1} \\
0 \\
0 \\
\Xi \\
\tilde{n}\end{array}$ & 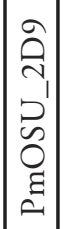 & 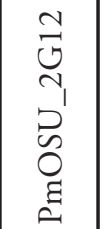 & 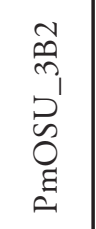 & $\begin{array}{l}\text { के } \\
\text { ले } \\
\text { Dे } \\
\text { on } \\
\text { घี }\end{array}$ & $\left|\begin{array}{c}n \\
\hat{n} \\
\vdots \\
\tilde{n} \\
0 \\
\vdots \\
\tilde{n}\end{array}\right|$ & 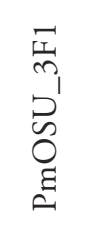 & 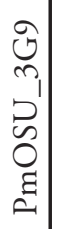 & 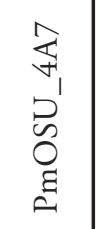 & 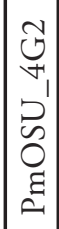 \\
\hline
\end{tabular}




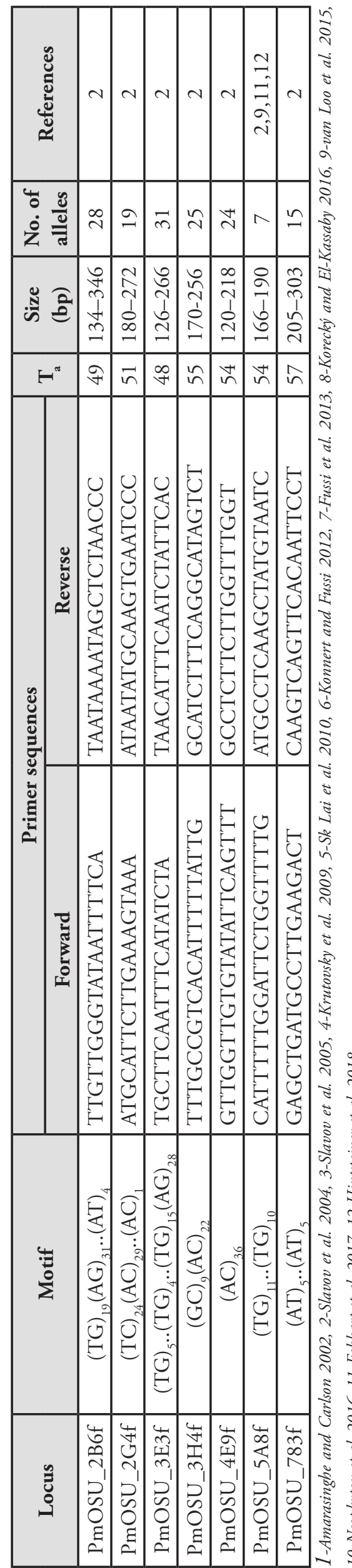

\section{Material for DNA-extraction}

DNA has been extracted from needles (Neophytou et al. 2016, Fussi et al. 2013, Slavov et al. 2004, 2005, van Loo et al. 2015, Eckhart et al. 2017, Hintsteiner et al. 2018), buds (Slavov et al. 2005, Sk Lai et al. 2010, Korecký and El-Kassaby (2016), seed (embryos, megagamethophytes) (Krutovskii et al. 2009, Sk Lai et al. 2010) and cambium (Neophytou et al. 2016, van Loo et al. 2015, Eckhart et al. 2017, Hintsteiner et al. 2018).

\section{DNA-extraction and amplification protocols}

Total DNA has been extracted from the mentioned tissue using:

- the commercial DNeasy Plant Maxi DNA-extraction Kit (QIAGEN) (Slavov et al. 2004, 2005) and DNeasy 96 plant kit (Qiagen) (Neophytou et al. 2016);

- the OMEGA E.Z.N.A Plant DNA Kit (OMEGA Bio-Tek, Inc., Norcross, Georgia, USA) (van Loo et al. 2015, Eckhart et al. 2017, Hintsteiner et al. 2018);

- the CTAB extraction protocol of Doyle and Doyle (1990) (Sk Lai et al. 2010), Korecký and El-Kassaby 2016);

- the ATMAB extraction protocol of Dumolin et al. (1995) (Fussi et al. 2013).

\section{Examples for amplification protocols}

In most studies, the following PCR conditions described by Slavov et al. (2004) have been used:

- seven cycles of touchdown PCR: $95{ }^{\circ} \mathrm{C}$ for 30 $s$, empirically determined optimal annealing temperature $(\mathrm{T})+7^{\circ} \mathrm{C}$ for $30 \mathrm{~s}$, then $72{ }^{\circ} \mathrm{C}$ for 45 s. The $\mathrm{T}_{\mathrm{a}}$ was decreased by $1{ }^{\circ} \mathrm{C}$ for each of the six subsequent touchdown cycles. Following touchdown PCR, the program continued with 32 cycles of $95^{\circ} \mathrm{C}$ for $30 \mathrm{~s}, \mathrm{~T}_{\mathrm{a}}^{\circ} \mathrm{C}$ for $30 \mathrm{~s}, 72{ }^{\circ} \mathrm{C}$ for $45 \mathrm{~s}$, and a final extension of $72{ }^{\circ} \mathrm{C}$ for $20 \mathrm{~min}$.

\section{Important results}

- Nuclear SSRs are useful tools for genetic studies in Douglas-fir. Especially the nSSRs developed by Slavov (2004) have been applied in several studies (e.g. Krutovsky et al. 2009, Sk Lai et al. 2010, Fussi et al. 2013, Neophytou et al. 2016, van Loo et al. 2015, Korecký and El-Kassaby 2016, Hintsteiner et al. 2018).

- Based on nSSRs, coastal and interior Douglas-fir can be clearly differentiated from each other (Fussi 
et al. 2013, van Loo et al. 2015, Neophytou et al. 2016). Following Fussi et al. (2013), the nSSR loci PmOSU_3B2 and PmOSU_4A7 are indicated to distinguish between the two varieties.

- Intra-varietal variation (division into different clusters within a variety) and hybridization patterns were described and related to the phylogeographic history (van Loo et al. 2015). Results were used to trace back the origin of numerous Douglas-fir stands in Europe (Austria and Germany) (Fussi et al. 2013, Eckhart et al. 2017, Hintsteiner et al. 2018).

- European seedlings have a lower genetic diversity than the American seedlings and native populations (Konnert and Ruetz 2006, Eckhart et al. 2017).

- Weak genetic differentiation among populations and high within population differentiation for coastal Douglas-fir, already observed for allozyme loci, has been confirmed by nSSRs. Genetic distance has been positively and significantly correlated with geographic distance (Krutovsky et al. 2009).

- nSSR markers are also powerful tools for a) studying the pollination dynamics in seed orchard, b) characterizing seed lots and c) improving the design and management of Douglas-fir seed orchard (Slavov et al. 2005, Sk Lai et al. 2010, Kess and ElKassaby 2015, Korecký and El-Kassaby 2016). For example, Korecký and El-Kassaby (2016) found that in the coastal Douglas-fir seed orchard $80 \%$ of parental gametes were produced by $52 \%$ of the parents, $13 \%$ of paternal gametes resulted from pollen contamination and $12 \%$ of the seed were the product of selfing. Slavov et al. (2005) discovered that levels of pollen contamination in a seed orchard in Oregon varied substantially among clones. These levels were higher in clones with early female receptivity (mean $=55.5 \%$ ) than in those with midand low-receptivity.

- Neophytou et al. (2016) assessed the link between genetic and phenotypic variation in the height growth. Statistical evidence was provided for a genetic component in the height growth.

\section{c) SNPs (single-nucleotide polymorphisms)}

\section{Loci and primers used}

Krutovsky and Neale (2005) studied single-nucleotide polymorphisms (SNPs) and linkage disequilibrium in 15 cold-hardiness- and 3 wood quality-related candidate genes in Douglas-fir. Their study, which primarily aimed to select SNPs for further association mapping, shed light on SNP frequency, haplotype and nucleotide diversities of studied candidate genes. Four years later Eckert et al. (2009a) analysed diversity and divergence for a set of 121 cold-hardiness candidate genes (classified by 933 SNPs) in coastal Douglas-fir trees. These represented 24 unrelated Douglas-fir individuals from six regions located across Washington and Oregon. In their following study, Eckert et al. (2009b) used a candidate gene-based approach to search for genetic associations between 384 SNPs from 117 candidate genes and 21 cold-hardiness related traits within natural populations (700 unrelated families) of coastal Douglas-fir from western Oregon and Washington. The SNPs were selected from the already existing sets of SNPs represented by 400 SNPs and 933 SNPs from Krutovsky and Neale (2005) and from Eckert et al. (2009a), respectively. A list of 154 candidate genes with SNPs used by Krutovsky and Neale (2005) and Eckert et al. $(2009 a, b)$ is presented in Table 7.

Applying transcriptome sequencing on cDNA from Douglas-fir seedlings included in a drought stress experiment Müller et al. (2012) identified about 1,000 candidate genes related to drought stress with a total number of 187,653 SNPs. They established a catalogue of putative unique transcripts (PUTs) and a large SNP database for Douglas-fir. Using targeted sequence capture, Müller et al. (2015) re-sequenced 72 trees of both coastal and interior variety in order to characterize genetic diversity in coding regions and to identify genes involved in local adaptation. To facilitate genomic selection in Douglas-fir breeding programs Howe et al. (2013) combined high-throughput sequencing technologies (454 pyrosequencing and Illuminasequencing-by-synthesis) to sequence the transcriptomes of diverse tissues of Douglas-fir genotypes. 


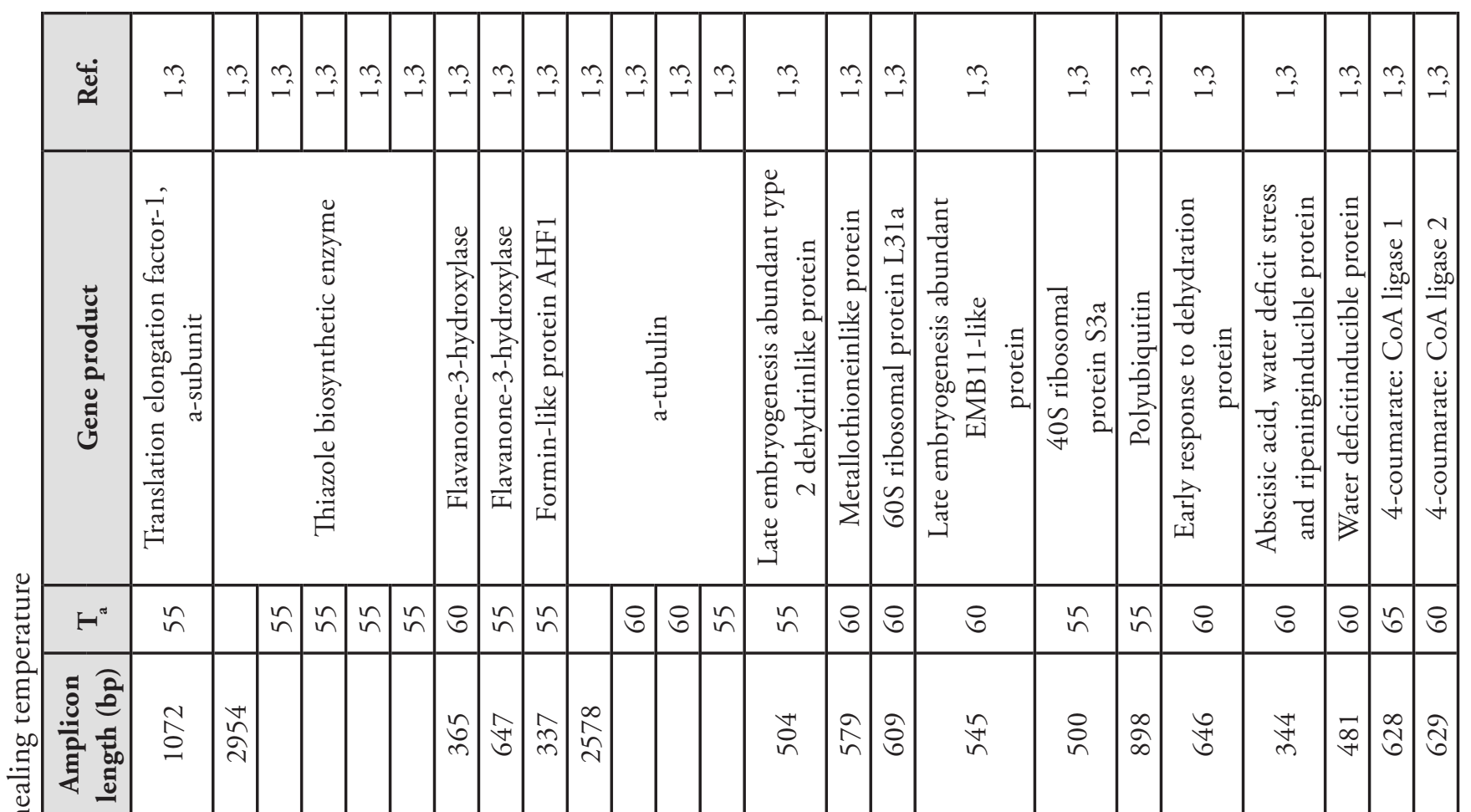

\begin{tabular}{|c|c|c|c|c|c|c|c|c|c|c|c|c|c|c|c|c|c|c|c|c|}
\hline 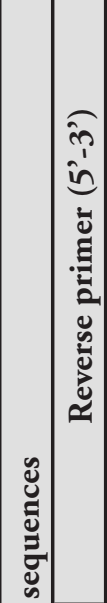 & 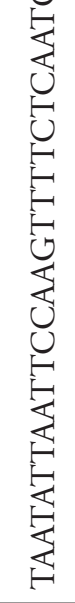 & & 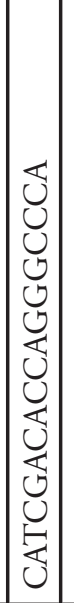 & 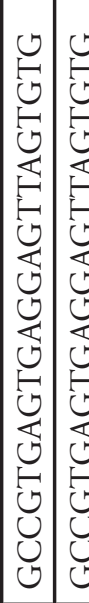 & 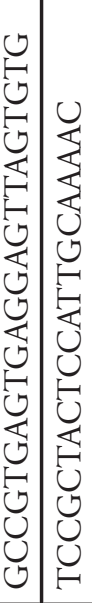 & 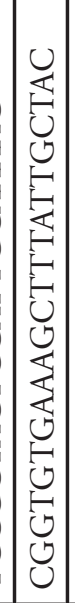 & 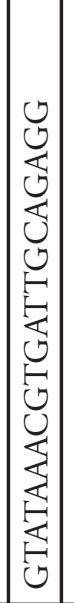 & 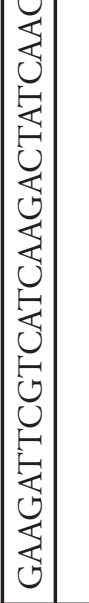 & 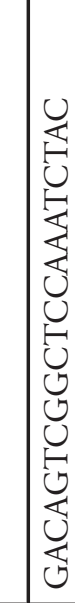 & 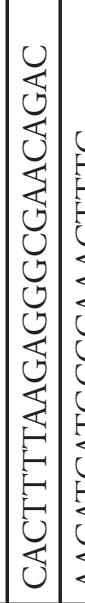 & 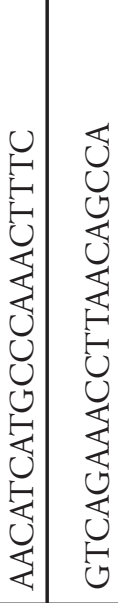 & 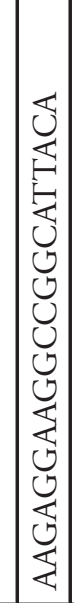 & 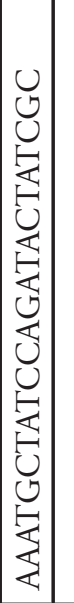 & 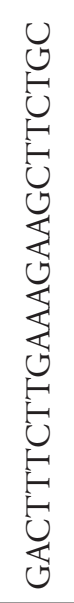 & 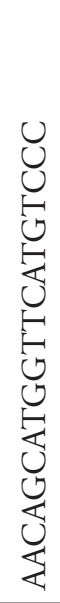 & 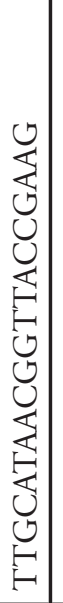 & 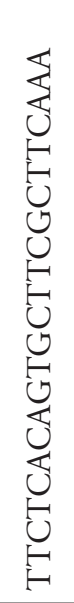 & 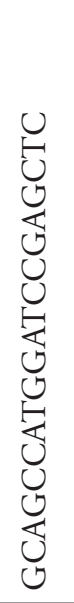 & 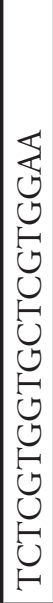 & 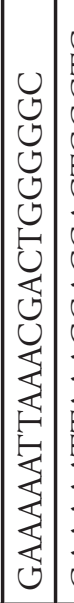 \\
\hline 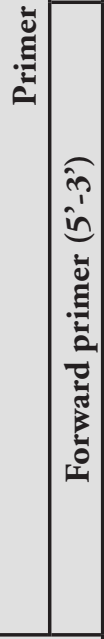 & 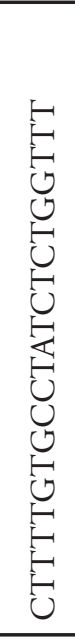 & & 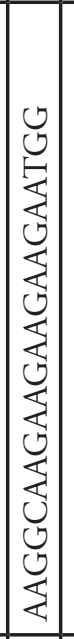 & 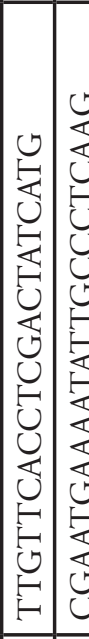 & 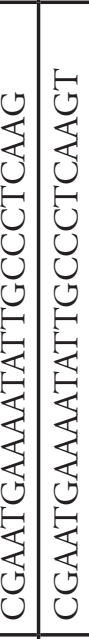 & 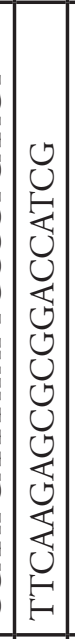 & 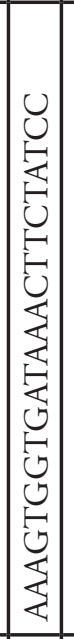 & 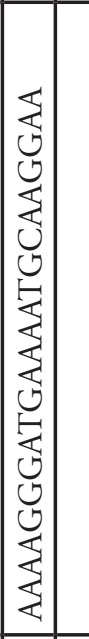 & 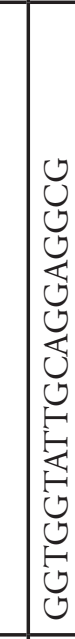 & 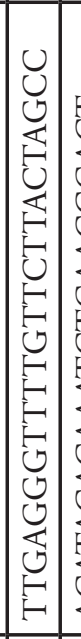 & 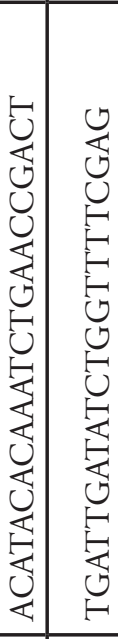 & 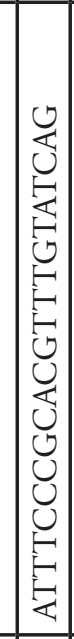 & 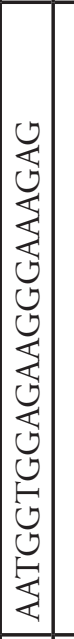 & 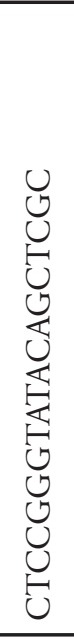 & 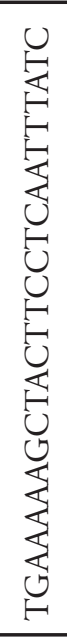 & 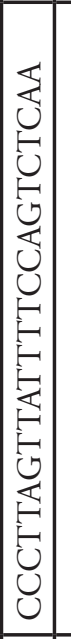 & 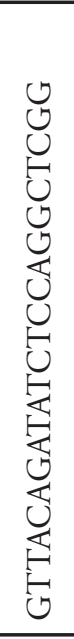 & 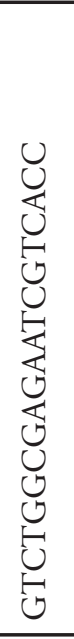 & 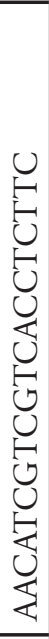 & 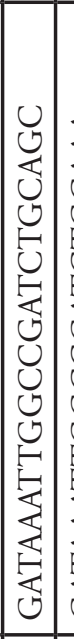 \\
\hline 氶芯 & \pm & $\stackrel{\infty}{n}$ & & & & \pm & \pm & $m \mid \tilde{\sigma}$ & & & $\stackrel{\infty}{\sim}$ & 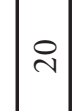 & $\vec{\sim}$ & $\stackrel{m}{n}$ & $\approx$ & $\approx$ & 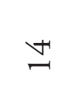 & $a$ & $\stackrel{\bullet}{\simeq}$ & $\infty$ \\
\hline 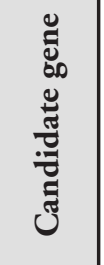 & 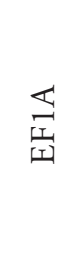 & 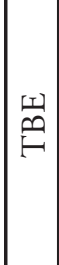 & 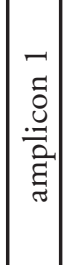 & 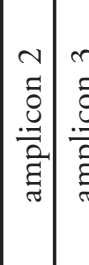 & 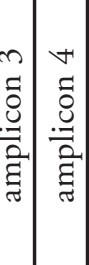 & 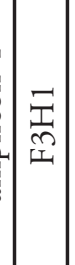 & $\left|\begin{array}{c}\mathcal{I} \\
\underset{I}{M} \\
I \\
I\end{array}\right|$ & 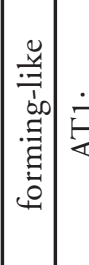 & 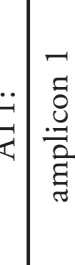 & 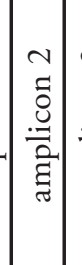 & 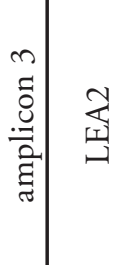 & 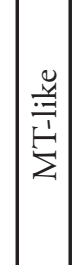 & 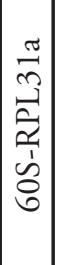 & 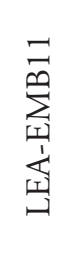 & 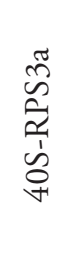 & $\mid \begin{array}{l}0 \\
0 \\
0 \\
2 \\
\underline{0} \\
0\end{array}$ & 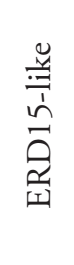 & 疍 & $\stackrel{y}{=}$ & $\underset{\vec{Y}}{\vec{\underbrace{}}}$ \\
\hline
\end{tabular}




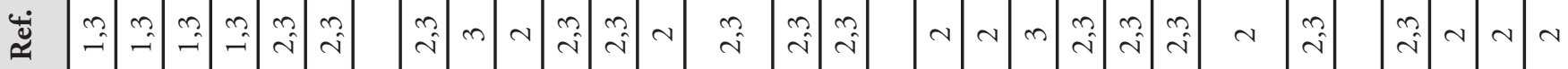

\begin{tabular}{|c|c|c|c|c|c|c|c|c|c|c|c|c|c|c|c|c|c|c|c|c|c|c|c|c|c|c|c|c|}
\hline 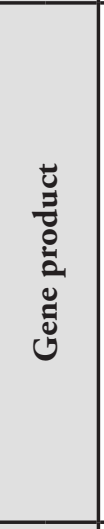 & & 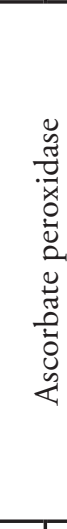 & & & 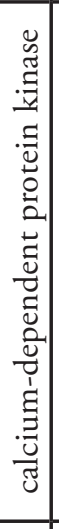 & 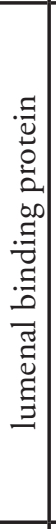 & & 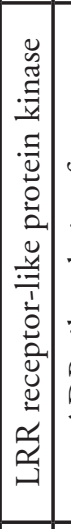 & 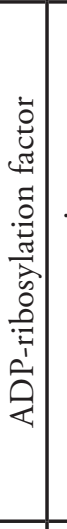 & & 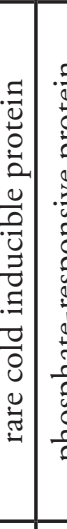 & 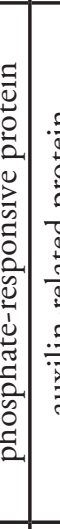 & & 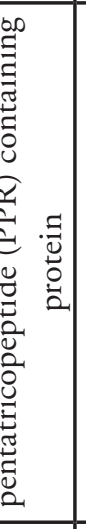 & 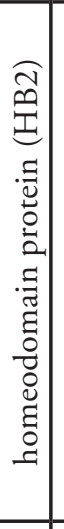 & 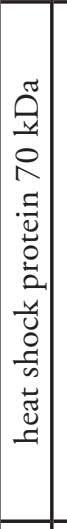 & & 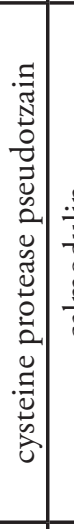 & 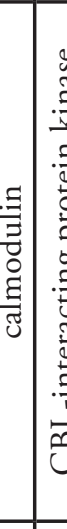 & 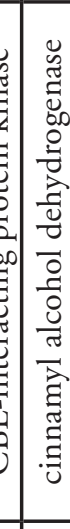 & 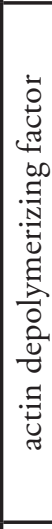 & 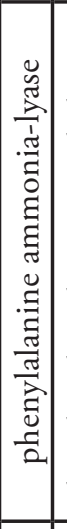 & 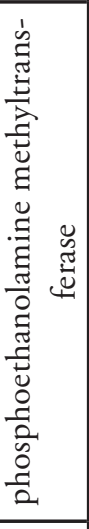 & 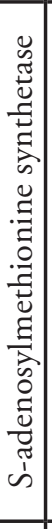 & & $\begin{array}{l}\stackrel{x}{\tilde{I}} \\
\stackrel{\widetilde{U}}{\Xi} \\
0\end{array}$ & 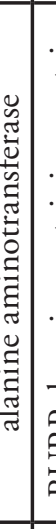 & . \\
\hline$F^{\prime \prime}$ & & $n$ & 8 & 8 & $\hat{n}$ & $n$ & $n$ & 8 & & $\approx$ & $n$ & \begin{tabular}{l|l}
8 & \\
6
\end{tabular} & & 8 & 8 & $n$ & $n \approx$ & $n \tilde{v}$ & 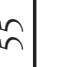 & $n$ & $i n$ & $i n$ & $n$ & $n$ & $n$ & in & $\hat{n}$ & 18 \\
\hline 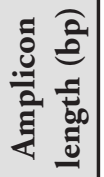 & $\stackrel{\infty}{\infty}$ & & & & 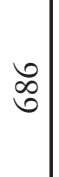 & $\widehat{N}$ & & $\left|\begin{array}{c}\infty \\
\infty \\
\sim\end{array}\right|$ & & $\stackrel{\infty}{\curvearrowright}$ & \begin{tabular}{l}
$\infty$ \\
\multirow{f}{*}{} \\
\end{tabular} & $\vec{n}=$ & & $\stackrel{0}{-}$ & $\hat{\widehat{\sigma}}$ & $\hat{n}$ & & $\stackrel{2}{\equiv}$ & & $\underset{m}{F}$ & 6 & $\stackrel{\sim}{\sigma}$ & 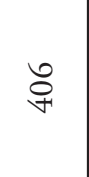 & $\infty$ & & & $\overbrace{n}^{\circ}$ & $m$ \\
\hline
\end{tabular}

\begin{tabular}{|c|c|c|c|c|c|c|c|c|c|c|c|c|c|c|c|c|c|c|c|c|c|c|c|c|c|}
\hline 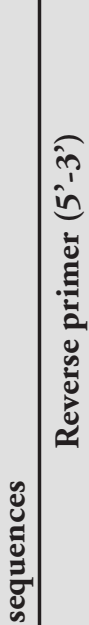 & & 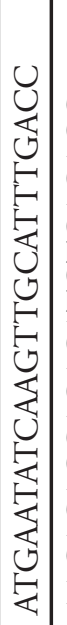 & 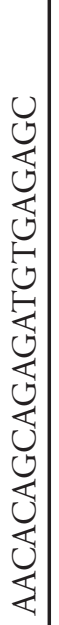 & 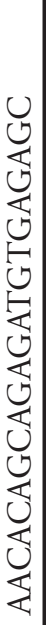 & 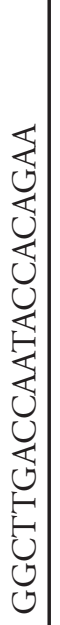 & 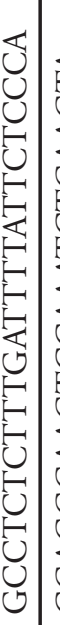 & 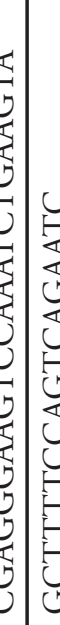 & & 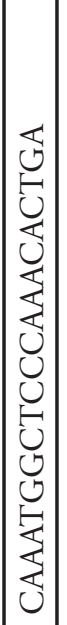 & 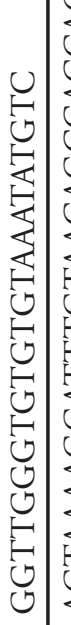 & 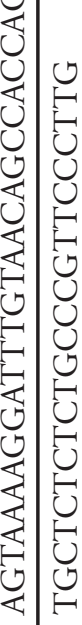 & 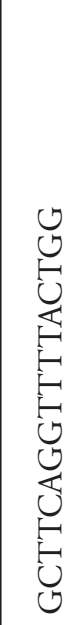 & 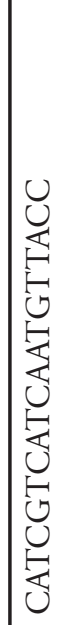 & 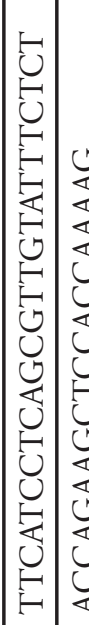 & 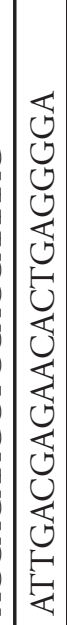 & 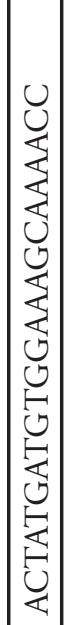 & 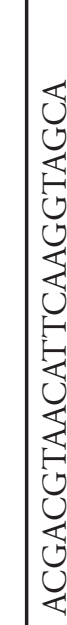 & 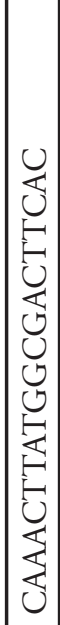 & 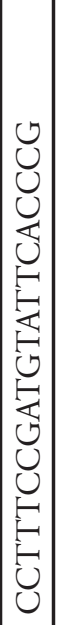 & 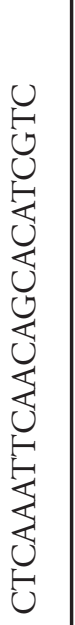 & 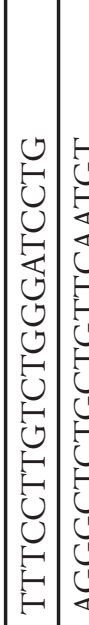 & 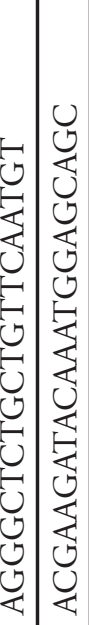 & 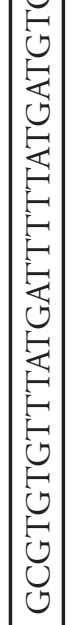 & 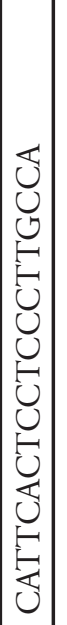 & 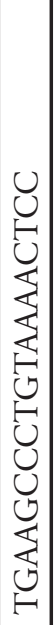 \\
\hline 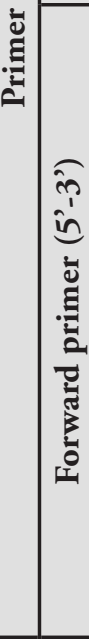 & & 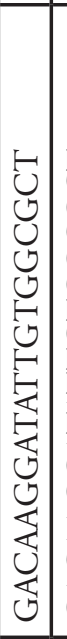 & 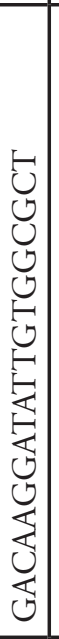 & 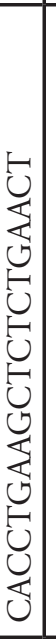 & 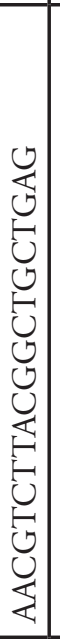 & 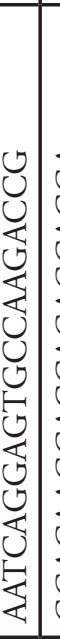 & 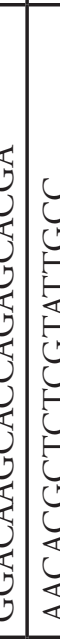 & 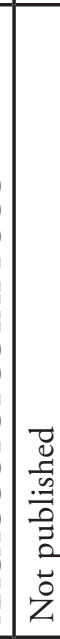 & $\begin{array}{l}0 \\
0 \\
0 \\
0 \\
5 \\
0 \\
0 \\
0 \\
0 \\
0 \\
0 \\
2 \\
0 \\
0 \\
0 \\
0 \\
0 \\
0 \\
0 \\
0 \\
0 \\
0\end{array}$ & 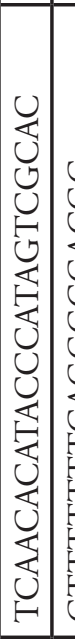 & 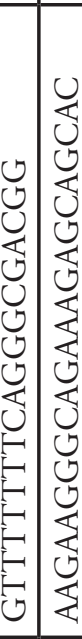 & 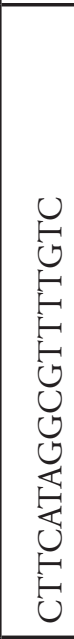 & 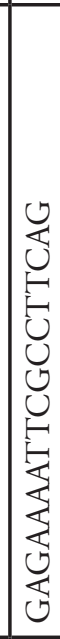 & 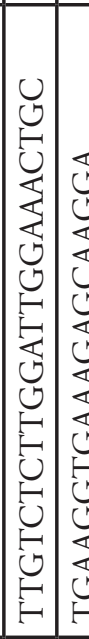 & 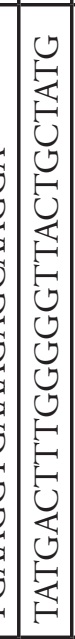 & 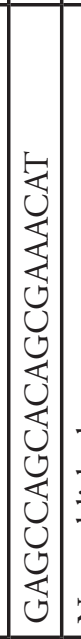 & 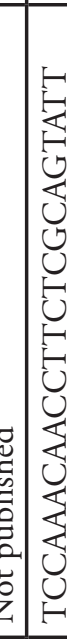 & 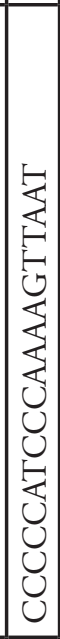 & 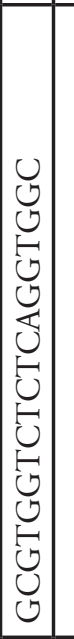 & 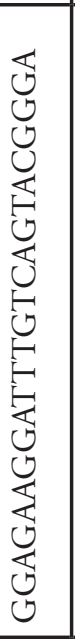 & 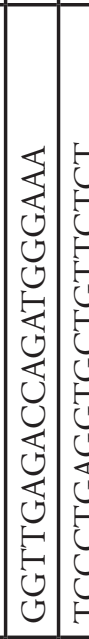 & 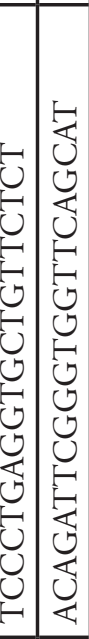 & 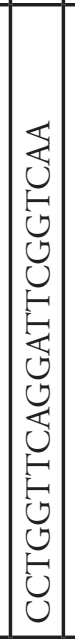 & 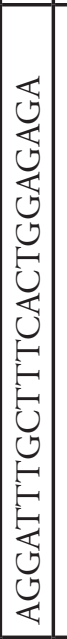 & 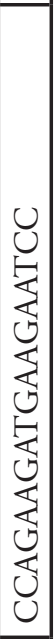 \\
\hline$\hat{z}$ & 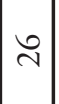 & & & & ণ & $\tilde{\sim}$ & C & & $\vec{\sim}$ & $\ddot{\sim}$ & $\stackrel{\sim}{\sim} \mid \stackrel{\sim}{ }$ & $\beth$ & $\ddot{\imath}$ & $\underset{\sim}{\sim}$ & तี & สิ & $\stackrel{\infty}{\sim}$ & $\tilde{\imath}$ & $\ddot{\imath}$ & ? & 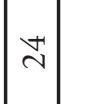 & $\tilde{\sim}$ & $\approx$ & $\approx$ & 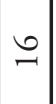 \\
\hline 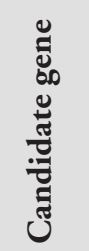 & 妾 & 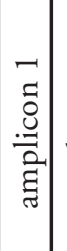 & 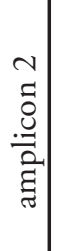 & 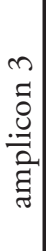 & 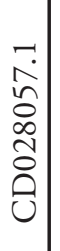 & 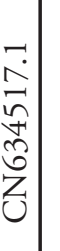 & N & 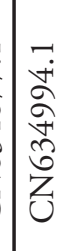 & $\mid \begin{array}{c}-1 \\
\hat{n} \\
\tilde{n} \\
\hat{\sigma} \\
z \\
0\end{array}$ & $\begin{array}{c}-1 \\
\dot{a} \\
\hat{+} \\
\hat{n} \\
\hat{0} \\
z \\
0\end{array}$ & 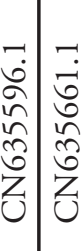 & 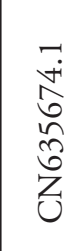 & 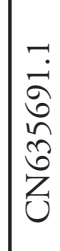 & 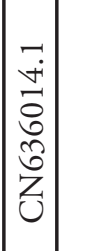 & 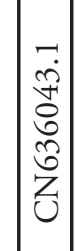 & 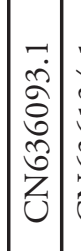 & 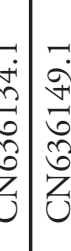 & 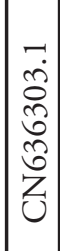 & 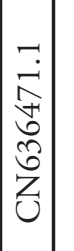 & 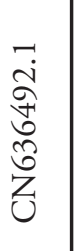 & 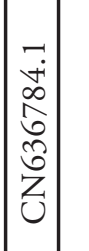 & 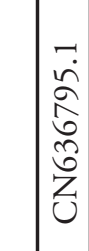 & 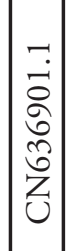 & 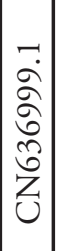 & 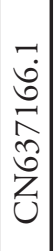 \\
\hline
\end{tabular}




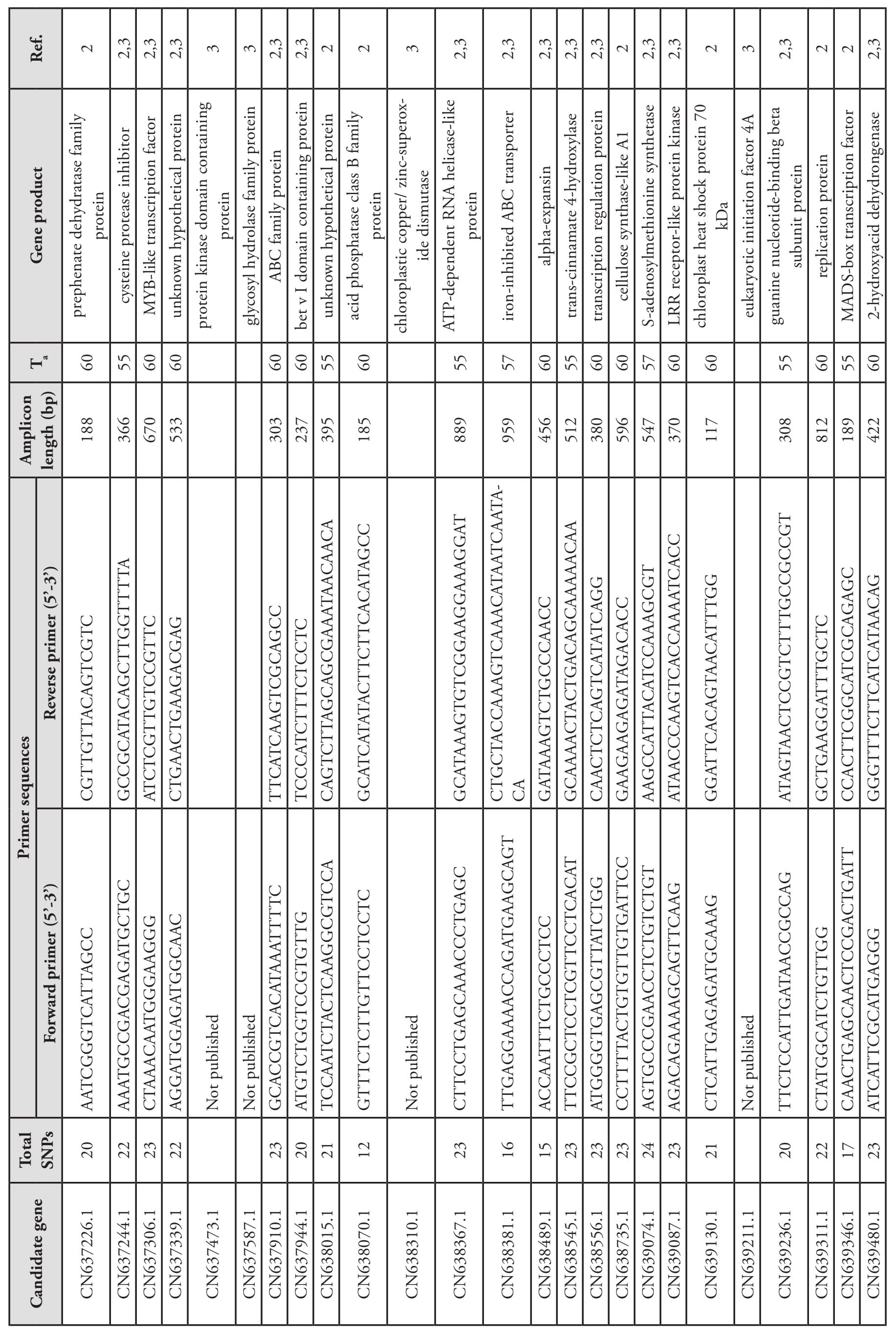




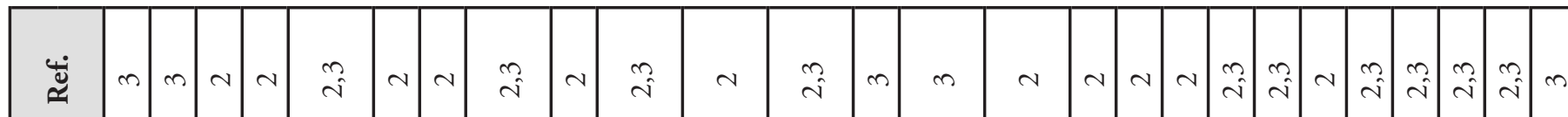

\begin{tabular}{|c|c|c|c|c|c|c|c|c|c|c|c|c|c|c|c|c|c|c|}
\hline 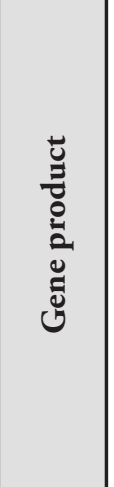 & 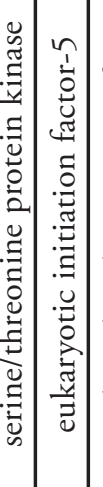 & 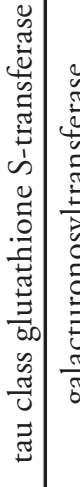 & 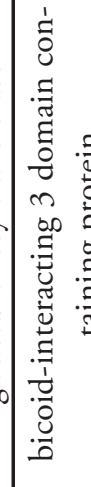 & 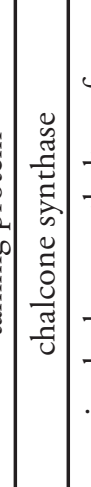 & 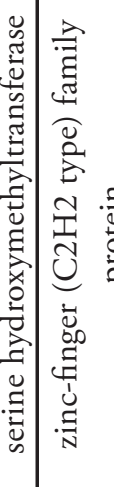 & 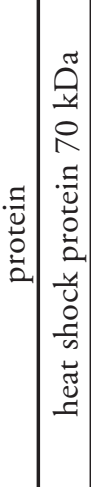 & 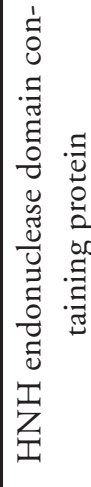 & 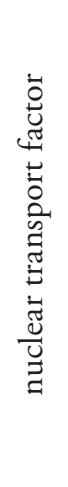 & 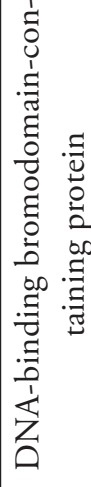 & 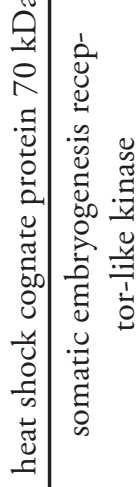 & 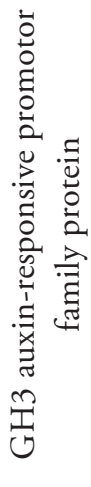 & 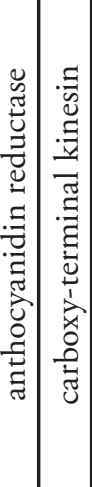 & & & & & & \\
\hline$F^{*}$ & & $8: S$ & 8 & in & $\approx 8$ & in & 8 & $i n$ & 8 & & 8 & $\approx 8$ & $i$ & 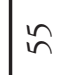 & 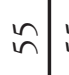 & & & $i n$ \\
\hline 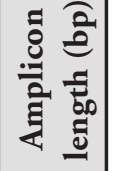 & & di & $\stackrel{\infty}{m}$ & 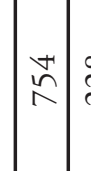 & 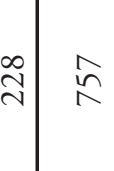 & $\frac{\infty}{6}$ & $\tilde{n}^{\infty}$ & 㼛 & $\underset{\forall}{\infty}$ & & 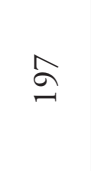 & & & & & & $\vec{\infty}$ & 命 \\
\hline
\end{tabular}

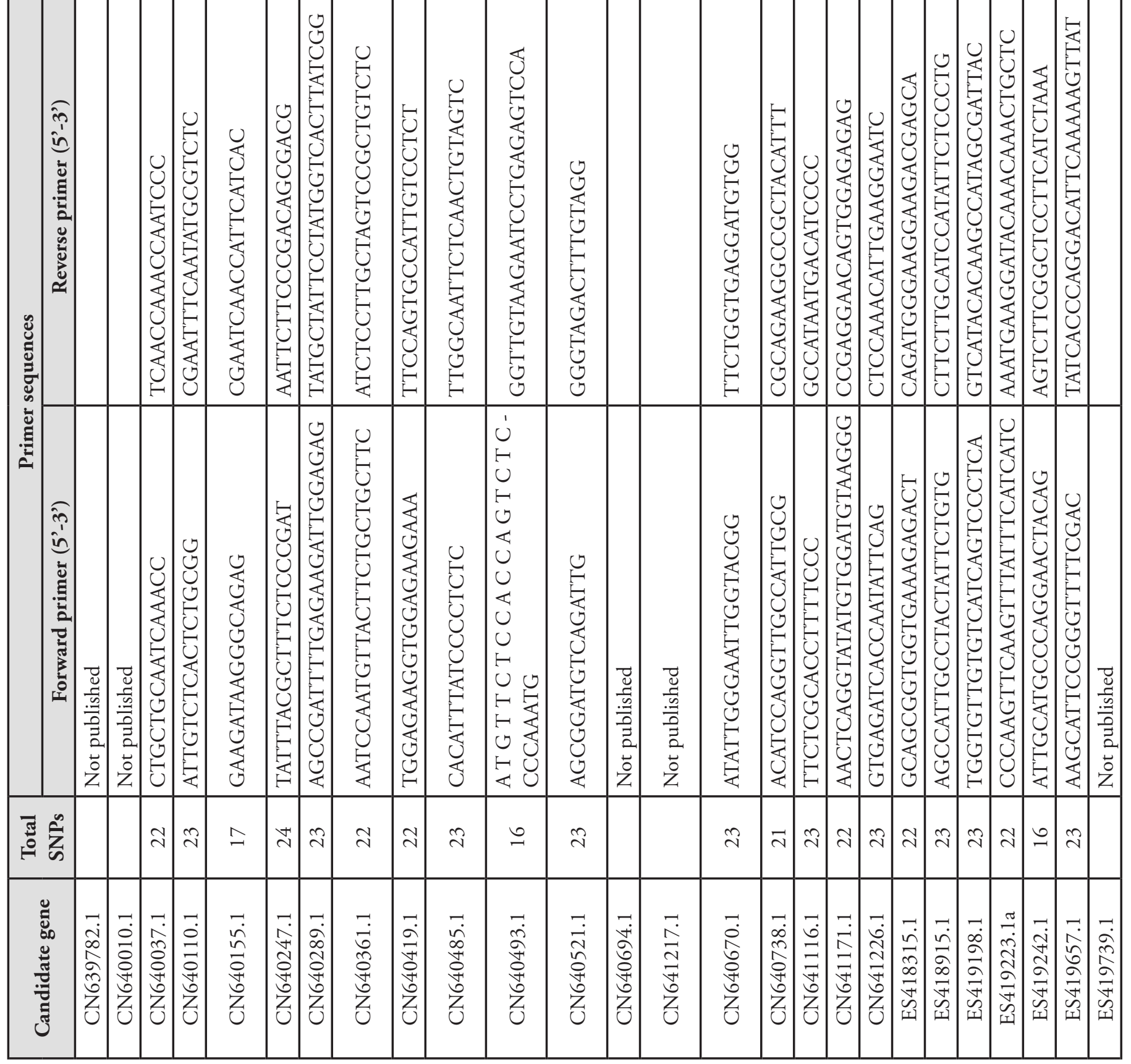




\begin{tabular}{|c|c|c|c|c|c|c|c|c|c|c|c|c|c|c|c|c|c|c|c|c|c|c|}
\hline$\widetilde{\mathscr{U}}$ & $n$ & $N$ & $\begin{array}{c}m \\
i\end{array}$ & $n$ & $\mid \begin{array}{c}n \\
i\end{array}$ & $\underset{\sim}{\sim}$ & $\stackrel{m}{i}$ & $m$ & $\hat{i}$ & $\hat{i}$ & $\begin{array}{l}m \\
i\end{array}$ & $\begin{array}{c}m \\
i\end{array} \mid$ & & $N$ & $\stackrel{m}{i}$ & $\stackrel{n}{\sim}$ & $m$ & $\stackrel{\sim}{\sim}$ & $\hat{i}$ & $\stackrel{n}{i}$ & $\sim$ & $\hat{i}$ \\
\hline 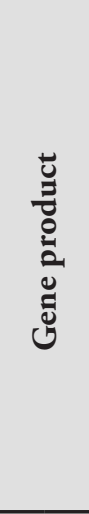 & 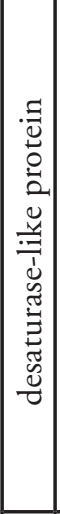 & 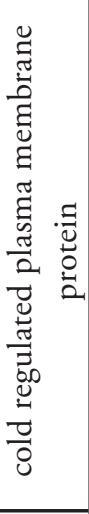 & 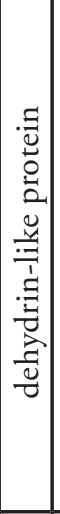 & 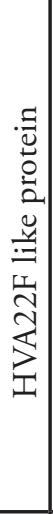 & 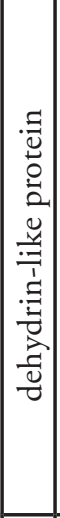 & 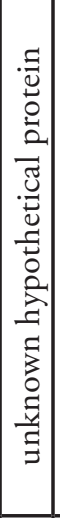 & 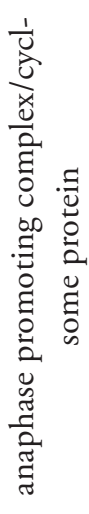 & 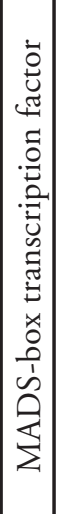 & 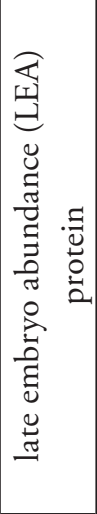 & 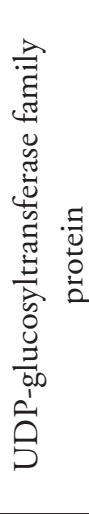 & 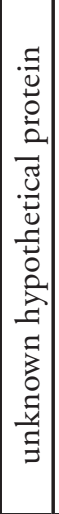 & 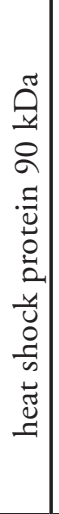 & & 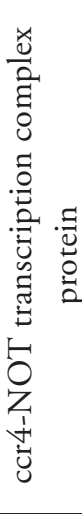 & 志 & 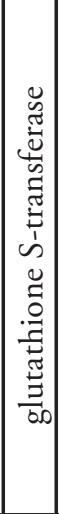 & 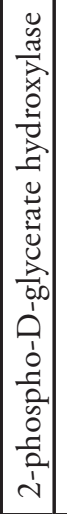 & 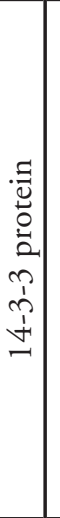 & 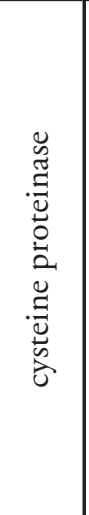 & 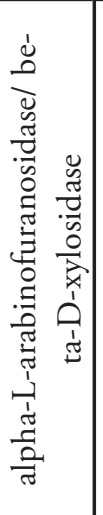 & 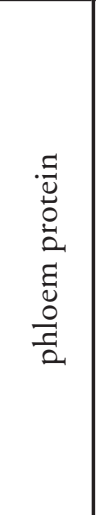 & 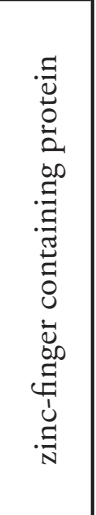 \\
\hline$H^{\infty}$ & & $n$ & $n$ & & $\approx$ & $n$ & $n$ & & $n$ & $i n$ & $n$ & $n$ & $\hat{n}$ & $\approx$ & $\approx$ & $\approx$ & & $\approx$ & 8 & 8 & 8 & 8 \\
\hline 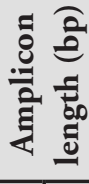 & & $\hat{\sim}$ & $\left|\begin{array}{l}+ \\
\infty \\
\infty\end{array}\right|$ & & 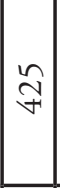 & 文 & సิ & & శิ & बे & $\stackrel{\mathrm{N}}{\mathrm{N}}$ & $\begin{array}{l}\infty \\
\sim \\
\sim\end{array}$ & & ñ & $\stackrel{\infty}{\infty}$ & $\hat{\bar{m}}$ & & $\begin{array}{l}: \\
\infty \\
0\end{array}$ & ईे & $\stackrel{\infty}{\infty}$ & $\stackrel{\sim}{n}$ & ڤn \\
\hline 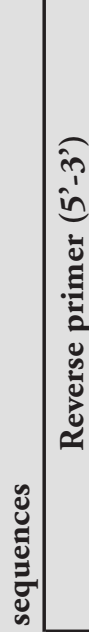 & & 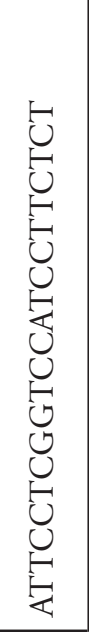 & 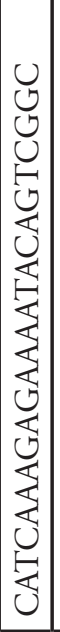 & & 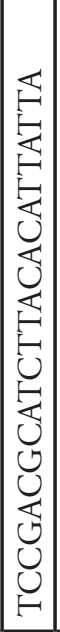 & 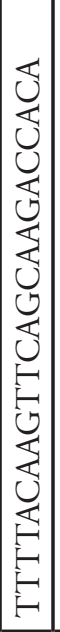 & 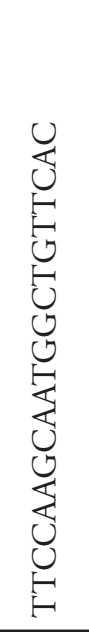 & & 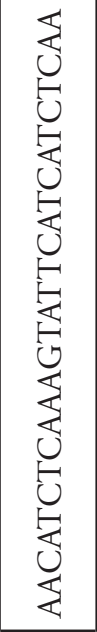 & 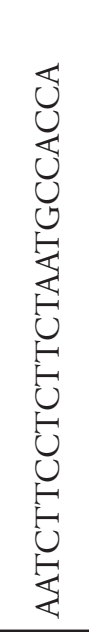 & 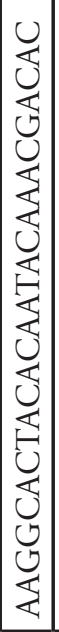 & \begin{tabular}{|l|} 
\\
0 \\
0 \\
0 \\
$=$ \\
0 \\
0 \\
0 \\
0 \\
0 \\
0 \\
0 \\
0 \\
0 \\
0 \\
0 \\
0 \\
0 \\
0 \\
0
\end{tabular} & 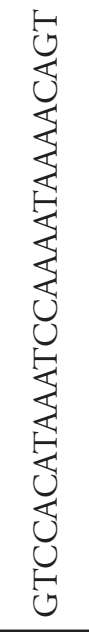 & 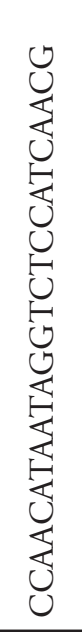 & 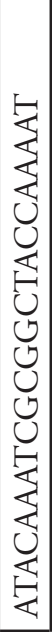 & 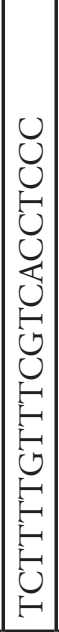 & & 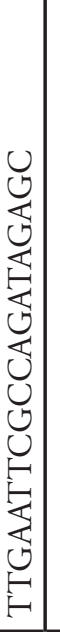 & 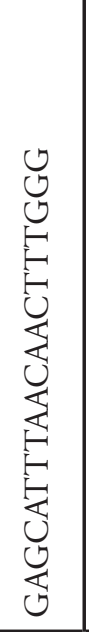 & 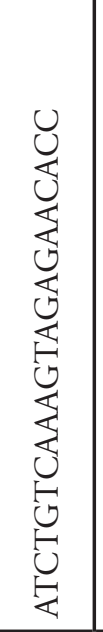 & 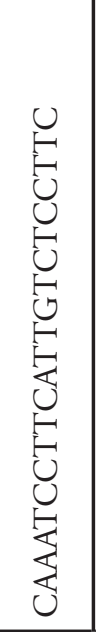 & 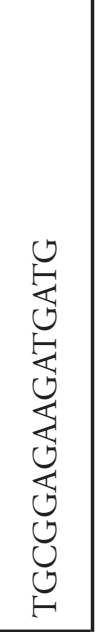 \\
\hline 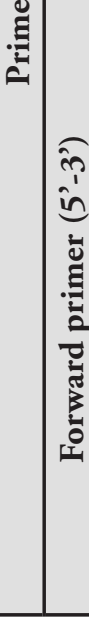 & 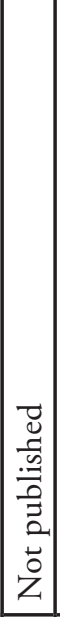 & 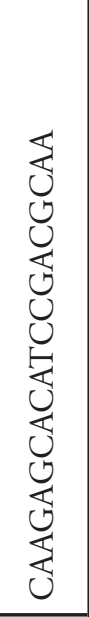 & 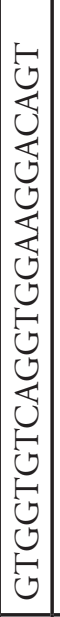 & 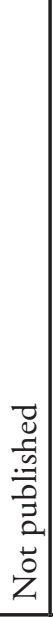 & 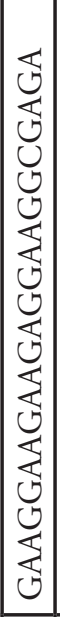 & 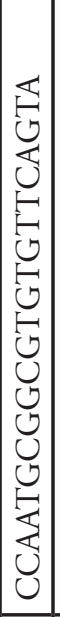 & 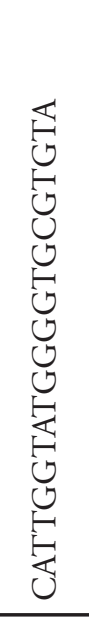 & 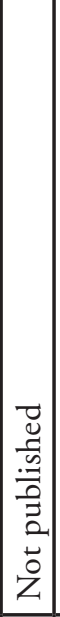 & 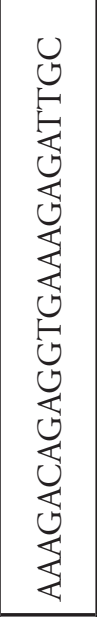 & 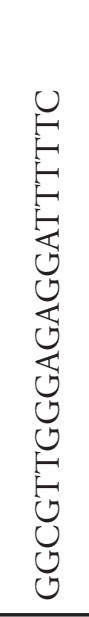 & 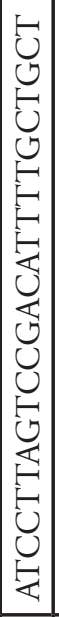 & $\begin{array}{l} \\
0 \\
0 \\
0 \\
0 \\
= \\
\sqcup \\
0 \\
5 \\
0 \\
0 \\
0 \\
0 \\
0 \\
0 \\
0 \\
0 \\
0 \\
0 \\
\end{array}$ & 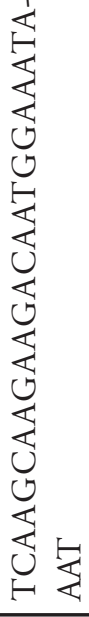 & 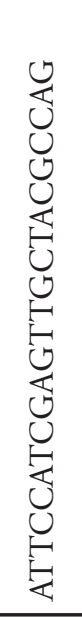 & 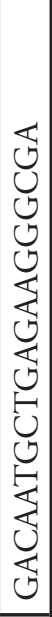 & 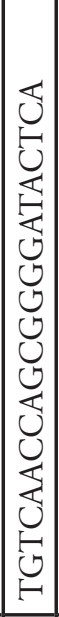 & 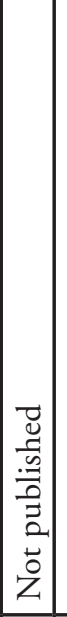 & 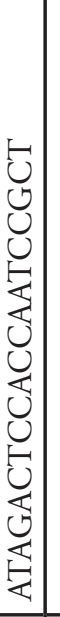 & 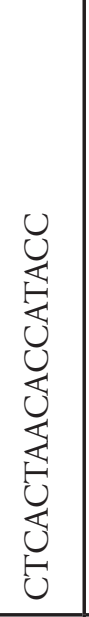 & 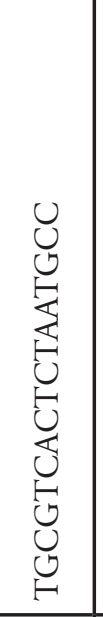 & 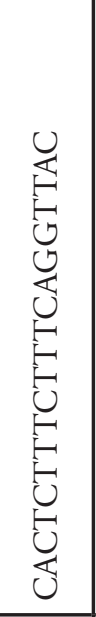 & 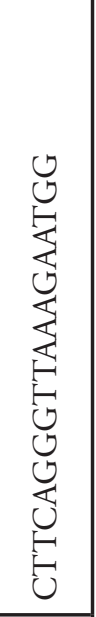 \\
\hline 䒢 & & $\stackrel{\sim}{\sim}$ & $\stackrel{\sim}{\sim}$ & & $\approx$ & $\approx$ & ิ & & $\stackrel{\sim}{\sim}$ & ปี & $\vec{\sim}$ & $\stackrel{\sim}{\sim}$ & & $\beth$ & 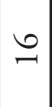 & $\vec{\sim}$ & & $\approx$ & $\infty$ & $\stackrel{\sim}{\sim}$ & $\stackrel{0}{-}$ & $\widetilde{\sim}$ \\
\hline 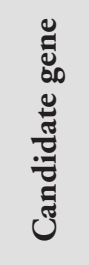 & 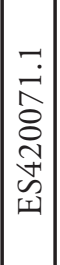 & 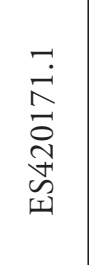 & 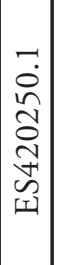 & 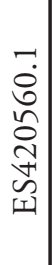 & 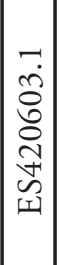 & 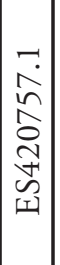 & 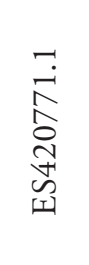 & 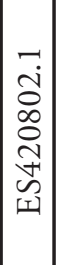 & 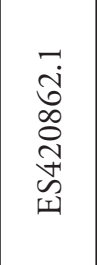 & $\begin{array}{l}\overrightarrow{\vec{a}} \\
\underset{\vec{I}}{\vec{I}} \\
\underset{\vec{J}}{\tilde{I}}\end{array}$ & 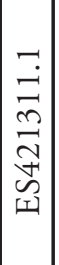 & 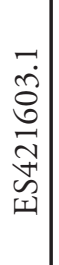 & & 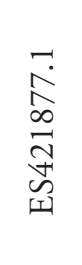 & 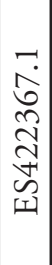 & 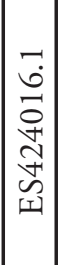 & 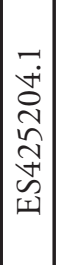 & 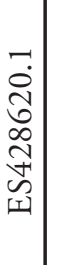 & 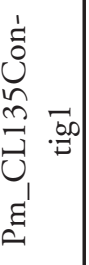 & 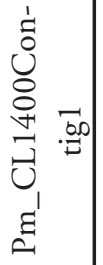 & 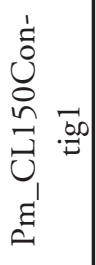 & 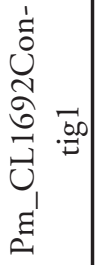 \\
\hline
\end{tabular}




\begin{tabular}{|c|c|c|c|c|c|c|c|c|c|c|c|c|c|c|c|c|c|c|}
\hline 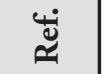 & $\stackrel{n}{\sim}$ & $\stackrel{n}{i}$ & $\stackrel{m}{i}$ & $N$ & $\stackrel{\text { r }}{\mathrm{r}}$ & $\stackrel{n}{\sim}$ & $\sim$ & $\stackrel{m}{i}$ & $\stackrel{\tilde{c}}{\sim}$ & $N$ & $N$ & $\stackrel{m}{\sim}$ & $N$ & $\stackrel{2}{\sim}$ & $\stackrel{m}{\sim}$ & $\stackrel{m}{\sim}$ & $\stackrel{m}{\sim}$ & $\stackrel{m}{i}$ \\
\hline 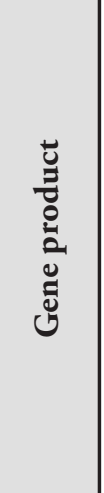 & 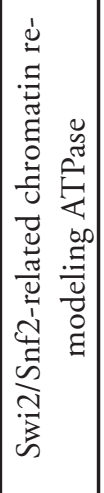 & 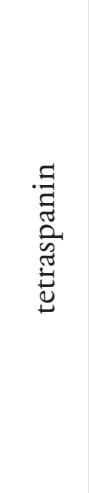 & 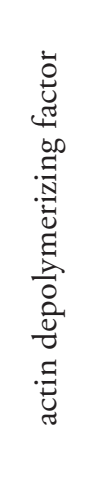 & 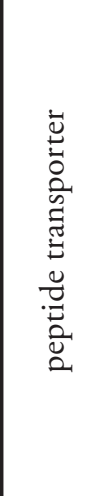 & 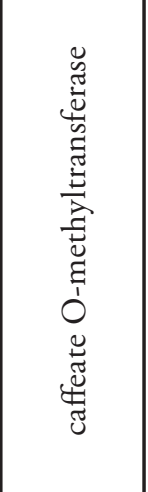 & 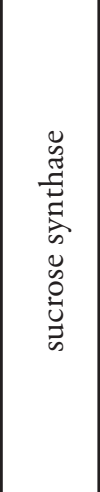 & 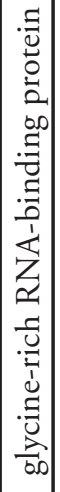 & 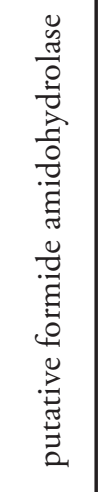 & 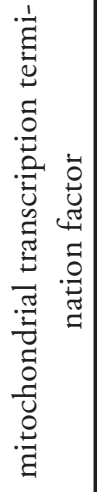 & 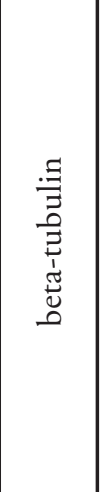 & 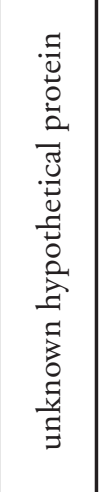 & 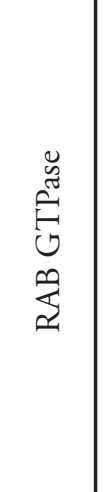 & 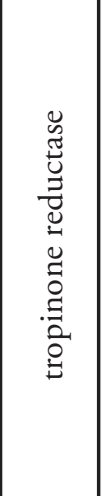 & 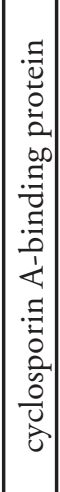 & 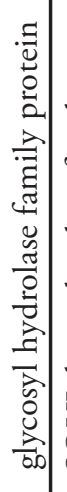 & 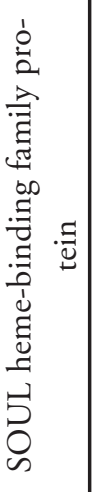 & 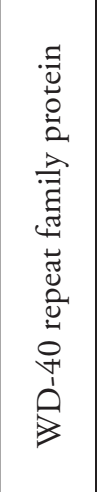 & 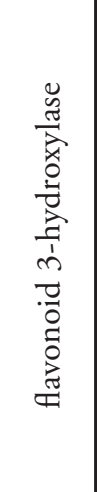 \\
\hline$H^{\circ}$ & 8 & 8 & 8 & 8 & 8 & 8 & 8 & 8 & 8 & 8 & 8 & 8 & 8 & 8 & 8 & 8 & 8 & 8 \\
\hline 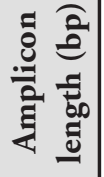 & $\sqrt{\sigma}$ & 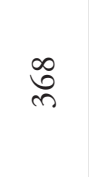 & $\stackrel{\infty}{\sim}$ & ત્ & $\vec{\infty}$ & $\vec{\nabla}$ & $\vec{\sigma}$ & \&্ণ & 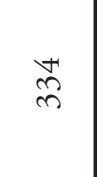 & $\begin{array}{l}\text { Dे } \\
\text { n. }\end{array}$ & $\bar{n}$ & $\stackrel{n}{n}$ & $\begin{array}{l}\infty \\
\text { +े }\end{array}$ & స్ & $\begin{array}{l}\text { To } \\
+\end{array}$ & ঃ̊ & $\vec{F}$ & চे \\
\hline 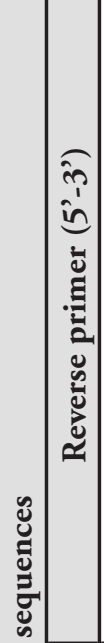 & 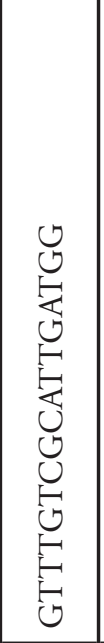 & 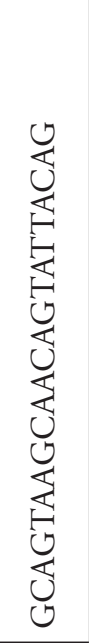 & 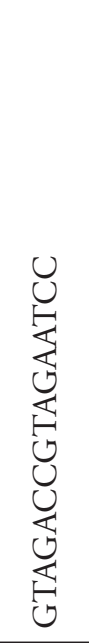 & 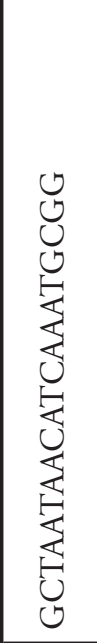 & 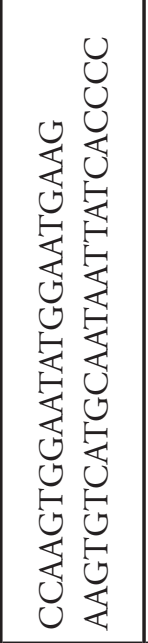 & 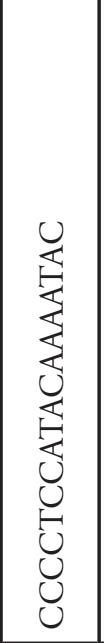 & 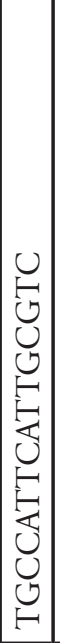 & 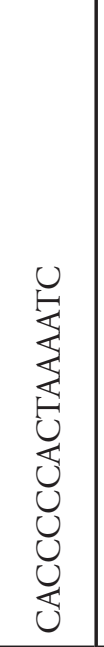 & 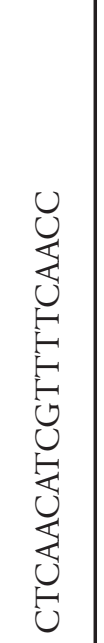 & 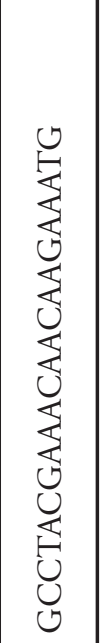 & 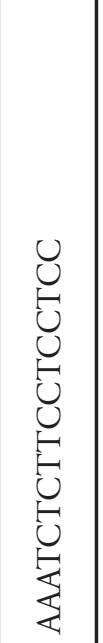 & 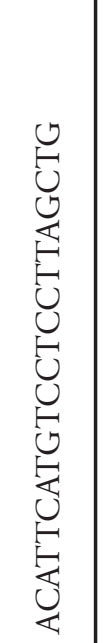 & 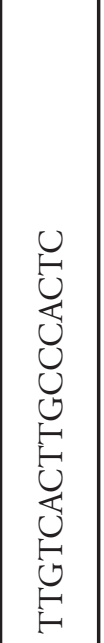 & 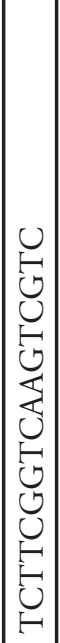 & 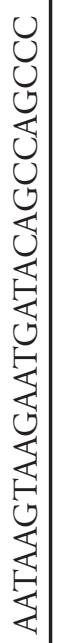 & 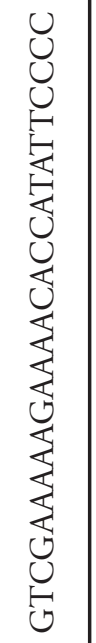 & 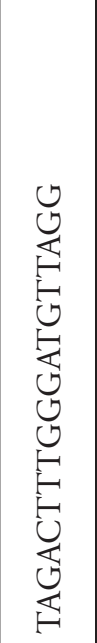 & 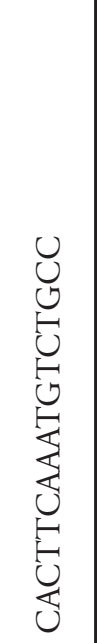 \\
\hline 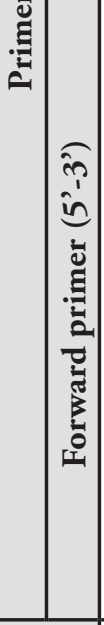 & 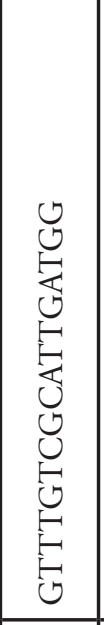 & 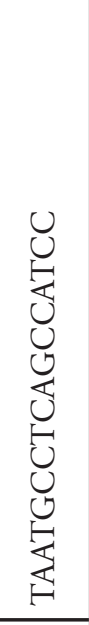 & 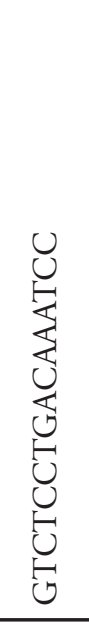 & 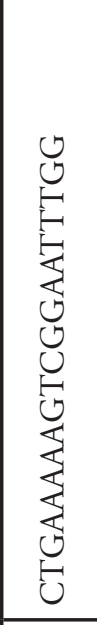 & 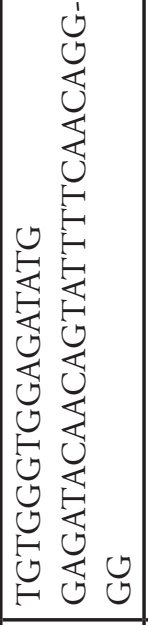 & 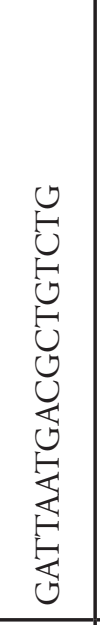 & 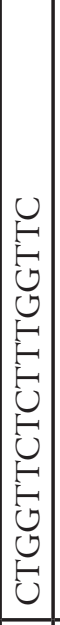 & 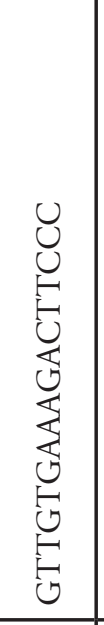 & 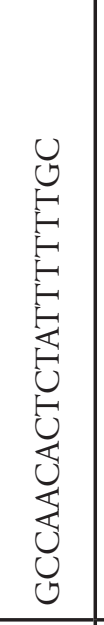 & 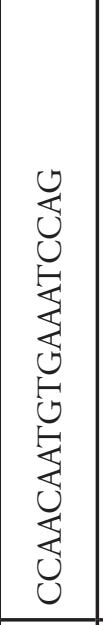 & 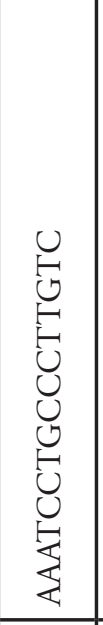 & 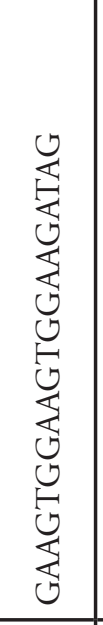 & 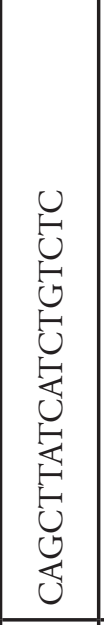 & 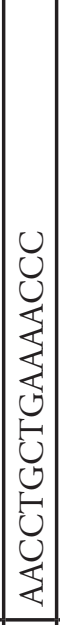 & 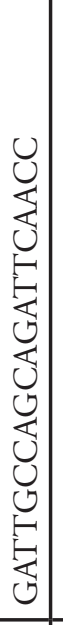 & 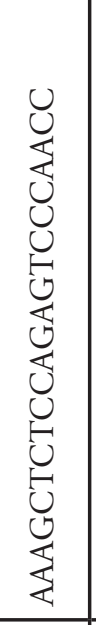 & 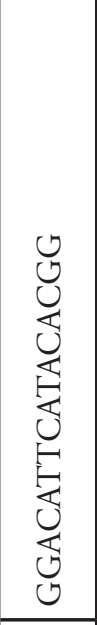 & 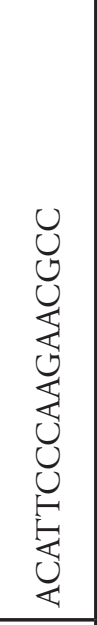 \\
\hline 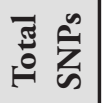 & $\stackrel{\bullet}{\ddots}$ & $\stackrel{\sim}{\sim}$ & $\vec{\sim}$ & $\ddot{\sim}$ & $\stackrel{\sim}{\sim}$ & $\vec{\sim}$ & 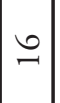 & $\ddot{\sim}$ & $\stackrel{\sim}{\sim}$ & तี & $\stackrel{\sim}{\sim}$ & $\stackrel{\infty}{-}$ & $\stackrel{\sim}{\sim}$ & $\vec{\sim}$ & $\approx$ & ปี & $\ddot{\sim}$ & $\stackrel{\sim}{\sim}$ \\
\hline 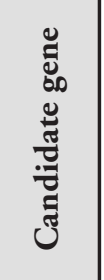 & 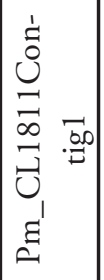 & 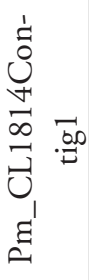 & 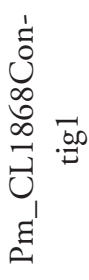 & 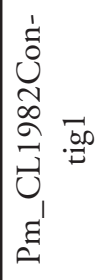 & 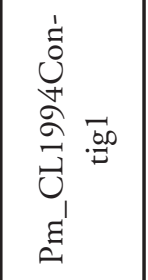 & 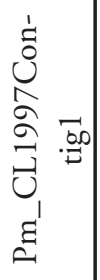 & 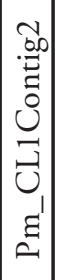 & 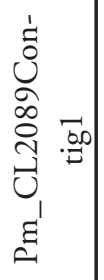 & 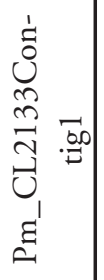 & 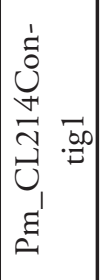 & 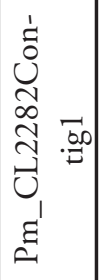 & 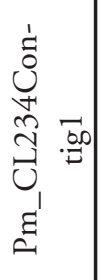 & 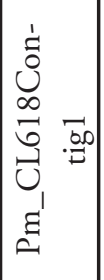 & 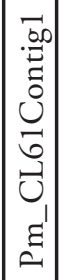 & 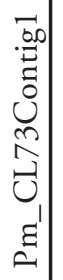 & 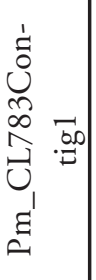 & 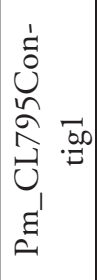 & 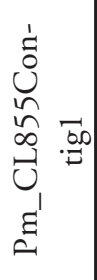 \\
\hline
\end{tabular}




\begin{tabular}{|c|c|c|c|c|c|c|c|c|c|c|c|c|c|c|c|c|c|c|}
\hline$\underset{\approx}{\mathscr{\Xi}}$ & $N$ & $\stackrel{n}{i}$ & $\hat{i}$ & $\stackrel{m}{\sim}$ & $\stackrel{n}{\sim}$ & $\sim$ & $\approx$ & $\stackrel{m}{\sim}$ & $\sim$ & $\hat{i}$ & $\stackrel{n}{i}$ & $\stackrel{n}{\sim}$ & $\hat{n}$ & $\tilde{n}$ & $\hat{v}$ & $\hat{i}$ & $\hat{i}$ & $\hat{i}$ \\
\hline 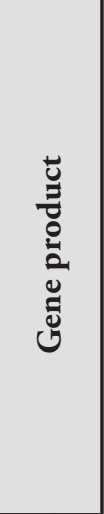 & 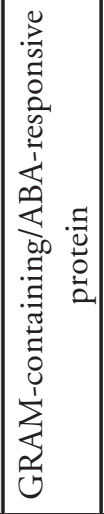 & 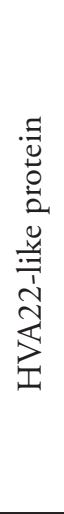 & 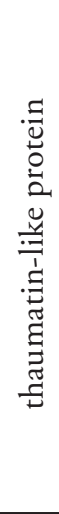 & 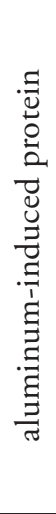 & 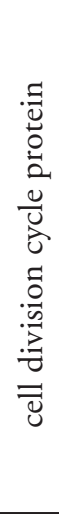 & 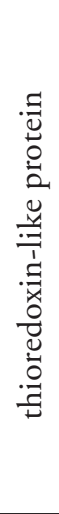 & 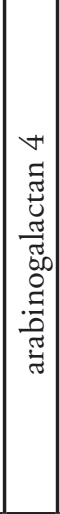 & 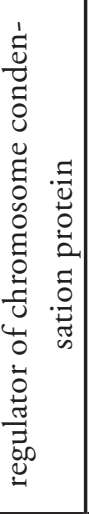 & 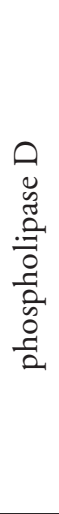 & 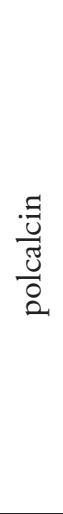 & 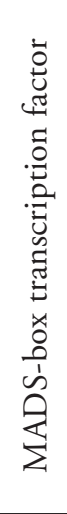 & 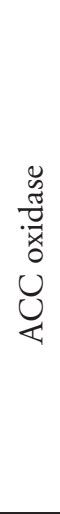 & 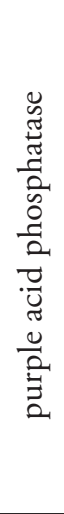 & 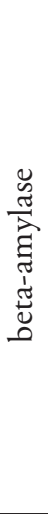 & 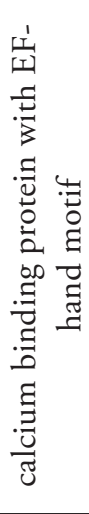 & 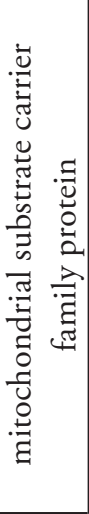 & 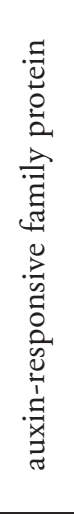 & 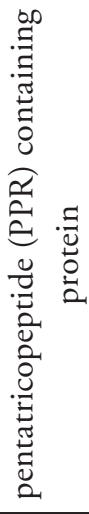 \\
\hline$F^{\prime \prime}$ & \& & 8 & 8 & 8 & 8 & 8 & $\approx$ & 8 & \& & 8 & 8 & 8 & 8 & 8 & 8 & 8 & 8 & 8 \\
\hline 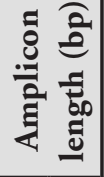 & in & $\stackrel{\infty}{\curvearrowright}$ & $\stackrel{\widetilde{F}}{*}$ & $\stackrel{\sim}{+}$ & 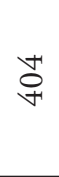 & $\hat{n}$ & $\begin{array}{l}\infty \\
\sim \\
\sim\end{array}$ & î & $\approx$ & $\underset{m}{\infty}$ & $\stackrel{\infty}{\infty}$ & 음 & $\stackrel{\mathscr{\sigma}}{*}$ & $\stackrel{\infty}{f}$ & $\infty$ & $\tilde{n}$ & $\stackrel{\infty}{n}$ & \\
\hline
\end{tabular}

\begin{tabular}{|c|c|c|c|c|c|c|c|c|c|c|c|c|c|c|c|c|c|c|}
\hline 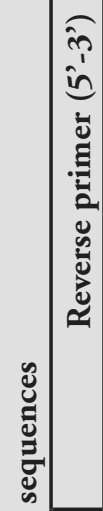 & 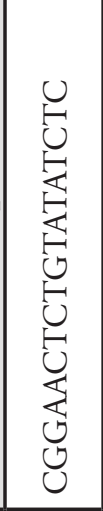 & 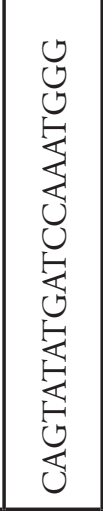 & 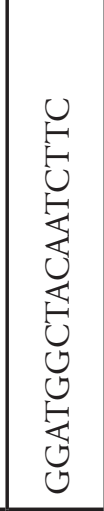 & 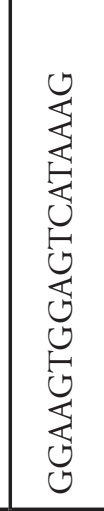 & 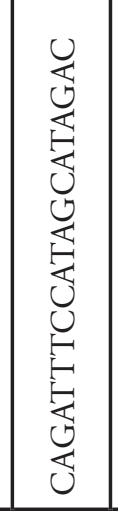 & 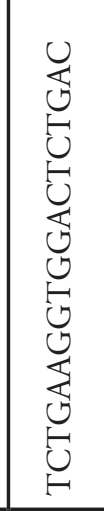 & 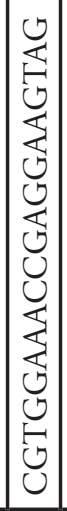 & 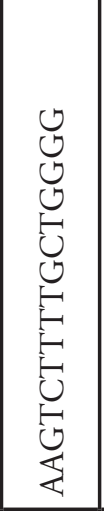 & 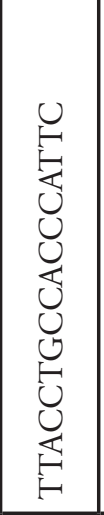 & 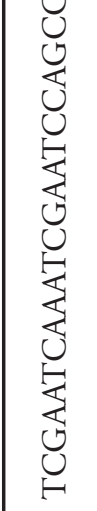 & 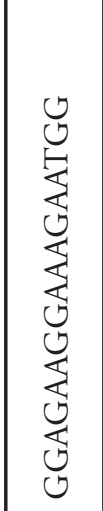 & 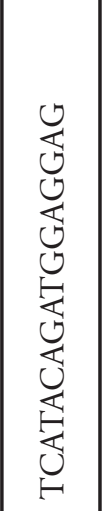 & 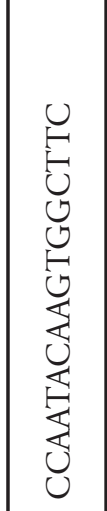 & 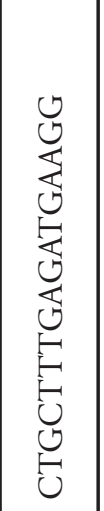 & 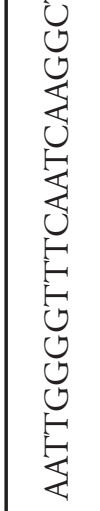 & 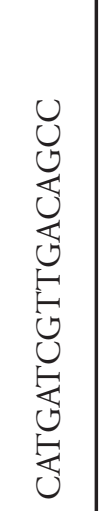 & 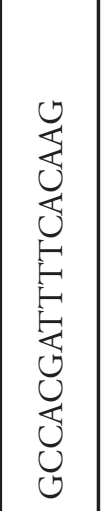 & 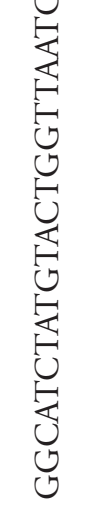 \\
\hline 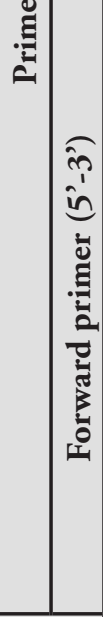 & 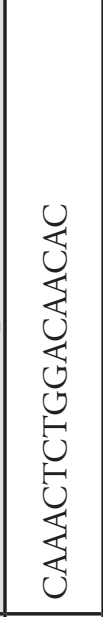 & 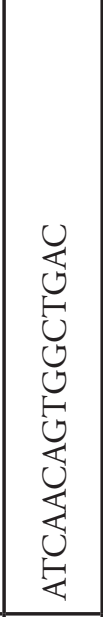 & 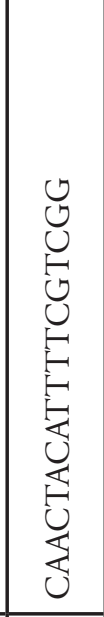 & 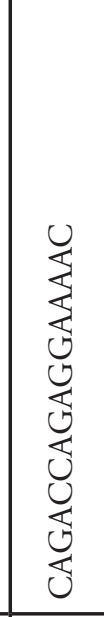 & 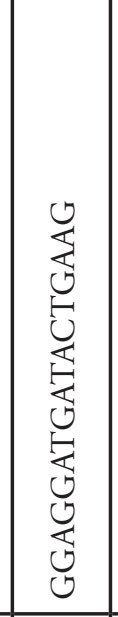 & 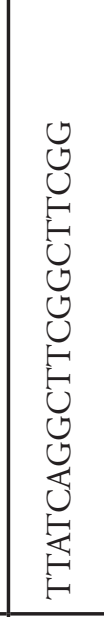 & 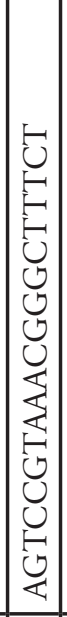 & 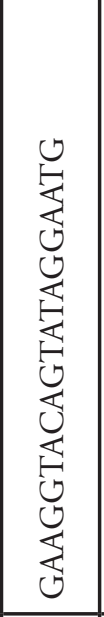 & 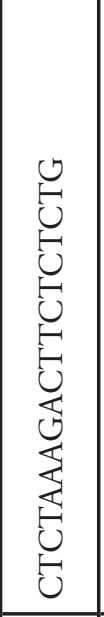 & 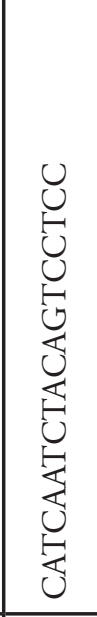 & 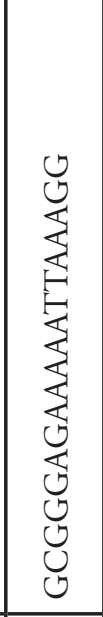 & 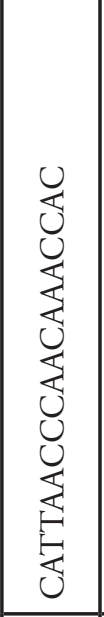 & 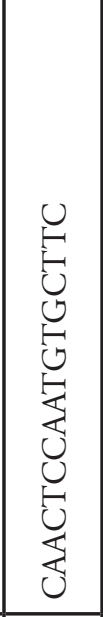 & 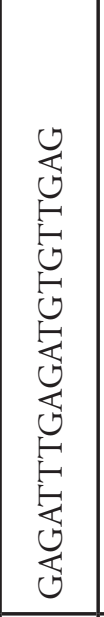 & 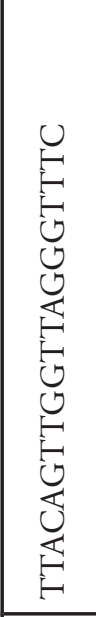 & 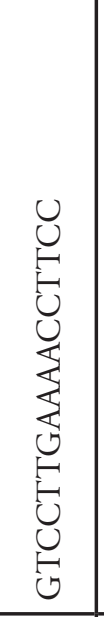 & 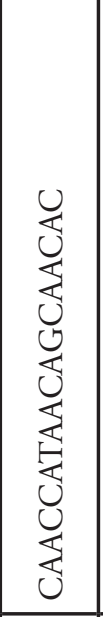 & 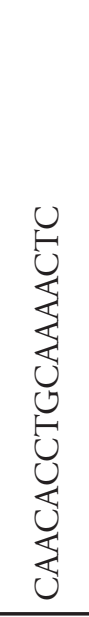 \\
\hline 胥 & $\stackrel{\infty}{\sim}$ & ส & ส & $\vec{\sim}$ & $\stackrel{\sim}{\sim}$ & $\stackrel{\sim}{\sim}$ & 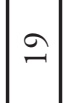 & $\vec{\sim}$ & $\stackrel{\vartheta}{\sim}$ & $\stackrel{\sim}{\sim}$ & ปี & 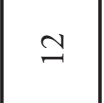 & $\stackrel{m}{=}$ & $\tilde{N}$ & $\stackrel{\sim}{\sim}$ & $\stackrel{\sim}{\sim}$ & $\vec{\lambda}$ & ন \\
\hline 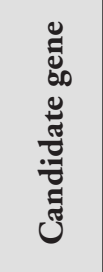 & 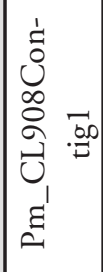 & 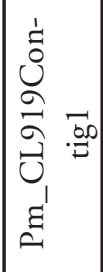 & 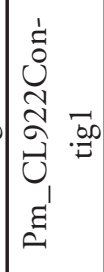 & 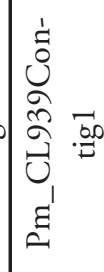 & 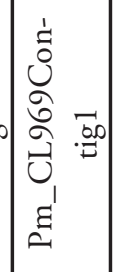 & 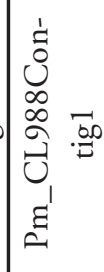 & 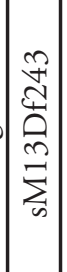 & 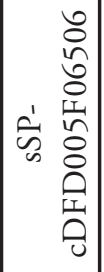 & 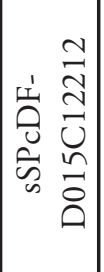 & 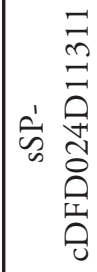 & 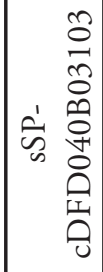 & 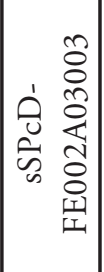 & 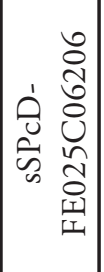 & 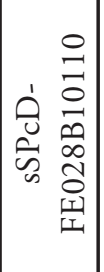 & 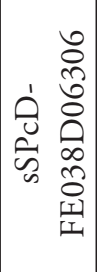 & 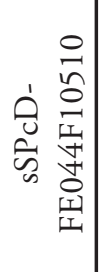 & 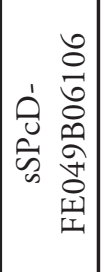 & 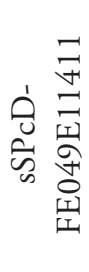 \\
\hline
\end{tabular}




\begin{tabular}{|c|c|c|c|c|c|c|}
\hline 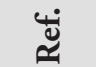 & $\stackrel{m}{i}$ & $\stackrel{m}{i}$ & $\stackrel{n}{i}$ & $\sim$ & $\stackrel{n}{i}$ & \\
\hline 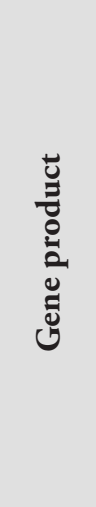 & 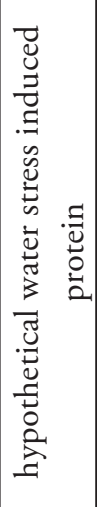 & 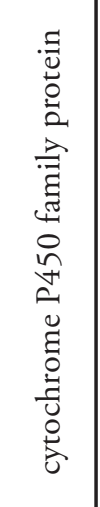 & 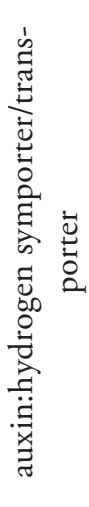 & 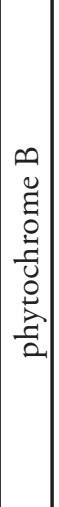 & 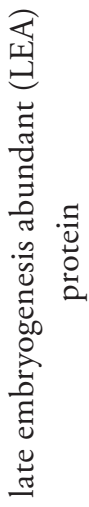 & \\
\hline$H^{\circ}$ & 8 & 8 & 8 & $\approx$ & $\hat{n}$ & $\hat{n}$ \\
\hline 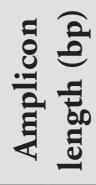 & શे & 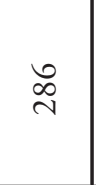 & 芯 & 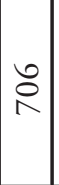 & $\stackrel{+}{\infty}$ & \\
\hline 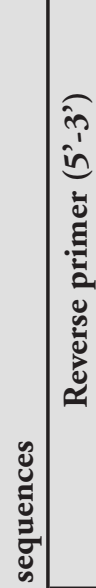 & 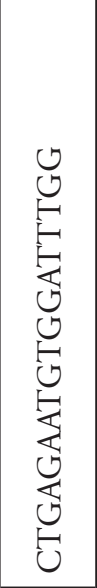 & 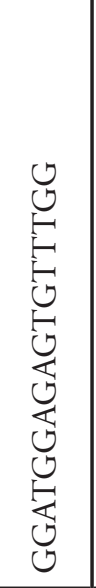 & 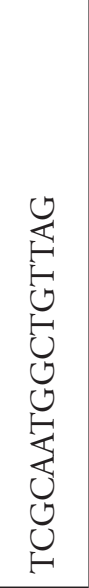 & 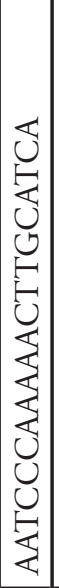 & 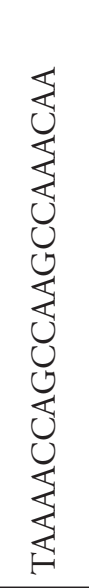 & 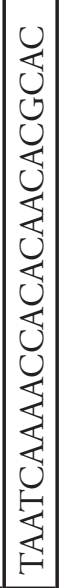 \\
\hline 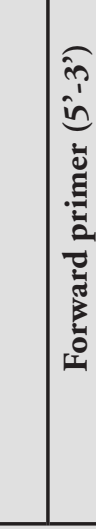 & 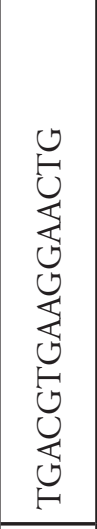 & 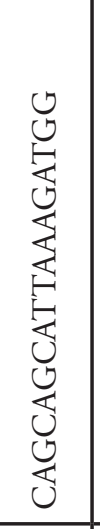 & 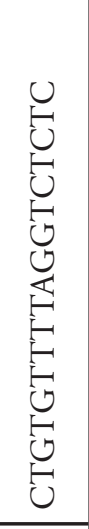 & 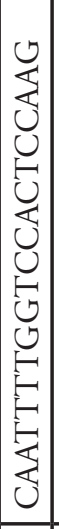 & 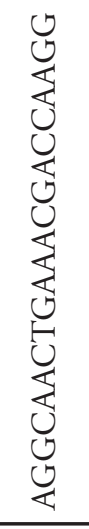 & 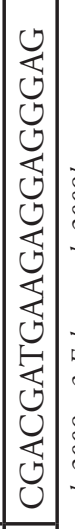 \\
\hline 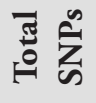 & 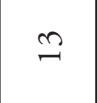 & $\ddot{\sim}$ & $\stackrel{0}{-}$ & $\stackrel{\sim}{\sim}$ & ㄱ. & \\
\hline 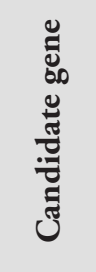 & 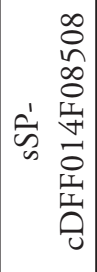 & 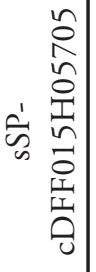 & 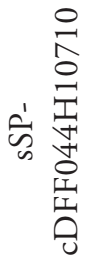 & 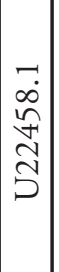 & 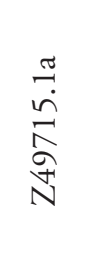 & \\
\hline
\end{tabular}

\section{Material for DNA-extraction}

The cited studies (Krutovsky and Neale 2005, Eckert et al. 2009a,b) used haploid seed megagametophytes for DNA extraction.

\section{DNA-extraction protocols}

DNA was isolated using commercial DNeasy kits (QIAGEN, Valencia, CA) such as DNeasy plant mini kit (Krutovsky and Neale 2005), or DNeasy 96 plant kit (Eckert et al. 2009b).

\section{Material and protocols for RNA-extraction and analysis}

Detailed information on the material used, RNA isolation, preparation of cDNA libraries as well as the background and technical procedure of SNPdevelopment and analysis are given in all previously mentioned publications and in Müller et al. (2012), Howe et al. (2013) and Müller et al. (2015).

\section{Important results}

- The large SNP database for Douglas-fir and the large number of putative unique transcripts (PUTs) are useful resources for the further characterization of the genome and transcriptome of Douglas-fir, for the analysis of genetic variation using genotyping or resequencing methods and for breeding applications (Eckert 2009b, Müller at al. 2012, Müller 2015, Howe et al. 2013).

- Howe et al. (2013) developed a reference transcriptome for Douglas-fir and identified 278,979 unique SNPs across both varieties. This set of SNPs was deposited in the dbSNP database with submitted SNP ID numbers (ss\#) ranging from $523,746,501$ to $524,245,331$. 183,380 SNPs were detected in more than one dataset (sort of plant material). 151,014 SNPs were detected in 17,361 isogroups in both the coastal and interior datasets. On average, the shared SNPs represented $74 \%$ of all coastal SNPs and 67\% of all interior SNPs. 8067 SNPs were validated on 260 trees using an Illumina Infinium SNP genotyping array. Out of these SNPs, $5847(72.5 \%)$ were called successfully and were polymorphic.

- Müller et al. (2015) found 79,910 SNPs, whose genotypes were called in all individuals. In their data, genetic differentiation between interior and coastal provenances as well as little differentiation 
between coastal provenances was confirmed. 58 highconfidence candidate genes for directional selection with a broad functional diversity were identified. Genes involved in drought tolerance showed a significantly higher genetic differentiation between interior and coastal Douglas-fir suggesting a different evolution despite a low level of polymorphism.

- The analysed single-nucleotide polymorphisms (SNPs) refer to candidate genes for:

1. cold hardiness (Krutovsky and Neale 2005-15 cold hardiness related genes; Eckert et al. 2009a - 121 cold hardiness candidate genes in coastal Douglas-fir),

2. drought resistance (Müller et al. 2012, Müller et al. 2015) - about 1,000 candidate genes related to drought stress with a total number of 187,653 single nucleotide polymorphisms (SNPs),

3. wood quality (Krutovsky and Neale (2005) - 3 wood quality-related genes).

\section{References}

Aas, G. (2008): Die Douglasie (Pseudotsuga menziesii) in Nordamerika: Verbreitung, Variabilität und Ökologie. LWF-Wissen, 59: 7-11.

Aagaard, J.E. (1997): Genetic diversity and differentiation in Douglas-fir from RAPD markers of nuclear and mitochondrial origin. MSc Thesis, Oregon State University, Corvallis, USA.

Aagaard, J.E., Krutovskii, K.V., Strauss, S.H. (1998a): RAPDs and allozymes exhibit similar levels of diversity and differentiation among populations and races of Douglas-fir. Heredity, 81(1): 69-78.

Aagaard, J., Krutovskii, K.V., Strauss, S. H. (1998b): RAPD markers of mitochondrial origin exhibit lower population diversity and higher differentiation than RAPDs of nuclear origin in Douglas-fir. Molecular Ecology, 7: 801-812.

Aagaard, J.E., Vollmer, S.S., Sorensen, F.C., Strauss, S.H. (1995): Mitochondrial DNA products among RAPD profiles are frequent and strongly differentiated between races of Douglas-fir. Molecular Ecology, 4(4): 441-446.

Adams, W.T., Joly, R.J. (1980): Genetics of allozyme variants in loblolly pine. Journal of Heredity, 1:33-40.

Adams, W.T., Neale, D.B., Doerksen, A.H., Smith, D.B. (1990): Inheritance and linkage of Isozyme Variants from Seed and Vegetative Bus Tissue in Coastal Douglas fir. Silvae Genetica, 39,:153-167.

Adams, W.T., Hipkins, V.D., Burczyk, J., Randall, W.K.
(1997): Pollen contamination trends in a maturing Douglas-fir seed orchard. Canadian Journal of Forest Research, 27(1):131-134.

Adams W.T., Zuo J., Shimizu, J.Y., Tappeiner J.C. (1998). Impact of alternative regeneration methods on genetic diversity in coastal Douglas-fir. For. Sci., 44(3): 390-396.

Aitken, S.N., Adams, W.T., Schermann, N.; Fuchigami, L.H. (1996): Family variation for fall cold hardiness in two Washington populations of coastal Douglas-fir (Pseudotsuga menziesii var. menziesii [Mirb.] Franco). Forest Ecology and Management, 80: 187-195.

Amarasinghe, V., Carlson, J.E. (2002): The development of microsatellite DNA markers for genetic analysis in Douglas-fir. Canadian Journal of Forest Research, 32(11):1904-1915.

Angelier, A., Archevèque, G., Aspe, P., Bastien, J.C., Boussaïd, O., Brahic, P., Durin, A. , Gauvin, J., Guérinet, S., Le Guerroué, B., Klein, E., Lamant, T., Landon, S., Lévèque, L., Lorme, P., Martin, S., Montagnon, F., Oddou-Muratorio, S., Pâques, L., Plas, G., Poursat, P., Raimbault, J.P., Rochas, D., Simmer, P., Valadon, A., Vauthier, D. (2011). Qualité génétique d'une régénération naturelle dans un peuplement mélangé de Douglas vert, Mélèze du Japon et Sapin pectiné. Internal report - conservatoire Génétique des Arbres Forestiers Office National des Forêts, pp. 184.

Carlson, J.E., Tulsieram, L.K., Glaubitz, J.C., Luk, V.W.K., Kauffeldt, C., Rutledge, R. (1991): Segregation of random amplified DNA markers in F1 progeny of conifers. Theoretical and Applied Genetics, 83(2): 194200.

Chen, Z., Kolb, T.E., Clancy, K.M., Hipkins, V.D., DeWald, L.E. (2001): Allozyme variation in interior Douglas-fir: association with growth and resistance to western spruce budworm herbivory. Canadian Journal of Forest Research, 31(10): 1691-1700.

Cheliak, W.M., Pitel, J.A. (1984): Techniques for starch electrophoresis of enzymes from forest tree species. Petawawa National Forestry Institute, Information Report PI-X-42, 49 pp.

Conkle, M.T., Hodgskiss, P.D., Nunnally, L., B., Hunter, S.C. (1982): Starch Gel Electrophoresis of Conifer Seeds: a Laboratory Manual. USDA Forest Service, General Technical Report, PSW-64, Pacific Southwest Forest and Range Experiment Station, Berkeley, CA, pp.18.

Copes, D.L. (1978): Isoenzyme activities differ in compatible and incompatible Douglas-fir graft unions. Forest Science, 24(2): 297-303. 
Davis, M. (1981): Habitat diversity and its effect on the genetic and ecological structures of two successional population of Douglas fir. $\mathrm{PhD}$ thesis, University of Colorado, $97 \mathrm{pp}$.

Doyle, J., Doyle, J. (1990): Isolation of small amount of plant tissues. Focus, 12: 13-15.

Doyle, J.J., Doyle, J.L. (1987): A rapid DNA isolation procedure for small quantities of fresh leaf tissue. Phytochemical Bulletin, 19: 11-15.

Dumolin S., Demesure B., Petit R.J., 1995. Inheritance of chloroplast and mitochondrial genomes in pedunculate oak investigated with an efficient PCR method. Theoretical and Applied Genetics, 91: 1253-1256.

Eckert, A.J., Wegrzyn, J.L., Pande, B., Jermstad, K.D., Lee, J.M., Liechty, J.D., Tearse, B.R., Krutovsky, K.V., Neale, D.B. (2009a): Multilocus patterns of nucleotide diversity and divergence reveal positive selection at candidate genes related to cold hardiness in coastal Douglas fir (Pseudotsuga menziesii var. menziesii). Genetics, 183(1):289-298.

Eckert, A.J., Bower, A.D., Wegrzyn, J.L., Pande, B., Jermstad, K.D., Krutovsky, K.V., St. Clair, J.B., Neale, D.B. (2009b): Association genetics of coastal Douglas fir (Pseudotsuga menziesii var. menziesii, Pinaceae). I. Coldhardiness related traits. Genetics, 182(4):1289-1302.

Eckhart, T., Walcher, S., Hasenauer, H., van Loo, M. (2017): Genetic diversity and adaptive traits of European versus American Douglas-fir seedlings. European Journal of Forest Research, 136: 811-825.

El-Kassaby, Y.A., Ritland, K. (1986a): Low levels of pollen contamination in a Douglas-fir seed orchard as detected by allozyme markers. Silvae Genetica, 35: 224229.

El-Kassaby, Y.A., Ritland, K. (1986b): The relation of outcrossing and contamination to reproductive phenology and supplemental mass pollination in a Douglas-fir seed orchard. Silvae Genetica, 35 (5-6): 240244.

El-Kassaby, Y.A., Davidson, R. (1990): Impact of crop management Practices on the Seed Crop genetic quality in a Douglas-fir seed orchard. Silvae Genetica, 39:230237.

El-Kassaby, Y.A., Davidson, R. (1991): Impact of pollination environment manipulation on the apparent outcrossing rate in a Douglas-fir seed orchard. Heredity, 66(1):55.

El-Kassaby, Y.A., Ritland, K. (1996a): Genetic variation in low elevation Douglas-fir of British Columbia and its relevance to gene conservation. Biodiversity and
Conservation, 5(6):779-794.

El-Kassaby, Y.A., Ritland, K. (1996b): Impact of selection and breeding on the genetic diversity in Douglas-fir. Biodiversity and Conservation, 5(6):795-813.

El-Kassaby, Y.A., Yeh, F.C., Sziklai, O. (1981): Estimation of the outcrossing rate of Douglas-fir (Pseudotsuga menziesii (Mirb.) Franco) using allozyme polymorphisms. Silvae Genetica, 30(6):182.

El-Kassaby, Y.A., Yeh, F.C., Sziklai, O. (1982): Inheritance of allozyme variants in coastal Douglas-fir (Pseudotsuga menziesii var. menziesii). Canadian Journal of Genetics and Cytology, 24(3):325-335.

Erickson, V.J., Adams, W.T. (1989): Mating success in a coastal Douglas-fir seed orchard as affected by distance and floral phenology. Canadian Journal of Forest Research, 19, 1248-1255.

Fontes, L., Savill, P., Luis, J.S., Harris, S. (2003): Identification of the origin of Portuguese Douglas-fir [Pseudotsuga menziesii (Mirb.) Franco] provenances. Silvae Genetica, 52: 266-273.

Fussi, B., Konnert, M., Dounavi, A. (2013): Identification of varieties and gene flow in Douglas fir exemplified in artificially established stands in Germany. Annals of Forest Research, 56(2): 249-268.

Konnert, M., Fussi, B. (20 I 2): Natürliche und künstliche Verjüngung der Douglasie in Bayern aus genetischer Sicht. Schweizerische Zeitschrift für Forstwesen 163, 3: 79-87

Gould, P.J., Harrington, C.A., Clair, J.B.S (2012): Growth phenology of coast Douglas-fir seed sources planted in diverse environments. Tree Physiology, 32:1482-1496.

Guillemaut, P., Maréchal-Drouard, L. (1992): Isolation of plant DNA: a fast, inexpensive, and reliable method. Plant. Mol. Biol. Rep.10:60-65.

Gugger, P.F., González-Rodríguez, A., RodríguezCorrea, H., Sugita, S., Cavender-Bares, J. (2011): Southward Pleistocene migration of Douglas-fir into Mexico: phylogeography, ecological niche modeling, and conservation of 'rear edge' populations. New Phytologist, 189(4): 1185-1199.

Gugger, P.F., Sugita, S., Cavender-Bares, J. (2010): Phylogeography of Douglas-fir based on mitochondrial and chloroplast DNA sequences: testing hypotheses from the fossil record. Molecular Ecology, 19(9): 1877-1897.

Hermann, R.K., Lavender, D.P. (1990): In: Burns, R.M., Barbara, H., Honkala, B.H. (Eds.). Silvics of North America: 1.Conifers. Agriculture Handbook 654, 
Washington DC, 527-540.

Hintsteiner, W.J., van Loo, M., Neophytou, Ch., Schueler, S., Hasenauer, H. (2018): The geographic origin of old Douglas-fir stands growing in Central Europe. European Journal of Forest Research 05/2018.

Hipkins, V.D., Marshall, K.A., Neale, D.B., Rottmann, W.H., Strauss, S.H. (1995): A mutation hotspot in the chloroplast genome of a conifer (Douglas-fir: Pseudotsuga) is caused by variability in the number of direct repeats derived from a partially duplicated tRNA gene. Current Genetics, 27(6): 572-579.

Howe, G.T., Yu, J., Knaus, B., Cronn, R., Kolpak, S., Dolan, P., Lorenz, W.W., Dean, J.F.D. (2013): A SNP resource for Douglas-fir: de novo transcriptome assembly and SNP detection and validation. BMC Genomics, 14(1): 137.

Jermstad, K.D., Reem, A.M., Henifin, J.R., Wheeler, N.C., Neale, D.B. (1994): Inheritance of restriction fragment length polymorphisms and random amplified polymorphic DNAs in coastal Douglas-fir. Theoretical and Applied Genetics, 89(6): 758-766.

Kess, T., El-Kassaby, Y.A. (2015): Estimates of pollen contamination and selfing in a coastal Douglas-fir seed orchard. Scandinavian Journal of Forest Research, 30(4), 266-275.

Klumpp, R. (1999): Untersuchungen zur Genökologie der Douglasie (Pseudotsuga menziesii (Mirb.) Franco). [Gene-ecological investigations of Douglas fir (Pseudotsuga menziesii (Mirb.) Franco)]. Dissertation, University of Göttingen, p. 289.

Konnert M. (2004): Handbücher für Isoenzymanalyse. Isoenzymuntersuchungen bei Douglasie (Pseudotsuga menziesii)- Anleitungen zur Trennmethodik und Auswertung der Zymogramme. [Handbook for isoenzyme analysis. Isoenzyme investigations in Douglas fir (Pseudotsuga menziesii) - Guide on separation methodology and evaluation of zymograms] Web: https://blag-fgr.genres.de/ausgewaehlte-informationen/ handbuecher-fuer-isoenzymanalysen/. Accessed 201803-08.

Konnert, M., Fussi, B. (2012): Natürliche und künstliche Verjüngung der Douglasie in Bayern aus genetischer Sicht. Schweizerische Zeitschrift für Forstwesen, 163(3): 79-87.

Konnert, M., Ruetz, W. (2006): Genetic aspects of artificial regeneration of Douglas-fir (Pseudotsuga menziesii) in Bavaria. European Journal of Forest Research, 125(3): 261-270.

Korecký J., El-Kassaby Y.A. (2016): Pollination dynamics variation in a Douglas-fir seed orchard as revealed by microsatellite analysis. Silva Fennica, 50(4): 1-12.

Krutovskii, K.V., Vollmer, S.S., Sorensen, F.C., Adams, W.T., Knapp, S.J., Strauss, S.H. (1998): RAPD genome maps of Douglas-fir. Journal of Heredity, 89(3): $197-$ 205.

Krutovsky, K.V., Clair, J.B.S., Saich, R., Hipkins, V.D., Neale, D.B. (2009): Estimation of population structure in coastal Douglas-fir [Pseudotsuga menziesii (Mirb.) Franco var. menziesii] using allozyme and microsatellite markers. Tree Genetics \& Genomes, 5(4): 641-658.

Krutovsky, K.V., Neale, D.B. (2005): Nucleotide diversity and linkage disequilibrium in cold-hardinessand wood quality-related candidate genes in Douglas fir. Genetics, 171(4): 2029-2041.

Lavender, D. P., Hermann, R. K. (2014): Douglas-fir: the genus Pseudotsuga. Corvallis, OR: Forest Research Publications Office, Oregon State University.

Leinemann, L. (1996): Genetic differentiation of damaged and healthy Douglas-fir stands in RheinlandPfalz with respect to their origin. Silvae Genetica, 45: 250-256.

Leinemann, L., Maurer, W. (1999): Bedeutung von Isoenzymgenmarkern für den Anbau der Douglasie. AFZ/Der Wald, 5: 242-243.

Li, P., Adams W.T., (1989): Range wide patterns of allozyme variation in Douglas-fir (Pseudotsuga menziesii). Canadian Journal of Forest Research, 19: 149-161.

Merkle, S.A., Adams, W.T. (1987): Patterns of allozyme variation within and among Douglas fir breeding zones in southwest Oregon. Canadian Journal of Forest Research, 17, 402-407,

Merkle, S.A., Adams, W.T. (1988): Multivariate analysis of allozyme variation patterns in coastal Douglas-fir from southwest Oregon. Canadian Journal of Forest Research,18: 181-187.

Mitton, J.B., Linhart, Y.B., Sturgeon, K.B., Hamrick, J.Ll (1979): Allozyme polymorphism detected in mature needle tissue of ponderosa pine. Journal of Heredity, 70: 86-89.

Milenkova, A., Konnert, M., Fusi, B., Petkova, K. (2018): Identification of varieties and genetic diversity of Douglas fir stands in the region of Osogovo, Southwest Bulgaria. Forest Ideas, (accepted for publication).

Moran, G.F., Adams, W.T. (1989): Microgeographical patterns of allozyme differentiation in Douglas-fir from southwest Oregon. Forest Science, 35: 3-15.

Müller, T., Ensminger, I., Schmid, K.J. (2012): A 
catalogue of putative unique transcripts from Douglasfir (Pseudotsuga menziesii) based on 454 transcriptome sequencing of genetically diverse, drought stressed seedlings, BMC Genomics 13: 673.

Müller, T., Freund, F., Wildhagen H., Schmid, K.J. (2015): Targeted re-sequencing of five Douglas-fir provenances reveals population structure and putative target genes of positive selection. Tree Genetics \& Genomes, 11: 816.

Neale, D.B. (1984): Population genetic structure of the Douglas-fir shelterwood regeneration system in southwest Oregon. PhD Dissertation, Oregon State University, Corvallis. Diss Abstr LD4330-1984D-N4.

Neale D.B. (1985). Genetic implication of shelterwoord regeneration of Douglas-fir in southwest Oregon. For. Sci., 3:995-1005.

Neale, D.B., Weber, J.C., Adams, W.T. (1984): Inheritance of needle tissue isozymes in Douglas-fir. Canadian Journal of Genetics and Cytology, 26: 459468.

Neale, D.B., Adams, W.T. (1985): The mating system in natural and shelterwood stands of Douglas-fir. Theoretical and Applied Genetics, 71(2): 201-207.

Nelson, R.J., Stoehr, M., Cooper, G., Smith, C., Mehl, H. (2003): High levels of chloroplast genetic variation differentiate coastal and interior Douglas-fir (Pseudotsuga menziesii) lineages in southern British Columbia. Forest Genetics, 10(2):153-158.

Neophytou, Ch., Weisser, A.M., Landwehr, D., et al. (2016): Assessing the relationship between height growth and molecular genetic variation in Douglas-fir (Pseudotsuga menziesii) provenances. European Journal of Forest Research, 135: 465-481

Ponoy, B. (1993): Genetic variability in Douglas-fir based on molecular genetic markers and morphological traits. Doctoral dissertation, University of British Columbia, p. 187.

Prat, D., Arnal, S. (1994): Allozyme variation and mating system in three artificial stands of Douglas fir (Pseudotsuga menziesii (Mirb.) Franco) planted in Europe. Silvae Genetica 43(4): 199-206.

Prat, D. (1995): Mating system in a clonal Douglas fir (Pseudotsuga menziesii (Mirb) Franco) seed orchard. II. Effective pollen dispersal. In Annales des sciences forestières, 52(3): 213-222. EDP Sciences.

Prat, D., Burczyk, J. (1998): Genetic variation and mating system in a native provenance and the derived seed orchard of Douglas-fir (Pseudotsuga menziesii (Mirb.) Franco). Forest Genetics, 5(4): 201-209.
Provan, J., Soranzo, N., Wilson, N.J., McNicol, J.W., Forrest, G.I., Cottrell, J., Powell, W. (1999): Gene pool variation in Caledonian and European Scots pine (Pinus sylvestris L.) revealed by chloroplast simple sequence repeat. Proceedings of the Royal Society of London, 265: 1697-1705.

Schober, R. (1954): Douglasien-Provenienzversuche, Teil I. Allgemeine Forst- und Jagdzeitung, 125: 160-178.

Shaw, C.R., Prasad, R. (1970): Starch gel electrophoresis of enzymes - A compilation of recipes. Biochemical Genetics, 4: 297-320.

Shaw, D.V., Allard, R.W. (1982): Estimation of outcrossing rates in Douglas-fir using isozyme markers. Theoretical and Applied Genetics, 62(2): 113-120.

Shaw, J., Lickey, E.B., Beck, J.T., Farmer, S.B., Liu, W., Miller, J., Siripun, K.C., Windel, C.T., Schilling, E.E., Small, R.L. (2005): The tortoise and the hare II: relative utility of 21 noncoding chloroplast DNA sequences for phylogenetic analysis. American Journal of Botany, 92: 142-166.

Sk Lai, B., Funda, T., Liewlaksaneeyanawin, C., Klápště, J., Van Niejenhuis, A., Cook, C., Stoeher, M.U., Woods, J., El-Kassaby, Y.A. (2010): Pollination dynamics in a Douglas-fir seed orchard as revealed by pedigree reconstruction. Annals of Forest Science, 67(8): 808808.

Slavov, G.T., Howe, G.T., Adams, W.T. (2005): Pollen contamination and mating patterns in a Douglas-fir seed orchard as measured by simple sequence repeat markers. Canadian Journal of Forest Research, 35(7): 1592-1603.

Slavov, G.T., Howe, G.T., Yakovlev, I., Edwards, K.J., Krutovskii, K.V., Tuskan, G.A., Carlson, J.E., Strauss, S.H., Adams, W.T. (2004): Highly variable SSR markers in Douglas-fir: Mendelian inheritance and map locations. Theoretical and Applied Genetics, 108(5): 873-880.

Stauffer H., Adams W.T. (1993): Allozyme variation and mating system of three Douglas-fir $\mathrm{r}$ stands in Switzerland. Silvae Genetica, 42: 254-258.

St. Clair, J.B., Mandel, N.L., Vance-Borland, K.W. (2005): Genecology of Douglas Fir in Western Oregon and Washington. Annals of Botany, 96: 1199-1214.

St. Clair, J.B. (2006): Genetic variation in fall cold hardiness in coastal Douglas-fir in western Oregon and Washington. Canadian Journal of Forest Research, 84: $1110-1121$.

Stoehr, M.U., Orvar, B.L., Vo, T.M., Gawley, J.R., Webber, J.E., Newton, C.H. (1998): Application of a chloroplast DNA marker in seed orchard management evaluations of Douglas-fir. Canadian Journal of Forest 
Research, 28(2): 187-195.

Tsai, C.H., Strauss, S.H. (1989): Dispersed repetitive sequences in the chloroplast genome of Douglas-fir. Current Genetics, 16(3): 211-218.

Tsumura, Y., Ohba, K., Strauss, S.H. (1996): Diversity and inheritance of inter-simple sequence repeat polymorphisms in Douglas-fir (Pseudotsuga menziesii) and sugi (Cryptomeria japonica). Theoretical and applied genetics, 92(1): 40-45.

van Loo, M., Hintsteiner, W., Pötzelsberger, E., Schüler, S., Hasenauer, H. (2015): Intervarietal and intravarietal genetic structure in Douglas-fir: nuclear SSRs bring novel insights into past population demographic processes, phylogeography, and intervarietal hybridization. Ecology and Evolution, 5(9): 1802-1817.

Vendramin, G.G., Lelli, L., Rossi, P., Morgante, M. (1996): A set of primers for the amplification of 20 chloroplast microsatellites in Pinaceae. Molecular Ecology, 5: 595-598.

Viard, F., El-Kassaby, Y.A., Ritland, K. (2001): Diversity and genetic structure in populations of Pseudotsuga menziesii (Pinaceae) at chloroplast microsatellite loci. Genome, 44(3): 336-344.

Wagner, D.B., Fumier, G.R., Saghai-Madroof, M.A., Williams, S.M., Cancik, B.P., Allard, R.W. (1987): Chloroplast DNA polymorphisms in lodgepole and jack pines and their hybrids. Proceedings of the National Academy of Sciences of the USA, 84: 2097-2100.

Wei, X.X., Beaulieu, J., Khasa, D.P., Vargas-Hernández, J., López-Upton, J., Jaquish, B., Bousquet, J. (2011): Range-wide chloroplast and mitochondrial DNA imprints reveal multiple lineages and complex biogeographic history for Douglas-fir. Tree Genetics \& Genomes, 7(5): 1025-1040.

Westfall, R.D., Conkle, M.T. (1992): Allozyme markers in breeding zone designation. In Population Genetics of Forest Trees, Springer, Dordrecht, pp. 279-309.

Williams, J.G.K., Kubelik, A.R., Livak, K.J., Rafalski, J.A., Tingey, S.V. (1990): DNA polymorphisms amplified by arbitrary primers are useful as genetic markers. Nucleic Acids Research, 18: 6531-6535.

Yang, J.C. (1974): Isoenzyme polymorphism in provenances of Douglas-fir (Pseudotsuga menziesii [Mirb.] Franco var. menziesii). Oregon State University, Doctoral dissertation.

Yeh, F.C., Morgan,K. (1987): Mating system and multilocus associations in a natural population of Pseudotsuga menziesii (Mirb.) Franco. Theoretical and
Applied Genetics 78: 799-808.

Yeh, F.C., O'Malley, D. (1980): Enzyme variations in natural populations of Douglas-fir, Pseudotsuga menziesii (Mirb.) Franco, from British Columbia. 1. Genetic variation patterns in coastal populations. Silvae Genetica, 29: 83-92.

Zietkiewicz, E., Rafalski A., Labuda, D. (1994): Genome fingerprinting by simple sequence repeat (SSR)-anchored polymerase chain reaction amplification. Genomics 20: 176-183. 


\title{
Molecular markers used for genetic studies in Sitka spruce (Picea sitchensis (Bong.) Carr.)
}

\author{
Branislav Cvjetković ${ }^{1}$, Jason Holliday ${ }^{2}$, Monika Konnert ${ }^{3}$, Anna-Maria Szasz-Len ${ }^{3}$ \\ ${ }^{1}$ University of Banja Luka, Faculty of Forestry, Department for Forest Genetics and Afforestation/Reforestation, Bullevar Vojvode Stepe \\ Stepanovića 75A, 78000 Banja Luka, Bosnia and Hercegovina \\ ${ }^{2}$ Department of Forest Resources and Environmental Conservation, Virginia Tech, 451 Latham Hall, USA \\ ${ }^{3}$ Bavarian Office for Forest Seeding and Planting, Forstamtsplatz 1, D-83317 Teisendorf (Germany)
}

\section{General remarks}

The native range of Sitka spruce (Picea sitchensis (Bong.) Carr.) occupies a narrow band along the northwest coast of North America, from Kodiak Island and Prince William Sound $\left(61^{\circ} 00 \mathrm{~N}\right)$, in the western Gulf of Alaska to California, Mendocino County (latitude $39^{\circ} 20$ $\mathrm{N})$. In total, its north to south natural distribution is approximately $2.900 \mathrm{~km}$, whereas the west to east extension is nowhere more than $400 \mathrm{~km}$ from the Pacific Ocean (Figure 1). The species reaches elevation up to $700 \mathrm{~m}$ a.s.l. The range of Sitka spruce is dependent on abundant moisture content during the growing season and its maximum development occurs when summer precipitation is high and there is no pronounced summer drought (Roche and Haddock, 1987).

The species was firstly described in 1787 by the Scottish botanist Archibald Menzies, who recorded it on the shores of Puget Sound. In Europe, it was first introduced

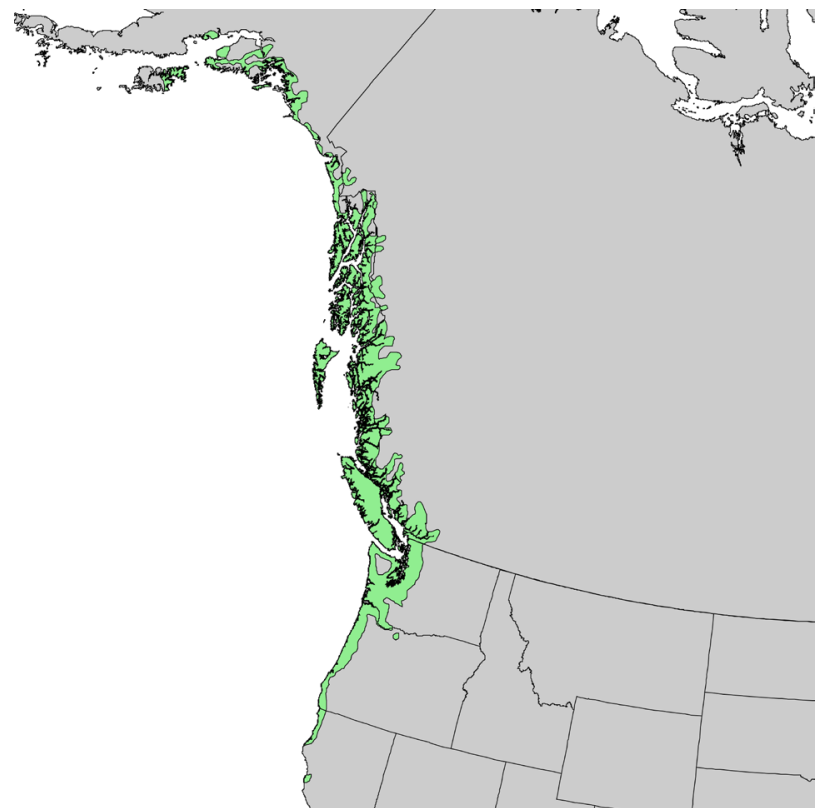

Figure 1. Native distribution range of Sitka spruce (source: USGS, USA) into Great Britain in 1831 by the Horticultural Society of London from seed collected by David Douglas, who named it Pinus menziesii (Mitchell 1978). The north part of Europe, especially Ireland, United Kingdom, Norway, France and Denmark was the main destination for Sitka spruce (Mason and Perks 2011). Sitka spruce is also grown in Iceland and southern Sweden and several stands have been established in New Zealand (Peterson et al. 1997), too. Hybridization with white spruce (Picea glauca) is frequent and well explored (e.g. Bennuah 2004; Hamilton et al. 2012; 2013).

Most of the genetic markers used for investigation on Sitka spruce had been developed for Norway spruce and white spruce and later transferred to Sitka spruce. The present overview on genetic markers used for genetic investigations in Sitka spruce might give better insight into marker efficiency and lead researchers to easier choice of the right marker depending on research goals.

\section{Isozymes}

First publications on isozymes for Sitka spruce appeared in the second half of the $1970^{\text {th }}$. Further on, isozymes were used to study:

- the genetic variation pattern of natural stands (Yeh and El-Kassaby, 1980) and to compare it with gene diversity of other species (Douglas fir, lodgepole pine) from the same region (Yeh, 1979);

- to differentiate Sitka spruce, white spruce and their hybrids and to analyse introgression aspects (Copes and Beckwith 1977, Yeh and Arnott, 1986);

- to describe diversity and outcrossing rates in Sitka spruce seed orchards (Cottrell and White 1995, Chaisurisri et al. 1994) in comparison with natural populations as well (Chaisurisri and El-Kassaby 1994).

The list of enzymes, number of loci and maximum number of alleles per locus found in the cited studies is presented in Table 1. 
Table 1: List of enzymes, scored loci and number of alleles for Sitka spruce

\begin{tabular}{|c|c|c|c|c|}
\hline Enzyme & $\begin{array}{c}\text { E.C. } \\
\text { Number }\end{array}$ & Scored loci & $\begin{array}{l}\text { No. of } \\
\text { alleles* }\end{array}$ & Reference \\
\hline Aspartate aminotransferase & 2.6.1.1 & AAT-1,-2 resp. GOT-1,-2 & 3,3 & $1,2,3,4,5$ \\
\hline Aconitase & 4.2 .1 .3 & ACO-1 & 3 & $1,3,4$ \\
\hline Aldolase & 4.1 .2 .13 & ALD-1 & 1 & 3,4 \\
\hline Alcohol dehydrogenase & 1.1.1.1 & $\mathrm{ADH}-1$ & 3 & 5 \\
\hline Diaphorase & 1.6.2.3 & DIA-1,-2,-3 & $3,2,2$ & $3,4,5$ \\
\hline Esterase & 3.1 .1 .2 & EST-1 & 4 & $1,4,5$ \\
\hline Glutamate dehydrogenase & 1.4 .1 .3 & GDH-1 & 3,3 & $1,2,3,4,5$ \\
\hline Glucose-6-phosphate dehydrogenase & 1.1 .1 .49 & G6PDH-1 & 3 & $1,3,4,5$ \\
\hline Isocitrate dehydrogenase & 1.1.1.42 & IDH-1 & 3 & $1,3,4,5$ \\
\hline Malate dehydrogenase & 1.1 .1 .37 & $\mathrm{MDH}-1,-2,-3$ & $4,3,4$ & $1,3,4,5$ \\
\hline Malic enzyme & 1.1 .1 .39 & ME-1 & 2 & 3,4 \\
\hline Peptidase & 3.4 .14 .5 & PEP-1,-2,-3 & $1,1,1$ & 3,4 \\
\hline Phosphoglucose isomerase & 5.3 .1 .9 & PGI-1,-2 & 4,3 & $1,2,3,4,5$ \\
\hline Phosphoglucomutase & 2.7 .5 .1 & PGM-1,-2 & 3,5 & $1,2,3,4,5$ \\
\hline 6-Phosphogluconate dehydrogenase & 1.1 .1 .44 & 6PGDH-1,-2,-3 & $3,4,3$ & $1,3,4,5$ \\
\hline
\end{tabular}

\section{Material for protein extraction}

Proteins were extracted from dormant germ buds (Copes and Beckwith, 1977) and megagametophytes (Yeh, 1979; Yeh and Kassaby, 1980; Chaisurisri and ElKassaby 1993, 1994; Yeh and Arnot, 1986).

\section{Protein extraction and separation protocols}

Isozyme extraction, separation by starch gel electrophoresis and staining of gels were carried out by procedures described in detail by Yeh and O'Malley (1980) (protocol developed first for Douglas fir) and Yeh and Layton (1979) (protocol developed first for lodgepole pine).

\section{Important results:}

- Some isozymes (e.g. GDH, 6PGDH) seem to be useful to distinguish between Sitka spruce and white spruce. Therefore, they can be used for taxonomic classification of spruce seed lots collected in areas where hybridization between the two species occurs (Copes and Beckwith 1977, Yeh and Arnott, 1986).

- For populations in the natural range only little differentiation at isozyme loci has been found. The genetic diversity within populations is high (92\%). Only $8 \%$ of the gene diversity was attributed to differentiation between populations (Yeh and ElKassaby, 1980).

- Significant differences in gene diversity and heterozygosity estimates were found between a seed orchard in British Columbia and natural populations. The seed orchard population showed a significantly higher number of alleles per locus and percentage of polymorphic loci (Chaisurisri and ElKassaby 1994).

- In seed orchards outcrossing rates and pollen allelic frequencies differ between trees. Minor differences in pollen allelic frequencies were observed in the upper vs. the lower crown, whereas outcrossing rate of the upper crown exceeds that of the lower crown (Chaisurisri et al. 1994).

- For a Sitka spruce seed orchard in Scotland, high estimates of outcrossing rates were found in a year with intensive flowering. The attempt to identify all clones by means of four enzyme systems failed. Due to the comparatively low degree of polymorphism of the enzymes GOT, PGM, PGI and GDH used, only $42 \%$ of the clones could be identified (Cotrell and White 1995). 


\section{Organelle DNA markers (chloroplast (cp) DNA, mitochondrial (mt)DNA)}

\section{Loci and primers used}

Studies dealing with genetic analysis of organelle DNA markers in Sitka spruce are rare. As mitochondrial DNA is maternally inherited, whereas chloroplast DNA paternally, organelle DNA markers are suitable to analyze the introgression between coastal Sitka spruce (Picea sitchensis) and interior spruce species (Picea glauca and Picea engelmanii) (Szmidt et al. 1988, Sutton et al. 1991, Hamilton and Aitken 2013). Organelle markers have been also used in a phylogenetic study of the genus Picea, which includes also Picea sitchensis (Ran et al. 2006). Besides nuclear SSRs, Hamilton and Aitken (2013) applied one chloroplast PCR-RFLP marker and one mitochondrial marker to study the mechanisms involved in tree migration and adaptation in response to past environmental changes.

Coombe et al. (2016) published the first Sitka spruce chloroplast genome assembled exclusively from Picea sitchensis genomic libraries prepared using the 10X Genomics protocol.

Szmidt et al. (1988) and Sutton et al. (1991) analyzed restriction fragment length polymorphisms (RFLP) from the chloroplast DNA (cpDNA). According to Szmidt (1988), cpDNA was digested with four restriction endonucleases - Bam-HI, Bcl-I, Kpn-I and Sac-I - each of which recognizes a specific six base pair nucleotide sequence for cleavage. Sutton et al. (1991) digested mtDNA with Bam-HI before cloning. In the aforementioned papers, morphologically pure trees of white spruce (Picea glauca), western white spruce (Picea glauca var. albertiana) and Engelmann spruce (Picea engelmannii) were also analyzed in addition to Sitka spruce (Picea sitchensis).
Hamilton and Aitken (2013) amplified one universal cpDNA locus and one mtDNA locus and sequenced the products to find polymorphisms. Ran et al. (2006) also sequenced the maternally inherited mitochondrial nad5 intron and two paternally inherited chloroplast regions (see Table 2).

\section{Material for DNA-extraction}

DNA was extracted from fresh buds (Hamilton and Aitken 2013) or needles from adult trees (Szmidt et al. 1988, Sutton et al. 1991, Coombe et al. 2016, Ran et al. 2006) and seedlings four months after germination (Szmidt et al. 1988).

\section{DNA-extraction and amplification protocols}

Total DNA was extracted from ground, frozen needle tissue using the Doyle and Doyle (1990) CTAB protocol (Ran et al. 2006, Hamilton and Aitken 2013) and the method of Wagner et al. (1987) (Sutton at al. 1991).

For the extraction of chloroplast DNA from frozen needles, Szmidt et al. (1988) applied a method described by Szmidt et al. (1986), which represents a modified version of White's (1986) extraction protocol.

Amplification of the two cpDNA regions and the nad5 region of mtDNA was done using the following PCRprotocol:

- one cycle of $4 \mathrm{~min}$ at $70{ }^{\circ} \mathrm{C}, 4$ cycles of $2 \mathrm{~min}$ at $94^{\circ} \mathrm{C}, 20 \mathrm{~s}$ at $55^{\circ} \mathrm{C}(\operatorname{trnC}-\operatorname{trn} \mathrm{D})$ or $50^{\circ} \mathrm{C}(\operatorname{trn} \mathrm{T}-$ trnF and nad5), and 2 min at $72{ }^{\circ} \mathrm{C}$, followed by 36 cycles of $20 \mathrm{~s}$ at $94^{\circ} \mathrm{C}, 20 \mathrm{~s}$ at $55^{\circ} \mathrm{C}$ (trnC-trnD) or $50^{\circ} \mathrm{C}\left(\operatorname{trn} \mathrm{T}-\operatorname{trnF}\right.$ and nad5), and $2 \mathrm{~min}$ at $72^{\circ} \mathrm{C}$, with a final extension step for $10 \mathrm{~min}$ at $72{ }^{\circ} \mathrm{C}$ (Ran et al. 2006, Hamilton and Aitken 2013).

Table 2: Primer information of the chloroplast microsatellite markers used in genetic analysis of Sitka spruce $\left(T_{a}=\right.$ annealing temperature)

\begin{tabular}{|c|c|c|c|c|c|c|}
\hline Type & Locus & $\begin{array}{l}\text { Primer sequence }\left[5^{6}-3^{6}\right] \\
F=\text { Forward, R= Reverse }\end{array}$ & $\begin{array}{c}T_{a} \\
\left({ }^{\circ} \mathrm{C}\right)\end{array}$ & $\begin{array}{l}\text { Size } \\
(\mathbf{b p})\end{array}$ & Ref. & $\begin{array}{c}\text { Source of primer } \\
\text { pairs }\end{array}$ \\
\hline \multirow[t]{2}{*}{ cpDNA sequencing } & $\operatorname{trn} T-\operatorname{trnF}$ & $\begin{array}{l}\text { F:CATTACAAATGCGATGCTCT } \\
\text { R:ATTTGAACTGGTGACACGAG }\end{array}$ & 50 & 500 & 1,2 & $\begin{array}{l}\text { Taberlet et al. } \\
\text { (1991) }\end{array}$ \\
\hline & $\operatorname{trnC}-\operatorname{trn} \mathrm{D}$ & $\begin{array}{l}\text { F:CCAGTTCGAATCCGGGTGTC } \\
\text { R:GGGATTGTAGCTCAATTGGT }\end{array}$ & 55 & 2324 & 1 & $\begin{array}{c}\text { Demesure et al. } \\
\text { (1995) }\end{array}$ \\
\hline mtDNA sequencing & nad5a.1 & $\begin{array}{l}\text { F:CGCATATGGGTAGCAAGAGGGC } \\
\text { R:GAGGTTTCCCATCACACGGCTCACC }\end{array}$ & 50 & 500 & 1,2 & $\begin{array}{c}\text { Wang et al. 2000, } \\
\text { Jaramillo-Correa } \\
\text { and Bousquet, } \\
(2003)\end{array}$ \\
\hline
\end{tabular}

1-Ran et al. 2006, 2-Hamilton and Aitken 2013 


\section{Important results}

- Coombe et al (2016) found out that the chloroplast genome is 124,049 base pair long. It shares high sequence similarity with the related white spruce and Norway spruce chloroplast genomes.

- The cpDNA restriction patterns generated by Bam$H$ I, $B c l$-I from individual trees of Sitka spruce, white spruce and Engelmann spruce were species-specific. Based on this finding, Szmidt et al. (1988) used cpDNA restriction polymorphism successfully for classifying seed lots in regions where hybridization between the two species occurs.

- Based on sequencing of two chloroplast and one mitochondrial fragment, Ran et al. (2006) found out that Picea sitchensis were basal to other North American spruces that were further divided into three clusters in the cpDNA phylogeny.

- According to Hamilton and Aitken (2013) only two chloroplast haplotypes were observed within the -500-bp region $\operatorname{Trnf}-\operatorname{Trn} \mathrm{L}$ region among 255 individuals sequenced. One haplotype was fixed in the reference Haida Gwaii (Queen Charlotte Island) Sitka spruce population and another one in the reference Fort Nelson white spruce population. These haplotypes were distinguished by singlenucleotide base-pair mutations at five sites across the sequenced region (at 87, 188, 193, 268, and $285 \mathrm{bp}$ ) and a single base-pair deletion 339 bp from the 5' end of the sequenced region.

- Within the -500-bp region sequenced of the nad5a gene only two haplotypes were observed. "A fournucleotide tandem base repeat (CTTGACTTG) at 276 base pairs from the $5^{\prime}$ end of the sequenced nad5a region distinguishes white spruce; Sitka spruce mitotypes lack the repeat. The white spruce mitotype was found only in the 11 individuals sequenced within the reference white spruce population. The Sitka spruce mitotype was fixed in all other populations, including the hybrid and reference Sitka spruce populations" (Hamilton and Aitken 2013).

\section{Nuclear DNA markers (nSSR, EST-SSR, SNP)}

a) nSSRs (putatively neutral microsatellites), ESTSSRs (expressed sequence tag derived microsatellites)

\section{Loci and primers used}

The first nuclear microsatellite markers for Sitka spruce were developed by van de Van and McNicol (1996). They used nSSRs to screen 58 Sitka spruce clones. Later on A'Hara and Cottrell $(2004,2007,2009)$ developed additional microsatellite markers for this species as tools for genetic characterization of Sitka spruce populations and distinction from other related spruce species (e.g. white spruce).

Due to frequent introgression between Picea species, the problem of species identification and characterization of introgression zones is of high importance. Therefore, the search for species specific markers, among them highly polymorphic microsatellites, is central in Picea sitchensis research. Besides development of new markers, the transferability of already developed nSSR markers from other species to Sitka spruce was tested by many research teams (Hodgetts et al. 2001; Rungis et al. 2004, Bennuah, et al. 2004, Bérubé et al, 2009; Ralph et al. 2008). For example, Hodgetts et al. (2001) developed 13 nSSRs for Picea glauca, 10 of which he amplified successfully in Picea sitchensis. Similarly, from eight primer pairs developed by Rajora et al. (2001) for Picea glauca, five amplified also in Picea sitchensis. Rungis et al. (2004) developed 25 polymorphic EST-SSR markers which could be amplified in three spruce species namely Sitka spruce (Picea sitchensis), white spruce (Picea glauca) and black spruce or interior spruce (Picea glauca $\times$ Picea engelmannii).

EST-SSR-primers developed for black spruce by Perry and Bousquet (1998b) were successfully used to reveal sequence tag polymorphisms in Sitka spruce and to characterise the genetic diversity of natural populations (Bennuah et al. 2004, Gapare et al. 2005). 


\begin{tabular}{|c|c|c|c|c|c|c|c|c|c|c|c|c|c|c|}
\hline 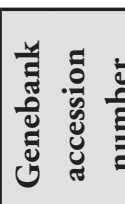 & & , & & & & & & 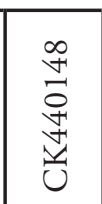 & $\begin{array}{l}\text { : } \\
\text { : } \\
0.0 \\
0 \\
0\end{array}$ & 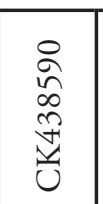 & 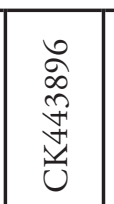 & 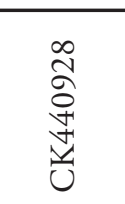 & 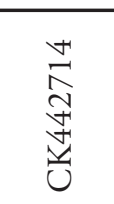 & 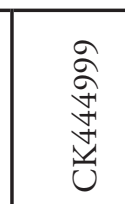 \\
\hline$\dot{\Xi}$ & - & - & - & - & - & - & - & $\triangle$ & $\sim$ & 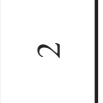 & N & N & $\mathrm{N}$ & \\
\hline$z^{\prime}$ & 0 & $\approx$ & $\cong$ & $\forall$ & $n$ & & 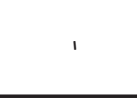 & . & & & 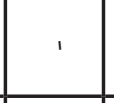 & & ' & \\
\hline 总 & 品 & $\stackrel{\circ}{\dddot{9}}$ & वे & I & $\widehat{\overbrace{}}$ & $\cong$ & $\tilde{z}$ & $\frac{4}{2}$ & 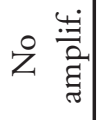 & 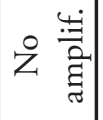 & 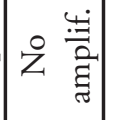 & 岸 & $\bar{g}$ & $\sigma_{0}^{\frac{\pi}{2}}$ \\
\hline$F^{\circ} a^{a}$ & 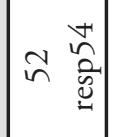 & 啇 & 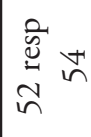 & 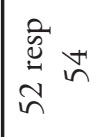 & $\begin{array}{l}\text { 害 } \\
\text { in in }\end{array}$ & 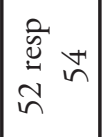 & 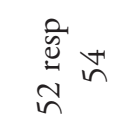 & $n$ & $n$ & $n$ & $n$ & $n$ & $i n$ & \\
\hline 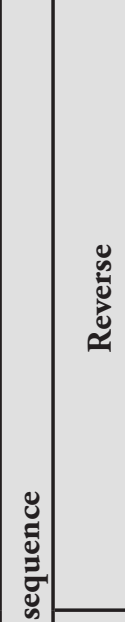 & 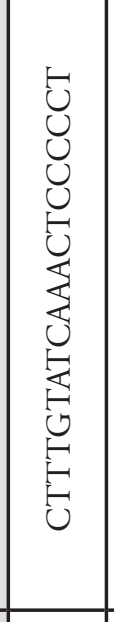 & 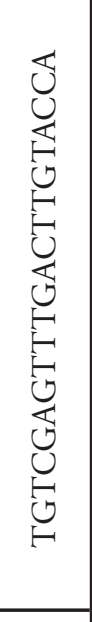 & 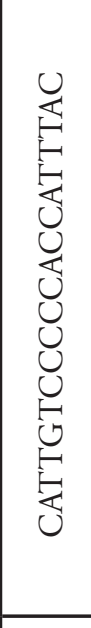 & 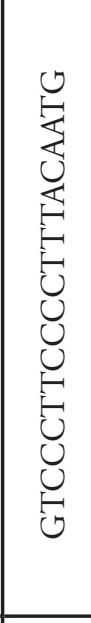 & 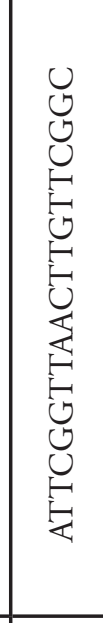 & 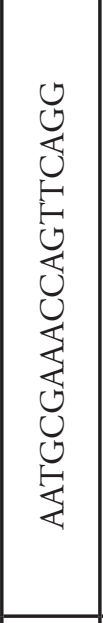 & 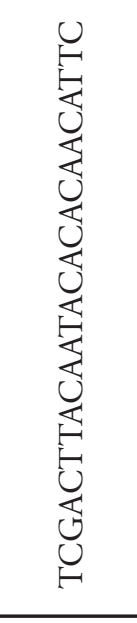 & 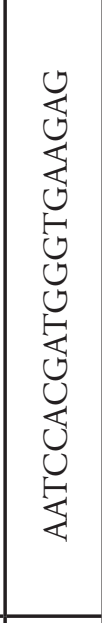 & 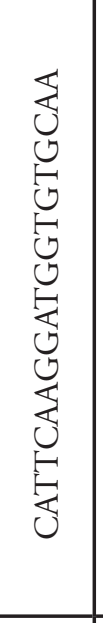 & 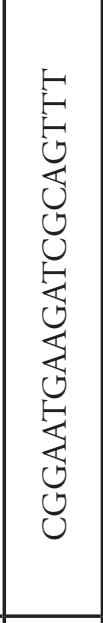 & 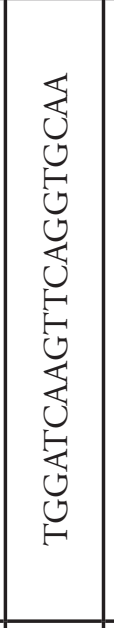 & 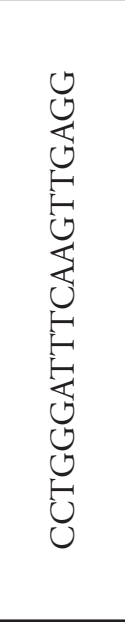 & 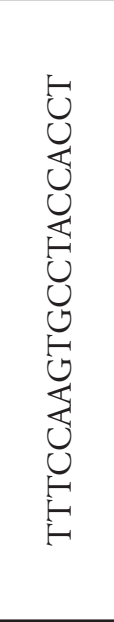 & 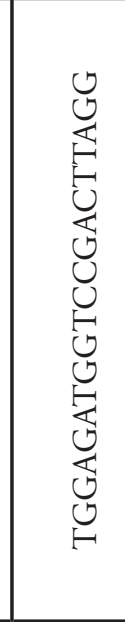 \\
\hline 荧 & 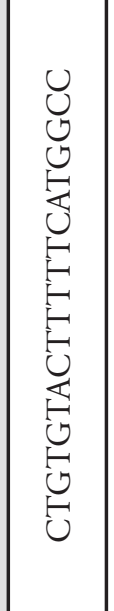 & 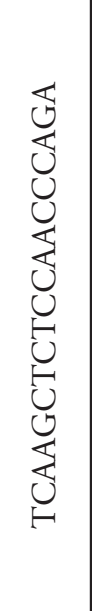 & 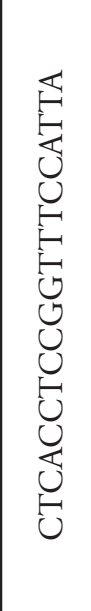 & 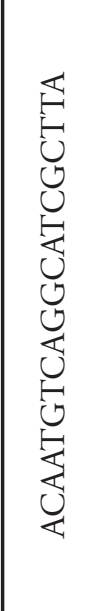 & 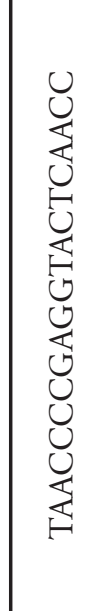 & 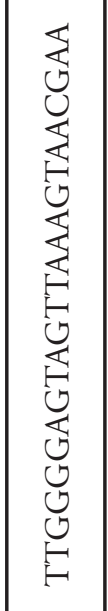 & 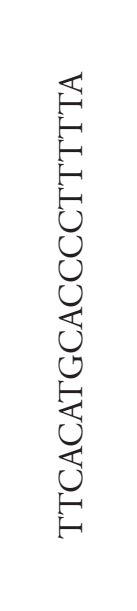 & 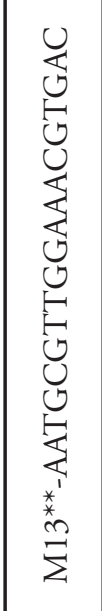 & 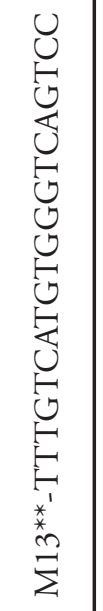 & 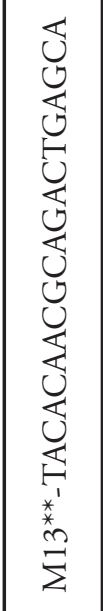 & 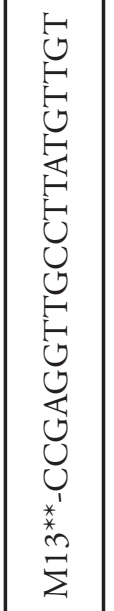 & 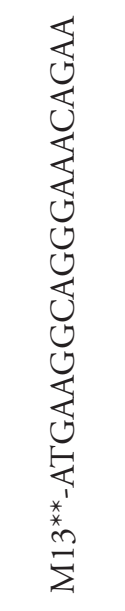 & 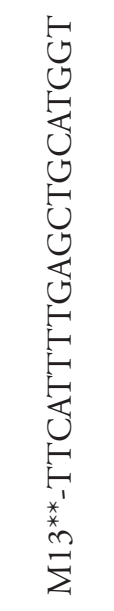 & 燎 \\
\hline$\frac{u}{2}$ & 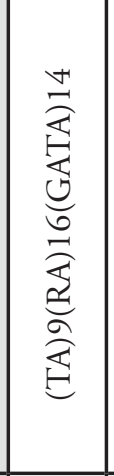 & 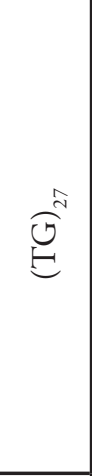 & 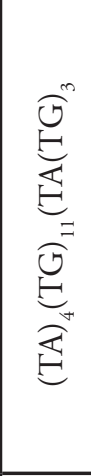 & 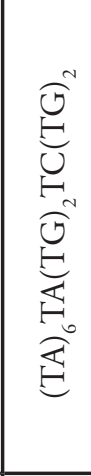 & 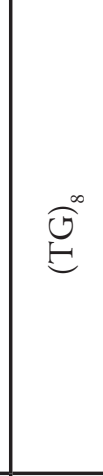 & 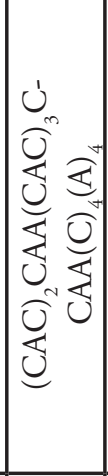 & 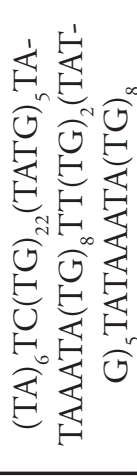 & Eी & 吾 & $\stackrel{\Theta}{E}^{\infty}$ & है & 是 & 吾 & \\
\hline$\frac{a}{\bar{z}}$ & 笼 & 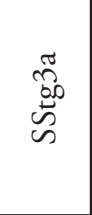 & 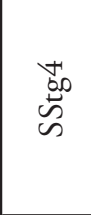 & 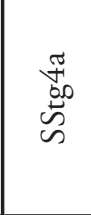 & 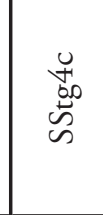 & 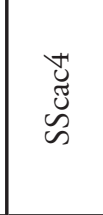 & 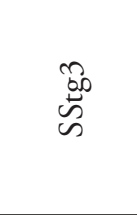 & $\overrightarrow{0}$ & 芯 & 悤 & 莣 & 㤐 & 岕 & \\
\hline
\end{tabular}




\begin{tabular}{|c|c|c|c|c|c|c|c|c|c|c|c|c|c|c|c|c|c|}
\hline 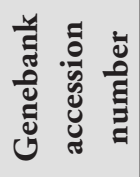 & 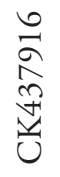 & 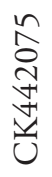 & 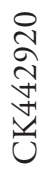 & \begin{tabular}{l}
$\infty$ \\
$\sim$ \\
$\infty$ \\
$\infty$ \\
\multirow{2}{*}{} \\
$v$
\end{tabular} & 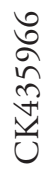 & 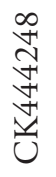 & 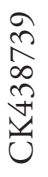 & 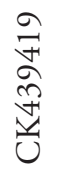 & 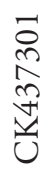 & 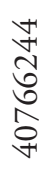 & 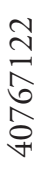 & \begin{tabular}{l}
$\stackrel{\infty}{N}$ \\
$\underset{n}{\hat{N}}$ \\
$\hat{o}$ \\
\multirow{+}{1}{}
\end{tabular} & 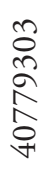 & 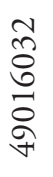 & $\begin{array}{l}\vec{n} \\
\frac{n}{a} \\
\stackrel{2}{+}\end{array}$ & 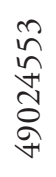 & 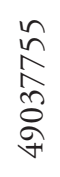 \\
\hline & $N$ & $\sim$ & $N$ & $\sim$ & $\sim$ & $\sim$ & $N$ & $N$ & $N$ & $n$ & $n$ & $n$ & $n$ & $n$ & $n$ & $n$ & $n$ \\
\hline$z^{4}$ & $N$ & $\triangleq$ & 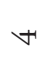 & $\beth$ & $\wedge$ & $n$ & $\stackrel{m}{=}$ & $a$ & $\stackrel{\infty}{\sim}$ & & 1 & , & , & & , & 1 & \\
\hline
\end{tabular}

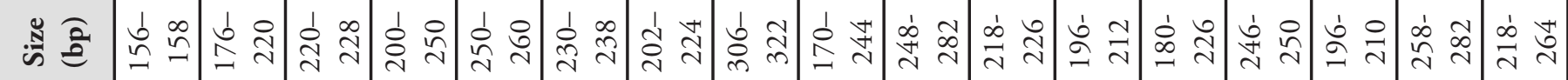

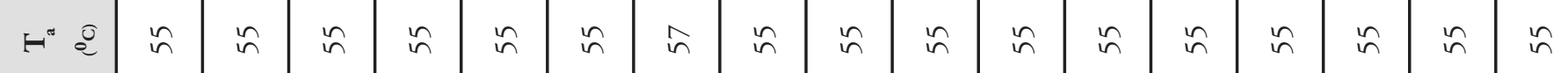

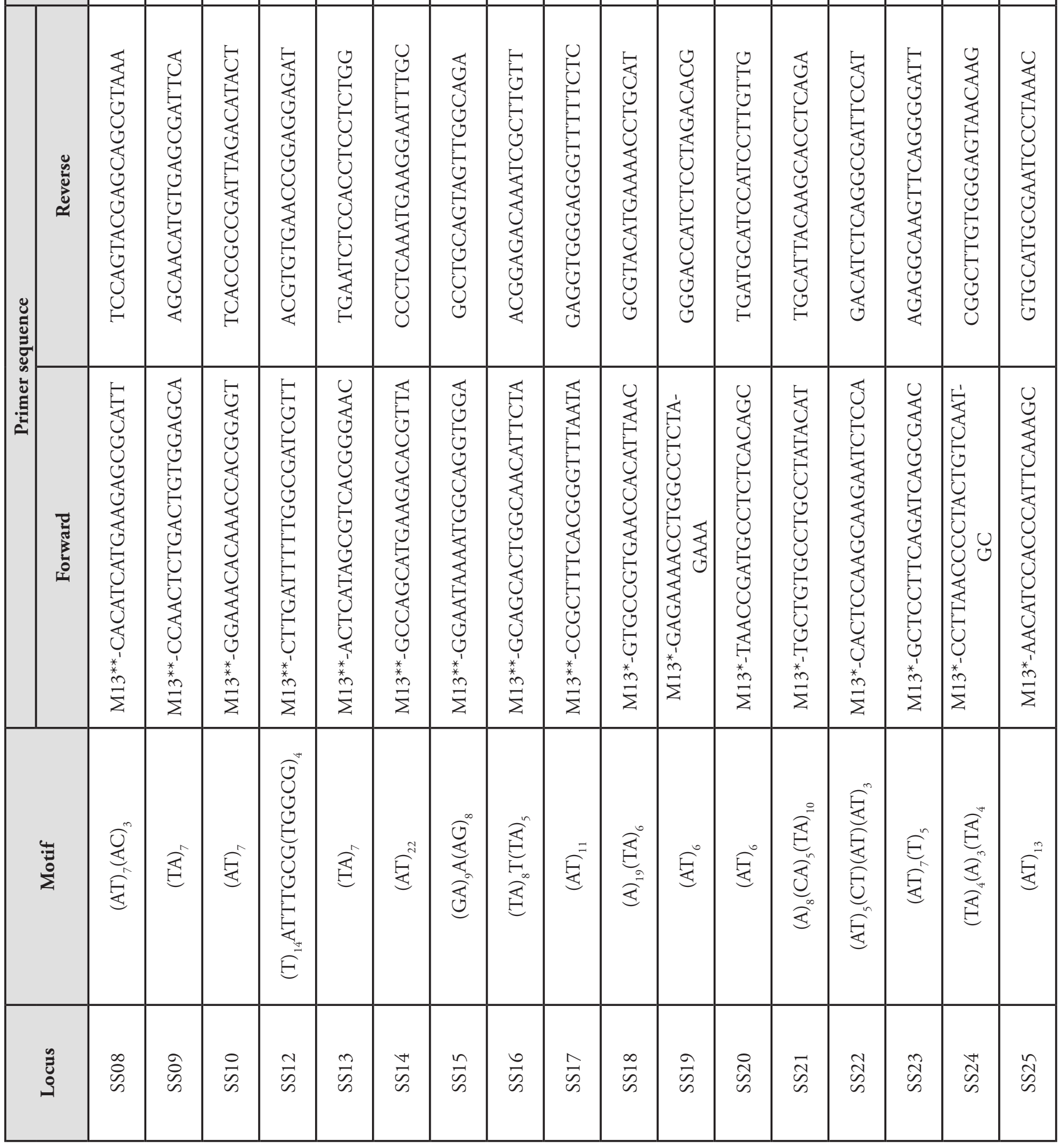




\begin{tabular}{|c|c|c|c|c|c|c|c|c|c|c|c|c|c|c|c|c|c|}
\hline 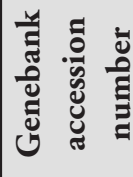 & $\begin{array}{l}\infty \\
\stackrel{\infty}{\infty} \\
\stackrel{+}{\$} \\
\stackrel{\sigma}{\sigma}\end{array}$ & $\begin{array}{l}\stackrel{2}{1} \\
\infty \\
\overrightarrow{+} \\
\stackrel{+}{\alpha} \\
\overrightarrow{+}\end{array}$ & 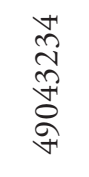 & 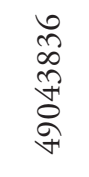 & $\begin{array}{l}\stackrel{+}{0} \\
\stackrel{+}{+} \\
\stackrel{+}{+} \\
\stackrel{+}{+}\end{array}$ & 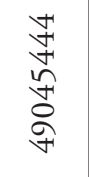 & 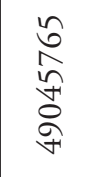 & 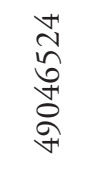 & 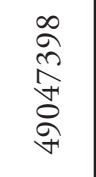 & \begin{tabular}{l}
$\vec{\delta}$ \\
o \\
\multirow{+}{*}{} \\
$\stackrel{2}{+}$ \\
$\forall$
\end{tabular} & 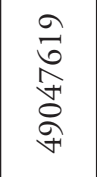 & 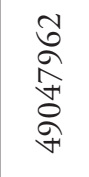 & 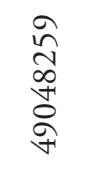 & 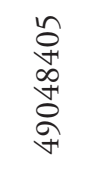 & 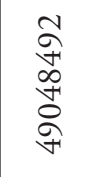 & 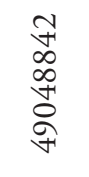 & \begin{tabular}{l}
$\overrightarrow{0}$ \\
$\stackrel{1}{a}$ \\
\multirow{2}{+}{} \\
$\stackrel{2}{+}$ \\
$\forall$
\end{tabular} \\
\hline 苞 & $n$ & $n$ & $n$ & $n$ & $n$ & $m$ & $n$ & $n$ & $n$ & $n$ & $n$ & $n$ & $n$ & $m$ & $n$ & $n$ & $n$ \\
\hline$z^{4}$ & ' & ' & ' & ' & ' & ' & 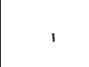 & ' & ' & ' & ' & ' & ' & ' & ' & ' & \\
\hline in है & $\stackrel{\infty}{\stackrel{\infty}{\rightleftharpoons}} \underset{\sim}{\sim}$ & 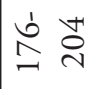 & 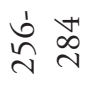 & 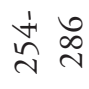 & $\stackrel{1}{\stackrel{\overbrace{}}{\curvearrowright}}$ & $\underset{ \pm}{ \pm}$ & 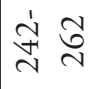 & $\begin{array}{ll}1 & +1 \\
\infty & \stackrel{2}{二}\end{array}$ & 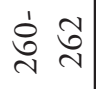 & 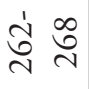 & 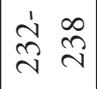 & $\begin{array}{l}\dot{亠} \\
\stackrel{\sim}{\sim}\end{array}$ & 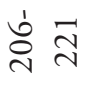 & $\underset{⿱ 亠}{\stackrel{\sim}{\sim}} \stackrel{\infty}{\curvearrowleft}$ & 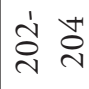 & $\begin{array}{l}\dot{1}+\infty \\
\stackrel{\infty}{\sim}\end{array}$ & $\stackrel{\dot{\sim}}{\stackrel{\infty}{\sim}}$ \\
\hline$H^{\circ} \approx$ & $i n$ & $n$ & $n$ & $i n$ & $i n$ & $n$ & $n$ & $i n$ & $n$ & $n$ & $n$ & $i n$ & $n$ & $n$ & $n$ & $n$ & $n$ \\
\hline 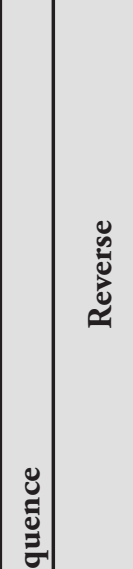 & 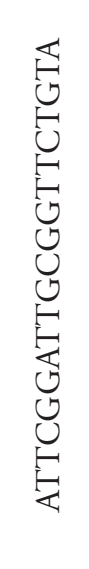 & 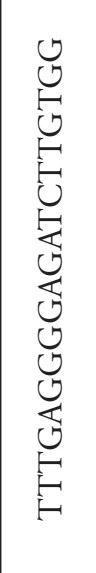 & 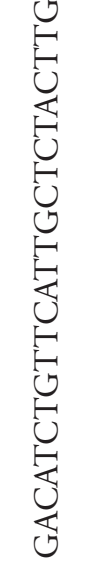 & 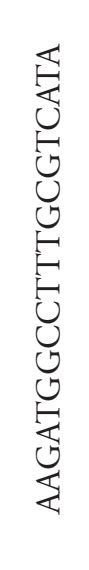 & 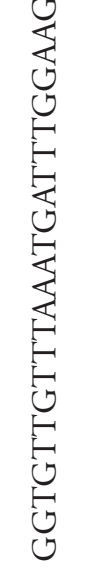 & 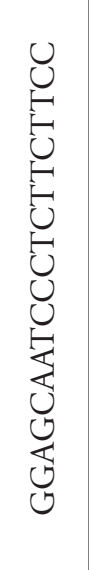 & 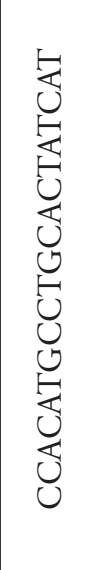 & 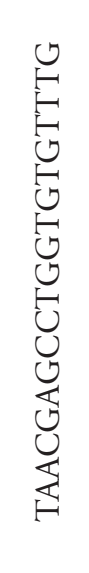 & 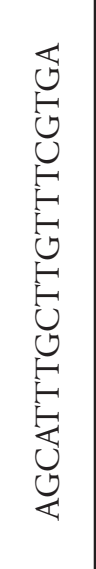 & 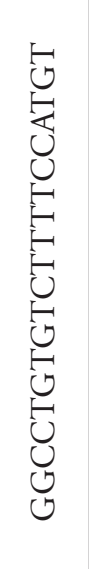 & 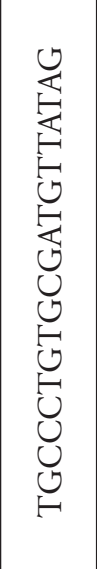 & 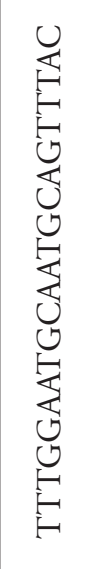 & 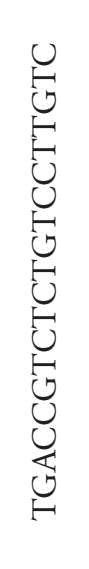 & 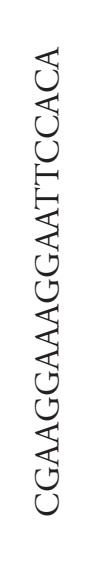 & 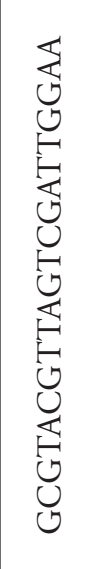 & 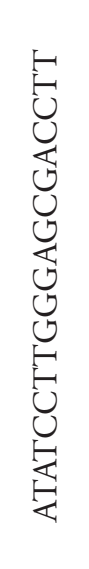 & 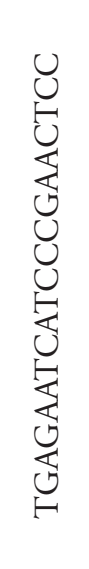 \\
\hline 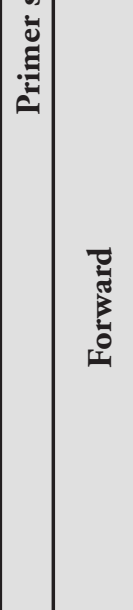 & 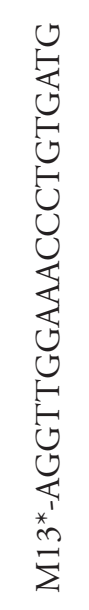 & 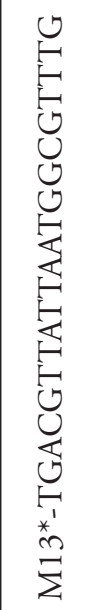 & 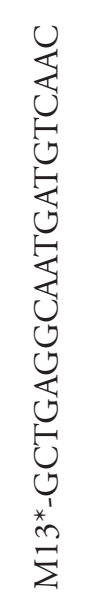 & 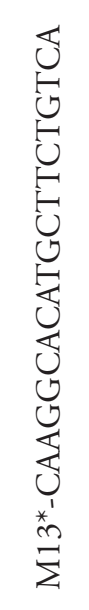 & 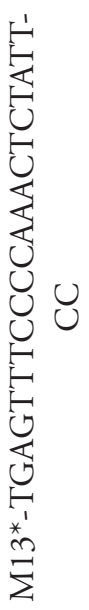 & 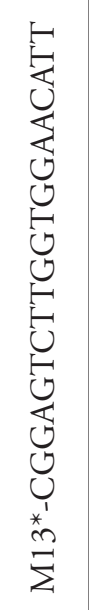 & 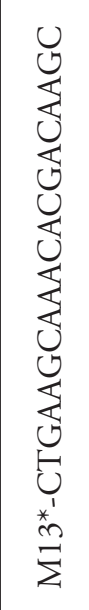 & 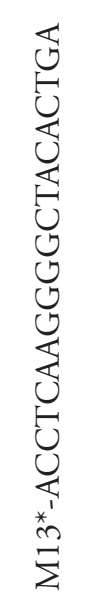 & 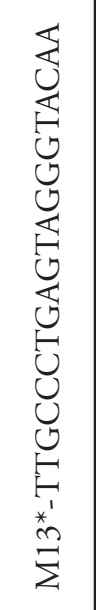 & 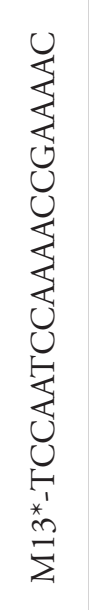 & 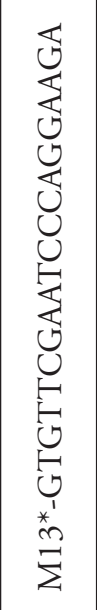 & 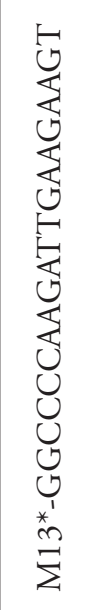 & 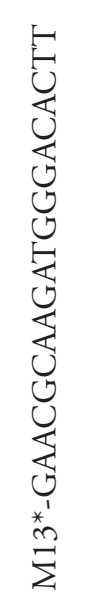 & 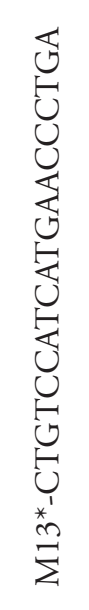 & 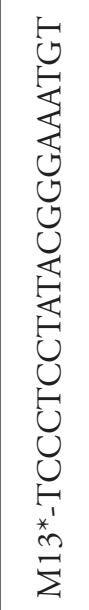 & 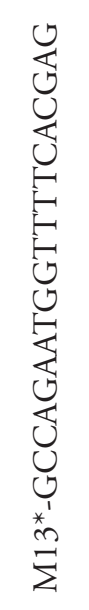 & 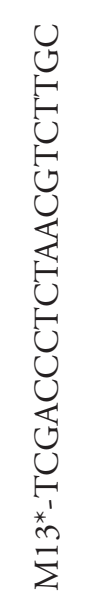 \\
\hline : & $\stackrel{\rightleftarrows}{\stackrel{\Xi}{E}}$ & $\stackrel{\stackrel{\leftrightarrow}{\rightleftarrows}}{E}$ & 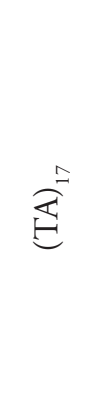 & 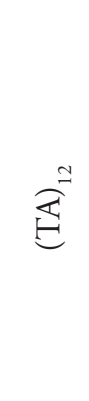 & $\stackrel{\Xi}{\Xi}$ & $\stackrel{\Xi}{\rightleftarrows}$ & 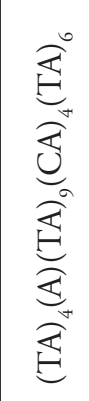 & $\underbrace{\infty}$ & 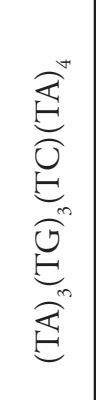 & $\stackrel{\Xi}{\boxminus}$ & $\overbrace{}^{\infty}$ & $\overbrace{}^{n}$ & 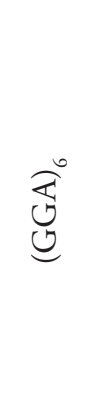 & $\stackrel{0}{\underbrace{\natural}}$ & $\widetilde{\theta}$ & $\begin{array}{l}\underset{\mathbb{E}}{\rightleftarrows} \\
\underset{\mathbb{E}}{\mathbb{E}} \\
\underset{\mathbb{E}}{\mathbb{E}}\end{array}$ & 蒫 \\
\hline 苞 & Wू. & $\begin{array}{l}\hat{w} \\
\hat{\omega}\end{array}$ & $\begin{array}{l}\infty \\
\tilde{\omega}\end{array}$ & సे & $\begin{array}{l}\hat{N} \\
\tilde{n}\end{array}$ & $\overrightarrow{\tilde{n}}$ & $\begin{array}{l}\tilde{\tilde{N}} \\
\tilde{\omega}\end{array}$ & $\stackrel{\widetilde{n}}{\tilde{n}}$ & $\begin{array}{l}\vec{N} \\
\tilde{n}\end{array}$ & $\tilde{\tilde{n}}$ & $\tilde{n}$ & $\hat{\tilde{n}}$ & $\begin{array}{l}\infty \\
\tilde{n}\end{array}$ & $\hat{\tilde{n}}$ & $\begin{array}{l}\stackrel{P}{+} \\
\stackrel{\infty}{n}\end{array}$ & $\underset{\omega}{\vec{w}}$ & Fै \\
\hline
\end{tabular}




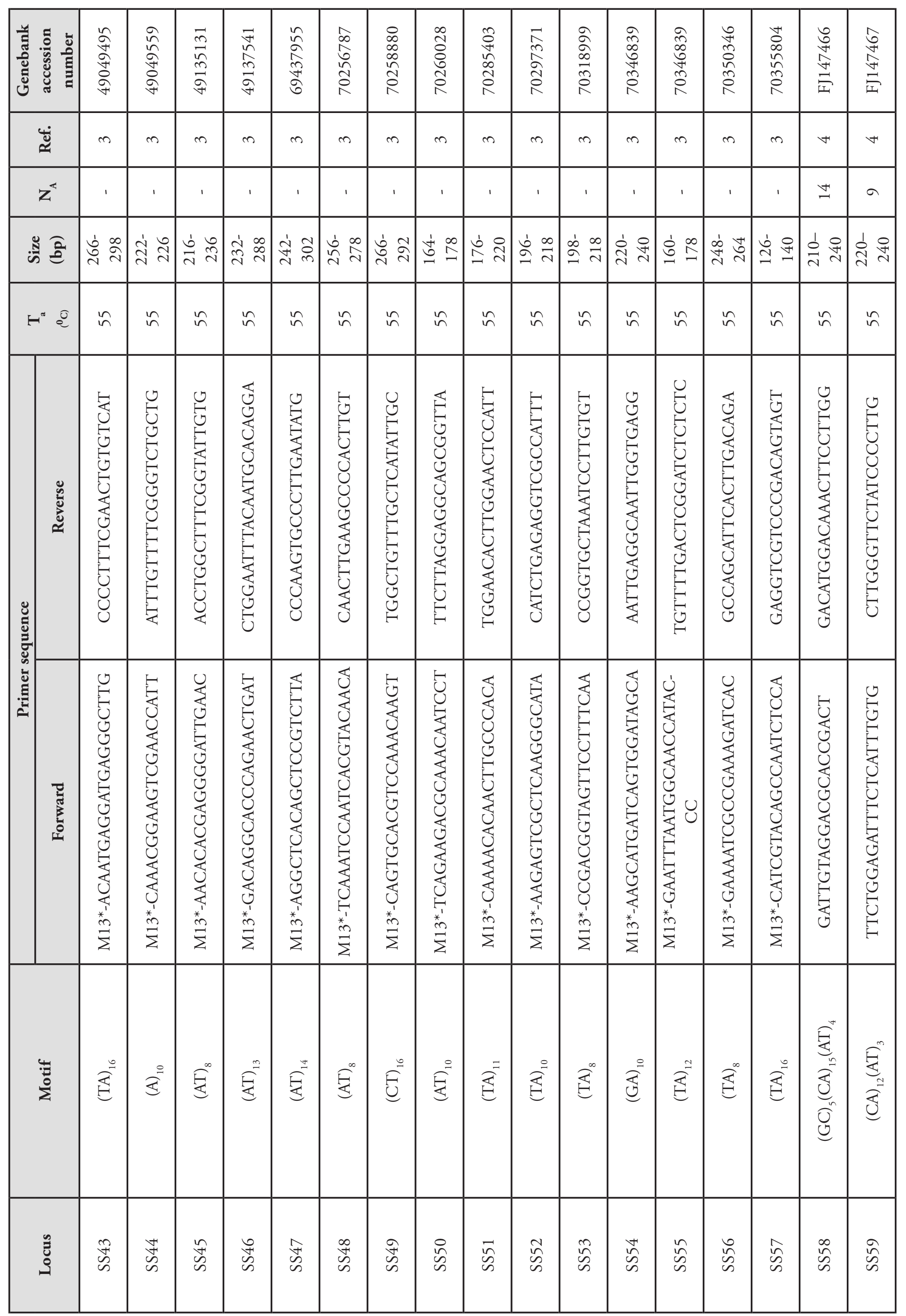




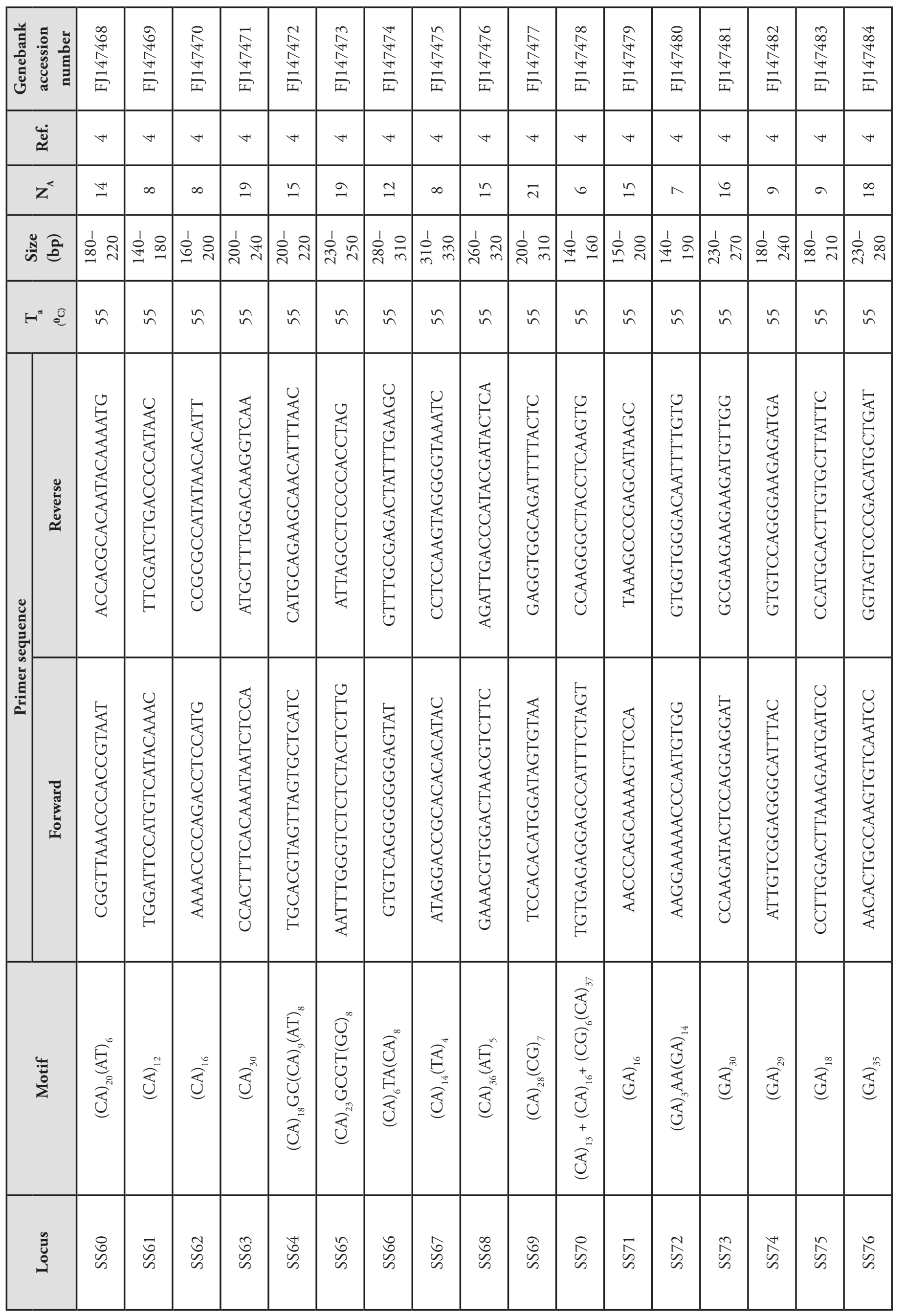




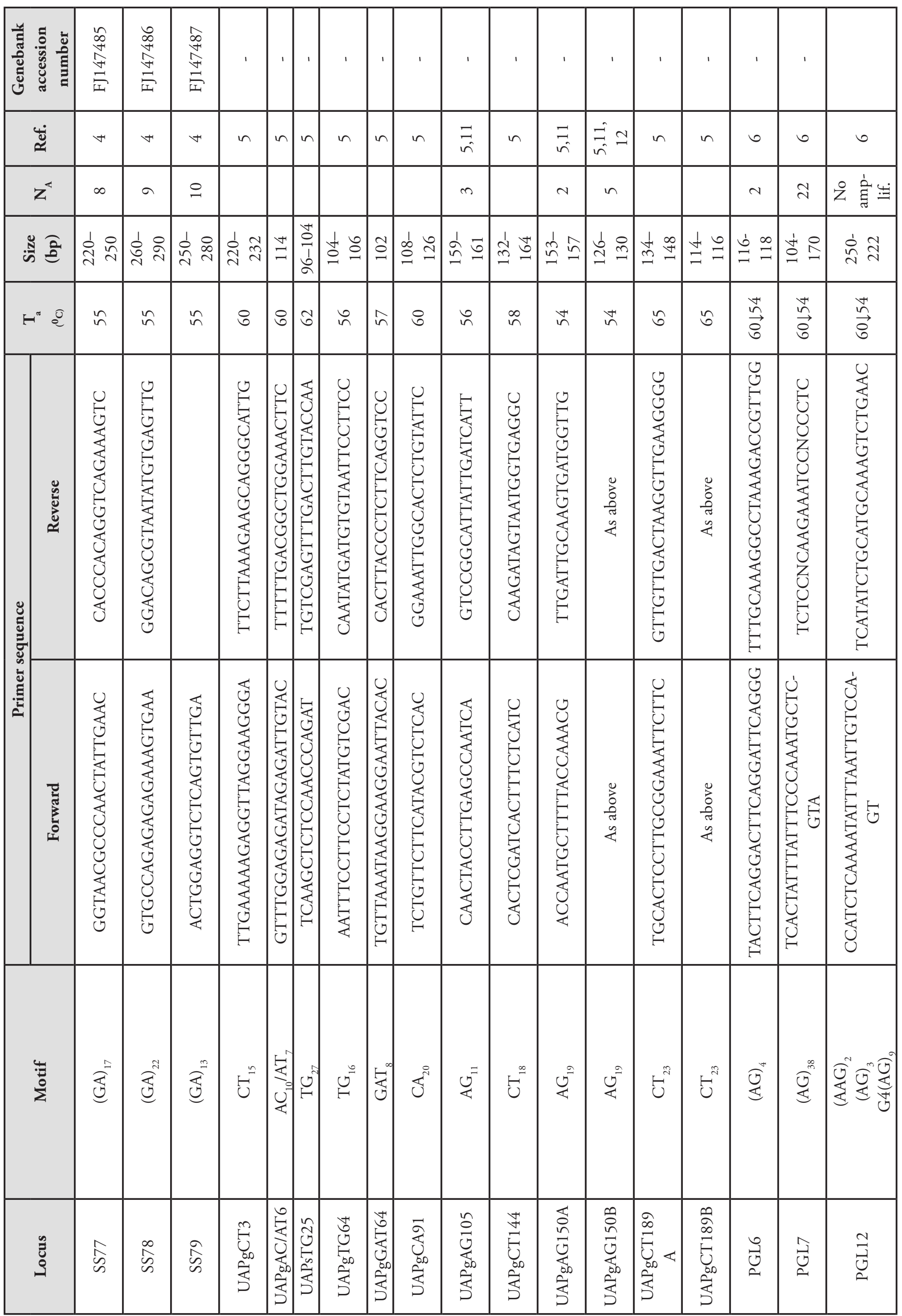




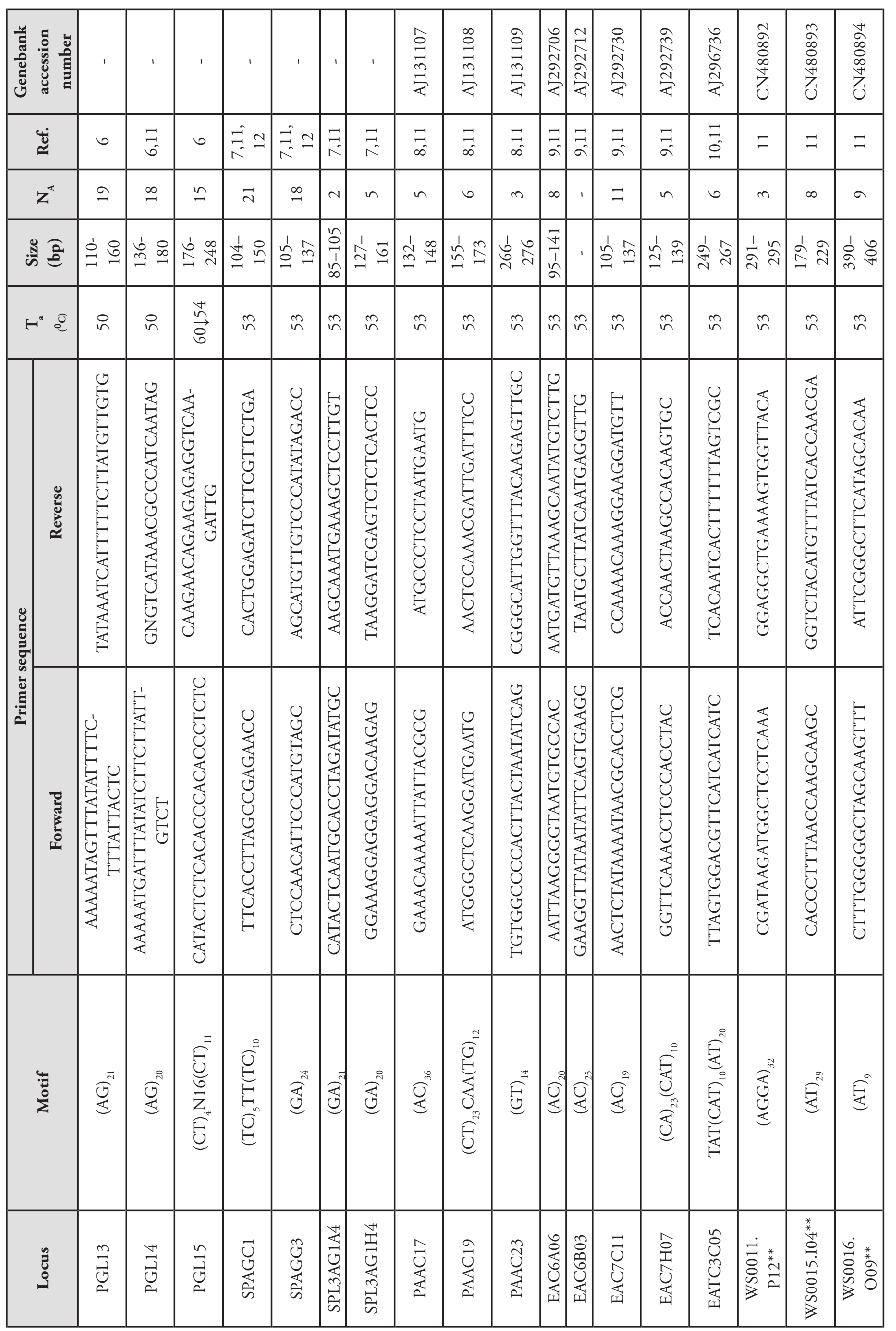




\begin{tabular}{|c|c|c|c|c|c|c|c|c|c|c|c|c|c|c|c|c|c|}
\hline 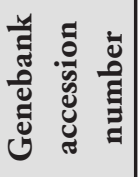 & $\begin{array}{l}n \\
\alpha \\
\infty \\
\infty \\
\stackrel{+}{+} \\
z\end{array}$ & 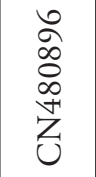 & 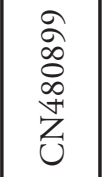 & $\begin{array}{l}8 \\
\& \\
\circ \\
\infty \\
\stackrel{+}{Z} \\
Z\end{array}$ & 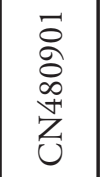 & 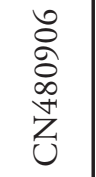 & $\begin{array}{l}\hat{\circ} \\
\circ \\
o \\
\infty \\
\stackrel{+}{u} \\
Z\end{array}$ & $\begin{array}{l}\infty \\
\stackrel{2}{\circ} \\
\stackrel{2}{0} \\
\infty \\
\stackrel{+}{U} \\
Z\end{array}$ & $\begin{array}{l}\vec{\sigma} \\
\infty \\
0 \\
\infty \\
+1 \\
z\end{array}$ & $\begin{array}{l}\infty \\
\infty \\
\infty \\
\infty \\
\infty \\
\stackrel{+}{Z}\end{array}$ & $\begin{array}{l}0 \\
\infty \\
\infty \\
\infty \\
\infty \\
⿱ 亠 亠 \\
Z\end{array}$ & $\begin{array}{l}\infty \\
\infty \\
\infty \\
\infty \\
\infty \\
⿱ 亠 凶 \\
Z\end{array}$ & 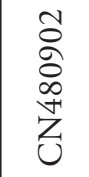 & 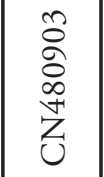 & 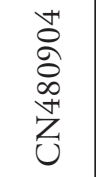 & 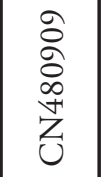 & 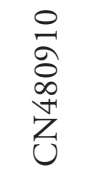 \\
\hline జుّ & $=$ & $\exists$ & $\Xi$ & $=$ & $\exists$ & $\Xi$ & $=$ & $=$ & $=$ & $=$ & $=$ & $\begin{array}{l}\cong \\
=\end{array}$ & $\exists$ & $\begin{array}{l}\cong \\
\Rightarrow\end{array}$ & $=$ & $\exists$ & $\exists$ \\
\hline$z^{4}$ & $m$ & $\bullet$ & $\checkmark$ & $a$ & $n$ & $\infty$ & $n$ & $\sim$ & - & n & n & 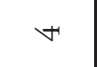 & $=$ & $\simeq$ & N & $\forall$ & 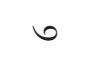 \\
\hline के है & $\underset{d}{\stackrel{1}{d}} \stackrel{\sim}{m}$ & $\begin{array}{cc}1 & 0 \\
\sim & 0 \\
n & \stackrel{n}{n}\end{array}$ & 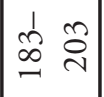 & $\stackrel{+}{\stackrel{\infty}{\beth}} \stackrel{\infty}{\sim}$ & \begin{tabular}{ll}
1 & $\infty$ \\
0 & $\infty$ \\
\hdashline & $\infty$ \\
- & 0
\end{tabular} & $\begin{array}{ll}1 & \infty \\
\stackrel{\infty}{v} & \stackrel{n}{~}\end{array}$ & $\stackrel{1}{\stackrel{1}{\rightleftarrows}} \underset{\exists}{ت}$ & \begin{tabular}{ll}
1 & 0 \\
$\infty$ & 0 \\
\hdashline & $n$ \\
-1
\end{tabular} & $\stackrel{1}{\stackrel{1}{\infty}} \stackrel{\hat{\sim}}{\sim}$ & $\frac{1}{\vec{i}} \vec{\sim}$ & $\stackrel{1}{\widehat{\vartheta}} \widehat{\curvearrowright}$ & $\stackrel{1}{\stackrel{\nu}{\vec{v}}} \overrightarrow{\vec{v}}$ & $\stackrel{1}{\stackrel{1}{\sim}} \underset{\sim}{\sim}$ & $\left|\begin{array}{ll}1 & \infty \\
\infty & \vec{\sim} \\
- & \vec{\sim}\end{array}\right|$ & 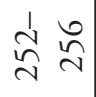 & ळે & 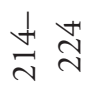 \\
\hline$H \approx 0$ & $n$ & 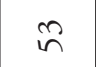 & $n$ & $n$ & $n$ & $n$ & $n$ & $n$ & $n$ & $n$ & $n$ & $\stackrel{n}{n}$ & $n$ & $n$ & $n$ & $n$ & 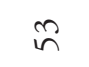 \\
\hline 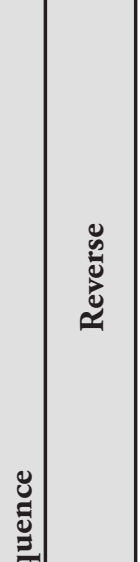 & 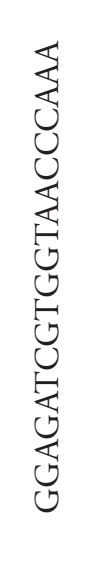 & 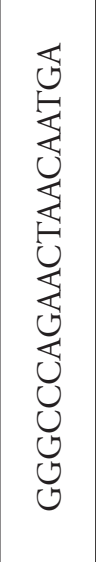 & 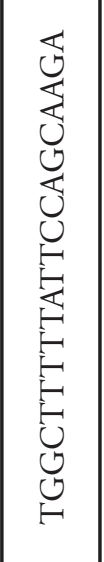 & 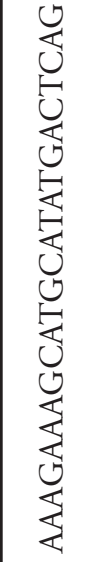 & 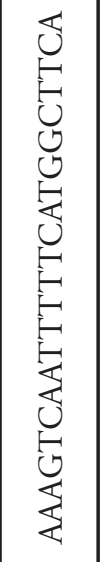 & 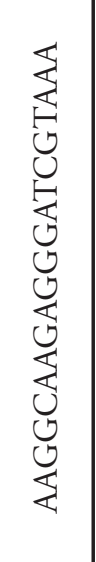 & 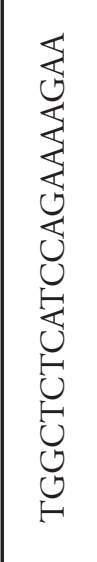 & 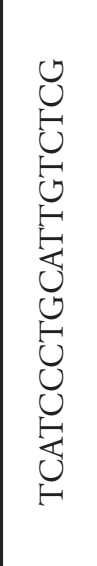 & 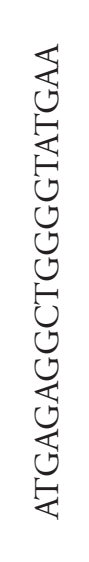 & 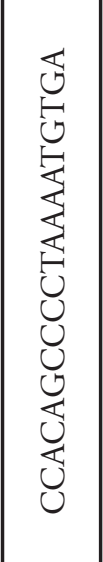 & 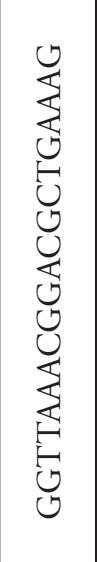 & 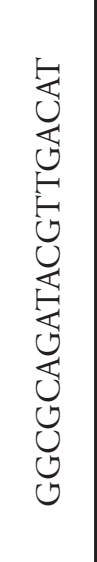 & 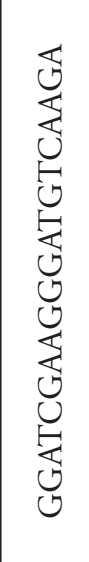 & 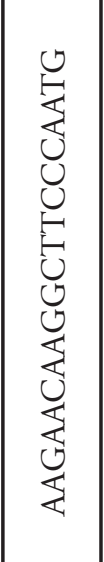 & 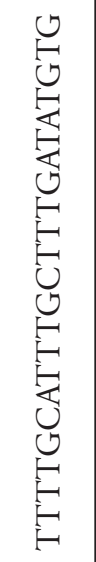 & 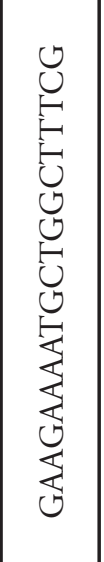 & 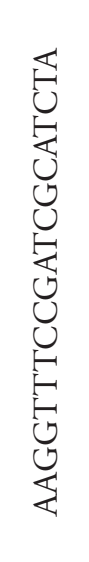 \\
\hline 苞 & 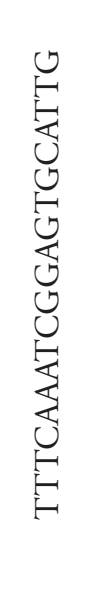 & 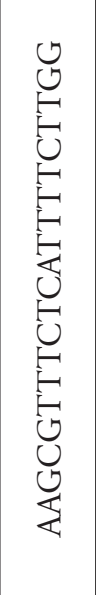 & 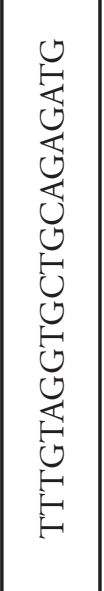 & 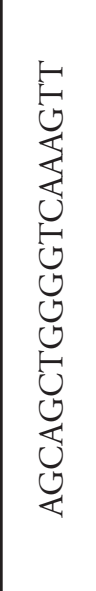 & 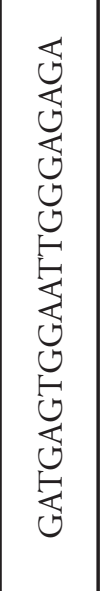 & 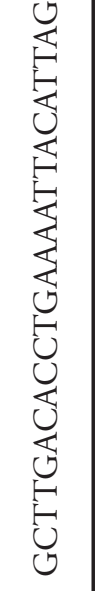 & 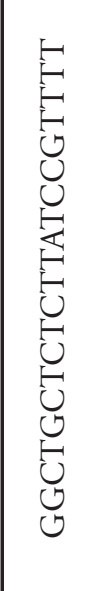 & 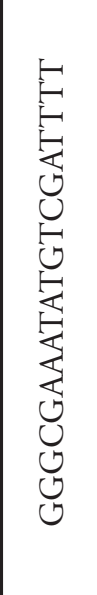 & 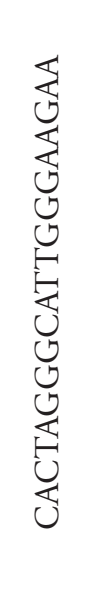 & 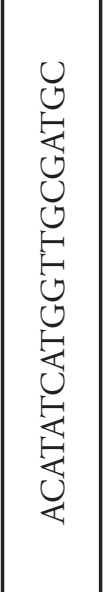 & 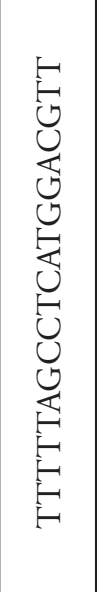 & 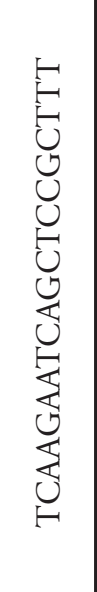 & 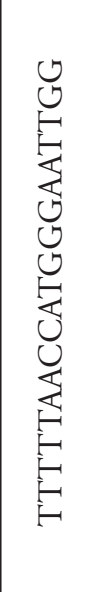 & 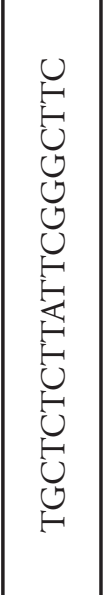 & 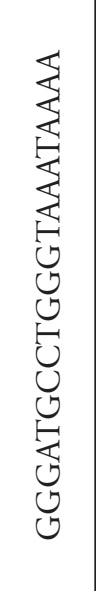 & 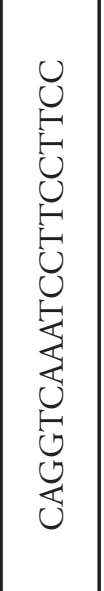 & 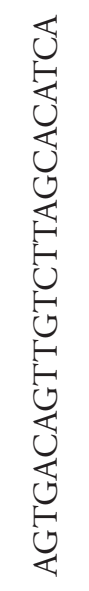 \\
\hline 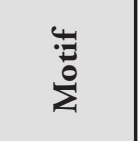 & 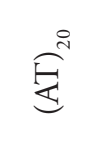 & ${\stackrel{2}{E^{2}}}^{2}$ & $\stackrel{\mathbb{Z}}{\mathbb{S}}$ & $\stackrel{8}{E^{2}}$ & $\underset{\Xi}{\stackrel{2}{E}}$ & $\stackrel{0}{E^{0}}$ & 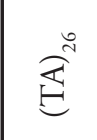 & $\stackrel{=}{\stackrel{E}{E}}$ & 莛 & 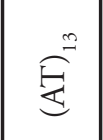 & $\begin{array}{l}\hat{E}^{n} \\
\stackrel{E^{n}}{U}\end{array}$ & 实 & $\overbrace{\mathbb{E}}^{\pi}$ & $\stackrel{+}{E^{ \pm}}$ & 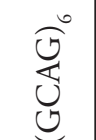 & $\stackrel{\vec{\xi}}{E}$ & $\stackrel{n}{\rightleftarrows}$ \\
\hline לِّ & 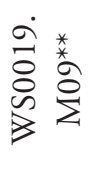 & 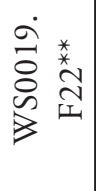 & 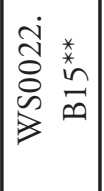 & 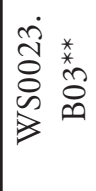 & 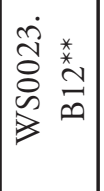 & 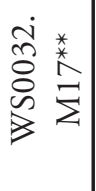 & 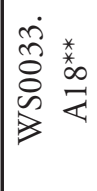 & 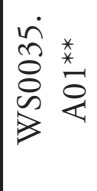 & 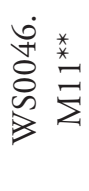 & 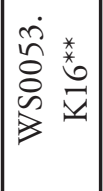 & 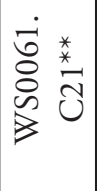 & 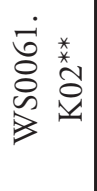 & 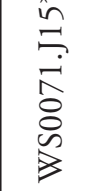 & 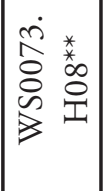 & 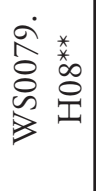 & 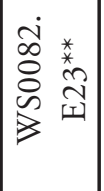 & 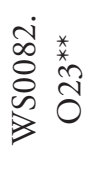 \\
\hline
\end{tabular}




\begin{tabular}{|c|c|c|c|c|c|c|c|c|c|c|c|c|c|c|c|c|c|}
\hline 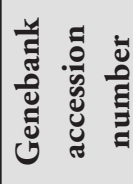 & $\begin{array}{l}\infty \\
\infty \\
\infty \\
0 \\
\infty \\
+ \\
⿱ 亠 乂 \\
u\end{array}$ & $\begin{array}{l}\infty \\
\infty \\
\infty \\
\infty \\
\infty \\
\stackrel{+}{Z} \\
u\end{array}$ & $\begin{array}{l}\stackrel{2}{\circ} \\
\infty \\
0 \\
\infty \\
\stackrel{+}{Z} \\
Z\end{array}$ & 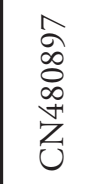 & $\begin{array}{l}n \\
o \\
o \\
o \\
o+1 \\
\stackrel{+}{Z} \\
u\end{array}$ & ' & 1 & 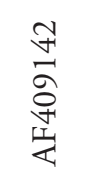 & 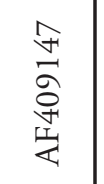 & 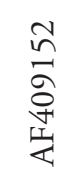 & 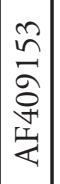 & $\begin{array}{l}\vec{\sigma} \\
\vec{a} \\
\text { oे } \\
\text { ț }\end{array}$ & 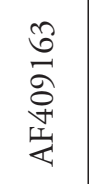 & 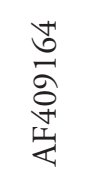 & 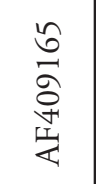 & 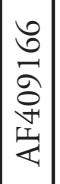 & 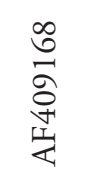 \\
\hline$\ddot{\mathscr{\varpi}}$ & $\exists$ & $\exists$ & $\exists$ & $=$ & $\exists$ & $=$ & $\exists$ & $\mathscr{n}$ & 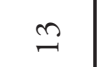 & $\ddot{2}$ & 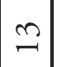 & 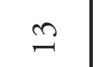 & 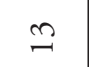 & $\stackrel{m}{=}$ & 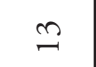 & 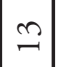 & $\stackrel{m}{=}$ \\
\hline$z^{4}$ & $n$ & $m$ & $n$ & $n$ & $\wedge$ & $\sigma$ & 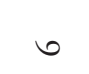 & & & & & & & & & & \\
\hline के है & $\stackrel{1}{\stackrel{1}{\sim}} \underset{\sim}{\sim}$ & $\stackrel{1}{\stackrel{1}{\sim}} \stackrel{\infty}{\vec{\sim}}$ & సે & $\stackrel{1}{\stackrel{n}{\sim}} \underset{\sim}{\sim}$ & $\underset{\sim}{1} \stackrel{\hat{n}}{\stackrel{\infty}{n}}$ & $\stackrel{1}{\stackrel{2}{N}} \underset{\sim}{\sim}$ & $\stackrel{1}{\stackrel{1}{\beth}}$ & $\frac{1}{n} \stackrel{\infty}{\sim}$ & 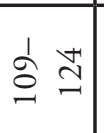 & $\stackrel{1}{\stackrel{t}{t}} \stackrel{n}{=}$ & \begin{tabular}{|l|} 
\\
+ \\
+1 \\
$b$ \\
$\curvearrowleft$
\end{tabular} & 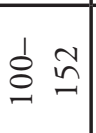 & $\frac{1}{\sigma} \stackrel{\sim}{\sim}$ & 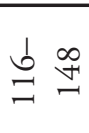 & $\frac{1}{\lesssim} \underset{\sim}{\sim}$ & $\begin{array}{l}0 \\
\cdots \\
1 \\
\sigma \\
\sigma\end{array}$ & 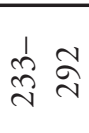 \\
\hline$H^{*} Q$ & $\approx$ & 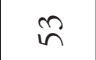 & $n$ & $n$ & $n$ & $n$ & $n$ & $\stackrel{\bullet}{n}$ & $\stackrel{\gtrless}{\curvearrowleft}$ & in & $\stackrel{\circ}{n}$ & in & 요 & 요 & ำ & in & $n$ \\
\hline 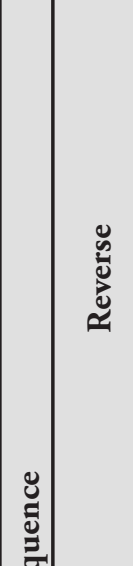 & 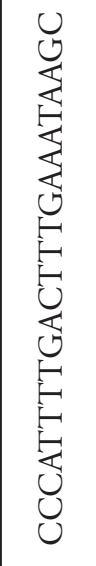 & 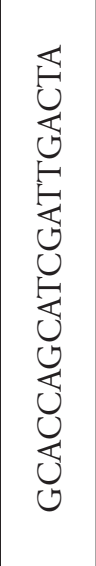 & 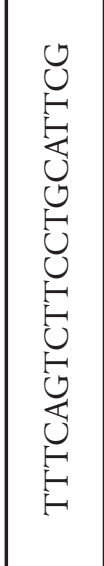 & 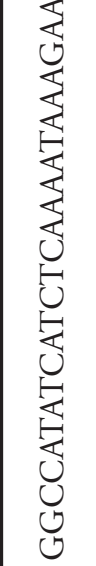 & 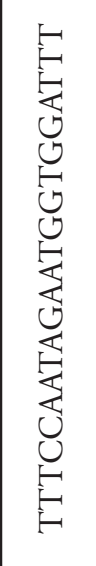 & 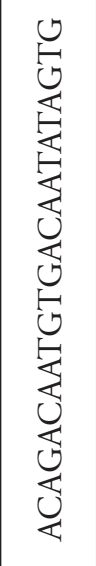 & 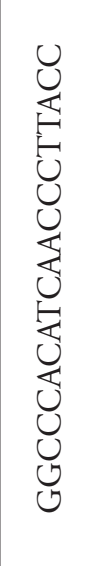 & 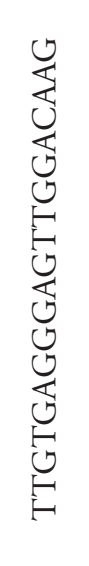 & 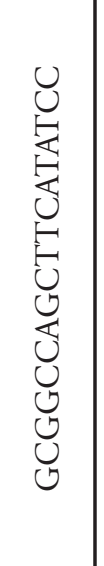 & 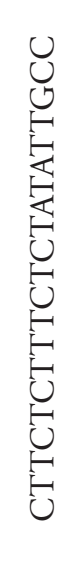 & 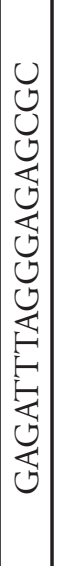 & 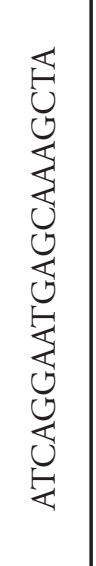 & 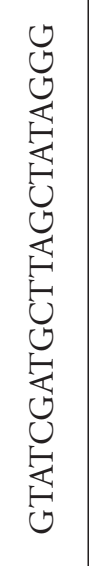 & 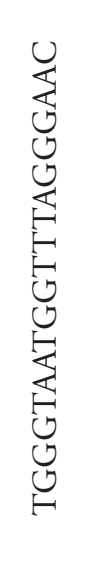 & 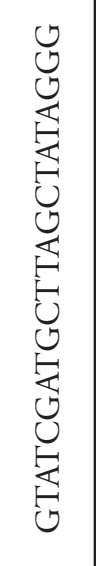 & 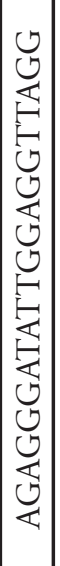 & 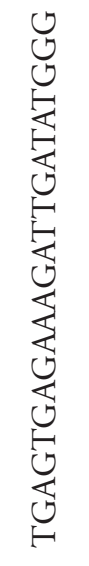 \\
\hline 范 & 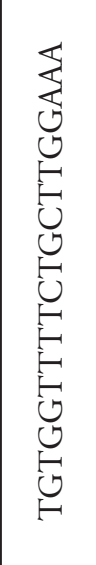 & 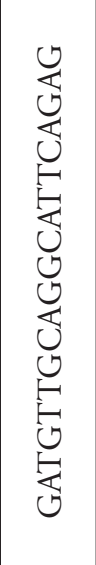 & 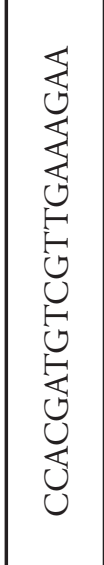 & 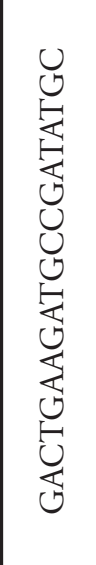 & 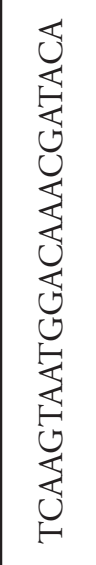 & 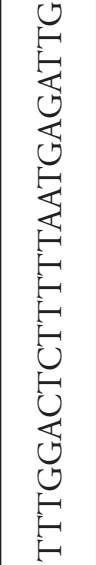 & 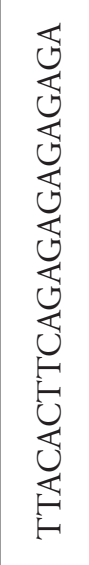 & 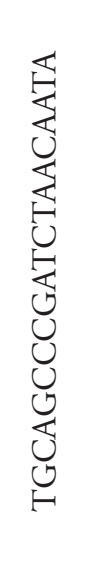 & 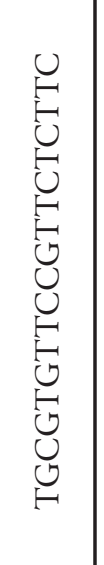 & 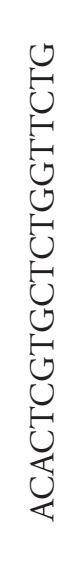 & 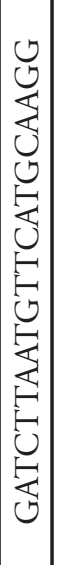 & 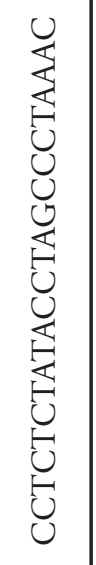 & 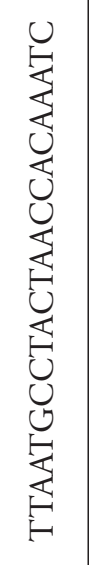 & 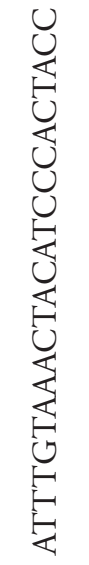 & 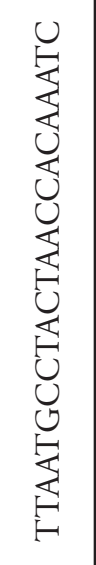 & 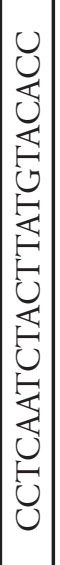 & 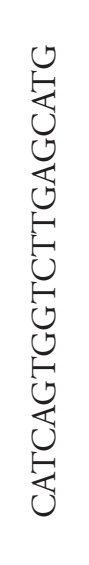 \\
\hline 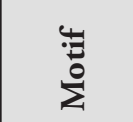 & 这 & $\begin{array}{l}\hat{U}^{0} \\
\stackrel{E}{U}\end{array}$ & 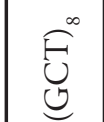 & 度 & 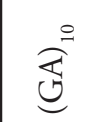 & $\overrightarrow{0}^{n}$ & $\mathbb{G}^{a}$ & & & & & & & & & & \\
\hline 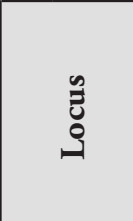 & 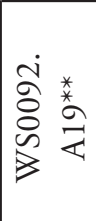 & 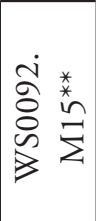 & 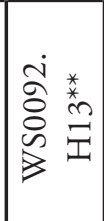 & 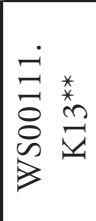 & 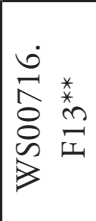 & $N$ & $\underset{\forall}{*}$ & 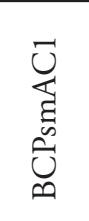 & 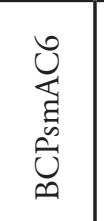 & 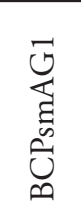 & 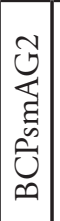 & 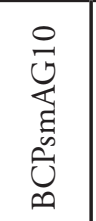 & 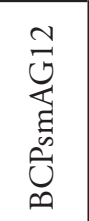 & 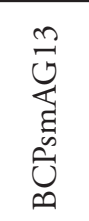 & 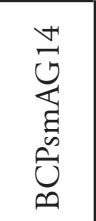 & 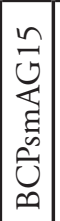 & 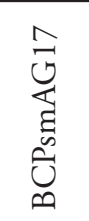 \\
\hline
\end{tabular}




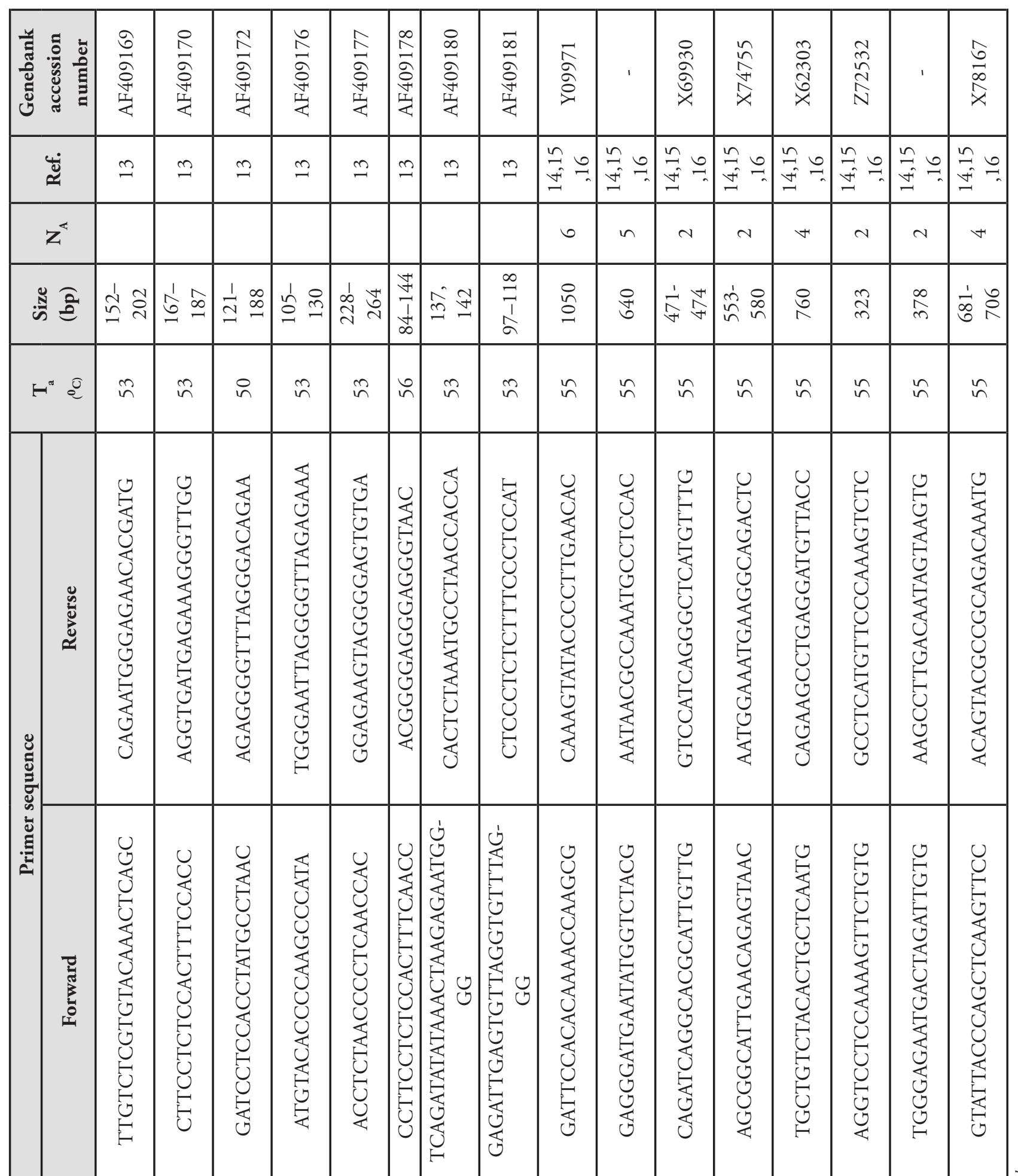

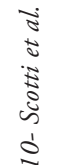
ปี 竝 के 


\section{Material for extraction}

DNA was extracted from:

- needles (fresh and frozen) (van de Ven and McNicol 1997. Hodgetts et al. 2001, Rajora et al. 2001, Bennuah et al. 2004, Gapare et al. 2005, Gapare and Aitken 2005, Mimura and Aitken 2007a, 2007b);

- embryos (van de Ven and McNicol 1997) and germinants (Rungis et al. 2004, Mimura and Aitken 2007a, 2007b);

- megagametophyte from the seed (van de Ven and McNicol 1997, Hodgetts et al. 2001, Mimura and Aitken 2007a, 2007b).

\section{DNA-extraction and amplification protocols}

DNA was extracted from the above mentioned tissues using:

- the DNeasy 96 Plant Kit from QIAGEN (A'Hara and Cottrell 2004, 2007, 2009);

- the Doyle and Doyle (1990) CTAB protocol (Gapare et al. 2005, Gapare and Aitken 2005, Bennuah et al. 2004, Rungis et al. 2004);

- a modified protocol of Murray and Thompson (1980) which is described in detail in Hodgetts et al. (2001).

\section{Examples of amplification protocols}

- Initial denaturation step of $95^{\circ} \mathrm{C}$ for $2 \mathrm{~min} ; 30$ cycles of $95^{\circ} \mathrm{C}$ for $20 \mathrm{~s}, 53^{\circ} \mathrm{C}$ for $20 \mathrm{~s}$, and $72^{\circ} \mathrm{C}$ for $30 \mathrm{~s}$; followed by a final extension step of $72^{\circ} \mathrm{C}$ for $3 \mathrm{~min}$ (Rungis et al. 2004).

- Initial denaturation step of $94^{\circ} \mathrm{C}$ for $3 \mathrm{~min}$, followed by 10 cycles of $94^{\circ} \mathrm{C}$ for $30 \mathrm{~s}, 57^{\circ} \mathrm{C}$ for $1 \mathrm{~min}$ and $72{ }^{\circ} \mathrm{C}$ for $30 \mathrm{~s}$. This was followed by 28 cycles of 94 ${ }^{\circ} \mathrm{C}$ for $30 \mathrm{~s}, 55^{\circ} \mathrm{C}$ for $1 \mathrm{~min}$ then $72{ }^{\circ} \mathrm{C}$ for $30 \mathrm{~s}$. A final elongation step at $72{ }^{\circ} \mathrm{C}$ for 6 min was then carried out (A'Hara and Cottrell 2007, 2009).

- Initial denaturation step at $94^{\circ} \mathrm{C}$ for $5 \mathrm{~min}$ was followed by 25 cycles consisting of $94^{\circ} \mathrm{C}$ (denaturation) for $30 \mathrm{~s}$, the appropriate annealing temperatures (see Table 3) for $30 \mathrm{~s}$ and a final elongation step at $72^{\circ} \mathrm{C}$ for 30 s (Hodgetts et al. (2001).

\section{Important results}

- A considerable number of highly polymorphic nSSR markers are available for genetic analysis of Sitka spruce (see Table 3). A part of them were developed especially for Sitka spruce; others were transferred from related species.
- $\mathrm{nSSR}$ were successfully applied to species and clone identification, hybridization studies, analysis of genetic diversity along the natural distribution range and in introgression zones.

- Comparison of central and peripheral Sitka spruce populations within the natural distribution range shows pronounced genetic differences. While core populations of Sitka spruce have little withinpopulation genetic structure, peripheral populations are strongly spatially structured at distances up to $500 \mathrm{~m}$. Higher allelic richness and gene diversity was observed in central populations in comparison with peripheral ones. Also, in the central and continuous populations, more private alleles were identified. Gene diversity was lowest in isolated peripheral populations. Selfing rate increased from $7.3 \%$ in central populations to as high as $35.2 \%$ in a northern, isolated population from Kodiak Island (Mimura and Aitken 2007a, 2007b). Inbreeding is higher in peripheral populations. In core populations, the number of migrants is significantly higher (Gapare et al. 2005, Gapare and Aitken 2005, Gapare et al. 2008).

- Given the above findings, different sampling protocols are needed for central and peripheral populations. Because of stronger within-population spatial genetic structure, a higher number of samples is needed in peripheral areas (indication: 180 samples from at least $324 \mathrm{ha}$ ) (Gapare et al. 2008).

- Following Rajora et al. (2001), the closely related spruce species Picea sitchensis and Picea glauca can be distinguished by the PGL12 SSR marker. PGL12 amplifies only in Picea glauca, while in Picea sitchensis no amplification products were observed.

- Bennuah et al. (2004) developed a useful hybrid index for classifying individual families and populations in introgressed populations between Sitka and white spruce based on EST-SSR markers developed by Perry and Bosquet (1998a, 1998b). Results led to the conclusion that in the middle of the introgression zone no pure individuals of one species are present.

- In the introgression zone, little differentiation between populations was found. Hamilton and Aitken (2013) attribute this to widespread gene flow between the two species.

\section{b) SNPs (single-nucleotide polymorphisms)}

Holliday et al. (2010) investigated single-nucleotide polymorphisms (SNPs) in Sitka spruce using an Illumina 
GoldenGate genotyping array. These SNPs were developed from re-sequencing of coding genes that were chosen partially on the basis of gene expression differences during autumn cold acclimation (Holliday et al 2008) and partially on the basis of functional information from model species (e.g., Arabidopsis thaliana, Populus trichocarpa). In addition, -100 SNPs were genotyped from randomly chosen genes to serve as a set of neutral markers. Among 768 SNPs designed for the array, 339 gave high quality genotypes and were polymorphic, which is similar to contemporaneous studies in other conifers. These SNPs were used to investigate demographic history and local adaptation. For the latter, numerous genotypephenotype relationships were uncovered, demonstrating the utility of SNP markers for understanding adaptation (Holliday et al. 2010). This same SNP panel served as a basis for studies related to introgression between Sitka spruce and white spruce (Hamilton et al. 2012; Hamilton et al. 2013), and between Sitka spruce and Engelmann spruce (De La Torre et al. 2015).

Pavy et al. (2013) designed two high-density SNP arrays relying on the Infinium iSelect technology (Illumina) for use in white spruce (Picea glauca), one with 7338 segregating SNPs representative of 2814 genes of various molecular functional classes, the other one with 9559 segregating SNPs representative of 9543 genes. $22.4 \%$ of these SNPs were segregating in Sitka spruce, too.

These studies demonstrate the efficiency and quality of array-based genotyping, and we expect such methods to continue to be useful. At the same time, the field is moving toward next generation sequence based genotyping. Of particular utility for conifers such as Sitka spruce, with their large genomes, is genotyping by sequencing (GBS) and especially sequence capture. For example, Suren et al. (2016) showed that sequence capture can be successfully used in both interior spruce (Picea glauca $x$ engelmanii) and lodgepole pine (Pinus contorta), and while we are not aware of related efforts in Sitka spruce, it should be feasible to use the same capture baits in this species (based on Suren et al's successful capture and sequencing of DNA from several congeners).

\section{Material for extraction}

For DNA extraction, needles were used (Holliday et al. 2010).

\section{DNA-extraction methods}

DNA extraction was performed using a modified CTAB protocol (Doyle and Doyle, 1990) (Holliday et al. 2010).

\section{Important results}

- According to Holliday et al. (2010), SNPs reveal three clusters for Sitka spruce:

1. Populations in California, Oregon, and British Columbia;

2. Populations in Alaska;

3. Populations from Kodiak Island.

- Genotype-phenotype associations for cold hardiness and bud set timing were found in 28 of the candidate genes described above. Interestingly, co-variance between tests of selective neutrality and latitudinal population origin suggest that postglacial history has impacted variation across the range of Sitka spruce, and suggests that caution is warranted in genotypeenvironment tests, as spurious relationships are likely if population structure is not effectively controlled (Holliday et al. 2010).

- The high number of SNPs developed can be useful in studies of genetic association, population genetics, genomic prediction and genome linkage mapping (Pavy et al. 2013).

\section{References}

A'Hara, S.W., Cottrell, J.E. (2004): A set of microsatellite markers for use in Sitka spruce (Picea sitchensis) developed from Picea glauca ESTs. Molecular Ecology Notes, 4: 659-663.

A'Hara, S.W., Cottrell, J.E. (2007): Characterization of a Suite of 40 EST-derived Microsatellite Markers for Use in Sitka Spruce (Picea sitchensis (Bong.) Carr.). Silvae Genetica, 56: 3-4.

A'Hara, S.W., Cottrell, J.E. (2009): Development of a set of highly polymorphic genomic microsatellites (gSSRs) in Sitka spruce (Picea sitchensis (Bong.) Carr.). Mol. Breeding, 23: 349-355.

Amarasinghe, V., Carlson, J.R. (2002): The development of microsatellite DNA markers for genetic analysis in Douglas-fir. Canadian Journal of Forest Research, 32: 1904-1915.

Bennuah, S.Y., Wang, T., Aitken, S.N. (2004): Genetic analysis of the Picea sitchensis $\mathrm{x}$ glauca introgression zone in British Columbia. Forest Ecology and Management, 197: 65-77.

Bérubé, Y., Zhuang, J., Rungis, D., Ralph, S., Bohlmann, J., Ritland, K. (2009): Characterization of EST-SSRs in loblolly pine and spruce. Tree Genetics and Genomes. 
Chaisurisri, K., El-Kassaby, Y. A. (1993): Genetic Control of Isoenzymes in Sitka Spruce. Journal of Heredity, 84(3): 206-211.

Chaisurisri, K., El-Kassaby, Y.A. (1994): Genetic diversity in a seed production population vs. natural populations of Sitka Spruce. Biodiversity and Conservation, 3: 512523.

Chaisurisri, K., Mitton, J.B., El-Kassaby, Y.A. (1994): Variation in the mating system of Sitka spruce (Picea sitchensis): Evidence for positive assortative mating. American Journal of Botany, 81(11): 1410-1415.

Coombe, L., Warren, R.L., Jackman, S.D., Yang, C., Vandervalk, B.P., Moore, R.A., et al. (2016): Assembly of the complete Sitka Spruce Chloroplast Genome Using 10X Genomics' GemCode SequencingData. PLoS ONE, 11(9): e0163059. doi:10.1371/journal. pone.0163059.

Copes, D.L., Beckwith, R.C. (1977): Isoenzyme identification of Picea glauca, P. sitchensis, and P. lutzii populations. Botanical Gazette, 138(4): 512-521.

Cottrell, J .E., White, I. M. S. (1995): The use of isozyme genetic markers to estimate the rate of outcrossing in a Sitka spruce (Picea sitchensis (Bong.) Carr.) seed orchard in Scotland. New Forests, 10:,111- 122.

De La Torre, A., Ingvarsson, P.K., Aitken, S.N. (2015): Genetic architecture and genomic patterns of gene flow between hybridizing species of Picea, Heredity, 115:,153-164.

Demesure, B., Sodzi, N., Petit, R.J. (1995): A set of universal primers for amplification of polymorphic noncoding regions of mitochondrial and chloroplast DNA in plants. Molecular Ecology, 4: 129-131.

Doyle, J.J., Doyle, J.L. (1990): Isolation of plant DNA from fresh tissue. Focus, 23: 13-15.

Gapare W.J., Aitken, S.N., Ritland, C.E. (2005): Genetic diversity of core and peripheral Sitka spruce (Picea sitchensis (Bong.) Carr.) populations: implications for conservation of widespread species. Biological Conservation, 123:,113-123.

Gapare, W. J., Aitken, S.N. (2005): Strong spatial genetic structure in peripheral but not core populations of Sitka spruce [Picea sitchensis (Bong.) Carr.]. Molecular Ecology, 14: 2659-2667.

Gapare, W.J., Yanchuk, A.D., Aitken, S.N. (2008): Optimal sampling strategies for capture of genetic diversity differ between core and peripheral populations of Picea sitchensis (Bong.) Carr. Conservation Genetics, 9: 411-418.

Hamilton J.A., Aitken, S.N. (2013): Genetic and morphological structure of a spruce hybrid (Picea sitchensis $\times P$. glauca) zone along a climatic gradient. American Journal of Botany, 100(8): 1651-1662.

Hamilton, J. A., Lexer, C., Aitken, S.N. (2012): Genomic and phenotypic architecture of a spruce hybrid zone (Picea sitchensis x P. glauca). Molecular Ecology, doi: 10.1111/mec. 12007.

Hamilton, J.A., Ralph, S.G., White, R., Bohlmann, J., Aitken, S.N. (2008): Global monitoring of autumn gene expression within and among phenotypically divergent populations of Sitka spruce (Picea sitchensis). New Phytologist, 178: 103-122.

Hodgetts, R.B., Aleksiuk, M.A., Brown, A., Clarke, C., Macdonald, E., Nadeem, S. Khasa, D. (2001): Development of microsatellite markers for white spruce (Picea glauca) and related species. Theoretical and Applied Genetics, 102: 1252-1258.

Holliday, J.A., Ritland, K., Aitken, S.N. (2010): Widespread, ecologically relevant genetic markers developed from association mapping of climate-related traits in Sitka spruce (Picea sitchensis)., New Phytologist, 188(2): 501-514.

Holliday, J.A., Tongli W., Aitken, S.A. (2012): Predicting Adaptive Phenotypes From Multilocus Genotypes in Sitka Spruce (Picea sitchensis) Using Random Forest. G3 Genes, Genomes, Genetics, 2: 1085-1093.

Holliday, J.A., Yuen, M., Ritland, R., Aitken, S.N. (2010): Postglacial history of a widespread conifer produces inverse clines in selective neutrality tests. Molecular Ecology, 19: 3857-3864.

Jaramillo-Correa, J.P., Bousquet, J. (2003): New evidence from mitochondrial DNA of a progenitor-derivative species relationship between black spruce and red spruce (Pinaceae). American Journal of Botany, 90: 1801-1806.

Mason, B., Perks, M.P. (2011). Sitka spruce (Picea sitchensis) forests in Atlantic Europe: changes in forest management and possible consequences for carbon sequestration. Scandinavian Journal of Forest Research, 26 (Suppl 11): 72-81.

Mimura, M., Aitken, S.N. (2007a): Adaptive gradients and isolation-by-distance with postglacial migration in Picea sitchensis. Heredity, 99: 224-232.

Mimura, M., Aitken, S.N. (2007b): Increased selfing and decreased effective pollen donor number in peripheral relative to central populations in Picea sitchensis (Pinaceae). American Journal of Botany, 94(6): 991-998.

Mitchell, A. (1978): Trees of Britain and Northern Europe. Collins Field Guide. Harper Collins. London. 
Pavy, N., Gagnon, F., Rigault, Ph. BLAIS, S., Deschênes, A., Boyle, B., Pelgas, B., Deslauriers, M., Clement, S., Lavigne, P., Lamothe, M., Cooke, J.E.K., JaramilloCorrea, J.P., Beaulieu, J., Isabel, N., Mackay. J., Bousquet, J. (2013): Development of high-density SNP genotyping arrays for white spruce (Picea glauca) and transferability to subtropical and nordic congeners. Molecular Ecology Resources. doi: 10.1111/1755-0998.12062.

Rajora, O.P., Rahman, M.H., Dayanandan, S., Mosseler, A. (2001): Isolation, characterization inheritance and linkage of microsatelite markers in white spruce (Picea glauca) and their usefullnes in other spruce species. Molecular Genetics and Genomics, 246: 871-882.

Roche, L., Haddok, P.G. (1987). Sitka spruce (Picea sitchensis) in North America with special reference to its role in British forestry. In: (eds.) Henderson, D.M., Faulkner, R. Proceedings, Section B (Biological Sciences), The Royal Society of Edinburgh, 1-13.

Ralph, S.G., Chun, H.J.E, Kolosova, N., Cooper, D., Oddy, C., Ritland, C.E., Kirkpatrick, R., Moore, R., Barber, S., Holt, R.A., Jones, S.J.M., Marra, M.A., Douglas, C.J., Ritland, K., Bohlmann, J. (2008): A conifer genomics resource of 200,000 spruce (Picea spp.) ESTs and 6,464 high-quality, sequence-finished fulllength cDNAs for Sitka spruce (Picea sitchensis). BMC Genomics, 9: 484.

Ran, J.H., Wei, X.-X., Wang, X.-Q. (2006): Molecular phylogeny and biogeography of Picea (Pinaceae): Implications for phylogeographical studies using cytoplasmic haplotypes. Molecular Phylogenetics and Evolution, 41: 405-419.

Rungis, D., Bérubé, Y., Zhang, J., Ralph, S., Ritland, C. E., Ellis, B.E., Douglas, C., Bohlmann, J., Ritland, K. (2004): Robust simple sequence repeat markers for spruce (Picea spp.) from expressed sequence tags. Theoretical and Applied Genetics, 109: 1283-1294.

Scotti, I., Magni, F., Fink, R., Powell, W., Binelli, G., Hedley, P.E. (2000): Microsatellite repeats are not randomly distributed within Norway spruce (Picea abies K.) expressed sequences. Genome, 43: 41-46.

Scotti, I., Magni, F., Paglia, G., Morgante, M. (2002a): Trinucleotide microsatellites in Norway spruce (Picea abies): their features and the development of molecular markers. Theoretical and Applied Genetics, 106: 40-50.

Scotti, I., Paglia, G., Magni, F., Morgante, M. (2002b): Efficient development of dinucleotide microsatellite markers in Norway spruce (Picea abies Karst.) through dot-blot selection. Theoretical and Applied Genetics, 104: 1035-1041.

Soltis, D.E., Gitzendanner, M. A., Strenge, D.D.,
Soltis, P.S. (1997): Chloroplast DNA intraspecific phylogeography of plants from the Pacific Northwest of North America. Plant Systematics and Evolution, 206: 353-373.

Suren, H., Hodgins, K.A., Yeaman, S., Nurkowski, K.A., Smets, P., Rieseberg, L.H., Aitken, S.N., Holliday, J.A. (2016): Exome capture from the spruce and pine gigagenomes. Molecular Ecology Resources, 16(5): 11361146.

Sutton, B.C., Flanagan, D.J., Gawley, J.R., Newton, C., Lester, D.T., El-Kassaby, Y.A. (1991): Inheritance of chloroplast and mitochondrial DNA in Picea and composition of hybrids from introgression zones. Theoretical and Applied Genetics, 82: 242-248.

Szmidt, A.E., Lidholm, J., Hällgren, J.E. (1986): DNA extraction and preliminary characterization of chloroplast DNA from Pinus sylvestris and Pinus contorta. In: Lindgren D (ed):Provenances and forest tree breeding for high latitudes. Proceedings of the Frans Kempe Symp, Umea, Sweden, pp 269-280.

Szmidt, A.E., El-Kassaby, Y.A., Sigurgeirsson, A., Alden, T., Lindgren, D., Hällgren, J.E. (1988): Classifying seedlots of Picea sitchensis and P. glauca in zones of introgression using restriction analysis of chloroplast DNA. Theoretical and Applied Genetics, 76(6): 841-845

VandeVen, W.T.G., McNicol,R.G.(1996):Microsatellites as DNA markers in Sitka spruce. Theor. Appl. Genet., 93: 613-617.

Peterson, E.B., Peterson, N.M., Weetman, G.F., Martin, P.J. (1997). Ecology and management of Sitka spruce, emphasizing its natural range in British Columbia. UBC Press, Vancouver, Canada.

Perry, D.J., Bousquet, J. (1998a):Sequence-taggedsite (STS) markers of arbitrary genes: development, characterization and analysis of linkage in black spruce. Genetics, 149: 1089-1098.

Perry, D.J., Bousquet, J. (1998b): Sequence-tagged-site (STS) markers of arbitrary genes: the utility of black spruce-derived STS primers in other conifers. Theoretical and Applied Genetics, 97: 735-743.

Pfeiffer, A., Olivieri, A.M., Morgante, M. (1997):Identification and characterization of microsatellites in Norway spruce (Picea abies K.). Genome, 40: 411-419.

van de Ven, W.T.G., McNicol, R.J. (1996): Microsatellites as DNA markers in Sitka spruce. Theoretical and Applied Genetics, 93: 613-617.

Wang, X.-Q., Tank, D.C., Sang, T., 2000. Phylogeny and divergence times in Pinaceae: evidence from three 
genomes. Molecular Biology and Evolution, 17: 773781.

Wagner, DB, Furnier GR, Saghai-Maroof MA, Williams SM, Dancik BP, Allard RW (1987) Chloroplast DNA polymor- phisms in lodgepole and jack pines and their hybrids. Proceedings of the National Academy of Sciences USA, 84: 2097-2100.

White, E.E. (1986): A method for extraction of chloroplast DNA from conifers. Plant Molecular Biology Reporter, 4: 98-101.

Yeh, F.C. (1979): Analyses of Gene Diversity in Some Species of Conifers. Presented at the Symposium on Isozymes of North American Forest Trees and Forest Insects, Berkeley, Calif.

Yeh, F.C., Layton, C: (1979): The organization of genetic variability in central and marginal populations of lodgepole pine Pinus contorta ssps. Latifolia. Canadian Journal of Genetics and Cytology, 21: 487-503.

Yeh, F.C., El-Kassaby, Y.A. (1980): Enzyme variation in natural populations of Sitka spruce (Picea sitchensis). I. Genetic variation patterns among trees from 10 IUFRO provenances. Canadian Journal of Forest Research, 10: 415-422.

Yeh, F.C., O’Malley, D. (1980): Enzyme variations in natural populations of Douglas-fir, Pseudotsuga Menziesii (Mirb.) Franco, from British Columbia. 1. Genetic variation patterns in coastal populations. Silvae Genetica, 29: 83-92.

Yeh, F.C., Arnott, J.T. (1986): Electrophoretical and morphological differentiation of Picea sitchesis, Picea glauca and their hybrids. Canadian Journal of Forest Research, 16: 791-718. 


\title{
Molecular markers used for genetic studies in tree of heaven (Ailanthus altissima (Mill.) Swingle)
}

\author{
Marcela van Loo ${ }^{1}$ and Vlatko Andonovski ${ }^{2}$
}

\begin{abstract}
${ }^{1}$ University of Vienna, Department of Botany and Biodiversity Research, Rennweg 14, 1030 Vienna, Austria
${ }^{2}$ University Ss. Cyril and Methodius - Faculty of Forestry in Skopje, P.O.Box 235, 1000 Skopje, FYR Macedonia
\end{abstract}

\section{General remarks}

The genus Ailanthus consists of 5-15 species (depending on the authors and cited Flora), which have a wide distribution ranging from Asia to north Oceania (Engler 1931, Nooteboom 1962, eFloras 2008).

Ailanthus altissima (Mill.) Swingle (tree of heaven), with its largest native distribution in China, was in the middle of $18^{\text {th }}$ century mistakenly introduced to France and later to England when the seed sent from China was believed to be that of Chinese lacquer tree (Burch and Zedaker 2003). The tree of heaven soon became a popular planted species in Europe because of its attractive foliage, rapid growth, timber qualities and as forage for Samia cynthia (Drurvy), a species of silk-producing caterpillar (Huemer and Rabitsch 2002).

Three varieties of $A$. altissima have been described. Two of them (Ailanthus altissima var. altissima and var. sutchuensis (Dode) Rehd. \& Wilson) are distributed in China and North Vietnam (see Figure 1), whereas the third variety, var. tanakae (Hayata) Kanehira et Sasaki, was reported for Taiwan (eFloras 2008).

The tree of heaven is a diploid, dioecious species (Kowarik and Säumel 2007), with reported 80 and 64 chromosomes (Slavik 1997). It reproduces both sexually and asexually. Asexual reproduction takes place by vegetative sprouting from stumps or root portions $(\mathrm{Hu}$ 1979) forming clones smaller than 50 meters (Kowarik and Säumel 2007, Chuman et al. 2015). Flowering occurs rather late in spring (June). Seeds ripen in large crowded clusters from September to October of the same year and may persist on the tree through the following winter (Little 1974, Hu 1979). Not only can individual Ailanthus produce $>1$ million seeds per year, but a significant relationship exists between seed production and tree diameter (Wickert et al. 2017, Martin and Canham 2010). In addition, it can already produce seed as young as four years of age. Seedlings grow quickly in full sunlight and average a meter of growth in height per year for at least the first 4 years (Adamik and Brauns 1957).

Prolific fruiting, ready germination, adaptability to infertile sites and rapid growth rate make $A$. altissima a noxious weed in many countries where it has been introduced (Feret 1985, Shah 1997). This species is often invasive in its introduced range, where it can colonize native plant communities and greatly reduce their species richness (Kowarik 1995, Knapp and Canham 2000, Merriam 2003, Wickert et al. 2017).

\section{Isozymes}

Only one publication - Feret and Bryant (1974) - dealing with isozyme analysis is known for Ailanthus altissima. Fifteen peroxidase isozymes were investigated for their presence/absence in five Chinese- (native range) and five American- seed sources (introduced range). Isoenzymes

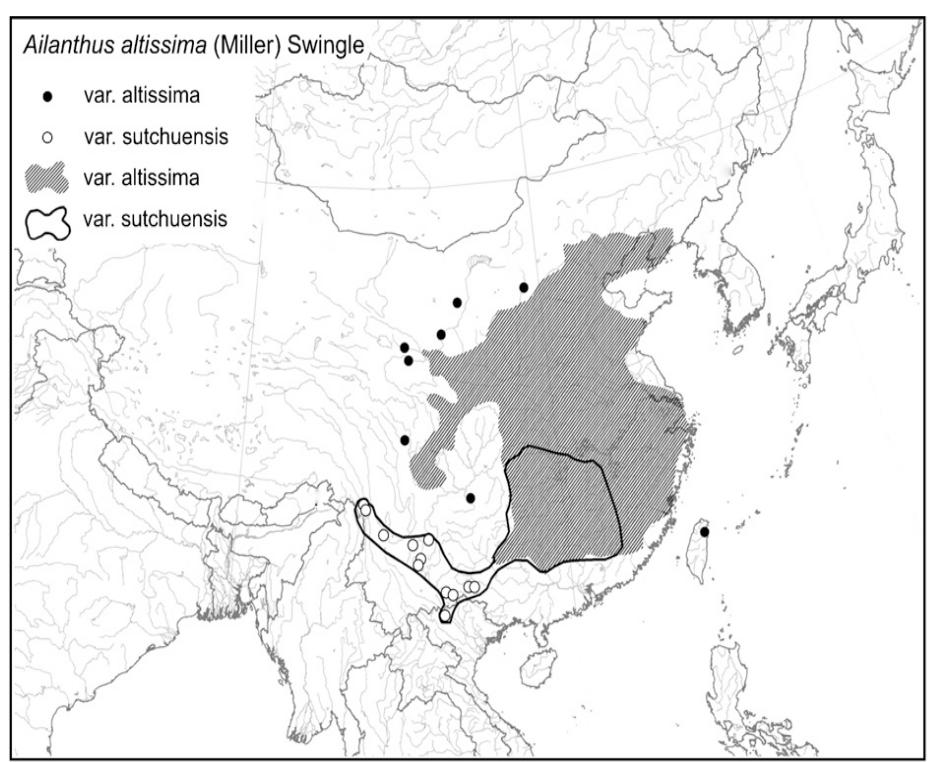

Figure 1. Native distribution range of tree of heaven (source: Kowarik and Sämuel 2007) 
were numbered from 1 to 15 . Authors didn't present the names of isozymes (including E.C. numbers), nor scored loci or alleles. They refer only to presence-absence of peroxidase isozymes 1 to 15 .

\section{Material for protein extraction}

Enzymes were extracted from leaf material (Feret and Bryant 1974).

\section{Protein extraction and separation protocols}

For isozyme separation electrophoresis on polyacrylamide disc gels was used. Methodological details are published in Feret (1970).

\section{Important results}

- No unique isozymes exist for the North American and Chinese seed sources.

- The studied isozymes were similar in frequency in both ranges. According to the authors, this indicates that the gene pool of Ailanthus in North America is not necessarily depauperate and probably is nearly as diverse as native populations with regard to "allelic variability".
- From the practical view, authors (Feret and Bryant 1974, Feret 1985) assumed that these results probably means that if you want to improve the species in North America, you might not gain much by importing additional genotypes from China.

\section{Organelle markers (chloroplast (cp)DNA)}

Four studies have so far used chloroplast DNA-markers in Ailanthus altissima: Liao et al. (2014), Chuman et al. (2015), Kurokochi et al. (2013, 2015).

Loci and primers used (see Table 1)

Analysis of Liao et al (2014)

- 3 Plastid DNA markers psbA-trnH, trnD-trnT, trnL-trnF

\section{Analysis of Kurokochi et al. (2013)}

- 4 Plastid DNA markers trnL5'F - 3'trnL R, 3'trnG5'trnG2 G, matKM - A6_inner, ycf6-R - E2_inner

- 3 Plastid DNA markers psbA-trnH, $\operatorname{trn} \mathrm{D}-\operatorname{trn} \mathrm{T}$, trnL-trnF

Table 1: Primer information of the chloroplast DNA-markers used for DNA sequencing in Ailanthus altissima

\begin{tabular}{|c|c|c|c|}
\hline Locus & $\begin{array}{c}\text { Primer sequence } \\
F=\text { Forward, } R=\text { Reverse }\end{array}$ & kind of marker & References \\
\hline psbA-trnH & $\begin{array}{l}\text { F:GTTATGCATGAACGTAATGCTC (psbAF) } \\
\text { R:CGCGCATGGTGGATTCACAAATC (trnHR) }\end{array}$ & \multirow{10}{*}{$\begin{array}{c}\text { Plastid } \\
\text { m.* sequencing }\end{array}$} & 1,9 \\
\hline $\operatorname{trnD}-\operatorname{trn} T$ & $\begin{array}{l}\text { F:ACCAATTGAACTACAATCCC }\left(\operatorname{trnD}^{\mathrm{GUC}} \mathrm{F}\right) \\
\text { R:CTACCACTGAGTTAAAAGGG }\left(\operatorname{trnT}^{\mathrm{GGU}} \mathrm{R}\right) \\
\end{array}$ & & 2,9 \\
\hline $\operatorname{trnL} \mathrm{t} \operatorname{trnF}$ & $\begin{array}{l}\text { F:ATTTGAACTGGTGACACGAG }\left(\operatorname{trnF}^{\mathrm{GAA}}=\mathrm{TabF}\right) \\
\text { R:GGTTCAAGTCCCTCTATCCC }\left(\operatorname{trnL}^{\mathrm{UAA}} \mathrm{F}=\mathrm{TabE}\right)\end{array}$ & & 3,9 \\
\hline $\operatorname{trnL} 5^{\prime} F-3^{\prime} \operatorname{trnL} R$ & $\begin{array}{l}\text { F:CGAAATCGGTAGACGCTACG (TabC) } \\
\text { R:GGGGATAGAGGGACTTGAAC (TabD) }\end{array}$ & & 3,6 \\
\hline $3^{\prime} \operatorname{trnG}-5^{\prime} \operatorname{trn} G 2 \mathrm{G}$ & $\begin{array}{l}\text { F:GTAGCGGGAATCGAACCCGCATC } \\
\text { R:GCGGGTATAGTTTAGTGGTAAAA }\end{array}$ & & 4,6 \\
\hline matKM-A6_inner & $\begin{array}{l}\text { F:TCGACTTTCTGGGCTATC } \\
\text { R:CGTGCTTGCATTTTTCATTGC }\end{array}$ & & 5,6 \\
\hline ycf6-R-E2_inner & $\begin{array}{l}\text { F:GCCCAAGCRAGACTTACTATATCCAT } \\
\text { R:CGAAACAGCTGGGGTTTCTG }\end{array}$ & & 4,6 \\
\hline Aacp01 & $\begin{array}{l}\text { F:CGCTTATCCTTCATCCCTTTT } \\
\text { R:GGTGCAGAGACTCAATGGAGG }\end{array}$ & & 7,8 \\
\hline Aacp02 & $\begin{array}{l}\text { F:CGACAACCCAATCTGTAGTTC } \\
\text { R:CGATCAGATTATGGAGTGAATG }\end{array}$ & & 7,8 \\
\hline Aаср03 & $\begin{array}{l}\text { F:GACACCCTTGATGAAAGACT } \\
\text { R:GGGCAACAAAAAAACGAATAGGTC }\end{array}$ & & 7,8 \\
\hline
\end{tabular}

*maternally inherited, 1-Sang et al. 1997, 2-Demesure et al. 1995, 3-Taberlet et al. 1991, 4-Shaw et al. 2005, 5-Tate and Simpson 2003, 6-Kurokochi et al. 2013, 7-Kurokochi et al. 2015, 8-Chuman et al. 2015, 9-Liao et al 2014 
Analysis of Kurokochi et al. (2015) and Chuman et al. (2015)

- 3 Plastid DNA markers Aacp01, Aacp02, Aacp03

\section{Material for DNA-extraction}

DNA was extracted from leaves dried in silica (Kurokochi et al. 2013, Liao et al. 2014, Kurokochi et al. 2015, Chuman et al. 2015).

\section{DNA-extraction protocols}

Total genomic DNA extraction followed the method of Kurokochi et al. (2013) or Doyle and Doyle (1987).

\section{Important results}

- $\quad$ By sequencing of 4 non-coding plastid regions in 449 A. altissima trees sampled in 64 non-native Japanese populations and four native Chinese populations, six haplotypes were identified. Three plastid haplotypes were observed in Japan, whereas four were detected in Chinese populations. Most A. altissima trees in Japan harbored two different haplotypes. These two haplotypes were not genetically similar. Analysis of molecular variation showed some genetic differentiation among populations. Twenty-two Japanese populations contained two haplotypes within each population, whereas the other 42 Japanese populations were composed of only one haplotype indicating that Japanese populations may have originated from limited number of seed introductions (Kurokochi et al. 2013).

- Biogeographic history (refugia and patterns of migration during past climatic changes) in native range, in China, was revealed by sequencing three cpDNA markers and 440 individuals. Identification and geographical distribution of twelve haplotypes led to the hypothesis that multiple glacial refugia existed in mainland China during the Quaternary oscillations, out of which Ailanthus spread by three main dispersal routes (Liao et al. 2014).

- For results of Kurokochi et al. (2015) and Chuman et al. (2015) see paragraph 4 a) where analyses of cpDNA were combined with nSSRs.

\section{Nuclear DNA markers (nSSRs, SNPs) \\ a) nSSRs (putatively neutral microsatellites)}

Dallas et al. (2005) developed nine $(C T)_{n}$ nuclear microsatellite markers using European Ailanthus trees collected across Mediterranean region (Corsica, Crete, Lesbos, Mallorca, Menorca, and Sardinia). Microsatellites were developed using a combination of published methods based on enrichment (Koblízková et al. 1998, Gardner et al. 1999, Hamilton et al. 1999, see the full protocol at www.abdn.ac.uk/ $\sim$ nhi571). These simple sequence repeats (SSRs) were further used together with plastid markers by Kurokochi et al. (2015) and Chuman et al. (2015). Only five nSSRs were used in a study of American Ailanthus by Aldrich et al. (2010).

\section{Material for DNA-extraction}

Genomic DNA was extracted from silica-gel-dried leaf tissue (Dallas et al. 2005, Aldrich et al. 2010, Chuman et al. 2015).

\section{DNA-isolation protocols}

Protocols for DNA-isolation are published in Dallas et al. (2005). Aldrich et al (2010) extracted DNA from leaf tissue using the DNeasy Plant Minikit (Qiagen).

\section{Important results}

- Developed nSSRs are useful for genetic analyses of tree of heaven and its close relatives as they were polymorphic in each of three studied varieties of tree of heaven ( $A$. altissima var. erythrocarpa, A. altissima var. sutchuensis, $A$. altissima var. tanakai) and two other Ailanthus species (A. giraldii, A. vilmariniana) (Dallas et al. 2005).

- In the USA, where this species was introduced from both sides of the continent, small but significant genetic differences were found between populations with little correspondence between geographic and genetic distance (Aldrich et al. 2010). These conclusions are consistent with a model of multiple introductions followed by high rates of genetic exchange between cities and regions.

- In Japan, the mechanism of range expansion was estimated by $9 \mathrm{nSSR}$ and 3 plastid markers (see the paragraph on plastid markers for detail) in 35 patches located within three sites (Chuman et al. 2015). Analyses with markers showed limited asexual reproduction up to 45 meters. Most related genotypes were detected within the same patch with an extent of nonrandom spatial genetic structure up to $2 \mathrm{~km}$ indicating natural regeneration from seeds.

- In Japan, also genetic structure of planted and naturalized populations was estimated by 
Table 2: Primer information of nSSR-markers used for genetic analysis of Ailanthus altissima $\left(\mathrm{N}_{\mathrm{a}}=\right.$ number of alleles scored)

\begin{tabular}{|c|c|c|c|c|c|c|}
\hline Locus & Motif & $\begin{array}{l}\text { Primer sequence }\left[5^{6}-3^{c}\right]^{a} \\
F=\text { Forward, R= Reverse }\end{array}$ & Size (bp) & $\mathbf{N}_{\mathrm{a}}$ & Ref. & $\begin{array}{c}\text { GenBank } \\
\text { Accession no. }\end{array}$ \\
\hline $\mathrm{Aa} 22$ & $(\mathrm{CT})_{20}$ & $\begin{array}{l}\text { F:CTGGTATCTGAATTTGAGCAGTAGC } \\
\text { R:GAACAAATTAATCCCAAGTGAAGC }\end{array}$ & $171-211$ & 11 & 1,4 & AY750965 \\
\hline Aa68 & $\begin{array}{l}(\mathrm{CT})_{24} \text { ATCT } \\
(\mathrm{AT})_{9}\end{array}$ & $\begin{array}{l}\text { F:AACTTGATTAGTTTATATTAGGCGTGAC } \\
\text { R:AAGTCCGATTGAAATTACAAGTCC } \\
\end{array}$ & $206-233$ & 12 & 1 & AY750966 \\
\hline $\mathrm{Aa} 69$ & $\begin{array}{l}(\mathrm{CT}) 15 \\
(\mathrm{CA}) 17\end{array}$ & $\begin{array}{l}\text { F:CATGGAAGCCTCTTGGAAAC } \\
\text { R:TGAAGCAAATATGTGAAACAACC }\end{array}$ & $152-214$ & 8 & 1,4 & AY750967 \\
\hline Aa75 & $(\mathrm{CT})_{25}$ & \begin{tabular}{|l|} 
F:CTCTTGCATCTGAAATAGTGAACG \\
R:GTTTGTTTTGGCTAAATGCTATTACC \\
\end{tabular} & $93-138$ & 10 & 1,4 & AY750968 \\
\hline Aa76 & $(\mathrm{CT})_{24}$ & $\begin{array}{l}\text { F:AAGCAAAGTCAAGGCCAGAC } \\
\text { R:CCATTTCACCСACСTTCTTC } \\
\end{array}$ & $141-194$ & 8 & 1,4 & AY750969 \\
\hline Aa79 & $\begin{array}{l}(\mathrm{CT})_{14} \mathrm{CC} \\
(\mathrm{CT})_{14} \\
\end{array}$ & $\begin{array}{l}\text { F:TGCTGCCAATGTCAGTGATG } \\
\text { R:TTCACACAAAGAAACCCATGTC }\end{array}$ & $122-172$ & 12 & 1 & AY750970 \\
\hline Aa80 & $(\mathrm{CT})_{26}$ & $\begin{array}{l}\text { F:GAAGAAATGAATTGACAGTTGACC } \\
\text { R:ATTTACACTAGGGCTACCAACACC } \\
\end{array}$ & $167-225$ & 7 & 1 & AY750971 \\
\hline Aa82 & $(\mathrm{CT})_{29}$ & $\begin{array}{l}\text { F:CAACATTCCGTGATTCACACTC } \\
\text { R:CTTGCACGCTTCAGTGAAAG }\end{array}$ & $109-170$ & 7 & 1,4 & AY750972 \\
\hline Aa92 & $\begin{array}{l}(\mathrm{CT}){ }_{16} \mathrm{CC} \\
(\mathrm{CT})_{8}(\mathrm{AT})_{11}\end{array}$ & $\begin{array}{l}\text { F:CTTGAACAGAAACAAATGCAAAG } \\
\text { R:GAAATTGTTTAAATGCCACTACCTG }\end{array}$ & $157-195$ & 11 & $1,2,3$ & AY750973 \\
\hline
\end{tabular}

$T_{a}^{*}=$ annealing temperature, in touch down PCR first $60^{\circ} \mathrm{C}-49^{\circ} \mathrm{C}$ (decreasing by 0.5 per cycle), then $50^{\circ} \mathrm{C}$.

1- Dallas et al. 2005, 2-Kurokochi et al. 2015, 3-Chuman et al. 2015, 4-Aldrich et al. 2010.

combination of 9 nSSRs and 3 plastid markers (see the paragraph on plastid markers for details) (Kurokochi et al. 2015). There was no obvious genetic differentiation between planted and naturalized populations. Nevertheless, two main plastid haplotypes were recognized within trees allowing to separate studied individuals into two groups. Within each haplotype group most trees were strictly assigned to one cluster indicating two distinct provenances. An admixture between the two lineages has occurred, but remained limited.

\section{b) SNPs (single-nucleotide polymorphisms)}

No publications on SNP analysis for Ailanthus altissima exist.

\section{References}

Adamik, K.J., Brauns, F.E. (1957): Ailanthus glandulosa (tree of heaven) as a pulpwood. Part II. Tappi, 40(7): 522-527.

Aldrich, P.R., Briguglio, J.S., Kapadia, S.N., Morker,
M.U., Rawal, A., Kalra, P., Huebner, C.D., Greer, G.K. (2010): Genetic structure of the invasive tree Ailanthus altissima in eastern United States cities. Journal of Botany, 1-9.

Burch, P.L., Zedaker, S.M. (2003): Removing the invasive tree Ailanthus altissima and restoring natural cover. Journal of Arboriculture, 29: 18-24.

Chuman, M., Kurokochi, H., Saito, Y., Ide, Y. (2015): Expansion of an invasive species, Ailanthus altissima, at a regional scale in Japan. Journal of Ecology and Environment, 38(1): 47-56.

Dallas, J.F., Leitch, M.J.B., Hulme, P.E. (2005): Microsatellites for tree of heaven (Ailanthus altissima). Molecular Ecological Notes, 5: 340-342.

Demesure, B., Sodzi, N., Petit, R.J. (1995): A set of universal primers for amplification of polymorphic noncoding regions of mitochondrial and chloroplast DNA in plants. Molecular Ecology, 4: 129-134.

Doyle, J.J., Doyle, J.L. (1987): A rapid DNA isolation procedure for small quantities of fresh leaf material. Phytochemistry, 19: 11-15.

eFloras (2008): Published on the Internet http://www. efloras.org. Missouri Botanical Garden, St. Louis, MO \& 
Harvard University Herbaria, Cambridge, MA. Accessed 01/12/2017.

Engler, A. (1931): In: Engler, A., Prantl, K. (Eds.) Die natürlichen Pflanzenfamilien, 2(19a), Wilhelm Engelmann, Leipzig.

Feret, P.P. (1970): Disc electrophoresis of enzymes in Ulmus spp. and Picea glauca- PhD. Disseration, Univ. of Wisconsin, Madison, Wis., pp. 119.

Feret, P.P. (1985): Ailanthus: Variation, cultivation, and frustration. Journal of Arboriculture, 11: 361-368.

Feret, P.P., Bryant, R.L. (1974): Genetic differences between American and Chinese Ailanthus seedlings. Silvae Genetica, 23(5): 144-148.

Gardes, M., Bruns, T.D. (1993): ITS primers with enhanced specificity for basidiomycetes: application to the identification of mycorrhizae and rusts. Mol. Ecol., 2: 113-118.

Hamilton, M.B., Pincus, E.L., Di Fiore, A., Fleischer, R.C. (1999): Universal linker and ligation procedures for construction of genomic DNA libraries enriched for microsatellites. BioTechniques, 27: 500-507.

Hu, S.Y. (1979): Ailanthus. Arnoldia, 39(2): 29-50.

Huemer, P., Rabitsch, W. (2002): 6.3.19 Schmetterlinge (Lepidoptera) pp. 354-362. In: Essl, F., Rabitsch, W.: Neobiota in Österreich. Umweltbundesamt, Wien, pp. 355.

Knapp, L.B., Canham, C.H.D. (2000): Invasion of an Old-Growth Forest in New York by Ailanthus altissima: Sapling Growth and Recruitment in Canopy Gaps. Journal of the Torrey Botanical Society, 127(4): 307315.

Koblízková, A., Dolezel, J., Macas, J. (1998): Subtraction with 3囚 modified oligonucleotides eliminates amplification artifacts in DNA libraries enriched for microsatellites. BioTechniques, 25: 32-38.

Kowarik, I. (1995): Clonal growth in Ailanthus altissima on a natural site in West Virginia. Journal of Vegetation Science, 6: 853-856.

Kowarik, I., Säumel, I. (2007): Biological flora of Central Europe: Ailanthus altissima (Mill.) Swingle. Perspectives in Plant Ecology, Evolution and Systematics, 8(4): 207237.

Kurokochi, H., Saito, Y., Chuman, M., Ide, Y. (2013): Low chloroplast diversity despite of phylogenetically divergent haplotypes in Japanese populations of Ailanthus altissima (Simaroubaceae). Botany, 91: 148-154.

Kurokochi, H., Saito, Y., Ide, Y. (2015): Genetic structure of the introduced Heaven Tree (Ailanthus altissima) in Japan: Evidence for two distinct origins with limited admixture. Botany, 93(3): 133-139.

Liao, Y.Y., Guo, Y.H., Chen, J.M., Wang, Q.F. (2014): Phylogeography of the widespread plant Ailanthus altissima (Simaroubaceae) in China indicated by three chloroplast DNA regions. Journal of Systematics and Evolution, 52(2):175-185.

Little, S. (1974): Ailanthus altissima. In Schopmeyer, C.S. (ed.), Seeds of Woody plants in the United States. USDA Forest Service Agriculture Handbook, No. 450.

Martin, P.H., Canham, C.D. (2010): Dispersal and recruitment limitation in native versus exotic tree species: Life-history strategies and Janzen-Connell effects. Oikos, 119: 807-824.

Merriam, R.W. (2003): The abundance, distribution and edge associations of six non-indigenous harmful plants across North Carolina. Journal of the Torrey Botanical Society, 130: 283-291.

Noteboom, H.P. (1962): Simaroubaceae. Flora Malesiana, Ser. 1.6: 193-226.

Sang, T., Crawford, D.J., Stuessy, T.F. (1997): Chloroplast DNA phylogeny, reticulate evolution, and biogeography of Paeonia (Paeoniaceae). American Journal of Botany, 84: 1120-1136.

Shah, B. (1997): The checkered career of Ailanthus altissima. Arnoldia, 57: 20-27.

Shaw, J., Lickey, E.B., Beck, J.T., Farmer, S.B., Liu, W., Miller, J., Siripun, K.C., Winder, C.T., Schilling, E.E., Small, R.L. (2005): The tortoise and the hare II: relative utility of 21 noncoding chloroplast DNA sequences for phylogenetic analysis. American Journal of Botany, 92: 142-166.

Slavík, B. (Ed.)(1997): Květena České republiky [Flora of the Czech Republic], vol. 5, Academia, Praha

Taberlet, P., Gielly, L., Pautou, G., Bouvet, J. (1991): Universal primers for amplification of three non-coding regions of chloroplast DNA. Plant Molecular Biology Reporter, 17: 1105-1109.

Tate, J.A., Simpson, B.B. (2003): Paraphyly of Tarasa (Malvaceae) and diverse origins of the polyploid species. Systematic Botany, 28: 723-737.

Wickert, K.L., O’Neal, E.S., Davis, D.D., Kasson, M.T. (2017): Seed Production, Viability, and Reproductive Limits of the Invasive Ailanthus altissima (Tree-ofHeaven) within Invaded Environments. Forests, 8(7): 226. 


\title{
Molecular markers used for genetic studies in Northern red oak (Quercus rubra L.)
}

\author{
Charalambos Neophytou ${ }^{1}$ and Branislav Cvjetković ${ }^{2}$ \\ ${ }^{1}$ University of Natural Resources and Life Sciences (BOKU), Department of Forest and Soil Sciences, Institute of Silviculture, \\ Peter-Jordan-Straße 82, 1190 Vienna, Austria \\ ${ }^{2}$ University of Banja Luka, Faculty of Forestry, Department for Forest Genetics and Afforestation/Reforestation, \\ Bulevar Vojvode Stepe Stepanovića 75A, 78000 Banja Luka, Bosnia and Hercegovina
}

\section{General remarks}

Northern red oak (Quercus rubra L.), also known as common red oak, eastern red oak, gray oak or mountain red oak, is widespread in the eastern part of North America. Northern red oak is the only native oak extending northeast to Nova Scotia (Figure 1). It grows from Cape Breton Island, Nova Scotia, Prince Edward Island, New Brunswick, and the Gaspé Peninsula of Quebec, to Ontario, in Canada; from southern Minnesota to eastern Nebraska and Oklahoma; east to Arkansas, southern Alabama, Georgia, and North Carolina. Outliers are found in Louisiana and Mississippi and almost the entire USA is a potential planting range (Gilman and Watson 1994).

Northern red oak grows on a variety of soils and topography. Pure stands are the most common plant community. Moderate to fast growing, this tree is one of

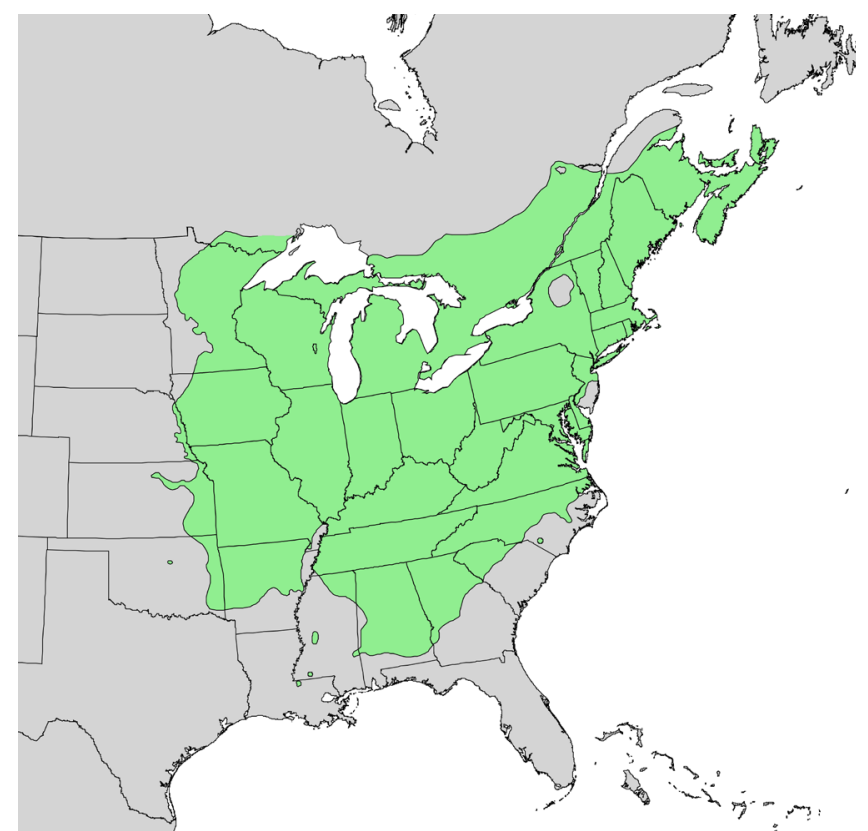

Figure 1. Native distribution range of northern red oak (source: USGS, USA) the most important lumber species of red oak and is an easily transplanted, popular shade tree with good form and dense foliage.

According to Sander (1965), in the area where northern red oak grows, mean annual precipitation varies from about $760 \mathrm{~mm}$ in the Northwest to about $2030 \mathrm{~mm}$ in the South. Annual snowfall ranges from a trace in southern Alabama to $254 \mathrm{~cm}$ or more in the Northern States and Canada. Mean annual temperature is about $4^{\circ} \mathrm{C}$ in the northern part of the range and $16^{\circ} \mathrm{C}$ in the extreme southern part. The frost-free period averages 100 days in the North and 220 days in the South (Sander 1965).

Mature northern red oaks are usually from 20 to $30 \mathrm{~m}$ tall and 61 to $91 \mathrm{~cm}$ in dbh (diameter at breast height) in undisturbed stands on good sites. Forest-grown trees develop a tall, straight columnar bole and large crowns. Open grown trees tend to have short boles and spreading crowns (Sander 1965).

Northern red oak hybridizes with other species in the subgenus Erythrobalanus and the following hybrids have been named: Quercus x columnaris Laughlin (Q. palustris $x$ rubra); Q. x fernaldii Trel. (Q. ilicifolia x rubra); $Q . x$ heterophylla Michx. f. (Q. phellos x rubra); Q. x hawkinsiae Sudw. (Q. velutina $x$ rubra); Q. $x$ riparia Laughlin $(Q$. shumardii x rubra); and Q. x runcinata (A. DC.) Engelm. (Q. imbricaria $\times$ rubra). It also hybridizes with blackjack oak (Q. marilandica) and with northern pin oak (Q. ellipsoidalis) (Little 1979).

Up to date, several molecular markers have been developed (e.g. Aldrich et al. 2002, Magni et al. 2005, Sullivan et al. 2013, Konar et al. 2017) and used to investigate phylogeographic patterns (Birchenko et al. 2009, Magni et al. 2005) and population genetic variation at the local (Aldrich et al. 2003b), regional (Gerwein and Kesseli 2006) and range-wide scale (Daubree and Kremer 1993, Sork et al. 1993, Borkowski et al. 2017, Merceron et al. 
2017). A lack of pronounced large-scale phylogeographic structure revealed by chloroplast DNA markers could be due to the species' biogeographic history (putative recolonization from one single glacial population) or due to low resolution of the universal markers used (Magni et al. 2005).

For long time, only isozyme studies from the 90s had provided some range-wide data from indigenous populations (Daubree and Kremer 1993, Sork et al. 1993). Such data are particularly useful in order to trace the origin of introduced populations. Recently, genetic structure across the whole natural range of northern red oak was studied based on microsatellites (Borkowski et al. 2017) and SNPs (Merceron et al. 2017). In addition, new chloroplast DNA markers may increase the resolution of population genetic analyses (Alexander and Woeste 2014, Borkowski et al. 2014). These studies may open up new perspectives in traceability of introduced populations and forest reproductive material of northern red oak.

Here, we provide a review of available laboratory techniques which can be used to investigate the origin of northern red oak, as well as its genetic diversity and differentiation.

\section{Isozymes}

In the period from 1980 to 1995 , investigation of isozymes took place in order to:

- determine species identity due to hybridization among the Quercus species (Tobolski 1978, Manos and Fairbrothers 1987, Hokanson et al. 1993);

- investigate genetic diversity among natural and planted populations (Manos and Fairbrothers 1987, Schwarzmann and Gerhold 1991, Daubree and Kremer 1993, Hokanson et al. 1993, Sork et al. 1993).

A summary of the isozyme systems, scored loci and number of alleles found in the cited studies is presented in Table 1.

\section{Material for protein extraction}

Proteins were extracted from mature leaves (Manos and Fairbrothers 1987, Sork et al. 1993, Jones et al. 2006), young leaves (Hokanson et al. 1993, Daubree and Kremer 1993), buds (Tobolski 1978, Hokanson et al. 1993, Daubree and Kremer 1993), acorns (Tobolski 1978, Hokanson et al. 1993, Daubree and Kremer 1993) and embryos (Schwarzmann and Gerhold 1991).

Table 1: List of enzymes, scored loci, number of alleles for northern red oak (Quercus rubra)

\begin{tabular}{|l|c|c|c|c|}
\hline \multicolumn{1}{|c|}{ Enzyme system } & $\begin{array}{c}\text { E.C. } \\
\text { Number }\end{array}$ & Scored loci & $\begin{array}{c}\text { No. of } \\
\text { alleles* }\end{array}$ & References \\
\hline Alcohol dehydrogenase & 1.1 .1 .1 & ADH-1 & 5 & 6,7 \\
\hline Acid phosphatase & 3.1 .3 .2 & ACP-1,-2 & 3,2 & 7 \\
\hline Fluorescent esterase & 3.1 .1 .1 & FEST-1,-2 & n.a.; 2 & 5 \\
\hline Glutamate dehydrogenase & 1.4 .1 .2 & GDH-1 & 3 & 1 \\
\hline Glutamate oxalacetate transaminase & 2.6 .1 .1 & GOT-1 & 1 & 2,7 \\
\hline Isocitrate dehydrogenase & 1.1 .1 .42 & IDH-1,-2,-3 & 5,4 & $1,2,4,5,6$ \\
\hline Leucine aminopeptidase & 3.4 .11 .1 & LAP-1,-2 & 4,2 & $1,2,4,5,6,7$ \\
\hline Malate dehydrogenase & 1.1 .1 .37 & MDH-1,-2,-3 & 5,3 & $1,2,3,4,6$ \\
\hline Malic enzyme & 1.1 .1 .40 & ME-1 & 3 & 6 \\
\hline Menadione reductase & 1.6 .5 .2 & MNR-1 & 2 & 6 \\
\hline Phosphoglucose isomerase & 5.3 .1 .9 & PGI-1,-2 & 1,8 & $1,2,3,4,5,6$ \\
\hline Peroxidase & 1.11 .1 & PER-1,-2 & 4,3 & 1,5 \\
\hline Phosphoglucomutase & 2.7 .5 .1 & PGM-1,-2 & 5,6 & $1,2,5,6$ \\
\hline Shikimate dehydrogenase & 1.1 .1 .25 & SKDH -1 & n.a.;6 & $1,3,4,5,6$ \\
\hline Triose-phosphate isomerase & 5.3 .1 .1 & TPI-1 & n.a. & 5 \\
\hline 6-Phosphogluconate dehydrogenase & 1.1 .1 .44 & 6-PGDH-1,-2 & 3,5 & $1,3,4,5,6$ \\
\hline
\end{tabular}

*only the highest number of alleles reported in at least one reference is given; 1-Manos and Fairbrothers 1987, 2-Schwarzmann and Gerhold 1991, 3-Daubree and Kremer 1993, 4-Hokanson et al. 1993, 5-Sork et al. 1993, 6-Jones et al. 2006, 7-Tobolski 1978. 


\section{Protein extraction and separation protocols}

Separation protocols are described in: Manos and Fairbrothers (1987). Tobolski (1978) followed the separation protocols described by Scandalios (1969).

\section{Important results}

- The similarity in isozyme patterns was high among Quercus species. Most isozyme bands were found to be common to two or more oak species (Tobolski 1978).

- The diversity among natural populations of red oak was low (Manos and Fairbrothers 1987).

- Schwarzmann and Gerhold (1991) confirmed little differentiation of isozyme gene frequencies among northern red oak populations in Pennsylvania.

- Most of the genetic variation which was observed for isozyme loci was found to reside within populations.

- Pronounced genetic differentiation of European populations in comparison to native ones was found for loci PGI and PGM. Frequency of rare alleles was increased in European populations resulting in a higher genetic diversity (mean number of alleles). This was interpreted as a possible result of weaker selective pressure on northern red oak regeneration in Europe (Daubree and Kremer 1993).

\section{Organelle DNA markers (chloroplast (cp) DNA, mitochondrial (mt)DNA)}

\section{Loci and primers used}

Magni et al. (2005) applied PCR-RFLP markers in order to study phylogeography of northern red oak across its natural range. They used universal primers (DumolinLapègue et al. 1997, Taberlet et al. 1991) to amplify 13 chloroplast DNA (cpDNA) fragments and two different restriction enzymes (Hinfl and TaqI) independently to digest the PCR products. Among them, only 5 primer pairs combined with TaqI provided informative polymorphisms (Table 2).

Further cpDNA studies based on PCR-RFLPs were carried out by Romero-Severson et al. (2003) and Birchenko et al. (2009). These authors amplified three universal cpDNA loci (Demesure et al. 1995) and digested the fragments with a mix of seven restriction enzymes BamHI, EcoRI, AluI, HhaI, MspI, HaeIII and RsaI using compatible buffers (Table 2).

\section{Material for DNA-extraction}

Plant tissue used for extraction included leaves (RomeroSeverson et al. 2003, Magni et al. 2005), buds (Magni et al. 2005), twigs or cambium (inner bark; Birchenko et

Table 2: PCR-RFLP markers (chloroplast DNA) for northern red oak

\begin{tabular}{|c|c|c|c|c|}
\hline Locus & $\begin{array}{c}\text { Primer sequences } \\
\mathrm{F}=\text { Forward, } \mathrm{R}=\text { Reverse }\end{array}$ & $\begin{array}{c}\text { Restriction } \\
\text { enzymes }\end{array}$ & $\begin{array}{c}\text { No. of } \\
\text { polymorphic } \\
\text { bands }\end{array}$ & References \\
\hline $\begin{array}{c}\mathrm{CD} \\
(\operatorname{trnC} / \operatorname{trnD})\end{array}$ & $\begin{array}{l}\text { F:CCAGTTCAAATCTGGGTGTC } \\
\text { R:GGGATTGTAGTTCAATTGGT }\end{array}$ & \multirow{5}{*}{ TaqI } & 2 & $3,4,6$ \\
\hline $\begin{array}{c}\mathrm{DT} \\
(\operatorname{trnD} / \operatorname{trnT}) \\
\end{array}$ & $\begin{array}{l}\text { F:ACCAATTGAACTACAATCCC } \\
\text { R:CTACCACTGAGTTAAAAGGG }\end{array}$ & & 1 & $3,4,6$ \\
\hline $\begin{array}{c}\mathrm{VL} \\
(\operatorname{trnV} / \mathrm{rbcL})\end{array}$ & $\begin{array}{l}\text { F:CGAACCGTAGACCTTCTCGG } \\
\text { R:GCTTTAGTCTCTGTTTGT }\end{array}$ & & 1 & $3,4,6$ \\
\hline $\operatorname{trnH} / \mathrm{psbA}$ & $\begin{array}{l}\text { F:ACTGCCTTGATCCACTTGGC } \\
\text { R:CGAAGCTCCATCTACAAATGG }\end{array}$ & & 1 & $3,4,6$ \\
\hline $\operatorname{trnS} / \operatorname{trnG}$ & $\begin{array}{l}\text { F:GCCGCTTTAGTCCACTCAGC } \\
\text { R:GAACGAATCACACTTTTACCAC }\end{array}$ & & 1 & $3,4,6$ \\
\hline $\begin{array}{c}\mathrm{CD} \\
(\operatorname{trnC} / \operatorname{trnD})\end{array}$ & $\begin{array}{l}\text { F:CCAGTTCAAATCTGGGTGTC } \\
\text { R:GGGATTGTAGTTCAATTGGT }\end{array}$ & \multirow{3}{*}{$\begin{array}{c}\text { Bam } \mathrm{HI}, \\
\text { EcoRI, } \\
\text { AluI }, H h a \mathrm{I}, \\
\text { MspI, } \\
\text { HaeIII and } \\
\text { RsaI }\end{array}$} & 2 & $1,2,5$ \\
\hline $\begin{array}{c}\mathrm{FV} \\
(\mathrm{trnF} / \mathrm{trnV})\end{array}$ & $\begin{array}{l}\text { F:CTCGTGTCACCAGTTCAAAT } \\
\text { R:CCGAGAAGGTCTACGGTTCG }\end{array}$ & & 3 & $1,2,5$ \\
\hline $\begin{array}{c}\mathrm{TC} \\
(\operatorname{trnT} / \mathrm{psbC})\end{array}$ & $\begin{array}{l}\text { F:GCCCTTTTAACTCAGTGGTA } \\
\text { R:GAGCTTGAGAAGCTTCTGGT }\end{array}$ & & 2 & $1,2,5$ \\
\hline
\end{tabular}

1-Birchenko et al. 2009, 2-Demesure et al. 1995, 3-Dumolin-Lapègue et al. 1997, 4-Magni et al. 2005, 5-Romero-Severson et al. 2003, 6-Taberlet et al. 1991 
al. 2009).

\section{DNA-isolation protocols}

For DNA isolation, Magni et al. (2005) used an ATMAB (Acryltrimethylammonium bromide) protocol following Dumolin et al. (1995). Romero-Severson et al. (2003), as well as Birchenko et al. (2009) used a commercial DNA-extraction kit (Dneasy, Qiagen).

\section{Important results}

- In the study of Magni et al. (2005), twelve different haplotypes (A through G) were described. One of them, haplotype $\mathrm{E}$ was present in $75 \%$ of the trees throughout the distribution range, whereas all other haplotypes were rare $(<8 \%)$ and displayed only local distribution. For these reasons, the diagnostic power of this marker set for traceability of introduced populations and forest reproductive material is limited.

- Romero-Severson et al. (2003) detected five different haplotypes (I through V) in the State of Indiana (USA) by analyzing a limited number of individuals and populations. Using the same marker set, Birchenko et al. (2009) found four of these haplotypes in the northwestern part of the native range. Haplotypic diversity was found to decline poleward. Towards the centre of the range, high admixture and no spatial patterns were observed. Given this high admixture, the utility of this marker set to trace the origin of introduced northern red oak might be limited, too.

\section{Nuclear DNA markers (nSSR, EST-SSR, SNP)}

\section{a) nSSRs (putatively neutral microsatellites), EST- SSRs (expressed sequence tag derived microsatellites)}

\section{Loci and primers used}

Development of SSR markers for red oak was done by Aldrich et al. (2002; 2003a). In addition, transferability of nSSR (genomic) and EST-SSR-loci (derived from expressed sequence tags) from pedunculate oak $(Q$. robur; initial primer notes by Steinkellner et al. 1997 and Durand et al. 2010) into northern red oak was tested by Sullivan et al. (2013). Moreover, Gerwein and Kesseli (2006) successfully used loci initially developed for Quercus myrsinifolia (Isagi and Suhadono 1997), Quercus robur (Steinkellner et al. 1997) and Quercus macrocarpa (Dow et al. 1995) in their study with northern red oak. Finally, Konar et al. (2017) used 116 published or newly developed microsatellites in their genetic mapping study in Q. rubra. A list of 169 microsatellite loci (nSSRs and EST-SSRs) available for genotyping of northern red oak, as well as studies where these were used is presented in Table 3 . 


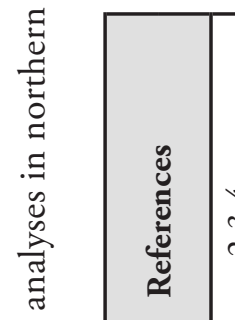

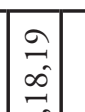

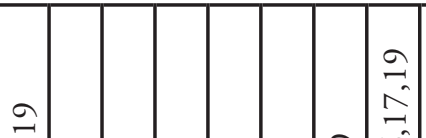

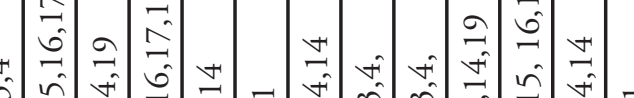
. ป ثุ

$\frac{0}{\frac{\pi}{\pi}}$

कั ํํำ

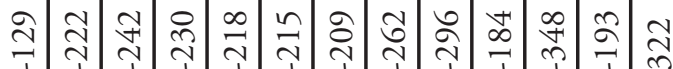
.

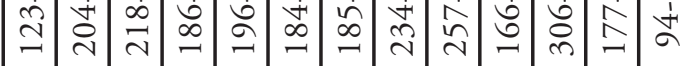

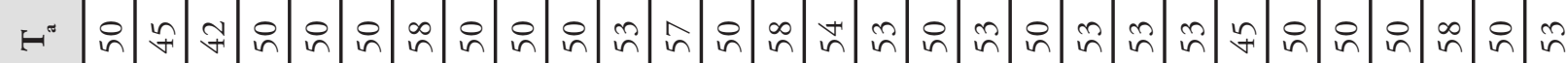

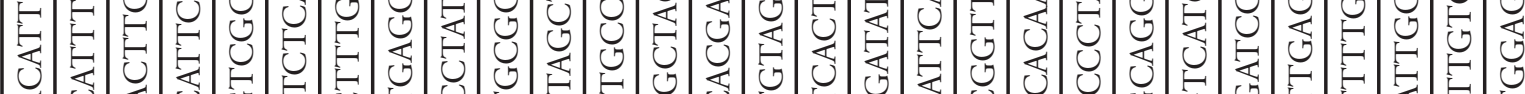

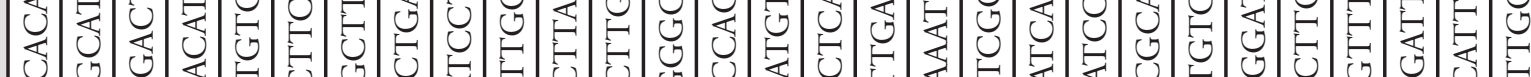

อิ

悹 年 U 盯

ฮี

离

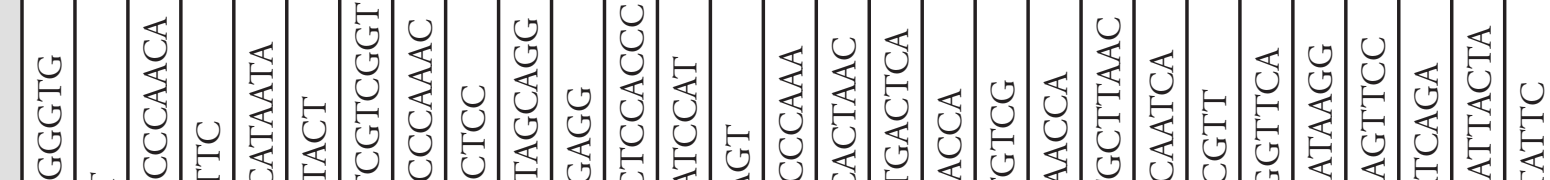
至至

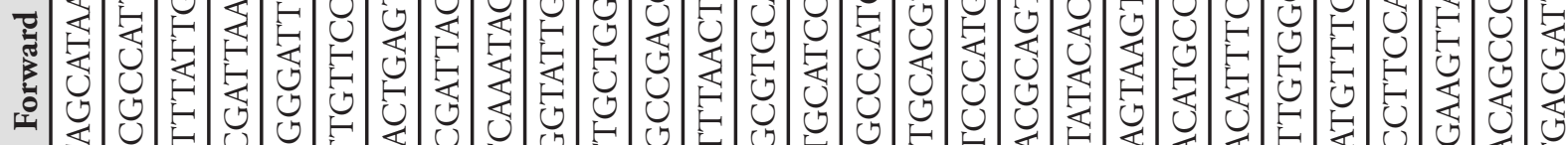
勿 过

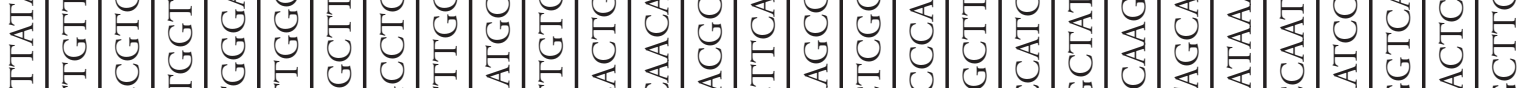

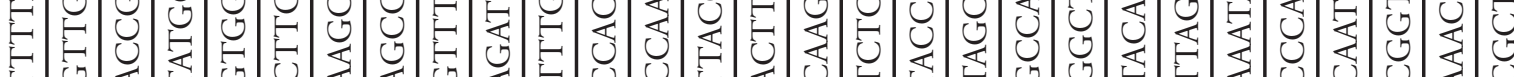
安苦

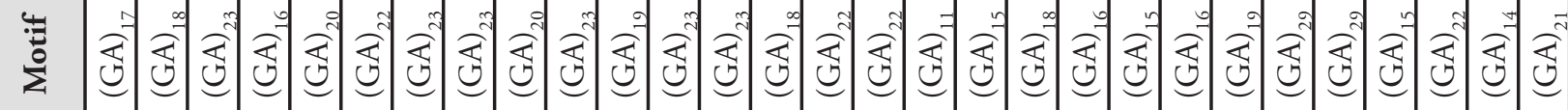

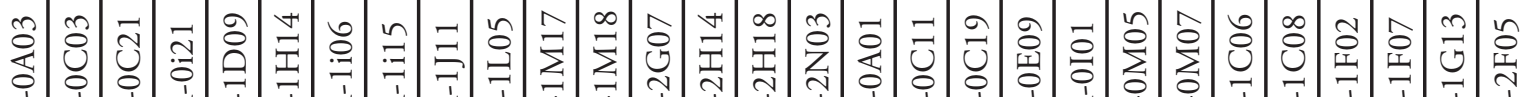

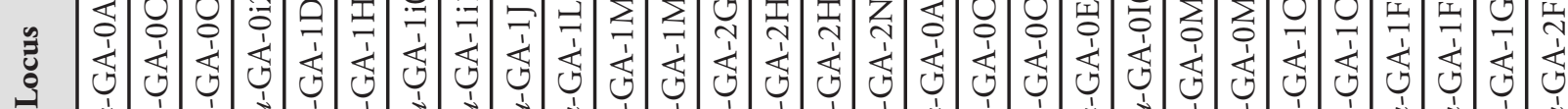
范

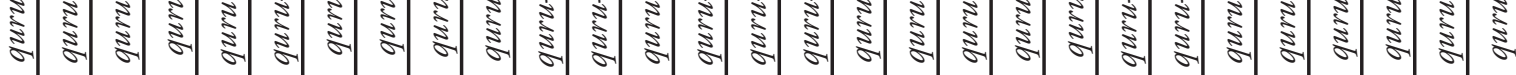




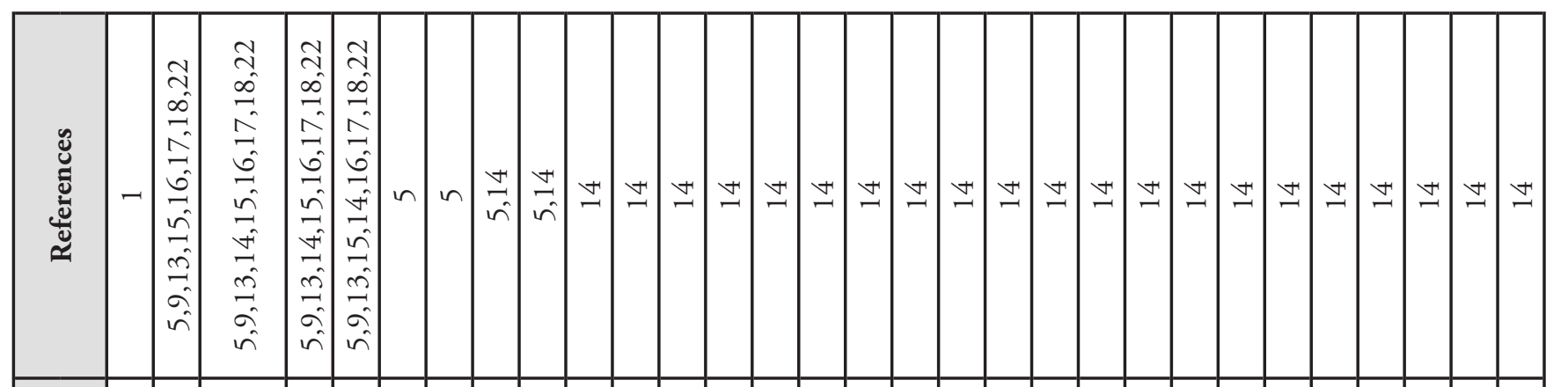

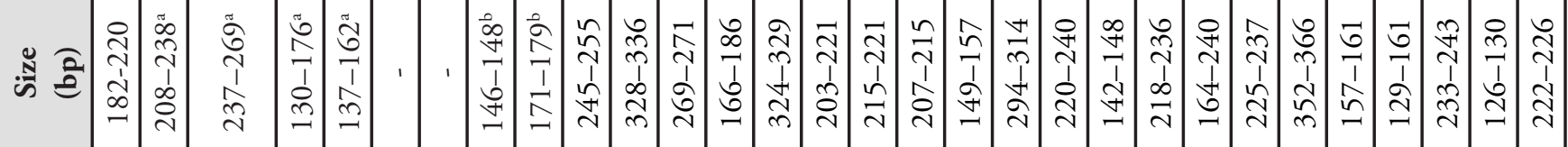

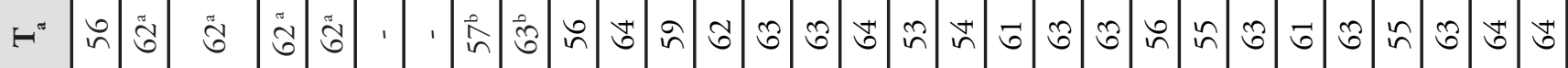

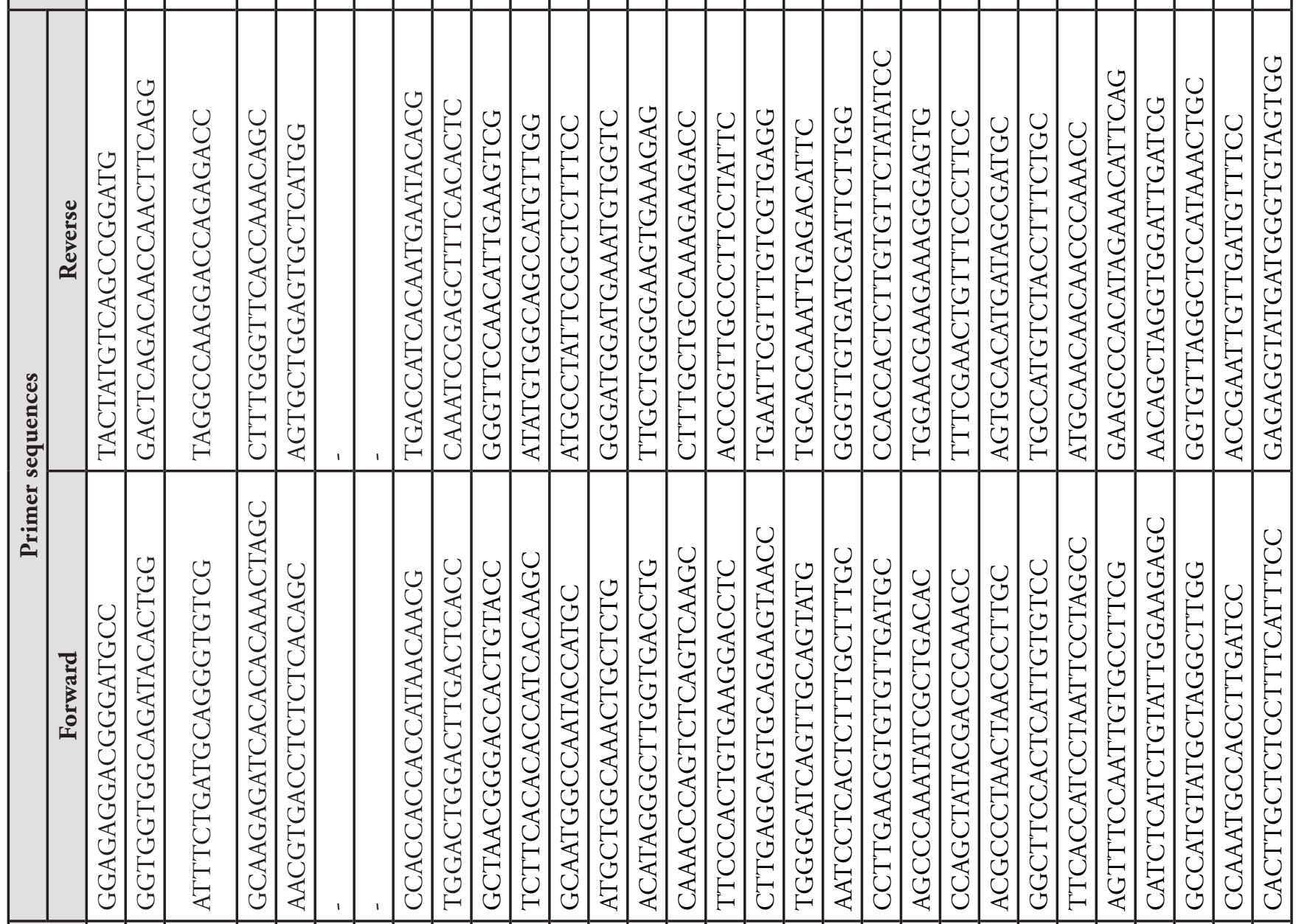

之.

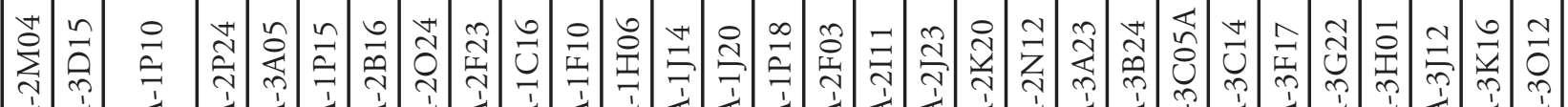

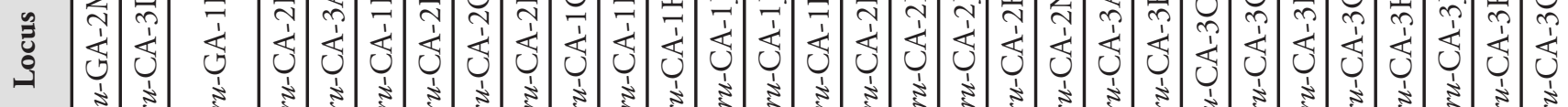

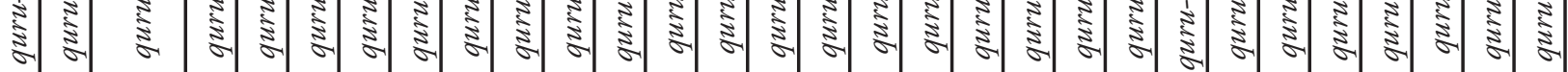




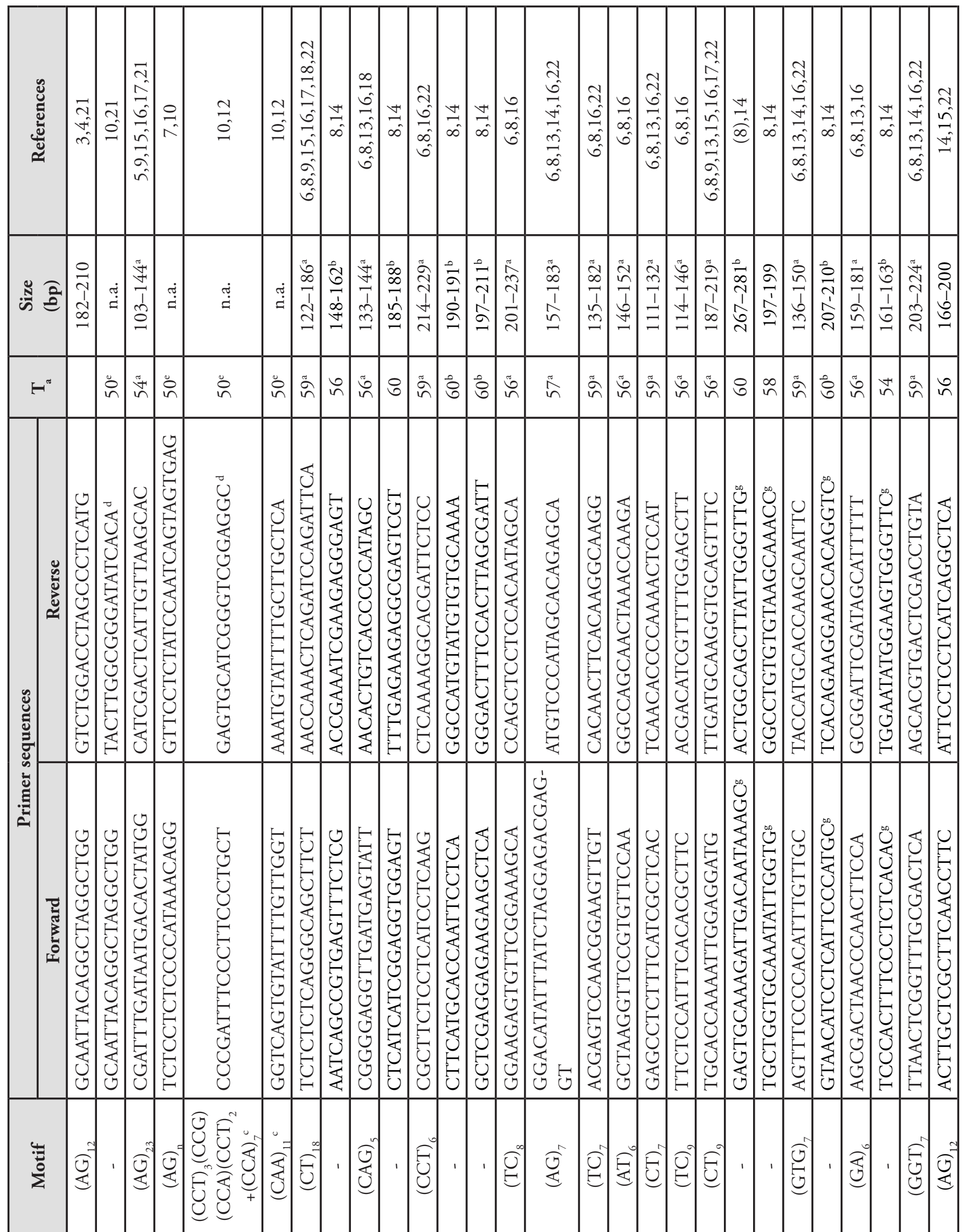

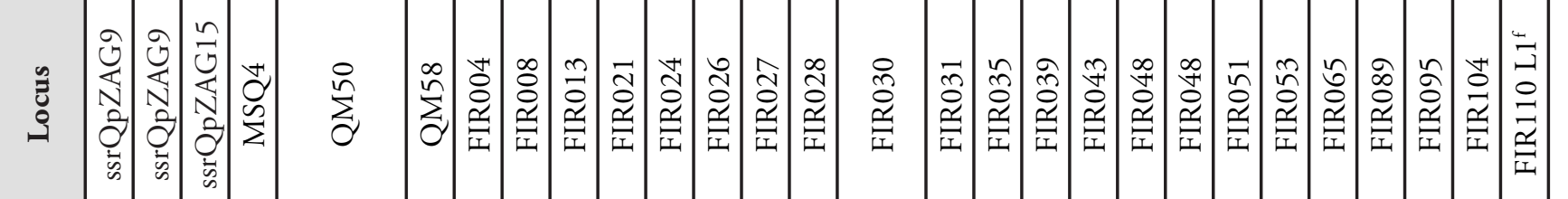




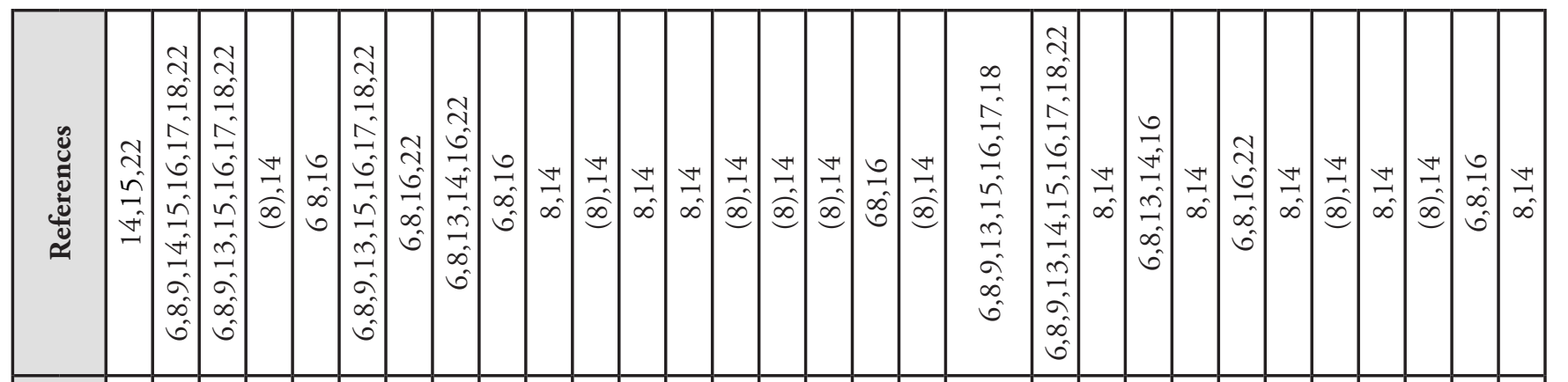

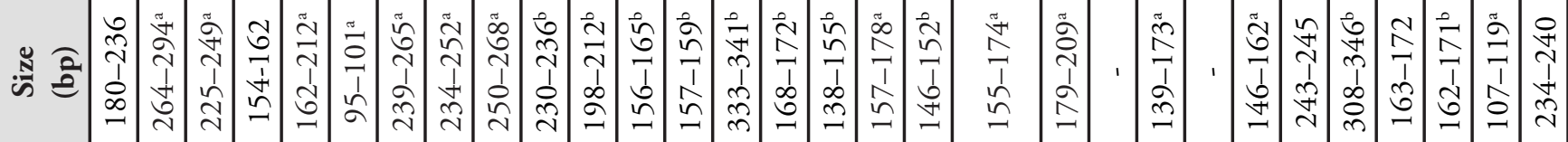

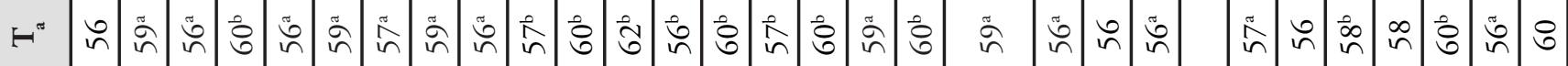

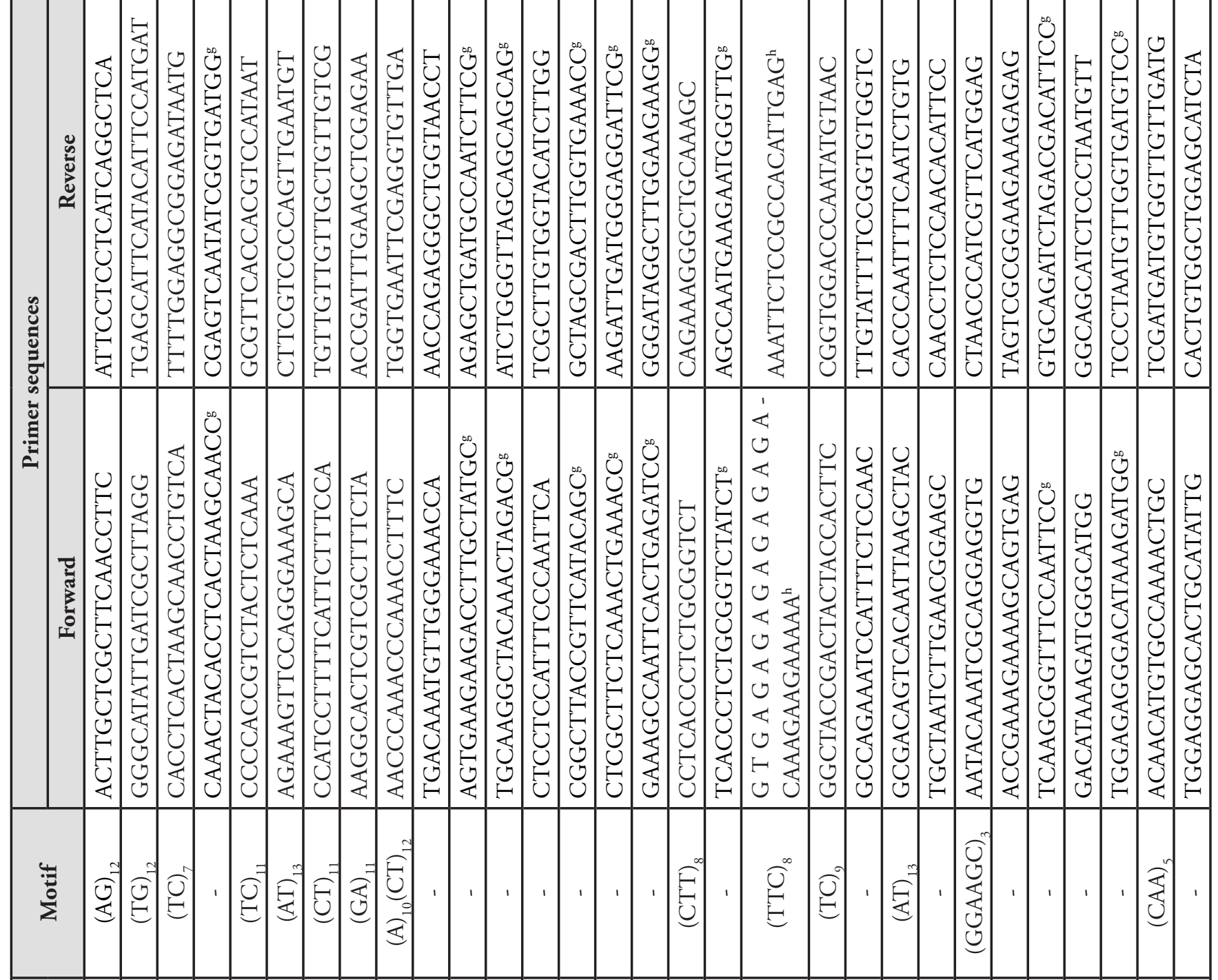

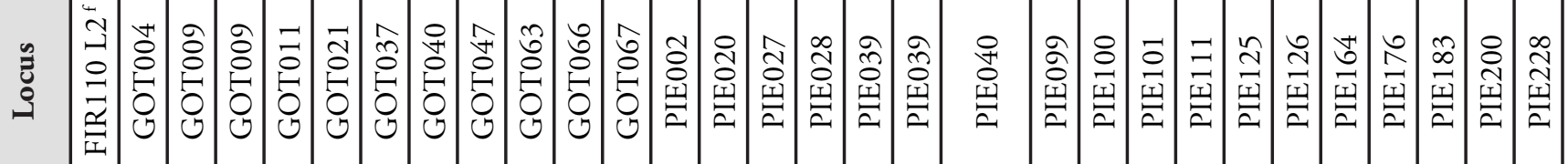




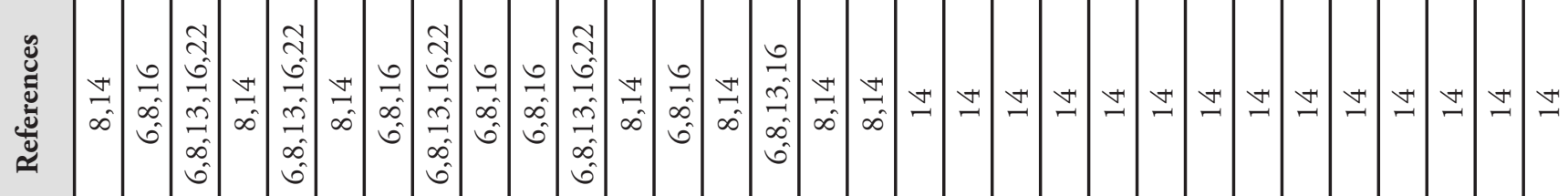

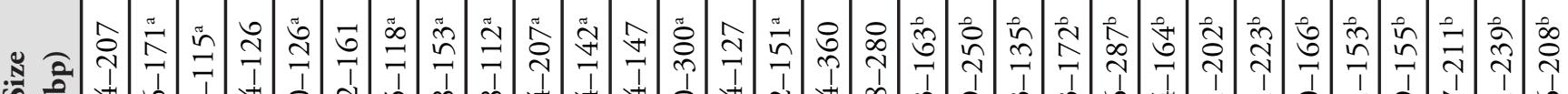

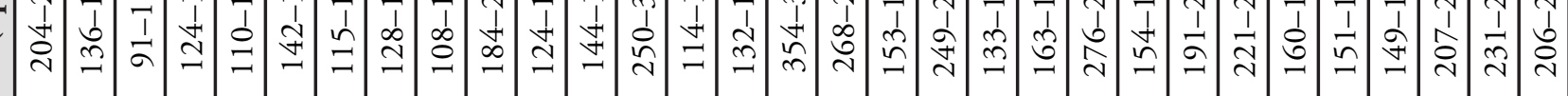

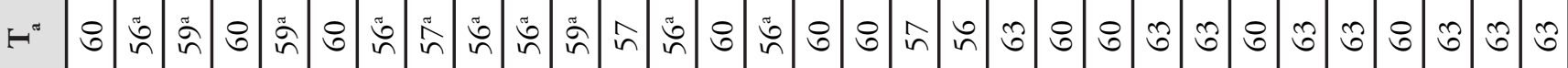

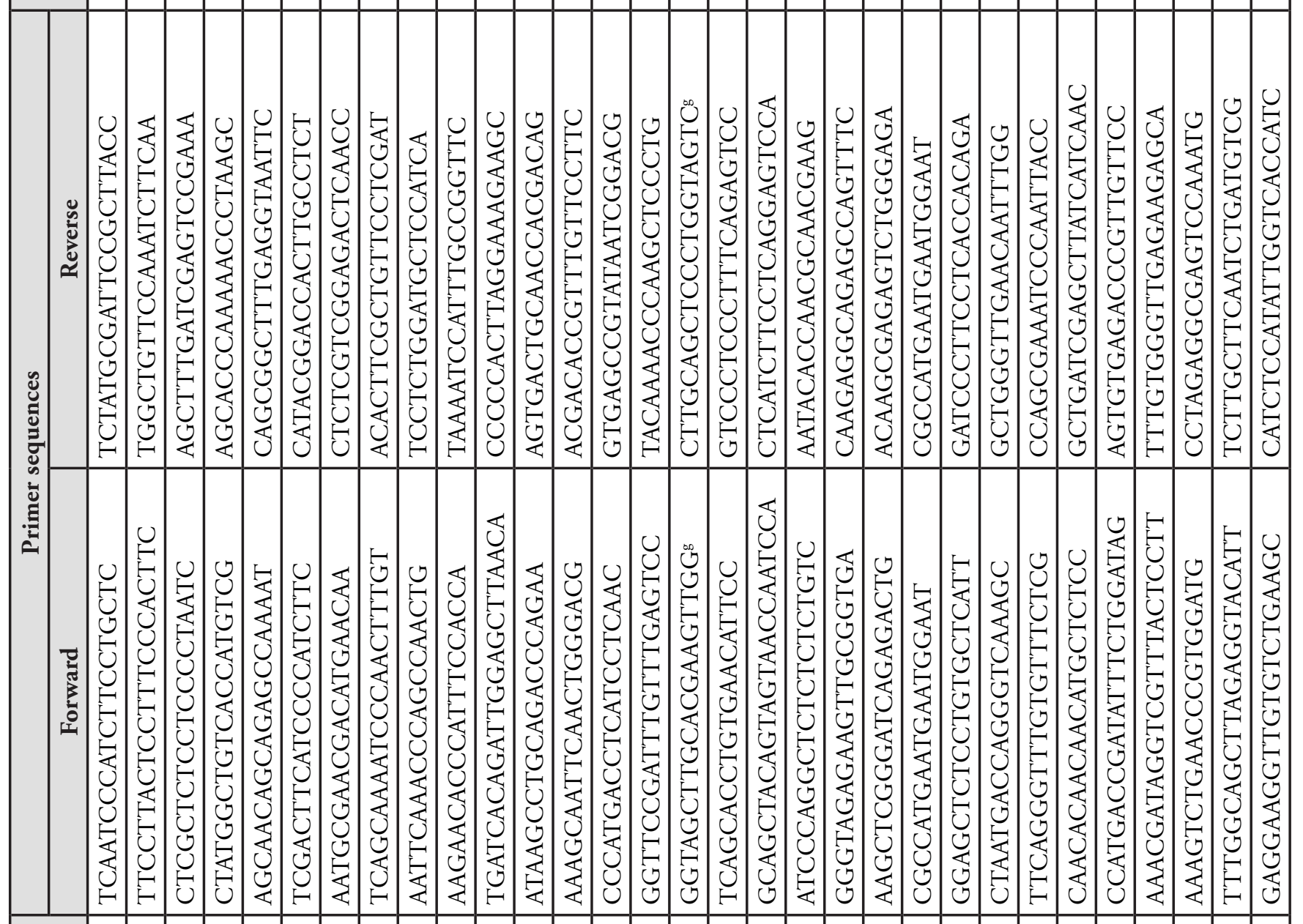

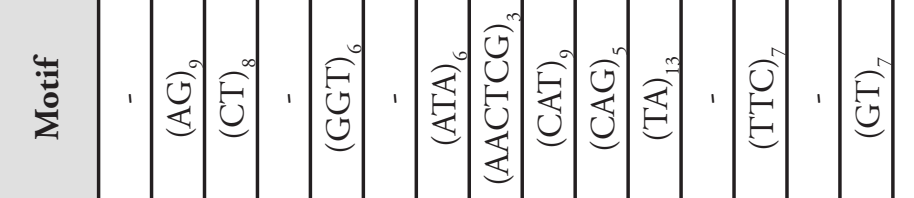

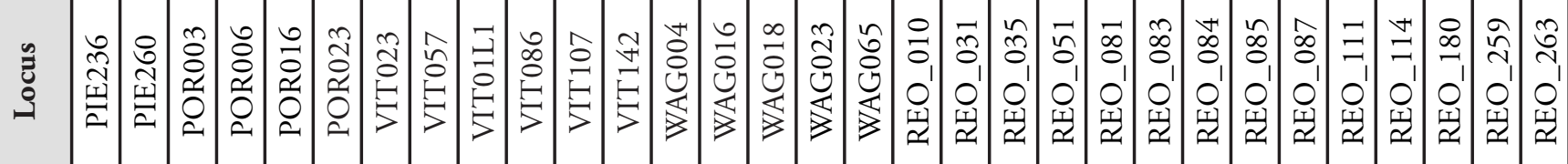




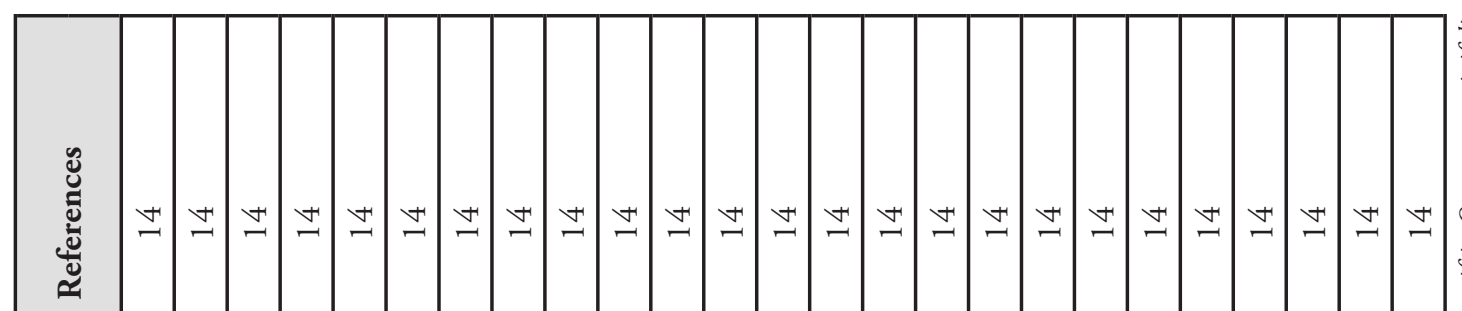

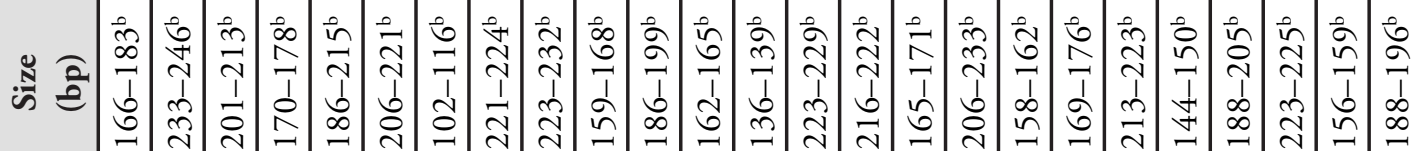

$H^{\circ}$ ชర

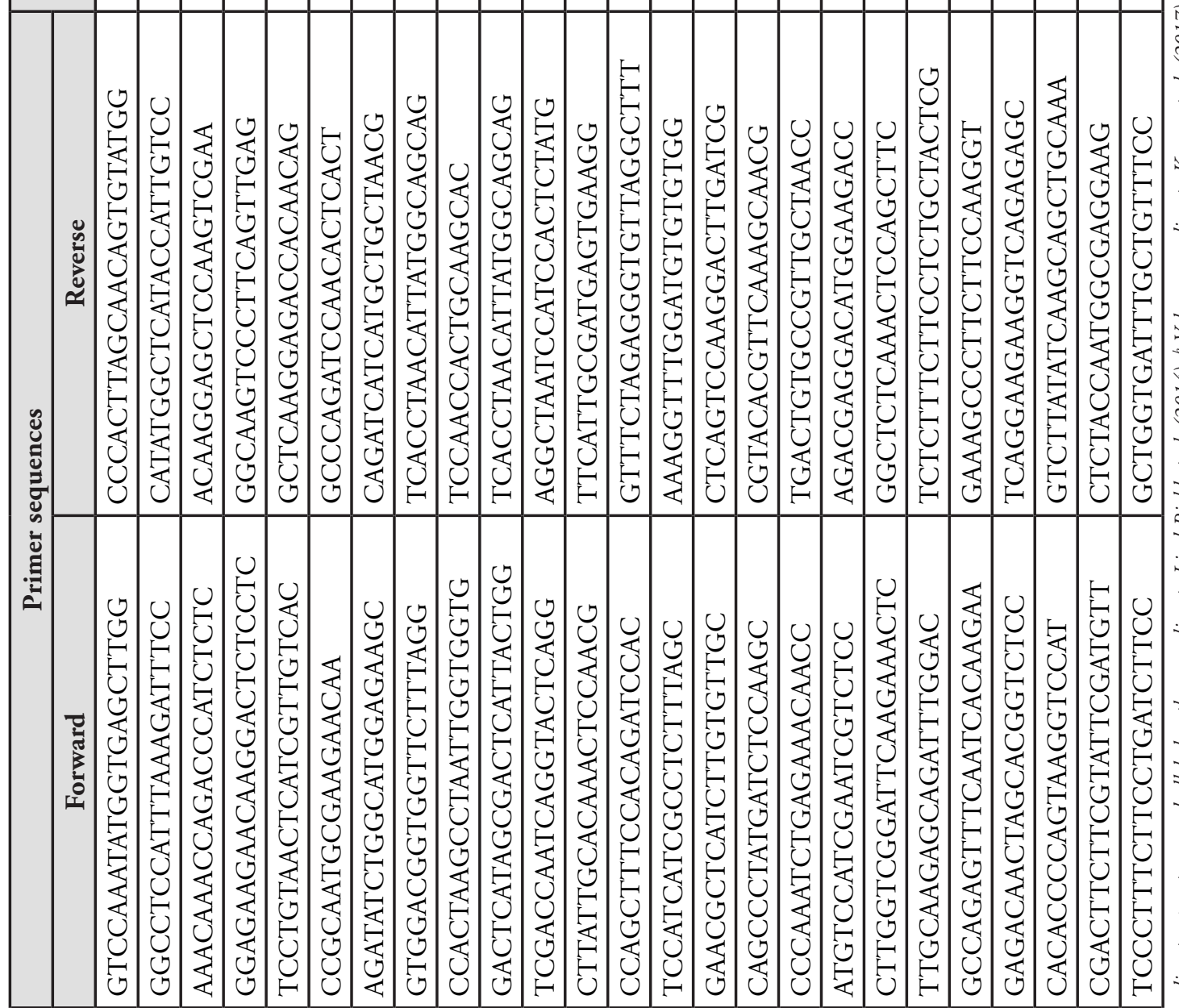

$\sum^{0}$

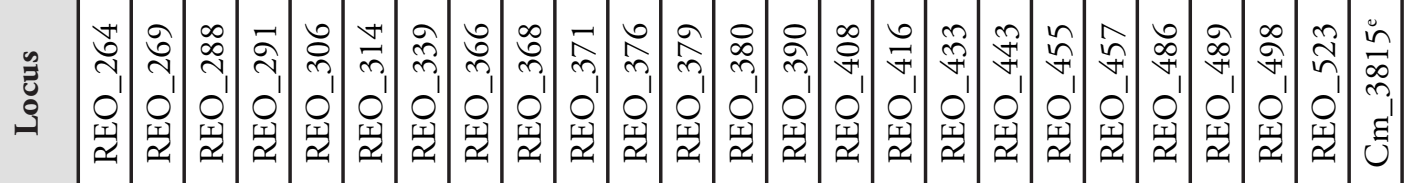




\section{Material for DNA-extraction}

Leaves were the most common tissue used for extraction (Aldrich et al. 2002, Collins et al. 2015, Gailing et al. 2012, Gerwein and Kesseli 2006, Khodwekar and Gailing 2017, Lind and Gailing 2013, Lind-Riehl et al. 2014, 2015, 2017, Moran et al. 2012, Sullivan et al. 2013) followed by cambium from the base of trunk (Aldrich et al. 2003a, 2003b, 2005). Khodwekar and Gailing (2017) additionally extracted DNA from embryos (from acorns) in order to carry out a paternity analysis. In one case, tissue used for extraction was not defined (Borkowski et al. 2017).

\section{DNA-extraction protocols}

In most studies, a commercial DNA-extraction kit (DNeasy 96, Qiagen) was used (Aldrich et al. 2003a, Collins et al. 2015, Gailing et al. 2012, Lind and Gailing 2013, Lind-Riehl et al. 2014, 2015, 2017, Sullivan et al. 2013). Some authors extracted DNA using a CTABprotocol (Borkowski et al. 2017, Gerwein et al. 2006, Moran et al. 2012). Borkowski et al. (2017) refers to Hoban et al. (2009) for modifications to the CTABprotocol used. Finally, Aldrich et al. (2002) refer to Murray and Pitas (1996) for the octanol-chlorophorm based protocol used for DNA-isolation in their study. Aldrich et al. (2003b, 2005) cite Aldrich et al. (2002) for DNA-extraction.

\section{Important results}

- Differences between northern red oak and other oak species, as well as hybridization were investigated by Aldrich et al. (2003b), Moran et al. (2012), Sullivan et al. (2013) and Lind-Riehl and Gailing (2015).

- Until recently, microsatellite-based genetic studies within northern red oak were of regional or local scope (e.g. Aldrich et al. 2003b, Gerwein and Kesseli 2006). Aldirch et al. (2005) used a panel of 14 loci to screen for diversity in 10 adult Q. rubra from two old-growth stand at the Davis-Purdue Research Forest in east-central Indiana. They found slight but significant differentiation among stands but also weak isolation by distance within large stands. In total, 105 alleles were detected with a mean of 7,5 alleles per locus (range, 4-13 alleles). Gailing et al. (2012) found that genetic distance at the 15 microsatellite markers developed by Durand et al. (2010) for $Q$. robur, seven simple sequence repeat (SSR) markers developed for Q. rubra (Aldrich et al. 2002, Sullivan et al. 2013) and the Q. robur microsatellite QpZAG15 (Steinkellner et al. 1997) was not correlated with geographic distance.

- Lind and Gailing (2013) identified a small but significant differentiation between managed and unmanaged populations of Quercus rubra. LindRiehl et al. (2014) showed that locus FIR013 displayed a high differentiation between Q. rubra and Q. ellipsoidalis, which might be due to divergent selection (outlier approach). This marker is located within a CONSTANS-like gene which is involved in photoperiodic control of growth, which might pose a prezygotic barrier between these two species (Collins et al. 2015). In subsequent publications, Khodwekar and Gailing (2017) and Lind-Riehl and Gailing (2017) suggest adaptive introgression between the two species in sympatric populations based on results from allelic frequencies and sequences of locus FIR013.

- In a recent study, Borkowski et al. (2017) investigated genetic differentiation across the native range of northern red oak. By performing a Bayesian cluster analysis (STRUCTURE-method; Pritchard et al. 2000; Falush et al. 2003) based on genotypic data from 10 unlinked microsatellites, they found a well-defined genetic cluster including populations from the area around the Lake Superior in the northwestern part of the species' native range. They also detected a cluster with regional distribution in the northeastern part and another one covering most of the species' range which is spread across southern and central areas. There was a continuous gradient between those two clusters. Finally, a fourth cluster with a local occurrence in only one population in Massachusetts was identified.

\section{b) SNPs (single-nucleotide polymorphisms)}

\section{Loci and primers used}

Restriction site associated DNA sequencing (RADsequencing) was used in a recent genome mapping study conducted by Konar et al. (2017) using a full-sib family in Q. rubra. In total, 78725 SNPs were called by applying RAD-sequencing. Various quality filters (e.g. for missing data and deviation from the Hardy-Weinberg Equilibrium) were applied resulting in 1413 SNPs along with 116 SSRs available for mapping. A list of all SNPloci finally used for mapping (849 in total), along with sequence reads where the SNP occurred, position in linkage groups and distances in centimorgans is provided as supplementary material by Konar et al. (2017).

In a following study, Merceron et al. (2017) published 
their results about range-wide variation of northern red oak at SNP-loci using Sequenom ${ }^{\bullet}$ technology. A total of 1410 bi-allelic SNPs already described in Konar et al. (2017) were used to design 3 multiplexes with a total of 115 SNP markers (40, 40 and 35, respectively). After removal of monomorphic or non-amplifiable loci, 80 SNPs were usable for population genetic analysis. However, among them 69 were included in the final population genetic analysis after filtering for missing values.

The 115 primer sequences from Merceron et al. (2017) including primer tags are presented in Table 4. These were organized in 3 multiplexes based on Sequenom ${ }^{\circ}$ methodology (Table 4). Initial PCR reaction produced amplicons of length between 72 and $85 \mathrm{bp}$. For more details about the Sequenom method the reader is referred to Bradić et al. (2011).

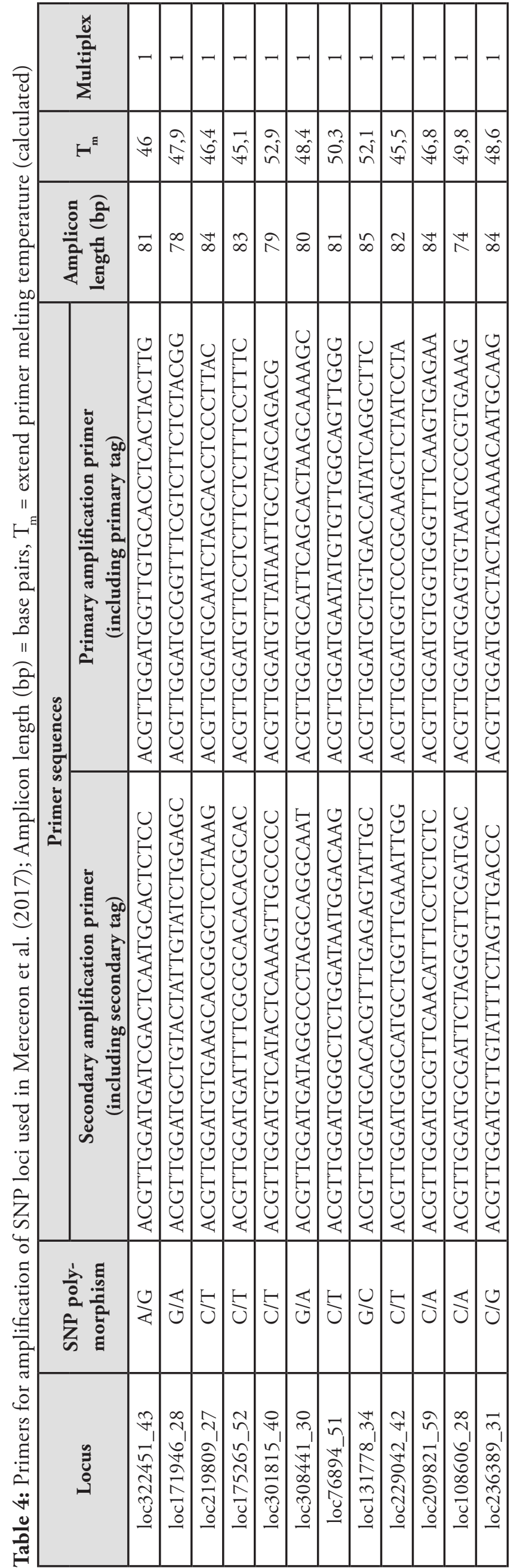




\begin{tabular}{|c|c|c|c|c|c|c|c|c|c|c|c|c|c|c|c|c|c|c|c|c|c|c|c|c|c|c|c|c|c|c|c|}
\hline$\frac{\sqrt{0}}{3}$ & $\neg$ & -1 & -1 & -1 & - & - & - & - & - & -1 & $\neg$ & - & $\neg$ & $\neg$ & - & - & - & $\neg$ & -1 & $\neg$ & $\neg$ & 一 & - & - & $\neg$ & $\neg$ & $\neg$ & $\neg$ & $N$ & $\sim$ & $\sim$ \\
\hline$H^{\sharp}$ & $\stackrel{\infty}{\hat{F}^{n}}$ & $\tilde{n}$ & $\stackrel{n}{f}$ & $\begin{array}{l}n \\
\stackrel{n}{f}\end{array}$ & $\begin{array}{l}n \\
\infty \\
n\end{array}$ & 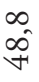 & $\stackrel{n}{\infty}$ & 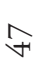 & $\stackrel{0}{\overbrace{}^{\circ}}$ & $\stackrel{\infty}{n}$ & $\stackrel{\vec{\infty}}{\stackrel{\infty}{+}}$ & $\stackrel{N}{n}$ & $\vec{n}$ & $\hat{n}$ & $\stackrel{\infty}{\infty}$ & $\hat{\wp}$ & $\stackrel{n}{\stackrel{n}{F}}$ & $\begin{array}{c}\sim \\
\text { ñ }\end{array}$ & $\hat{n}$ & N & $\stackrel{\Im}{\Im}$ & బ઼ & $\hat{\tilde{n}}$ & 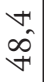 & $\hat{n}$ & $\begin{array}{l}\stackrel{r}{n} \\
\hat{n}\end{array}$ & $\begin{array}{c}\Omega_{\mathfrak{n}} \\
\stackrel{n}{n}\end{array}$ & ñ & 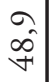 & $\stackrel{N}{N}$ & \begin{tabular}{l}
$\forall$ \\
\multirow{F}{*}{}
\end{tabular} \\
\hline 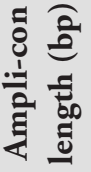 & $n$ & $\underset{\infty}{ঋ}$ & $\infty$ & $\curvearrowright$ & 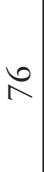 & $\infty$ & $\Re$ & $n$ & $\cong$ & $\stackrel{+}{\bowtie}$ & $\underset{\infty}{+}$ & $\infty$ & $\underset{\infty}{+}$ & $\curvearrowleft$ & 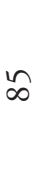 & $\approx$ & 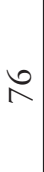 & $\hat{\lambda}$ & 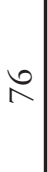 & 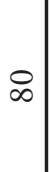 & $\curvearrowleft$ & $\underset{\infty}{+}$ & $\underset{\infty}{+}$ & N & $\infty$ & 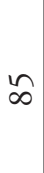 & $\infty$ & 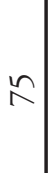 & $N$ & $\infty$ & $\stackrel{m}{n}$ \\
\hline
\end{tabular}

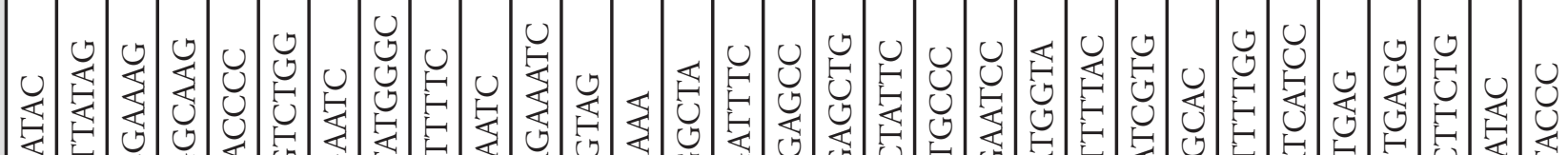

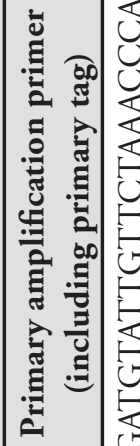

ن 


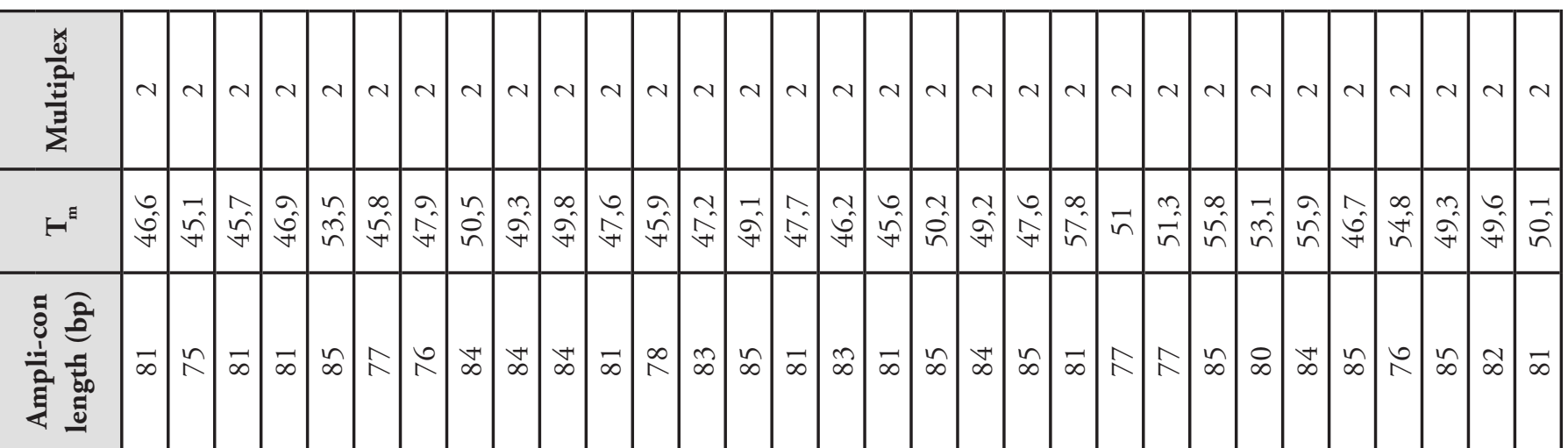

U

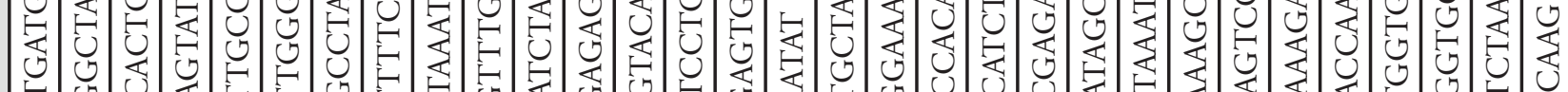
兽 司至

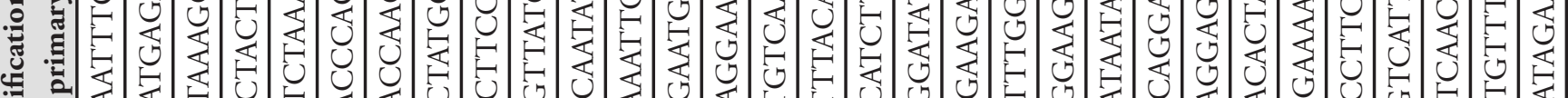

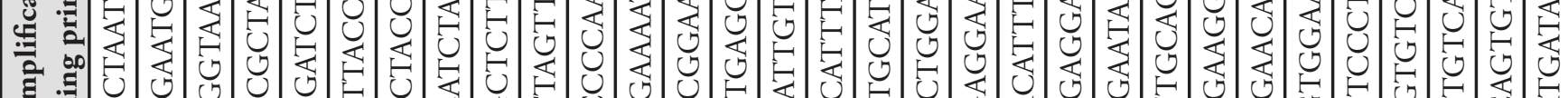

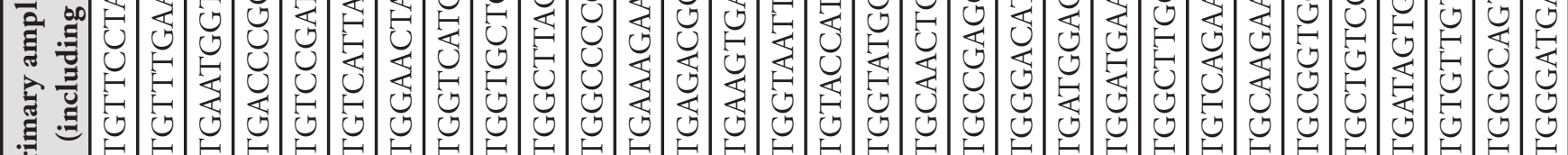

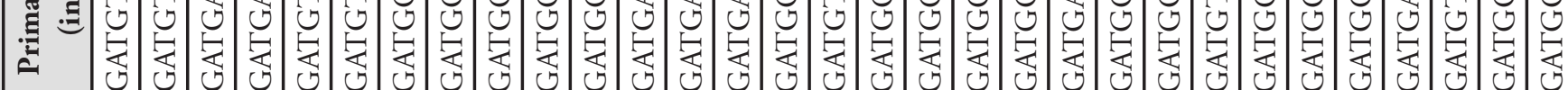

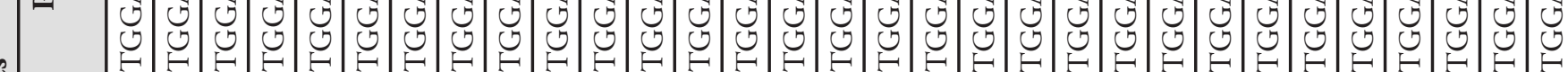

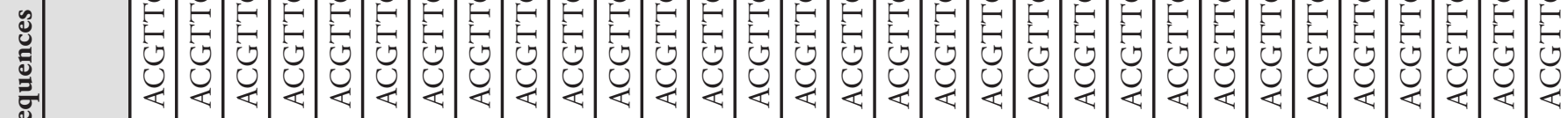

:

等 喜 至

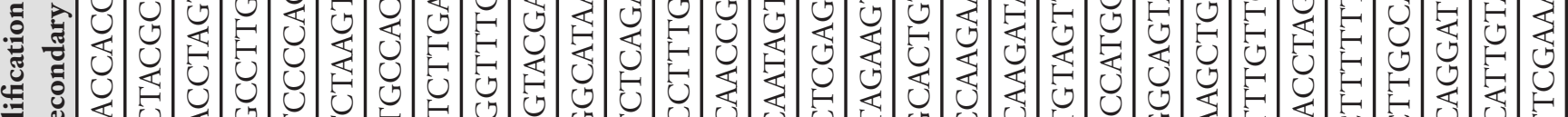
至 菏

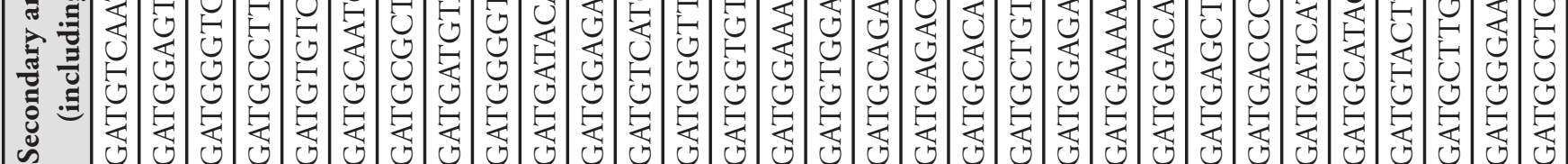
J 至 Y 安

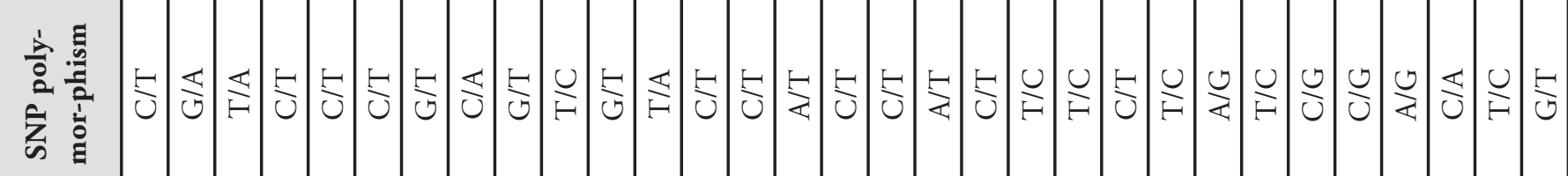

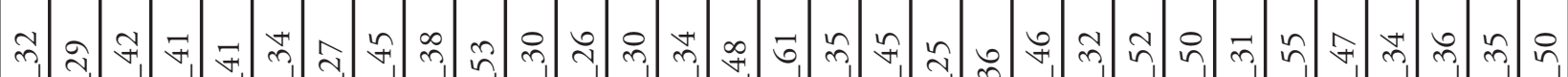

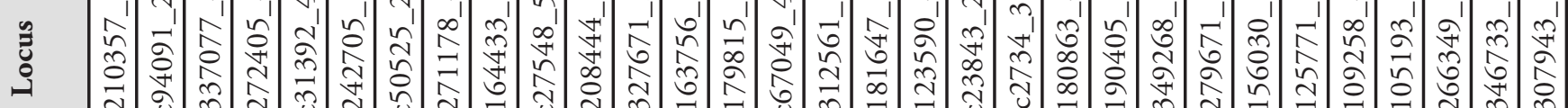

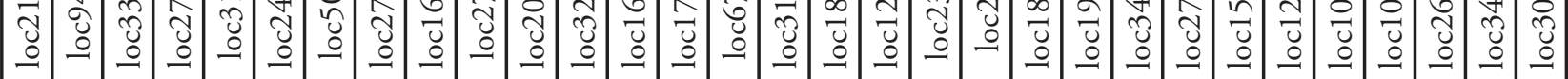




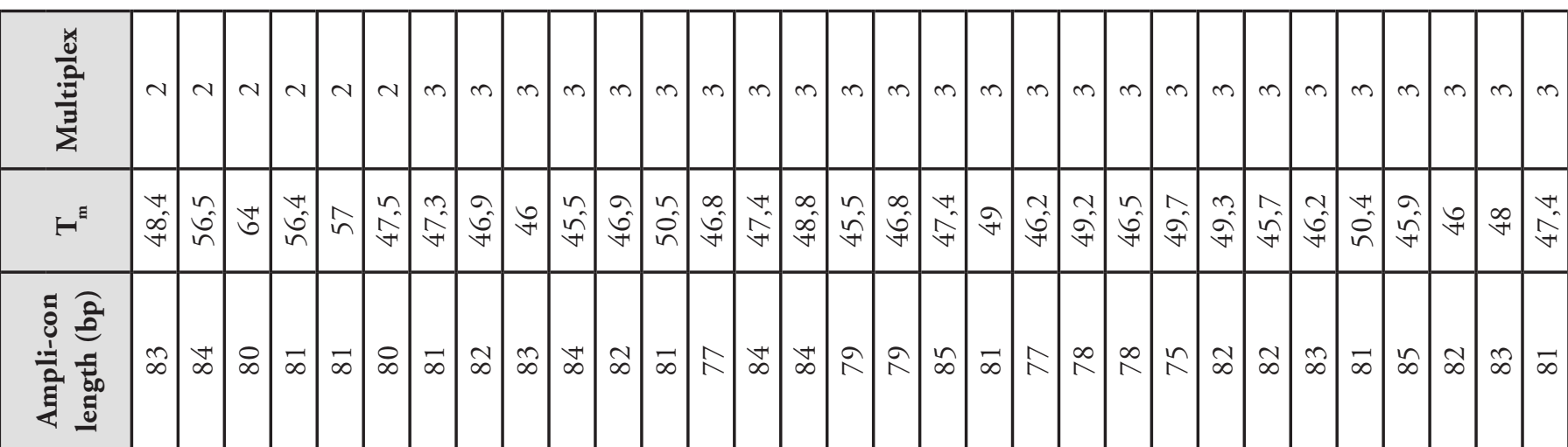

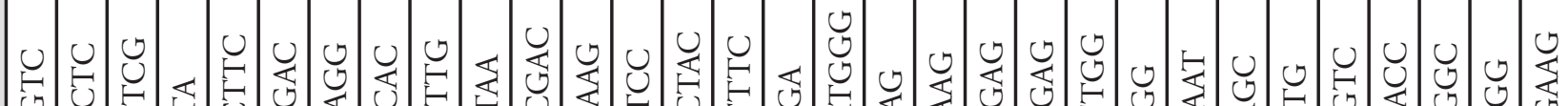

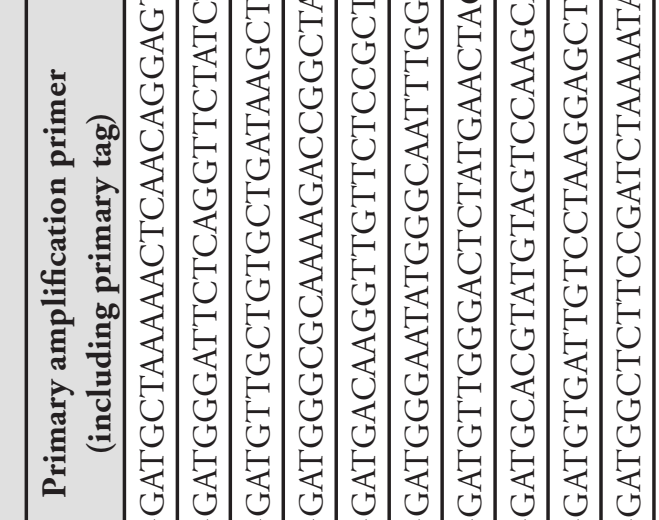

|

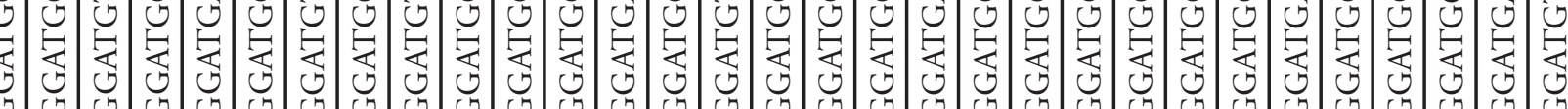

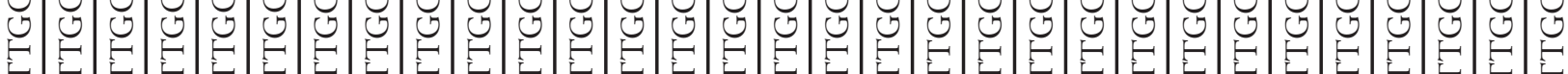

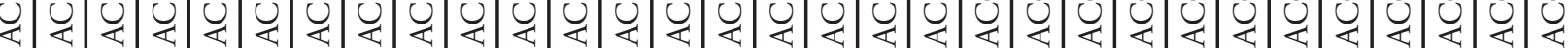

离

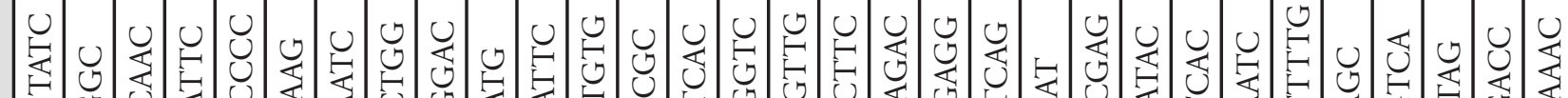

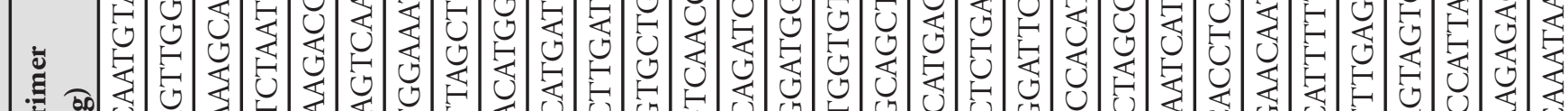

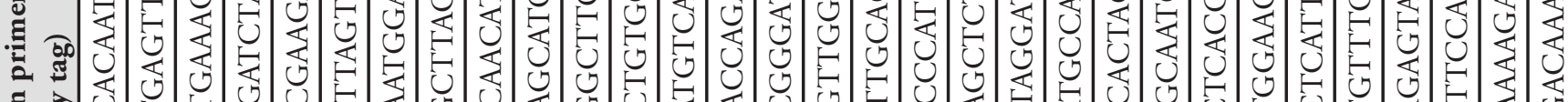

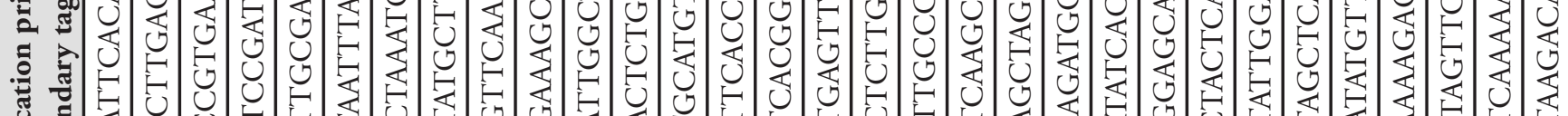
吾

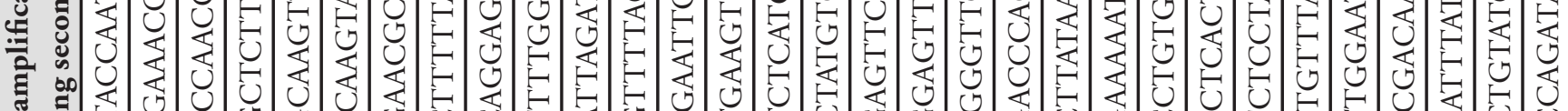

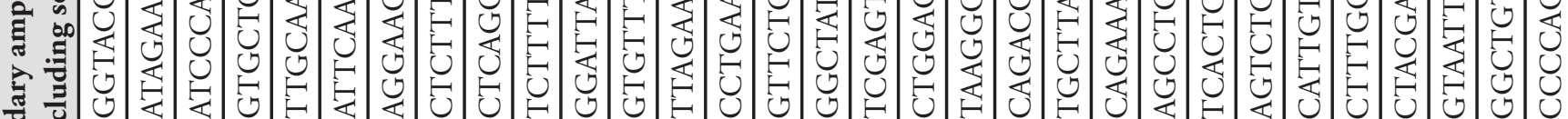

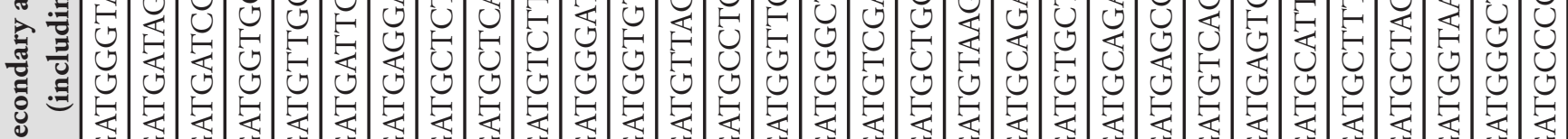

ڤ J ○

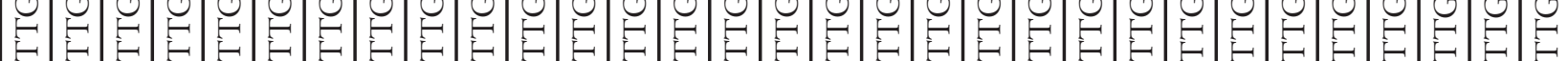
Y

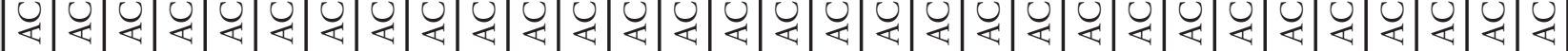

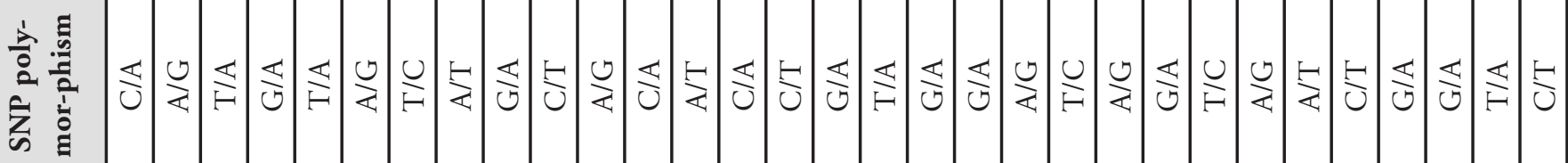

불

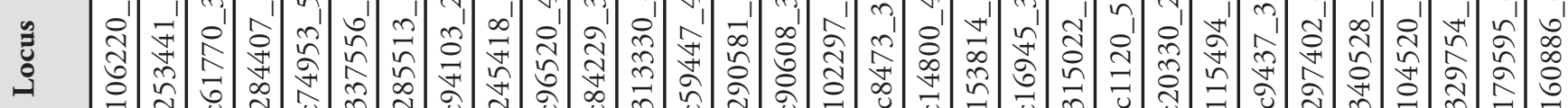

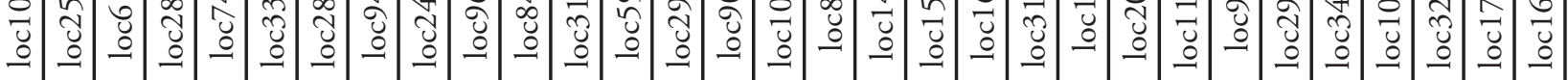




\begin{tabular}{|c|c|c|c|c|c|c|c|c|c|c|}
\hline 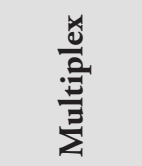 & $m$ & $n$ & $n$ & $n$ & $n$ & $n$ & $n$ & $n$ & $n$ & $n$ \\
\hline$H^{\Xi}$ & $\begin{array}{l}0 \\
\stackrel{2}{f} \\
F\end{array}$ & $\begin{array}{l}\vec{r} \\
\hat{n}\end{array}$ & $\begin{array}{l}n \\
n \\
n\end{array}$ & $\frac{n}{n}$ & $\frac{n}{n}$ & 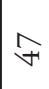 & $\begin{array}{l}n \\
n \\
n\end{array}$ & $\stackrel{\vec{F}}{\mathrm{~F}}$ & 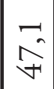 & $\stackrel{N}{n}$ \\
\hline 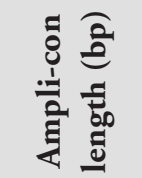 & $\infty$ & $\infty$ & $\infty$ & $\infty$ & $\stackrel{\infty}{\infty}$ & $\infty$ & $\infty$ & $\curvearrowright$ & 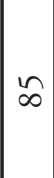 & $\stackrel{\infty}{\wedge}$ \\
\hline 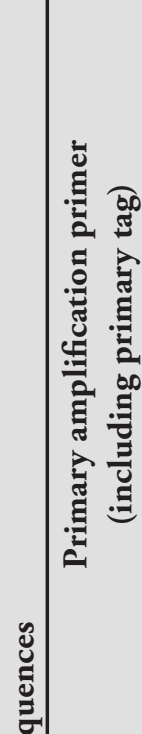 & 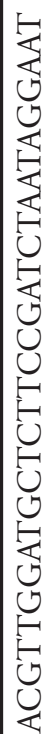 & 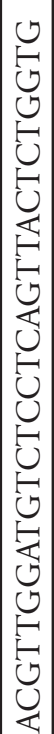 & 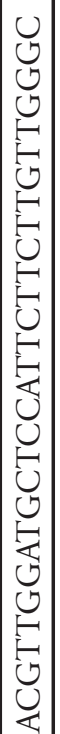 & 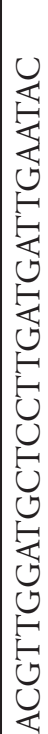 & 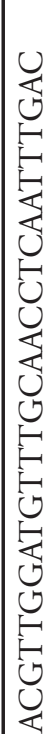 & 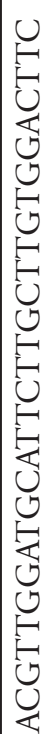 & 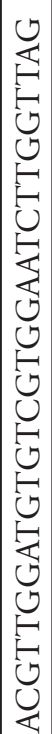 & 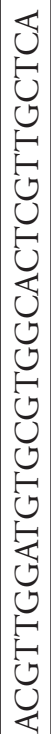 & 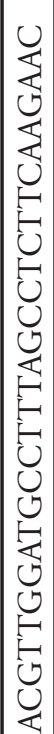 & 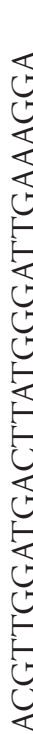 \\
\hline 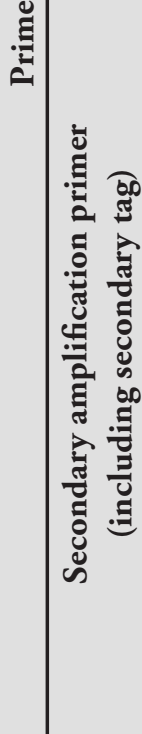 & 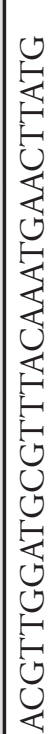 & 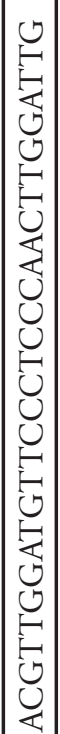 & 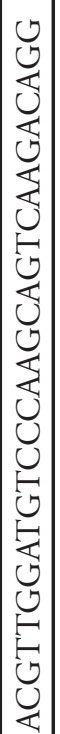 & 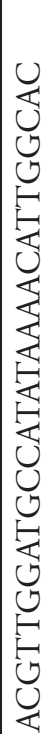 & 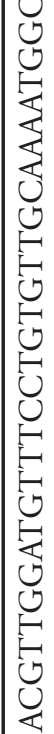 & 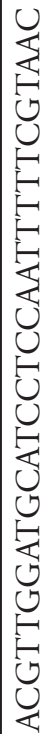 & 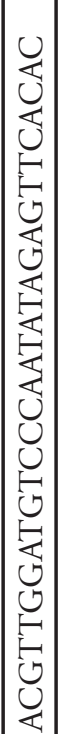 & 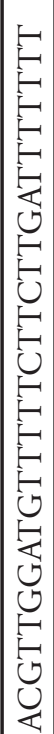 & 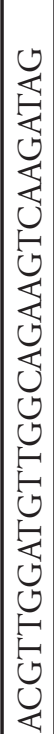 & 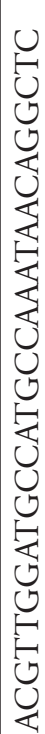 \\
\hline 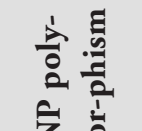 & $\frac{F}{0}$ & $\frac{\mathbb{U}}{\circlearrowleft}$ & $\stackrel{5}{\gtrless}$ & $\stackrel{\cup}{\gtrless}$ & $\frac{F}{\circlearrowleft}$ & $\frac{\mathbb{U}}{0}$ & $\frac{\mho}{\circlearrowright}$ & $\frac{\mathbb{U}}{0}$ & $\stackrel{5}{\gtrless}$ & $\frac{\mathbb{S}}{U}$ \\
\hline •] & 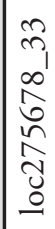 & 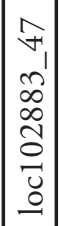 & $\begin{array}{c}\sqrt{1} \\
m \\
n \\
n \\
\infty \\
0 \\
0 \\
0 \\
0 \\
0\end{array}$ & 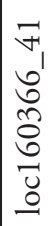 & 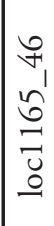 & 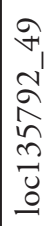 & 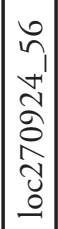 & $\mid \begin{array}{c}n \\
\hat{n} \\
\hat{n} \\
\hat{n} \\
\hat{n} \\
y \\
0 \\
0\end{array}$ & 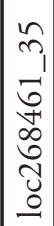 & 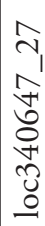 \\
\hline
\end{tabular}

\section{Material for DNA-extraction}

Both cited studies (Konar et al. 2017, Merceron et al. 2017) used both leaves and buds for DNA extraction.

\section{DNA-extraction protocols}

Konar et al. (2017) isolated DNA following a CTAB protocol described in Hoban et al. (2009) from the parental trees, whereas they used the commercial kit DNeasy (Qiagen) for DNA extraction from seedlings. Merceron et al. (2017) used a commercial DNA extraction kit (HTS 96 kit, STRATEC Molecular GmbH).

\section{Important results}

The population genetic analysis of Merceron et al. (2017) included both native provenances and introduced populations from Europe, which were represented in a provenance-progeny test. The population genetic analysis revealed three ancestral clusters in the native range with a predominant latitudinal differentiation which was rather gradual. In Europe, most introduced populations show an affinity to clusters dominating the northern part of the native range. Moreover, results suggest recent admixture among different origins after species' introduction to Europe.

\section{References}

Aldrich, P.R., Glaubitz, J.C., Parker, G.R., Rhodes Jr., O.E., Michler, C.H. (2005): Genetic Structure Inside a Declining Red Oak Community in Old-Growth Forest. Journal of Heredity, 96(6): 627-634.

Aldrich, P.R. Jagtap, M., Michler, C.H., RomeroSeverson, J. (2003a): Amplification of North American Red Oak Microsatellite Markers in European White Oaks and Chinese Chestnut. Silvae Genetica, 52: 3-4.

Aldrich, P.R., Michler, C.H., Sun, W., Romero-Severson, J. (2002): Microsatellite markers for northern red oak (Fagaceae: Quercus rubra) Molecular Ecology Notes, 2: 472-474.

Aldrich, P.R., Parker, G.R., Michler, C.H., RomeroSeverson, J. (2003b): Whole-tree silvic identifications and the microsatellite genetic structure of a red oak species complex in an Indiana old-growth forest. Canadian Journal of Research, 33: 2228-2237.

Alexander, L.W., Woeste, K.E. (2014): Pyrosequencing of the northern red oak (Quercus rubra L.) chloroplast genome reveals high quality polymorphisms for population management. Tree Genetics \& Genomes, 10(4): 803-812. 
Birchenko, I., Feng, Y., Romero-Severson, J., (2009): Biogeographical distribution of chloroplast diversity in Northern Red Oak (Quercus rubra L.). The American Midland Naturalist, 161(1): 134-145.

Borkowski, D.S., Hoban, S.M., Chatwin, W., Romero-Severson, J. (2017): Rangewide population differentiation and population substructure in Quercus rubra L. Tree Genetics \& Genomes, 13(3): 67.

Borkowski, D.S., McCleary, T., McAllister, M., RomeroSeverson, J. (2014): Primers for 52 polymorphic regions in the Quercus rubra chloroplast, 47 of which amplify across 11 tracheophyte clades. Tree Genetics \& Genomes, 10(4): 885-893.

Bradić, M., Costa, J., Chelo, I.M. (2011): Genotyping with sequenom. Molecular Methods for Evolutionary Genetics, Human Press, p. 193-210.

Collins, E., Sullivan, A.R., Gailing, O. (2015): Limited effective gene flow between two interfertile red oak species. Trees, 29(4): 1135-1148.

Daubree, J.B., Kremer, A. (1993): Genetic and phenological differentiation between introduced and natural populations of Quercus rubra L. Annales des Sciences Forestieres, 50 (Suppl1): 271-280.

Demesure, B., Sodzi, N., Petit, R.J. (1995): A set of universal primers for amplification of polymorphic noncoding regions of mitochondrial and chloroplast DNA in plants. Molecular Ecology, 4: 129-131.

Dow, B.D., Ashley, M.V., Howe, H.F. (1995): Characterization of highly variable (GA/CT) n microsatellites in the bur oak, Quercus macrocarpa. TAG Theoretical and Applied Genetics, 91(1): 137-141.

Dumolin, S., Demesure, B., Petit, R.J. (1995): Inheritance of chloroplast and mitochondrial genomes in pedunculated oak investigated with an efficient PCR method. Theoretical Applied Genetics, 91: 1253-1256.

Dumolin-Lapègue, S., Pemonge, M.-H., Petit, R.J. (1997): An enlarged set of consensus primers for the study of organelle DNA in plants. Molecular Ecology, 6: 393-397.

Durand, J., Bodénès, C., Chancerel, E., Frigerio, J.M., Vendramin, G., Sebastiani, F., Buonamici, A., Gailing, O., Koelewijn, H.-P., Villani, F., Mattioni, C., Cherubini, M., Goicoechea, P.G., Herrán, A., Ikaran, Z., Cabané, C., Ueno, S., Alberto, F., Dumoulin, P.-Y., Guichoux, E., de Daruvar, A., Kremer, A., Plomion, C. (2010): A fast and cost-effective approach to develop and map EST-SSR markers: oak as a case study. BMC Genomics, 11: 570.

Falush, D., Stephens, M., Pritchard, J.K. (2003): Inference of population structure using multilocus genotype data: linked loci and correlated allele frequencies. Genetics, 164(4): 1567-1587.

Gailing, O., Lind, J., Lilleskov, E. (2012): Leaf morphological and genetic differentiation between Quercus rubra L. and Q. ellipsoidalis In E.J. Hill populations in contrasting environments. Plant Systematics and Evolution, 298: 1533-1545.

Gerwein, J.B., Kesseli, R.V. (2006): Genetic diversity and population structure of Quercus rubra (Fagaceae) in old-growth and secondary forests in southern New England. Rhodora, 108(933): 1-18.

Gilman, E.F., Watson, D.G. (1994): Quercus rubra Northern red oak. Fact Sheet ST-560 Environmental Horticulture Department, Florida Cooperative Extension Service, Institute of Food and Agricultural Sciences, University of Florida.

Hoban, S.M., McCleary, T.S., Schlarbaum, S.E., Romero-Severson, J. (2009): Geographically extensive hybridization between the forest trees American butternut and Japanese walnut. Biology Letters, rsbl2009.0031

Hokanson, S.C., Isebrands, J.G., Jensen, R.J., Hancock, J.F. (1993): Isozyme variation in oaks Apostle Islands in Wisconsin: Genetic structure and level of inbreeding in Quercus rubra and Quercus elipsoidalis (Fagaceae). American Journal of Botany, 80(11): 1349-1357.

Isagi, Y., Suhandono, S. (1997): PCR primers amplifying microsatellite loci of Quercus myrsinifolia Blume and their conservation between oak species. Molecular Ecology, 6(9): 897-899.

Jones, F.A., Hamrick, J.L., Peterson, C.J., Squiers, E.S. (2006): Inferring colonization history from analyses of spatial genetic structure within populations of Pinus strobus and Quercus rubra. Molecular Ecology, 15: 851861.

Khodwekar, S., Gailing, O. (2017): Evidence for environment-dependent introgression of adaptive genes between two red oak species with different drought adaptations. American Journal of Botany, 104(7): 10881098.

Konar, A., Choudhury, O., Bullis, R., Fiedler, L., Kruser, J.M., Stephens, M.T., Gailing, O., Schlarbaum, S., Coggeshall, M.V., Staton, M.E., Carlson, J.E., Emrich, S., Romero-Severson, J. (2017): High-quality genetic mapping with ddRADseq in the non-model tree Quercus rubra. BMC Genomics, 18(1): 417.

Lind, J.F., Gailing, O. (2013): Genetic structure of Quercus rubra L. and Quercus ellipsoidalis E.J. Hill 
populations at gene-based EST-SSR and nuclear SSR markers. Tree Genetics \& Genomes, 9: 707-722.

Lind-Riehl, J., Gailing, O. (2015): Fine-scale spatial genetic structure of two red oak species, Quercus rubra and Quercus ellipsoidalis. Plant Systematics and Evolution, 301:1601-1612.

Lind-Riehl, J.F., Gailing, O. (2017): Adaptive Variation and Introgression of a CONSTANS-Like Gene in North American Red Oaks. Forests, 8(1): 3.

Lind-Riehl, J.F., Sullivan, A.R., Gailing, O. (2014): Evidence for selection on a CONSTANS-like gene between two red oak species. Annals of Botany, 113: 967-975.

Little, E.L., Jr. (1979): Checklist of United States trees (native and naturalized). U.S. Department of Agriculture, Agriculture Handbook 541. Washington, DC, pp. 375.

Magni, C.R., Ducousso, A., Caron, H., Petit, R.J., Kremer, A., (2005): Chloroplast DNA variation of Quercus rubra L. in North America and comparison with other Fagaceae. Molecular Ecology, 14(2): 513-524.

Manos, P.S., Fairbrothers, D.E. (1987): Allozyme Variation in Populations of Six Northeastern American Red Oaks (Fagaceae: Quercus subg. Erythrobalanus). Sistematic Botany, 12(3): 365-373.

Merceron, N.R., Leroy, T., Chancerel, E., RomeroSeverson, J., Borkowski, D.S., Ducousso, A., Monty, A., Porté, A., Kremer, A. (2017): Back to America: tracking the origin of European introduced populations of Quercus rubra L. Genome, 60(9): 778-790.

Moran, E.V., Willis, J., Clark, J.S. (2012): Genetic evidence for hybridization in red oaks (Quercus Sect. Lobatae, Fagaceae), American Journal of Botany, 99(1): 92-100.

Murray, M.G., Pitas, J.W. (1996): Plant DNA from alcohol-preserved samples. Plant Molecular Biology Reporter, 14(3): 261-265.

Pritchard, J.K., Stephens, M., Donnelly, P. (2000): Inference of population structure using multilocus genotype data. Genetics, 155(2): 945-959.

Romero-Severson, J., Aldrich P., Feng, Y., Weilin, S., Michler C. (2003). Chlorplast DNA variation of northern red oak (Quercus rubra L.) in Indiana. New Forests, 26(1): 43-49.

Sander, I.L. (1965): Northern red oak Quercus rubra L. In: Fowells, H.A. (Ed.), Silvics of Forest Trees of the United States. Agriculture Handbook, 271, Comp. U.S. Department of Agriculture, Washington, DC, pp. 588592.
Scandalios, J.G. (1969): Genetic control of multiple molecular forms of enzymes in plants: a review. Biochemical, 3: 37-79.

Schwarzmann, J.F., Gerhold, H.D. (1991): Genetic Structure and Mating System of Northern Red Oak (Quercus rubra L.) in Pennsylvania. Forest Science, 37(5): 1376-1389.

Sork, V.L., Huang, S., Wiener, E. (1993): Macrogeographic and fine-scale genetic structure in a North American oak species, Quercus rubra. Annales des Sciences Forestieres, 50 (Suppl1): 261-270.

Steinkellner, H., Fluch, S., Turetschek, E., Lexer, C., Streiff, R., Kremer, A., Burg, K., Glössl, J. (1997): Identification and characterization of $(\mathrm{GA} / \mathrm{CT})_{\mathrm{n}}$ microsatellite loci from Quercus petraea. Plant Molecular Biology, 33: 1093-1096.

Sullivan, A.R., Lind, J.F., McCleary, T.S., RomeroSeverson, J., Gailing, O. (2013): Development and Characterization of Genomic and Gene-Based Microsatellite Markers in North American Red Oak Species. Plant Molecular Biology Reporter, 31: 231-239.

Taberlet, P., Gielly, L., Pautou, G., Bouvet, J. (1991): Universal primers for amplification of three non-coding regions of chloroplast DNA. Plant Molecular Biology, 17(5): 1105-1109.

Tobolski, J.J. (1978): Isozyme variation in several species of oaks. Central hardwood Forest Conference, Purdue University, West Lafayette, LN, p. 456-478. 


\title{
Molecular markers used for genetic studies in black locust (Robinia pseudoacacia L.)
}

\author{
Charalambos Neophytou ${ }^{1}$, Marcela van Loo $^{2}$ and Marcin Klisz ${ }^{3}$ \\ ${ }^{1}$ University of Natural Resources and Life Sciences (BOKU), Department of Forest and Soil Sciences, Institute of Silviculture, Peter-Jordan- \\ Straße 82, 1190 Vienna, Austria \\ ${ }^{2}$ University of Vienna, Department of Botany and Biodiversity Research,Rennweg 14, 1030 Vienna, Austria \\ ${ }^{3}$ Department of Silviculture and Forest Tree Genetics, Forest Research Institute, Braci Leśnej 3, Sękocin Stary, 05-090 Raszyn, Poland
}

\section{General remarks}

Black locust (Robinia pseudoacacia L.) has its origin in eastern North America. Other known names for this tree species are false acacia or robinia. Its native range consists of two distinct areas and several outlying populations. The larger of these two distribution areas coincides with the Appalachian Mountains; the smaller one is located in the states Arkansas, Missouri and Oklahoma (Little 1976; see Figure 1). The precise extent of the native range, however, is not accurately known as the black locust has been widely planted and has become naturalized throughout North America. At the present, it is spread in every state of the contiguous USA and also in British Columbia, Québec, Newfoundland and Labrador in Canada (map of the naturalized / invaded area in the N. America can be found at http://plants. usda.gov/core/profile?symbol=ROPS), and many areas with temperate and Mediterranean climate across the world (Schütt 1994).

Black locust grows on a wide variety of soils; from acidic to base-rich, from nutrient-poor to nutrient-rich and from moist to dry. However, it avoids compacted, not well-aerated soils (Huntley 1990). Due to its nitrogenfixation ability, it has been widely planted outside its native range for soil amelioration and for restoration of disturbed sites (Huntley 1990, Schütt 1994). It is fast growing and its wood is hard and durable. However, wood quality is often reduced by its crooked stem form (Hanover et al. 1991, Schütt 1994). Other uses include energy fuel production (Rédei \& Veperdi 2009) and bee honey production (Schütt 1994).

Mature trees can reach a height from 15 to $30 \mathrm{~m}$ and a diameter at breast height (dbh) of $60 \mathrm{~cm}$ at the age of 40 . Forest-grown trees develop a taller and straighter stem (Schütt 1994). In natural populations, clonal structures are present due to the black locust's ability to reproduce also asexually by root suckering (Chang et al. 1998).
In total, the genus Robinia is represented by ca. 20 species (depending on the cited Flora; Schütt 1994), which are native to North America and northern Mexico. Robinia pseudoacacia is the only species to produce white flowers. In the native range, natural hybrids have been described between the black locust and the following three Robinia species:

1. Clammy locust, $R$. viscosa Vent. Their hybrid: $R . \mathrm{x}$ ambigua Poir;

2. New Mexico locust, $R$. neomexicana Gray. Their hybrid: $R$. x hodltii Beissn;

3. Kelsey locust, $R$. kelseyi Cowell ex Hutch. Their hybrid: $R$. x slavinii Rehder (Isely and Peabody 1984, Isely 1998).

In Europe, no reports on spontaneous hybridization are available. However, hybrids are available for cultivation (e.g. Royal Horticultural Society 2018). Many cultivars

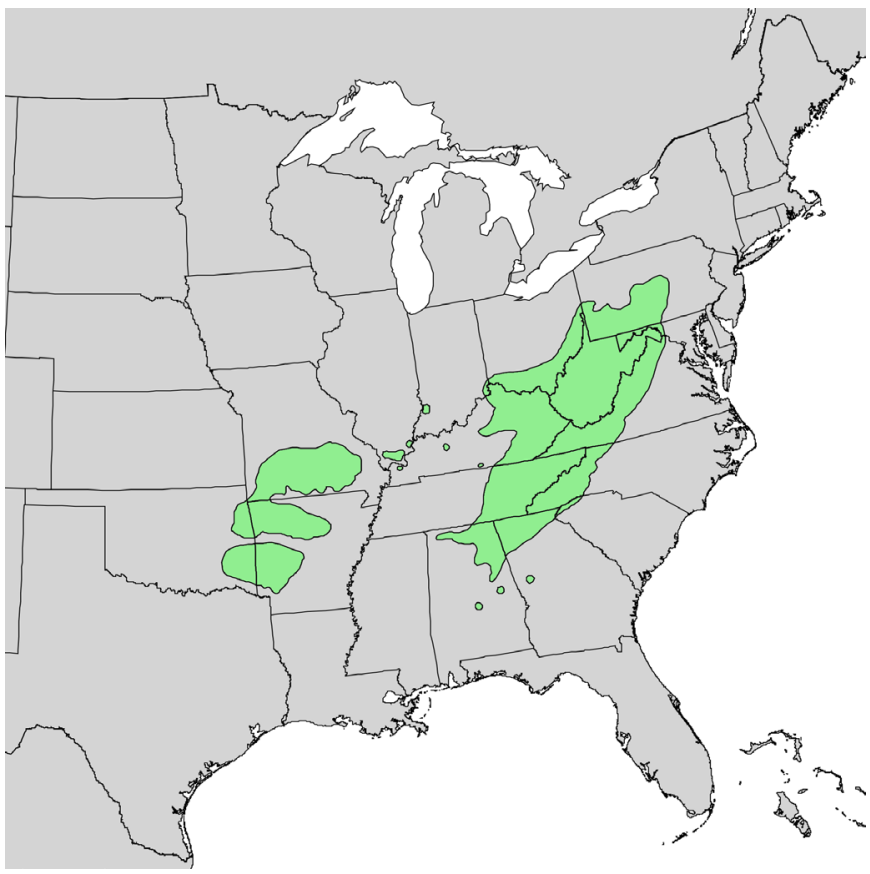

Figure 1. Native distribution range of black locust (source: USGS, USA) 
do exist: they vary in crown and stem form, growth rate, growth habit (upright vs. prostrate), leaf shape, thorniness, flowering characteristics and phenology.

Until now, several different marker systems have been developed (e.g. nuclear microsatellites; Lian and Hogetsu 2002, Mishima et al. 2009) and used for population genetic studies in native and introduced populations. Molecular genetic studies have focused on population genetic variation at the local (Chang et al. 1998) and range-wide scale (Surles et al. 1989, Houser 2014), as well as on clonal structures (Chang et al. 1998). The species' phylogeography in its native range has not been studied in detail. Chloroplast DNA markers have been developed (Kimura et al. 2013) but not widely used in native populations. Use of these markers in rangewide studies within the natural distribution could enable tracing the origin of introduced populations. In addition, recently developed nuclear DNA markers (e.g. SNPs; Verdu et al. 2016) may increase the performance of population genetic studies.

Here, we provide a review of existing laboratory techniques which can be used to investigate the origin of black locust, as well as its genetic diversity and differentiation.

\section{Isozymes}

Earlier studies based on isozymes focused on:

- Investigation of genetic diversity within and between populations (Gu et al. 2010);

- study of clonal structures (Chang et al. 1998, Liesebach et al. 2004);

- spatial (range-wide) patterns of genetic variation (Surles et al. 1989).

\section{Material for protein extraction}

Proteins were extracted from leaves (Chang et al. 1998, Gu et al. 2010, Liesebach et al. 2004, Surles et al. 1989) or root tissue (Surles et al. 1989).

\section{Protein extraction and separation protocols}

The extraction procedure and separation protocols are described in Liesebach et al. (2004) and Surles et al. (1989).

\section{Important results}

- In an early research paper, Surles et al. (1989) described range-wide patterns of genetic variation. They investigated seedlings of 23 seed sources

Table 1: List of enzymes, scored loci and number of alleles for black locust

\begin{tabular}{|l|c|l|c|c|}
\hline \multicolumn{1}{|c|}{ Enzyme system } & E.C. Number & \multicolumn{1}{c|}{ Scored loci } & $\begin{array}{c}\text { No. of } \\
\text { alleles }\end{array}$ & References \\
\hline Amylase & 3.2 .1 .1 & AMY-1,-2 & 5,3 & 2,3 \\
\hline Adenylate kinase & 2.7 .4 .3 & AK-1 & 4 & 4 \\
\hline Aldolase & 4.1 .2 .13 & ALD-1 & 3 & 4 \\
\hline Alpha-galactosidase & 3.2 .1 .22 & a-GAL-1 & 3 & 4 \\
\hline Colorimetric esterase & 3.1 .1$. & CE-1,-2 & 3,3 & 4 \\
\hline Diaphorase & 1.6 .2 .2 & DIA-1,-2,-3 & $3,3,2$ & 4 \\
\hline Fluorescent esterase & 3.1 .1 .56 & FEST-1,-2,-3 & $4,4,4$ & $1,2,3,4$ \\
\hline Fructose 1-6 phosphate & 3.1 .3 .11 & Fl-6-1,-2,-3 & $4,3,2$ & 4 \\
\hline Glutamate dehydrogenase & 1.4 .1 .2 & GDH-1 & 3 & 4 \\
\hline Glutamate oxaloacetate transaminase & 2.6 .1 .1 & GOT-1,-2 & 3,4 & 4 \\
\hline Isocitrate dehydrogenase & 1.1 .1 .42 & IDH-1 & 4 & $1,3,4$ \\
\hline Leucine aminopeptidase & 3.4 .11 .1 & LAP-1,-3 & 4,5 & $1,3,4$ \\
\hline Malate dehydrogenase & 1.1 .1 .37 & MDH-1,-2,-3 & $4,3,4$ & $1,2,3,4$ \\
\hline Peroxidase & 1.11 .1 & PER-1,-2,-3,-4 & $4,3,4,3$ & 4 \\
\hline Phosphoglucose isomerase & 5.3 .1 .9 & PGI-1 & 2 & 4 \\
\hline Phosphoglucomutase & 2.7 .5 .1 & PGM-1,-2,-3 & $3,4,3$ & 1,4 \\
\hline 6-Phosphogluconate dehydrogenase & 1.1 .1 .44 & 6-PGDH-1,-2,-3,-4 & $3,3,3,3$ & $2,3,4$ \\
\hline Shikimate dehydrogenase & 1.1 .1 .25 & SKDH-1,-2 & 3,3 & $1,2,3,4$ \\
\hline Triose-phosphate isomerase & 5.3 .1 .1 & TPI-1,-2,-3,-4 & $2,1,3,3$ & 4 \\
\hline
\end{tabular}

1-Chang et al. 1998, 2-Gu et al. 2010, 3-Liesebach et al. 2004, 4-Surles et al. 1989 
(populations) within the native range and scored 40 loci across 18 allozyme systems. Most of the genetic diversity $(88 \%)$ resided within seed sources. The most differentiated sources were located on disjunct sites of marginal populations in Georgia and the Ozark Mountains of Arkansas and Missouri, and in Pennsylvania. No patterns of geographic differentiation were determined, which the authors attributed to widespread plantings from different (even European) seed sources.

- In their study, Chang et al. (1998) investigated fine-scale population structure and clonality in two populations of black locust in North Carolina, within the species' native range. They showed that native populations maintain very high levels of genetic diversity, however no noticeable geographic patterns could be recognized. Out of 200 and 420 plants analysed within each population, 13 and 15 unique genotypes were distinguished, respectively. These genotypes represented one clone each, represented by several ramets. The largest clones (genets) in both study sites covered more than $100 \mathrm{~m} \times 100 \mathrm{~m}$.

- Liesebach et al. (2004) compared genetic diversity between progenies of two native North American populations of black locust and 16 introduced European populations collected in Germany, Hungary and Slovakia. All seedlings were assayed for 11 enzyme systems. In Europe, Hungarian populations had high within population genetic variation (diversity) and low among population genetic variation. Conversely, in Germany one progeny had low within population genetic variation and there was high differentiation between populations. Slovak populations were similar to Hungarian with regard to the within population diversity. In the two populations from the USA, genetic diversity was similar to German stands.
The authors attributed the contrasting patterns of diversity and differentiation found in Hungary and Germany to differences in management of black locust in Europe.

- Finally, a more recent study (Gu et al. 2010) described genetic diversity of 19 black locust populations distributed in China and evaluated them using seven allozyme systems. Most of the genetic variation resided within populations. Moreover, a significant pattern of isolation by distance was found.

\section{Organelle DNA markers (chloroplast (cp) DNA, mitochondrial (mt)DNA)}

\section{Loci and primers used}

Liesebach and Schneck (2012) applied PCR-RFLP techniques to investigate polymorphisms in a set of populations analyzed previously with isozymes. Mostly using universal primers, they amplified five chloroplast DNA (cpDNA) loci by means of PCR and digested the amplicons with eight restriction enzymes (Table 2).

In their technical report, Kimura et al. (2013) developed a set of five polymorphic chloroplast microsatellites (cpSSRs) for genetic analyses in black locust. Locus description, primer sequences and annealing temperatures are presented in Table 3.

\section{Material for DNA-extraction}

In both cited studies, leaves were used for DNA extraction (Liesebach and Schneck 2012, Kimura et al. 2013).

Table 2: PCR-RFLP markers (cpDNA) for black locust (Liesebach and Schneck 2012)

\begin{tabular}{|c|c|c|c|}
\hline Locus & $\begin{array}{c}\text { Primer sequences } \\
\mathrm{F}=\text { Forward, } \mathrm{R}=\text { Reverse }\end{array}$ & Restriction enzymes & $\begin{array}{l}\text { No. of polymorphic } \\
\text { bands }\end{array}$ \\
\hline $\mathrm{DT}(\operatorname{trnD} / \operatorname{trnT})$ & $\begin{array}{l}\text { F:ACCAATTGAACTACAATCCC } \\
\text { R:CTACCACTGAGTTAAAAGGG }\end{array}$ & \multirow{5}{*}{$\begin{array}{c}\text { BamHI, DraI, HinfI, } \\
\text { MboI, MspI, } \\
\text { RsaI, SspI, TaqI }\end{array}$} & 2 \\
\hline $\mathrm{HK}(\operatorname{trnH} / \operatorname{trnK})$ & $\begin{array}{l}\text { F:ACGGGAATTGAACCCGCGCA } \\
\text { R:CCGACTAGTTCCGGGTTCGA }\end{array}$ & & 5 \\
\hline $\mathrm{K} 1 \mathrm{~K} 2(\operatorname{trnK} / \operatorname{trnK})$ & $\begin{array}{l}\text { F:GGGTTGCCCGGGACTCGAAC } \\
\text { R:CAACGGTAGAGTACTCGGCTTTTA }\end{array}$ & & 3 \\
\hline BB (psbB/petB) & $\begin{array}{l}\text { F:CAGAAGCTTGGTCTAAAATTCC } \\
\text { R:GRTCCCAAGGGAARGAATAACCAGT }\end{array}$ & & 3 \\
\hline $\mathrm{ED}(\operatorname{trnE} / \mathrm{psbD})$ & $\begin{array}{l}\text { F:GTCCCGACGTAACCAGTCAT } \\
\text { R:TGAACCACTAGACGATGGGG }\end{array}$ & & 2 \\
\hline
\end{tabular}


Table 3: List of markers and primer sequences ( $\mathrm{T}_{\mathrm{a}}$-annealing temperature, $\mathrm{N}_{\mathrm{A}}$-number of alleles scored) (Kimura et al. 2013)

\begin{tabular}{|c|c|l|l|c|c|c|}
\hline \multirow{2}{*}{ Locus } & \multirow{2}{*}{ Motif } & \multicolumn{2}{|c|}{ Primer sequences } & \multirow{2}{*}{ Size $(\mathbf{b p})$} & $\mathbf{T}_{\mathbf{a}}$ & \multirow{2}{*}{$\mathbf{N}_{\mathbf{A}}$} \\
\cline { 3 - 7 } & & \multicolumn{2}{|c}{ Forward } & Reverse & \\
\hline Ropscp03 & $\mathrm{A}_{10}$ & GGATCTTTCTGAATTCCGTAG & CAGCAAATCAATCATTTCCTG & $155-164$ & 54 & 6 \\
\hline Ropscp04 & $\mathrm{T}_{5} \mathrm{AT}_{10}$ & TACGATCTTGTAGTAATTTCC & ACTACTCTCCTTTCATCAAAG & $99-113$ & 54 & 3 \\
\hline Ropscp06 & $\mathrm{T}_{10} \mathrm{AT}_{5}$ & CCATGGTATTTGATTTACCAA & TCAAGGTCGAGAGTGAATTTC & $137-140$ & 54 & 4 \\
\hline Ropscp07 & $\mathrm{T}_{10}$ & AGTACCGGACCAATTATTTGG & TTAACCGATCAACTTGCTTTG & $171-176$ & 54 & 6 \\
\hline Ropscp08 & $\mathrm{A}_{11}$ & GAACACGAGTCGAGGCTATC & GATACCATGGATTCTGGTATC & $186-201$ & 54 & 8 \\
\hline
\end{tabular}

\section{DNA-extraction protocols}

For DNA isolation, the cetyltrimethyl ammonium bromide, the (CTAB) protocol was used in both cited studies (Liesebach and Schneck 2012 according to Dumolin et al. 1995; Kimura et al. 2013 according to Lian et al. 2003).

\section{Important results}

Liesebach and Schneck (2012) used PCR-RFLP techniques to study polymorphisms in a set of European populations. They additionally analysed four American populations from the native range with the identical markers. They found eleven cpDNA haplotypes belonging to two clearly separated groups of related haplotypes. The four US populations from the native range (representing the four US States: Illinois, Tennessee, Virginia and Georgia) displayed a low average number of haplotypes per population and were significantly differentiated from each other. In Europe, no spatial distribution pattern was found. Genetic variation within populations was higher than in the native range, whereas the opposite was observed for the genetic variation among populations. This result was attributed to repeated seed introductions from arbitrarily selected sources.

\section{Nuclear DNA markers (AFLPs, nSSRs, ISSRs, SNPs)}

a) AFLPs (Amplified Fragment Length Polymorphism)

\section{Loci and primers used}

In Huo et al. (2009), 10 populations of black locust collected in China were analyzed by AFLP using 10 primer combinations. Primer combination, total number of bands, number of polymorphic markers, and polymorphic rate are presented in Table 4 .

\section{Material for DNA-extraction}

Leaves were used for DNA extraction (Huo et al. 2009).

\section{DNA-extraction protocols}

Huo et al. (2009) used a CTAB-protocol for DNA extraction. Authors provide all details for modification of the Vos et al. (1995) protocol.

\section{Important results}

Huo et al. (2009) used 10 AFLP primer pairs to detect genetic diversity of 10 black locust populations originated

Table 4: Primer combinations, number of bands and degree of polymorphism

\begin{tabular}{|c|c|c|c|}
\hline Primer combination & $\begin{array}{c}\text { Total } \\
\text { bands }\end{array}$ & $\begin{array}{c}\text { Polymorphic } \\
\text { bands }\end{array}$ & $\begin{array}{c}\text { Polymorphism } \\
\text { \% }\end{array}$ \\
\hline E-GGA / M-TAT & 91 & 46 & 50.0 \\
\hline E-GGA / M-CTC & 87 & 37 & 42.5 \\
\hline E-CGG / M-GGA & 77 & 38 & 49.4 \\
\hline E-CGG / M-CAG & 83 & 31 & 37.3 \\
\hline E-AGC / M-TAT & 88 & 39 & 44.3 \\
\hline E-AGC / M-CAC & 38 & 17 & 44.7 \\
\hline E-AGC / M-CTT & 78 & 37 & 47.4 \\
\hline E-AAG / M-CAA & 97 & 44 & 45.4 \\
\hline E-ACA / M-CAA & 74 & 44 & 59.5 \\
\hline E-AAC / M-CAC & 39 & 19 & 48.7 \\
\hline
\end{tabular}


from China. Genetic diversity within populations $(66.10 \%)$ was higher than among populations $(33.90 \%)$. The UPGMA cluster analysis resulted in three major groups, where the most individuals of the same population clustered together. Furthermore, no significant correlation between the genetic diversity parameters $\left(D, I_{N}, P, N e, H\right.$, and $\left.I\right)$ and geographic (longitude, latitude) and climatic factors (annual mean temperature, and annual mean precipitation) was found.

\section{b) nSSRs (putatively neutral microsatellites)}

\section{Loci and primers used}

Lian and Hogetsu (2002) developed seven and Mishima et al. (2009) $11 \mathrm{nSSRs}$ in black locust which have been used for population genetic studies. Primer sequences, repeat motifs and allele length range are presented in Table 5.

Table 5: Primer sequences, allele length in base pairs (bp), annealing temperatures $\left(T_{a}\right)$ and references for nSSRs available for genetic analyses in black locust.

\begin{tabular}{|c|c|c|c|c|c|}
\hline Locus & Repeat motif & $\begin{aligned} & \text { Primer sequences } \\
\mathrm{F}= & \text { Forward, } \mathrm{R}=\text { Reverse }\end{aligned}$ & $\begin{array}{l}\text { Size } \\
\text { (bp) }\end{array}$ & $\mathbf{T}_{\mathrm{a}}$ & $\begin{array}{c}\text { Referen } \\
\text { ces }\end{array}$ \\
\hline Rops02 & $(\mathrm{AC})_{13}(\mathrm{AT})_{4}$ & $\begin{array}{l}\text { F:CAGAACTGTGGAGAATAATTCTGAACCG } \\
\text { R:CGCCATCTGTTAGTTTGTTGC }\end{array}$ & $107-138$ & 60 & 1,2 \\
\hline Rops04 & $(\mathrm{AC})_{10}$ & $\begin{array}{l}\text { F:GTCTAATTTCACTTTTCTCACGAG } \\
\text { R:GGACACCACCRAAATTCTACC }\end{array}$ & $105-110$ & 56 & 2 \\
\hline Rops05 & $(\mathrm{AC})_{2} \mathrm{GC}(\mathrm{AC})_{7}$ & $\begin{array}{l}\text { F:TGGTGATTAAGTCGCAAGGTG } \\
\text { R:GTTGTGACTTGTACGTAAGTC }\end{array}$ & $120-138$ & 56 & 2 \\
\hline Rops06 & $(\mathrm{GT})_{3} \mathrm{ACA}(\mathrm{GT})_{11}$ & $\begin{array}{l}\text { F:CTAAGGAGGTGCTGACCCTC } \\
\text { R:TTAATCTGTGATGGGACACTG }\end{array}$ & $117-144$ & 56 & 2 \\
\hline Rops08 & $(\mathrm{CA})_{8} \mathrm{TA}(\mathrm{CA})_{3}$ & $\begin{array}{l}\text { F:TTCTGAGGAAGGGTTCCGTGG } \\
\text { R:GTTAAAGCAACAGGCACATGG }\end{array}$ & $191-205$ & 56 & 1,2 \\
\hline Rops09 & $(\mathrm{TA})_{6} \mathrm{~A}_{4}(\mathrm{TA})_{2}(\mathrm{TG})$ & $\begin{array}{l}\text { F:CTCCAGGTCACTCGATTGAGG } \\
\text { R:TTTCTCATTTGATACGACCCC }\end{array}$ & $89-150$ & 56 & 2 \\
\hline Rops10 & $\mathrm{T}_{12} \mathrm{AAT}_{4}$ & $\begin{array}{l}\text { F:AACTTTTTCCGTATAGGGGTC } \\
\text { R:GAGTTTTACACTTGGTCAAACC }\end{array}$ & $182-187$ & 56 & 2 \\
\hline RP035 & $(\mathrm{TC})_{15}$ & $\begin{array}{l}\text { F:GGAGTGGAATGCATGCTCTCATG } \\
\text { R:TCCAAATGGAAACTCCCTTGAAACAGC }\end{array}$ & $89-112$ & $63-53^{*}$ & 1,3 \\
\hline RP102 & $(\mathrm{GA})_{12}$ & $\begin{array}{l}\text { F:CCAAATCTCAAAATGTGCTAAGTAGC } \\
\text { R:ACTTGGGCTATGGTATTGCA }\end{array}$ & $205-211$ & $63-53^{*}$ & 3 \\
\hline RP106 & $(\mathrm{GT})_{9}$ & $\begin{array}{l}\text { F:AAACTGAATTATATCCCTTTACGGC } \\
\text { R:GCATATATCCACCAGATACCCG }\end{array}$ & $143-154$ & $63-53^{*}$ & 1,3 \\
\hline RP109 & $(\mathrm{AG})_{17}$ & $\begin{array}{l}\text { F:GAGGAATCACAAAACCGTTTGG } \\
\text { R:TGGGATTTGAGAGAGTGGTGGTG }\end{array}$ & $136-160$ & $63-53^{*}$ & 1,3 \\
\hline $\mathrm{RP} 150$ & $(\mathrm{TC})_{3} \mathrm{TT}(\mathrm{TC})_{12}$ & $\begin{array}{l}\text { F:TCGTTGGATCAACATGCATGG } \\
\text { R:ACAGAACCCTAACCCTAGCA }\end{array}$ & $199-217$ & $63-53^{*}$ & 1,3 \\
\hline RP206 & $(\mathrm{GT})_{9}$ & $\begin{array}{l}\text { F:GCCAAATCCCATTAGATCACAGTTGA } \\
\text { R:AGAAGTTAGACTTACGTGCTGC }\end{array}$ & $222-246$ & $63-53^{*}$ & 1,3 \\
\hline RP200 & $(A G)_{23}$ & $\begin{array}{l}\text { F:GGTTTCTTTGTTCACCTGCTCTGG } \\
\text { R:ACCTACGTGTCCACGGCTCT }\end{array}$ & $160-198$ & $63-53^{*}$ & 1,3 \\
\hline RP032 & $(\mathrm{TG})_{13}$ & $\begin{array}{l}\text { F:GCATATTGCATATGCGCTTGTG } \\
\text { R:TCCCTGAAGCTCATAACTGTCATGTG }\end{array}$ & $109-135$ & $63-53^{*}$ & 3 \\
\hline RP211 & $(\mathrm{TC})_{7} \mathrm{~A}(\mathrm{AC})_{8}$ & $\begin{array}{l}\text { F:TGTAATCCATGTAGTTGACCCCAC } \\
\text { R:TGATTACTCTGCATGCATGTG }\end{array}$ & $196-201$ & $63-53^{*}$ & 3 \\
\hline RP165 & $(\mathrm{TG})_{8}$ & $\begin{array}{l}\text { F:TTAGATGTTGCAAGTGCTGAGG } \\
\text { R:ACAATGCCTCAATGCAGC }\end{array}$ & $146-159$ & $63-53^{*}$ & 3 \\
\hline RP01B & $(\mathrm{CT})_{16}$ & $\begin{array}{l}\text { F:ACCAATTAGGTAACGTCAGC } \\
\text { R:TGTTCACTGACAAAGCTG }\end{array}$ & $172-192$ & $63-53^{*}$ & 3 \\
\hline
\end{tabular}

*touch down PCR

1-Houser (2014), 2-Lian and Hogetsu (2002), 3-Mishima et al. (2009) 


\section{Material for DNA-extraction}

Leaves have been used for DNA extraction (Lian and Hogetsu 2002, Houser 2014).

\section{DNA-isolation protocols}

Lian and Hogetsu (2002) used a CTAB-protocol for DNA extraction, whereas Mishima et al. (2009) and Houser (2014) used a commercial extraction kit (DNEasy, Qiagen). All papers provide details on: (i) chemical concentrations, (ii) PCR programs and (iii) allele scoring methods.

\section{Important results}

Houser (2014) compared the genetic diversity between tree regions: throughout the native Appalachian region and in two invaded/naturalized regions in the Northeast and Midwest regions of the US using the microsatellites developed by Lian and Hogetsu (2002) and Mishima et al. (2009). Several genets with multiple ramets were identified in the study populations. In a total of 369 sampled trees, only 142 unique genotypes were found. Genetic differentiation among regions was low (1-3\%) and no obvious genetic structure was obtained by using clustering methods. The highest genetic diversity was found in the native Appalachian region.

\section{c) EST-SSRs (expressed sequence tag derived microsatellites)}

\section{Loci and primers used}

In a recent study, Guo et al. (2017) developed a new set of 45 EST-derived microsatellite loci for population genetic analyses in black locust. Primer sequences, repeat motifs and allele length range are presented in Table 6.

\section{Material for DNA-extraction}

Leaves were used for DNA extraction (Guo et al. 2017).

\section{DNA-extraction protocols}

A commercial kit (Tiangen, Beijing, China) was used in the study of Guo et al. (2017).

Details on: (i) chemical concentrations, (ii) PCR programs and (iii) allele scoring methods are provided in Guo et al. (2017).

\section{Important results}

Due to their high polymorphism, EST-SSRs developed by Guo et al. (2017) may provide a valuable tool for investigating genetic diversity and population structure of black locust, constructing a DNA fingerprint database and performing quantitative trait locus mapping.

\section{d) ISSRs (inter-simple sequence repeats)}

Guo et al. (2006) applied 32 inter-simple sequence repeat (ISSR) markers to 41 micropropagated plants of Robinia ambiqua var. idahoensis and their donor plant, from which they have been propagated by tissue culture system. Robinia ambiqua is known for its tolerance to drought and pests and doesn't possess roots characteristics as aggressive as $R$. pseudoacacia, one of its parental species. The authors assessed the occurrence and the extent of genomic changes in the in vitro micropropagated plants in order to study genomic stability / instability of the developed micropropagation protocol.

\section{Loci and primers used}

Loci and primers used are listed in Table 7.

\section{Material for DNA-extraction}

For DNA extraction, young leaves were used.

\section{DNA-isolation protocols}

Total genomic DNA was extracted using a modified CTAB method of Kidwell and Osborn (1992) and purified by phenol extraction.

\section{Important results}

Low genomic variation within the micropropagated plants (only $10.6 \%$ of the bands were polymorphic) was observed. The occurrence of genomic changes and their possible effect on morphological and physiological traits have to be, however, taken into consideration when the developed in vitro protocol will be further commercially used for micropropagation of the hybrid Robinia ambiqua.

\section{e) SNPs (single-nucleotide polymorphisms)}

\section{Loci and primers used}

Restriction site associated DNA sequencing (RADsequencing) was used by Verdu et al. (2016) to develop 377 SNP-markers. These were organized in 12 multiplexes based on Sequenom ${ }^{\ominus}$ technology. For more details about the Sequenom method the reader is referred to Bradić et al. (2011). Thus far, no case studies using these markers have been published. 


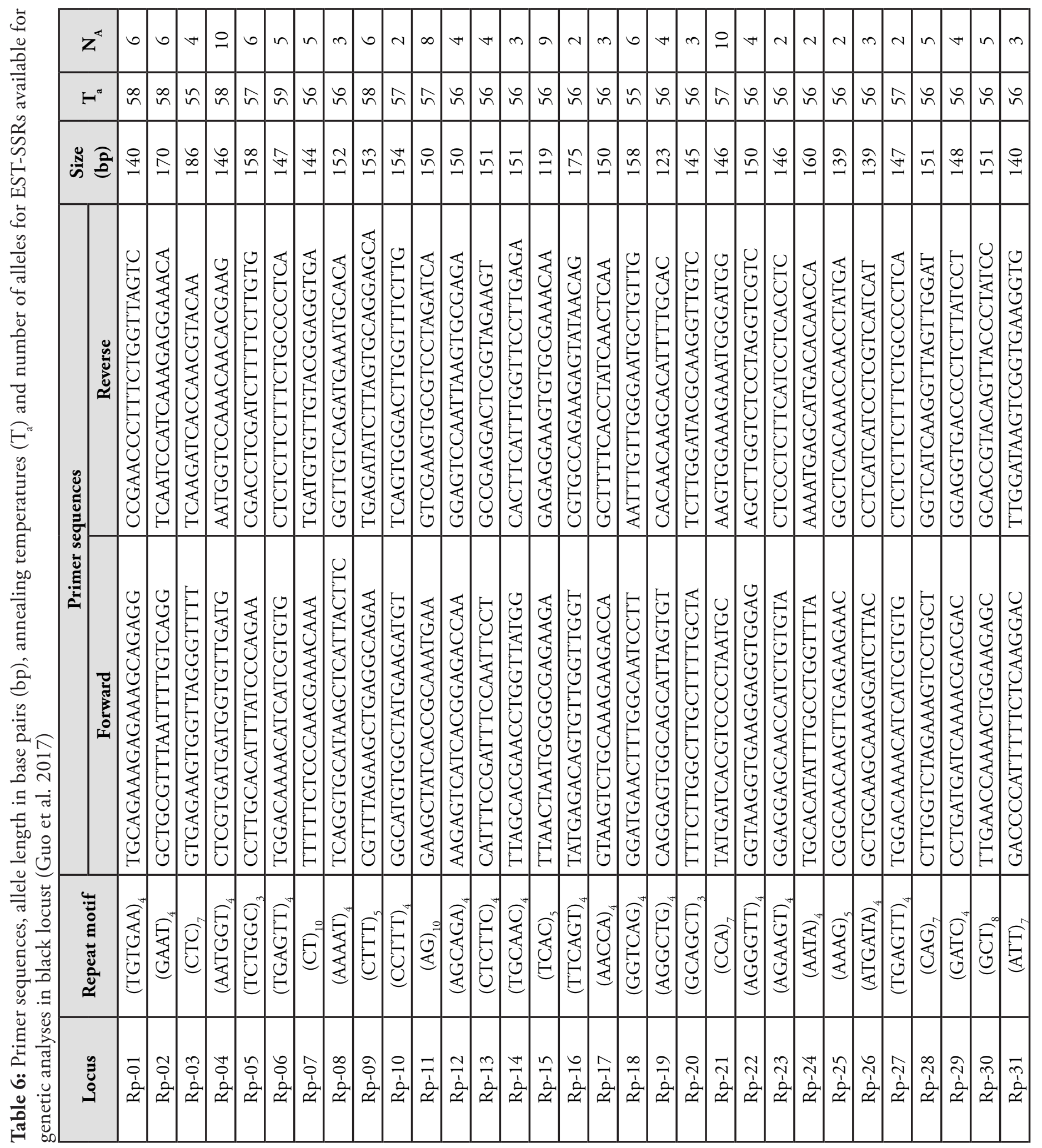




\begin{tabular}{|c|c|c|c|c|c|c|c|c|c|c|c|c|c|c|}
\hline$z^{4}$ & 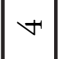 & 0 & $m$ & $m$ & 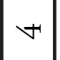 & 6 & $\stackrel{0}{\circ}$ & $\checkmark$ & $\sim$ & ${ }^{*}$ & 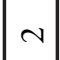 & 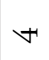 & $N$ & T \\
\hline$H^{\circ}$ & $\hat{n}$ & $\stackrel{2}{*}$ & $\hat{n}$ & $\stackrel{\infty}{\curvearrowleft}$ & $i n$ & $\stackrel{\circ}{\sim}$ & $\stackrel{2}{n}$ & $\stackrel{\imath}{n}$ & $\stackrel{\circ}{\curvearrowleft}$ & $\stackrel{\ddots}{\curvearrowleft}$ & $i$ & in & $\hat{n}$ & 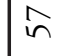 \\
\hline$\hat{\partial}$ & $\begin{array}{l}\tilde{0} \\
- \\
-1\end{array}$ & $F$ & 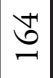 & $\simeq$ & 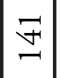 & $\tilde{n}$ & $\cong$ & $\cong$ & $\underline{m}$ & \pm & (2) & $\stackrel{n}{\approx}$ & $\stackrel{\sim}{ \pm}$ & $\cong$ \\
\hline 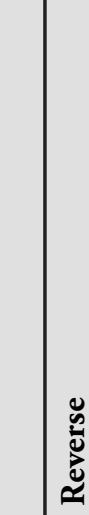 & 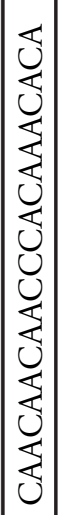 & 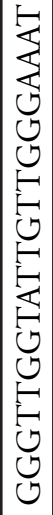 & 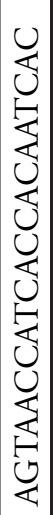 & 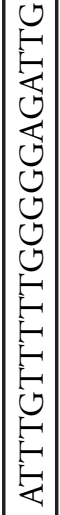 & 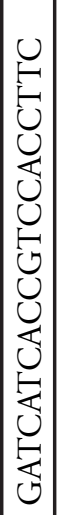 & 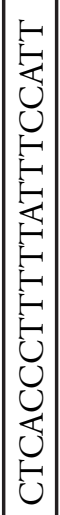 & 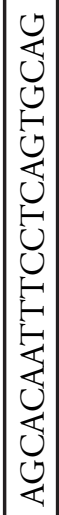 & 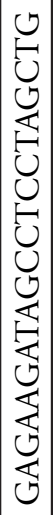 & 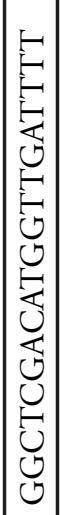 & 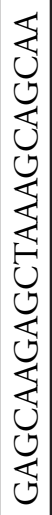 & 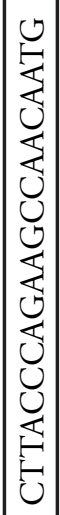 & 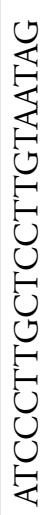 & 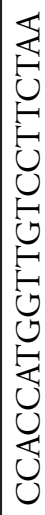 & 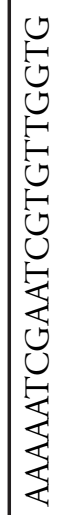 \\
\hline 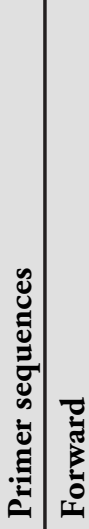 & 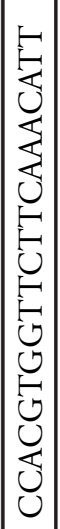 & 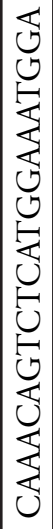 & 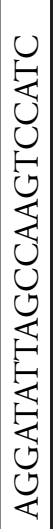 & 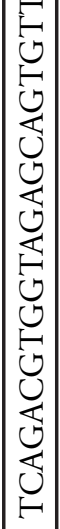 & 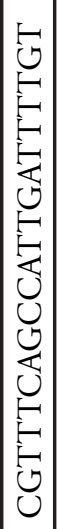 & 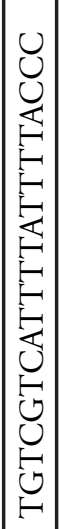 & 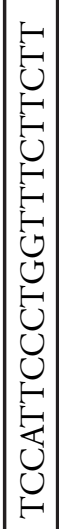 & 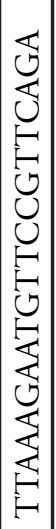 & 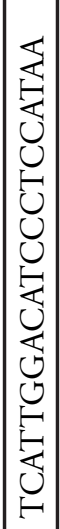 & 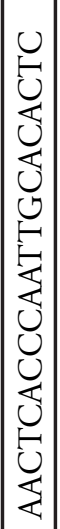 & 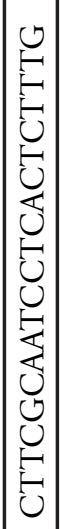 & 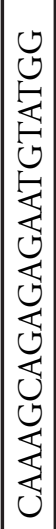 & 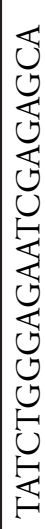 & 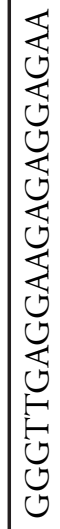 \\
\hline 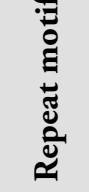 & $\left|\begin{array}{|c}0 \\
0 \\
0 \\
0\end{array}\right|$ & 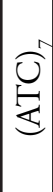 & $\begin{array}{l}0 \\
0 \\
0\end{array}$ & 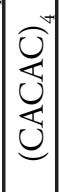 & 安 & 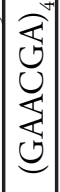 & $\begin{array}{l}0 \\
E\end{array}$ & 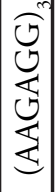 & 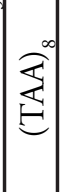 & 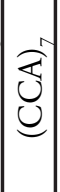 & $\begin{array}{l}0 \\
0 \\
\\
\end{array}$ & 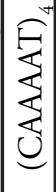 & 胥 & 5 \\
\hline 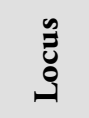 & $\mid \begin{array}{l}\hat{1} \\
\grave{\alpha} \\
\mid\end{array}$ & & & $\mid \begin{array}{l}\hat{\imath} \\
\hat{\imath} \\
\end{array}$ & & $\left|\begin{array}{l}n \\
\hat{1} \\
\hat{\alpha}\end{array}\right|$ & 4 & 4 & 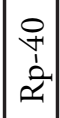 & $\mid \begin{array}{l}1 \\
\text { za }\end{array}$ & 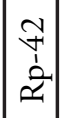 & ح. & 亲 & 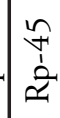 \\
\hline
\end{tabular}

Table 7: Primer sequences, allele length in base pairs (bp), annealing temperatures $(T)$ and number of alleles for ISSRs available for genetic analyses in black locust (Guo et al. 2017)

\begin{tabular}{|c|c|c|c|c|}
\hline Locus & Sequence $\left(5^{\prime}-3^{\prime}\right)$ & Size (bp) & $\mathrm{T}_{a}\left({ }^{\circ} \mathrm{C}\right)$ & $\mathbf{N}_{\mathrm{A}}$ \\
\hline W1 & $(\mathrm{AG})_{8} \mathrm{G}$ & $200-1000$ & 58 & 8 \\
\hline W3 & $(\mathrm{AC})_{8} \mathrm{C}$ & $100-1100$ & 58 & 7 \\
\hline W6 & $(\mathrm{GA})_{8} \mathrm{YC}$ & $100-1300$ & 58 & 8 \\
\hline W7 & GGG(TGGGG) ${ }_{2} \mathrm{TG}$ & $300-2500$ & 55 & 7 \\
\hline W8 & $(\mathrm{CT})_{8} \mathrm{G}$ & $300-1500$ & 55 & 6 \\
\hline W9 & $(\mathrm{CA})_{8} \mathrm{G}$ & $200-600$ & 55 & 7 \\
\hline W11 & $(\mathrm{TC})_{8} \mathrm{~A}$ & $400-1500$ & 55 & 8 \\
\hline $\mathrm{W} 12$ & $(\mathrm{TC})_{8} \mathrm{C}$ & $300-1800$ & 55 & 9 \\
\hline W14 & $(\mathrm{AC})_{8} \mathrm{~T}$ & $100-1200$ & 55 & 9 \\
\hline W18 & $(\mathrm{CA})_{8} \mathrm{RC}$ & $100-900$ & 55 & 7 \\
\hline W19 & $(\mathrm{CA})_{8} \mathrm{RG}$ & $100-1100$ & 55 & 9 \\
\hline W20 & $(\mathrm{GT})_{8} \mathrm{YA}$ & $400-1300$ & 55 & 5 \\
\hline W22 & $(\mathrm{TC})_{8} \mathrm{RT}$ & $800-1300$ & 52 & 3 \\
\hline W23 & $(\mathrm{AC})_{8} \mathrm{YT}$ & $400-1500$ & 58 & 5 \\
\hline W24 & $(\mathrm{AC})_{8} \mathrm{YA}$ & $100-1500$ & 58 & 8 \\
\hline W25 & $(\mathrm{AC})_{8} \mathrm{YG}$ & $300-1600$ & 58 & 8 \\
\hline W26 & $(\mathrm{TG})_{8} \mathrm{RC}$ & $300-900$ & 58 & 6 \\
\hline W27 & $(\mathrm{TG})_{8} \mathrm{RA}$ & $700-900$ & 55 & 2 \\
\hline W28 & $(\mathrm{ATG})_{6}$ & $400-1500$ & 55 & 6 \\
\hline W29 & $\left(\mathrm{GACA}_{4}\right.$ & $300-1200$ & 55 & 4 \\
\hline W30 & $(\text { GGAGA })_{3}$ & $400-2000$ & 58 & 11 \\
\hline 4 & $\mathrm{BDB}(\mathrm{CA})_{6}$ & $400-1200$ & 58 & 9 \\
\hline 15 & $\mathrm{CCC}(\mathrm{GT})_{6}$ & $300-900$ & 58 & 8 \\
\hline 20 & CCAG(TGG) ${ }_{3} \mathrm{TG}$ & $300-1800$ & 52 & 9 \\
\hline 22 & $\mathrm{SSWN}_{(\mathrm{GACA})_{3}}$ & $200-1200$ & 52 & 9 \\
\hline 31 & $(\mathrm{AG})_{8} \mathrm{~T}$ & $100-900$ & 55 & 6 \\
\hline 32 & $(\mathrm{AG})_{8} \mathrm{C}$ & $200-1400$ & 58 & 6 \\
\hline 33 & $(\mathrm{GA})_{8} \mathrm{~T}$ & $100-1000$ & 55 & 9 \\
\hline 34 & $(\mathrm{GA})_{8} \mathrm{C}$ & $100-800$ & 52 & 7 \\
\hline 36 & $(\mathrm{AG})_{8} \mathrm{YT}$ & $100-1400$ & 52 & 8 \\
\hline 37 & $(\mathrm{AG})_{8} \mathrm{YC}$ & $200-1300$ & 52 & 6 \\
\hline 39 & $(\mathrm{CTTCA})_{3}$ & $200-1400$ & 52 & 6 \\
\hline
\end{tabular}




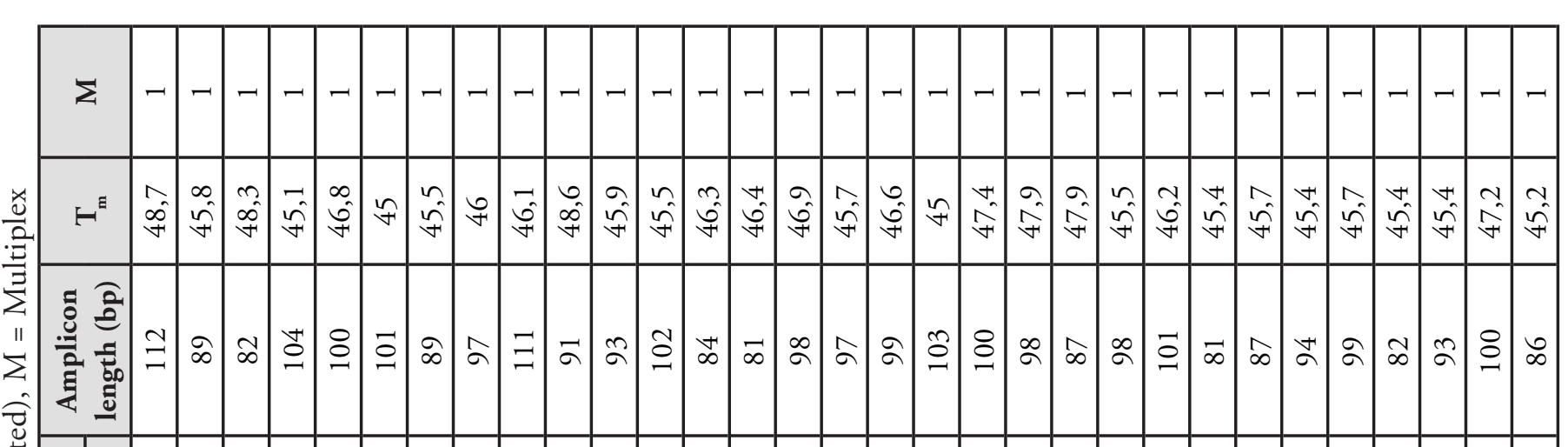

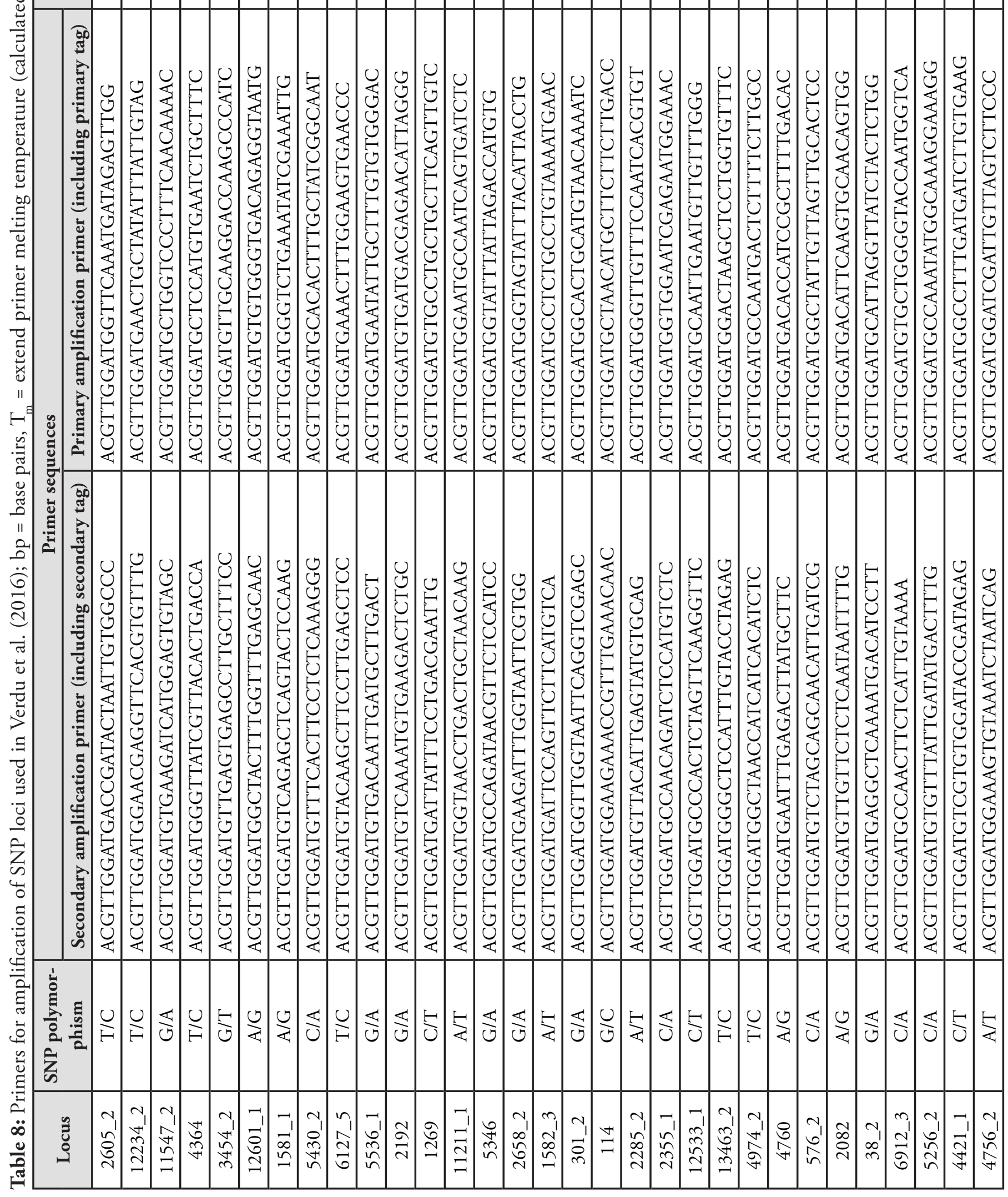


$\Sigma$

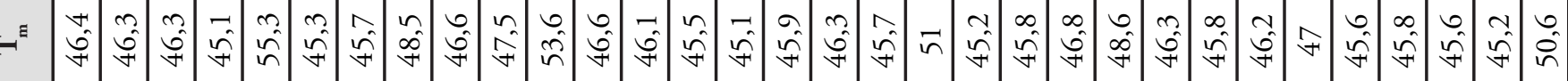

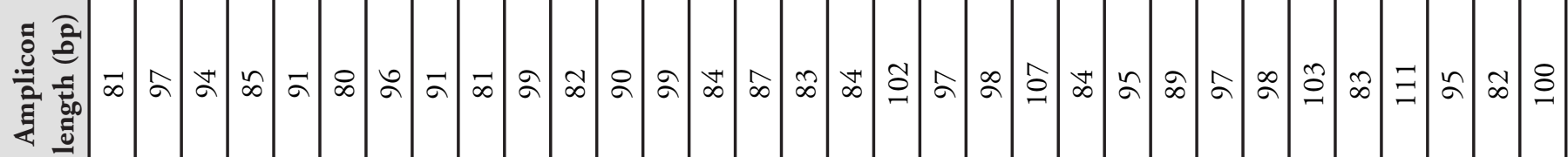

๘ొ

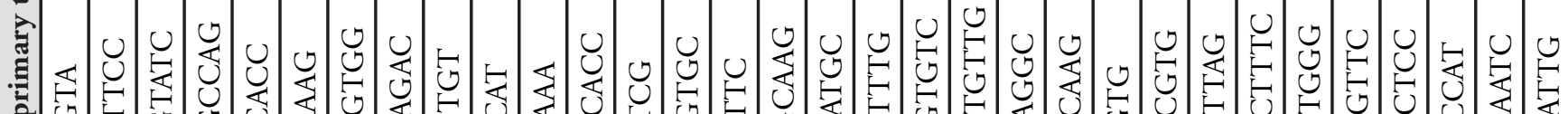

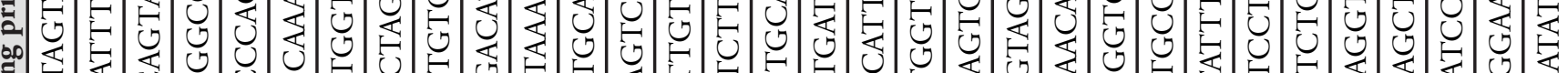

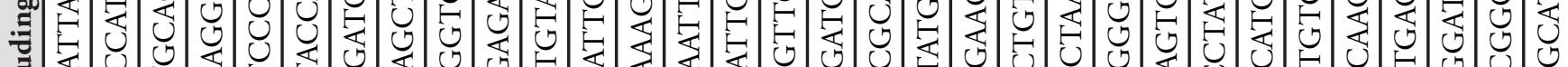

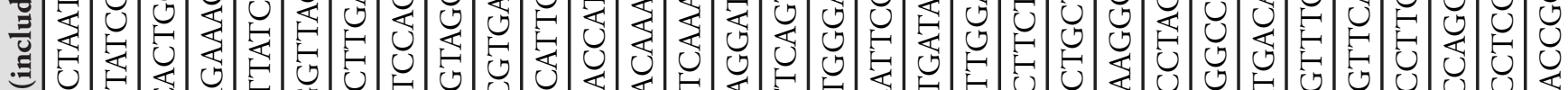

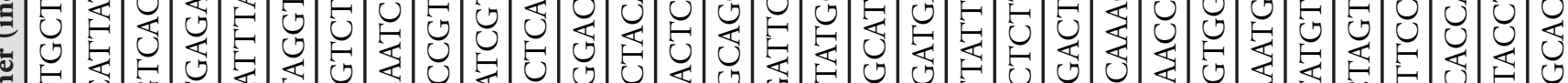
目 豆

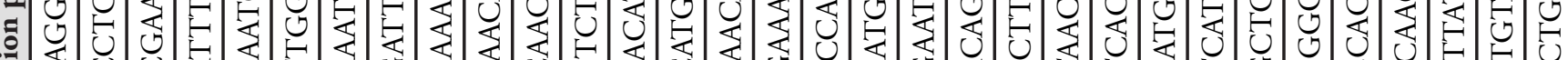

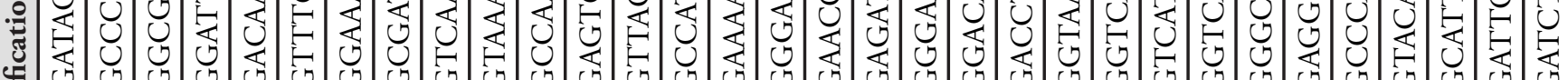

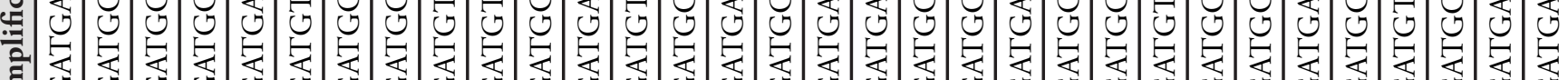

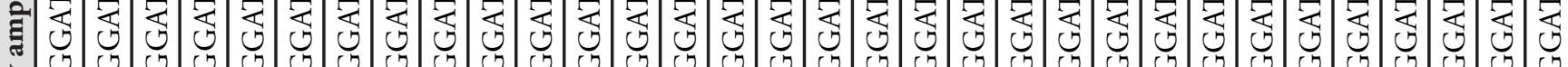
党 \&

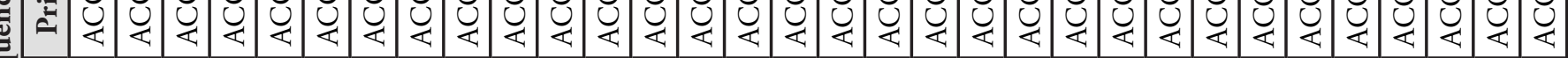

竞

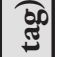

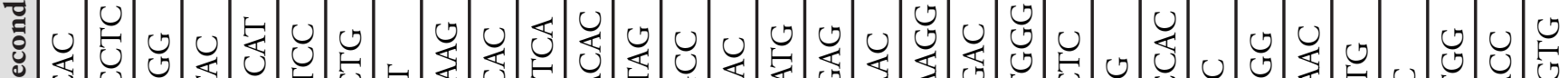
م

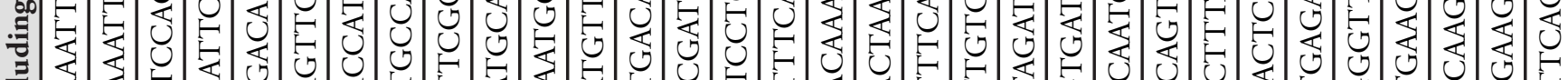

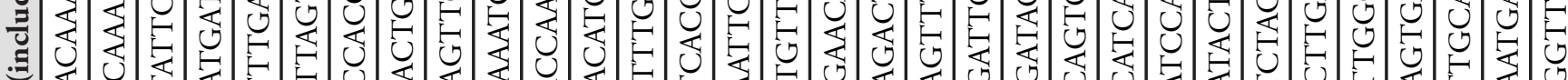

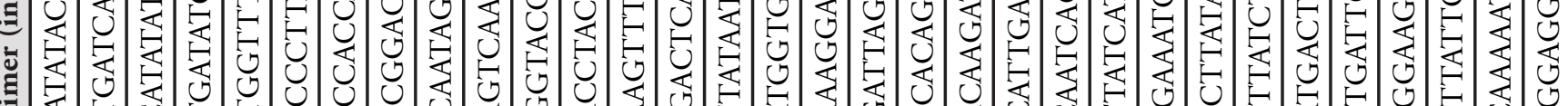

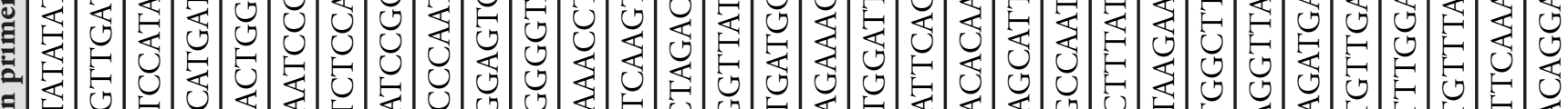

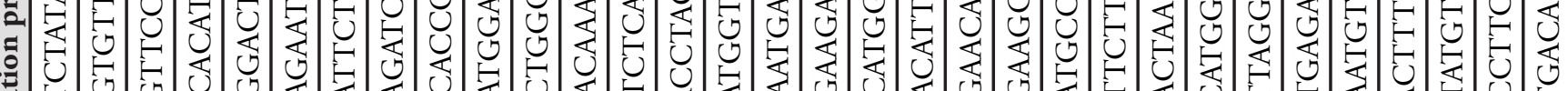
至 :

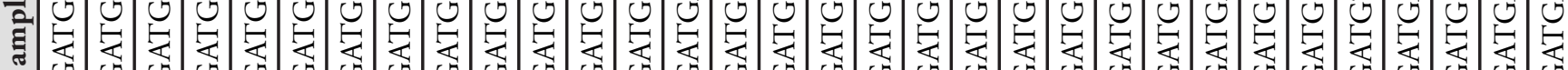
ف

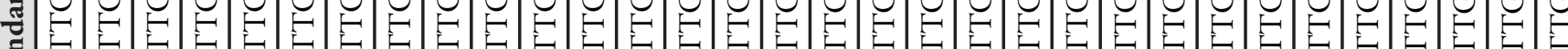
:

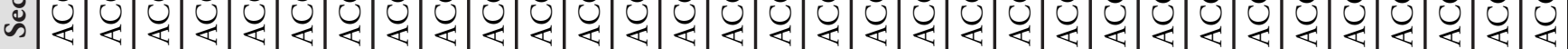

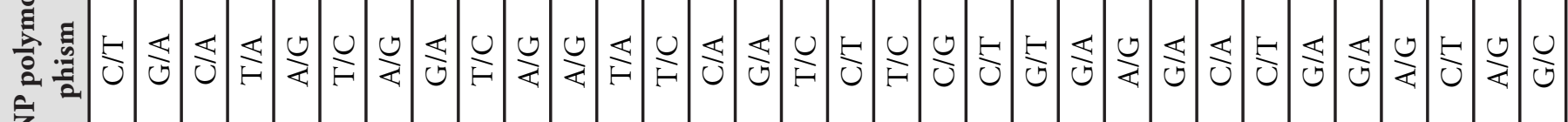

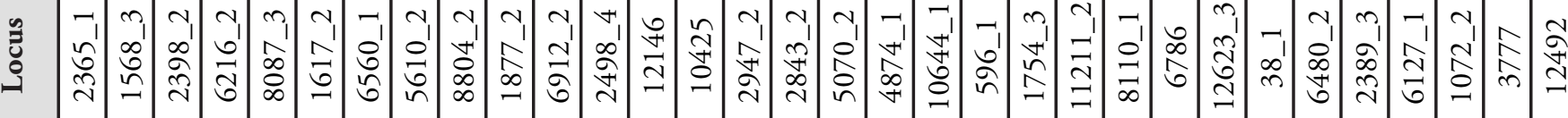




\section{$\Sigma$}

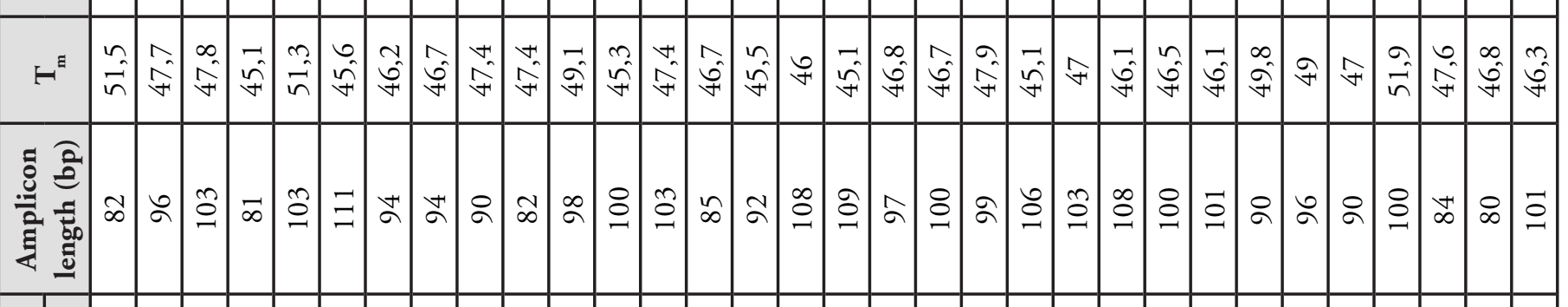

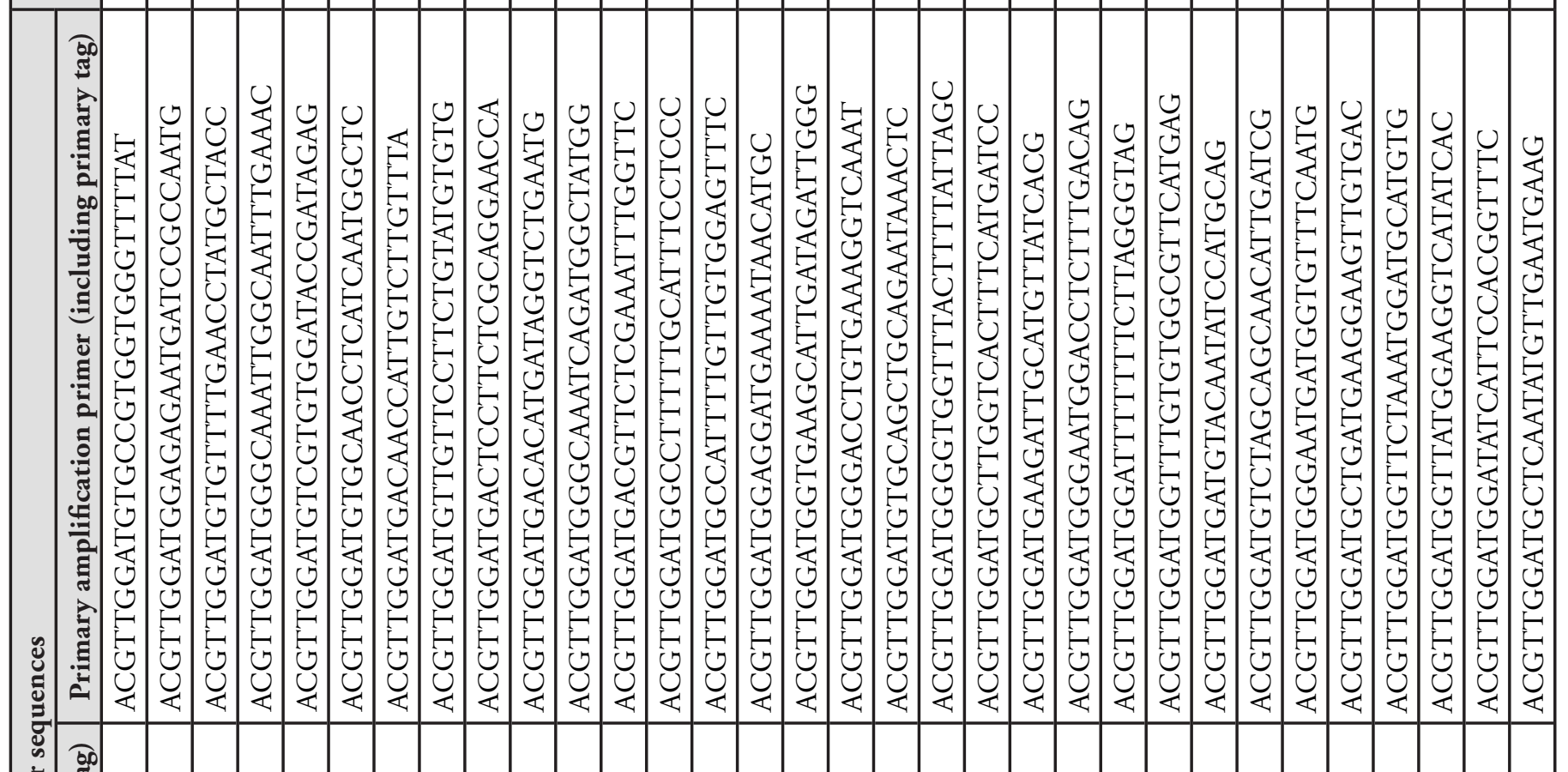

尝

(2)

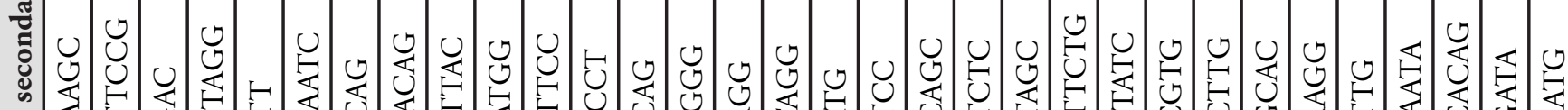

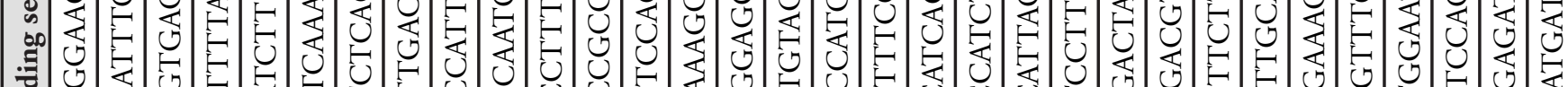

至

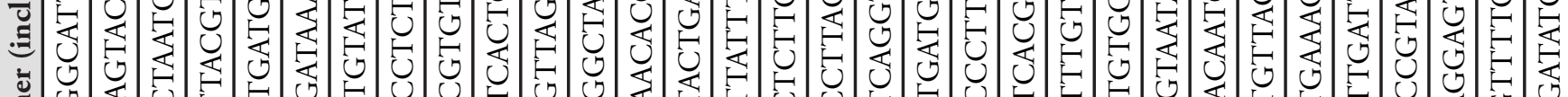

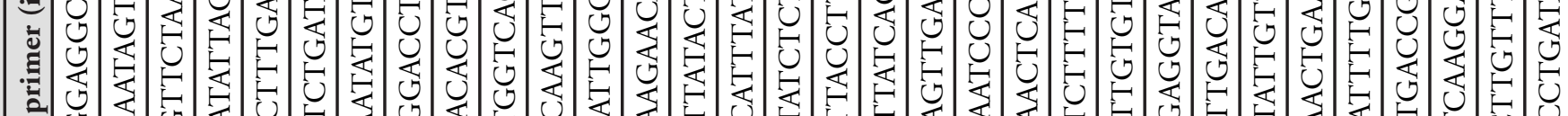

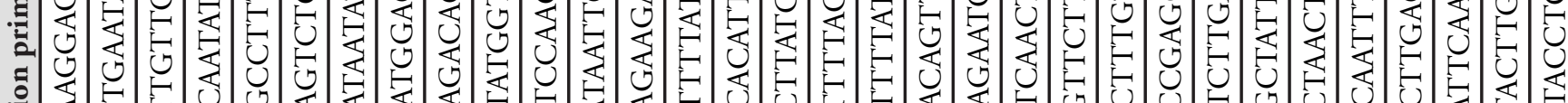

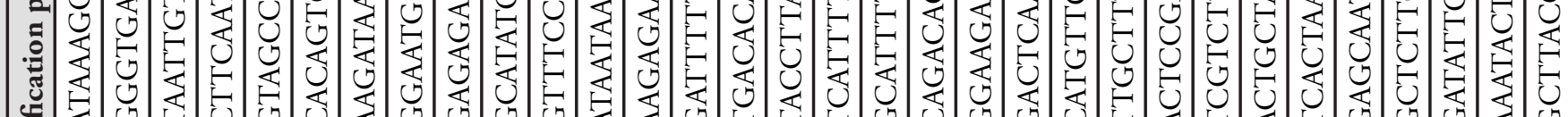

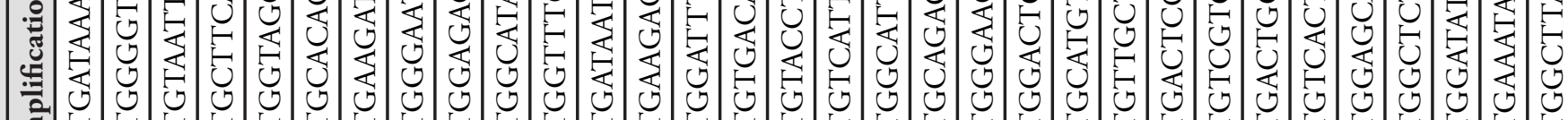

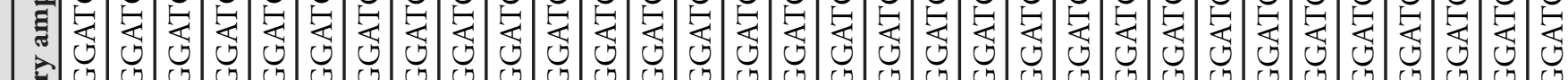

:

苔

œ

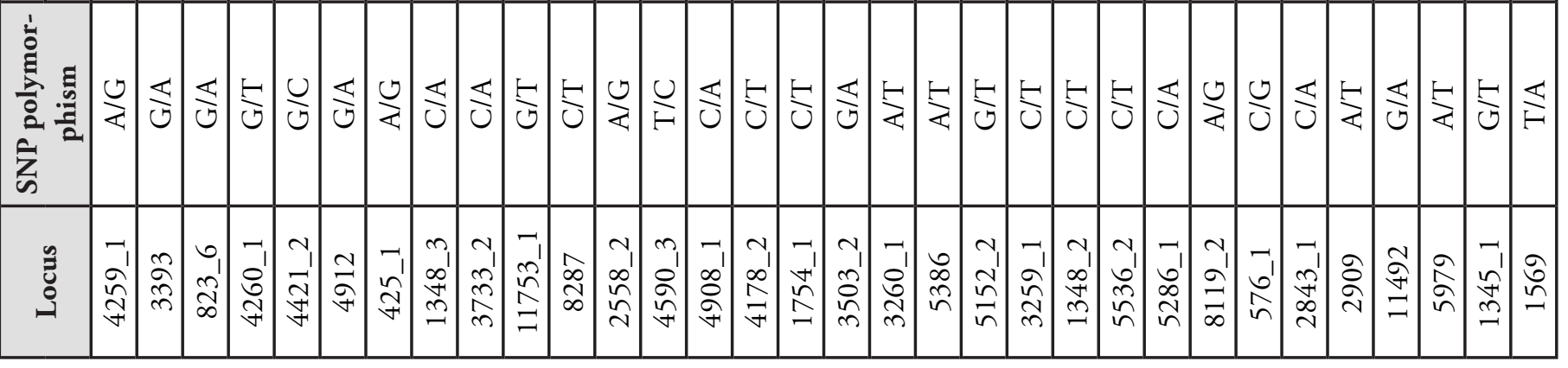


$\Sigma$

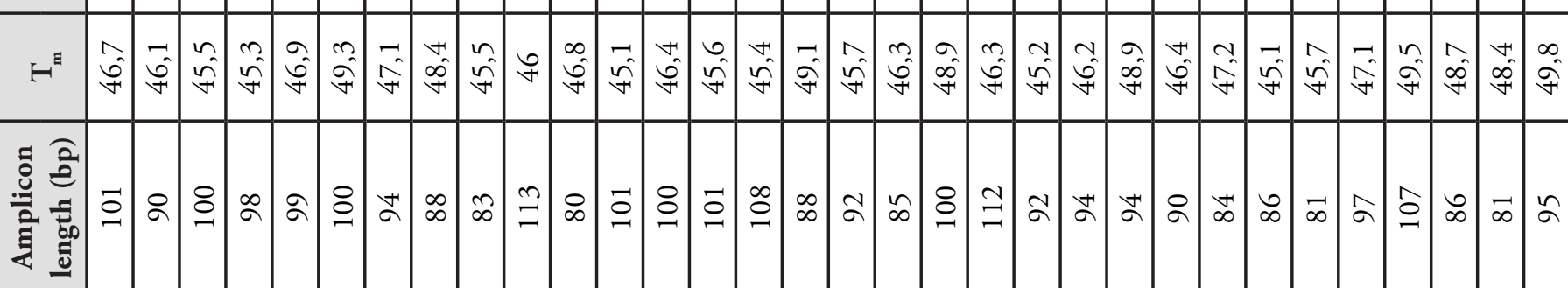

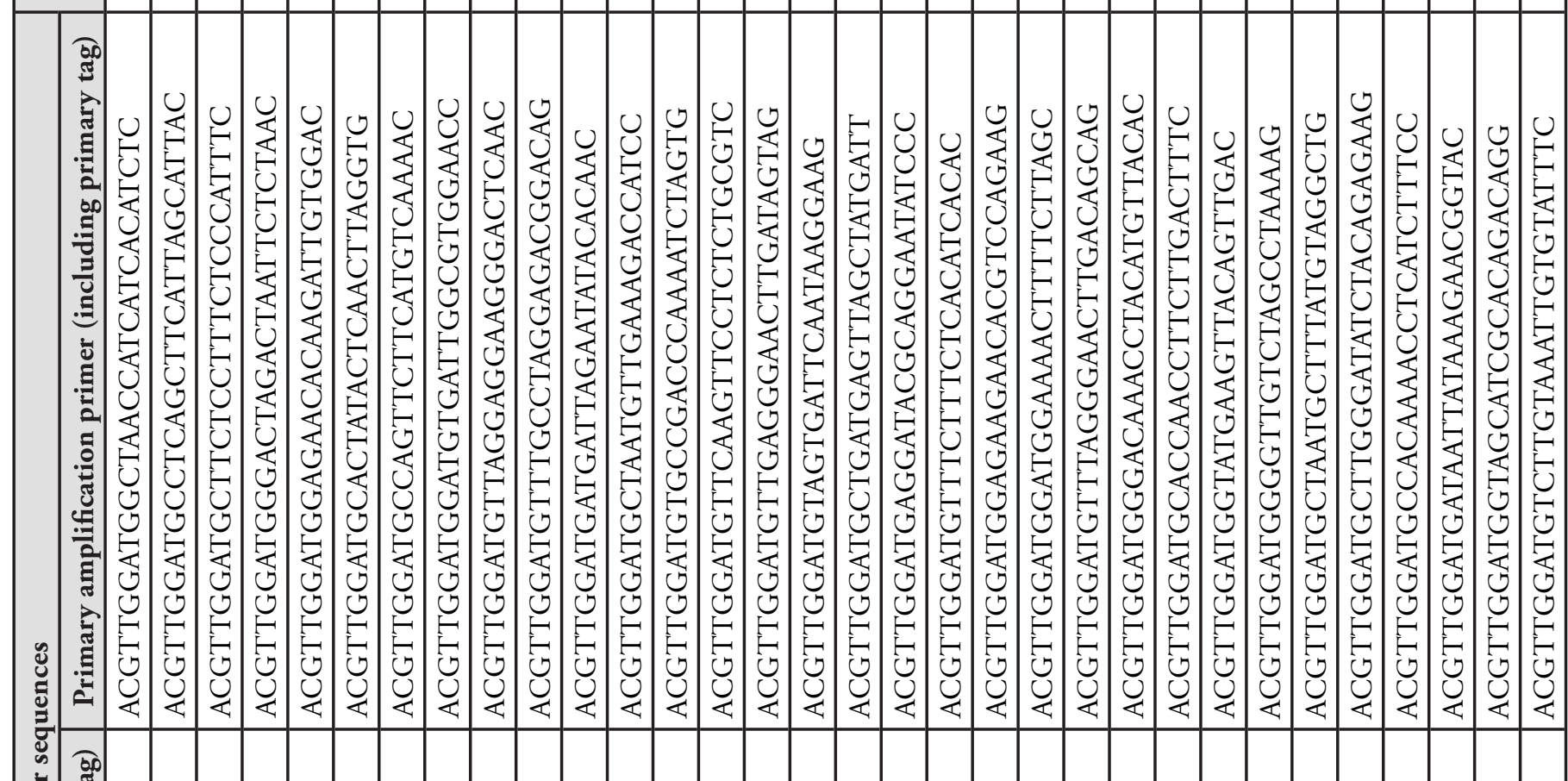
总

苟

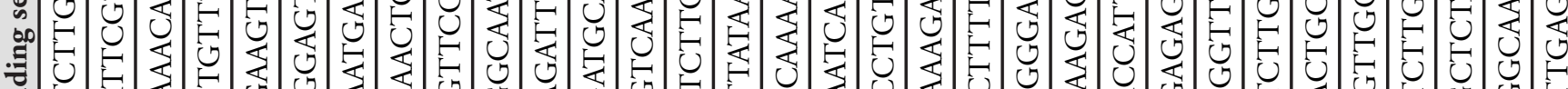
当卢

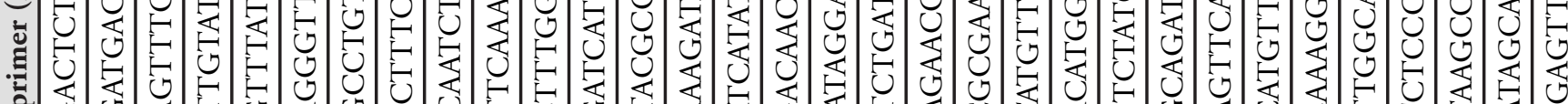
ప

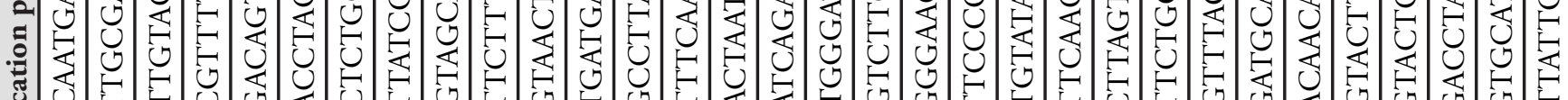
苞

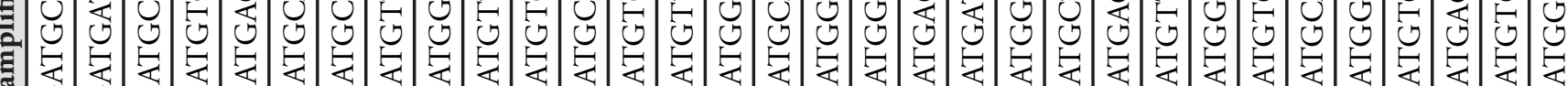

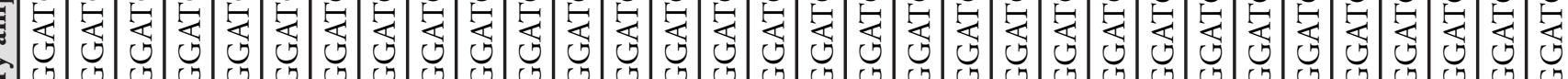

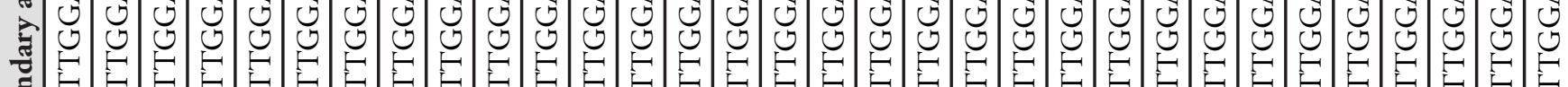
こே ウ

$\stackrel{1}{\circ}$

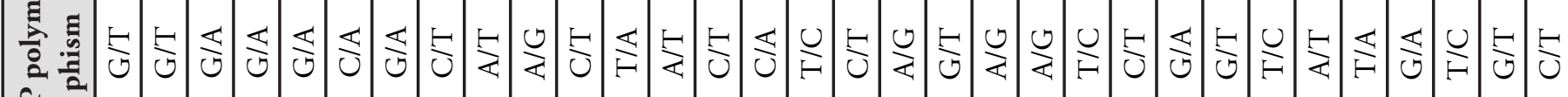
言

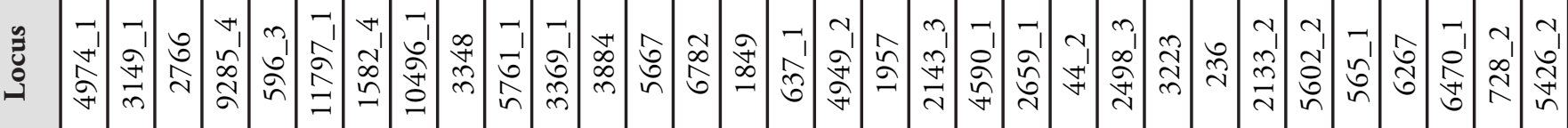


$\Sigma$

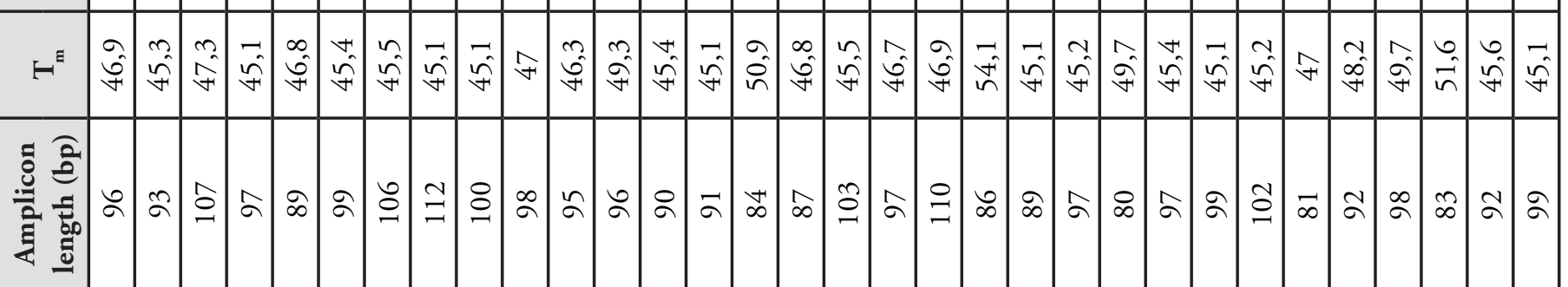

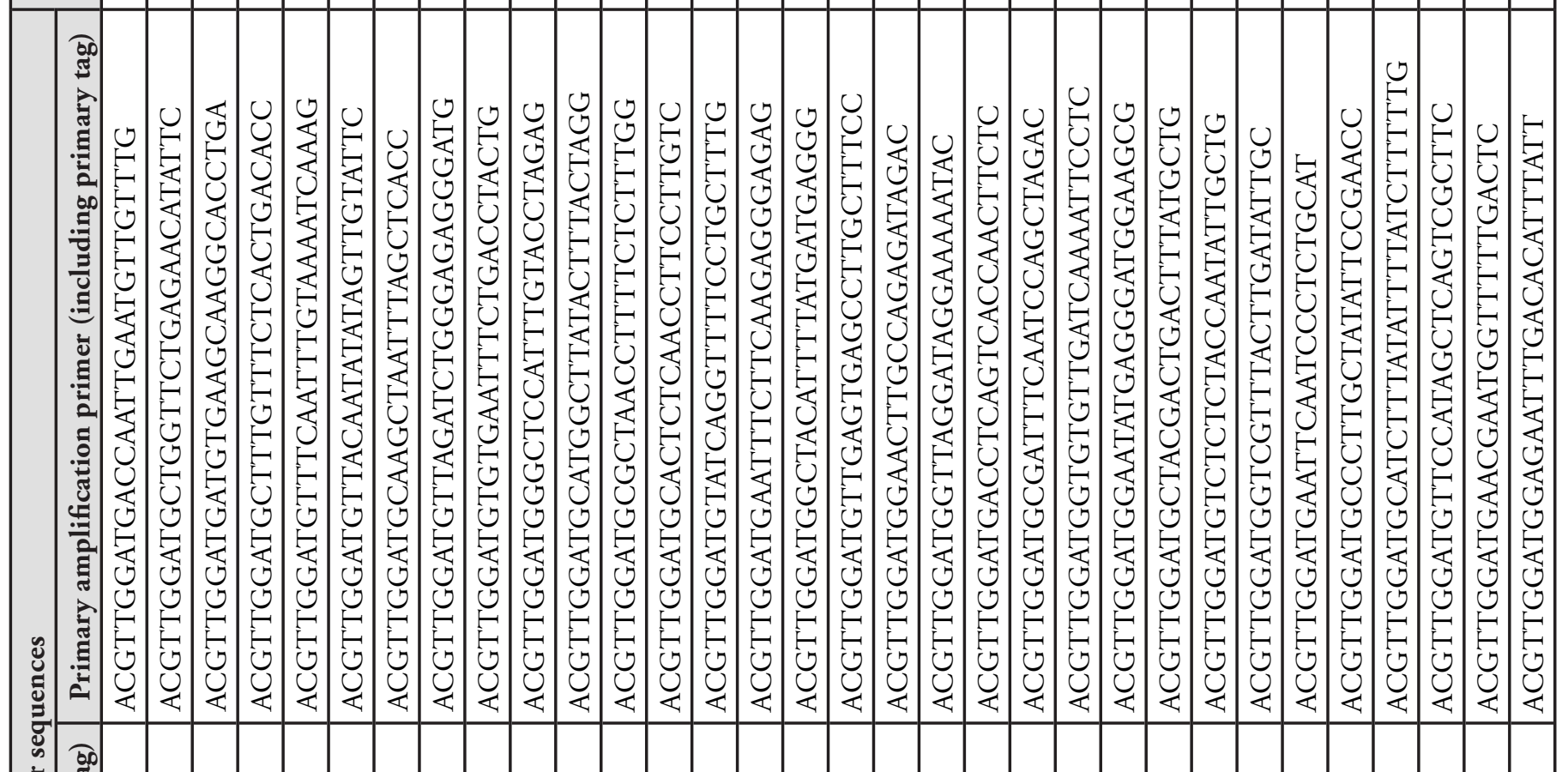
离

ป

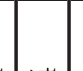

$\checkmark$

$\checkmark$

(

.

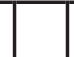

传

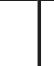

$\longrightarrow$

$\longrightarrow$

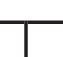
$\ln \mid \frac{}{n}$ 
$\Sigma$

$\sum$

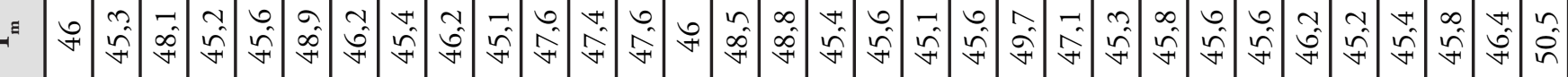

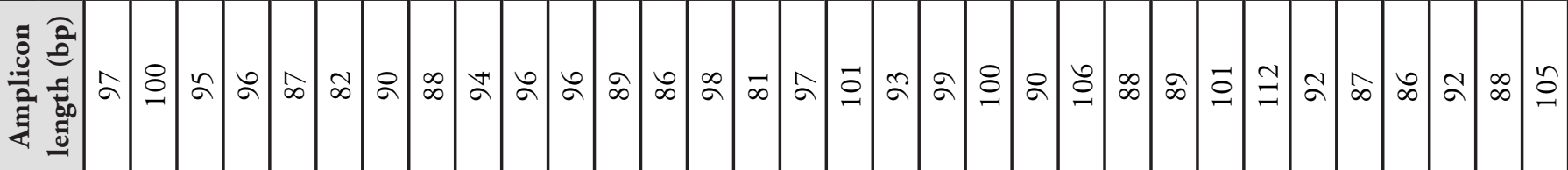

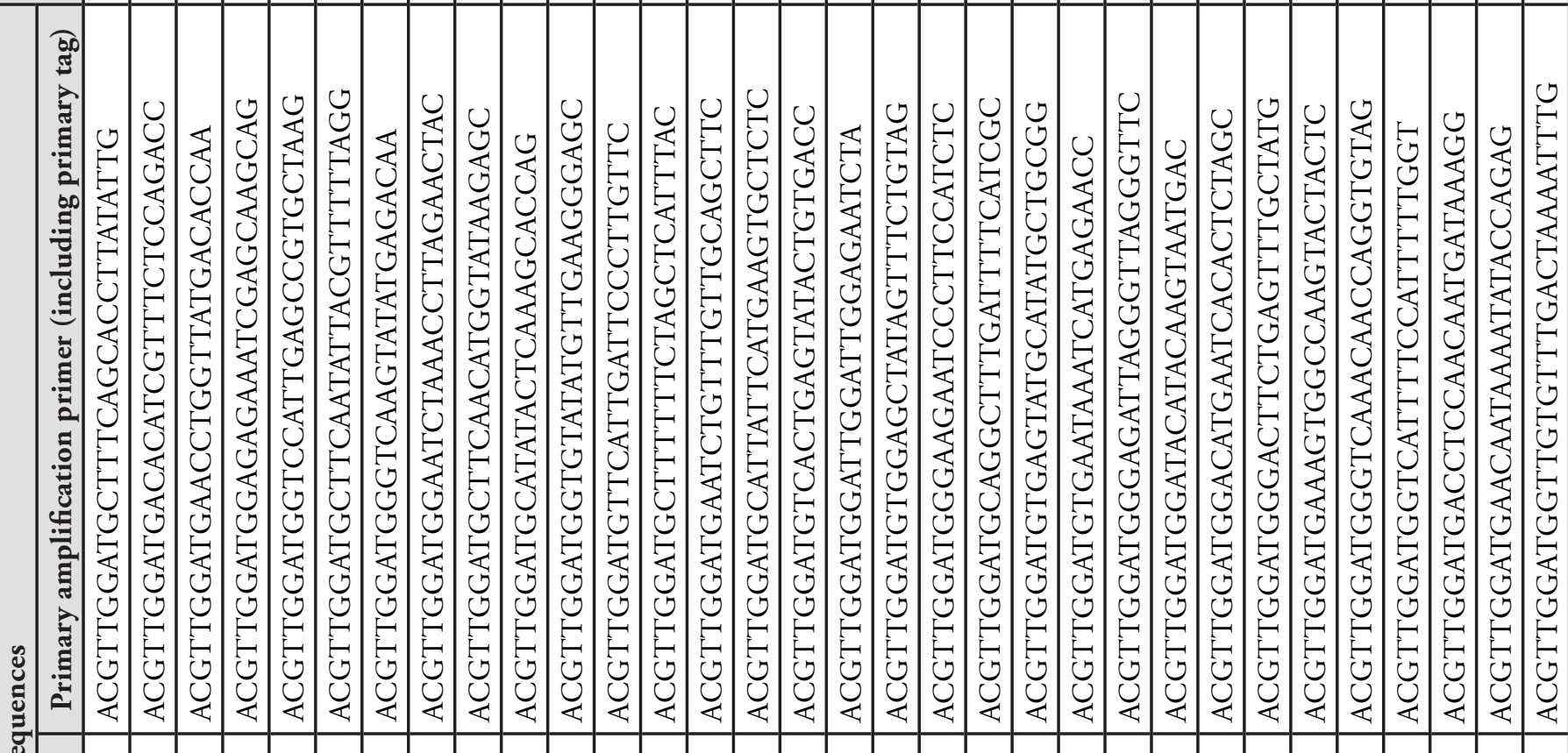
至

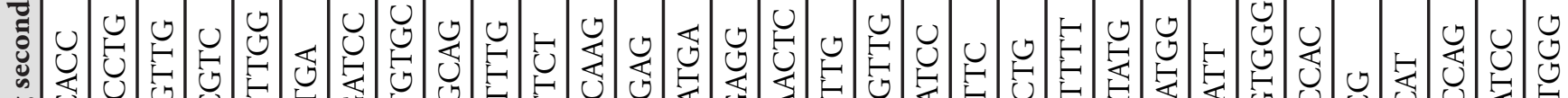
م

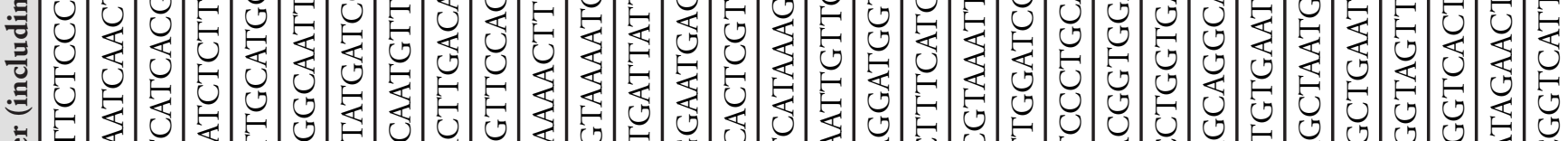

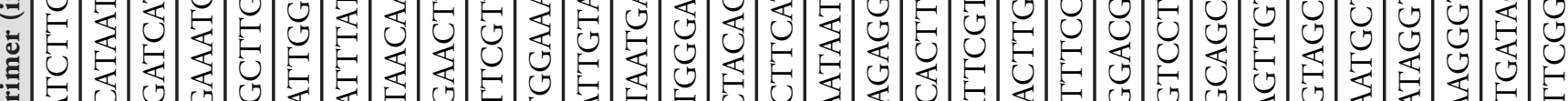

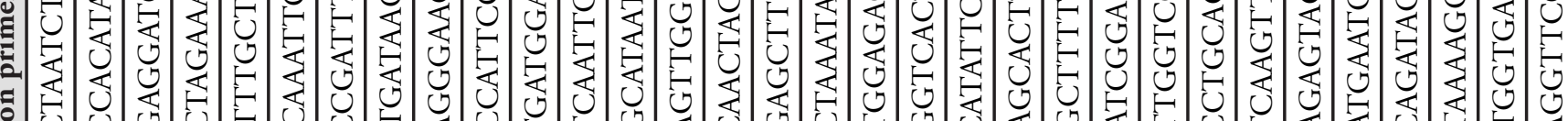
.

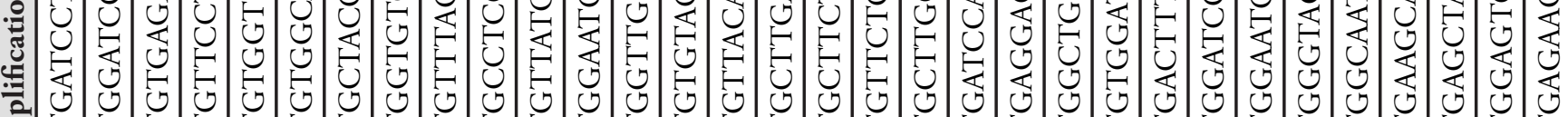
帝安 ف 焉 : ம

突

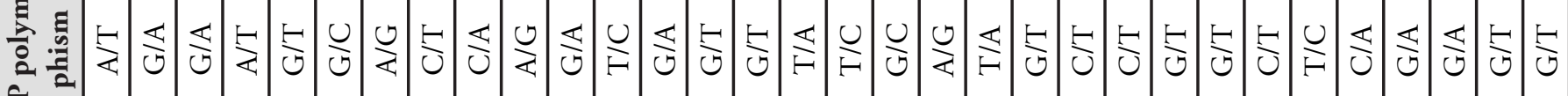
空

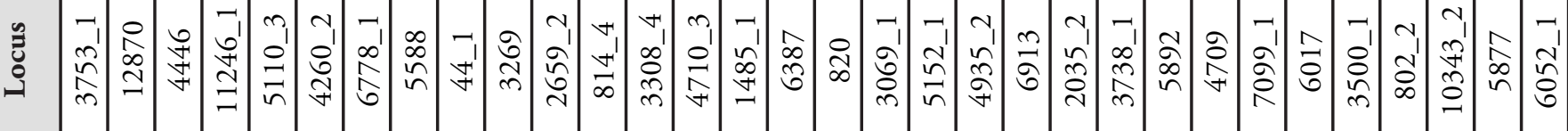




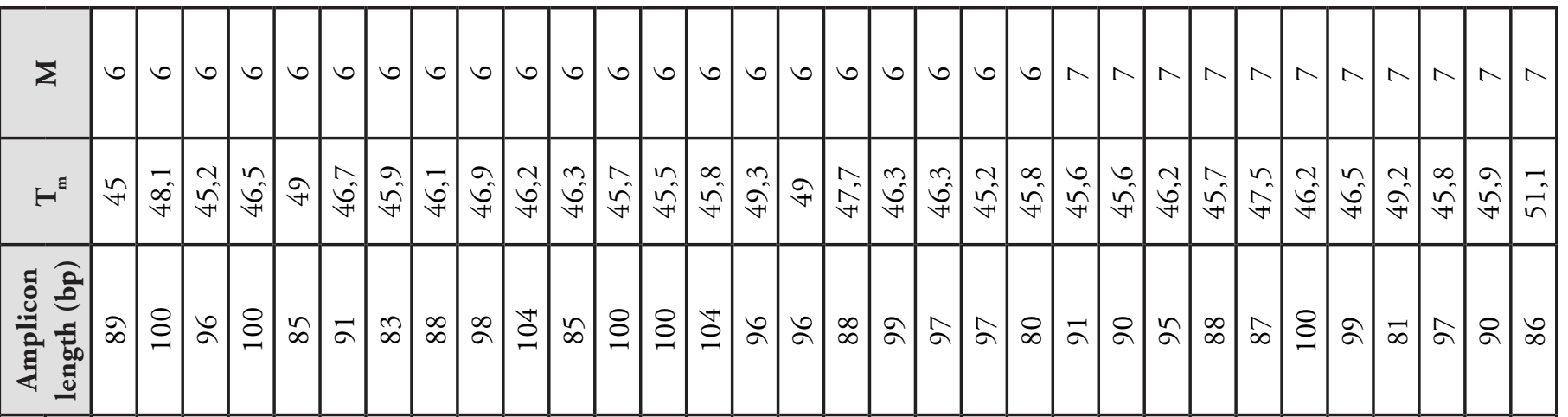

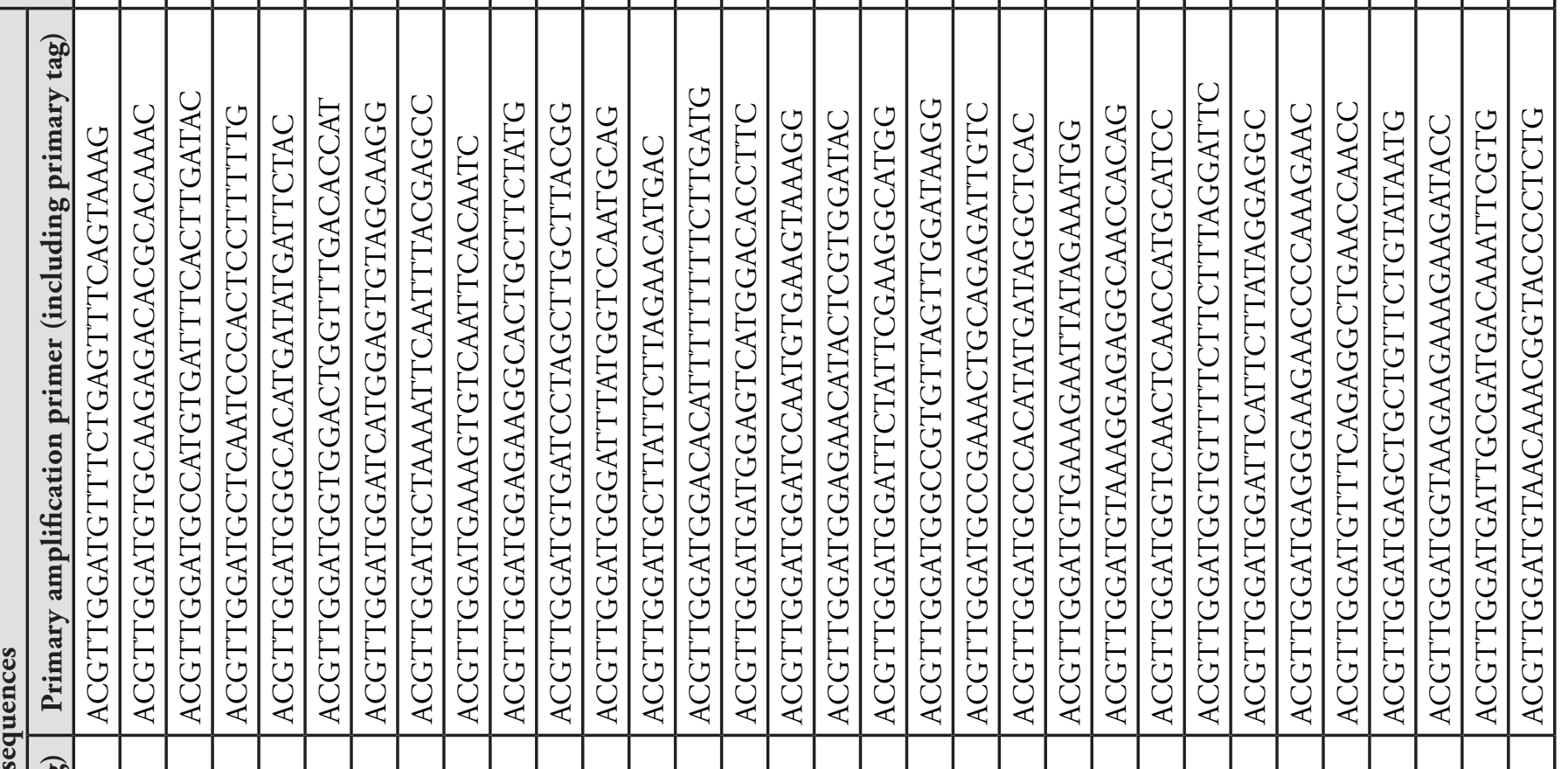
:

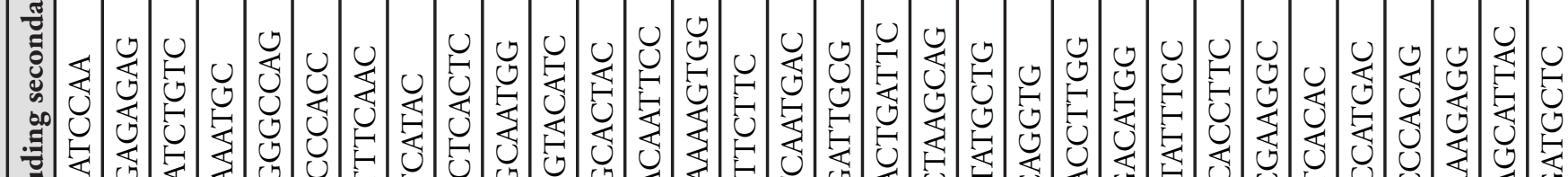

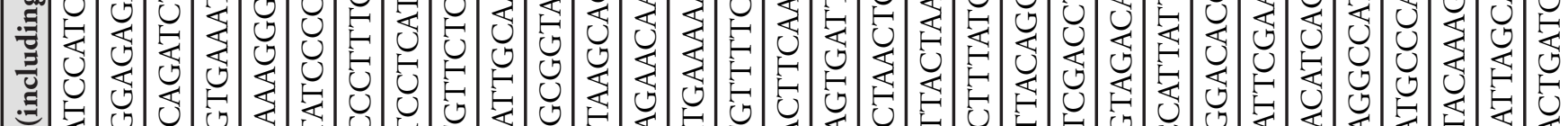

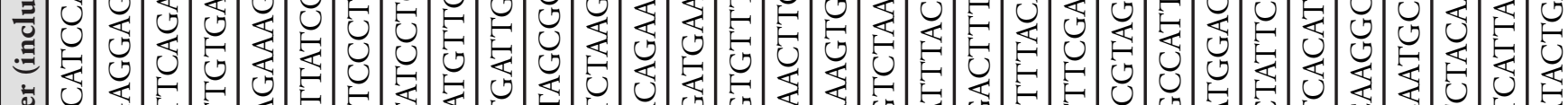

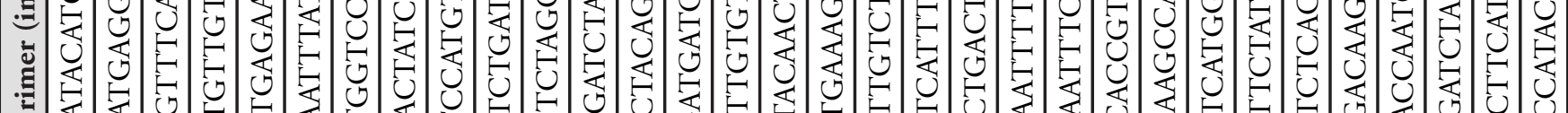

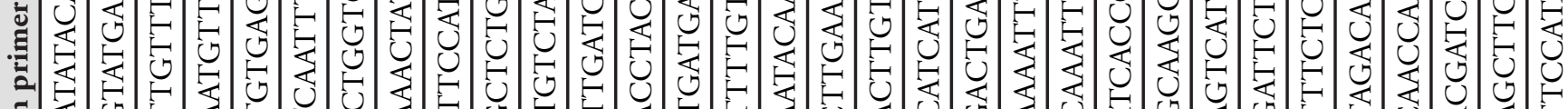

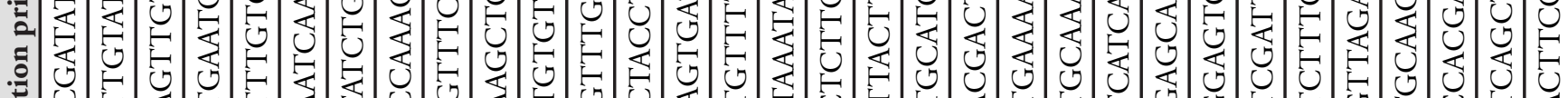

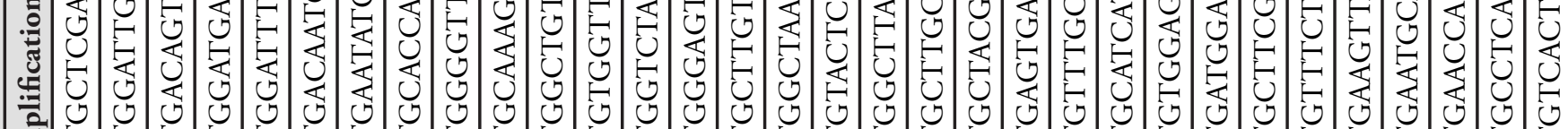

音

包

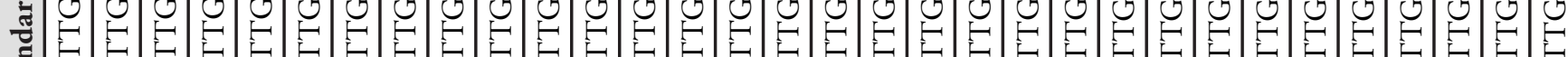

总

ڤ

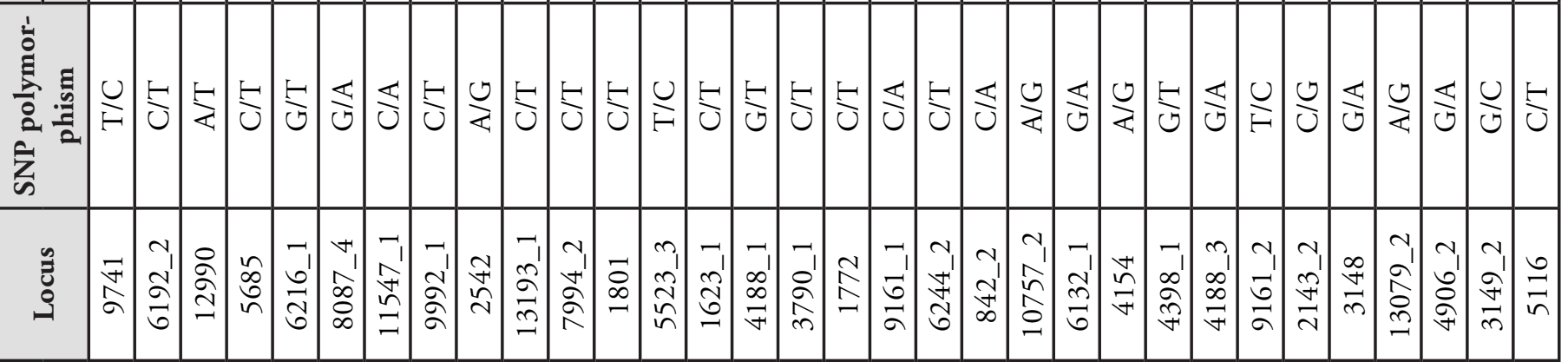




\section{$\Sigma$}

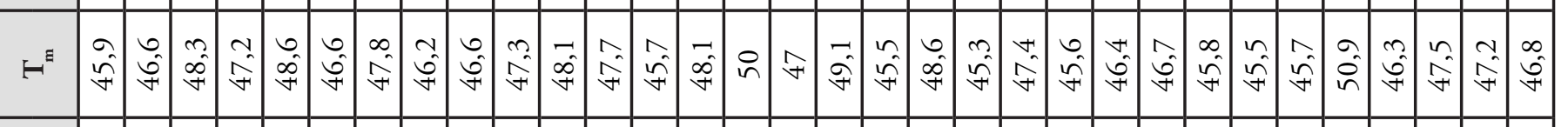

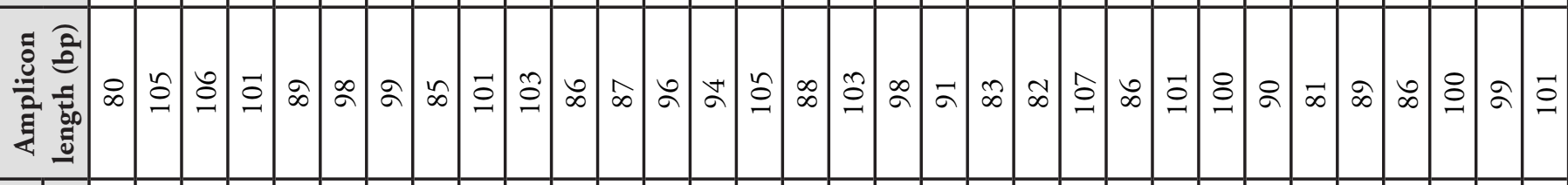

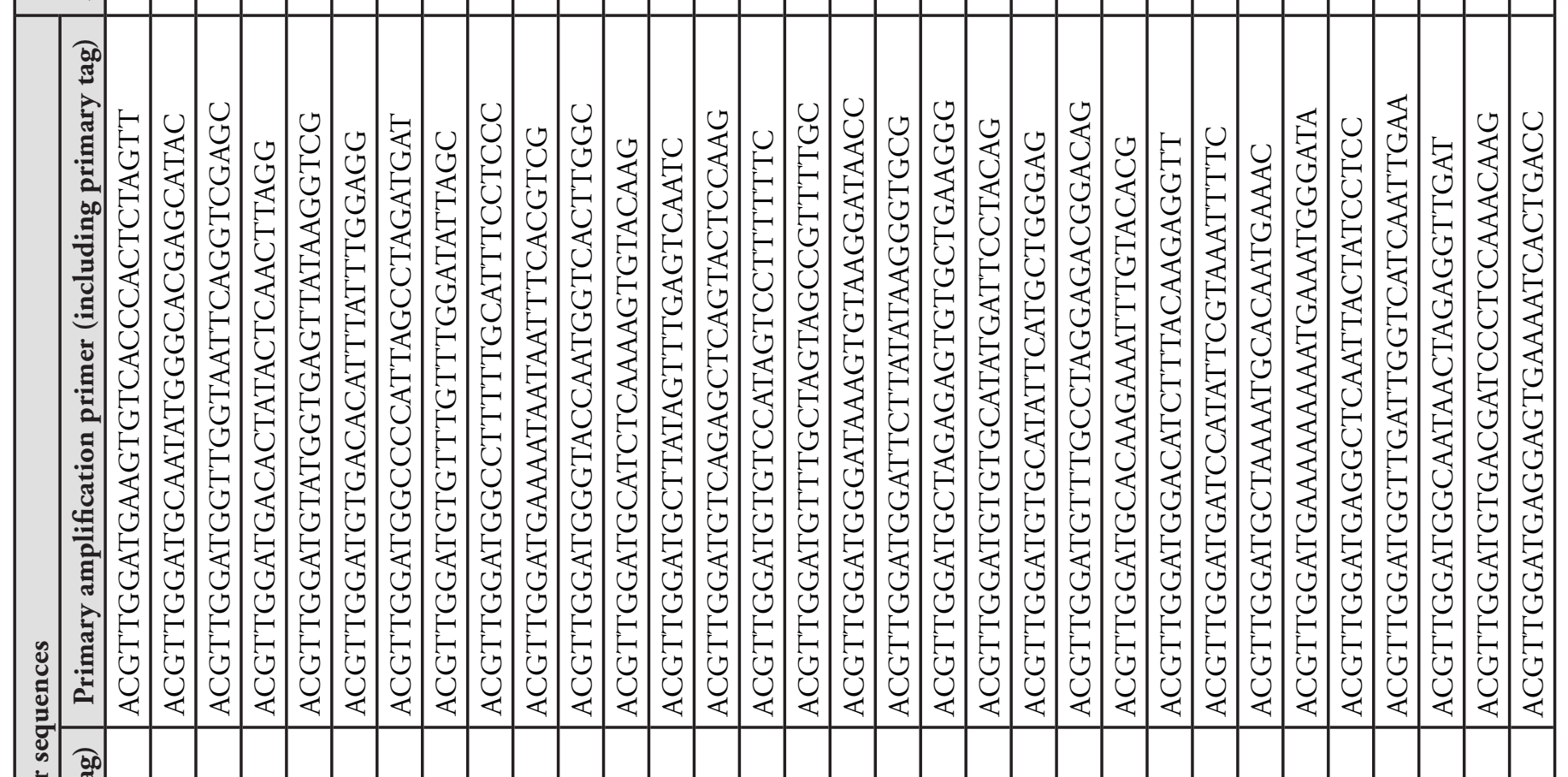
焉

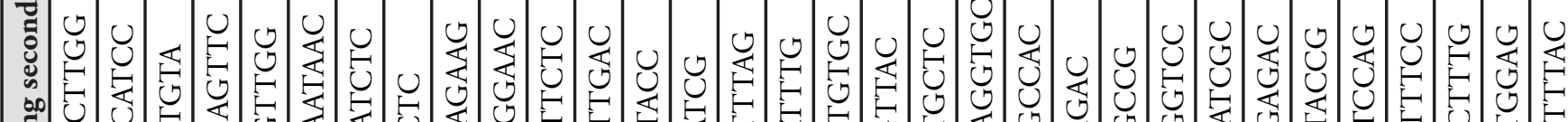

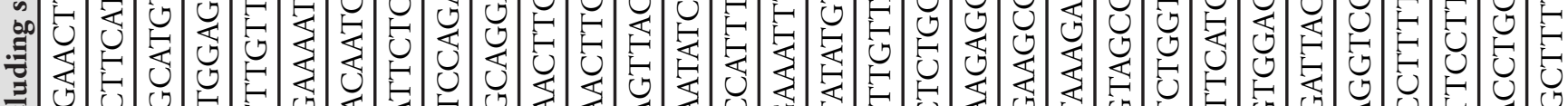
可至

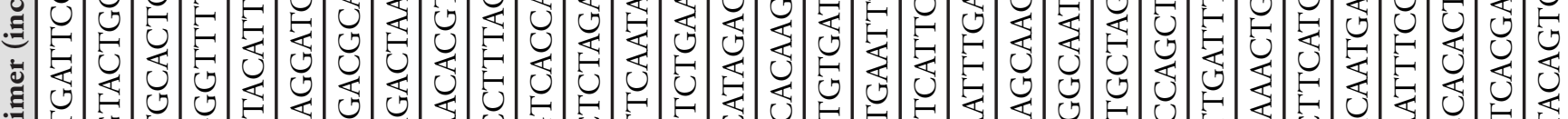
司

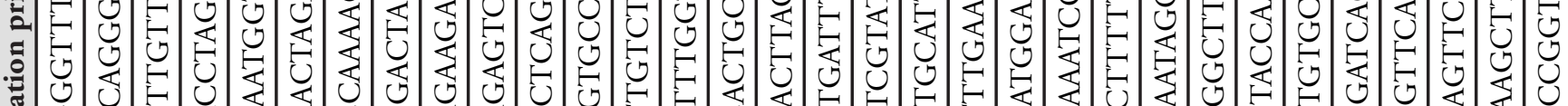

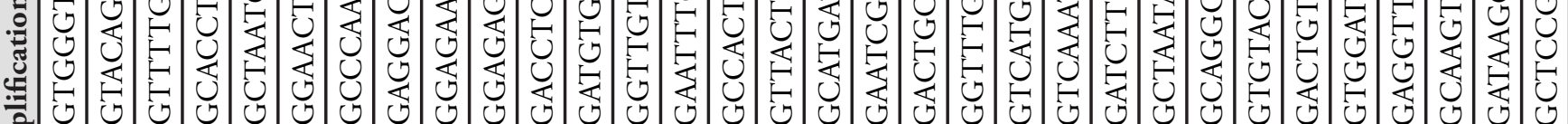

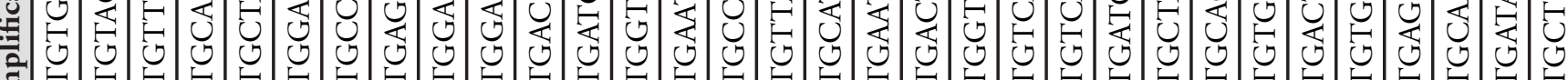

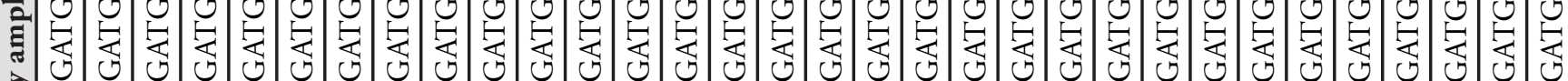

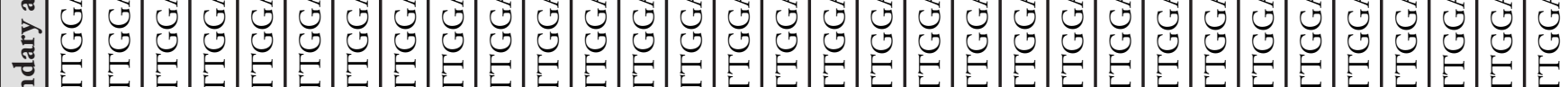
至

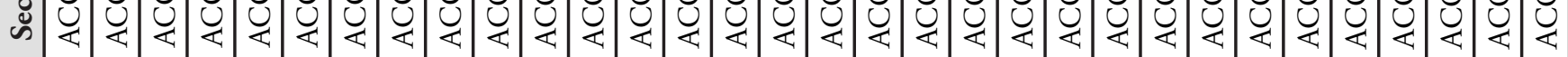

范

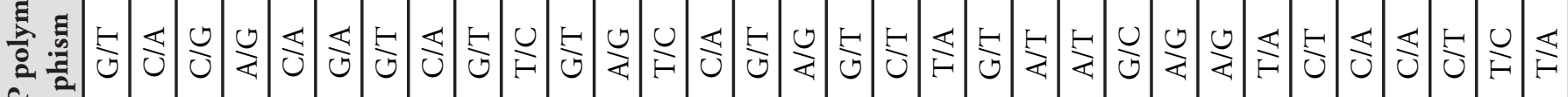
灾

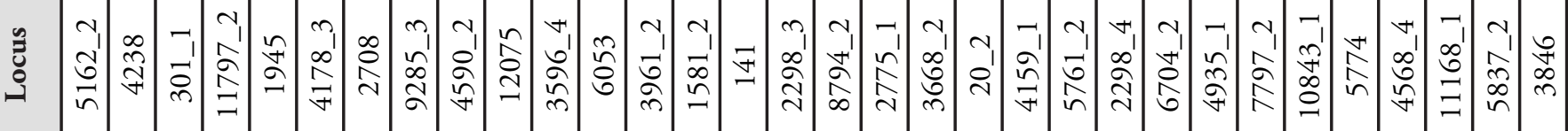




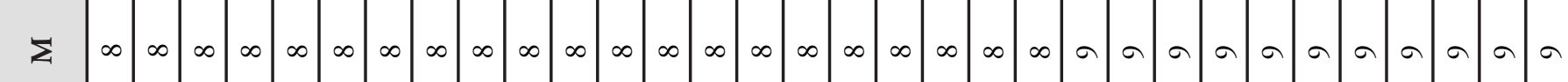

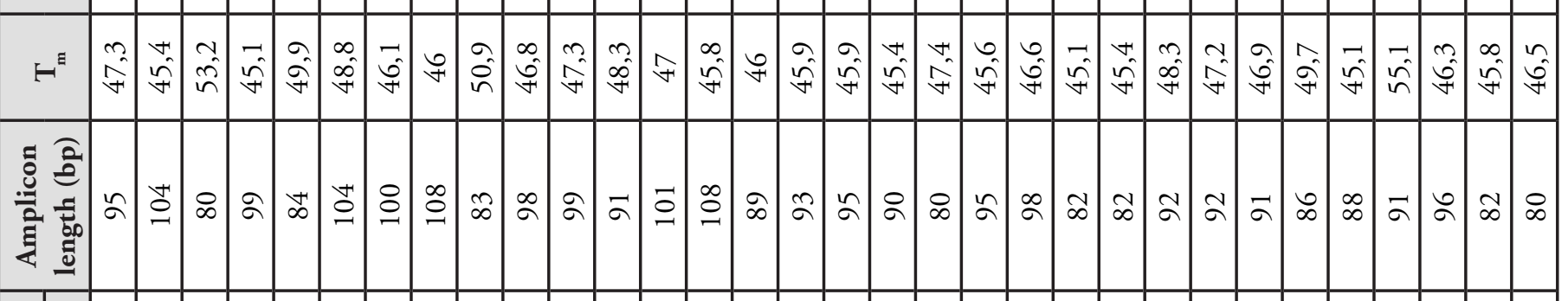

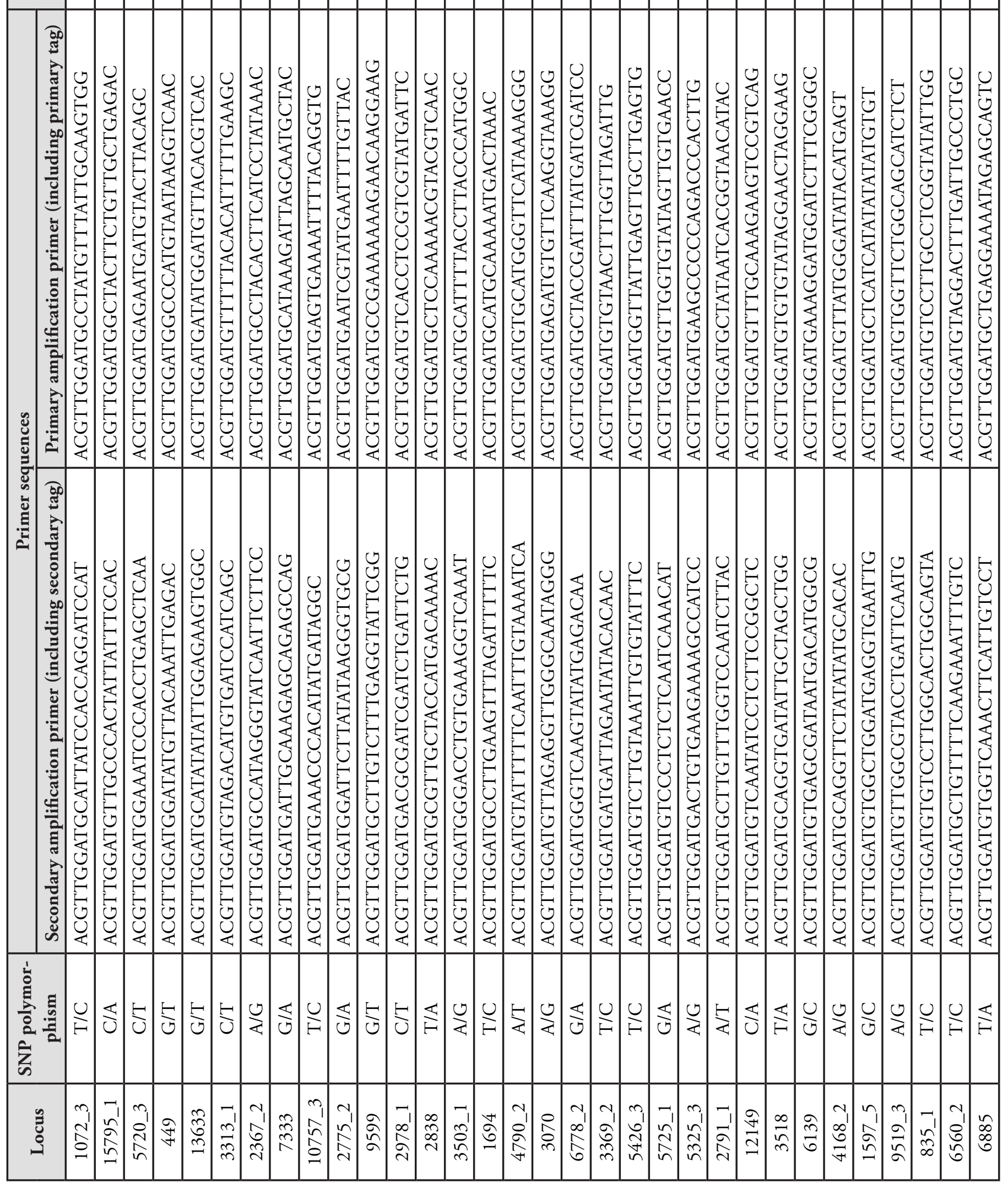




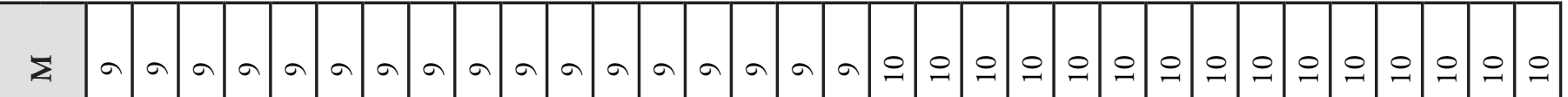

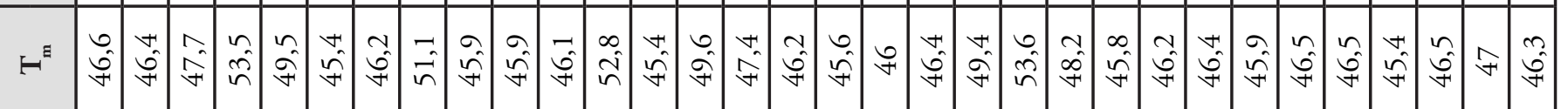

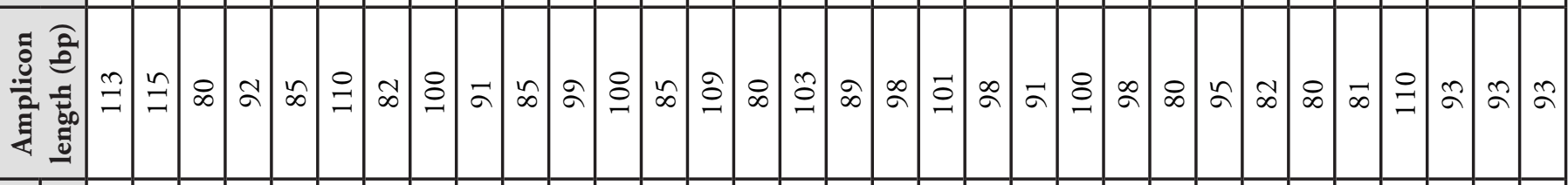

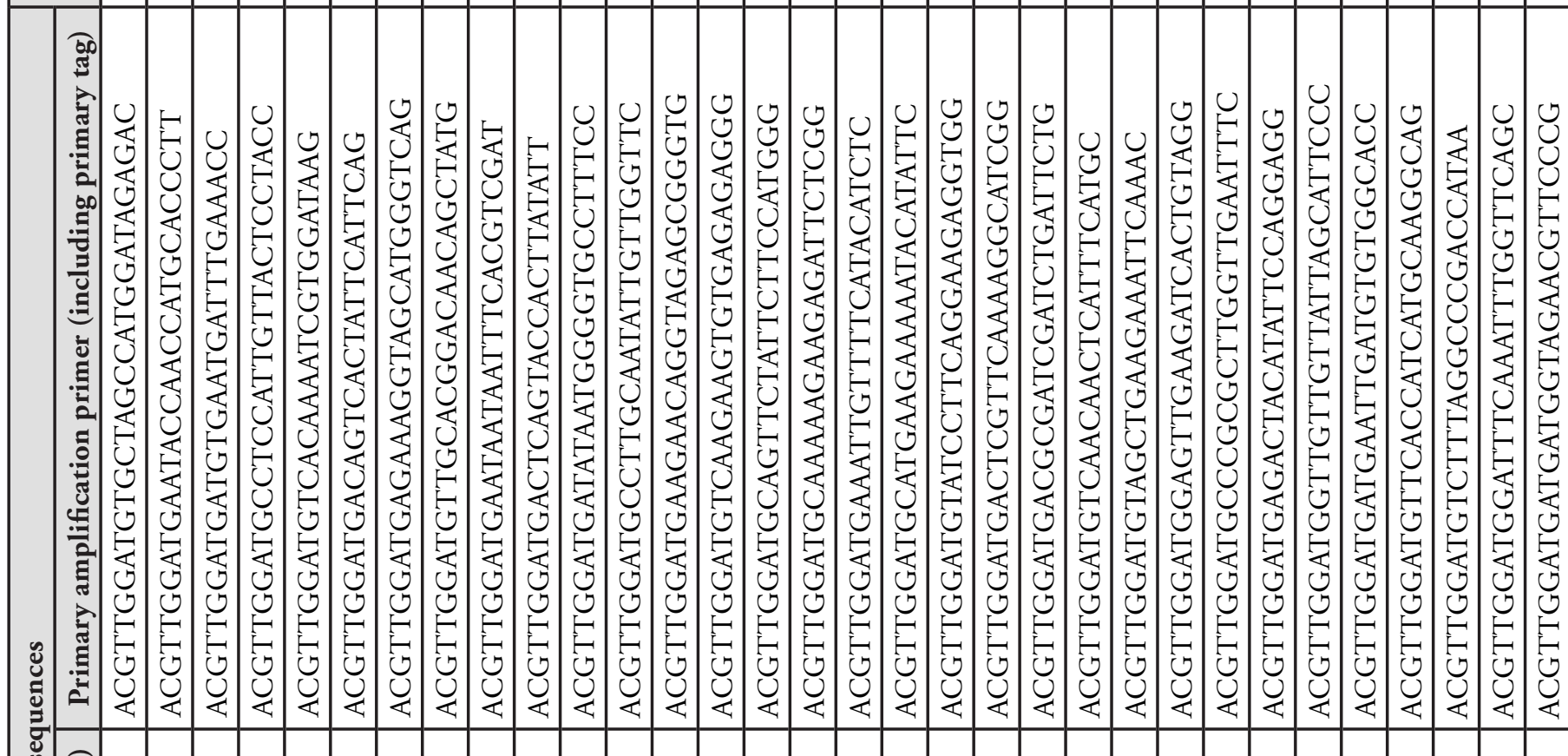

焉

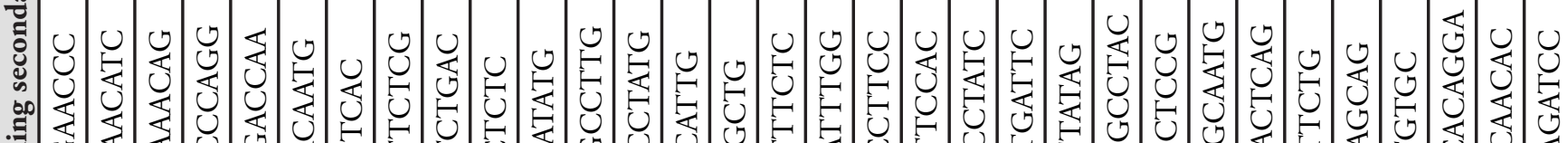

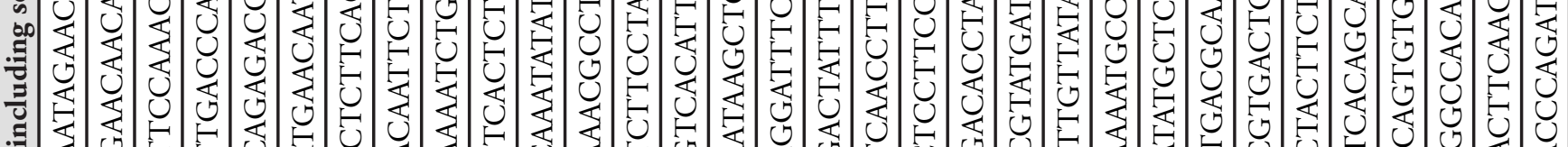

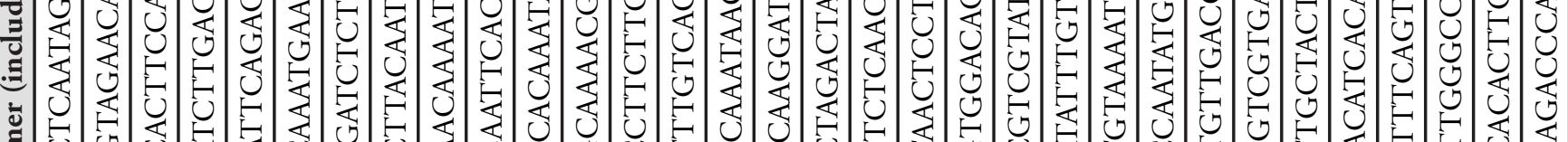

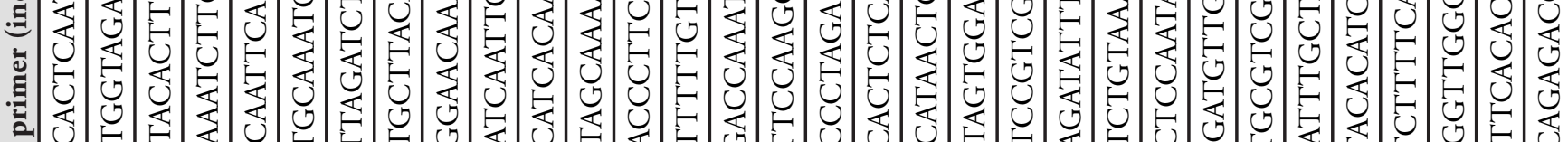

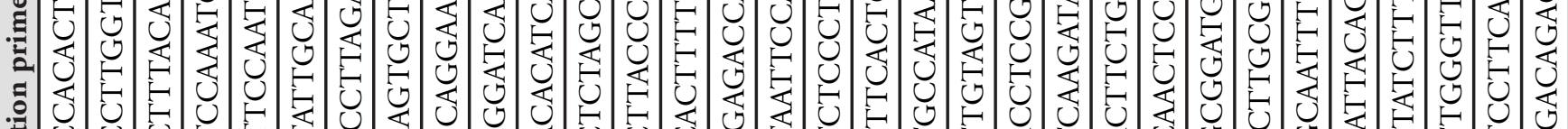

.

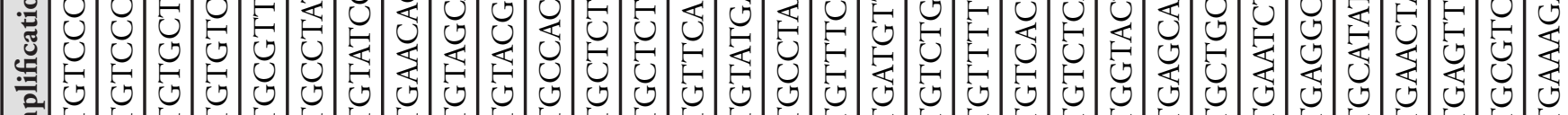

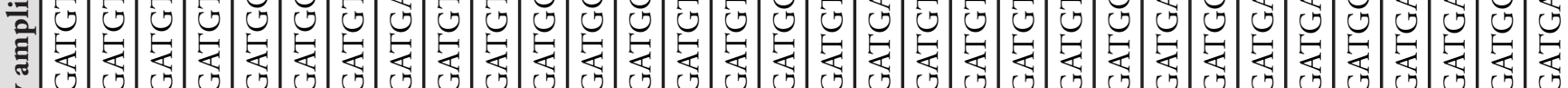

司

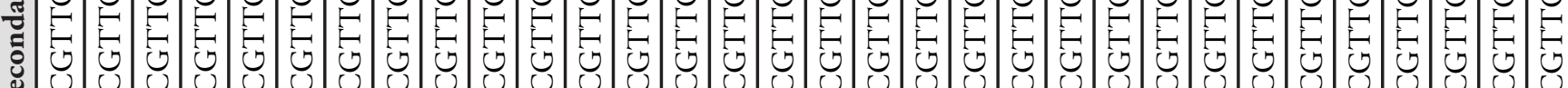

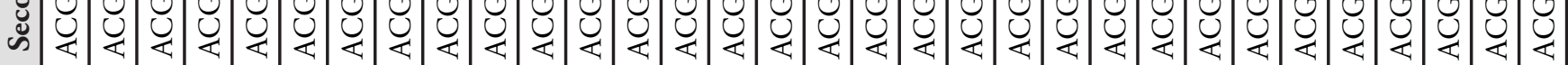

官

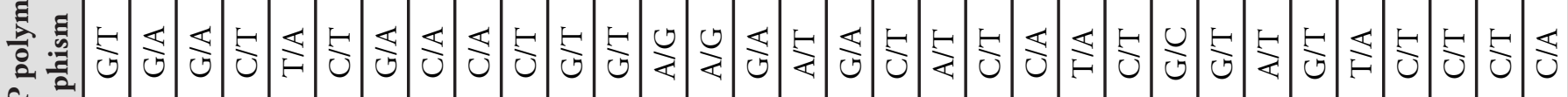
空

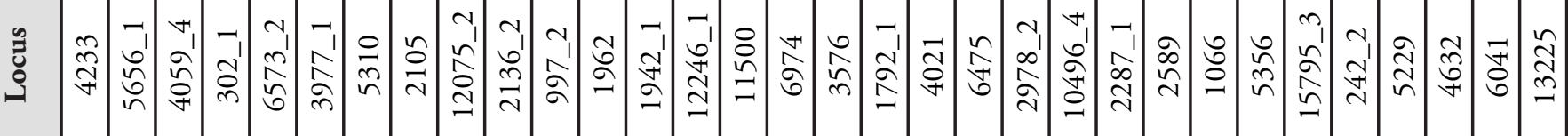




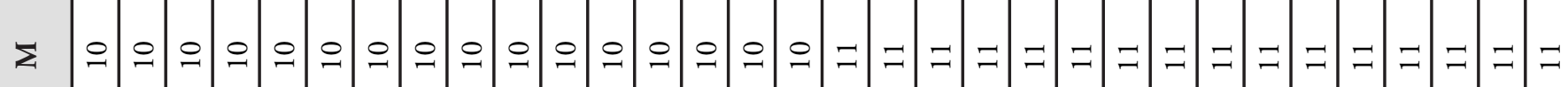

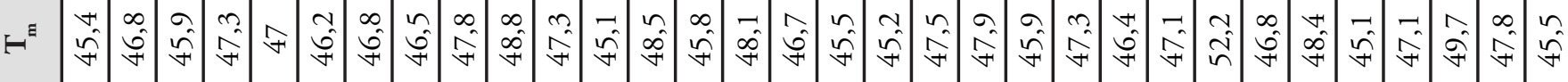

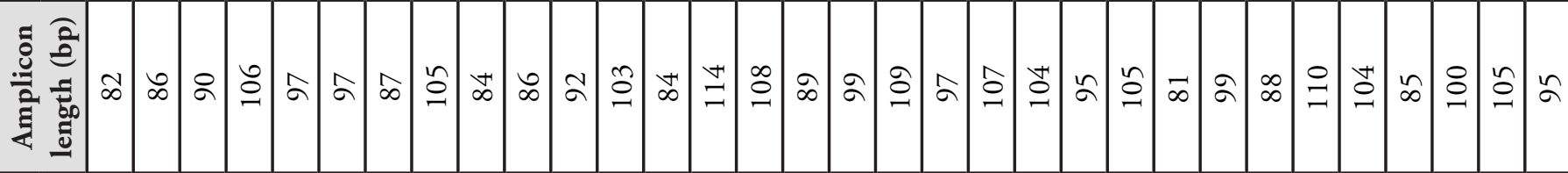

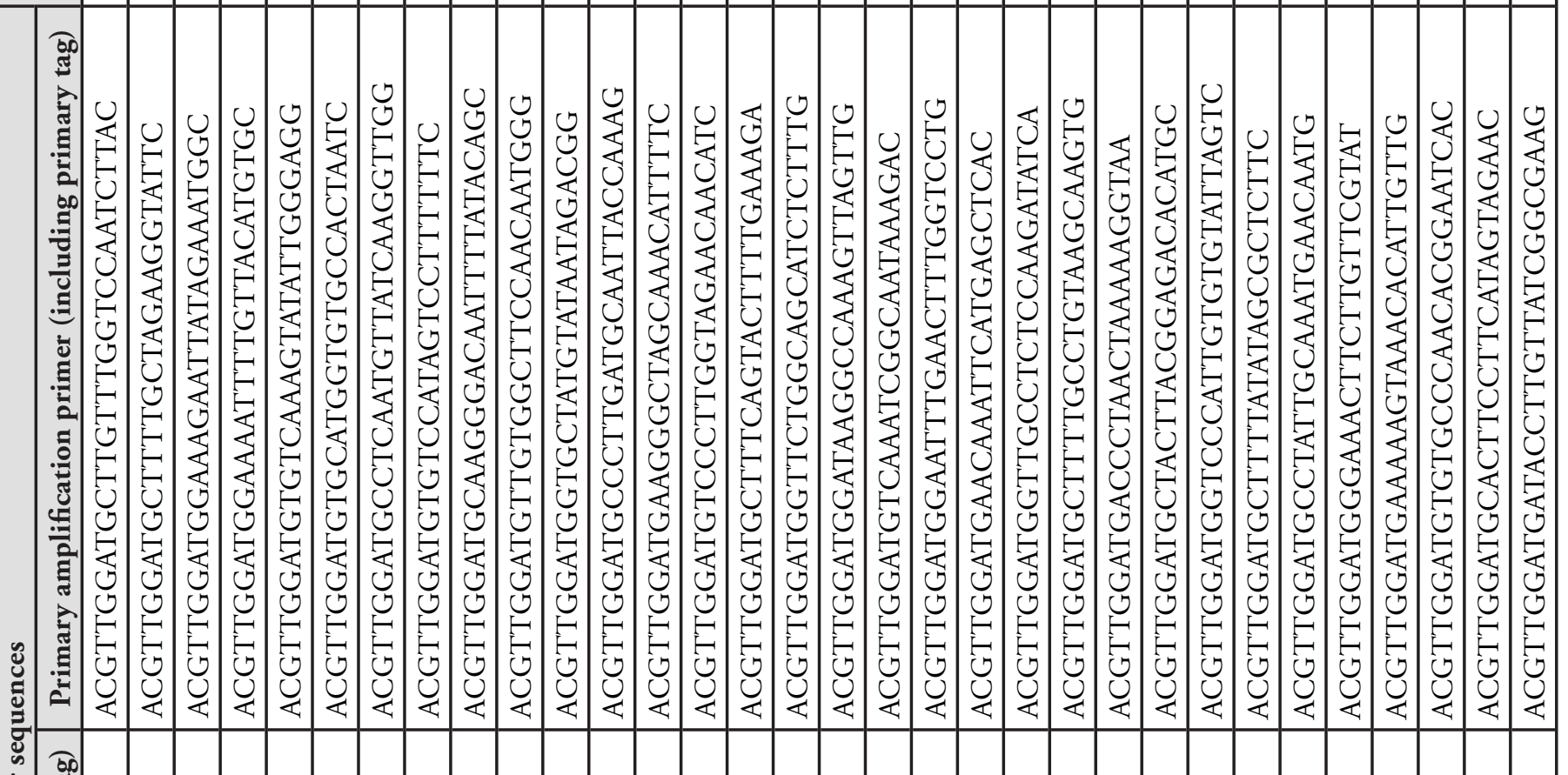
: (3)

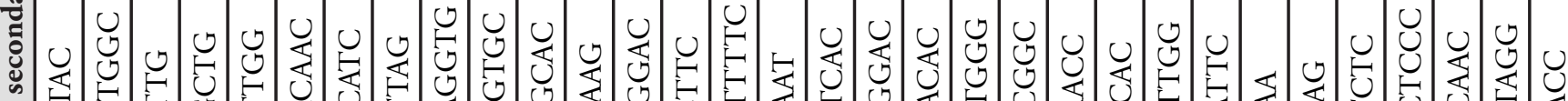

至

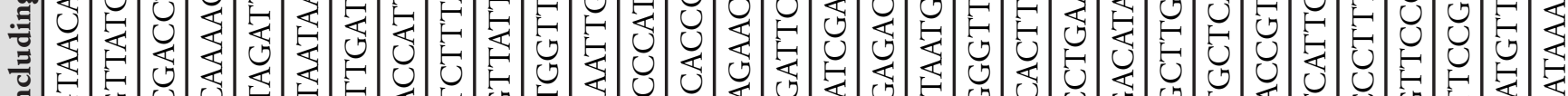

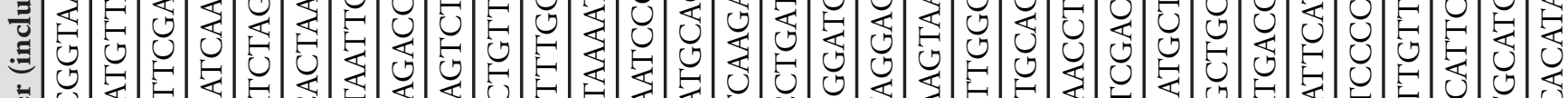

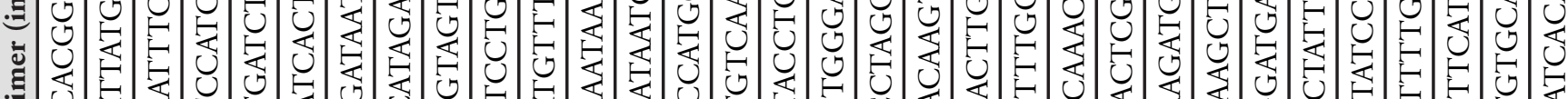

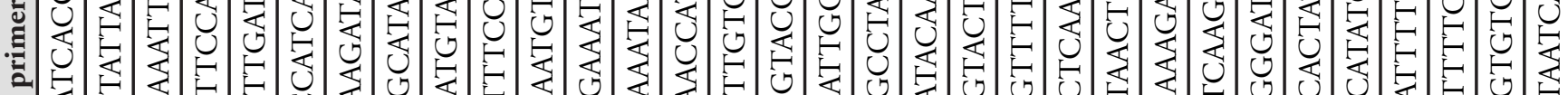

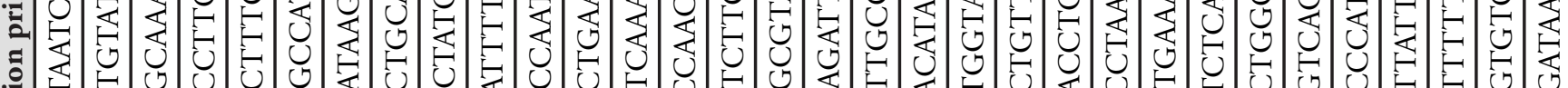

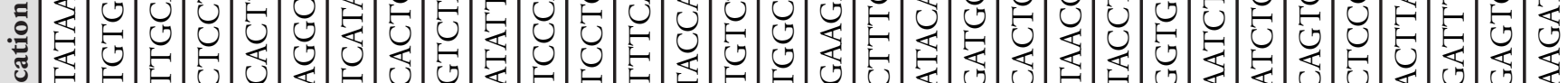

至

音

恶究

。 心

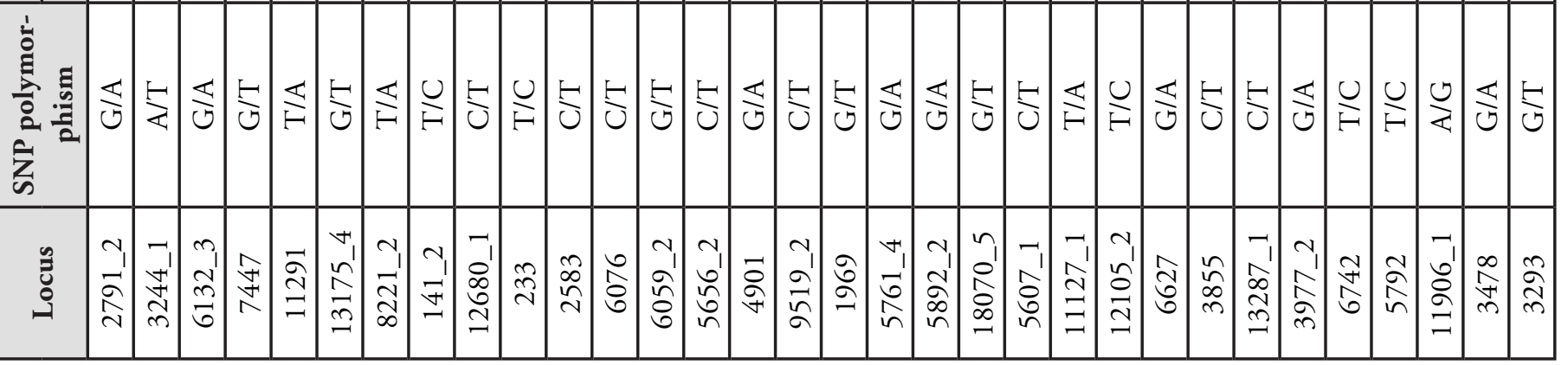




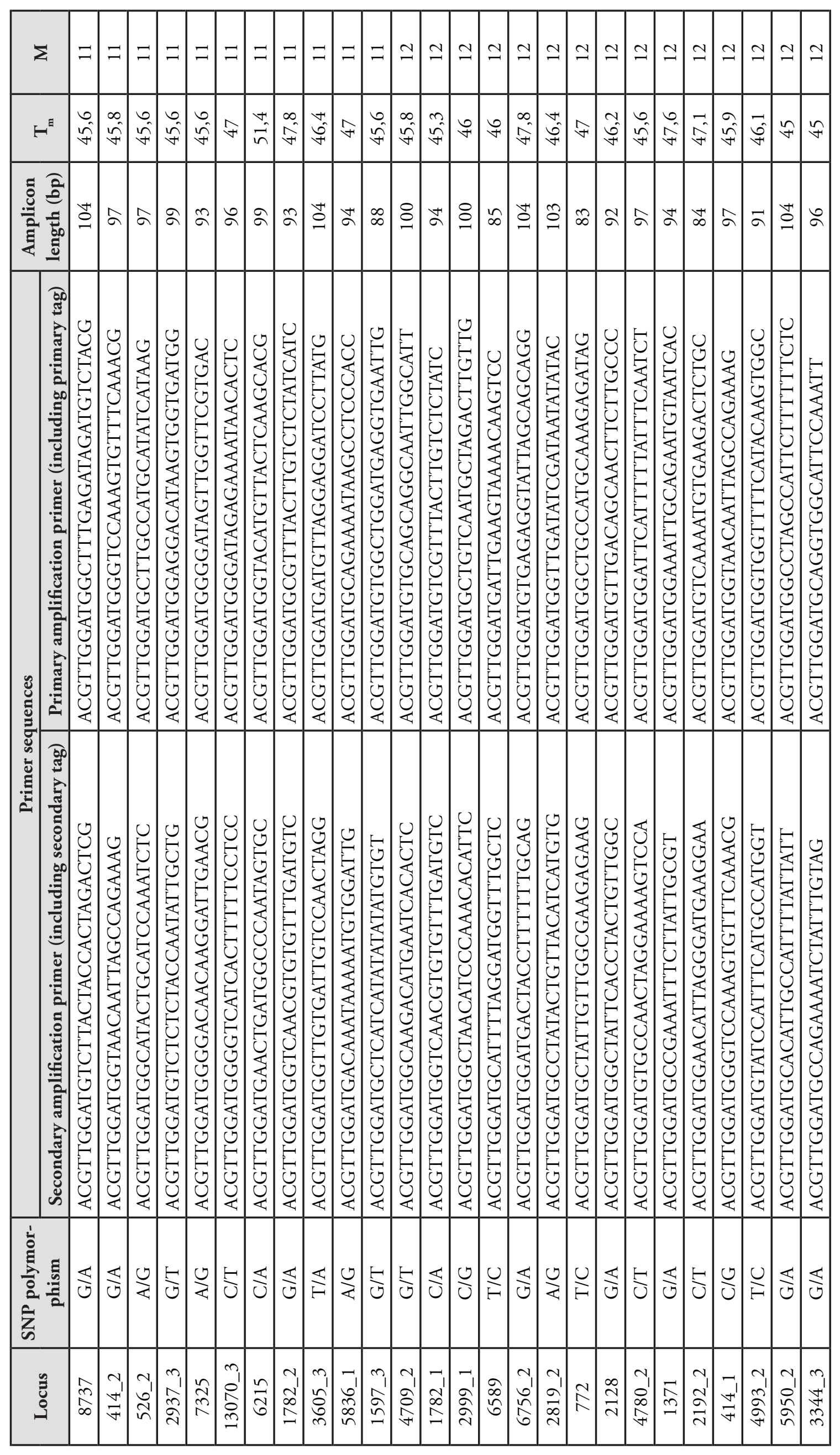




\section{Material for DNA-extraction}

Seedlings were grown in a growth chamber and leaves were collected and used for DNA isolation (Verdu et al. 2016).

\section{DNA-extraction protocols}

A commercial DNA extraction kit was used for DNA isolation (Dneasy, Qiagen) (Verdu et al. 2016).

\section{Important results}

The study was rather methodological and focused on the effects of paralogy on marker quality. Putative paralogy was shown to inflate the observed diversity values. Moreover, a rather low ( $\mathrm{x} 4)$ threshold of sequencing depth resulted in better SNP validation (Verdu et al. 2016). Taking these factors into account, the authors developed a set of 377 SNPs ready to use in population genetic analyses.

\section{References}

Bradić, M., Costa, J., Chelo, I.M. (2011): Genotyping with Sequenom. In: Orgogozo V., Rockman, M. (eds.) Molecular Methods for Evolutionary Genetics. Humana Press, New York.

Chang, C.S., Bongarten, B., Hamrick, J. (1998): Genetic structure of natural populations of black locust (Robinia pseudoacacia L.) at Coweeta, North Carolina. Journal of Plant Research, 111(1): 17-24.

Dumolin, S., Demesure, B., Petit, R.J. (1995): Inheritance of chloroplast and mitochondrial genomes in pedunculated oak investigated with an efficient PCR method. Theoretical and Applied Genetics, 91: 12531256 .

Gu, J., Yang, M., Wang, J., Zhang, J., Liang, H., Jia, L. (2010): Genetic diversity analysis of black locust (Robinia pseudoacacia L.) distributed in China based on allozyme markers approach. Frontiers of Agriculture in China, 4(3): 366-374.

Guo, Q., Wang, J.X., Su, L.Z., Lv, W., Sun, Y.H., Li, Y. (2017): Development and Evaluation of a Novel Set of EST-SSR Markers Based on Transcriptome Sequences of Black Locust (Robinia psendoacacia L.).Genes, 8(7): 177.

Guo, W., Li, Y., Gong, L., Li, F., Dong, Y., Liu, B. (2006): Efficient micropropagation of Robinia ambigua var. idahoensis (Idaho Locust) and detection of genomic variation by ISSR markers. Plant cell, tissue and organ culture, 84(3): 343-351.

Hanover, J.W., Mebrathu, T., Bloese, P. (1991): Genetic improvement of black locust: a prime agroforestry species. The Forestry Chronicle, 67(3): 227-231.

Houser, C. (2014): Genetically-mediated leaf chemistry in invasive and native black locust (Robinia pseudoacacia L.) ecosystems. Doctoral dissertation, Appalachian State University.

Huntley, J.C. (1990): Robinia pseudoacacia L., black locust. Hardwoods, 755-761.

Huo, X., Han, H., Zhang, J., Yang, M. (2009): Genetic diversity of Robinia pseudoacacia populations in China detected by AFLP markers. Frontiers of Agriculture in China, 3(3): 337-345.

Isely, D. (1998): Native and naturalized Leguminosae (Fabaceae) of the United States (exclusive of Alaska and Hawaii). Provo, Utah: Monte L. Bean Life Science Museum xi, 1007p.

Isely, D., Peabody, F.J. (1984): Robinia (Leguminosae: Papilionoidea). Castanea, 187-202.

Kidwell, K.K., Osborn, T.C. (1992): Simple plant DNA isolation procedures. In: Beckman JS and Osborn TC (eds) Plant Genomes: Methods for Genetic and Physical Mapping. Kluwer Academic Publishers, Amsterdam, The Netherlands (pp. 1-13).

Kimura, M.K., Lian, C., Hogetsu, T. (2013): Isolation and characterization of chloroplast microsatellite markers in the invasive tree species Robinia pseudoacacia L. L. Silvae Genetica, 62: 4-5.

Lian, C., Hogetsu, T. (2002): Development of microsatellite markers in black locust (Robinia pseudoacacia) using a dual-supression-PCR technique. Molecular Ecology Notes, 2(3): 211-213.

Lian, C., Oishi R., Miyashita N., Nara K., Nakaya H., Wu B., Zhou Z., Hogetsu T. (2003): Genetic structure and reproduction dynamics of Salix reinii during primary succession on Mount Fuji, as revealed by nuclear and chloroplast microsatellite analysis. Molecular Ecology, 12: 609-618.

Liesebach, H., Schneck, V. (2012): Chloroplast DNA variation in planted and natural regenerated stands of black locust (Robinia psendoacacia L.). Silvae Genetica, 61(1-2): 27-35.

Liesebach, H., Yang, M.S., Schneck, V. (2004): Genetic diversity and differentiation in a black locust (Robinia pseudoacacia L.) progeny test. Forest Genetics, 2: 151161.

Little, E.L. (1976): Atlas of United States Trees. vol. 
3, Minor Western Hardwoods. U.S. Department of Agriculture Miscellaneous Publication 1314. U.S. Department of Agriculture, Washington D.C.

Mishima, K., Hirao, T., Urano, S., Watanabe, A., Takata, K. (2009): Isolation and characterization of microsatellite markers from Robinia pseudoacacia L. Molecular Ecology Resources, 9(3): 850-852.

Rédei, K., \& Veperdi, I. (2009). The role of black locust (Robinia pseudoacacia L.) in establishment of shortrotation energy plantations in Hungary. International Journal of Horticultural Science, 15(3): 41-44.

Royal Horticultural Society (2018): Robinia. URL: $<$ https://www.rhs.org.uk/Search? query=Robinia> Accessed 9 January 2018.

Schütt, P. (1994): Robinia pseudoacacia. In: Enzyklopädie der Holzgewächse: Handbuch und Atlas der Dendrologie. Wiley-VCH.

Surles, S.E., Hamrick, J.L., Bongarten, B.C. (1989): Allozyme variation in black locust (Robinia pseudoacacia). Canadian Journal of Forest Research, 19(4): 471-479.

Verdu, C.F., Guichoux, E., Quevauvillers, S., De Thier, O., Laizet, Y.H., Delcamp, A., Gévaudent F., Monty A., Porté A., Lejeune P., Ludivine L., Mariette, S. (2016): Dealing with paralogy in RADseq data: in silico detection and single nucleotide polymorphism validation in Robinia pseudoacacia L. Ecology and Evolution, 6(20): 7323-7333.

Vos, P., Hogers, R., Blecker, M., Reijans, M., Lee, T.V.D., Hornes, M., Frijters, A., Pot, J., Peleman, J., Kuiper, M., Zabeau, M. (1995): AFLP: a new technique for DNA fingerprinting. Nucleic Acids Research, 23(21): 44074414. 
Non-native Tree Species for European Forests Experiences, Risks and Opportunities (FP1403)
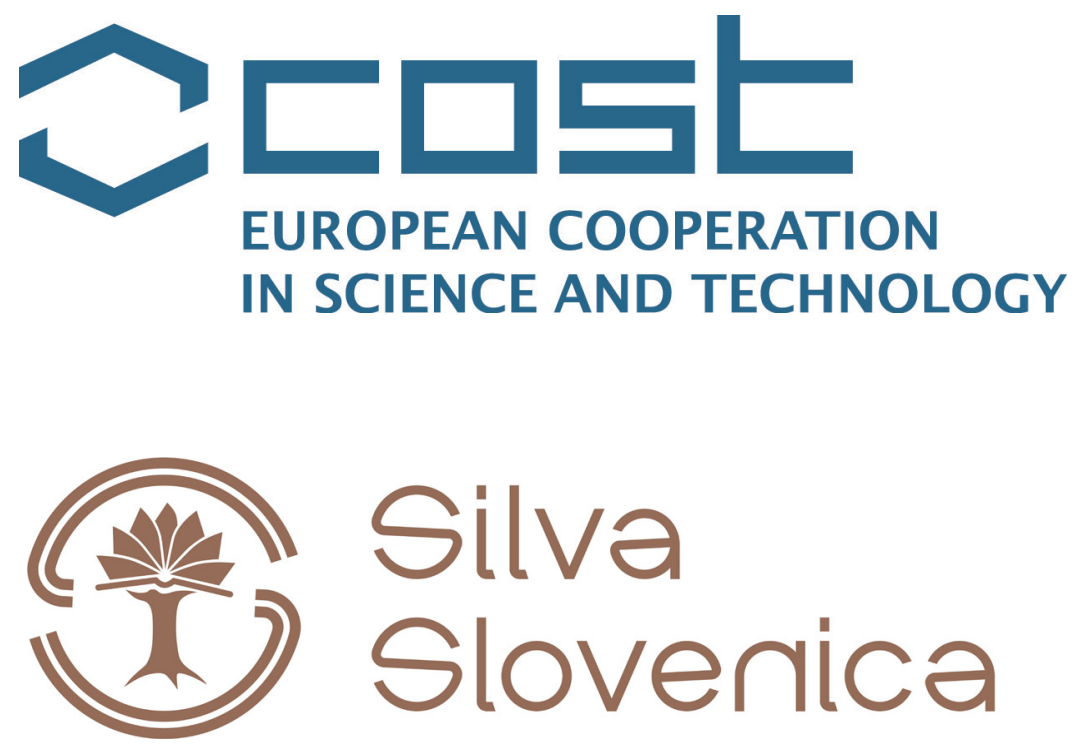


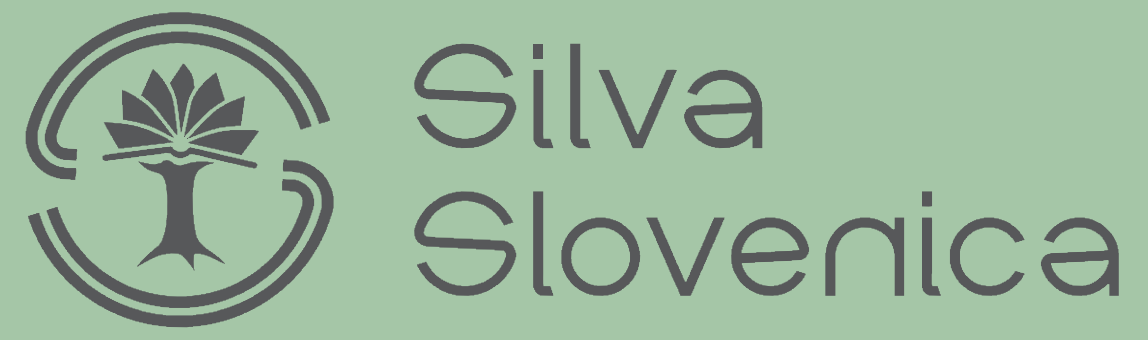

